\title{
La conjecture de Baum-Connes à coefficients pour les groupes hyperboliques
}

\author{
Vincent Lafforgue
}

Résumé. Cet article montre la conjecture de Baum-Connes à coefficients pour les groupes hyperboliques. Plus précisément l'injectivité de l'application de Baum-Connes a été établie par Kasparov et Skandalis et on montre la surjectivité.

\begin{abstract}
This paper gives a proof of the Baum-Connes conjecture with coefficients for hyperbolic groups. More precisely the injectivity of the Baum-Connes map was etablished by Kasparov and Skandalis and we prove the surjectivity.
\end{abstract}

Mathematics Subject Classification (2010). 19K35; 19L47, 20F67, 46L80.

Keywords. Kasparov theory, hyperbolic groups, Baum-Connes conjecture.

\section{Table des matières}

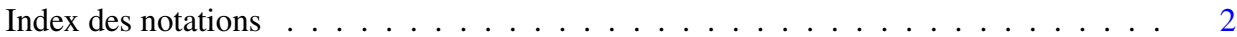

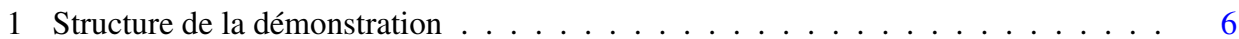

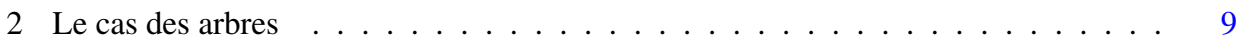

3 Construction des espaces et des opérateurs . . . . . . . . . . . . . 16

3.1 Construction du premier parametrix $H_{x} \ldots \ldots \ldots \ldots \ldots \ldots$

3.2 Une conséquence de la construction de $H_{x} \ldots \ldots \ldots \ldots \ldots \ldots$. . . . . . . 32

3.3 Construction d'un deuxième paramétrix $u_{x} \ldots \ldots \ldots \ldots$. . . . . . . 33

3.4 Construction du paramétrix définitif $J_{x} \ldots \ldots \ldots \ldots$. . . . . . . . 42

3.5 Construction d'une distance moyennée pour conjuguer les opérateurs . . . . . 50

4 Construction des normes . . . . . . . . . . . . . . . . . . . 65

4.1 Formule pour la norme . . . . . . . . . . . . . . . . . . 65

4.2 Enoncé des résultats . . . . . . . . . . . . . . . . . . . . 69

4.3 Premières propriétés de la norme . . . . . . . . . . . . . . 70

4.4 Autres propriétés de la norme . . . . . . . . . . . . . . . 82

4.5 Continuité de $\partial$ et $J_{x} \ldots \ldots \ldots \ldots \ldots \ldots$

4.6 Continuité de $e^{\tau \theta_{x}^{\mathrm{b}}} \partial e^{-\tau \theta_{x}^{\mathrm{b}}}$ et $e^{\tau \theta_{x}^{\mathrm{b}}} J_{x} e^{-\tau \theta_{x}^{\mathrm{b}}} \ldots \ldots \ldots \ldots \ldots \ldots \ldots \ldots$

4.7 Propriétés d'équivariance de la norme . . . . . . . . . . . . . . . . . . 124

4.8 Equivariance des opérateurs à compacts près . . . . . . . . . . . . . . . . . 160

5 Fin de l'homotopie . . . . . . . . . . . . . . . . . . . . . . 192

Références . . . . . . . . . . . . . . . . . . . . . 196 


\section{Index des notations}

Page 3: $H_{\delta}(x, y, z, t)$

Page $3: B(x, r)$

Page $16: d(A, B), d_{\max }(A, B)$

Page $17: N,\left(H_{N}\right), \Delta, \Delta_{p}, \mathbb{C}^{\left(\Delta_{p}\right)}, p_{\max }$

Page $17: \partial$

Page $21:\left(H_{\delta}^{\beta}(x, a, b, c)\right),\left(H_{\delta}^{0}(x, a, b, c)\right), U_{S}$

Page $23: A_{S, x}, Y_{S, x, r}, A_{\emptyset, x}, Y_{\emptyset, x, r}$

Page $25: \chi_{A}, v_{A}, \psi_{S, x}, \psi_{S, x, t}, \mathrm{E}(\cdot)$

Page $28: h_{x}, h_{x, t}$

Page 28: $H_{x}, \zeta_{x}(S)$

Page $32: \Phi_{p}$

Page $34: A_{x, a, r, k}, \mu_{r}(x, a), \mu_{r, t}(x, a)$

Page $36: u_{x}, u_{x, r, t}, u_{x, r, t}^{p}, v_{x, r, t}^{p}$

Page $43:\left(H_{Q}\right), \tilde{H}_{x}, K_{x}$

Page $43: K_{x, q},\left(t_{1}, \ldots, t_{q}\right), \widetilde{H}_{x, q,\left(t_{1}, \ldots, t_{q}\right)}, B(A, r)$

Page $45: J_{x}$

Page $48: F$

Page $55: Y_{x, y}^{r}, \Lambda_{x, r}^{y, s}, \alpha_{r^{\prime} \leftarrow r}^{s^{\prime} \leftarrow s}$

Page $56: \Lambda_{x, r^{\prime}, r}^{y, s^{\prime}, s}$

Page $57: \Lambda_{x, r_{1}, r_{2}, r_{3}}^{y, s_{1}, s_{2}, s_{3}}, \beta_{x, r}^{y, s}, \tilde{\Lambda}_{x, r_{1}, r_{2}, r_{3}}^{y, s_{1}, s_{2}, s_{3}}$

Page 58: $A_{x, u_{1}, u_{2}, u_{3}}^{y, v_{1}, v_{2}, v_{3}}, d^{\mathrm{b}}(x, y), d^{\mathrm{b}}{ }_{u_{1}, u_{2}, u_{3}}^{v_{1}, v_{2}, v_{3}}(x, y)$

Page $65: \rho_{x}^{\mathrm{b}}(a), \rho_{x}^{\mathrm{b}}(S)$

Page $65:\left(H_{P}\right), Y_{x}^{p, k, m,\left(l_{0}, \ldots, l_{m}\right)}, y_{i}^{j}$

Page $67:\left(H_{M}\right), \bar{Y}_{x}^{p, k, m,\left(l_{0}, \ldots, l_{m}\right)}, \pi_{x}^{p, k, m,\left(l_{0}, \ldots, l_{m}\right)}, r_{0}(Z), \ldots, r_{m}(Z), s_{0}(Z), \ldots, s_{m}(Z)$

Page $67:\left(H_{B}\right),\left(H_{\alpha}\right), \xi_{Z}, \mathscr{H}_{x, s}\left(\Delta_{p}\right)$

Page $69: \theta_{x}^{b}$

Page $70: \Psi$

Page $86: Y_{x} \rightarrow, p, k, m,\left(l_{0}, \ldots, l_{m}\right), \bar{Y}_{x} \rightarrow, p, k, m,\left(l_{0}, \ldots, l_{m}\right), \pi_{x}, p, k, m,\left(l_{0}, \ldots, l_{m}\right)$

Page $86:\|\cdot\|_{\mathscr{H}_{x, S}\left(\Delta_{p}\right)}$

Page $88: \mathcal{P}$

Page $91: Y_{x}^{\natural, p, k, m,\left(l_{0}, \ldots, l_{m}\right), \lambda_{0}, \lambda_{1}, Z_{i}^{j}}$

Page $92: \bar{Y}_{x}^{\natural, p, k, m,\left(l_{0}, \ldots, l_{m}\right), \lambda_{0}, \lambda_{1}}, \pi_{x}^{\natural, p, k, m,\left(l_{0}, \ldots, l_{m}\right), \lambda_{0}, \lambda_{1}}, r_{i, j}^{\max }(Z), t_{i}^{j}(Z)$

Page $93: \mathscr{H}_{x, s}^{\natural, \mu_{0}, \mu_{1}}\left(\Delta_{p}\right)$

Page $96: \kappa_{\sigma}, \bar{\kappa}_{\sigma, \infty}, \bar{\kappa}_{\sigma, i}$

Page $118: \rho_{x}, \theta_{x}, \rho_{x}^{0}(Z), \rho_{x}^{1}(Z)$

Page 119: $\left(\rho_{x}^{\mathrm{b}}\right)_{u_{1}, u_{2}, u_{3}}^{v_{1}, v_{2}, v_{3}}$

Page $125: \pi_{x, x^{\prime}}^{p, k,\left(l_{0}, \ldots, l_{m}\right)}, \bar{Y}_{x, x^{\prime}}^{p, k, m,\left(l_{0}, \ldots, l_{m}\right)}, r_{0}^{\prime}(Z), k^{\prime}(Z)$

Page $127: \bar{Y}_{x, x^{\prime}, \star}^{p, k,\left(l_{0}, \ldots, l_{m}\right)}, \pi_{x, x^{\prime}, \star}^{p, k},\left(l_{0}, \ldots, l_{m}\right)$

Page $161: \mathcal{P}_{n}$ 
Le but de cet article est de montrer la conjecture de Baum-Connes à coefficients pour les groupes hyperboliques au sens de Gromov [Gro87], [CDP90], [GdlH90].

Soit $G$ un groupe localement compact. La conjecture de Baum-Connes, formulée en 1982 [BC82] affirme que

$$
\mu_{\mathrm{red}}^{G}: K_{*}^{\mathrm{top}}(G) \rightarrow K_{*}\left(C_{\mathrm{red}}^{*}(G)\right)
$$

est un isomorphisme de groupes abéliens. La conjecture de Baum-Connes à coefficients [BCH94] affirme que pour toute $G-C^{*}$-algèbre $A$,

$$
\mu_{\mathrm{red}}^{G, A}: K_{*}^{\mathrm{top}}(G, A) \rightarrow K_{*}\left(C_{\mathrm{red}}^{*}(G, A)\right)
$$

est un isomorphisme de groupes abéliens.

Pour les groupes hyperboliques, l'injectivité de $\mu_{\text {red }}^{G, A}$ a été montrée par Kasparov et Skandalis [KS94], [KS03]. La conjecture sans coefficients a été démontrée dans [Laf02] et [MY02]. La conjecture à coefficients commutatifs a été démontrée dans [Laf07] (mais la méthode de [Laf07] ne permet pas de montrer la conjecture de Baum-Connes à coefficients commutatifs pour un produit de deux groupes hyperboliques).

Pour énoncer le théorème principal nous avons besoin de quelques définitions.

Définition 0.1. Soit $\delta \geq 0$. Un espace métrique $(X, d)$ est dit $\delta$-hyperbolique si pour tout quadruplet $(x, y, z, t)$ de points de $X$ on a

$$
d(x, z)+d(y, t) \leq \max (d(x, y)+d(z, t), d(x, t)+d(y, z))+\delta .\left(H_{\delta}(x, y, z, t)\right)
$$

Définition 0.2. Soit $\delta \geq 0$. Un espace métrique $(X, d)$ est dit faiblement $\delta$-géodésique si pour tous $x, y \in X$ et pour tout $s \in[0, d(x, y)+\delta]$ il existe $z \in X$ tel que $d(x, z) \leq s$ et $d(z, y) \leq d(x, y)-s+\delta$.

Un espace métrique $(X, d)$ est dit hyperbolique (resp. faiblement géodésique) s'il existe $\delta \geq 0$ tel que $(X, d)$ soit $\delta$-hyperbolique (resp. faiblement $\delta$-géodésique).

Lorsque $x \in X$ et $r \in \mathbb{R}_{+}$, on note $B(x, r)=\{y \in X \mid d(x, y) \leq r\}$.

Définition 0.3. Un espace métrique $(X, d)$ est dit uniformément localement fini si pour tout $r \in \mathbb{R}_{+}$il existe $K \in \mathbb{N}$ tel que, pour tout $x \in X, B(x, r)$ contienne au plus $K$ points.

Le théorème principal est le suivant.

Théorème 0.4. Soit $G$ un groupe localement compact agissant de façon isométrique, continue et propre sur un espace métrique hyperbolique, faiblement géodésique et uniformément localement fini. Alors $G$ vérifie la conjecture de Baum-Connes à coefficients, c'est-à-dire que pour toute $G$-C $C^{*}$-algèbre $A, \mu_{\mathrm{red}}^{G, A}: K_{*}^{\mathrm{top}}(G, A) \rightarrow$ $K_{*}\left(C_{\text {red }}^{*}(G, A)\right)$ est une bijection. 
On rappelle que l'injectivité de $\mu_{\text {red }}^{G, A}$ est démontrée dans [KS94], [KS03].

Tout groupe hyperbolique $\Gamma$ muni de la métrique $d$ invariante à gauche associée à la longueur des mots déterminée par un système fini de générateurs est un espace métrique hyperbolique, faiblement géodésique et uniformément localement fini. Donc le théorème 0.4 implique la conjecture de Baum-Connes à coefficients pour les groupes hyperboliques.

On aimerait remplacer dans le théorème 0.4 l'hypothèse "uniformement localement fini” par l'hypothèse "à géométrie grossière bornée" qui est strictement plus faible (on renvoie à [KS03] pour cette notion et on note que dans [KS03] l'injectivité de $\mu_{\text {red }}^{G, A}$ est démontrée sous cette hypothèse plus faible). Cependant cela rendrait la démonstration encore plus technique et nous y avons renoncé.

On aimerait aussi traiter le cas général des groupoïdes hyperboliques, au moins à base compacte, mais cela serait très difficile, car il faudrait adapter la construction de cet article (qui est assez combinatoire) aux techniques de Jean-Louis Tu dans [Tu99], consistant à pondérer par des coefficients tendant vers 0 les éléments du groupoïde qui sont près de disparaître. En revanche, la conjecture sans coefficients pour ces groupoïdes est beaucoup plus accessible par les méthodes de [Laf07], elle est d'ailleurs démontrée dans certains cas dans [Laf07].

D'après [HLS02] la conjecture de Baum-Connes à coefficients est fausse pour certains groupes aléatoires construits par Gromov dans [Gro03]. Ces groupes sont des limites inductives de groupes hyperboliques, la limite étant indexée par $\mathbb{N}$, et les morphismes de transition étant surjectifs. Comme le membre de gauche de la conjecture de Baum-Connes commute aux limites inductives, on voit bien que ces contre-exemples sont "dus" au fait que $C_{\text {red }}^{*}$ n'est pas fonctoriel en les morphismes de groupes (non nécessairement injectifs). Plus précisément si $G \rightarrow H$ est un morphisme de groupes, et $A$ une $H$-C $\mathrm{C}^{*}$-algèbre, donc aussi une $G$-C*-algèbre, le morphisme $C_{\mathrm{c}}(G, A) \rightarrow C_{\mathrm{c}}(H, A)$ ne se prolonge pas en général par continuité en un morphisme $C_{\text {red }}^{*}(G, A) \rightarrow C_{\text {red }}^{*}(H, A)$.

Voici maintenant quelques indications sur la démonstration du théorème 0.4 , qui occupe tout l'article. On commence par des rappels sur la méthode "Dirac-dual Dirac”, inventée par Kasparov puis développée par Kasparov et Skandalis, et Higson et Kasparov. Cette méthode s'applique à une très large classe $\mathcal{C}$ de groupes localement compacts, dont la définition est rappelée dans l'introduction de [Laf02], et qui contient en particulier les groupes hyperboliques, les sous-groupes fermés des groupes réductifs sur un corps local, et les groupes ayant la propriété de Haagerup, c'est-à-dire possédant une action affine continue et propre sur un espace de Hilbert. Pour tout groupe $G$ dans la classe $\ell$, on possède un idempotent $\gamma \in \mathrm{KK}_{G}(\mathbb{C}, \mathbb{C})$ tel que pour toute $G$-C*-algèbre $A, \mu_{\text {red }}^{G, A}$ soit injectif et que l'image de l'action de $\gamma$ sur $K_{*}\left(C_{\text {red }}^{*}(G, A)\right)$ soit égale à l'image de $\mu_{\text {red }}^{G, A}$. Donc si $G$ appartient à $C$, la conjecture de Baum-Connes à coefficients pour $G$ équivaut au fait que $\gamma$ agit par l'identité sur $K_{*}\left(C_{\text {red }}^{*}(G, A)\right)$. Si $G$ a la propriété de Haagerup, Higson et Kasparov ont montré dans $[\mathrm{HK} 01]$ que $\gamma=1$ dans $\mathrm{KK}_{G}(\mathbb{C}, \mathbb{C})$. 
Soit $G$ un groupe hyperbolique. Comme certains groupes hyperboliques ont la propriété $(\mathrm{T})$ de Kazhdan, on ne peut pas espérer montrer que $\gamma=1$ dans $\mathrm{KK}_{G}(\mathbb{C}, \mathbb{C})$. D'un autre côté on sait d'après [Laf02] que $\gamma$ est égal à 1 dans $\operatorname{KK}_{G, s \ell}^{\text {ban }}(\mathbb{C}, \mathbb{C})$ pour tout $s>0$ : cela permet de montrer la conjecture sans coefficients grâce à la propriété (RD) de Jolissaint [Laf02], et aussi à coefficients commutatifs grâce à un autre argument de stabilité par calcul fonctionnel holomorphe un peu plus subtil [Laf07], mais cela ne permet pas de montrer la conjecture à coefficients arbitraires.

L'idée pour montrer la conjecture de Baum-Connes à coefficients pour les groupes hyperboliques est que ceux-ci, même s'ils ont la propriété (T), ne vérifient pas la propriété $(\mathrm{T})$ renforcée au sens de la définition 0.1 de [Laf08]. De façon un peu imprécise un groupe localement compact $G$ n'a pas la propriété (T) renforcée s'il existe une longueur $\ell$ sur $G$ (comme dans la définition 1.1) telle que pour tout $s>0$ il existe $C \in \mathbb{R}_{+}$tel que la représentation triviale ne soit pas isolée parmi les représentations continues $\pi$ de $G$ dans des espaces de Hilbert vérifiant $\|\pi(g)\| \leq e^{C+s \ell(g)}$ pour tout $g \in G$. Le théorème 1.4 de [Laf08] affirme que si un groupe localement compact possède la propriété $(\mathrm{T})$ renforcée toute action continue et isométrique de ce groupe sur un espace métrique hyperbolique, faiblement géodésique et uniformément localement fini a des orbites bornées. La démonstration du théorème 1.4 de [Laf08] montre même que pour un groupe hyperbolique $\Gamma$ muni de la longueur $\ell$ associée à un système fini de générateurs, il existe un polynôme $P$ tel que la représentation triviale ne soit pas isolée parmi les représentations $\pi$ de $\Gamma$ dans des espaces de Hilbert vérifiant $\|\pi(g)\| \leq P(\ell(g))$ pour tout $g \in \Gamma$. Notons que Ozawa [Oza08] a montré que les groupes hyperboliques sont faiblement moyennables, ce qui amène à se demander si dans la phrase précédente on ne pourrait pas prendre pour $P$ un polynôme constant. Cependant cela n'apporterait rien pour la conjecture de Baum-Connes car la seule chose qui compte est que $P$ soit une fonction sous-exponentielle.

Pour montrer le théorème 0.4 nous construisons une homotopie de 1 à $\gamma$ en utilisant des représentations (continues) de $G$ dans des espaces de Hilbert qui ne sont pas unitaires mais à croissance exponentielle arbitrairement petite. Plus précisément nous fixons une longueur $\ell$ sur $G$ et nous montrons que pour tout $s>0$ il existe $C \in \mathbb{R}_{+}$ tel que l'on puisse construire une homotopie de 1 à $\gamma$ en utilisant des représentations $\pi$ de $G$ dans des espaces de Hilbert qui vérifient

$$
\|\pi(g)\| \leq e^{C+s \ell(g)} \quad \text { pour tout } g \in G .
$$

Le théorème 1.3 affirme l'existence pour tout $s>0$ d'une telle homotopie et le théorème 1.2 (qui repose sur des idées de Nigel Higson) montre que cela implique la conjecture de Baum-Connes à coefficients pour $G$. La preuve du théorème 1.3 est ramenée à celle du théorème 1.5 où l'on suppose que $G$ agit proprement sur un espace hyperbolique $X$ vérifiant certaines propriétés supplémentaires (essentiellement que la métrique est associée à une structure de graphe). La preuve du théorème 1.5 repose sur l'acyclicité du complexe d'homologie simpliciale

$$
0 \leftarrow \mathbb{C}^{\left(\Delta_{0}\right)} \stackrel{\partial}{\leftarrow} \mathbb{C}^{\left(\Delta_{1}\right)} \stackrel{\partial}{\leftarrow} \mathbb{C}^{\left(\Delta_{2}\right)} \stackrel{\partial}{\leftarrow} \cdots \stackrel{\partial}{\leftarrow} \mathbb{C}^{\left(\Delta_{p_{\max }}\right)} \leftarrow 0
$$


du complexe de Rips, où l'ensemble $\Delta_{p}$ des faces de dimension $p-1$ est formé des parties de $X$ de cardinal $p$ et de diamètre $\leq N$, pour $N$ assez grand. On fixe un point base $x \in X$. La partie difficile est la construction de $J_{x}: \mathbb{C}^{\left(\Delta_{p}\right)} \rightarrow \mathbb{C}^{\left(\Delta_{p+1}\right)}$ tel que $\partial J_{x}+J_{x} \partial=1$ et de normes de Hilbert sur $\mathbb{C}^{\left(\Delta_{p}\right)}$ vérifiant (1) et telles que $\partial$ et $J_{x}$ soient continus. L'homotopie de 1 vers $\gamma$ se fait alors en conjuguant $\partial+J_{x}$ par $e^{t \rho^{\mathrm{b}}}$, où $\rho^{\mathrm{b}}(a)$ est égal à $d(x, a)$ à une constante près et est obtenu par un procédé de moyenne garantissant l'équivariance à compacts près des opérateurs conjugués. Le lecteur qui voudrait se faire une idée rapide de la construction est invité à lire les paragraphes 1 et 2, l'introduction du paragraphe 3, et les sous-paragraphes 4.1 et 4.2.

La méthode que nous utilisons est semblable à celle utilisée par Pierre Julg pour montrer la conjecture de Baum-Connes à coefficients pour $\operatorname{Sp}(n, 1)$ (voir [Jul02]). En fait l'idée de Julg d'utiliser des représentations non unitaires dans des espaces de Hilbert est très ancienne : dans [Ju197] Julg proposait de construire une homotopie de 1 à $\gamma$ en utilisant des représentations uniformément bornées de $\operatorname{Sp}(n, 1)$. L'idée d'utiliser des représentations non pas uniformément bornées mais à petite croissance exponentielle a été dégagée lors de discussions avec Julg et Higson en 1999.

Pour conclure voici un petit aperçu du statut actuel de la conjecture de Baum-

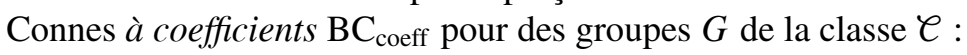

- "non T" : si $G$ a la propriété de Haagerup, $G$ vérifie $\mathrm{BC}_{\text {coeff }}$ d'après [HK01];

- "T possible mais non T renforcé": $\mathrm{BC}_{\text {coeff }}$ est vrai si $G=\operatorname{Sp}(n, 1)$ d'après [Jul02] ou si $G$ est un groupe hyperbolique par le présent article ;

- "T renforcé" : dans ce cas, qui comprend probablement tous les groupes simples sur des corps locaux de rang déployé $\geq 2$ (et au moins ceux qui contiennent un $\mathrm{SL}_{3}$ d'après [Laf08]), $\mathrm{BC}_{\text {coeff }}$ est totalement ouvert et ne pourra être résolu qu'avec des idées nouvelles comme le principe d'Oka (on renvoie à [Laf09] pour plus de détails).

Je remercie Georges Skandalis pour son aide et toutes les discussions que j' ai eues avec lui. Je remercie aussi Miguel Bermudez pour m' avoir indiqué le logiciel JPicEdit, avec lequel les dessins ont été réalisés, et Thomas Delzant pour m'avoir parlé du lemme d'approximation par les arbres, qui simplifie certaines démonstrations. Enfin je remercie vivement le rapporteur qui a tout lu en détail et indiqué de nombreuses corrections.

\section{Structure de la démonstration}

Le but de ce paragraphe est de ramener la démonstration du théorème 0.4 à celle du théorème 1.5. Les paragraphes 3,4 et 5 seront consacrés à la démonstration du théorème 1.5 .

Le théorème 1.2 ci-dessous affirme en gros que pour un groupe $G$ agissant proprement sur un espace hyperbolique, l'existence d'homotopies de 1 à $\gamma$, utilisant 
des représentations dans des espaces de Hilbert dont la croissance est contrôlée par une exponentielle arbitrairement petite, implique la surjectivité de l'application de Baum-Connes à coefficients (l'injectivité est déjà connue grâce à [KS03]).

Définition 1.1. Soit $G$ un groupe localement compact. On appelle longueur sur $G$ une fonction continue $\ell: G \rightarrow \mathbb{R}_{+}$vérifiant $\ell\left(g^{-1}\right)=\ell(g)$ et $\ell\left(g_{1} g_{2}\right) \leq \ell\left(g_{1}\right)+\ell\left(g_{2}\right)$ pour tous $g, g_{1}, g_{2} \in G$.

Soit $G$ un groupe localement compact et $\ell$ une longueur sur $G$. Pour toutes $G-C^{*}$ algèbres $A$ et $B$ on définit $E_{G, \ell}(A, B)$ comme l'ensemble des classes d'isomorphisme de $(E, \pi, T)$ où $E$ est un $(A, B)$-bimodule hilbertien $\mathbb{Z} / 2 \mathbb{Z}$-gradué muni d'une action continue de $G$ vérifiant $\|\pi(g)\| \leq e^{\ell(g)}$ pour tout $g \in G$, et d'un opérateur $T$ borné impair tel que pour tout $a \in A$ les opérateurs $[a, T]$ et $a\left(T^{2}-1\right)$ soient compacts et que l'application $g \mapsto a(g(T)-T)$ soit une application normiquement continue de $G$ dans $\mathcal{K}_{B}(E)$. On définit ensuite $\mathrm{KK}_{G, \ell}(A, B)$ comme l'ensemble des classes d'homotopie dans $E_{G, \ell}(A, B)$ : deux éléments sont homotopes si ils sont les évaluations en 0 et 1 d'un élément de $E_{G, \ell}(A, B[0,1])$. On rappelle que $B[0,1]=C([0,1], B)$ muni de la norme du sup. On peut montrer que la somme directe munit $\mathrm{KK}_{G, \ell}(A, B)$ d'une structure de groupe abélien.

En particulier $E_{G, \ell}(\mathbb{C}, \mathbb{C})$ est l'ensemble des classes d'isomorphisme de $(H, \pi, T)$ où $H$ est un espace de Hilbert $\mathbb{Z} / 2 \mathbb{Z}$-gradué muni d'une action continue de $G$ vérifiant $\|\pi(g)\| \leq e^{\ell(g)}$ pour tout $g \in G$, et d'un opérateur $T$ borné impair tel que $\left(T^{2}-1\right)$ soit compact et que l'application $g \mapsto g(T)-T$ soit une application normiquement continue de $G$ dans $\mathcal{K}(H)$.

Théorème 1.2. Soit $G$ un groupe localement compact agissant de façon isométrique, continue et propre sur un espace métrique $(X, d)$ hyperbolique, faiblement géodésique et uniformément localement fini. Soit $\gamma \in \mathrm{KK}_{G}(\mathbb{C}, \mathbb{C})$ l'élément défini sous ces hypothèses par Kasparov et Skandalis [KS03]. Soit $x$ un point de X et $\ell$ la longueur sur $G$ définie par $\ell(g)=d(x, g x)$. Supposons que pour tout $s>0$ il existe $C \in \mathbb{R}_{+}$ tel que l'image de $1-\gamma$ dans $\mathrm{KK}_{G, s \ell+C}(\mathbb{C}, \mathbb{C})$ soit nulle. Alors $G$ vérifie la conjecture de Baum-Connes à coefficients, c'est-à-dire que pour toute $G$-C ${ }^{*}$-algèbre $A$, $\mu_{\text {red }}^{G, A}: K_{*}^{\text {top }}(G, A) \rightarrow K_{*}\left(C_{\text {red }}^{*}(G, A)\right)$ est une bijection.

Ce théorème est le corollaire 2.12 de [Laf09], dont la preuve repose sur des idées de Higson. En fait nous avons remplacé l'hypothèse "à géométrie grossière bornée" du corollaire 2.12 de [Laf09] par l'hypothèse "uniformément localement fini” qui est strictement plus forte, car nous appliquerons ce théorème à des espaces uniformément localement finis et qu'il n'est donc pas nécessaire de rappeler la notion de géométrie grossière bornée.

Grâce au théorème 1.2 , le théorème 0.4 est une conséquence du théorème suivant. 
Théorème 1.3. Soit $G$ un groupe localement compact agissant de façon isométrique, continue et propre sur un espace métrique $(X, d)$ hyperbolique, faiblement géodésique et uniformément localement fini. Soit $\gamma \in \mathrm{KK}_{G}(\mathbb{C}, \mathbb{C})$ l'élément défini sous ces hypothèses par Kasparov et Skandalis [KS03]. Soit $x \in X$ et $\ell$ la longueur sur $G$ définie par $\ell(g)=d(x, g x)$. Alors pour tout $s>0$ il existe $C \in \mathbb{R}_{+}$tel que l'image de $1-\gamma$ dans $\mathrm{KK}_{G, s \ell+C}(\mathbb{C}, \mathbb{C})$ est nulle.

Remarque. Le théorème 1.3 est encore vrai sans l'hypothèse de propreté de l'action de $G$ sur $X$ (nous avons inclus cette hypothèse pour que $\gamma$ soit un "élément $\gamma$ " pour $G)$. De toute façon le théorème avec l'hypothèse de propreté de l'action implique le théorème sans cette hypothèse car le groupe des automorphismes de $(X, d)$ est localement compact et agit proprement sur $X$.

Nous allons voir maintenant que le théorème 1.3 résulte du théorème 1.5 cidessous qui utilise des hypothèses plus fortes sur l'espace métrique $(X, d)$.

Définition 1.4. On dit qu'un espace métrique $(X, d)$ est un bon espace hyperbolique discret si

- $d$ prend ses valeurs dans $\mathbb{N}$ et est géodésique, c'est-à-dire vérifie

$$
\forall a, b \in X, \forall k \in\{0, \ldots, d(a, b)\}, \exists c \in X, d(a, c)=k, d(c, b)=d(a, b)-k,
$$

autrement dit $d$ provient d'une structure de graphe connexe sur $X$,

- $(X, d)$ est uniformément localement fini (comme $d$ est géodésique, cela équivaut à dire que le nombre de points à distance $1 \mathrm{~d}$ 'un point, est borné indépendamment du point),

$-(X, d)$ est hyperbolique.

Remarquons que si $G$ est un groupe hyperbolique, et $d$ est la distance invariante à gauche associée à la longueur des mots, pour un système fini de générateurs, $(G, d)$ est un bon espace hyperbolique discret, muni d'une action isométrique de $G$ par translations à gauche ( $d$ provient de la structure de graphe de Cayley sur $G$ associée à ce système de générateurs).

Soit $\delta \in \mathbb{R}_{+}^{*}$, et $(X, d)$ un espace métrique $\delta$-hyperbolique, faiblement $\delta$-géodésique, uniformément localement fini, et muni d'une action isométrique d'un groupe $G$. Munissons $X$ de la distance suivante :

$$
\begin{aligned}
d^{\prime}(a, b)=\min \{i \in \mathbb{N} \mid & \exists a_{0}, \ldots, a_{i}, \text { tels que } a_{0}=a, a_{i}=b, \\
& \text { et } \left.\forall j \in\{0, \ldots, i-1\}, d\left(a_{j}, a_{j+1}\right) \leq \delta+1\right\} .
\end{aligned}
$$

Autrement dit $d^{\prime}$ provient de la structure de graphe sur $X$ pour laquelle deux points distincts $x, y \in X$ sont voisins si $d(x, y) \leq \delta+1$. On a alors les propriétés suivantes :

- l'action de $G \operatorname{sur}\left(X, d^{\prime}\right)$ est isométrique, 
- $d^{\prime}$ est quasi-isométrique à $d$ : on a $d \leq(\delta+1) d^{\prime}$, et, en utilisant le fait que $(X, d)$ est faiblement $\delta$-géodésique, on montre facilement que $d^{\prime} \leq d+1$,

- $\left(X, d^{\prime}\right)$ est un bon espace hyperbolique discret.

L'hyperbolicité de $\left(X, d^{\prime}\right)$ résulte de la conservation de l'hyperbolicité par quasiisométrie pour des espaces faiblement géodésiques. Pour les espaces géodésiques la démonstration figure dans [GdlH90], [CDP90] et pour les espaces faiblement géodésiques il n'y a pas grand chose à modifier. On peut aussi invoquer le théorème 3.18 de [Vai05] ainsi que la remarque 3.19 de [Vai05] appliquée à $(X, d)$ et à l'espace total du graphe considéré précédemment (je remercie Yves Stalder qui m'a indiqué cette référence).

Donc le théorème 1.3 pour $(X, d)$ résulte du théorème 1.5 ci-dessous appliqué à $\left(X, d^{\prime}\right)$.

Théorème 1.5. Soit $G$ un groupe localement compact agissant de façon isométrique, continue et propre sur un espace métrique $(X, d)$ qui est un bon espace hyperbolique discret. Soit $\gamma \in \mathrm{KK}_{G}(\mathbb{C}, \mathbb{C})$ l'élément défini sous ces hypothèses par Kasparov et Skandalis [KS03]. Soit $x \in X$ et $\ell$ la longueur sur $G$ définie par $\ell(g)=d(x, g x)$. Alors pour tout $s>0$ il existe $C \in \mathbb{R}_{+}$tel que l'image de $1-\gamma$ dans $\mathrm{KK}_{G, s \ell+C}(\mathbb{C}, \mathbb{C})$ est nulle.

Pour montrer le théorème 0.4 nous sommes donc ramenés à montrer le théorème 1.5. Les paragraphes 3,4 et 5 sont consacrés à la démonstration du théorème 1.5 .

\section{Le cas des arbres}

Le but de ce paragraphe est de démontrer le théorème 1.5 dans le cas où $X$ est un arbre, afin d'introduire dans un cas simple les idées de la démonstration du théorème 1.5. Soit $X$ un arbre et $q$ un entier tel que chaque sommet de l' arbre ait au plus $q+1$ voisins. Soit $G$ un groupe localement compact agissant de façon isométrique, continue et propre sur $X$. Soit $\gamma \in \mathrm{KK}_{G}(\mathbb{C}, \mathbb{C})$ l'élément $\gamma$ de Julg et Valette, dont la construction est rappelée ci-dessous. Soit $x \in X$ et $\ell$ la longueur sur $G$ définie par $\ell(g)=d(x, g x)$. La proposition suivante est le théorème 1.5 dans le cas où $X$ est un arbre.

Proposition 2.1. Pour tout $s>0$ il existe $C \in \mathbb{R}_{+}$tel que l'image de $1-\gamma$ dans $\mathrm{KK}_{G, s \ell+C}(\mathbb{C}, \mathbb{C})$ est nulle.

Bien sûr Julg et Valette ont montré dans [JV84] que $\gamma=1$ dans $\mathrm{KK}_{G}(\mathbb{C}, \mathbb{C})$, sans aucune hypothèse sur l'arbre (c'est-à-dire un résultat plus fort en partant d'une hypothèse plus faible).

Rappelons la construction de l'élément $\gamma$ de Julg et Valette. Notons $\Delta_{1}=X$ l'ensemble des sommets de l'arbre et $\Delta_{2}$ l'ensemble des arêtes. Notons $\mathbb{C}^{\left(\Delta_{1}\right)}$ l'ensemble des combinaisons finies d'éléments de $\Delta_{1}$. Pour simplifier les notations, nous 
choisissons pour chaque arête une orientation, ce qui permet d'identifier $\mathbb{C}^{\left(\Delta_{2}\right)}$ au sous-espace vectoriel de $\Lambda^{2}\left(\mathbb{C}^{\left(\Delta_{1}\right)}\right)$ engendré par les $e_{a} \wedge e_{b}$ pour $\{a, b\} \in \Delta_{2}$. De même $\ell^{2}\left(\Delta_{2}\right)$ s'identifie au sous-espace vectoriel fermé de $\Lambda^{2}\left(\ell^{2}\left(\Delta_{1}\right)\right)$ engendré par les $e_{a} \wedge e_{b}$ pour $\{a, b\} \in \Delta_{2}$.

L'élément $\gamma \in \mathrm{KK}_{G}(\mathbb{C}, \mathbb{C})$ (associé au choix de $x$ comme origine) est représenté par l'espace de Hilbert $\mathbb{Z} / 2 \mathbb{Z}$-gradué $\ell^{2}\left(\Delta_{1}\right) \oplus \ell^{2}\left(\Delta_{2}\right)$ et par l'opérateur $T=\left(\begin{array}{ll}0 & u \\ v & 0\end{array}\right)$, où $u: \ell^{2}\left(\Delta_{2}\right) \rightarrow \ell^{2}\left(\Delta_{1}\right)$ et $v: \ell^{2}\left(\Delta_{1}\right) \rightarrow \ell^{2}\left(\Delta_{2}\right)$ sont définis de la façon suivante : si $\{a, b\}$ est une arête telle que $b$ se trouve sur la géodésique entre $x$ et $a$, alors $u\left(e_{a} \wedge e_{b}\right)=-e_{a}$ et $v\left(e_{a}\right)=-e_{a} \wedge e_{b}$ et enfin $v\left(e_{x}\right)=0$. On voit que $1-v \circ u=0$ et que $1-u \circ v$ est le projecteur orthogonal sur $e_{x}$. De plus, si $g \in G, g(u)-u$ et $g(v)-v$ sont de rang fini. Dans la suite nous écrirons toujours $T=u+v$ au lieu de $T=\left(\begin{array}{ll}0 & u \\ v & 0\end{array}\right)$.

Démonstration de la proposition 2.1. Nous allons montrer que pour tout $s>0$ il existe $C \in \mathbb{R}_{+}$tel que l'image de $1-\gamma$ dans $\mathrm{KK}_{G, 2 s \ell+C}(\mathbb{C}, \mathbb{C})$ soit nulle. Soit $s \in \mathbb{R}_{+}^{*}$. En fait nous allons montrer qu'il existe un polynôme $P$ et une homotopie de 1 à $\gamma$ faisant intervenir des représentations $\pi$ de $G$ dans des espaces de Hilbert tels que $\|\pi(g)\| \leq P(\ell(g)) e^{s \ell(g)}$.

Notons $\partial: \mathbb{C}^{\left(\Delta_{2}\right)} \rightarrow \mathbb{C}^{\left(\Delta_{1}\right)}$ l'opérateur bord de l'homologie simpliciale. Pour toute arête $\{a, b\}$ on a $\partial\left(e_{a} \wedge e_{b}\right)=e_{b}-e_{a}$. Alors $\partial$ est injectif et son image est de codimension 1. Pour le montrer définissons $h: \mathbb{C}^{\left(\Delta_{1}\right)} \rightarrow \mathbb{C}^{\left(\Delta_{2}\right)}$ de la façon suivante : pour tout point $a \in X$, notons $n=d(a, x)$ et $a_{0}, \ldots, a_{n}$ la suite de points reliant $a$ à $x$ (c'est-à-dire que $a_{0}=a, a_{n}=x$ et $a_{i}$ est voisin de $a_{i-1}$ pour tout $i \in\{1, \ldots, n\}$ ) et posons $h\left(e_{a}\right)=-\left(e_{a_{0}} \wedge e_{a_{1}}+\cdots+e_{a_{n-1}} \wedge e_{a_{n}}\right)$. En particulier $h\left(e_{x}\right)=0$. Alors $h \circ \partial=\operatorname{Id}_{\mathbb{C}^{\left(\Delta_{2}\right)}}$ et $\operatorname{Id}_{\mathbb{C}^{\left(\Delta_{1}\right)}}-\partial \circ h$ est de rang 1 .

Plus généralement pour tout $t \in[0,1]$ on définit des opérateurs $u_{t}: \mathbb{C}^{\left(\Delta_{2}\right)} \rightarrow$ $\mathbb{C}^{\left(\Delta_{1}\right)}$ et $v_{t}: \mathbb{C}^{\left(\Delta_{1}\right)} \rightarrow \mathbb{C}^{\left(\Delta_{2}\right)}$ de sorte que $u_{1}=\partial, v_{1}=h$, et $u_{0}$ et $v_{0}$ soient les restrictions de $u$ et $v$ à $\mathbb{C}^{\left(\Delta_{2}\right)} \subset \ell^{2}\left(\Delta_{2}\right)$ et $\mathbb{C}^{\left(\Delta_{1}\right)} \subset \ell^{2}\left(\Delta_{1}\right)$. La formule est la suivante : pour toute arête $\{a, b\}$ telle que $b$ se trouve sur la géodésique entre $x$ et $a$ on pose $u_{t}\left(e_{a} \wedge e_{b}\right)=-\left(e_{a}-t e_{b}\right)$. Pour tout point $a \in X$, on note $n=d(a, x)$ et $a_{0}, \ldots, a_{n}$ la suite de points reliant $a$ à $x$ (c'est-à-dire que $a_{0}=a, a_{n}=x$ et $a_{i}$ est voisin de $a_{i-1}$ pour tout $\left.i \in\{1, \ldots, n\}\right)$ et on pose

$$
v_{t}\left(e_{a}\right)=-\left(e_{a_{0}} \wedge e_{a_{1}}+t e_{a_{1}} \wedge e_{a_{2}}+t^{2} e_{a_{2}} \wedge e_{a_{3}}+\cdots+t^{n-1} e_{a_{n-1}} \wedge e_{a_{n}}\right) .
$$

Nous allons compléter $\mathbb{C}^{\left(\Delta_{2}\right)}$ et $\mathbb{C}^{\left(\Delta_{1}\right)}$ pour certaines normes de Hilbert telles que $\|\pi(g)\| \leq P(\ell(g)) e^{s \ell(g)}$ pour un certain polynôme $P$ et telles que les opérateurs $u_{t}$ et $v_{t}$ soient continus, uniformément en $t \in[0,1]$.

Rappelons que dans [Laf02] nous introduisons les espaces $\ell_{x, s}^{1}\left(\Delta_{1}\right)$ et $\ell_{x, s}^{1}\left(\Delta_{2}\right)$ comme les complétés de $\mathbb{C}^{\left(\Delta_{1}\right)}$ et $\mathbb{C}^{\left(\Delta_{2}\right)}$ pour les normes $\ell^{1}$ pondérées suivantes :

$$
\left\|\sum_{a \in \Delta_{1}} f(a) e_{a}\right\|_{\ell_{x, s}^{1}\left(\Delta_{1}\right)}=\sum_{a \in \Delta_{1}}|f(a)| e^{s d(x, a)}
$$


et

$$
\left\|\sum_{\substack{\{a, b\} \in \Delta_{2} \\ b \in \operatorname{géod}(x, a)}} f(a, b) e_{a} \wedge e_{b}\right\|_{\ell_{x, s}^{1}\left(\Delta_{2}\right)}=\sum_{\substack{\{a, b\} \in \Delta_{2} \\ b \in \operatorname{geod}(x, a)}}|f(a, b)| e^{s d(x, a)} .
$$

On voit que pour tout $t \in[0,1]$ on a $\left\|u_{t}\right\|_{\mathscr{L}\left(\ell_{x, s}^{1}\left(\Delta_{2}\right), \ell_{x, s}^{1}\left(\Delta_{1}\right)\right)}=1+t e^{-s}$ et $\left\|v_{t}\right\|_{\mathscr{L}\left(\ell_{x, s}^{1}\left(\Delta_{1}\right), \ell_{x, s}^{1}\left(\Delta_{2}\right)\right)}=\left(1-t e^{-s}\right)^{-1}$.

Rappelons la construction de [Laf02] qui montre que l'image de $1-\gamma$ dans $\mathrm{KK}_{G, s \ell}^{\mathrm{ban}}(\mathbb{C}, \mathbb{C})$ est nulle. On note $E_{x, s}\left(\Delta_{1}\right)$ la $\mathbb{C}$-paire $\left(c_{0, x, s}\left(\Delta_{1}\right), \ell_{x, s}^{1}\left(\Delta_{1}\right)\right)$, où $c_{0, x, s}\left(\Delta_{1}\right)$ est le complété de $\mathbb{C}^{\left(\Delta_{1}\right)}$ pour la norme $\|f\|=\sup _{a \in \Delta_{1}}|f(a)| e^{-s d(x, a)}$ et où le crochet entre $c_{0, x, s}\left(\Delta_{1}\right)$ et $\ell_{x, s}^{1}\left(\Delta_{1}\right)$ est donné par $\left\langle f, f^{\prime}\right\rangle=\sum_{a \in \Delta_{1}} f(a) f^{\prime}(a)$. On introduit de la même façon la $\mathbb{C}$-paire $E_{x, s}\left(\Delta_{2}\right)$. Pour toute $\mathbb{C}$-paire $E=$ $\left(E^{<}, E^{>}\right) \quad$ on note $E[0,1]$ la $\left(E^{<}[0,1], E^{>}[0,1]\right)$ (où $E^{<}[0,1]=C\left([0,1], E^{<}\right)$muni de la norme du sup, et de même pour $\left.E^{>}[0,1]\right)$. Alors $\left(E_{x, s}\left(\Delta_{1}\right)[0,1] \oplus E_{x, s}\left(\Delta_{2}\right)[0,1],\left(u_{t}+v_{t}\right)_{t \in[0,1]}\right)$ définit un élément de $E_{G, s \ell}^{\text {ban }}(\mathbb{C}, \mathbb{C}[0,1])$. D'autre part $\left(E_{x, s}\left(\Delta_{1}\right) \oplus E_{x, s}\left(\Delta_{2}\right), u_{1}+v_{1}\right)$ est égal à 1 dans $\mathrm{KK}_{G, s \ell}^{\text {ban }}(\mathbb{C}, \mathbb{C})$ grâce au lemme 1.4.2 de [Laf02] et $\left(E_{x, s}\left(\Delta_{1}\right) \oplus\right.$ $\left.E_{x, s}\left(\Delta_{2}\right), u_{0}+v_{0}\right)$ est égal à $\gamma$ dans $\mathrm{KK}_{G, s \ell}^{\text {ban }}(\mathbb{C}, \mathbb{C})$ (pour l'homotopie entre ces deux éléments, on garde les opérateurs, on complète $\mathbb{C}^{\left(\Delta_{1}\right)}$ pour la norme $t\|\cdot\|_{E_{x, s}\left(\Delta_{1}\right)}+$ $(1-t)\|\cdot\|_{\ell^{2}\left(\Delta_{1}\right)}$, on considère la $\mathbb{C}$-paire formée de cet espace de Banach et du complété de $\mathbb{C}^{\left(\Delta_{1}\right)}$ pour la norme duale et on fait de même pour $\left.\Delta_{2}\right)$. Par conséquent on voit que l'image de $1-\gamma$ dans $\operatorname{KK}_{G, s \ell}^{\text {ban }}(\mathbb{C}, \mathbb{C})$ est nulle.

Pour montrer que l'image de $1-\gamma$ dans $\mathrm{KK}_{G, 2 s \ell+C}(\mathbb{C}, \mathbb{C})$ est nulle (avec $C$ une constante assez grande), la première idée qui vient est de remplacer les normes $\ell^{1}$ par des normes $\ell^{2}$ pour avoir des espaces de Hilbert.

Nous définissons donc $\ell_{x, s}^{2}\left(\Delta_{1}\right)$ et $\ell_{x, s}^{2}\left(\Delta_{2}\right)$ comme les complétés de $\mathbb{C}^{\left(\Delta_{1}\right)}$ et $\mathbb{C}^{\left(\Delta_{2}\right)}$ pour les normes $\ell^{2}$ pondérées suivantes :

$$
\left\|\sum_{a \in \Delta_{1}} f(a) e_{a}\right\|_{\ell_{x, s}^{2}\left(\Delta_{1}\right)}^{2}=\sum_{a \in \Delta_{1}}|f(a)|^{2} e^{2 s d(x, a)}
$$

et

$$
\left\|\sum_{\substack{\{a, b\} \in \Delta_{2} \\ b \in \operatorname{géod}(x, a)}} f(a, b) e_{a} \wedge e_{b}\right\|_{\ell_{x, s}^{2}\left(\Delta_{2}\right)}^{2}=\sum_{\substack{\{a, b\} \in \Delta_{2} \\ b \in \operatorname{géod}(x, a)}}|f(a, b)|^{2} e^{2 s d(x, a)} .
$$

Il est clair que $u_{t}: \ell_{x, s}^{2}\left(\Delta_{2}\right) \rightarrow \ell_{x, s}^{2}\left(\Delta_{1}\right)$ est continu (et que sa norme est majorée de façon uniforme en $t$ ), mais ce n'est pas le cas de $v_{t}$, si $s$ est petit et $t$ proche de 1 , pour la raison suivante.

Soit $\left\{z, z^{\prime}\right\}$ une arête de $X$ (telle que $\left.z^{\prime} \in \operatorname{géod}(x, z)\right)$ et

$$
\xi_{z, z^{\prime}}: \sum_{\substack{\{a, b\} \in \Delta_{2} \\ b \in \operatorname{géod}(x, a)}} f(a, b) e_{a} \wedge e_{b} \mapsto f\left(z, z^{\prime}\right)
$$


la forme linéaire sur $\ell_{x, s}^{2}\left(\Delta_{2}\right)$ qui donne le coefficient de cette arête. Cette forme linéaire est de norme $e^{-s d(x, z)}$. Or la forme linéaire ${ }^{t} v_{t}\left(\xi_{z, z^{\prime}}\right)=\xi_{z, z^{\prime}} \circ v_{t} \operatorname{sur} \ell_{x, s}^{2}\left(\Delta_{1}\right)$ est

$$
\sum_{a} f(a) e_{a} \mapsto-\left(\sum_{\substack{a \text { tel que } \\ z \in \operatorname{geod}(x, a)}} t^{d(z, a)} f(a)\right) .
$$

Si chaque sommet de l'arbre a exactement $q+1$ voisins, en notant $k=d(x, z)$ on a $\left\|^{t} v_{t}\left(\xi_{z, z^{\prime}}\right)\right\|_{\ell_{x, s}^{2}\left(\Delta_{1}\right)^{*}}^{2}=\sum_{n \geq k} t^{2(n-k)} e^{-2 s n} q^{n-k}$. On voit donc que cette forme linéaire ${ }^{t} v_{t}\left(\xi_{z, z^{\prime}}\right)$ n'est bornée que si $e^{-s} t \sqrt{q}<1$.

Nous allons compléter $\mathbb{C}^{\left(\Delta_{1}\right)}$ et $\mathbb{C}^{\left(\Delta_{2}\right)}$ pour d'autres normes de Hilbert de telle sorte que les opérateurs $u_{t}$ et $v_{t}$ soient continus pour $t \in[0,1]$.

Pour tout $n \in \mathbb{N}$, on note $S_{x}^{n}=\{a \in X \mid d(x, a)=n\}$ la sphère de rayon $n$ et de centre $x$. Pour $k, n \in \mathbb{N}$ avec $k \leq n$ et pour tout $z \in S_{x}^{k}$ on note $I_{z}^{n, k, x}=\left\{a \in S_{x}^{n} \mid\right.$ $z \in \operatorname{géod}(x, a)\}$ de sorte que $S_{x}^{n}=\bigcup_{z \in S_{x}^{k}} I_{z}^{n, k, x}$ est une partition de la sphère $S_{x}^{n}$. Lorsque $k=n$ c'est la partition par les singletons et lorsque $k=0$ c'est la partition grossière.

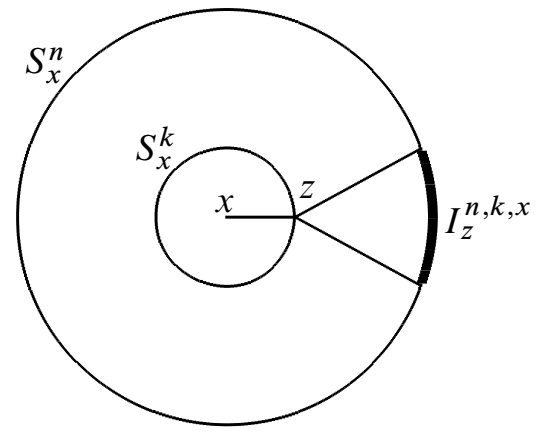

On définit alors $H_{x, s}\left(\Delta_{1}\right)$ comme l'espace de Hilbert, complétion de $\mathbb{C}^{\left(\Delta_{1}\right)}$ pour la norme

$$
\left\|\sum_{a} f(a) e_{a}\right\|_{H_{x, s}\left(\Delta_{1}\right)}^{2}=\sum_{n \in \mathbb{N}} e^{2 s n} \sum_{k=0}^{n} \sum_{z \in S_{x}^{k}}\left|\sum_{a \in I_{z}^{n, k, x}} f(a)\right|^{2} .
$$

Alors pour tout $t \in[0,1], v_{t}: H_{x, s}\left(\Delta_{1}\right) \rightarrow \ell_{x, s}^{2}\left(\Delta_{2}\right)$ est continu. En effet $v_{t}=$ $\sum_{j \in \mathbb{N}} v_{t, j}$ où $v_{t, j}$ est défini de la manière suivante : pour tout point $a \in X$, en notant $n=d(a, x)$ et $a_{0}, \ldots, a_{n}$ la suite de points reliant $a$ à $x$ (c'est-à-dire que $a_{0}=a, a_{n}=x$ et $a_{i}$ est voisin de $a_{i-1}$ pour tout $\left.i \in\{1, \ldots, n\}\right)$, on pose $v_{t, j}\left(e_{a}\right)=$ $-t^{j} e_{a_{j}} \wedge e_{a_{j+1}}$ si $j \leq n-1$ et $v_{t, j}\left(e_{a}\right)=0$ si $j \geq n$. On vérifie facilement que $v_{t, j}$ est continu de $H_{x, s}\left(\Delta_{1}\right)$ vers $\ell_{x, s}^{2}\left(\Delta_{2}\right)$ de norme inférieure ou égale à $t^{j} e^{-s j}$. La raison est que $\|\cdot\|_{\ell_{x, s}^{2}\left(\Delta_{2}\right)}^{2}$ est une somme pondérée des carrés des formes linéaires $\xi_{z, z^{\prime}}$, qu'en notant $k=d(x, z),{ }^{t} v_{t, j}\left(\xi_{z, z^{\prime}}\right)$ est le produit par $-t^{j}$ de la forme linéaire $f \mapsto \sum_{a \in I_{z}^{j}+k, k, x} f(a)$ et que $\|\cdot\|_{H_{x, s}\left(\Delta_{1}\right)}^{2}$ est une somme pondérée des carrés de telles formes linéaires. 
Bien sûr $\partial$ et plus généralement $u_{t}: \ell_{x, s}^{2}\left(\Delta_{2}\right) \rightarrow H_{x, s}\left(\Delta_{1}\right)$ ne sont plus continus et nous devons remplacer $\ell_{x, s}^{2}\left(\Delta_{2}\right)$ par un espace analogue à $H_{x, s}\left(\Delta_{1}\right)$.

Pour $k \in \mathbb{N}, n \in \mathbb{N}^{*}$ avec $k \leq n$ et $z \in S_{x}^{k}$ on pose

$$
J_{z}^{n, k, x}=\left\{(a, b) \mid\{a, b\} \in \Delta_{2}, a \in S_{x}^{n}, b \in S_{x}^{n-1}, z \in \operatorname{géod}(x, a)\right\} .
$$

On remarque que $\bigcup_{z \in S_{x}^{k}} J_{z}^{n, k, x}$ est une partition de l'ensemble des arêtes à distance $n-1$ de $x$. Lorsque $k=n$ c'est la partition par les singletons et lorsque $k=0$ c'est la partition grossière.

On définit alors $H_{x, s}\left(\Delta_{2}\right)$ comme la complétion de $\mathbb{C}^{\left(\Delta_{2}\right)}$ pour la norme

$$
\left\|\sum_{\substack{\{a, b\} \in \Delta_{2} \\ b \in \operatorname{ged}(x, a)}} f(a, b) e_{a} \wedge e_{b}\right\|_{H_{x, s}\left(\Delta_{2}\right)}^{2}=\sum_{n \in \mathbb{N}^{*}} e^{2 s n} \sum_{k=0}^{n} \sum_{z \in S_{x}^{k}}\left|\sum_{(a, b) \in J_{z}^{n, k, x}} f(a, b)\right|^{2} .
$$

Il est alors très facile de voir que pour tout $t \in[0,1]$, les opérateurs $u_{t}: H_{x, s}\left(\Delta_{2}\right) \rightarrow$ $H_{x, s}\left(\Delta_{1}\right)$ et $v_{t}: H_{x, s}\left(\Delta_{1}\right) \rightarrow H_{x, s}\left(\Delta_{2}\right)$ sont continus, de normes majorées uniformément en $t$. Pour $v_{t}$ la raison est la suivante : l'image $\operatorname{par}^{t} v_{t, j}$ de la forme linéaire $f \mapsto \sum_{(a, b) \in J_{z}^{n, k, x}} f(a, b)$ qui apparaît dans la formule ci-dessus est égale au produit par $-t^{j}$ de la forme linéaire $f \mapsto \sum_{a \in I_{z}^{n+j, k, x}} f(a)$ qui apparaît dans la formule pour $\|\cdot\|_{H_{x, s}\left(\Delta_{1}\right)}^{2}$ et on en déduit facilement $\left\|v_{t, j}\right\|_{\mathscr{L}\left(H_{x, s}\left(\Delta_{1}\right), H_{x, s}\left(\Delta_{2}\right)\right)} \leq t^{j} e^{-s j}$.

Pour conclure il ne reste donc plus qu'à établir le résultat suivant : il existe un polynôme $P$ tel que pour tout $s>0$ les représentations de $G$ sur $H_{x, s}\left(\Delta_{1}\right)$ et $H_{x, s}\left(\Delta_{2}\right)$ vérifient $\|\pi(g)\| \leq P(\ell(g)) e^{s \ell(g)}$ pour tout $g \in G$.

Démontrons ce résultat pour $H_{x, s}\left(\Delta_{1}\right)$. Soit $g \in G$. On pose $x^{\prime}=g(x)$, si bien que $d\left(x, x^{\prime}\right)=\ell(g)$. Il résulte de la définition de $H_{x, s}\left(\Delta_{1}\right)$ que l'on a une isométrie $\Theta_{x, s}: H_{x, s}\left(\Delta_{1}\right) \rightarrow \ell^{2}\left(\left\{(n, k, z), 0 \leq k \leq n, z \in S_{x}^{k}\right\}\right)$ définie par

$$
\Theta_{x, s}\left(\sum_{a} f(a) e_{a}\right)=\left(e^{s n} \sum_{a \in I_{z}^{n, k, x}} f(a)\right)_{(n, k, z)} .
$$

Pour tout $f \in \mathbb{C}^{\left(\Delta_{1}\right)}$ on a $\|\pi(g) f\|_{H_{x^{\prime}, s}\left(\Delta_{1}\right)}=\|f\|_{H_{x, s}\left(\Delta_{1}\right)}$. D'autre part on a une isométrie $\Theta_{x^{\prime}, s}$ de $H_{x^{\prime}, s}\left(\Delta_{1}\right)$ dans $\ell^{2}\left(\left\{\left(n^{\prime}, k^{\prime}, z^{\prime}\right), 0 \leq k^{\prime} \leq n^{\prime}, z^{\prime} \in S_{x^{\prime}}^{k^{\prime}}\right\}\right)$.

Le lemme suivant permet de comparer les normes de $H_{x, s}$ et de $H_{x^{\prime}, s}$.

Lemme 2.2. Pour $(n, k, z) \in\left\{(n, k, z) \mid 0 \leq k \leq n, z \in S_{x}^{k}\right\}$ on peut écrire $I_{z}^{n, k, x}$ comme une réunion disjointe finie d'au plus $(q-1)\left(d\left(x, x^{\prime}\right)+1\right)+2$ parties $I_{z^{\prime}}^{n^{\prime}, k^{\prime}, x^{\prime}}$ pour $\left(n^{\prime}, k^{\prime}, z^{\prime}\right) \in\left\{\left(n^{\prime}, k^{\prime}, z^{\prime}\right) \mid 0 \leq k^{\prime} \leq n^{\prime}, z^{\prime} \in S_{x^{\prime}}^{k^{\prime}}\right\}$, de sorte que pour chaque $\left(n^{\prime}, k^{\prime}, z^{\prime}\right)$ la partie $I_{z^{\prime}}^{n^{\prime}, k^{\prime}, x^{\prime}}$ intervienne au plus $d\left(x, x^{\prime}\right)+2$ fois dans ces décompositions (c'est-à-dire que l'ensemble des $(n, k, z)$ tels que la partie $I_{z^{\prime}}^{n^{\prime}, k^{\prime}, x^{\prime}}$ intervienne dans la décomposition de $I_{z}^{n, k, x}$ est fini et de cardinal inférieur ou égal $\left.\grave{a} d\left(x, x^{\prime}\right)+2\right)$. 
Ce lemme est le lemme 1.5 de [Laf08] mais nous en rappelons la démonstration (avec une petite correction).

Démonstration. Si $z$ n'appartient pas à géod $\left(x, x^{\prime}\right)$, on a $I_{z}^{n, k, x}=I_{z^{\prime}}^{n^{\prime}, k^{\prime}, x^{\prime}}$ avec $z^{\prime}=z, k^{\prime}=d\left(x^{\prime}, z\right), n^{\prime}=n+k^{\prime}-k$. Si $z$ appartient à $\operatorname{géod}\left(x, x^{\prime}\right)$, on peut écrire $I_{z}^{n, k, x}$ comme la réunion disjointe

- des $I_{z^{\prime}}^{n^{\prime}, k^{\prime}, x^{\prime}}$, avec $z^{\prime}$ n'appartenant pas à géod $\left(x, x^{\prime}\right)$ mais à distance 1 d'un point de géod $\left(z, x^{\prime}\right), k^{\prime}=d\left(x^{\prime}, z^{\prime}\right)$ et $n^{\prime}=n-d\left(x, x^{\prime}\right)+2\left(k^{\prime}-1\right)$, sous réserve que $n^{\prime} \geq 0$,

- et du singleton $I_{z^{\prime}}^{k^{\prime}, k^{\prime}, x^{\prime}}=\left\{z^{\prime}\right\}$, où $z^{\prime} \in \operatorname{géod}\left(z, x^{\prime}\right)$ vérifie $d\left(x, z^{\prime}\right)=n$, et avec $k^{\prime}=d\left(x^{\prime}, z^{\prime}\right)$, si $n \leq d\left(x, x^{\prime}\right)$.
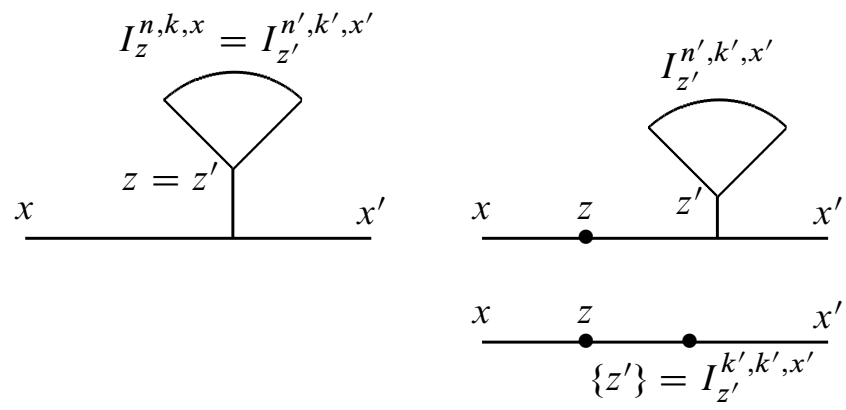

Le dessin illustre les deux cas envisagés dans la démonstration (le dessin de gauche correspond au cas où $z \notin$ géod $\left(x, x^{\prime}\right)$ et les deux dessins de droite correspondent au cas où $\left.z \in \operatorname{géod}\left(x, x^{\prime}\right)\right)$.

Fin de la démonstration de la proposition 2.1 en admettant le lemme 2.3. Soit $A$ la matrice de

$$
\ell^{2}\left(\left\{\left(n^{\prime}, k^{\prime}, z^{\prime}\right) \mid 0 \leq k^{\prime} \leq n^{\prime}, z^{\prime} \in S_{x^{\prime}}^{k^{\prime}}\right\}\right)
$$

dans

$$
\ell^{2}\left(\left\{(n, k, z) \mid 0 \leq k \leq n, z \in S_{x}^{k}\right\}\right)
$$

dont le coefficient vaut $e^{s\left(n-n^{\prime}\right)}$ si $I_{z^{\prime}}^{n^{\prime}, k^{\prime}, x^{\prime}}$ intervient dans la décomposition de $I_{z}^{n, k, x}$ dans le lemme précédent, et 0 sinon. On a alors $A \circ \Theta_{x^{\prime}, s}=\Theta_{x, s}$. Dans chaque ligne de $A$ il y a au plus $(q-1)\left(d\left(x, x^{\prime}\right)+1\right)+2$ coefficients non nuls, dans chaque colonne au plus $d\left(x, x^{\prime}\right)+2$ et ces coefficients ont une valeur absolue inférieure ou égale à $e^{s d\left(x, x^{\prime}\right)}$ (car pour $n$ et $n^{\prime}$ comme dans le lemme avec $I_{z^{\prime}}^{n^{\prime}, k^{\prime}, x^{\prime}}$ intervenant dans la décomposition de $I_{z}^{n, k, x}$, on a $\left.\left|n-n^{\prime}\right| \leq d\left(x, x^{\prime}\right)\right)$. D'après le lemme 2.3 ci-dessous, une matrice telle que dans chaque ligne il y ait au plus $C_{1}$ coefficients non nuls, dans 
chaque colonne au plus $C_{2}$ et que ses coefficients aient une norme inférieure ou égale à $C_{3}$ a une norme d'opérateur inférieure ou égale à $\sqrt{C_{1} C_{2}} C_{3}$. Donc

$$
\|A\| \leq \sqrt{(q-1)\left(d\left(x, x^{\prime}\right)+1\right)+2} \sqrt{d\left(x, x^{\prime}\right)+2} e^{s d\left(x, x^{\prime}\right)} .
$$

Pour tout $f \in \mathbb{C}^{\left(\Delta_{1}\right)}$, on a $\|f\|_{H_{x, s}} \leq\|A\|\|f\|_{H_{x^{\prime}, s}}$ et donc

$$
\|\pi(g) f\|_{H_{x, s}} \leq\|A\|\|\pi(g) f\|_{H_{x^{\prime}, s}}=\|A\|\|f\|_{H_{x, s}} .
$$

On voit que $\pi(g)$ est continu de $H_{x, s}$ dans lui-même, de norme inférieure ou égale à $\sqrt{(q-1)(\ell(g)+1)+2} \sqrt{\ell(g)+2} e^{s \ell(g)}$.

On termine maintenant la démonstration de la proposition 2.1. On décide que $H_{x, s}\left(\Delta_{1}\right)$ est pair et $H_{x, s}\left(\Delta_{2}\right)$ impair, et alors

$$
\left(\left(H_{x, s}\left(\Delta_{1}\right) \oplus H_{x, s}\left(\Delta_{2}\right)\right)[0,1],\left(u_{t}+v_{t}\right)_{t \in[0,1]}\right)
$$

appartient à $\mathrm{KK}_{G, 2 s \ell+C}(\mathbb{C}, \mathbb{C}[0,1])$ pour une constante $C$ assez grande, et réalise l'homotopie entre 1 et $\gamma$ : en effet $\left(H_{x, s}\left(\Delta_{1}\right) \oplus H_{x, s}\left(\Delta_{2}\right), u_{1}+v_{1}\right)$ est égal à 1 dans $\mathrm{KK}_{G, 2 s \ell+C}(\mathbb{C}, \mathbb{C})$ par la proposition 1.4.2 de [Laf02] et $\left(H_{x, s}\left(\Delta_{1}\right) \oplus\right.$ $\left.H_{x, s}\left(\Delta_{2}\right), u_{0}+v_{0}\right)$ est homotope à $\left(\ell^{2}\left(\Delta_{1}\right) \oplus \ell^{2}\left(\Delta_{2}\right), u_{0}+v_{0}\right)$ qui représente $\gamma$. Comme d'habitude cette homotopie est réalisée en introduisant les normes intermédiaires $\sqrt{t\|\cdot\|_{H_{x, s}}^{2}+(1-t)\|\cdot\|_{\ell^{2}}^{2}}$, et en conservant l'opérateur $u_{0}+v_{0}$ pendant l'homotopie.

Lemme 2.3. Soit $B$ une $C^{*}$-algèbre, $\left(E_{i}\right)_{i \in I}$ des $B$-modules hilbertiens et $E=$ $\bigoplus E_{i}$. Pour $i, j \in I$ on se donne $a_{i j} \in \mathscr{L}_{B}\left(E_{j}, E_{i}\right)$. Alors $s i$

$$
\sup _{i \in I}\left(\sum_{j \in I}\left\|a_{i j}\right\|_{\mathscr{L}_{B}\left(E_{j}, E_{i}\right)}\right) \quad \text { et } \sup _{j \in I}\left(\sum_{i \in I}\left\|a_{i j}\right\|_{\mathscr{L}_{B}\left(E_{j}, E_{i}\right)}\right)
$$

sont finies, $A=\left(a_{i j}\right)_{i, j \in I}$ appartient à $\mathscr{L}_{B}(E)$ et on a

$$
\|A\|_{\mathscr{L}_{B}(E)}^{2} \leq\left(\sup _{i \in I}\left(\sum_{j \in I}\left\|a_{i j}\right\|_{\mathscr{L}_{B}\left(E_{j}, E_{i}\right)}\right)\right)\left(\sup _{j \in I}\left(\sum_{i \in I}\left\|a_{i j}\right\|_{\mathscr{L}_{B}\left(E_{j}, E_{i}\right)}\right)\right) .
$$

En particulier si A, considérée comme une matrice par blocs, possède au plus $C_{1}$ blocs non nuls dans chaque ligne et $C_{2}$ blocs non nuls dans chaque colonne et si ces

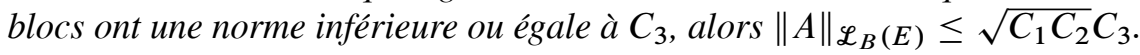

Démonstration. Il suffit de montrer l'inégalité (2) pour I fini. Comme

$$
A \mapsto \sup _{i \in I}\left(\sum_{j \in I}\left\|a_{i j}\right\|_{\mathscr{L}_{B}\left(E_{j}, E_{i}\right)}\right)
$$

est une norme d'algèbre $\operatorname{sur} \mathscr{L}_{B}(E)$, on a alors pour tout $n \in \mathbb{N}^{*}$, en notant 


$$
\begin{aligned}
\left(A^{*} A\right)^{n}= & \left(b_{i j}\right)_{i, j \in I}, \\
\|A\|_{\mathscr{L}_{B}(E)}^{2 n} & =\left\|\left(A^{*} A\right)^{n}\right\|_{\mathscr{L}_{B}(E)} \\
& \leq|I|\left(\sup _{i \in I}\left(\sum_{j \in I}\left\|b_{i j}\right\|_{\mathscr{L}_{B}\left(E_{j}, E_{i}\right)}\right)\right) \\
& \leq|I|\left(\sup _{i \in I}\left(\sum_{j \in I}\left\|a_{i j}\right\|_{\mathscr{L}_{B}\left(E_{j}, E_{i}\right)}\right)\right)^{n}\left(\sup _{j \in I}\left(\sum_{i \in I}\left\|a_{i j}\right\|_{\mathscr{L}_{B}\left(E_{j}, E_{i}\right)}\right)\right)^{n},
\end{aligned}
$$

d'où l'inégalité (2) en faisant tendre $n$ vers l'infini.

Remarque. Les espaces $H_{x, s}\left(\Delta_{1}\right)$ ressemblent aux espaces de Hilbert introduits par Julg et Valette dans [JV84] pour construire l'homotopie entre $\gamma$ et 1. Plus précisément on rappelle que pour $\lambda \in] 0,+\infty\left[\right.$ Julg et Valette notent $H_{\lambda}$ le complété de $\mathbb{C}^{\left(\Delta_{1}\right)}$ pour le produit scalaire $\left\langle\xi_{a}^{\lambda}, \xi_{b}^{\lambda}\right\rangle=e^{-\lambda d(a, b)}$, où $\xi_{a}^{\lambda}$ est la fonction qui vaut 1 en $a$ et 0 ailleurs. Nous écrirons ici $H_{\lambda}^{J V}$ au lieu de $H_{\lambda}$ pour éviter les confusions. Alors pour tout $\lambda>0$ et tout $n \in \mathbb{N}$, et $f \in \mathbb{C}^{\left(\Delta_{1}\right)}$ supporté sur $S_{x}^{n}$, on a

$$
\begin{aligned}
\|f\|_{H_{\lambda}^{J V}}^{2} & =\sum_{a, b \in S_{x}^{n}} e^{-\lambda d(a, b)} \overline{f(a)} f(b) \\
& =\left(1-e^{-2 \lambda}\right) e^{-2 \lambda n} \sum_{1 \leq k \leq n} e^{2 \lambda k} \sum_{z \in S_{x}^{k}}\left|\sum_{a \in I_{z}^{n, k, x}} f(a)\right|^{2}+e^{-2 \lambda n}\left|\sum_{a \in S_{x}^{n}} f(a)\right|^{2}
\end{aligned}
$$

et comme $S_{x}^{n}=I_{x}^{n, 0, x}$ on voit que cette formule ressemble à la formule pour $\|f\|_{H_{x, s}\left(\Delta_{1}\right)}^{2}$, bien que la présence des facteurs $\left(1-e^{-2 \lambda}\right)$ et $e^{2 \lambda k}$ empêche de les identifier, quelles que soient les valeurs de $s$ et de $\lambda$. Plus précisément les restrictions de $\|\cdot\|_{H_{\lambda}^{J V}}^{2}$ et $\|\cdot\|_{H_{x, s}\left(\Delta_{1}\right)}^{2}$ à l'espace des fonctions supportées sur $S_{x}^{n}$ s'expriment comme des sommes des carrés des mêmes formes linéaires $f \mapsto \sum_{a \in I_{z}^{n, k, x}} f(a)$, mais avec des pondérations un peu différentes. D'autre part les opérateurs $u_{t}+v_{t}$ que nous utilisons sont quasiment les mêmes que ceux introduits par Julg et Valette. On notera cependant que, dans [JV84], $\mathbb{C}^{\left(\Delta_{2}\right)}$ est complété pour la norme de $\ell^{2}\left(\Delta_{2}\right)$ alors que nous le complétons pour la norme de $H_{x, s}\left(\Delta_{2}\right)$.

\section{Construction des espaces et des opérateurs}

Ce paragraphe et les deux suivants sont consacrés à la démonstration du thérorème 1.5.

Soit $G$ un groupe localement compact agissant de façon isométrique, continue et propre sur un espace métrique $(X, d)$ qui est un bon espace hyperbolique discret. Soit $\delta \in \mathbb{N}^{*}$ tel que $(X, d)$ soit $\delta$-hyperbolique. Dans toute la suite de cet article on utilisera les notations suivantes : si $A$ et $B$ sont des parties finies de $X$, on note $d(A, B)=\min _{a \in A, b \in B} d(a, b)$ et $d_{\max }(A, B)=\max _{a \in A, b \in B} d(a, b)$. Si $A$ est un singleton $\{x\}$ on note $d(x, B)$ et $d_{\max }(x, B)$ au lieu de $d(\{x\}, B)$ et $d_{\max }(\{x\}, B)$. 
Soit $x \in X$ et $\ell$ la longueur sur $G$ définie par $\ell(g)=d(x, g x)$. Le point $x$ sert d'origine dans la construction. Cependant on utilisera en d'autres points que $x$ les constructions faites pour $x$ (notamment pour vérifier les propriétés d'équivariance). On utilisera aussi $x$ comme variable dans certains lemmes.

Soit $\gamma \in \mathrm{KK}_{G}(\mathbb{C}, \mathbb{C})$ l'élément défini sous ces hypothèses par Kasparov et Skandalis [KS03]. Soit $s \in] 0,1]$. On va construire, pour une certaine constante $C$ assez grande, une homotopie de 1 à $\gamma$ dans $E_{G, 2 s \ell+C}(\mathbb{C}, \mathbb{C}[0,1])$. Cette homotopie sera composée d'une partie difficile (partant de 1), et d'une partie facile (aboutissant à $\gamma$ ) dont la construction est reléguée au paragraphe 5. Le but de ce paragraphe est de construire des espaces vectoriels et des opérateurs pour la partie difficile de l'homotopie. Dans le paragraphe 4 nous construirons les normes sur ces espaces.

On commence par fixer un entier $N$ tel que

$N$ est assez grand en fonction de $\delta$.

$\left(H_{N}\right)$

Plus précisément nous utiliserons un nombre fini de fois l'inégalité $N \geq C \delta$, avec $C$ un entier.

On note $K$ un entier tel que pour tout point $a$ de $X$ le nombre de points de $X$ à distance 1 de $a$ soit inférieur ou égal à $K$. Dans toute la suite on notera $C(\delta), C(\delta, K)$, $C(\delta, K, N), \ldots$ des constantes qui ne dépendent que des variables indiquées, mais varient d'une formule à l'autre.

On note $\Delta$ l'ensemble des parties de $X$ dont le diamètre est inférieur ou égal à $N$. On note $p_{\max }$ le cardinal maximal d'une partie de $X$ de diamètre $\leq N$. On a $p_{\max } \leq C(\delta, K, N)$. Pour $p \in\left\{1, \ldots, p_{\max }\right\}$ on note $\Delta_{p}$ l'ensemble des parties de $X$ à $p$ éléments de diamètre $\leq N$. On note $\mathbb{C}^{(X)}$ le $\mathbb{C}$-espace vectoriel formé des fonctions à support fini de $X$ dans $\mathbb{C}$, dont la base canonique est notée $\left(e_{a}\right)_{a \in X}$. On choisit une orientation de chaque simplexe associé à un élément de $\Delta \backslash\{\emptyset\}$, ce qui permet de noter, pour tout $p \in\left\{1, \ldots, p_{\max }\right\}, \mathbb{C}^{\left(\Delta_{p}\right)}$ le sous-espace vectoriel de $\Lambda^{p}\left(\mathbb{C}^{(X)}\right)$ engendré par les $e_{a_{1}} \wedge \cdots \wedge e_{a_{p}}$ pour $\left\{a_{1}, \ldots, a_{p}\right\} \in \Delta_{p}$. Pour $S=\left\{a_{1}, \ldots, a_{p}\right\} \in \Delta_{p}$ on notera aussi $e_{S}= \pm e_{a_{1}} \wedge \cdots \wedge e_{a_{p}}$ (suivant l'orientation choisie pour ce simplexe). Ces choix d'orientation ont simplement pour but d'alléger les notations. On a $\mathbb{C}^{\left(\Delta_{1}\right)}=\mathbb{C}^{(X)}$. On note encore $\Delta_{0}=\{\emptyset\}, \mathbb{C}^{\left(\Delta_{0}\right)}=\mathbb{C}=\Lambda^{0}\left(\mathbb{C}^{(X)}\right)$ et $e_{\emptyset}=1$.

On note $\mathbb{C}^{X}$ l'espace des fonctions de $X$ dans $\mathbb{C}$, qui est le dual algébrique de $\mathbb{C}^{(X)}$. Pour tout $p \in\left\{0, \ldots, p_{\max }-1\right\}$ on note $\partial: \mathbb{C}^{\left(\Delta_{p+1}\right)} \rightarrow \mathbb{C}^{\left(\Delta_{p}\right)}$ la contraction à gauche $\operatorname{par}(\ldots, 1,1, \ldots) \in \mathbb{C}^{X}$, c'est-à-dire que pour $\left\{a_{0}, \ldots, a_{p}\right\} \in \Delta_{p+1}$, on a

$$
\partial\left(e_{a_{0}} \wedge \cdots \wedge e_{a_{p}}\right)=\sum_{i=0}^{p}(-1)^{i} e_{a_{0}} \wedge \cdots \wedge e_{a_{i-1}} \wedge e_{a_{i+1}} \wedge \cdots \wedge e_{a_{p}} .
$$

En d'autres termes $\partial: \mathbb{C}^{\left(\Delta_{1}\right)} \rightarrow \mathbb{C}^{\left(\Delta_{0}\right)}=\mathbb{C}$ est défini par $\partial\left(\sum_{a \in X} f(a) e_{a}\right)=$ $\sum_{a \in X} f(a)$ et $\left(\mathbb{C}^{\left(\Delta_{1}\right)} \stackrel{\partial}{\leftarrow} \mathbb{C}^{\left(\Delta_{2}\right)} \stackrel{\partial}{\leftarrow} \cdots \stackrel{\partial}{\leftarrow} \mathbb{C}^{\left(\Delta_{p_{\max }}\right)}\right)$ est le complexe d'homologie simpliciale. 
Le complexe suivant est exact :

$$
0 \leftarrow \mathbb{C}^{\left(\Delta_{0}\right)} \stackrel{\partial}{\leftarrow} \mathbb{C}^{\left(\Delta_{1}\right)} \stackrel{\partial}{\leftarrow} \mathbb{C}^{\left(\Delta_{2}\right)} \stackrel{\partial}{\leftarrow \cdots} \cdots \stackrel{\partial}{\leftarrow} \mathbb{C}^{\left(\Delta_{p_{\max }}\right)} \leftarrow 0 .
$$

En fait nous allons construire, pour tout point $x \in X$, un premier parametrix $H_{x}$ pour ce complexe (le même que dans [Laf02]), puis un deuxième parametrix $u_{x}$, puis un troisième parametrix $J_{x}$ qui est un mélange de $H_{x}$ et $u_{x}$, et c'est celui-là qui nous servira. Ici comme dans la suite on emploie le terme "parametrix" plutôt que "homotopie" pour éviter que ce mot ait un double sens. Pour construire l'homotopie de 1 à $\gamma$ on commencera par représenter l'image de 1 dans $\mathrm{KK}_{G, 2 s \ell+C}(\mathbb{C}, \mathbb{C})$ (avec $C$ assez grand) par l'opérateur $\partial+J_{x}$ agissant sur le complété de $\bigoplus_{p=1}^{p_{\max }} \mathbb{C}^{\left(\Delta_{p}\right)}$ pour certaines normes de Hilbert $\|\cdot\|_{\mathscr{H}_{x, s}}$ très compliquées construites au paragraphe suivant, puis on déformera l'opérateur $\partial+J_{x}$ en le conjuguant par $e^{\tau \theta_{x}^{\mathrm{b}}}$ où $\theta_{x}^{\mathrm{b}}: \bigoplus_{p=1}^{p_{\max }} \mathbb{C}^{\left(\Delta_{p}\right)} \rightarrow \bigoplus_{p=1}^{p_{\max }} \mathbb{C}^{\left(\Delta_{p}\right)}$ est défini par $\theta_{x}^{\mathrm{b}}\left(e_{S}\right)=\rho_{x}^{\mathrm{b}}(S) e_{S}$ et où $\rho_{x}^{\mathrm{b}}$ est une variante moyennée de la distance à $x$ : c'est la partie difficile de l'homotopie, qui est l'objet de ce paragraphe et du suivant. Pour $\tau$ assez grand l'opérateur $\partial+J_{x}$ conjugué par $e^{\tau \theta_{x}^{\mathrm{b}}}$ est continu sur $\bigoplus_{p=1}^{p_{\max }} \ell^{2}\left(\Delta_{p}\right)$, ce qui permet de remplacer les normes compliquées par les normes $\ell^{2}$ et d'arriver ensuite à $\gamma: c^{\prime}$ 'est la partie facile de l'homotopie, qui est traitée au paragraphe 5.

L'opérateur $J_{x}$ vérifiera les deux conditions suivantes, qui ne sont pas formulées de manière précise et servent seulement d'heuristique pour la construction de $J_{x}$. Il existe une constante $C$ telle que

(C1) $J_{x}$ rapproche de l'origine, plus précisément si $S_{0}, S_{1}, \ldots, S_{n}$ est une suite sans répétition d'éléments de $\Delta$ telle que $e_{S_{i+1}}$ apparait avec un coefficient non nul dans $\partial\left(e_{S_{i}}\right)$ ou dans $J_{x}\left(e_{S_{i}}\right)$, la suite $S_{0}, S_{1}, \ldots, S_{n}$ se rapproche de $x$ en restant à distance $\leq C$ de la réunion des géodésiques entre $x$ et les points de $S_{0}$,

(C2) $J_{x}$ est une intégrale sur un paramètre $\alpha$ d'opérateurs $J_{x, \alpha}$ tels qu'il existe des parties $Y_{x, \alpha, S}$ (pour $S \in \Delta$ ) vérifiant les propriétés suivantes :

- $Y_{x, \alpha, S}$ est une partie finie de $X$, ne dépendant que de $x, \alpha, S$, de cardinal $\leq C$, et contenant $x$ et $S$,

- tous les points de $Y_{x, \alpha, S}$ sont à distance $\leq C$ de la réunion des géodésiques entre $x$ et les points de $S$,

- les distances entre les points de $Y_{x, \alpha, S}$ sont déterminées à $C$ près par $x, \alpha, S$ (ce qui fait que le nombre de possibilités pour l'ensemble des distances entre les points de $Y_{x, \alpha, S}$ est borné par une constante)

- pour $T \in \Delta$, le coefficient de $e_{T}$ dans $J_{x, \alpha}\left(e_{S}\right)$ est nul si $T$ n'est pas inclus dans $Y_{x, \alpha, S}$ et il ne dépend que de la connaissance des distances entre les points de $Y_{x, \alpha, S}$, c'est-à-dire, plus précisément : si on se donne

$$
\begin{aligned}
& S=\left\{a_{1}, \ldots, a_{p-1}\right\}, T=\left\{b_{1}, \ldots, b_{p}\right\} \subset Y_{x, \alpha, S}, x^{\prime}, \\
& S^{\prime}=\left\{a_{1}^{\prime}, \ldots, a_{p-1}^{\prime}\right\} \text { et } T^{\prime}=\left\{b_{1}^{\prime}, \ldots, b_{p}^{\prime}\right\} \subset Y_{x^{\prime}, \alpha, S^{\prime}}
\end{aligned}
$$


tels qu'il existe une isométrie de $Y_{x, \alpha, S}$ dans $Y_{x^{\prime}, \alpha, S^{\prime}}$ qui envoie

$$
x, a_{1}, \ldots, a_{p-1}, b_{1}, \ldots, b_{p} \text { sur } x^{\prime}, a_{1}^{\prime}, \ldots, a_{p-1}^{\prime}, b_{1}^{\prime}, \ldots, b_{p}^{\prime},
$$

alors le coefficient de $e_{b_{1}^{\prime}} \wedge \cdots \wedge e_{b_{p}^{\prime}}$ dans $J_{x^{\prime}, \alpha}\left(e_{a_{1}^{\prime}} \wedge \cdots \wedge e_{a_{p-1}^{\prime}}\right)$ est égal au coefficient de $e_{b_{1}} \wedge \cdots \wedge e_{b_{p}}$ dans $J_{x, \alpha}\left(e_{a_{1}} \wedge \cdots \wedge e_{a_{p-1}}\right)$.

Dans la condition (C2) la donnée des points de $S$ sert à lever l'ambiguïté de signe sur $e_{S}$ et de même pour $T, S^{\prime}, T^{\prime}$.

Ces propriétés $(\mathrm{C} 1)$ et $(\mathrm{C} 2)$ serviront pour montrer la continuité de $J_{x}$. D'après la formule (31) ci-dessous, pour $f \in \mathbb{C}^{\left(\Delta_{p}\right)}$, on aura

$$
\|f\|_{\mathscr{H}_{x, s}}^{2}=\sum_{Z} \kappa_{Z}\left|\xi_{Z}(f)\right|^{2}
$$

où la somme porte sur certaines classes d'équivalences $Z$ d'uplets $\left(a_{1}, \ldots, a_{p}, Y\right)$, avec $\left\{a_{1}, \ldots, a_{p}\right\} \in \Delta$ et $Y$ une partie finie de $X$ dont tous les points sont à distance $\leq C$ de la réunion des géodésiques entre $x$ et les points de $\left\{a_{1}, \ldots, a_{p}\right\}$ (la somme sur $Z$ est infinie et le cardinal de $Y$ ne dépend que de $Z$ mais n'est pas borné indépendamment de $Z$ ). La relation d'équivalence est telle que $Z$ détermine les distances entre les points de $\{x\} \cup\left\{a_{1}, \ldots, a_{p}\right\} \cup Y$. Enfin $\kappa_{Z} \in \mathbb{R}_{+}^{*}$ est une pondération et

$$
\xi_{Z}(f)=\sum_{\left(a_{1}, \ldots, a_{p}, Y\right) \in Z} f\left(a_{1}, \ldots, a_{p}\right)
$$

où $f\left(a_{1}, \ldots, a_{p}\right)$ désigne le coefficient de $e_{a_{1}} \wedge \cdots \wedge e_{a_{p}}$ dans $f$. De plus si $Z$ est comme ci-dessus et $J_{x, \alpha}$ est comme dans (C2), ${ }^{t} J_{x, \alpha}\left(\xi_{Z}\right)$ sera une combinaison finie de formes linéaires $\xi_{Z^{\prime}}$, avec $Z^{\prime}$ une classe d'équivalence de $\left(a_{1}^{\prime}, \ldots, a_{p-1}^{\prime}, Y^{\prime}\right)$ tels qu'il existe $\left(a_{1}, \ldots, a_{p}, Y\right) \in Z$ vérifiant

$-e_{a_{1}} \wedge \cdots \wedge e_{a_{p}}$ peut apparaître dans $J_{x, \alpha}\left(e_{a_{1}^{\prime}} \wedge \cdots \wedge e_{a_{p-1}^{\prime}}\right)$, en particulier $a_{1}, \ldots, a_{p}$ sont à distance $\leq C$ de la réunion des géodésiques entre $x$ et les points de $\left\{a_{1}^{\prime}, \ldots, a_{p-1}^{\prime}\right\}$,

- $Y^{\prime}$ contient $Y \cup\left\{a_{1}, \ldots, a_{p}\right\} \cup Y_{x, \alpha,\left\{a_{1}^{\prime}, \ldots, a_{p-1}^{\prime}\right\}}$.

Donc $\|\cdot\|_{\mathscr{H}_{x, s}}^{2}$ sera choisie de telle sorte que $\mathrm{si} \xi_{Z}$ apparaît dans la formule pour $\|\cdot\|_{\mathscr{H}_{x, s}}^{2}$, ces nouvelles formes linéaires $\xi_{Z^{\prime}}$ apparaissent aussi dans la formule pour $\|\cdot\|_{\mathscr{H}_{x, s}}^{2}$. La condition (C2) fournit une constante $C^{\prime}$ telle que ${ }^{t} J_{x, \alpha}\left(\xi_{Z}\right)$ soit une combinaison d'au plus $C^{\prime}$ formes linéaires $\xi_{Z^{\prime}}$, ce qui permettra d'appliquer Cauchy-Schwarz pour majorer $\left|\xi_{Z}\left(J_{x, \alpha}(f)\right)\right|^{2}$ par une combinaison des $\left|\xi_{Z^{\prime}}(f)\right|^{2}$. Comme $J_{x}$ sera une intégrale de tels opérateurs $J_{x, \alpha}$ on montrera, en utilisant encore Cauchy-Schwarz,

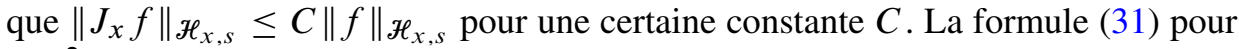
$\|\cdot\|_{\mathscr{H}_{x, s}}^{2}$ que nous donnerons plus loin est plus ou moins déterminée par la condition que si une forme linéaire $\xi_{Z}$ figure dans la formule pour $\|\cdot\|_{\mathscr{H}_{x, s}}^{2}$, les formes linéaires $\xi_{Z^{\prime}}$ servant à décomposer ${ }^{t} J_{x, \alpha}\left(\xi_{Z}\right)$ doivent apparaître dans $\|\cdot\|_{\mathscr{H}_{x, s}}^{2}$ (ainsi que la même condition pour les autres opérateurs comme $e^{\tau \theta_{x}^{\mathrm{b}}} \partial e^{-\tau \theta_{x}^{\mathrm{b}}}$ et $\left.e^{\tau \theta_{x}^{\mathrm{b}}} J_{x} e^{-\tau \theta_{x}^{\mathrm{b}}}\right)$. 
La condition (1) garantit que ce procédé ne diverge pas, en particulier que pour $f \in \mathbb{C}^{\left(\Delta_{p}\right)}, \sum_{Z} \kappa_{Z}\left|\xi_{Z}(f)\right|^{2}<\infty$.

Nous allons voir que $H_{x}$ satisfait (C1) mais pas (C2). Inversement $u_{x}$ satisfera (C2) mais pas (C1). Heureusement $J_{x}$ vérifiera (C1) et (C2).

3.1. Construction du premier parametrix $\boldsymbol{H}_{\boldsymbol{x}}$. Dans toute la suite nous aurons besoin de la notation suivante.

Définition 3.1. Si $(Y, d)$ est un espace métrique, $\varepsilon \in \mathbb{R}_{+}$, et $x, y \in Y$, on note $\varepsilon$ - géod $(x, y)$ l'ensemble des points $z$ de $Y$ tels que $d(x, z)+d(z, y) \leq d(x, y)+\varepsilon$. On note $\operatorname{géod}(x, y)$ au lieu de 0 - $\operatorname{gé} o d(x, y)$.

Donc géod $(x, y)$ désigne simplement l'ensemble des points $z$ de $Y$ tels que $d(x, z)+d(z, y)=d(x, y)$.

Les deux lemmes suivants sont valables pour tout espace métrique car ils n'utilisent que l'inégalité triangulaire.

Lemme 3.2. Soient $(Y, d)$ un espace métrique, $x, x^{\prime}, y, y^{\prime}, z, z^{\prime} \in Y$ et $\alpha \in \mathbb{R}_{+}$tels que $z \in \alpha$ - géod $(x, y)$. Alors

$$
z^{\prime} \in\left(\alpha+2 d\left(x, x^{\prime}\right)+2 d\left(y, y^{\prime}\right)+2 d\left(z, z^{\prime}\right)\right)-\operatorname{géod}\left(x^{\prime}, y^{\prime}\right) .
$$

Démonstration. Cela résulte des inégalités évidentes

$d\left(x^{\prime}, z^{\prime}\right) \leq d(x, z)+d\left(x, x^{\prime}\right)+d\left(z, z^{\prime}\right), d\left(z^{\prime}, y^{\prime}\right) \leq d(z, y)+d\left(y, y^{\prime}\right)+d\left(z, z^{\prime}\right)$

et $d\left(x^{\prime}, y^{\prime}\right) \geq d(x, y)-d\left(x, x^{\prime}\right)-d\left(y, y^{\prime}\right)$.

Lemme 3.3. Soient $(Y, d)$ un espace métrique, $x, a, b, c \in Y$ et $\alpha, \beta \in \mathbb{R}_{+}$tels que $b \in \alpha$-géod $(x, a)$ et $c \in \beta$ - $\operatorname{géod}(x, b)$. Alors

a) $c \in(\alpha+\beta)$ - $\operatorname{géod}(x, a)$,

b) $b \in(\alpha+\beta)-\operatorname{géod}(a, c)$,

c) $\operatorname{si} d(b, c) \geq \alpha+\beta, d(a, c) \geq d(a, b)$.

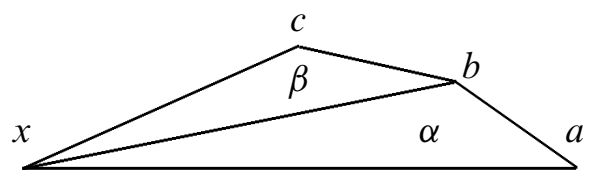

Démonstration. On a

$$
d(a, b)+d(b, c)+d(c, x) \leq d(a, b)+d(b, x)+\beta \leq d(a, x)+\alpha+\beta,
$$

d'où l'on déduit a) et b) à l'aide des inégalités triangulaires pour les triangles $a b c$ et $a c x$ respectivement. Enfin c) résulte immédiatement de b). 
Voici maintenant un lemme qui utilise le fait que $(X, d)$ est $\delta$-hyperbolique.

Lemme 3.4. Soient $x, a, b, c \in X$.

a) Soit $\beta \in \mathbb{N}$ tel que $b \in \beta$-géod $(a, c)$. Alors

$$
d(x, b) \leq \max (d(x, a)-d(a, b), d(x, c)-d(c, b))+\beta+\delta . \quad\left(H_{\delta}^{\beta}(x, a, b, c)\right)
$$

b) En particulier si b appartient à géod $(a, c)$ on $a$

$$
d(x, b) \leq \max (d(x, a)-d(a, b), d(x, c)-d(c, b))+\delta . \quad\left(H_{\delta}^{0}(x, a, b, c)\right)
$$

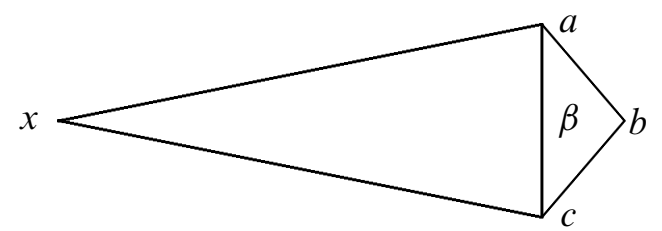

Démonstration. Le a) est une conséquence directe de la propriété d'hyperbolicité $\left(H_{\delta}(x, a, b, c)\right)$ de la définition 0.1 et b) est le cas particulier de a) où $\beta=0$.

Nous devons commencer par rappeler certains lemmes de [KS03] et [Laf02]. Comme nous avons affaire à des espaces hyperboliques et géodésiques, et non pas seulement boliques et faiblement géodésiques, les énoncés et les démonstrations des lemmes se simplifient, et nous repartirons donc de zéro. En plus nous éliminerons les paramètres $k$ et $t$ de [Laf02].

On rappelle que $\Delta$ est l'ensemble des parties de $X$ dont le diamètre est inférieur ou égal à $N$. Pour $S \in \Delta \backslash\{\varnothing\}$ on note

$$
U_{S}=\bigcap_{a \in S} B(a, N)=\{z \in X \mid\{z\} \cup S \in \Delta\} .
$$

Lemme 3.5 (cf. le lemme 6.2 de [KS03], et le lemme 2.1.4 de [Laf02]). Soient $x \in X$ et $S \in \Delta \backslash\{\emptyset\}$. Pour $z, z^{\prime} \in U_{S}$ on $a$

$$
d\left(z, z^{\prime}\right) \leq\left(d(x, z)-d\left(x, U_{S}\right)\right)+\left(d\left(x, z^{\prime}\right)-d\left(x, U_{S}\right)\right)+4 \delta .
$$

En particulier le diamètre de $\left\{z \in U_{S} \mid d(x, z) \leq d\left(x, U_{S}\right)+\delta\right\}$ est inférieur ou égal à $6 \delta$.

Grâce à $\left(H_{N}\right)$ on peut supposer $N \geq 6 \delta$, ce que l'on fait. Le lemme 3.5 implique alors que le diamètre de $\left\{z \in U_{S} \mid d(x, z) \leq d\left(x, U_{S}\right)+\delta\right\}$ est inférieur ou égal à $N$.

Démonstration. On commence par un résultat trivial. 
Lemme 3.6. Soit $x \in X, r \in \mathbb{N}, z_{1}, z_{3} \in B(x, r)$ et $z_{2} \in \operatorname{géod}\left(z_{1}, z_{3}\right)$ avec $d\left(z_{1}, z_{2}\right) \geq \delta$ et $d\left(z_{2}, z_{3}\right) \geq \delta$. Alors $z_{2} \in B(x, r)$.

Démonstration. Par $\left(H_{\delta}^{0}\left(x, z_{1}, z_{2}, z_{3}\right)\right)$ on a

$$
d\left(x, z_{2}\right) \leq \max \left(d\left(x, z_{1}\right)-d\left(z_{1}, z_{2}\right), d\left(x, z_{3}\right)-d\left(z_{2}, z_{3}\right)\right)+\delta .
$$

Fin de la démonstration du lemme 3.5. Soient $z_{1}, z_{3} \in U_{S}$ avec

$$
d\left(z_{1}, z_{3}\right) \geq\left(d\left(x, z_{1}\right)-d\left(x, U_{S}\right)\right)+\left(d\left(x, z_{3}\right)-d\left(x, U_{S}\right)\right)+4 \delta .
$$

Nous allons aboutir à une contradiction. Il existe $z_{2} \in \operatorname{géod}\left(z_{1}, z_{3}\right)$ tel que

$$
d\left(z_{1}, z_{2}\right)=\left(d\left(x, z_{1}\right)-d\left(x, U_{S}\right)\right)+2 \delta .
$$

Alors on a $d\left(z_{3}, z_{2}\right) \geq\left(d\left(x, z_{3}\right)-d\left(x, U_{S}\right)\right)+2 \delta$. D'après le lemme 3.6 , on a $z_{2} \in U_{S}$. Mais par $\left(H_{\delta}^{0}\left(x, z_{1}, z_{2}, z_{3}\right)\right)$ on a $d\left(x, z_{2}\right) \leq d\left(x, U_{S}\right)-\delta$, d'où une contradiction.

Lemme 3.7 (cf. le lemme 6.3 de [KS03] et le lemme 2.1.5 de [Laf02]). Soient $x \in X$ et $S, T \in \Delta \backslash\{\emptyset\}$. Supposons que tout point a dans la différence symétrique de $S$ et $T$ vérifie $d(x, a) \leq d\left(x, U_{S}\right)+N-5 \delta$. Alors

a) $d\left(x, U_{T}\right)=d\left(x, U_{S}\right)$,

b) pour tout b dans la différence symétrique de $U_{S}$ et $U_{T}$,

$$
d(x, b)>d\left(x, U_{S}\right)+\delta .
$$

Démonstration. Nous suivons la preuve du lemme 6.3 de [KS03]. Par récurrence sur le cardinal de la différence symétrique de $S$ et $T$ on peut supposer que la différence symétrique de $S$ et $T$ est un singleton $\{a\}$.

Supposons d'abord $a \in T$. Alors $U_{T} \subset U_{S}$. Comme $T=S \cup\{a\}$ appartient à $\Delta, a \in U_{S}$. Par le lemme 3.5, $d\left(a,\left\{z \in U_{S} \mid d(x, z) \leq d\left(x, U_{S}\right)+\delta\right\}\right) \leq N$. Donc

$$
\left\{z \in U_{S} \mid d(x, z) \leq d\left(x, U_{S}\right)+\delta\right\} \subset U_{T} \subset U_{S}
$$

et les assertions a) et b) du lemme en résultent.

Supposons maintenant $a \in S$. Alors $U_{S} \subset U_{T}$ et comme $S=T \cup\{a\}$ appartient à $\Delta, a \in U_{T}$. Il suffit de montrer a) car en échangeant les rôles de $S$ et $T$, b) en résulte. Raisonnons par l'absurde et supposons $d\left(x, U_{T}\right)<d\left(x, U_{S}\right)$. Soit $b \in U_{T}$ tel que $d(x, b)=d\left(x, U_{T}\right)$. On a alors $b \notin U_{S}$ donc $d(a, b)>N$. On rappelle que $N \geq 6 \delta$. Soit $c \in \operatorname{géod}(a, b), d(a, c)=N-3 \delta$. Par le lemme 3.6, comme $a, b \in U_{T}$, $d(a, c) \geq \delta, d(b, c) \geq \delta$ et $c \in \operatorname{géod}(a, b)$, on a $c \in U_{T}$. Mais $d(a, c) \leq N$ donc $c \in U_{S}$. Or $\left(H_{\delta}^{0}(x, a, c, b)\right)$ donne

$$
d(x, c) \leq \max (d(x, a)-d(a, c), d(x, b)-d(b, c))+\delta<d\left(x, U_{S}\right),
$$

ce qui est contradictoire. 
Pour $x \in X, S \in \Delta \backslash\{\emptyset\}$, on définit

$$
A_{S, x}=\left\{z \in U_{S} \mid d(x, z) \leq d\left(x, U_{S}\right)+\delta\right\}
$$

et pour $r \in \mathbb{N}$,

$$
Y_{S, x, r}=\left\{z \in U_{S} \mid \exists y \in \delta-\operatorname{géod}(x, z), d(x, y) \leq r, d(y, z)=d\left(y, U_{S}\right)\right\} .
$$

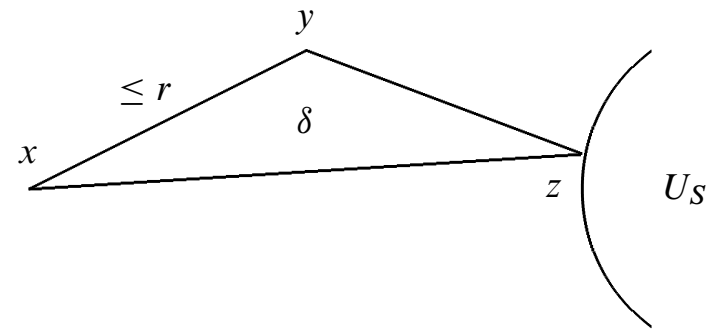

Il est clair que $r \mapsto Y_{S, x, r}$ est une application croissante, c'est-à-dire que si $r \leq r^{\prime}$ on a $Y_{S, x, r} \subset Y_{S, x, r^{\prime}}$. De plus $Y_{S, x, 0}$ est non vide. Si $r \leq d\left(x, U_{S}\right)$ on a

$$
Y_{S, x, r}=\left\{z \in U_{S} \mid \exists y \in \delta-\operatorname{géod}(x, z), d(x, y)=r, d(y, z)=d\left(y, U_{S}\right)\right\} .
$$

En effet pour $z \in Y_{S, x, r}$ et $y \in \delta$-géod $(x, z)$ vérifiant $d(x, y) \leq r$ et $d(y, z)=$ $d\left(y, U_{S}\right),\left\{d\left(x, y^{\prime}\right) \mid y^{\prime} \in \operatorname{géod}(y, z)\right\}$ est un intervalle contenant $d(x, y)$ et $d(x, z)$, et comme $d(x, y) \leq r$ et $d(x, z) \geq d\left(x, U_{S}\right) \geq r$, il existe $y^{\prime} \in \operatorname{géod}(y, z)$ tel que $d\left(x, y^{\prime}\right)=r$ et alors $d\left(y^{\prime}, z\right)=d\left(y^{\prime}, U_{S}\right)$ et $y^{\prime} \in \delta$ - $\operatorname{géod}(x, z)$ par le a $) \mathrm{du}$ lemme 3.3.

Plus tard on aura besoin des conventions : $A_{\emptyset, x}=A_{\{x\}, x}=B(x, \delta)$ et $Y_{\emptyset, x, r}=$ $Y_{\{x\}, x, r}$, qui est égal à $\{x\}$ pour $r=0$.

Cet ensemble $Y_{S, x, r}$ est très proche de celui défini par Kasparov et Skandalis dans [KS03], il sert pour l'astuce des ensembles emboîtés.

Comme le diamètre de $U_{S}$ est inférieur ou égal à $2 N$, il est clair que $U_{S}$ donc aussi $A_{S, x}$ et $Y_{S, x, r}$, ont des cardinaux bornés par $C(\delta, K, N)$. En fait le lemme 3.5 montre que le diamètre de $A_{S, x}$ est inférieur ou égal à $6 \delta$, donc son cardinal est borné $\operatorname{par} C(\delta, K)$.

Lemme 3.8. Si $r \leq d\left(x, U_{S}\right)-N$ ou si $r=0$, on a $Y_{S, x, r} \subset A_{S, x}$ et

$$
Y_{S, x, r}=\left\{z \in A_{S, x} \mid \exists y \in \delta-\operatorname{géod}(x, z), d(x, y)=r, d(y, z)=d\left(y, A_{S, x}\right)\right\} .
$$

Démonstration. Si $r=0$, c'est évident. Supposons donc $r \leq d\left(x, U_{S}\right)-N$. Soit $z \in Y_{S, x, r}$. Grâce à (3) on a $z \in U_{S}$ et il existe $y \in \delta$ - $\operatorname{géod}(x, z)$, tel que $d(x, y)=r$ et $d(y, z)=d\left(y, U_{S}\right)$. On a $d(x, z) \geq d\left(x, U_{S}\right) \geq r+N$ donc $d(y, z) \geq N$. 


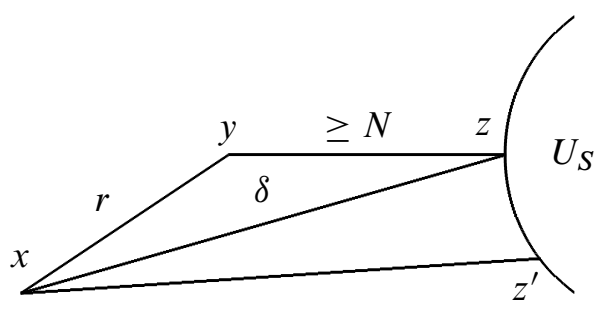

On va montrer que $d(x, z) \leq d\left(x, U_{S}\right)+\delta$. Soit $z^{\prime} \in U_{S}$. On a $d\left(z, z^{\prime}\right) \leq 2 N$ et $d\left(y, z^{\prime}\right) \geq d(y, z) \geq N$. Par $\left(H_{\delta}\left(x, y, z, z^{\prime}\right)\right)$ on a

$$
d(x, z) \leq \max \left(d\left(x, z^{\prime}\right)+d(y, z)-d\left(y, z^{\prime}\right), d(x, y)+d\left(z, z^{\prime}\right)-d\left(y, z^{\prime}\right)\right)+\delta .
$$

Mais $d\left(x, z^{\prime}\right)+d(y, z)-d\left(y, z^{\prime}\right) \leq d\left(x, z^{\prime}\right)$ et

$$
d(x, y)+d\left(z, z^{\prime}\right)-d\left(y, z^{\prime}\right) \leq r+2 N-N \leq d\left(x, U_{S}\right) .
$$

Donc $d(x, z) \leq d\left(x, z^{\prime}\right)+\delta$ pour tout $z^{\prime} \in U_{S}$. On a montré $Y_{S, x, r} \subset A_{S, x}$.

Soit maintenant $z \in A_{S, x}$ et $y \in \delta$ - $\operatorname{géod}(x, z)$ tels que $d(x, y)=r$ et $d(y, z)=$ $d\left(y, A_{S, x}\right)$. On veut montrer $d\left(y, A_{S, x}\right)=d\left(y, U_{S}\right)$. Si ce n'est pas vrai, il existe $z^{\prime} \in U_{S} \backslash A_{S, x}$ tels que $d\left(y, z^{\prime}\right)=d\left(y, U_{S}\right)$. Comme $d\left(x, z^{\prime}\right) \geq d\left(x, U_{S}\right)+\delta \geq$ $d(x, z)$ et $d\left(y, z^{\prime}\right) \leq d(y, z)$, on a $y \in \delta$ - $\operatorname{géod}\left(x, z^{\prime}\right)$. Alors $z^{\prime} \in Y_{S, x, r} \subset A_{S, x}$, ce qui amène une contradiction.

L'astuce des ensembles emboîtés repose sur le lemme suivant.

Lemme 3.9. Si $r \leq d\left(x, U_{S}\right)-d\left(x, x^{\prime}\right)-\delta$, on a $Y_{S, x, r} \subset Y_{S, x^{\prime}, r+2 d\left(x, x^{\prime}\right)+\delta}$.

Démonstration du lemme 3.9 en admettant le lemme 3.11. Soit $z \in Y_{S, x, r}$. Par hypothèse il existe $y \in \delta$ - $\operatorname{géod}(x, z), d(x, y) \leq r, d(y, z)=d\left(y, U_{S}\right)$. On a

$$
d(y, z) \geq d(x, z)-d(x, y) \geq d\left(x, U_{S}\right)-r \geq d\left(x, x^{\prime}\right)+\delta .
$$

Soit $y^{\prime} \in \operatorname{géod}(y, z), d\left(y, y^{\prime}\right)=d\left(x, x^{\prime}\right)+\delta$.

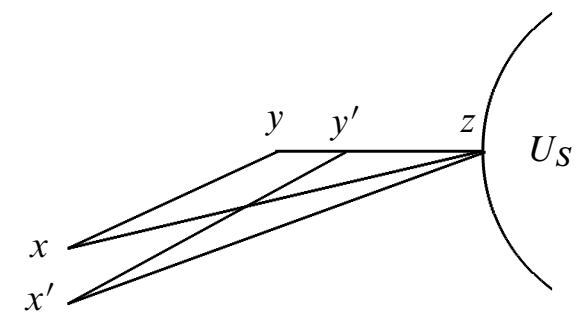


Alors $d\left(y^{\prime}, z\right)=d\left(y^{\prime}, U_{S}\right)$, et par le lemme 3.11 (avec $\varepsilon=\delta$ ) on a $y^{\prime} \in$ $\delta$ - géod $\left(x^{\prime}, z\right)$. Enfin

$$
d\left(x^{\prime}, y^{\prime}\right) \leq d\left(x^{\prime}, x\right)+d(x, y)+d\left(y, y^{\prime}\right) \leq r+2 d\left(x, x^{\prime}\right)+\delta .
$$

Lemme 3.10. Pour tout $\varepsilon>0$ et $x, z, y, y^{\prime} \in X$, si $y \in \varepsilon$-géod $(x, z), y^{\prime} \in$ géod $(y, z), d\left(y, y^{\prime}\right) \geq \varepsilon / 2$, alors $y^{\prime} \in \delta$ - $\operatorname{géod}(x, z)$.

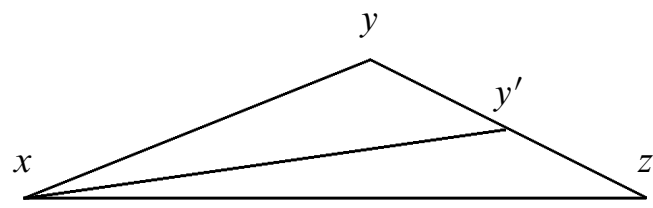

Démonstration. Par $\left(H_{\delta}^{0}\left(x, y, y^{\prime}, z\right)\right)$ on a

$$
d\left(x, y^{\prime}\right) \leq \max \left(d(x, y)-d\left(y, y^{\prime}\right), d(x, z)-d\left(z, y^{\prime}\right)\right)+\delta .
$$

D'où $d\left(x, y^{\prime}\right)+d\left(y^{\prime}, z\right) \leq \max \left(d(x, y)-d\left(y, y^{\prime}\right)+d\left(y^{\prime}, z\right), d(x, z)\right)+\delta$. Or

$$
\begin{aligned}
d(x, y)-d\left(y, y^{\prime}\right)+d\left(y^{\prime}, z\right) & =(d(x, y)+d(y, z))-2 d\left(y, y^{\prime}\right) \\
& \leq d(x, z)+\varepsilon-2 d\left(y, y^{\prime}\right) .
\end{aligned}
$$

Donc $d\left(x, y^{\prime}\right)+d\left(y^{\prime}, z\right) \leq d(x, z)+\delta$.

Lemme 3.11. Pour tous $\varepsilon>0, x, x^{\prime}, z, y, y^{\prime} \in X$, si $y \in \varepsilon$-géod $(x, z), y^{\prime} \in$ $\operatorname{géod}(y, z), d\left(y, y^{\prime}\right) \geq \varepsilon / 2+d\left(x, x^{\prime}\right)$, alors $y^{\prime} \in \delta-\operatorname{géod}\left(x^{\prime}, z\right)$.

Démonstration. D'après le lemme 3.2, $y \in\left(\varepsilon+2 d\left(x, x^{\prime}\right)\right)$ - géod $\left(x^{\prime}, z\right)$. On applique alors le lemme 3.10 à $\left(x^{\prime}, z, y, y^{\prime}\right)$ au lieu de $\left(x, z, y, y^{\prime}\right)$ et $\varepsilon+2 d\left(x, x^{\prime}\right)$ au lieu de $\varepsilon$.

Dans [KS03] Kasparov et Skandalis définissent une mesure $\psi_{S, x}$ de masse 1 en normalisant une moyenne paramétrée par $r$ des fonctions caractéristiques de $Y_{S, x, r}$. Nous allons faire de même, à ceci près que nous prendrons plutôt une moyenne sur $r$ de la fonction caractéristique de $Y_{S, x, r}$ normalisée. Pour tout ensemble non vide $A$ on note $v_{A}=\frac{1}{\sharp A} \chi_{A}$, où $\chi_{A}$ est la fonction caractéristique de $A$.

On pose alors

$$
\psi_{S, x}=\frac{1}{\max \left(1, d\left(x, U_{S}\right)-N\right)} \sum_{r=0}^{\max \left(0, d\left(x, U_{S}\right)-N-1\right)} v_{Y, x, r} .
$$

D'après le lemme 3.8 le support de $\psi_{S, x}$ est inclus dans $A_{S, x}$. 
Plus loin nous aurons besoin de la notation suivante : on définit

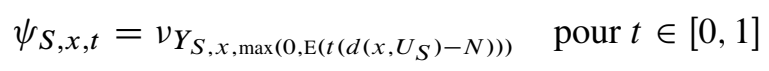

de sorte que $\psi_{S, x}=\int_{0}^{1} \psi_{S, x, t} d t$. Dans tout cet article on note $\mathrm{E}(\cdot)$ la partie entière.

Lemme 3.12. Pour tout $t \in[0,1]$, le support de $\psi_{S, x, t}$ est inclus dans $A_{S, x}$.

Démonstration. Cela résulte immédiatement du lemme 3.8.

Pour $r \in\left\{0, \ldots, \max \left(0, d\left(x, U_{S}\right)-N\right)\right\}$, on a, grâce au lemme 3.8,

$$
Y_{S, x, r}=\left\{z \in A_{S, x} \mid \exists y \in \delta-\operatorname{géod}(x, z), d(x, y)=r, d(y, z)=d\left(y, A_{S, x}\right)\right\} .
$$

Donc $Y_{S, x, r}$ ne dépend que de la connaissance des points de

$$
S \cup A_{S, x} \cup\left\{y \mid \exists z \in A_{S, x}, y \in \delta-\operatorname{géod}(x, z), d(x, y)=r\right\} \cup\{x\}
$$

et des distances mutuelles entre tous ces points. De façon plus précise, si $x^{\prime}$ et $S^{\prime}$ sont tels qu'il existe une isométrie de (4) vers l'ensemble correspondant pour $x^{\prime}$ et $S^{\prime}$, qui envoie $x$ et $S$ sur $x^{\prime}$ et $S^{\prime}$, alors $Y_{S^{\prime}, x^{\prime}, r} \subset A_{S^{\prime}, x^{\prime}}$ est l'image de $Y_{S, x, r} \subset A_{S, x}$ par cette isométrie.

En anticipant un peu justifions l'intérêt de cette propriété. D'après la démonstration du lemme 3.20, l'ensemble (4) est inclus dans l'ensemble figurant dans l'énoncé du lemme 3.20 et on déduit du lemme 3.13 que le cardinal de (4) est borné par une constante $C(\delta, K, N)$, et de plus la connaissance de $r$ et de $d\left(x, U_{S}\right)$ détermine les distances entre les points de (4) à une constante $C(\delta, N)$ près. Donc connaissant $r$ et $d\left(x, U_{S}\right)$, il n'y a qu'un nombre fini, borné par $C(\delta, K, N)$, de possibilités pour la donnée de toutes les distances entre les points de (4). Enfin $\psi_{S, x}$ est une moyenne entre 0 et $\max \left(0, d\left(x, U_{S}\right)-N-1\right)$ de $v_{Y_{S, x, r}}$. Au contraire avec la définition de Kasparov et Skandalis, où la normalisation est faite après la moyennne, le calcul de $\psi_{S, x}$ nécessiterait la connaissance simultanée de tous les points $y$ tels qu'il existe $z \in A_{S, x}$ vérifiant $y \in \delta$ - $\operatorname{géod}(x, z)$.

Le lemme suivant, que nous venons d'utiliser, servira à de nombreuses reprises.

Lemme 3.13. Pour tout $r \in \mathbb{N}$ il existe $C$ dépendant seulement de $\delta, K$ et $r$ tel que pour $x, y \in X$, on ait,

- pour tout $l \in\{0, \ldots, d(x, y)+r\}, \sharp\{z \in r-\operatorname{géod}(x, y) \mid d(x, z)=l\} \leq C$,

- et $\sharp(r-\operatorname{géod}(x, y)) \leq C(d(x, y)+1)$.

Démonstration. Pour $x, y \in X$ on pose $d=d(x, y)$ et on choisit $x_{0}=x$, $x_{1}, \ldots, x_{d}=y$ des points de géod $(x, y)$ tels que $d\left(x, x_{i}\right)=i$. Soit $z \in r-\operatorname{géod}(x, y)$ et $i=\min (d, d(x, z))$. Alors $\operatorname{par}\left(H_{\delta}^{0}\left(z, x, x_{i}, y\right)\right)$ on a

$$
d\left(z, x_{i}\right) \leq \max \left(d(z, x)-d\left(x, x_{i}\right), d(z, y)-d\left(y, x_{i}\right)\right)+\delta \leq r+\delta
$$


$\operatorname{car} d\left(x, x_{i}\right)=i, d\left(x_{i}, y\right)=d-i, d(x, z) \in[i, i+r]$ et $d(z, y) \in[d-i, d-i+r]$. On pose $C=1+K+K^{2}+\cdots+K^{r+\delta}$. Alors $\max _{x \in X} \sharp B(x, r+\delta) \leq C$. On voit que la première assertion est vraie et pour la deuxième assertion on remarque que $r-\operatorname{géod}(x, y) \subset \bigcup_{i=0}^{d} B\left(x_{i}, r+\delta\right)$.

Par l'astuce des ensembles emboîtés nous allons montrer que pour $x, x^{\prime} \in X$, $\left\|\psi_{S, x}-\psi_{S, x^{\prime}}\right\|_{1}$ tend vers 0 en dehors des parties finies de $\Delta$ (où $\|\cdot\|_{1}$ désigne la masse totale d'une mesure). Cela résulte du lemme suivant qui est plus fort.

Lemme 3.14. Il existe $C=C(\delta, K, N)$ tel que la mesure de l'ensemble des $t \in[0,1]$ tels que $\psi_{S, x, t} \neq \psi_{S, x^{\prime}, t}$ soit $\leq \frac{C d\left(x, x^{\prime}\right)}{1+d(x, S)}$.

Démonstration. Le lemme est vrai si $x=x^{\prime}$, donc on suppose $d\left(x, x^{\prime}\right) \geq 1$. On prendra $C \geq 2 N+2$ si bien que $\frac{C d\left(x, x^{\prime}\right)}{1+d(x, S)} \geq 1$ si $d(x, S) \leq d\left(x, x^{\prime}\right)+2 N$. Donc on suppose

$$
d(x, S) \geq d\left(x, x^{\prime}\right)+2 N+1,
$$

et il suffit de montrer le lemme sous cette hypothèse.

On a alors

$$
\min \left(d\left(x, U_{S}\right)-N, d\left(x^{\prime}, U_{S}\right)-N\right) \geq d(x, S)-2 N-d\left(x, x^{\prime}\right) \geq 1 .
$$

Comme le cardinal de $Y_{S, x, r}$ est non nul et borné par une constante $C_{0}=$ $C(\delta, K, N)$, et que l'application $r \mapsto Y_{S, x, r}$ est croissante, l'intervalle

$$
\left[0, d\left(x, U_{S}\right)-N-1\right]
$$

se découpe en au plus $C_{0}$ intervalles où l'application $r \mapsto Y_{S, x, r}$ est constante. Il résulte du lemme 3.9 que l'application $r \mapsto Y_{S, x^{\prime}, r}$ coïncide avec la précédente sur des intervalles (éventuellement vides) obtenus à partir des précédents en raccourcissant chaque extrémité de $2 d\left(x, x^{\prime}\right)+\delta$.

Il en résulte que l'application croissante

$$
t \mapsto Y_{S, x, \mathrm{E}\left(t\left(d\left(x, U_{S}\right)-N\right)\right)}
$$

prend au plus $C_{0}$ valeurs et pour chaque valeur l'image inverse est un intervalle, et l'image inverse de cette même valeur par l'application

$$
t \mapsto Y_{S, x^{\prime}, \mathrm{E}\left(t\left(d\left(x^{\prime}, U_{S}\right)-N\right)\right)}
$$

est un autre intervalle dont les extrémités diffèrent au plus de $\frac{3 d\left(x, x^{\prime}\right)+\delta+1}{d(x, S)-2 N}$ des extrémités du premier. En effet pour $t, t^{\prime} \in[0,1]$, l'inégalité

$$
\left|\mathrm{E}\left(t\left(d\left(x, U_{S}\right)-N\right)\right)-\mathrm{E}\left(t^{\prime}\left(d\left(x^{\prime}, U_{S}\right)-N\right)\right)\right| \leq 2 d\left(x, x^{\prime}\right)+\delta
$$

implique $\left|t-t^{\prime}\right| \leq \frac{3 d\left(x, x^{\prime}\right)+\delta+1}{d\left(x, U_{S}\right)-N} \operatorname{car}\left|d\left(x, U_{S}\right)-d\left(x^{\prime}, U_{S}\right)\right| \leq d\left(x, x^{\prime}\right)$ et on utilise le fait que $d\left(x, U_{S}\right)-N \geq d(x, S)-2 N$. 
On en déduit que la mesure de l'ensemble des $t \in[0,1]$ tels que $\mu_{r, t}(x, a) \neq$ $\mu_{r, t}\left(x^{\prime}, a\right)$ est inférieure ou égale à $\frac{2 C_{0}\left(3 d\left(x, x^{\prime}\right)+\delta+1\right)}{d(x, S)-2 N}$. Enfin on a

$$
\frac{2 C_{0}\left(3 d\left(x, x^{\prime}\right)+\delta+1\right)}{d(x, S)-2 N} \leq \frac{2 C_{0}(4+\delta)(2 N+2) d\left(x, x^{\prime}\right)}{1+d(x, S)}
$$

car on a supposé $d\left(x, x^{\prime}\right) \geq 1$ et $d(x, S) \geq 2 N+1$. On prend alors $C=$ $2 C_{0}(4+\delta)(2 N+2)$.

On définit un opérateur $h_{x}$ de degré $1 \operatorname{sur} \bigoplus_{p=0}^{p_{\max }} \mathbb{C}^{\left(\Delta_{p}\right)}$ par la formule suivante :

$$
h_{x}\left(e_{S}\right)=\psi_{S, x} \wedge e_{S} .
$$

Plus loin nous aurons besoin de la notation suivante : pour $t \in[0,1], h_{x, t}$ est l'opérateur défini par

$$
h_{x, t}\left(e_{S}\right)=\psi_{S, x, t} \wedge e_{S},
$$

de sorte que $h_{x}=\int_{0}^{1} h_{x, t} d t$. On note que pour tout $t \in[0,1], h_{x, t}\left(e_{\emptyset}\right)=e_{x}$.

Lemme 3.15. Il existe $C=C(\delta, K, N)$ tel que la mesure de l'ensemble des $t \in[0,1]$ tels que $\left(h_{x, t}-h_{x^{\prime}, t}\right)\left(e_{S}\right) \neq 0$ soit $\leq \frac{C d\left(x, x^{\prime}\right)}{1+d(x, S)}$.

Démonstration. Cela résulte immédiatement de (5) et du lemme 3.14.

Le lemme suivant servira tout à fait à la fin de l'article.

Lemme 3.16. On $a h_{x}^{2}=0$.

Démonstration. D'après les lemmes 3.7 et 3.12, si $e_{T}$ apparaît dans $h_{x}\left(e_{S}\right)$ on a $A_{T, x}=A_{S, x}$. Le lemme 3.8 montre alors que $\psi_{T, x}=\psi_{S, x}$.

Nous voulons montrer que l'opérateur $\partial h_{x}+h_{x} \partial$ est inversible de $\bigoplus_{p=0}^{p_{\max }} \mathbb{C}^{\left(\Delta_{p}\right)}$ dans lui-même. En effet $H_{x}=h_{x}\left(\partial h_{x}+h_{x} \partial\right)^{-1}$ sera alors un parametrix pour $\partial$, c'est-à-dire que l'on aura $\partial H_{x}+H_{x} \partial=1$. Pour cela nous introduisons, comme dans le paragraphe 2.5.1 de [Laf02], la "distance moyenne tronquée de $S$ à $x$ " :

$$
\zeta_{x}(S)=\frac{1}{p_{\max }}\left(\sum_{a \in S} \max \left(d\left(x, U_{S}\right), d(x, a)-\delta\right)+\left(p_{\max }-\sharp S\right) d\left(x, U_{S}\right)\right),
$$

si $S \neq \emptyset$ et $\zeta_{x}(\emptyset)=0$. Cette distance est tronquée au sens où tous les points de $S$ dont la distance à $x$ est comprise entre $d\left(x, U_{S}\right)$ et $d\left(x, U_{S}\right)+\delta$ contribuent de la même façon. On a clairement $d\left(x, U_{S}\right) \leq \zeta_{x}(S) \leq d\left(x, U_{S}\right)+N-\delta$.

Lemme 3.17. Pour tout $S \in \Delta$ on a

$$
\begin{gathered}
\partial\left(e_{S}\right) \in \underset{T \text { tel que } \zeta_{x}(T) \leq \zeta_{x}(S)}{\bigoplus_{T}} \mathbb{C} e_{T}, \quad h_{x}\left(e_{S}\right) \in \underset{T \text { tel que } \zeta_{x}(T) \leq \zeta_{x}(S)}{\bigoplus} \mathbb{C} e_{T}, \\
\left(1-\partial h_{x}-h_{x} \partial\right)\left(e_{S}\right) \in \underset{T \text { tel que } \zeta_{x}(T)<\zeta_{x}(S)-\frac{N-6 \delta}{p_{\max }}}{\mathbb{C} e_{T} .}
\end{gathered}
$$


Démonstration. La première assertion est évidente. La deuxième assertion est vraie car le support de $\psi_{S, x}$ est inclus dans $A_{S, x}$, en vertu du lemme 3.12 et pour $T=$ $S \cup\{a\}$ avec $a \in A_{S, x}$ on a $d\left(x, U_{T}\right)=d\left(x, U_{S}\right)$ d'après le lemme 3.7. Pour montrer la dernière assertion on remarque que

$$
\left(1-\partial h_{x}-h_{x} \partial\right)\left(e_{S}\right)=\sum_{a \in S} \pm\left(\psi_{S, x}-\psi_{S \backslash\{a\}, x}\right) \wedge e_{S \backslash\{a\}}
$$

Si $d(x, a) \leq d\left(x, U_{S}\right)+N-5 \delta$, on a $d\left(x, U_{S \backslash\{a\}}\right)=d\left(x, U_{S}\right)$ et $A_{S \backslash\{a\}, x}=A_{S, x}$ par le lemme 3.7, d'où $\psi_{S \backslash\{a\}, x}=\psi_{S, x}$ par le lemme 3.8. Si $d(x, a)>d\left(x, U_{S}\right)+$ $N-5 \delta$, comme les supports de $\psi_{S, x}$ et $\psi_{S \backslash\{a\}, x}$ sont inclus dans $A_{S, x}$ et $A_{S \backslash\{a\}, x}$, $\left(\psi_{S, x}-\psi_{S \backslash\{a\}, x}\right) \wedge e_{S \backslash\{a\}}$ est une combinaison de $e_{S \backslash\{a\} \cup\{b\}}$ avec

$$
d(x, b) \leq \max \left(d\left(x, U_{S \backslash\{a\}}\right)+\delta, d\left(x, U_{S}\right)+\delta\right)=d\left(x, U_{S}\right)+\delta,
$$

ce qui fait que $\zeta_{x}(S \backslash\{a\} \cup\{b\})<\zeta_{x}(S)-\frac{N-6 \delta}{p_{\max }}$.

Définition 3.18. Pour $p \in\left\{1, \ldots, p_{\max }\right\}$ et $f=\sum_{S \in \Delta_{p}} f(S) e_{S}$, on note

$$
\operatorname{supp}(f)=\bigcup_{S \text { tel que } f(S) \neq 0} S .
$$

On a $h_{x}=\int_{0}^{1} h_{x, t} d t$ et les lemmes suivants permettent, pour $t \in[0,1]$ et $S \in \Delta$, d'estimer le support de $h_{x, t}\left(e_{S}\right)$ et de savoir de quoi $h_{x, t}\left(e_{S}\right)$ dépend.

Lemme 3.19. Soit $x \in X$ et $S \in \Delta \backslash\{\emptyset\}$.

a) Pour tout $t \in[0,1]$ le support de $h_{x, t}\left(e_{S}\right)$ est inclus dans $S \cup A_{S, x}$.

b) On a

$$
\left.\left.A_{S, x} \subset\left(B(x, 2 \delta) \cap U_{S}\right) \cup \underset{\substack{a \in S \\ a \notin B(x, 2 \delta)}}{\bigcup}\{y \in 3 \delta-\operatorname{géod}(x, a) \mid d(y, a) \in] N-2 \delta, N\right]\right\} .
$$

En particulier on a toujours $A_{S, x} \subset \bigcup_{a \in S} 4 \delta$ - géod $(x, a)$.

Démonstration. Comme $h_{x, t}\left(e_{S}\right)=\psi_{S, x, t} \wedge e_{S}$, le a) résulte du lemme 3.12. Pour montrer le b) on rappelle que $A_{S, x}=\left\{y \in U_{S} \mid d(x, y) \leq d\left(x, U_{S}\right)+\delta\right\}$. Soit $y \in A_{S, x}$ n'appartenant pas à $B(x, 2 \delta)$. Soit $z \in \operatorname{géod}(x, y)$ tel que $d(y, z)=2 \delta$. Comme $d\left(x, U_{S}\right) \geq d(x, y)-\delta$ et $d(x, z)=d(x, y)-2 \delta$, on a $z \notin U_{S}$. Donc il existe $a \in S$ tel que $d(a, z)>N$. 


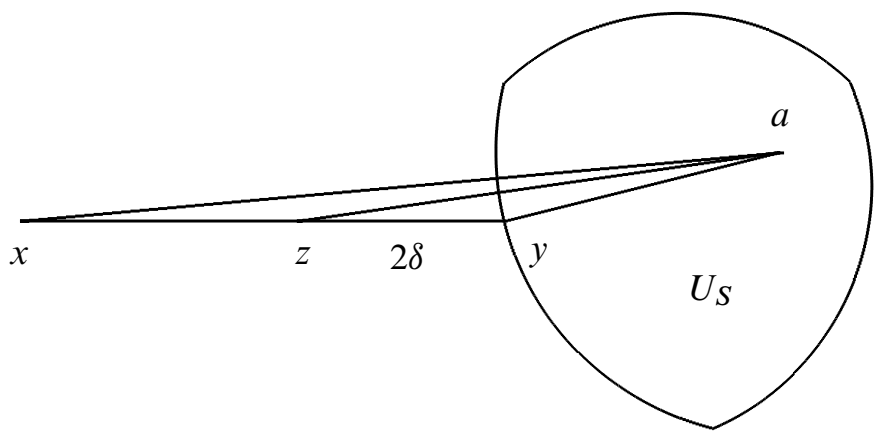

Comme $d(y, z)=2 \delta$, on a $d(a, y)>N-2 \delta$. Ensuite $\left(H_{\delta}^{0}(a, x, z, y)\right)$ donne

$$
d(a, z) \leq \max (d(a, y)-2 \delta, d(a, x)-d(x, y)+2 \delta)+\delta .
$$

Comme $d(a, z)>N$ et $d(a, y) \leq N$ on a nécessairement

$$
d(a, z) \leq d(a, x)-d(x, y)+3 \delta,
$$

et comme $d(a, y) \leq N<d(a, z)$ on en déduit $y \in 3 \delta$ - $\operatorname{géod}(x, a)$. Par (6) on a aussi

$$
d(x, a) \geq d(x, y)+N-3 \delta \geq N-3 \delta>2 \delta
$$

et $a \notin B(x, 2 \delta)$. La seconde assertion de b) résulte immédiatement de la première : comme $S$ est non vide, $B(x, 2 \delta) \subset \bigcup_{a \in S} 4 \delta$ - $\operatorname{géod}(x, a)$.

Lemme 3.20. Pour $t \in[0,1]$ et $S \in \Delta, h_{x, t}\left(e_{S}\right)$ ne dépend que de la connaissance des points de

$$
\begin{aligned}
\left.\left.B(x, 2 \delta) \cup S \cup \bigcup_{a \in S}\{y \in 3 \delta-\operatorname{géod}(x, a) \mid d(y, a) \in] N-2 \delta, N\right]\right\} \\
\cup \bigcup_{a \in S}\{y \in 5 \delta-\operatorname{géod}(x, a) \mid d(x, y) \in[t d(x, S)-2 N-1, t d(x, S)]\}
\end{aligned}
$$

et des distances entre tous ces points. Plus précisément, si on note $S=\left\{a_{1}, \ldots, a_{p-1}\right\}$, si $T=\left\{b_{1}, \ldots, b_{p}\right\}$ est inclus dans (7) (sans quoi le coefficient de e $e_{T}$ dans $h_{x, t}\left(e_{S}\right)$ est nul), et si $x^{\prime} \in X, S^{\prime}=\left\{a_{1}^{\prime}, \ldots, a_{p-1}^{\prime}\right\}$ et $T^{\prime}=\left\{b_{1}^{\prime}, \ldots, b_{p}^{\prime}\right\}$ sont tels qu'il existe une isométrie de (7) dans l'ensemble correspondant pour $x^{\prime}$ et $S^{\prime}$, qui envoie $x, a_{1}, \ldots, a_{p-1}, b_{1}, \ldots, b_{p}$ sur $x^{\prime}, a_{1}^{\prime}, \ldots, a_{p-1}^{\prime}, b_{1}^{\prime}, \ldots, b_{p}^{\prime}$, alors le coefficient de $e_{b_{1}} \wedge \cdots \wedge e_{b_{p}}$ dans $h_{x, t}\left(e_{a_{1}} \wedge \cdots \wedge e_{a_{p-1}}\right)$ est égal au coefficient de $e_{b_{1}^{\prime}} \wedge \cdots \wedge e_{b_{p}^{\prime}}$ dans $h_{x^{\prime}, t}\left(e_{a_{1}^{\prime}} \wedge \cdots \wedge e_{a_{p-1}^{\prime}}\right)$.

Démonstration. On rappelle que $\psi_{S, x, t}=v_{Y_{S, x, r}}$ avec

$$
r=\max \left(0, \mathrm{E}\left(t\left(d\left(x, U_{S}\right)-N\right)\right)\right)
$$

et que, d'après le lemme 3.8 ,

$$
Y_{S, x, r}=\left\{z \in A_{S, x} \mid \exists y \in \delta-\operatorname{géod}(x, z), d(x, y)=r, d(y, z)=d\left(y, A_{S, x}\right)\right\} .
$$


Donc $Y_{S, x, r}$ ne dépend que de la connaissance des points de

$$
S \cup A_{S, x} \cup\left\{y \mid \exists z \in A_{S, x}, y \in \delta-\operatorname{géod}(x, z), d(x, y)=r\right\} \cup\{x\}
$$

et des distances entre tous ces points. D'après le lemme 3.19,

$$
\left.\left.A_{S, x} \subset B(x, 2 \delta) \cup \bigcup_{a \in S}\{y \in 3 \delta-\operatorname{géod}(x, a) \mid d(y, a) \in] N-2 \delta, N\right]\right\} .
$$

Soit maintenant $z \in A_{S, x}$ et $y \in \delta$ - $\operatorname{géod}(x, z)$ tel que $d(x, y)=r$. D'après le lemme 3.19, il existe $a \in S$ tel que $z \in 4 \delta$ - $\operatorname{géod}(x, a)$. Or $y \in \delta$-géod $(x, z)$ et $z \in 4 \delta$-géod $(x, a)$ impliquent $y \in 5 \delta$-géod $(x, a)$ par le a) du lemme 3.3. Comme $d\left(x, U_{S}\right) \in[d(x, S)-N, d(x, S)]$ on a $r \in[t d(x, S)-2 N-1, t d(x, S)]$.

Le lemme suivant est un lemme général sur les espaces hyperboliques, qui généralise le lemme 3.10 .

Lemme 3.21. Soient $\alpha, \beta \in \mathbb{N}$ et $x, a, b, c \in X$ tels que $b \in \alpha$-géod $(a, x), c \in$ $\beta$ - géod $(b, x)$. Alors

$$
c \in(\max (\alpha+2 \beta-2 d(b, c), \beta)+\delta)-\operatorname{géod}(a, x),
$$

en particulier $c \in(\beta+\delta)-\operatorname{géod}(a, x)$ si $d(b, c) \geq \frac{\alpha+\beta}{2}$.

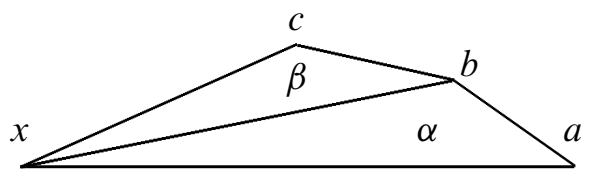

Démonstration. En effet $\left(H_{\delta}^{\beta}(a, b, c, x)\right)$ s'écrit

$$
d(a, c) \leq \max (d(a, b)-d(b, c), d(a, x)-d(x, c))+\beta+\delta,
$$

d'où l'on déduit

$d(a, c)+d(c, x)-d(a, x) \leq \max (d(a, b)-d(b, c)+d(c, x)-d(a, x), 0)+\beta+\delta$

et d'autre part

$$
d(a, b)+d(b, c)+d(c, x) \leq d(a, b)+d(b, x)+\beta \leq d(a, x)+\alpha+\beta .
$$

Nous allons montrer qu'en appliquant $\partial$ et $h_{x}$ de façon répétée à $e_{S}$ (pour $S \in$ $\Delta \backslash\{\emptyset\}$ ), on reste à distance bornée de la réunion des géodésiques reliant $x$ aux points de $S$.

Lemme 3.22. Soit $n \in \mathbb{N}$ et $S_{0}, \ldots, S_{n}$ une suite d'éléments de $\Delta$ telle que pour tout $i, e_{S_{i+1}}$ apparaît avec un coefficient non nul dans $\partial\left(e_{S_{i}}\right)$ ou dans $h_{x}\left(e_{S_{i}}\right)$. Alors pour tout point $y_{n} \in S_{n}$ n'appartenant ni à $B(x, 2 \delta)$ ni à $S_{0}$, il existe $y_{0} \in S_{0}$ $n$ 'appartenant pas à $B(x, 2 \delta)$ tel que $y_{n} \in 4 \delta-\operatorname{géod}\left(x, y_{0}\right)$ et $d\left(y_{0}, y_{n}\right)>N-2 \delta$. 
Démonstration. D'après le lemme 3.19 il existe $y_{n-1} \in S_{n-1}, \ldots, y_{0} \in S_{0}$ n'appartenant pas à $B(x, 2 \delta)$ tels que $y_{i}=y_{i+1}$ ou bien

$$
y_{i+1} \in 3 \delta-\operatorname{géod}\left(x, y_{i}\right) \quad \text { et } d\left(y_{i}, y_{i+1}\right)>N-2 \delta .
$$

Par récurrence sur $i \in\{1, \ldots, n\}$ on montre $y_{i} \in 4 \delta$ - $\operatorname{géod}\left(x, y_{0}\right)$ en appliquant le lemme 3.21 à $\left(y_{0}, y_{i-1}, y_{i}\right)$ au lieu de $(a, b, c)$ et $(4 \delta, 3 \delta)$ au lieu de $(\alpha, \beta)$ et en utilisant le fait que $N-2 \delta \geq 7 \delta / 2$. On suppose $N \geq 9 \delta$, ce qui est permis $\operatorname{par}\left(H_{N}\right)$. D'après le c) du lemme 3.3 appliqué à $\left(y_{0}, y_{i}, y_{i+1}\right)$ au lieu de $(a, b, c)$ et à $(4 \delta, 3 \delta)$ au lieu de $(\alpha, \beta)$, on a $d\left(y_{0}, y_{i}\right) \leq d\left(y_{0}, y_{i+1}\right)$ pour tout $i$, et comme $d\left(y_{0}, y_{i}\right)>N-2 \delta$ si $i$ est le plus petit entier tel que $y_{i} \neq y_{0}$ on en déduit $d\left(y_{0}, y_{n}\right)>N-2 \delta$.

Il résulte du lemme 3.20 que pour $x \in X$ et $S \in \Delta \backslash\{\emptyset\}$, la connaissance de $h_{x}\left(e_{S}\right)$ dépend seulement de celle des points de $\bigcup_{a \in S} 5 \delta$ - $\operatorname{géod}(x, a)$. Grâce au lemme 3.22, on en déduit le corollaire suivant.

Corollaire 3.23. Si $S_{0}, \ldots, S_{n}$ est une suite d'éléments de $\Delta$ telle que $S_{0} \neq \emptyset$ et que pour tout $i, e_{S_{i+1}}$ apparaît avec un coefficient $c_{i}$ non nul dans $\partial\left(e_{S_{i}}\right)$ ou dans $h_{x}\left(e_{S_{i}}\right)$, alors $S_{0} \cup \cdots \cup S_{n} \subset \bigcup_{a \in S_{0}} 4 \delta$ - géod $(x, a)$ et la connaissance de tous les $c_{i}$ ne dépend que de la connaissance des points de $\bigcup_{a \in S_{0}} 9 \delta$ - géod $(x, a)$ (et de leurs distances mutuelles).

Démonstration. Si $x, a, b, c$ sont tels que $b \in 4 \delta$ - $\operatorname{géod}(x, a)$ et $c \in 5 \delta$ - géod $(x, b)$ alors $c \in 9 \delta$ - $\operatorname{géod}(x, a)$ par le a) du lemme 3.3.

On rappelle que $\partial h_{x}+h_{x} \partial$ est inversible de $\bigoplus_{p=0}^{p_{\max }} \mathbb{C}^{\left(\Delta_{p}\right)}$ dans lui-même et que l'on a posé $H_{x}=h_{x}\left(\partial h_{x}+h_{x} \partial\right)^{-1}$.

Corollaire 3.24. Pour $p \in\left\{1, \ldots, p_{\max }\right\}$ et $S \in \Delta_{p}$, le support de $H_{x}\left(e_{S}\right)$ est inclus dans $\bigcup_{a \in S} 4 \delta-\operatorname{géod}(x, a)$ et $H_{x}\left(e_{S}\right)$ ne dépend que de la connaissance des points de $\bigcup_{a \in S} 9 \delta$ - $\operatorname{géod}(x, a)$ (et de leurs distances mutuelles).

Démonstration. C'est une conséquence immédiate du corollaire 3.23.

On note par ailleurs que $H_{x}\left(e_{\emptyset}\right)=e_{x}$.

3.2. Une conséquence de la construction de $\boldsymbol{H}_{\boldsymbol{x}}$. Pour la construction du deuxième parametrix $u_{x}$, nous aurons besoin du lemme suivant qui est une variante du lemme 2.5.6 de [Laf02]. On rappelle que pour $f=\sum_{S \in \Delta_{p}} f(S) e_{S}$, on note $\operatorname{supp}(f)=$ $\bigcup_{S \text { tel que } f(S) \neq 0} S$.

Lemme 3.25. Pour tout $p \in\left\{1, \ldots, p_{\max }\right\}$, il existe une application $G$-équivariante mais non nécessairement linéaire

$$
\Phi_{p}:\left\{f \in \mathbb{C}^{\left(\Delta_{p}\right)} \mid \partial_{p-1}(f)=0\right\} \rightarrow \mathbb{C}^{\left(\Delta_{p+1}\right)}
$$

telle que pour tout $f$ dans l'ensemble de départ de $\Phi_{p}$, 
- $\partial_{p}\left(\Phi_{p}(f)\right)=f$,

- $\Phi_{p}(\lambda f)=\lambda \Phi_{p}(f)$ pour $\lambda \in \mathbb{C}$,

- $\operatorname{supp}\left(\Phi_{p}(f)\right)$ est inclus dans $\bigcup_{y, z \in \operatorname{supp}(f)} 4 \delta$ - $\operatorname{géod}(y, z)$,

- $\Phi_{p}(f)$ ne dépend que de $f$ et de la connaissance des points de

$$
\bigcup_{y, z \in \operatorname{supp}(f)} 9 \delta-\operatorname{géod}(y, z)
$$

et de leurs distances mutuelles, autrement dit si $f^{\prime}$ est une autre fonction une isométrie de (8) vers $\bigcup_{y, z \in \operatorname{supp}\left(f^{\prime}\right)} 9 \delta$ - géod $(y, z)$ envoyant $f$ sur $f^{\prime}$ envoie $\Phi_{p}(f) \operatorname{sur} \Phi_{p}\left(f^{\prime}\right)$,

et telle que pour tout $R \in \mathbb{R}_{+}$il existe $C \in \mathbb{R}_{+}$ne dépendant que de $\delta, K, N$, $R$, tel que pour tout $f \in \mathbb{C}^{\left(\Delta_{p}\right)}$ avec $\partial_{p-1}(f)=0$ et $\operatorname{diam}(\operatorname{supp}(f)) \leq R$ on ait $\left\|\Phi_{p}(f)\right\|_{\ell^{1}\left(\Delta_{p+1}\right)} \leq C\|f\|_{\ell^{1}\left(\Delta_{p}\right)}$.

Démonstration. On donne une formule explicite pour $\Phi_{p}$, qui est la même que dans [Laf02] :

$$
\Phi_{p}(f)=\frac{1}{\sharp(\operatorname{supp}(f))} \sum_{z \in \operatorname{supp}(f)} H_{z}(f) .
$$

Pour montrer les propriétés de $\Phi_{p}$, on applique le corollaire 3.24.

Pour la suite de l'article, on fixe de telles applications $\Phi_{p}$.

3.3. Construction d'un deuxième paramétrix $\boldsymbol{u}_{\boldsymbol{x}}$. Le paramétrix $H_{x}$ ne nous convient pas car nous ne savons pas construire de normes sur $\bigoplus_{p=0}^{p_{\max }} \mathbb{C}^{\left(\Delta_{p}\right)}$ telles qu'il soit continu ainsi que $\partial$ et que $\|\pi(g)\| \leq P(\ell(g)) e^{s \ell(g)}$ pour un certain polynôme $P$. En fait nous avions déjà un problème dans [Laf02], puisqu'il avait fallu prendre l'opérateur $H_{x}$ associé à une valeur plus grande de $N$ et le modifier grâce au lemme 2.5.6. Ici le problème est encore aggravé : pour $a \in X$ le coefficient dans $H_{x}\left(e_{a}\right)$ d'une arête contenant $x$ dépend au moins de la connaissance de tous les points de $9 \delta$ - géod $(x, a)$, et le nombre de possibilités pour les distances entre tous ces points est une exponentielle en $d(x, a)$, comme le montre l'exemple suivant.

Exemple. Soit $\Gamma$ le produit libre de $\mathbb{Z} / 3 \mathbb{Z}$ avec $\mathbb{Z}$. On note $e_{1}$ et $e_{2}$ les générateurs de $\mathbb{Z} / 3 \mathbb{Z}$ et $\mathbb{Z}$. Soit $\ell$ la longueur des mots sur $\Gamma$ et soit $X=\Gamma$ muni de la distance $d(a, b)=\ell\left(a^{-1} b\right)$. Alors pour tout $a \in X$, la réunion des cycles de longueur 3 passant par $a$ contient $a e_{1}$ et $a e_{1}^{-1}$ mais ni $a e_{2}$ et $a e_{2}^{-1}$. Donc la classe d'isométrie de 98 - géod $(x, a)$ détermine l'emplacement de $e_{1}^{\mp 1}$ et $e_{2}^{\mp 1}$ dans l'écriture de $x^{-1} a$ comme mot réduit, et il y a $2^{d(x, a)}$ possibilités.

Autrement dit $H_{x}$ ne vérifie pas la condition (C2) (en revanche $H_{x}$ vérifie la condition (C1) grâce au lemme 3.17 et au corollaire 3.24). 
Nous allons maintenant construire un nouveau parametrix $u_{x}$ vérifiant la condition $(\mathrm{C} 2)$ (mais pas la condition $(\mathrm{C} 1)$ ). Nous commençons par définir, pour tous $x, a \in X$ et $r \in\{1, \ldots, d(x, a)-1\}$ une mesure $\mu_{r}(x, a)$ de masse 1 , supportée par $\{y \in \delta$ - $\operatorname{géod}(x, a) \mid d(a, y)=r\}$. D'après le lemme 3.13, le cardinal de cet ensemble est borné par une constante $C(\delta, K)$. Nous voulons que la propriété suivante soit satisfaite :

$$
\begin{aligned}
& \forall \rho>0, \forall \varepsilon>0, \exists R>0 \text {, si } d\left(x, x^{\prime}\right) \leq \rho \text { et } r \leq d(a, x)-R, \\
& \text { alors }\left\|\mu_{r}\left(x^{\prime}, a\right)-\mu_{r}(x, a)\right\|_{1} \leq \varepsilon .
\end{aligned}
$$

Dans la propriété ci-dessus, la condition que $d(a, x)-r$ est suffisamment grand est évidemment la plus faible possible, car $d(a, x)-r$ est essentiellement la distance entre $x$ et les points du support de $\mu_{r}(x, a)$ et cette distance doit nécessairement être grande pour que $\mu_{r}(x, a)$ varie peu en fonction de $x$.

On rappelle que pour toute partie $A$ non vide de $X$, on note $v_{A}$ la mesure de masse 1 égale au produit par $(\sharp A)^{-1}$ de la fonction caractéristique de $A$.

Voici la formule :

$\mu_{r}(x, a)=\frac{1}{d(a, x)-r} \sum_{k=0}^{d(a, x)-r-1} v_{A_{x, a, r, k}}$ où pour $k \leq d(a, x)-r$ on pose $A_{x, a, r, k}=\{y \mid d(a, y)=r, \exists z \in \delta-\operatorname{géod}(x, a), d(a, z)=r+k, y \in \operatorname{géod}(z, a)\}$.

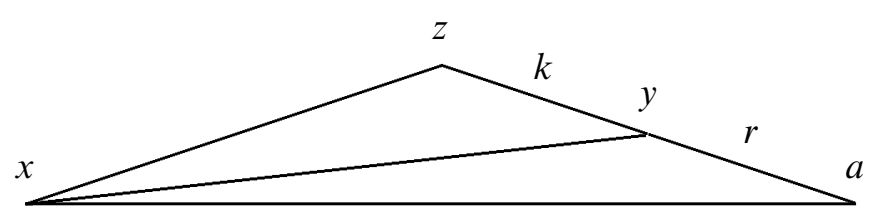

On a donc

$$
\mu_{r}(x, a)=\int_{0}^{1} \mu_{r, t}(x, a) d t \text { où } \mu_{r, t}(x, a)=v_{A_{x, a, r, \mathrm{E}(t(d(a, x)-r))}} .
$$

D'après le a) du lemme $3.3, A_{x, a, r, k} \subset\{y \in \delta$-géod $(x, a), d(a, y)=r\}$ et d'après le lemme 3.13 le cardinal de ces ensembles est borné par une constante de la forme $C(\delta, K)$. Pour tout $k \leq d(a, x)-r, A_{x, a, r, k}$ est non vide, puisque $X$ est géodésique.

Lemme 3.26. a) Pour tout $k \in\{1, \ldots, d(x, a)-r\}$ on a $A_{x, a, r, k} \subset A_{x, a, r, k-1}$.

b) Pour $x^{\prime} \in X$ et $k \in \mathbb{N}$ vérifiant $d\left(x, x^{\prime}\right)+\delta \leq k \leq d(x, a)-r$ on $a$ $A_{x, a, r, k} \subset A_{x^{\prime}, a, r, k-d\left(x, x^{\prime}\right)-\delta}$. 
Démonstration. Montrons a). Soient $y, z$ tels que

$$
d(a, y)=r, z \in \delta-\operatorname{géod}(x, a), \quad d(a, z)=r+k, y \in \operatorname{géod}(z, a) .
$$

Il existe un point $z^{\prime} \in \operatorname{géod}(y, z)$ à distance 1 de $z$. Alors $z^{\prime}$ vérifie

$$
d\left(a, z^{\prime}\right)=r+k-1, \quad y \in \operatorname{géod}\left(z^{\prime}, a\right)
$$

et $z^{\prime} \in \delta$ - géod $(x, a)$ par le a) du lemme 3.3.

Montrons b). Soient $y, z$ vérifiant (10). Comme $d(y, z)=k \geq d\left(x, x^{\prime}\right)+\delta$, il existe $z^{\prime} \in \operatorname{géod}(y, z)$ à distance $d\left(x, x^{\prime}\right)+\delta$ de $z$.

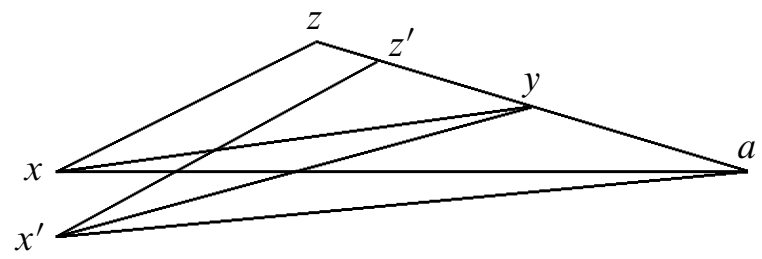

Alors $z^{\prime}$ vérifie $d\left(a, z^{\prime}\right)=r+k-d\left(x, x^{\prime}\right)-\delta, y \in \operatorname{géod}\left(z^{\prime}, a\right)$ de façon évidente et $z^{\prime} \in \delta$ - $\operatorname{géod}\left(x^{\prime}, a\right)$ d'après le lemme 3.11 appliqué à $\left(x, x^{\prime}, a, z, z^{\prime}\right)$ au lieu de $\left(x, x^{\prime}, z, y, y^{\prime}\right)$ et $\delta$ au lieu de $\varepsilon$.

La propriété (9) résulte immédiatement du lemme suivant.

Lemme 3.27. Il existe $C=C(\delta, K)$ tel que pour $a, x, x^{\prime} \in X$ et $r \in \mathbb{N}$ vérifiant $r<$ $\min \left(d(a, x), d\left(a, x^{\prime}\right)\right)$ la mesure de l'ensemble des $t \in[0,1]$ tels que $\mu_{r, t}(x, a) \neq$ $\mu_{r, t}\left(x^{\prime}, a\right)$ est $\leq \frac{C d\left(x, x^{\prime}\right)}{d(a, x)-r}$.

Démonstration. On utilise encore l'astuce des ensembles emboîtés. $\operatorname{Si} C_{0}=C(\delta, K)$ majore le cardinal des ensembles $A_{x, a, r, k}$ pour tous $a, x, r, k$, l'application décroissante $k \mapsto A_{x, a, r, k}$ de $\{0, \ldots, d(x, a)-r\}$ dans l'ensemble des parties non vides de $X$ prend au plus $C_{0}$ valeurs, et pour chaque valeur, l'image inverse est un intervalle, et l'image inverse de cette même valeur par l'application $k \mapsto A_{x^{\prime}, a, r, k}$ de $\left\{0, \ldots, d\left(x^{\prime}, a\right)-r\right\}$ dans l'ensemble des parties de $X$ est un autre intervalle dont les extrémités diffèrent au plus de $d\left(x, x^{\prime}\right)+\delta$ des extrémités du premier (il peut être vide si la longueur du premier intervalle est inférieure ou égale à $\left.2\left(d\left(x, x^{\prime}\right)+\delta\right)\right)$. Comme $\left|d(a, x)-d\left(a, x^{\prime}\right)\right| \leq d\left(x, x^{\prime}\right)$, il en résulte que l'application décroissante $t \mapsto A_{x, a, r, \mathrm{E}(t(d(a, x)-r))}$ prend au plus $C_{0}$ valeurs et pour chaque valeur l'image inverse est un intervalle, et l'image inverse de cette même valeur par l'application $t \mapsto A_{x^{\prime}, a, r, \mathrm{E}\left(t\left(d\left(a, x^{\prime}\right)-r\right)\right)}$ est un autre intervalle dont les extrémités diffèrent au plus de $\frac{2 d\left(x, x^{\prime}\right)+\delta+1}{d(a, x)-r}$ des extrémités du premier. En effet pour $t, t^{\prime} \in[0,1]$ vérifiant l'inégalité $\left|\mathrm{E}(t(d(a, x)-r))-\mathrm{E}\left(t^{\prime}\left(d\left(a, x^{\prime}\right)-r\right)\right)\right| \leq d\left(x, x^{\prime}\right)+\delta$ on a 
$\left|t-t^{\prime}\right| \leq \frac{2 d\left(x, x^{\prime}\right)+\delta+1}{d(a, x)-r}$. On en déduit que la mesure de l'ensemble des $t \in[0,1]$ tels que $\mu_{r, t}(x, a) \neq \mu_{r, t}\left(x^{\prime}, a\right)$ est inférieure ou égale à $\frac{2 C_{0}\left(2 d\left(x, x^{\prime}\right)+\delta+1\right)}{d(a, x)-r}$. Elle est donc inférieure ou égale à $\frac{2 C_{0}(\delta+3) d\left(x, x^{\prime}\right)}{d(a, x)-r}$ car elle est nulle si $x=x^{\prime}$. On peut prendre $C=2 C_{0}(\delta+3)$.

Cette formule pour $\mu_{r}(x, a)$ paraît artificiellement compliquée mais son gros avantage pour nous est que $\mu_{r}(x, a)$ est une certaine moyenne sur $k$ de $v_{A_{x, a, r, k}}$ et que $A_{x, a, r, k}$ ne dépend que de la connaissance des points de

$\{a, x\} \cup\{y \in \delta-\operatorname{géod}(x, a) \mid d(a, y)=r\} \cup\{z \in \delta-\operatorname{géod}(x, a) \mid d(a, z)=r+k\}$

et de leurs distances mutuelles. Or le nombre de ces points est borné par une constante $C(\delta, K)$ et les distances entre ces points sont égales à 0 ou $d(x, a)$ ou $d(x, a)-r$ ou $d(x, a)-r-k$ ou $r$ ou $k$ ou $r+k$ à une constante $C(\delta)$ près. Donc lorsque $d(a, x), r$ et $k$ sont fixés, le nombre de ces points et la donnée de leurs distances mutuelles n'admettent qu'un nombre fini de possibilités, borné par $C(\delta, K)$.

On pose enfin $\mu_{0, t}(x, a)=e_{a}$ et $\mu_{d(x, a), t}(x, a)=e_{x}$ pour tout $t \in[0,1]$.

Nous mettons la remarque précédente sous forme d'un lemme, qui servira ensuite.

Lemme 3.28. Pour $x, a \in X, r \in\{0, \ldots, d(a, x)\}$ et $t \in[0,1], \mu_{r, t}(x, a)$ est supporté par

$$
\{y \in \delta \text { - } \operatorname{géod}(x, a) \mid d(a, y)=r\}
$$

et dépend seulement de la connaissance des points de

$$
\begin{aligned}
&\{a, x\} \cup\{y \in \delta-\operatorname{géod}(x, a) \mid d(a, y)=r\} \\
& \cup\{z \in \delta-\operatorname{géod}(x, a) \mid d(a, z)=r+\mathrm{E}(t(d(a, x)-r))\}
\end{aligned}
$$

et de leurs distances mutuelles.

Démonstration. La preuve est incluse dans la remarque précédente.

Nous allons maintenant construire un nouveau parametrix $u_{x}$ pour $\partial$. Dans les trois pages qui suivent nous écrivons $u_{x}^{p}: \mathbb{C}^{\left(\Delta_{p-1}\right)} \rightarrow \mathbb{C}^{\left(\Delta_{p}\right)}$ au lieu de $u_{x}$ pour rendre plus claire la construction, qui se fait par récurrence sur $p$. Autrement dit on va construire des morphismes

$$
\mathbb{C}^{\left(\Delta_{0}\right)} \stackrel{u_{x}^{1}}{\longrightarrow} \mathbb{C}^{\left(\Delta_{1}\right)} \stackrel{u_{x}^{2}}{\longrightarrow} \mathbb{C}^{\left(\Delta_{2}\right)} \longrightarrow \cdots \stackrel{u_{x}^{p_{\max }}}{\longrightarrow} \mathbb{C}^{\left(\Delta_{p_{\max }}\right)}
$$

tels que $u_{x}^{p} \partial+\partial u_{x}^{p+1}=\operatorname{Id}_{\mathbb{C}^{\left(\Delta_{p}\right)}}$ pour tout $p \in\left\{0, \ldots, p_{\max }\right\}$.

En fait on aura

$$
u_{x}^{p}=\int_{t \in[0,1]} u_{x, t}^{p} d t
$$


et $u_{x, t}^{p}$ sera construit en même temps qu'une famille indéxée par $r \in \mathbb{N}$ d'endomorphismes $\left(v_{x, r, t}^{p}\right)_{p \geq 1}$ du complexe

$$
0 \leftarrow \mathbb{C}^{\left(\Delta_{0}\right)} \stackrel{\partial}{\leftarrow} \mathbb{C}^{\left(\Delta_{1}\right)} \stackrel{\partial}{\leftarrow} \mathbb{C}^{\left(\Delta_{2}\right)} \stackrel{\partial}{\leftarrow \cdots} \stackrel{\partial}{\leftarrow} \mathbb{C}^{\left(\Delta_{p_{\max }}\right)} \leftarrow 0
$$

qui consistent en gros à rapprocher de l'origine $x$ d'une longueur $r$, à l'aide des mesures $\mu_{r, t}(x, a)$. On aura $v_{x, 0, t}^{p}=\operatorname{Id}_{\mathbb{C}^{\left(\Delta_{p}\right)}}$ et quand $r$ tend vers l'infini $v_{x, r, t}^{p}: \mathbb{C}^{\left(\Delta_{p}\right)} \rightarrow$ $\mathbb{C}^{\left(\Delta_{p}\right)}$ tendra vers une limite $v_{x, \infty, t}^{p}$ égale à $\operatorname{Id}_{\mathbb{C}^{\left(\Delta_{0}\right)}}$ si $p=0$, au morphisme de rang 1 de $\mathbb{C}^{\left(\Delta_{1}\right)}$ donné par $e_{a} \mapsto e_{x}$ si $p=1$ et à 0 si $p>1$ (autrement dit $v_{x, \infty, t}^{p}$ est indépendant de $t$ et associé à la contraction de $X$ sur $x$ ). Pour $r \in \mathbb{N}^{*}$ et $p \geq 2$ on va construire $u_{x, r, t}^{p}: \mathbb{C}^{\left(\Delta_{p-1}\right)} \rightarrow \mathbb{C}^{\left(\Delta_{p}\right)}$ tel qu'en posant $u_{x, r, t}^{1}=0$ on ait $v_{x, r-1, t}^{p}-v_{x, r, t}^{p}=u_{x, r, t}^{p} \partial+\partial u_{x, r, t}^{p+1}$ pour tout $p$. On en déduira que

$$
\text { Id }-v_{x, \infty, t}^{p}=\left(\sum_{r=1}^{\infty} u_{x, r, t}^{p}\right) \partial+\partial\left(\sum_{r=1}^{\infty} u_{x, r, t}^{p+1}\right) \text { pour tout } p .
$$

On posera alors $u_{x, t}^{p}=\sum_{r=1}^{\infty} u_{x, r, t}^{p}$ pour $p \geq 2$ et $u_{x, t}^{1}\left(e_{\emptyset}\right)=e_{x}$, de sorte que l'on aura $\partial u_{x, t}^{p+1}+u_{x, t}^{p} \partial=\operatorname{Id}_{\left.\mathbb{C}^{\left(\Delta_{p}\right.}\right)}$ pour tout $p$ (grâce à (11) et au fait que $\partial u_{x, t}^{1}=v_{x, \infty, t}^{0}$ et $\left.u_{x, t}^{1} \partial=v_{x, \infty, t}^{1}\right)$.

On passe maintenant à la construction des opérateurs $u_{x}^{p}, u_{x, t}^{p}, u_{x, r, t}^{p}$ et $v_{x, r, t}^{p}$.

On définit $u_{x}^{1}: \mathbb{C}^{\left(\Delta_{0}\right)} \rightarrow \mathbb{C}^{\left(\Delta_{1}\right)}$ par $u_{x}^{1}\left(e_{\emptyset}\right)=e_{x}$ et on pose $u_{x, t}^{1}=u_{x}^{1}$.

Voici la formule pour $u_{x}^{2}: \mathbb{C}^{\left(\Delta_{1}\right)} \rightarrow \mathbb{C}^{\left(\Delta_{2}\right)}$ :

$$
u_{x}^{2}\left(e_{a}\right)=\int_{0}^{1} \sum_{r=1}^{d(x, a)} u_{x, r, t}^{2}\left(e_{a}\right) d t
$$

où

$$
u_{x, r, t}^{2}\left(e_{a}\right)=\Phi_{1}\left(\mu_{r-1, t}(x, a)-\mu_{r, t}(x, a)\right) .
$$

Il est évident que $\partial u_{x}^{2}\left(e_{a}\right)=e_{a}-e_{x}=\left(1-u_{x}^{1} \partial\right)\left(e_{a}\right)$.

Voici maintenant la formule pour $u_{x}^{3}: \mathbb{C}^{\left(\Delta_{2}\right)} \rightarrow \mathbb{C}^{\left(\Delta_{3}\right)}$. Par commodité nous étendons la fonction $r \mapsto \mu_{r, t}(x, a)$ par $\mu_{r, t}(x, a)=e_{x} \operatorname{si} r>d(x, a)$. La formule est, pour $\{a, b\} \in \Delta_{2}$ :

$$
u_{x}^{3}\left(e_{a} \wedge e_{b}\right)=\int_{0}^{1} \sum_{r=1}^{\max (d(x, a), d(x, b))} u_{x, r, t}^{3}\left(e_{a} \wedge e_{b}\right) d t
$$

où

$$
\begin{aligned}
u_{x, 1, t}^{3}\left(e_{a} \wedge e_{b}\right)= & \Phi_{2}\left(e_{a} \wedge e_{b}+\Phi_{1}\left(e_{a}-\mu_{1, t}(x, a)\right)-\Phi_{1}\left(e_{b}-\mu_{1, t}(x, b)\right)\right. \\
& \left.-\Phi_{1}\left(\mu_{1, t}(x, b)-\mu_{1, t}(x, a)\right)\right),
\end{aligned}
$$

et pour $r>1$,

$$
\begin{aligned}
u_{x, r, t}^{3}\left(e_{a}\right. & \left.\wedge e_{b}\right) \\
=\Phi_{2} & \left(\Phi_{1}\left(\mu_{r-1, t}(x, b)-\mu_{r-1, t}(x, a)\right)+\Phi_{1}\left(\mu_{r-1, t}(x, a)-\mu_{r, t}(x, a)\right)\right. \\
& \left.-\Phi_{1}\left(\mu_{r-1, t}(x, b)-\mu_{r, t}(x, b)\right)-\Phi_{1}\left(\mu_{r, t}(x, b)-\mu_{r, t}(x, a)\right)\right) .
\end{aligned}
$$


On vérifie que $\left(\partial u_{x}^{3}+u_{x}^{2} \partial\right)\left(e_{a} \wedge e_{b}\right)=e_{a} \wedge e_{b}$.

Afin de motiver la construction pour les plus grandes valeurs de $p$ on va réécrire les formules (12), (13), (14) à l'aide des opérateurs $v_{x, r, t}^{1}: \mathbb{C}^{\left(\Delta_{1}\right)} \rightarrow \mathbb{C}^{\left(\Delta_{1}\right)}$ et $v_{x, r, t}^{2}: \mathbb{C}^{\left(\Delta_{2}\right)} \rightarrow \mathbb{C}^{\left(\Delta_{2}\right)}$ définis par

$$
\begin{aligned}
v_{x, 0, t}^{1}\left(e_{a}\right) & =e_{a}, \\
v_{x, r, t}^{1}\left(e_{a}\right) & =\mu_{r, t}(x, a), \\
v_{x, 0, t}^{2}\left(e_{a} \wedge e_{b}\right) & =e_{a} \wedge e_{b}, \\
v_{x, r, t}^{2}\left(e_{a} \wedge e_{b}\right) & =\Phi_{1}\left(v_{x, r, t}^{1}\left(\partial\left(e_{a} \wedge e_{b}\right)\right)=\Phi_{1}\left(\mu_{r, t}(x, b)-\mu_{r, t}(x, a)\right) .\right.
\end{aligned}
$$

On a alors

$$
\begin{aligned}
u_{x, r, t}^{2}\left(e_{a}\right) & =\Phi_{1}\left(v_{x, r-1, t}^{1}\left(e_{a}\right)-v_{x, r, t}^{1}\left(e_{a}\right)\right), \\
u_{x, r, t}^{3}\left(e_{a} \wedge e_{b}\right) & =\Phi_{2}\left(v_{x, r-1, t}^{2}\left(e_{a} \wedge e_{b}\right)-v_{x, r, t}^{2}\left(e_{a} \wedge e_{b}\right)-u_{x, r, t}^{2}\left(\partial\left(e_{a} \wedge e_{b}\right)\right)\right) .
\end{aligned}
$$

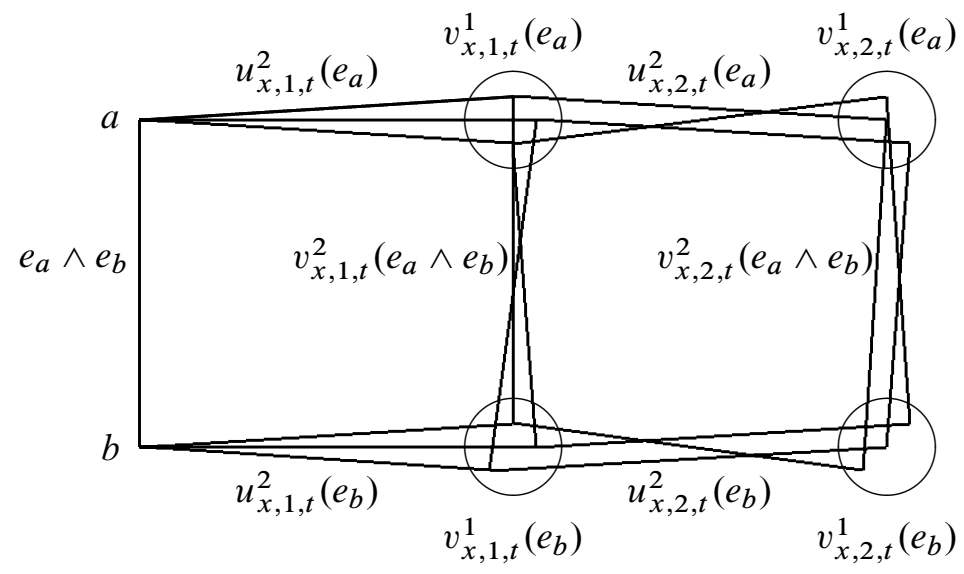

Le dessin illustre les calculs précédents. Les 4 petits cercles contiennent les supports de $v_{x, r, t}^{1}\left(e_{a}\right)=\mu_{r, t}(x, a)$ et $v_{x, r, t}^{1}\left(e_{b}\right)=\mu_{r, t}(x, b)$ pour $r=1,2$ (en réalité ces supports ne seront pas disjoints mais on l'a supposé pour la clarté du dessin). Les six éléments $v_{x, r, t}^{2}\left(e_{a} \wedge e_{b}\right), u_{x, r, t}^{2}\left(e_{a}\right), u_{x, r, t}^{2}\left(e_{b}\right)$ (pour $\left.r=1,2\right)$ sont des combinaisons d'arêtes comme il est indiqué sur le dessin. Enfin les deux éléments $u_{x, r, t}^{3}\left(e_{a} \wedge e_{b}\right)$ (pour $r=1,2$ ), qui ne sont pas représentés sur le dessin, sont des combinaisons de triangles remplissant les deux grands carrés.

En général on définit $u_{x}^{p}: \mathbb{C}^{\left(\Delta_{p-1}\right)} \rightarrow \mathbb{C}^{\left(\Delta_{p}\right)}$ pour $p \in\left\{1, \ldots, p_{\max }\right\}$ par récurrence ascendante sur $p$. La récurrence part de $p=1$ et fournit les formules ci-dessus 
pour $p=2,3$. On construit des applications linéaires

$$
u_{x, r, t}^{p}: \mathbb{C}^{\left(\Delta_{p-1}\right)} \rightarrow \mathbb{C}^{\left(\Delta_{p}\right)} \quad \text { pour } r \in \mathbb{N}^{*} \text { et } t \in[0,1]
$$

et

$$
v_{x, r, t}^{p}: \mathbb{C}^{\left(\Delta_{p}\right)} \rightarrow \mathbb{C}^{\left(\Delta_{p}\right)} \quad \text { pour } r \in \mathbb{N} \text { et } t \in[0,1]
$$

telles que $u_{x, r, t}^{p}\left(e_{a_{1}} \wedge \cdots \wedge e_{a_{p-1}}\right)$ soit nul si $r>\max \left(d\left(x, a_{1}\right), \ldots, d\left(x, a_{p-1}\right)\right)$ et $p \geq 2$ et $v_{x, r, t}^{p}\left(e_{a_{1}} \wedge \cdots \wedge e_{a_{p}}\right)$ soit nul si $r \geq \max \left(d\left(x, a_{1}\right), \ldots, d\left(x, a_{p}\right)\right)$ et $p \geq 2$. Ces applications sont définies par

$$
v_{x, 0, t}^{p}=\operatorname{Id}_{\mathbb{C}^{(\Delta p)}} \quad \text { pour } p \geq 1,
$$

et pour $r \geq 1$, par récurrence ascendante sur $p$ à l'aide des formules suivantes :

$$
u_{x, r, t}^{1}=0, \quad v_{x, r, t}^{1}\left(e_{a}\right)=\mu_{r, t}(x, a) \quad \text { pour } r \geq 1
$$

pour initialiser et

$$
\begin{aligned}
v_{x, r, t}^{p}\left(e_{a_{1}} \wedge \cdots \wedge e_{a_{p}}\right)= & \Phi_{p-1}\left(v_{x, r, t}^{p-1}\left(\partial\left(e_{a_{1}} \wedge \cdots \wedge e_{a_{p}}\right)\right)\right) \quad \text { pour } r \geq 1 \text { et } p \geq 2 \\
u_{x, r, t}^{p}\left(e_{a_{1}} \wedge \cdots \wedge e_{a_{p-1}}\right)= & \Phi_{p-1}\left(v_{x, r-1, t}^{p-1}\left(e_{a_{1}} \wedge \cdots \wedge e_{a_{p-1}}\right)\right. \\
& -v_{x, r, t}^{p-1}\left(e_{a_{1}} \wedge \cdots \wedge e_{a_{p-1}}\right) \\
& \left.-u_{x, r, t}^{p-1}\left(\partial\left(e_{a_{1}} \wedge \cdots \wedge e_{a_{p-1}}\right)\right)\right) \quad \text { pour } r \geq 1 \text { et } p \geq 2 .
\end{aligned}
$$

Notons que ces formules impliquent les deux égalités

$$
\partial \circ v_{x, r, t}^{p}=v_{x, r, t}^{p-1} \circ \partial \quad \text { pour } p \geq 2 \text { et } r \geq 0
$$

et

$$
\partial \circ u_{x, r, t}^{p}+u_{x, r, t}^{p-1} \circ \partial=v_{x, r-1, t}^{p-1}-v_{x, r, t}^{p-1} \quad \text { pour } p \geq 2 \text { et } r \geq 1 .
$$

Pour $p \in\left\{2, \ldots, p_{\max }\right\}$, on définit $u_{x}: \mathbb{C}^{\left(\Delta_{p-1}\right)} \rightarrow \mathbb{C}^{\left(\Delta_{p}\right)}$ par

$$
u_{x}=\int_{0}^{1} \sum_{r=1}^{\infty} u_{x, r, t}^{p} d t
$$

(la somme sur $r$ est toujours finie, plus précisément pour $u_{x}\left(e_{a_{1}} \wedge \cdots \wedge e_{a_{p-1}}\right)$ la somme s'arrête à $\left.\max \left(d\left(x, a_{1}\right), \ldots, d\left(x, a_{p-1}\right)\right)\right)$. Alors $u_{x}$ et $\partial$ sont des endomorphismes de degrés 1 et -1 de $\bigoplus_{p=0}^{p_{\max }} \mathbb{C}^{\left(\Delta_{p}\right)}$ et on a $\partial u_{x}+u_{x} \partial=1$.

Remarque. Comme les applications $\Phi_{i}$ ne sont pas linéaires, les formules précédentes pour $u_{x, r, t}$ en fonction de $\mu_{r, t}(x, a)$ ne donnent pas de formule pour $u_{x}$ en fonction de $\mu_{r}(x, a)$. Autrement dit $\mu_{r}(x, a)$ ne sert à rien. Plus loin pour montrer l'équivariance à compact près des opérateurs nous n'utiliserons pas la propriété (9) mais le lemme 3.27. 
Le gros avantage du paramétrix $u_{x}$ sur $H_{x}$ apparaît dans la proposition suivante, où l'on voit qu'il vérifie la condition (C2). Une propriété semblable sera vraie aussi pour le paramétrix définitif $J_{x}$ et jouera un rôle crucial dans la construction de normes telles que $J_{x}$ soit continu.

Proposition 3.29. Pour $p \in\left\{2, \ldots, p_{\max }\right\}, t \in[0,1], S=\left\{a_{1}, \ldots, a_{p-1}\right\} \in \Delta_{p-1}$,

$$
\operatorname{supp}\left(u_{x, r, t}\left(e_{a_{1}} \wedge \cdots \wedge e_{a_{p-1}}\right)\right) \subset \bigcap_{\substack{a \in S \\ y \in \delta \text { géod }(x, a) \\ d(y, a)=r}} B(y, N+5 p \delta)
$$

et $u_{x, r, t}\left(e_{a_{1}} \wedge \cdots \wedge e_{a_{p-1}}\right)$ ne dépend que de la connaissance des points de l'ensemble

$$
\begin{aligned}
S \cup\{x\} & \cup \bigcap_{\substack{a \in S \\
y \in \delta \text {-géd }(x, a) \\
d(y, a)=r}} B(y, N+5 \delta+5 p \delta) \\
& \cup \bigcap_{a \in S}\{y \in \delta-\operatorname{géod}(x, a) \mid d(y, a)=r+\mathrm{E}(t(d(x, a)-r))\} \\
& \cup \bigcap_{a \in S}\{y \in \delta-\operatorname{géod}(x, a) \mid d(y, a)=r-1+\mathrm{E}(t(d(x, a)-(r-1)))\}
\end{aligned}
$$

et des distances entre ces points.

Le nombre de points dans l'ensemble ci-dessus est borné par une constante $C(\delta, K, N)$ et les distances entre ces points sont elles-mêmes déterminées par la connaissance de $r, t$ et $d(x, S)$ à une constante $C(\delta, K, N)$ près. Donc le nombre total de possibilités pour le nombre de ces points et leurs distances mutuelles (c'està-dire le nombre de classes d'équivalence de (15) par les isométries qui préservent ses parties $S$ et $\{x\})$ est borné par $C(\delta, K, N)$.

Pour montrer la proposition 3.29 nous aurons besoin de trois lemmes.

Lemme 3.30. Soient $\alpha, \beta \in \mathbb{N}$ et $x, a, b, y, z \in X$ vérifiant $y \in \alpha$-géod $(x, a)$ et $z \in \beta$ - $\operatorname{géod}(x, b)$. Alors

$$
d(y, z) \leq d(a, b)+|d(a, y)-d(b, z)|+\alpha+\beta+2 \delta .
$$

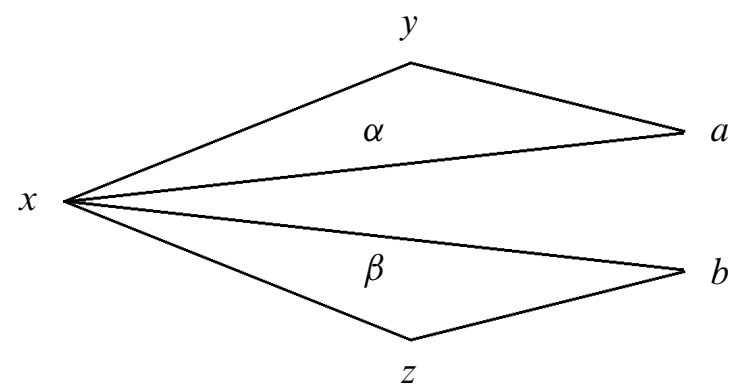


Démonstration. Par $\left(H_{\delta}^{\alpha}(b, a, y, x)\right)$, on a

$$
d(b, y) \leq \max (d(b, a)-d(a, y), d(b, x)-d(x, y))+\alpha+\delta
$$

et $\operatorname{par}\left(H_{\delta}^{\beta}(y, b, z, x)\right)$ on a

$$
\begin{aligned}
d(y, z) \leq & \max (d(y, b)-d(b, z), d(y, x)-d(x, z))+\beta+\delta \\
\leq & \max (d(a, b)-d(a, y)-d(b, z)+\alpha+\beta+2 \delta, \\
& d(b, x)-d(x, y)-d(b, z)+\alpha+\beta+2 \delta, d(x, y)-d(x, z)+\beta+\delta) \\
\leq & d(a, b)+|d(a, y)-d(b, z)|+\alpha+\beta+2 \delta
\end{aligned}
$$

car

$$
\begin{aligned}
d(b, x)-d(x, y)-d(b, z) & \leq d(a, b)+d(a, x)-d(x, y)-d(b, z) \\
& \leq d(a, b)+d(a, y)-d(b, z) \\
& \leq d(a, b)+|d(a, y)-d(b, z)|,
\end{aligned}
$$

et

$$
\begin{aligned}
d(x, y)-d(x, z) & \leq d(x, a)-d(a, y)+\alpha-d(x, b)+d(b, z) \\
& \leq d(a, b)+|d(a, y)-d(b, z)|+\alpha
\end{aligned}
$$

Lemme 3.31. Soit $R, \alpha \in \mathbb{R}_{+}$et $x, y, z, t \in X$ vérifiant $y, z \in B(x, R)$ et $t \in$ $\alpha$-géod $(y, z)$. Alors $t \in B(x, R+\alpha+\delta)$.

Démonstration. Par $\left(H_{\delta}^{\alpha}(x, y, t, z)\right)$, on a

$$
d(x, t) \leq \max (d(x, y)-d(y, t), d(x, z)-d(z, t))+\alpha+\delta \leq R+\alpha+\delta .
$$

Lemme 3.32. Pour tout $p \in\left\{1, \ldots, p_{\max }\right\}, y \in X, R \in \mathbb{R}_{+}$, et $f \in \mathbb{C}^{\left(\Delta_{p}\right)}$ telle que $\operatorname{supp}(f) \subset B(y, R)$, on a $\operatorname{supp}\left(\Phi_{p}(f)\right) \subset B(y, R+5 \delta)$ et $\Phi_{p}(f)$ ne dépend que de $f$ et de la connaissance des points de $B(y, R+10 \delta)$ et de leurs distances mutuelles.

Démonstration. Cela résulte des lemmes 3.25 et 3.31 .

Démonstration de la proposition 3.29. La réunion des supports des mesures

$$
\mu_{r, t}\left(x, a_{1}\right), \ldots, \mu_{r, t}\left(x, a_{p-1}\right), \mu_{r-1, t}\left(x, a_{1}\right), \ldots, \mu_{r-1, t}\left(x, a_{p-1}\right)
$$

est incluse dans

$$
\bigcup_{a \in S}\{y \in \delta-\operatorname{géod}(x, a) \mid d(y, a)=r\} \cup \bigcup_{a \in S}\{y \in \delta-\operatorname{géod}(x, a) \mid d(y, a)=r-1\} \text {. }
$$


Le lemme $3.30(\operatorname{avec} \alpha=\beta=\delta, a, b \in S, y \in \delta$ - $\operatorname{géod}(x, a)$ à distance $r$ ou $r-1$ de $a$, et $z \in \delta$ - géod $(x, b)$ à distance $r$ ou $r-1$ de $b$ ) implique que cet ensemble est de diamètre $\leq N+4 \delta+1 \leq N+5 \delta$ et qu'il est donc inclus dans

$$
\bigcap_{\substack{a \in S \\ y \in \text {-géd }(x, a) \\ d(y, a)=r}} B(y, N+5 \delta) .
$$

Grâce au lemme 3.32, on montre par récurrence sur $p$ l'assertion de la proposition 3.29 en même temps que l'assertion analogue pour $v_{x, r, t}$, à savoir que pour $p \in\left\{2, \ldots, p_{\max }\right\}, t \in[0,1], S=\left\{a_{1}, \ldots, a_{p}\right\} \in \Delta_{p}$,

$$
\operatorname{supp}\left(v_{x, r, t}\left(e_{a_{1}} \wedge \cdots \wedge e_{a_{p}}\right)\right) \subset \bigcap_{\substack{a \in S \\ y \in \delta \text {-géd }(x, a) \\ d(y, a)=r \text { ou } r+1}} B(y, N+5 p \delta)
$$

et $v_{x, r, t}\left(e_{a_{1}} \wedge \cdots \wedge e_{a_{p}}\right)$ ne dépend que de la connaissance des points de l'ensemble

$$
\begin{aligned}
S \cup\{x\} & \cup \bigcap_{\substack{a \in S \\
y \in \delta \text {-geod }(x, a) \\
d(y, a)=r \text { ou } r+1}} B(y, N+5 \delta+5 p \delta) \\
& \cup \bigcap_{a \in S}\{y \in \delta-\operatorname{géod}(x, a) \mid d(y, a)=r+\mathrm{E}(t(d(x, a)-r))\}
\end{aligned}
$$

et des distances entre tous ces points.

Lemme 3.33. Il existe $C=C(\delta, K, N)$ telle que pour tout $p \in\left\{1, \ldots, p_{\max }\right\}$ et pour tout $S \in \Delta_{p-1}, r \in \mathbb{N}$ et $t \in[0,1]$ on ait $\left\|u_{x, r, t}\left(e_{S}\right)\right\|_{\ell^{1}\left(\Delta_{p}\right)} \leq C$.

Démonstration. Cela résulte de la dernière assertion du lemme 3.25 et du fait que dans les formules pour $u_{x, r, t}$ on applique les $\Phi_{i}$ à des fonctions dont le support est de diamètre $\leq 2\left(N+5 \delta p_{\max }\right)$ d'après la démonstration de la proposition 3.29.

3.4. Construction du paramétrix définitif $\boldsymbol{J}_{\boldsymbol{x}}$. Nous allons introduire un opérateur $\tilde{H}_{x}$, sorte de troncature de $H_{x}$, et $J_{x}$ sera donné par la formule $J_{x}=\widetilde{H}_{x}+u_{x}(1-$ $\left.\partial \widetilde{H}_{x}-\widetilde{H}_{x} \partial\right)$.

En effet le gros inconvénient de $u_{x}$ est que l'on sait seulement, par la proposition 3.29 et le lemme 3.2, que, pour $S \in \Delta \backslash\{\emptyset\}, u_{x}\left(e_{S}\right)$ est supporté par $\bigcap_{a \in S}\left(2\left(N+5 p_{\max } \delta\right)+\delta\right)$ - géod $(x, a)$. Autrement dit $u_{x}$ ne vérifie pas la condition (C1) et la seule chose que l'on puisse affirmer est que $u_{x}$ "n'éloigne pas de $x$ de plus que de $N+5 p_{\max } \delta+\delta$ ". Cela compliquerait beaucoup la construction de normes pour lesquelles $\partial$ et $u_{x}$ soient continus, voire la rendrait impossible. Au contraire nous verrons dans la proposition 3.37 que l'opérateur $J_{x}$ vérifie les conditions $(\mathrm{C} 1)$ et (C2). L'idée pour montrer que $J_{x}$ vérifie $(\mathrm{C} 1)$ est que $\left(1-\partial \tilde{H}_{x}-\tilde{H}_{x} \partial\right)$ rapproche davantage de $x$ que $u_{x}$ n'en éloigne. L'idée pour montrer que $J_{x}$ vérifie (C2) est que, contrairement à $H_{x}, \widetilde{H}_{x}$ vérifie $(\mathrm{C} 2)$ grâce au fait que c'est une troncature. 
On peut écrire formellement $H_{x}$ sous la forme

$$
H_{x}=\sum_{q=1}^{+\infty} h_{x}\left(1-\left(\partial h_{x}+h_{x} \partial\right)\right)^{q-1}
$$

Cette somme est infinie (mais pour chaque $S \in \Delta, H_{x}\left(e_{S}\right)$ est donné par une somme finie, grâce au lemme 3.17).

On fixe un entier $Q$ tel que

$$
Q \text { soit assez grand en fonction de } \delta, K, N \text {. }
$$

Dans la suite nous utiliserons un nombre fini de fois l'inégalité $Q \geq C$ avec $C$ de la forme $C(\delta, K, N)$.

On pose

$$
\tilde{H}_{x}=\sum_{q=1}^{Q} h_{x}\left(1-\left(\partial h_{x}+h_{x} \partial\right)\right)^{q-1} \quad \text { et } \quad K_{x}=1-\left(\partial \tilde{H}_{x}+\tilde{H}_{x} \partial\right) .
$$

Il est clair que $K_{x}=\left(1-\left(\partial h_{x}+h_{x} \partial\right)\right)^{Q}$. Le lemme suivant résume les propriétés de $\widetilde{H}_{x}$ qui nous serviront ensuite. La propriété principale est que $K_{x}$ rapproche strictement de l'origine. On pose $K_{x, 0}=1$ et pour $q \in\{1, \ldots, Q\}$ et $\left(t_{1}, \ldots, t_{q}\right) \in$ $[0,1]^{q}$, on note

$$
K_{x, q,\left(t_{1}, \ldots, t_{q}\right)}=\left(1-\left(\partial h_{x, t_{q}}+h_{x, t_{q}} \partial\right)\right) \ldots\left(1-\left(\partial h_{x, t_{1}}+h_{x, t_{1}} \partial\right)\right)
$$

et

$$
\begin{aligned}
\tilde{H}_{x, q,\left(t_{1}, \ldots, t_{q}\right)} & =h_{x, t_{q}} K_{x, q-1,\left(t_{1}, \ldots, t_{q-1}\right)} \\
& =h_{x, t_{q}}\left(1-\left(\partial h_{x, t_{q-1}}+h_{x, t_{q-1}} \partial\right)\right) \ldots\left(1-\left(\partial h_{x, t_{1}}+h_{x, t_{1}} \partial\right)\right)
\end{aligned}
$$

de sorte que

$$
\tilde{H}_{x}=\sum_{q=1}^{Q} \int_{\left(t_{1}, \ldots, t_{q}\right) \in[0,1]^{q}} \tilde{H}_{x, q,\left(t_{1}, \ldots, t_{q}\right)} d t_{1} \ldots d t_{q}
$$

et

$$
K_{x}=\int_{\left(t_{1}, \ldots, t_{Q}\right) \in[0,1]} K_{x, Q,\left(t_{1}, \ldots, t_{Q}\right)} d t_{1} \ldots d t_{Q}
$$

Notation. Dans toute la suite de l'article, si $A$ est une partie de $X$ et $r \in \mathbb{N}$, on notera

$$
B(A, r)=\{y \in X \mid d(y, A) \leq r\}=\bigcup_{a \in A} B(a, r) .
$$

Lemme 3.34. 1) Pour $q \in\{1, \ldots, Q\},\left(t_{1}, \ldots, t_{q}\right) \in[0,1]^{q}$ et $S \in \Delta \backslash\{\emptyset\}$, 
a) $\tilde{H}_{x, q,\left(t_{1}, \ldots, t_{q}\right)}\left(e_{S}\right)$ est une combinaison de $e_{T}$ où $T$ vérifie :

$$
\begin{aligned}
T \subset S \cup(B(x, 2 \delta) \cap B(S, q N)) & \\
\cup & \left.\left.\bigcup_{\substack{a \in S \\
a \notin B(x, 2 \delta)}}\{y \in 4 \delta-\operatorname{géod}(x, a) \mid d(y, a) \in] N-2 \delta, q N\right]\right\},
\end{aligned}
$$

b) $\tilde{H}_{x, q,\left(t_{1}, \ldots, t_{q}\right)}\left(e_{S}\right)$ ne dépend que de la connaissance des points de

$$
\begin{aligned}
B(x, 7 \delta) & \cup \bigcup_{a \in S}\{y \in 4 \delta-\operatorname{géod}(x, a), d(y, a) \leq q N\} \\
& \cup \bigcup_{\substack{a \in S \\
i \in\{1, \ldots, q\}}}\left\{y \in 9 \delta-\operatorname{géod}(x, a)|| d(x, y)-t_{i} d(x, a) \mid \leq(q+2) N+1\right\}
\end{aligned}
$$

et des distances entre ces points.

2) $\operatorname{Pour}\left(t_{1}, \ldots, t_{Q}\right) \in[0,1]^{Q}$ et $S \in \Delta \backslash\{\emptyset\}$,

a) $K_{x, Q,\left(t_{1}, \ldots, t_{Q}\right)}\left(e_{S}\right)$ est une combinaison de $e_{T}$ où $T$ vérifie :

$$
T \subset \bigcup_{a \in S}\left\{y \in 4 \delta-\operatorname{géod}(x, a), d(y, a) \in\left[Q \frac{N-6 \delta}{p_{\max }}-2 N, Q N\right]\right\} .
$$

b) $K_{x, Q,\left(t_{1}, \ldots, t_{Q}\right)}\left(e_{S}\right)$ ne dépend que de la connaissance des points de

$$
\begin{aligned}
B(x, 7 \delta) & \cup \bigcup_{a \in S}\{y \in 4 \delta-\operatorname{géod}(x, a) \mid d(y, a) \leq Q N\} \\
& \cup \bigcup_{\substack{a \in S \\
i \in\{1, \ldots, Q\}}}\left\{y \in 9 \delta-\operatorname{géod}(x, a)|| d(x, y)-t_{i} d(x, a) \mid \leq(Q+2) N+1\right\}
\end{aligned}
$$

et des distances entre ces points.

Démonstration. Montrons 1a). C'est essentiellement le lemme 3.22 mais on doit en répéter les arguments parce qu'on a ici $h_{x, t_{i}}$ et non $h_{x}$. Soit $S_{0}=S$ et $S_{1}, \ldots, S_{q}$ tels que $e_{S_{i}}$ apparaisse avec un coefficient non nul dans $\left(1-\left(\partial h_{x, t_{i}}+h_{x, t_{i}} \partial\right)\right)\left(e_{S_{i-1}}\right)$ pour $i=1, \ldots, q-1$ et que $e_{S_{q}}$ apparaisse avec un coefficient non nul dans $h_{x, t_{q}}\left(e_{S_{q-1}}\right)$. Soit $y_{q} \in S_{q}$ n'appartenant pas à $B(x, 2 \delta)$. Par le lemme 3.19, il existe $y_{q-1} \in$ $S_{q-1}, \ldots, y_{0} \in S_{0}$ n'appartenant pas à $B(x, 2 \delta)$ tels que $y_{i}=y_{i+1}$ ou bien $y_{i+1} \in$ $3 \delta$ - $\operatorname{géod}\left(x, y_{i}\right)$ et $\left.\left.d\left(y_{i}, y_{i+1}\right) \in\right] N-2 \delta, N\right]$ pour $i \in\{0, \ldots, q-1\}$. En répétant la preuve du lemme 3.22 on montre que $y_{q} \in 4 \delta$ - $\operatorname{géod}\left(x, y_{0}\right)$ et $d\left(y_{0}, y_{q}\right)>N-2 \delta$ si $y_{0} \neq y_{q}$. Comme $d\left(y_{i}, y_{i+1}\right) \leq N$ pour $i \in\{0, \ldots, q-1\}$ on a $d\left(y_{0}, y_{q}\right) \leq q N$.

Montrons b). Soient $q \in\{1, \ldots, Q\}, S_{0}=S$ et $S_{1}, \ldots, S_{q-1}$ tels que $e_{S_{i}}$ apparaisse avec un coefficient non nul dans $\left(1-\left(\partial h_{x, t_{i}}+h_{x, t_{i}} \partial\right)\right)\left(e_{S_{i-1}}\right)$ pour $i=1, \ldots, q-1$. Soit $i \in\{1, \ldots, q\}$. Grâce au lemme 3.20, la connaissance de $\left(1-\left(\partial h_{x, t_{i}}+h_{x, t_{i}} \partial\right)\right)\left(e_{S_{i-1}}\right)$ si $i<q$ ou de $h_{x, t_{i}}\left(e_{S_{i-1}}\right)$ si $i=q$ ne dépend que de 
la connaissance des points de $B(x, 2 \delta)$ et de la réunion pour $b \in S_{i-1}$ des ensembles

$$
\begin{aligned}
\{b\} & \cup\{y \in 3 \delta-\operatorname{géod}(x, b) \mid d(y, b) \in] N-2 \delta, N]\} \\
& \cup\left\{y \in 5 \delta-\operatorname{géod}(x, b) \mid d(x, y) \in\left[t_{i} d\left(x, S_{i-1}\right)-2 N-1, t_{i} d\left(x, S_{i-1}\right)+N\right]\right\}
\end{aligned}
$$

(en effet si $T \in \Delta \backslash\{\emptyset\}$ est tel que $e_{T}$ apparaisse avec un coefficient non nul dans $\partial\left(e_{S_{i-1}}\right)$ on a $T \subset S_{i-1}$ donc $\left.d(x, T) \in\left[d\left(x, S_{i-1}\right), d\left(x, S_{i-1}\right)+N\right]\right)$. De plus la preuve de a) montre que pour tout $b \in S_{i-1}$, on a $b \in S_{0} \cup B(x, 2 \delta)$ ou il existe $a \in S_{0}$ tel que

$$
a \notin B(x, 2 \delta), \quad b \in 4 \delta-\operatorname{géod}(x, a) \quad \text { et } \quad d(a, b) \in] N-2 \delta,(q-1) N] .
$$

Si $b \in S_{0} \cup B(x, 2 \delta)$ on vérifie facilement que l'ensemble (18) est inclus dans l'ensemble (17) car $\left|d\left(x, S_{i-1}\right)-d(x, a)\right| \leq i N \leq q N$. Si $a \in S_{0}$ vérifie (19), l'ensemble (18) est également inclus dans l'ensemble (17) car

- si $y \in 3 \delta$ - $\operatorname{géod}(x, b)$ et $d(y, b) \in] N-2 \delta, N]$, en appliquant le lemme 3.21 avec $\alpha=4 \delta, \beta=3 \delta$ on a $y \in 4 \delta-\operatorname{géod}(x, a)$, et de plus $d(a, y) \leq q N$,

- si $y \in 5 \delta$ - géod $(x, b)$ et $d(x, y) \in\left[t_{i} d\left(x, S_{i-1}\right)-2 N-1, t_{i} d\left(x, S_{i-1}\right)+N\right]$ le a $)$ du lemme 3.3 montre $y \in 9 \delta$ - $\operatorname{géod}(x, a)$ et comme $\left|d\left(x, S_{i-1}\right)-d(x, a)\right| \leq q N$ on a $\left|d(x, y)-t_{i} d(x, a)\right| \leq(q+2) N+1$.

La preuve de 2) est tout à fait similaire à celle de 1), sauf que 2a) nécessite un argument supplémentaire. Si $e_{T}$ apparaît avec un coefficient non nul dans $K_{x, Q,\left(t_{1}, \ldots, t_{Q}\right)}\left(e_{S}\right)$, le lemme 3.17 implique $\zeta_{x}(T) \leq \zeta_{x}(S)-Q \frac{N-6 \delta}{p_{\max }}$. Le souslemme suivant, appliqué à $M=Q \frac{N-6 \delta}{p_{\max }}$, montre alors que pour tout $y \in T$ et $a \in S$ on a $d(a, y) \geq Q \frac{N-6 \delta}{p_{\max }}-2 N$.

Sous-lemme 3.35. Soient $M \in \mathbb{R}_{+}$et $S, T \in \Delta$. Si $\zeta_{x}(T) \leq \zeta_{x}(S)-M$, pour $y \in T$ et $a \in S$, on a $d(x, y) \leq d(x, a)-M+2 N$.

Démonstration. On a $d(x, y) \leq d\left(x, U_{T}\right)+N \leq \zeta_{x}(T)+N$ et $d(x, a) \geq d\left(x, U_{S}\right) \geq$ $\zeta_{x}(S)-N$.

Fin de la démonstration du lemme 3.34. On suppose $Q \frac{N-6 \delta}{p_{\max }}-2 N>N$, ce qui est permis par $\left(H_{Q}\right)$, d'où $y \notin S$. Enfin $S$ est non vide, donc

$$
B(x, 2 \delta) \subset \bigcup_{a \in S} 4 \delta-\operatorname{géod}(x, a) .
$$

Comme nous l'avons déjà dit nous posons

$$
J_{x}=\tilde{H}_{x}+u_{x} K_{x}
$$

Montrons que

$$
\partial J_{x}+J_{x} \partial=1 .
$$


D' abord comme $\partial^{2}=0, \partial$ commute à $K_{x}=1-\partial \tilde{H}_{x}-\tilde{H}_{x} \partial$ et donc

$$
\partial J_{x}+J_{x} \partial=\left(\partial \tilde{H}_{x}+\tilde{H}_{x} \partial\right)+\left(\partial u_{x}+u_{x} \partial\right) K_{x}=1
$$

puisque $\partial u_{x}+u_{x} \partial=1$. On a

$$
\begin{aligned}
J_{x}= & \sum_{q=1}^{Q} \int_{\left(t_{1}, \ldots, t_{q}\right) \in[0,1]^{q}} \tilde{H}_{x, q,\left(t_{1}, \ldots, t_{q}\right)} d t_{1} \ldots d t_{q} \\
& +\sum_{r=1}^{+\infty} \int_{t,\left(t_{1}, \ldots, t_{Q}\right) \in[0,1] Q+1} u_{x, r, t} K_{x, Q,\left(t_{1}, \ldots, t_{Q}\right)} d t d t_{1} \ldots d t_{Q} .
\end{aligned}
$$

Il résulte de tout ce qui précède que $\tilde{H}_{x, q,\left(t_{1}, \ldots, t_{q}\right)}\left(e_{S}\right)$ et $u_{x, r, t} K_{x, Q,\left(t_{1}, \ldots, t_{Q}\right)}\left(e_{S}\right)$ ne dépendent que de la connaissance d'un nombre fini de points (borné par $C(\partial t a, K, N, Q))$ et des distances entre ces points. De plus ces distances sont déterminées par $x, S, q, r, t$ et les $t_{i}$ à une constante $C(\delta, K, N, Q)$ près. Plus précisément le 1) du lemme 3.34 apporte toutes les informations relatives à $\widetilde{H}_{x, q,\left(t_{1}, \ldots, t_{q}\right)}\left(e_{S}\right)$, et le lemme suivant les donne pour $u_{x, r, t} K_{x, Q,\left(t_{1}, \ldots, t_{Q}\right)}\left(e_{S}\right)$.

Lemme 3.36. Pour $\left.t \in[0,1],\left(t_{1}, \ldots, t_{Q}\right) \in[0,1]\right]^{Q}, r \in \mathbb{N}^{*}$ et $S \in \Delta \backslash\{\emptyset\}$,

a) $u_{x, r, t} K_{x, Q,\left(t_{1}, \ldots, t_{Q}\right)}\left(e_{S}\right)$ est une combinaison de $e_{T}$ où $T$ vérifie :

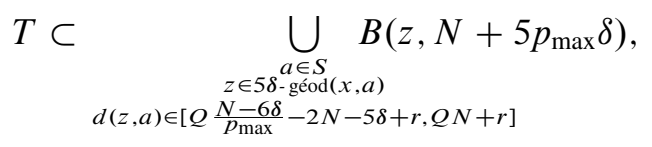

b) $u_{x, r, t} K_{x, Q,\left(t_{1}, \ldots, t_{Q}\right)}\left(e_{S}\right)$ ne dépend que de la connaissance des points de

$$
\begin{aligned}
& B(x, 7 \delta) \cup \bigcup_{a \in S}\{y \in 4 \delta-\operatorname{géod}(x, a) \mid d(y, a) \leq Q N\} \\
& \cup \underset{\substack{a \in S \\
i \in\{1, \ldots, Q\}}}{\bigcup}\left\{y \in 9 \delta-\operatorname{géod}(x, a)|| d(x, y)-t_{i} d(x, a) \mid \leq(Q+2) N+1\right\} \\
& \begin{array}{l}
\bigcup_{\substack{a \in S \\
z \in 5 \delta \text { géd }(x, a) \\
(z, a) \in[Q-6 \delta}} B\left(z, N+5 p_{\max } \delta+5 \delta\right) \\
\left.p_{\max }-2 N-5 \delta+r, Q N+r\right]
\end{array} \\
& \cup \bigcup_{a \in S}\{z \in 5 \delta-\operatorname{géod}(x, a), d(x, z) \\
& \in[(1-t)(d(x, a)-Q N-r),(1-t)(d(x, a)-r)+2+\delta]\}
\end{aligned}
$$

et des distances entre ces points.

Démonstration. Montrons a). D’après le 2)a) du lemme 3.34 et la proposition 3.29,

$$
u_{x, r, t} K_{x, Q,\left(t_{1}, \ldots, t_{Q}\right)}\left(e_{S}\right)
$$


est supporté par la réunion des $B\left(z, N+5 p_{\max } \delta\right)$ pour les $z$ tels que $z \in \delta$-géod $(x, y)$ et $d(y, z)=r$, avec $y \in 4 \delta$ - géod $(x, a), a \in S$ et $d(y, a) \in\left[Q \frac{N-6 \delta}{p_{\max }}-2 N, Q N\right]$. Alors $z \in 5 \delta$-géod $(x, a)$ par le a) du lemme 3.3. On a aussi $y \in 5 \delta$ - $\operatorname{géod}(z, a)$ par le b) du lemme 3.3 , d'où

$$
d(z, a) \in\left[Q \frac{N-6 \delta}{p_{\max }}-2 N-5 \delta+r, Q N+r\right] .
$$

Montrons b). D'après le $2 \mathrm{~b})$ du lemme $3.34, K_{x, Q,\left(t_{1}, \ldots, t_{Q}\right)}\left(e_{S}\right)$ dépend de la connaissance des points de

$$
\begin{aligned}
B(x, 7 \delta) & \cup \bigcup_{a \in S}\{y \in 4 \delta \text { - géod }(x, a) \mid d(y, a) \leq Q N\} \\
& \cup \bigcup_{\substack{a \in S \\
i \in\{1, \ldots, Q\}}}\left\{y \in 9 \delta-\operatorname{géod}(x, a)|| d(x, y)-t_{i} d(x, a) \mid \leq(Q+2) N+1\right\} .
\end{aligned}
$$

D’après le 2)a) du lemme 3.34 et la proposition 3.29 ,

$$
u_{x, r, t} K_{x, Q,\left(t_{1}, \ldots, t_{Q}\right)}\left(e_{S}\right)
$$

dépend de la connaissance de $K_{x, Q,\left(t_{1}, \ldots, t_{Q}\right)}\left(e_{S}\right)$ et des points de

$$
\begin{aligned}
& \{x\} \cup \bigcup_{a \in S}\left\{y \in 4 \delta-\operatorname{géod}(x, a) \mid d(y, a) \in\left[Q \frac{N-6 \delta}{p_{\max }}-2 N, Q N\right]\right\} \\
& \cup \bigcup_{\substack{a \in S \\
y \in 4 \delta-\operatorname{god}(x, a) \\
d(y, a) \in\left[Q \frac{N-6 \delta}{p-\max }-2 N, Q N\right] \\
z \in \delta \text {-geod }(x, y) \\
d(y, z)=r}} B\left(z, N+5 p_{\max } \delta+5 \delta\right)
\end{aligned}
$$

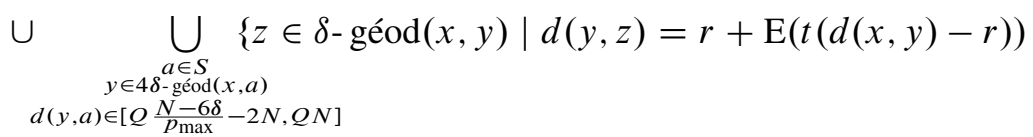

$$
\begin{aligned}
& \text { ou } d(y, z)=(r-1)+\mathrm{E}(t(d(x, y)-(r-1)))\} .
\end{aligned}
$$

Les deux derniers ensembles de la réunion ci-dessus sont inclus dans les deux derniers ensembles de la réunion figurant dans b) du lemme 3.36, pour les raisons suivantes. La preuve de a) montre que pour $a \in S$ les conditions

$y \in 4 \delta-\operatorname{géod}(x, a), d(y, a) \in\left[Q \frac{N-6 \delta}{p_{\max }}-2 N, Q N\right], z \in \delta-\operatorname{géod}(x, y), d(y, z)=r$ impliquent

$$
z \in 5 \delta-\operatorname{géod}(x, a), \quad d(z, a) \in\left[Q \frac{N-6 \delta}{p_{\max }}-2 N-5 \delta+r, Q N+r\right] .
$$

D'autre part pour $a \in S$ les conditions

$$
\begin{gathered}
y \in 4 \delta \text { - géod }(x, a), \quad d(y, a) \in\left[Q \frac{N-6 \delta}{p_{\max }}-2 N, Q N\right], \quad z \in \delta \text { - géod }(x, y), \\
d(y, z)=r+\mathrm{E}(t(d(x, y)-r)) \text { ou } d(y, z)=(r-1)+\mathrm{E}(t(d(x, y)-(r-1)))
\end{gathered}
$$


impliquent $z \in 5 \delta$ - $\operatorname{géod}(x, a)$,

$$
\begin{gathered}
r+t(d(x, y)-r)-2 \leq d(y, z) \leq r+t(d(x, y)-r), \\
d(x, z) \geq d(x, y)-d(y, z) \geq(1-t)(d(x, y)-r), \\
d(x, z) \leq d(x, y)-d(y, z)+\delta \leq(1-t)(d(x, y)-r)+2+\delta, \\
d(x, y) \geq d(x, a)-d(y, a) \geq d(x, a)-Q N
\end{gathered}
$$

et

$$
d(x, y) \leq d(x, a)-d(y, a)+4 \delta \leq d(x, a)-\left(Q \frac{N-6 \delta}{p_{\max }}-2 N\right)+4 \delta \leq d(x, a)
$$

car on suppose $\left(Q \frac{N-6 \delta}{p_{\max }}-2 N\right) \geq 4 \delta$ (ce qui est permis par $\left.\left(H_{Q}\right)\right)$.

Le 1) et le 2) de la proposition suivante récapitulent la partie des lemmes 3.34 et 3.36 qui nous sera utile ensuite sous une forme plus lisible. On suppose

$$
N \geq 6 \delta+1, Q \geq 2 \quad \text { et } \quad Q \frac{N-6 \delta}{p_{\max }} \geq 2\left(3 N+5 \delta p_{\max }+10 \delta\right),
$$

ce qui est permis par $\left(H_{N}\right)$ et $\left(H_{Q}\right)$. On pose

$$
F=15 \delta+2 N+10 \delta p_{\max } .
$$

On a donc $F \leq C(\delta, K, N)$. Il est important de souligner que $F$ est une simple notation permettant d'alléger les formules. Au contraire les constantes $N, Q$ et d'autres qui seront introduites ensuite, sont des paramètres dans notre construction et chacun de ces paramètres doit être choisi suffisamment grand par rapport à ceux introduits auparavant.

Proposition 3.37. 1) Pour $q \in\{1, \ldots, Q\},\left(t_{1}, \ldots, t_{q}\right) \in[0,1]^{q}$ et $S \in \Delta \backslash\{\emptyset\}$,

a) $\tilde{H}_{x, q,\left(t_{1}, \ldots, t_{q}\right)}\left(e_{S}\right)$ est une combinaison de $e_{T}$ où $T$ vérifie :

$$
\begin{aligned}
T \subset S \cup(B(x, 2 \delta) \cap B(S, q N)) & \\
& \left.\left.\cup \bigcup_{a \in S}\{y \in 4 \delta-\operatorname{géod}(x, a) \mid d(y, a) \in] N-2 \delta, q N\right]\right\},
\end{aligned}
$$

b) $\tilde{H}_{x, q,\left(t_{1}, \ldots, t_{q}\right)}\left(e_{S}\right)$ ne dépend que de la connaissance des points de

$$
\begin{aligned}
B(x, 7 \delta) \cup B(S, Q N) & \cup \underset{\substack{a \in S \\
i \in\{1, \ldots, q\}}}{\cup}\left\{y \in F-\operatorname{géod}(x, a)|| d(x, y)-t_{i} d(x, a) \mid \leq Q F\right\}
\end{aligned}
$$

et des distances entre ces points.

2) Pour $t \in[0,1],\left(t_{1}, \ldots, t_{Q}\right) \in[0,1]^{Q}, r \in \mathbb{N}^{*}$ et $S \in \Delta \backslash\{\emptyset\}$,

a) $u_{x, r, t} K_{x, Q,\left(t_{1}, \ldots, t_{Q}\right)}\left(e_{S}\right)$ est une combinaison de $e_{T}$ où $T$ vérifie :

$$
T \subset \bigcup_{a \in S}\left\{z \in F-\operatorname{géod}(x, a) \mid d(z, a) \in\left[\frac{Q}{F}+r, Q F+r\right]\right\},
$$


b) $u_{x, r, t} K_{x, Q,\left(t_{1}, \ldots, t_{Q}\right)}\left(e_{S}\right)$ ne dépend que de la connaissance des points de

$$
\begin{aligned}
B(x, F) & \cup B(S, Q N) \cup \bigcup_{a \in S}\{y \in F-\operatorname{géod}(x, a) \mid d(y, a) \in[r, r+Q F]\} \\
& \cup \bigcup_{\substack{a \in S \\
i \in\{1, \ldots, Q\}}}\left\{y \in F-\operatorname{géod}(x, a)|| d(x, y)-t_{i} d(x, a) \mid \leq Q F\right\} \\
& \cup \bigcup_{a \in S}\{y \in F-\operatorname{géod}(x, a)|| d(x, y)-(1-t)(d(x, a)-r) \mid \leq Q F\}
\end{aligned}
$$

et des distances entre ces points.

3) Il existe $C=C(\delta, K, N, Q)$ tel que dans les notations de 1) et 2) on ait

$$
\left\|\tilde{H}_{x, q,\left(t_{1}, \ldots, t_{q}\right)}\left(e_{S}\right)\right\|_{\ell^{1}} \leq C \quad \text { et } \quad\left\|u_{x, r, t} K_{x, Q,\left(t_{1}, \ldots, t_{Q}\right)}\left(e_{S}\right)\right\|_{\ell^{1}} \leq C .
$$

On remarque que le nombre de points des ensembles apparaissant dans l'énoncé est borné par $C(\delta, K, N, Q)$ et que les distances entre ces points sont déterminées à $C(\delta, K, N, Q)$ près par $d(x, S)$ et les divers paramètres (à savoir $q, t_{1}, \ldots, t_{q}$ dans 1 ) et $r, t, t_{1}, \ldots, t_{Q}$ dans 2$)$ ). Donc le nombre de combinaisons possibles pour le nombre de ces points et leurs distances mutuelles est borné par $C(\delta, K, N, Q)$.

Par des arguments similaires à ceux de la preuve du lemme 3.22 on peut montrer facilement, à l' aide de 1)a) et 2)a) du lemme précédent, que $J_{x}$ vérifie la condition (C1) (en fait ces arguments seront cachés dans l'étude des normes menée au paragraphe 4). D'autre part 1)b) et 2)b) garantissent que $J_{x}$ vérifie (C2).

Démonstration. Le 1) résulte du 1) du lemme 3.34 et le 2) résulte du lemme 3.36. En particulier pour montrer 2) on utilise les inégalités suivantes, qui découlent de (20) et $(21)$ :

$$
Q N+N+5 \delta p_{\max }+5 \delta \leq Q F, \quad(Q+2) N+1 \leq Q F
$$

et

$$
Q \frac{N-6 \delta}{p_{\max }}-2 N-5 \delta-N-5 \delta p_{\max }-5 \delta \geq Q \frac{N-6 \delta}{2 p_{\max }} \geq \frac{Q}{F} .
$$

On utilise aussi le fait que

$$
\begin{aligned}
& \begin{array}{r}
\bigcup_{\substack{a \in S \\
z \in 5 \delta \text {-géod }(x, a) \\
N-6 \delta}} B\left(z, N+5 p_{\max } \delta+5 \delta\right) \\
d(z, a) \in\left[Q \frac{N-6 \delta}{p_{\max }}-2 N-5 \delta+r, Q N+r\right]
\end{array} \\
& \subset \bigcup_{a \in S}\left\{z \in\left(5 \delta+2\left(N+5 p_{\max } \delta+5 \delta\right)\right)-\operatorname{géod}(x, a) \mid\right. \\
& \subset d(z, a) \in\left[Q \frac{N-6 \delta}{p_{\max }}-2 N-5 \delta+r-\left(N+5 p_{\max } \delta+5 \delta\right),\right. \\
& \left.\left.\subset Q N+r+\left(N+5 p_{\max } \delta+5 \delta\right)\right]\right\}
\end{aligned}
$$

grâce au lemme 3.2, ainsi que d'autres inclusions analogues ou plus faciles.

Enfin 3) résulte du lemme 3.33 et du fait que $\left\|h_{x, t}\left(e_{S}\right)\right\|_{\ell^{1}} \leq 1$ pour tout $S$ puisque $h_{x, t}\left(e_{S}\right)=\psi_{S, x, t} \wedge e_{S}$ et que $\psi_{S, x, t}$ est une mesure de probabilité. 
3.5. Construction d'une distance moyennée pour conjuguer les opérateurs. On montrera dans le paragraphe 4 que l'image de 1 dans $\mathrm{KK}_{G, 2 s \ell+C}(\mathbb{C}, \mathbb{C})$ est représenté par l'opérateur impair $\partial+J_{x}$ agissant sur le complété de $\bigoplus_{p=1}^{p_{\max }} \mathbb{C}^{\left(\Delta_{p}\right)}$ pour une certaine norme de Hilbert $\|\cdot\|_{\mathscr{H}_{x, s}}$. Pour construire la première partie de l'homotopie de 1 à $\gamma$ (qui fait l'objet de ce paragraphe et du suivant), on conjuguera $\partial+J_{x}$ par $e^{\tau \theta_{x}^{\mathrm{b}}}$ où $\theta_{x}^{\mathrm{b}}: \bigoplus_{p=1}^{p_{\max }} \mathbb{C}^{\left(\Delta_{p}\right)} \rightarrow \bigoplus_{p=1}^{p_{\max }} \mathbb{C}^{\left(\Delta_{p}\right)}$ est défini par $\theta_{x}^{\mathrm{b}}\left(e_{S}\right)=\rho_{x}^{\mathrm{b}}(S) e_{S}$, où $\rho_{x}^{\mathrm{b}}$ est une variante moyennée de la distance à $x$ et où $\tau$ varie de 0 à $T$ (avec $T$ assez grand). On doit moyenner la distance à $x$ pour qu'elle se comporte mieux quand on change l'origine $x$ en $g(x)$ pour $g \in G$. Cela est nécessaire pour que l'opérateur $\partial+J_{x}$ conjugué par $e^{\tau \theta_{x}^{b}}$ soit $G$-équivariant à compact près.

La suite de l'homotopie (qui est reléguée au paragraphe 5) sera facile : comme $T$ est assez grand, l'opérateur $e^{T \theta_{x}^{\mathrm{b}}}\left(\partial+J_{x}\right) e^{-T \theta_{x}^{\mathrm{b}}}$ est continu pour la norme $\ell^{2}$, donc par une homotopie et tout en gardant cet opérateur on peut remplacer la norme $\|\cdot\|_{\mathscr{H}_{x, s}}$ par la norme $\ell^{2} \operatorname{sur} \bigoplus_{p=1}^{p_{\max }} \mathbb{C}^{\left(\Delta_{p}\right)}$ et il est alors très simple de terminer l'homotopie en aboutissant à $\gamma$ dans $\mathrm{KK}_{G}(\mathbb{C}, \mathbb{C})$.

Le but de ce sous-paragraphe est la construction de $\rho_{x}^{b}$.

La proposition suivante renforce le théorème 17 de [MY02]. On rappelle que $(X, d)$ est un bon espace discret $\delta$-hyperbolique.

Proposition 3.38. Il existe une distance $G$-invariante $d^{\prime \prime}$ sur $X$ telle que $d-d^{\prime \prime}$ est borné et que

$$
\begin{gathered}
\forall r \in \mathbb{R}_{+}, \forall \varepsilon \in \mathbb{R}_{+}^{*}, \exists R \in \mathbb{R}_{+}, \forall x, x^{\prime}, y, y^{\prime} \in X, d\left(x, x^{\prime}\right) \leq r, \\
d\left(y, y^{\prime}\right) \leq r, d(x, y) \geq R, \text { on ait } \\
\left|d^{\prime \prime}(x, y)-d^{\prime \prime}\left(x, y^{\prime}\right)-d^{\prime \prime}\left(x^{\prime}, y\right)+d^{\prime \prime}\left(x^{\prime}, y^{\prime}\right)\right| \leq \varepsilon .
\end{gathered}
$$

La proposition 3.38 est une conséquence du lemme suivant.

Lemme 3.39. Il existe une famille de mesures positives $(\mu(x, y))_{(x, y) \in X \times X}$ de masse 1 sur $X$ de sorte que

- pour tout $g \in G, \mu(g x, g y)=g_{*} \mu(x, y)$,

- $\mu(x, y)=\mu(y, x)$,

- le support de $\mu(x, y)$ est inclus dans 78 - géod $(x, y)$,

- et

$$
\begin{aligned}
& \forall r \in \mathbb{R}_{+}, \forall \varepsilon \in \mathbb{R}_{+}^{*}, \exists R \in \mathbb{R}_{+}, \forall x, x^{\prime}, y, y^{\prime} \in X, \text { avec } d\left(x, x^{\prime}\right) \leq r, \\
& d\left(y, y^{\prime}\right) \leq r, d(x, y) \geq R, \text { on a }\left\|\mu(x, y)-\mu\left(x^{\prime}, y^{\prime}\right)\right\|_{1} \leq \varepsilon .
\end{aligned}
$$

La première condition est simplement la condition naturelle de $G$-équivariance.

Démonstration de la proposition 3.38 en admettant le lemme 3.39. On pose

$$
d^{\prime}(x, y)=\int_{X}(d(x, z)+d(z, y)) d \mu(x, y)(z) .
$$


On a $d(x, y) \leq d^{\prime}(x, y) \leq d(x, y)+7 \delta$ pour $x, y \in X$ et $d^{\prime}$ vérifie la condition (22) de la proposition 3.38. En effet soit $r \in \mathbb{R}_{+}, \varepsilon \in \mathbb{R}_{+}^{*}$. Soit $R$, et $x, y, x^{\prime}, y^{\prime}$ comme dans la dernière assertion du lemme 3.39. Pour $z \in 7 \delta$-géod $(x, y)$, on a $z \in(7 \delta+2 r)-\operatorname{géod}\left(x, y^{\prime}\right), z \in(7 \delta+2 r)$ - géod $\left(x^{\prime}, y\right)$, et $z \in(7 \delta+4 r)$ - géod $\left(x^{\prime}, y^{\prime}\right)$ par le lemme 3.2, donc

$$
\begin{aligned}
& \left|d^{\prime}\left(x^{\prime}, y\right)-\int_{X}\left(d\left(x^{\prime}, z\right)+d(z, y)\right) d \mu(x, y)(z)\right| \\
& \quad=\left|\int_{X}\left(d\left(x^{\prime}, z\right)+d(z, y)\right)\left(d \mu\left(x^{\prime}, y\right)-d \mu(x, y)\right)(z)\right| \\
& \quad=\left|\int_{X}\left(d\left(x^{\prime}, z\right)+d(z, y)-d\left(x^{\prime}, y\right)\right)\left(d \mu\left(x^{\prime}, y\right)-d \mu(x, y)\right)(z)\right| \leq \varepsilon(7 \delta+2 r)
\end{aligned}
$$

et de même

$$
\left|d^{\prime}\left(x, y^{\prime}\right)-\int_{X}\left(d(x, z)+d\left(z, y^{\prime}\right)\right) d \mu(x, y)(z)\right| \leq \varepsilon(7 \delta+2 r)
$$

et

$$
\left|d^{\prime}\left(x^{\prime}, y^{\prime}\right)-\int_{X}\left(d\left(x^{\prime}, z\right)+d\left(z, y^{\prime}\right)\right) d \mu(x, y)(z)\right| \leq \varepsilon(7 \delta+4 r) .
$$

D’autre part

$$
\begin{aligned}
(d(x, z)+d(z, y))-\left(d\left(x^{\prime}, z\right)+d(z, y)\right)-(d(x, z) & \left.+d\left(z, y^{\prime}\right)\right) \\
& +\left(d\left(x^{\prime}, z\right)+d\left(z, y^{\prime}\right)\right)=0
\end{aligned}
$$

pour tout $z \in X$. On en déduit

$$
\left|d^{\prime}(x, y)-d^{\prime}\left(x, y^{\prime}\right)-d^{\prime}\left(x^{\prime}, y\right)+d^{\prime}\left(x^{\prime}, y^{\prime}\right)\right| \leq \varepsilon(21 \delta+8 r) .
$$

Cependant $d^{\prime}$ n'est pas nécessairement une distance. Comme

$$
d(x, y) \leq d^{\prime}(x, y) \leq d(x, y)+7 \delta \quad \text { pour } x, y \in X
$$

on a $d^{\prime}(u, w) \leq d^{\prime}(u, v)+d^{\prime}(v, w)+7 \delta$ pour $u, v, w \in X$. On pose alors $d^{\prime \prime}(x, y)=$ 0 si $x=y$ et $d^{\prime \prime}(x, y)=d^{\prime}(x, y)+7 \delta$ si $x \neq y$. Alors $d^{\prime \prime}$ est une distance et on a montré la proposition 3.38 en admettant le lemme 3.39.

Avant de montrer le lemme 3.39 on commence par un lemme général très utile.

Lemme 3.40. Soient $x, y \in X, \alpha, \gamma \in \mathbb{N}, a \in \alpha-\operatorname{géod}(x, y), c \in \gamma$-géod $(x, y)$, et $b \in \operatorname{géod}(a, c)$, avec $d(a, b) \geq \frac{\alpha}{2}$ et $d(b, c) \geq \frac{\gamma}{2}$, alors $b \in 3 \delta-\operatorname{géod}(x, y)$.

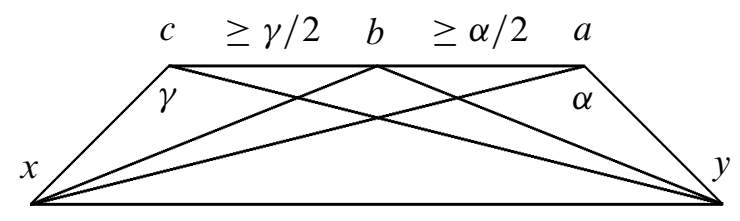


Démonstration. $\operatorname{Par}\left(H_{\delta}^{0}(x, a, b, c)\right)$ et $\left(H_{\delta}^{0}(y, a, b, c)\right)$ on a

$$
d(x, b) \leq \max (d(x, a)-d(a, b), d(x, c)-d(b, c))+\delta
$$

et

$$
d(y, b) \leq \max (d(y, a)-d(a, b), d(y, c)-d(b, c))+\delta .
$$

En additionnant ces deux inégalités on obtient

$$
\begin{aligned}
d(x, b)+d(b, y) \leq \max ( & d(x, a)+d(a, y)-2 d(a, b), \\
& d(x, c)+d(c, y)-2 d(b, c), \\
& d(x, a)+d(y, c)-d(a, c), \\
& d(x, c)+d(y, a)-d(a, c))+2 \delta .
\end{aligned}
$$

Comme $d(a, b) \geq \frac{\alpha}{2}$ on a $d(x, a)+d(a, y)-2 d(a, b) \leq d(x, y)$ et comme $d(b, c) \geq$ $\frac{\gamma}{2}$ on a $d(x, c)+d(c, y)-2 d(b, c) \leq d(x, y)$, donc

$$
\begin{aligned}
d(x, b)+d(b, y) \leq \max ( & d(x, y)+2 \delta, \\
& d(x, a)+d(y, c)-d(a, c)+2 \delta, \\
& d(x, c)+d(y, a)-d(a, c)+2 \delta) .
\end{aligned}
$$

Si $d(x, a)+d(y, c) \leq d(a, c)+d(x, y)+\delta$ et $d(x, c)+d(y, a) \leq d(a, c)+$ $d(x, y)+\delta$ on a fini. Dans le cas contraire, supposons par exemple

$$
d(x, a)+d(y, c)>d(a, c)+d(x, y)+\delta .
$$

Grâce à $\left(H_{\delta}(x, y, a, c)\right)$ qui s'écrit

$$
d(x, a)+d(y, c) \leq \max (d(x, y)+d(a, c), d(x, c)+d(y, a))+\delta
$$

on a alors $d(x, c)+d(y, a) \geq d(x, a)+d(y, c)-\delta>d(a, c)+d(x, y)$ d'où

$$
(d(x, a)+d(y, c))+(d(x, c)+d(y, a))>2 d(a, c)+2 d(x, y)+\delta .
$$

Or

$$
\begin{aligned}
(d(x, a)+d(y, c))+(d(x, c)+d(y, a)) & \leq 2 d(x, y)+\alpha+\gamma \\
& \leq 2 d(a, c)+2 d(x, y) .
\end{aligned}
$$

Cette contradiction achève la démonstration du lemme 3.40.

Démonstration du lemme 3.39. Comme le lemme 3.39 ne servira pas dans la suite nous abrégeons sa démonstration. On rappelle que pour toute partie $A$ non vide de $X, v_{A}$ désigne la mesure de masse 1 égale au produit par $(\sharp A)^{-1}$ de la fonction caractéristique de $A$. On pose alors $\phi(t)=\mathrm{E}(t / 8)$, et

$$
\mu(x, y)=\frac{1}{(\phi(d(x, y))+1)^{4}} \sum_{k, \tilde{k}, l, \tilde{l}=0}^{\phi(d(x, y))} v_{B_{x, y, k, \tilde{k}, l, \tilde{l}}}
$$



où pour $k, \tilde{k}, l, \tilde{l} \in\{0, \ldots, \phi(d(x, y))\}$ on note

$$
\begin{array}{r}
B_{x, y, k, \tilde{k}, l, \tilde{l}}=\left\{z \mid \exists(\tilde{x}, \tilde{y}) \in 3 \delta-\operatorname{géod}(x, y)^{2}, d(x, \tilde{x}) \leq k, d(\tilde{x}, z) \geq \tilde{k},\right. \\
d(y, \tilde{y}) \leq l, d(z, \tilde{y}) \geq \tilde{l}, z \in \operatorname{géod}(\tilde{x}, \tilde{y})\} .
\end{array}
$$

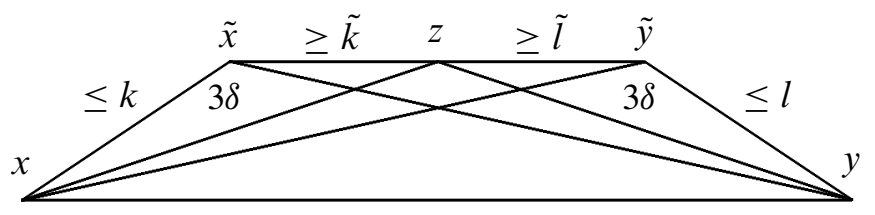

Il est clair que $\mu(x, y)=\mu(y, x)$. Montrons que le support de $\mu(x, y)$ est inclus dans $7 \delta$ - $\operatorname{géod}(x, y)$. Soient $z, \tilde{x}$ et $\tilde{y}$ comme dans la définition de $B_{x, y, k, \tilde{k}, l, \tilde{l}} \cdot \mathrm{Si}$ $d(\tilde{x}, z) \geq 2 \delta$ et $d(\tilde{y}, z) \geq 2 \delta$ le lemme 3.40 (avec $\alpha=\beta=3 \delta$ ) implique $z \in$ $3 \delta$ - $\operatorname{géod}(x, y)$. Si $d(\tilde{x}, z) \leq 2 \delta$ ou $d(\tilde{y}, z) \leq 2 \delta$ on a $z \in 7 \delta$ - $\operatorname{géod}(x, y)$ par le lemme 3.2. Pour montrer la dernière assertion du lemme 3.39 on utilise l'astuce des ensembles emboîtés, qui apparait dans la démonstration de la proposition 6.9 de [KS03]. Le cardinal de $B_{x, y, k, \tilde{k}, l, \tilde{l}}$ est toujours compris entre $\frac{d(x, y)}{C_{1}}$ et $C_{2} d(x, y)$ pour deux constantes $C_{1}$ et $C_{2}$ du type $C(\delta, K)$. De plus $(k, \tilde{k}, l, \tilde{l}) \mapsto B_{x, y, k, \tilde{k}, l, \tilde{l}}$ est une application croissante en $k$ et $l$ et décroissante en $\tilde{k}$ et $\tilde{l}$. Enfin étant donnés $x, y, x^{\prime}, y^{\prime} \in X$, en posant

$$
d=d\left(x, x^{\prime}\right)+d\left(y, y^{\prime}\right)+2 \delta
$$

on a

$$
B_{x, y, k, \tilde{k}, l, \tilde{l}} \subset B_{x^{\prime}, y^{\prime}, k+2 d, \tilde{k}-d, l+2 d, \tilde{l}-d}
$$

pour $\tilde{k}, \tilde{l} \geq d$. En effet soit $z \in B_{x, y, k, \tilde{k}, l, \tilde{l}}$ et $\tilde{x}, \tilde{y} \in 3 \delta$ - $\operatorname{géod}(x, y)^{2}$ tels que $d(x, \tilde{x}) \leq k, d(\tilde{x}, z) \geq \tilde{k}, d(y, \tilde{y}) \leq l, d(z, \tilde{y}) \geq \tilde{l}, z \in \operatorname{géod}(\tilde{x}, \tilde{y})$. Par le lemme $3.2, \tilde{x}$ et $\tilde{y}$ appartiennent à $\left(3 \delta+2 d\left(x, x^{\prime}\right)+2 d\left(y, y^{\prime}\right)\right)$ - $\operatorname{géod}\left(x^{\prime}, y^{\prime}\right)$. Soit $\tilde{x}^{\prime}$ un point de géod $(\tilde{x}, z)$ à distance $d$ de $\tilde{x}$ et $\tilde{y}^{\prime}$ un point de géod $(\tilde{y}, z)$ à distance $d$ de $\tilde{y}$. D'après le lemme $3.40, \tilde{x}^{\prime}$ et $\tilde{y}^{\prime}$ appartiennent à $3 \delta$ - $\operatorname{géod}\left(x^{\prime}, y^{\prime}\right)$ et donc $z$ appartient à $B_{x^{\prime}, y^{\prime}, k+2 d, \tilde{k}-d, l+2 d, \tilde{l}-d}$. Pour conclure la démonstration du lemme 3.39 on applique à $\Delta=d(x, y), \Delta^{\prime}=d\left(x^{\prime}, y^{\prime}\right), n_{k, \tilde{k}, l, \tilde{l}}=\sharp B_{x, y, k, \tilde{k}, l, \tilde{l}}$ et $n_{k, \tilde{k}, l, \tilde{l}}^{\prime}=\sharp B_{x^{\prime}, y^{\prime}, k, \tilde{k}, l, \tilde{l}}$ le sous-lemme suivant.

Sous-lemme 3.41. Soient $d \in \mathbb{N}, C_{1}, C_{2} \in \mathbb{R}_{+}^{*}$. Soit $\varepsilon>0$. Il existe $\Delta_{0} \in \mathbb{N}$ tel que pour $\Delta, \Delta^{\prime} \in \mathbb{N}$ vérifiant $\Delta \geq \Delta_{0},\left|\Delta^{\prime}-\Delta\right| \leq d$ et pour $\left(n_{k, \tilde{k}, l, \tilde{l}}\right)_{k, \tilde{k}, l, \tilde{l} \in\{0, \ldots, \phi(\Delta)\}}$ des éléments de $\mathbb{N} \cap\left[\frac{\Delta}{C_{1}}, C_{2} \Delta\right]$ et $\left(n_{k, \tilde{k}, l, \tilde{l}}^{\prime}\right)_{k, \tilde{k}, l, \tilde{l} \in\left\{0, \ldots, \phi\left(\Delta^{\prime}\right)\right\}}$ des éléments de $\mathbb{N} \cap$ $\left[\frac{\Delta^{\prime}}{C_{1}}, C_{2} \Delta^{\prime}\right]$, tels que

- $(k, \tilde{k}, l, \tilde{l}) \mapsto n_{k, \tilde{k}, l, \tilde{l}}$ soit croissant en $k$ et l et décroissant en $\tilde{k}$ et $\tilde{l}$, 
- $(k, \tilde{k}, l, \tilde{l}) \mapsto n_{k, \tilde{k}, l, \tilde{l}}^{\prime}$ soit croissant en $k$ et l et décroissant en $\tilde{k}$ et $\tilde{l}$,

$-n_{k, \tilde{k}, l, \tilde{l}} \leq n_{k+2 d, \tilde{k}-d, l+2 d, \tilde{l}-d}^{\prime}$ et $n_{k, \tilde{k}, l, \tilde{l}}^{\prime} \leq n_{k+2 d, \tilde{k}-d, l+2 d, \tilde{l}-d}$ quand ces nombres ont un sens,

alors la proportion de $(k, \tilde{k}, l, \tilde{l})$ dans $\{0, \ldots, \phi(\Delta)\}^{4}$ tels que l'expression $\frac{n_{k, \tilde{k}, l, \tilde{l}}}{n_{k+2 d, \tilde{k}-d, l+2 d, \tilde{l}-d}^{\prime}}$ ait un sens et appartienne à $[1-\varepsilon, 1]$ est supérieure ou égale $\grave{a} 1-\varepsilon$.

Démonstration. La démonstration est facile, et laissée au lecteur car ce sous-lemme ne servira pas dans la suite.

Fin de la démonstration du lemme 3.39. Si $A$ et $B$ sont deux parties finies de $X$ avec $A \subset B$ et $\sharp A / \sharp B \geq 1-\varepsilon$ on a $\left\|v_{A}-v_{B}\right\|_{1} \leq 2 \varepsilon$. Le lemme 3.39 en résulte facilement.

Nous pourrions poser $\rho_{x}^{b}(y)=d^{\prime}(x, y)$ mais cela ne nous convient pas car $d^{\prime}(x, y)$ fait intervenir une moyenne sur $B_{x, y, k, \tilde{k}, l, \tilde{l}}$, donc nécessite de connaître le cardinal de cet ensemble. Nous allons construire $d^{b}$ jouissant de propriétés analogues à celles de $d^{\prime}$ mais telle que $\left(d^{\mathrm{b}}-d\right)(x, y)$ soit une moyenne (sur un ensemble d'indices ne dépendant que de $d(x, y))$ d'une fonction à valeurs dans $[0,7 \delta]$, qui ne dépend (c'est là le point important) que de la connaissance d'un nombre de points borné par $C(\delta, K)$ et des distances entre eux. Plus précisément ces points seront $x, y$, les $z \in 3 \delta$-géod $(x, y)$ vérifiant $d(x, z)=r$ et les $t \in 3 \delta$ - géod $(x, y)$ vérifiant $d(y, t)=s$, pour $3(3 \delta+1)$ valeurs différentes de $r$ et de $s$. Nous définirons alors $\rho_{x}^{b}$ en posant $\rho_{x}^{b}(y)=d^{b}(x, y)$. Le lecteur peut donc oublier la construction de $d^{\prime}$ qui précède, c'est-à-dire la proposition 3.38 et les lemmes 3.39 et 3.41 (en revanche le lemme 3.40 sera réutilisé). L'idée de la construction de $d^{b}$ est de remplacer la moyenne par $\mu(x, y)$ qui sert à construire $d^{\prime}$ dans la formule (23) de la preuve de la proposition 3.38 par une moyenne sur un "point virtuel" défini comme la donnée de ses "distances" à certains autres points. Ces distances seront à valeurs dans $\mathbb{Z}$ et de plus on quotientera par une action naturelle de $\mathbb{Z}$ consistant à ajouter un entier relatif à certaines distances et à le retrancher aux autres. En particulier les distances d'un point virtuel à $x$ et $y$ ne seront pas bien définies, mais leur somme le sera. Les ensembles de points virtuels auront les deux propriétés suivantes : être de cardinal $\leq C(\delta, K)$ et même temps varier très peu souvent lorsqu'on bouge un peu $x$ ou $y$. Ces deux propriétés seraient contradictoires pour des points réels (dans la preuve du lemme 3.39 c'est la deuxième propriété qui est en défaut car le cardinal du support de $\mu(x, y)$ tend vers l'infini quand $d(x, y)$ tend vers l'infini).

La fonction $d^{b}: X \times X \rightarrow \mathbb{R}_{+}$sera définie dans la formule (26) ci-dessous. Jusqu'à (26) on suppose $d(x, y) \geq 6 \delta$.

Pour tout entier $r \in\left\{0, \ldots, \mathrm{E}\left(\frac{d(x, y)}{2}\right)-3 \delta\right\}$, on note $Y_{x, y}^{r}$ l'ensemble des points 
$z \in 3 \delta$ - géod $(x, y)$ tels que

$$
d(x, z) \in\{r, \ldots, r+3 \delta\} .
$$

Lemme 3.42. a) Etant donnés trois entiers $r, r^{\prime}, r^{\prime \prime} \in\left\{0, \ldots, \mathrm{E}\left(\frac{d(x, y)}{2}\right)-3 \delta\right\}$ avec $r \leq r^{\prime} \leq r^{\prime \prime}$, et $z \in Y_{x, y}^{r}$ et $z^{\prime \prime} \in Y_{x, y}^{r^{\prime \prime}}$, géod $\left(z, z^{\prime \prime}\right)$ rencontre $Y_{x, y}^{r^{\prime}}$.

b) Etant donnés deux entiers $r, r^{\prime} \in\left\{0, \ldots, \mathrm{E}\left(\frac{d(x, y)}{2}\right)-3 \delta\right\}$ avec $r \leq r^{\prime}$, et $z^{\prime} \in Y_{x, y}^{r^{\prime}}, \operatorname{géod}\left(x, z^{\prime}\right)$ rencontre $Y_{x, y}^{r}$.

Démonstration. Pour a), on doit montrer l'existence de $z^{\prime} \in \operatorname{géod}\left(z, z^{\prime \prime}\right)$ appartenant à $Y_{x, y}^{r^{\prime}}$. Si $z$ ou $z^{\prime \prime}$ appartient à $Y_{x, y}^{r^{\prime}}$ on prend $z^{\prime}=z$ ou $z^{\prime}=z^{\prime \prime}$. Supposons donc $d(x, z)<r^{\prime}$ et $d\left(x, z^{\prime \prime}\right)>r^{\prime}+3 \delta$. Soit $z^{\prime} \in \operatorname{géod}\left(z, z^{\prime \prime}\right)$ tel que $d\left(x, z^{\prime}\right)=$ $r^{\prime}+\mathrm{E}(3 \delta / 2)$ (un tel $z^{\prime}$ existe car lorsque $z^{\prime}$ parcourt géod $\left(z, z^{\prime \prime}\right)$ les valeurs prises par $d\left(x, z^{\prime}\right)$ forment un intervalle de $\left.\mathbb{N}\right)$. On a $d\left(z, z^{\prime}\right) \geq 3 \delta / 2$ et $d\left(z^{\prime}, z^{\prime \prime}\right) \geq 3 \delta / 2$ donc le lemme 3.40 implique $z^{\prime} \in 3 \delta$ - $\operatorname{géod}(x, y)$. Pour b) on doit montrer l'existence de $z \in \operatorname{géod}\left(x, z^{\prime}\right)$ appartenant à $Y_{x, y}^{r}$. Si $d\left(x, z^{\prime}\right) \leq r+3 \delta$ on prend $z=z^{\prime}$. Sinon soit $z \in \operatorname{géod}\left(x, z^{\prime}\right)$ tel que $d(x, z)=r$. Comme $d\left(z, z^{\prime}\right) \geq 3 \delta$, le lemme 3.10 montre que $z$ appartient à $\delta$ - géod $(x, y)$ et donc à $Y_{x, y}^{r}$.

Ensuite pour $r, s \in\left\{0, \ldots, \mathrm{E}\left(\frac{d(x, y)}{2}\right)-3 \delta\right\}$ on note $\Lambda_{x, r}^{y, s}$ l'ensemble des familles

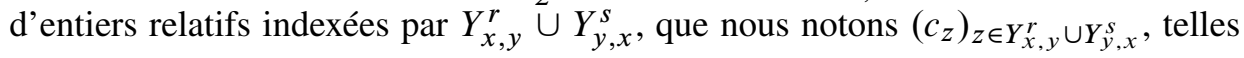
que

$$
\begin{aligned}
\forall z \in Y_{x, y}^{r}, \forall t & \in Y_{y, x}^{s}, \quad c_{z}+c_{t} \geq d(z, t), \\
\forall z, z^{\prime} \in Y_{x, y}^{r}, & \left|c_{z}-c_{z^{\prime}}\right| \leq d\left(z, z^{\prime}\right), \\
\forall t, t^{\prime} \in Y_{y, x}^{s}, & \left|c_{t}-c_{t^{\prime}}\right| \leq d\left(t, t^{\prime}\right) .
\end{aligned}
$$

En quelque sorte pour $z \in Y_{x, y}^{r} \cup Y_{y, x}^{s}, c_{z}$ est la "distance" d'un point virtuel à $z$ (on écrit "distance" car elle appartient à $\mathbb{Z}$ mais pas nécessairement à $\mathbb{N}$ ).

Maintenant nous allons imposer une condition qui impliquera en particulier que le point virtuel "appartient" à $3 \delta$ - géod $(x, y)$. L'idée naïve serait de demander qu'il existe $z \in Y_{x, y}^{r}$ et $t \in Y_{y, x}^{s}$ tels que $c_{z}+c_{t}=d(z, t)$, c'est-à-dire que le point virtuel "appartient" à géod $(z, t)$. Cependant cette condition nous empêcherait d'appliquer l'astuce des ensembles emboîtés. En effet on peut définir une application $\alpha_{r^{\prime} \leftarrow r}^{s^{\prime} \leftarrow s}: \Lambda_{x, r}^{y, s} \rightarrow \Lambda_{x, r^{\prime}}^{y, s^{\prime}}$, pour $r, r^{\prime}, s, s^{\prime}$ vérifiant

$$
0 \leq r^{\prime} \leq r \leq \mathrm{E}\left(\frac{d(x, y)}{2}\right)-3 \delta \quad \text { et } \quad 0 \leq s^{\prime} \leq s \leq \mathrm{E}\left(\frac{d(x, y)}{2}\right)-3 \delta,
$$

de la façon suivante : à $c \in \Lambda_{x, r}^{y, s}$ on associe $c^{\prime}=\alpha_{r^{\prime} \leftarrow r}^{s^{\prime} \leftarrow s}(c) \in \Lambda_{x, r^{\prime}}^{y, s^{\prime}}$ tel que

$$
\begin{aligned}
\text { pour } z^{\prime} \in Y_{x, y}^{r^{\prime}}, c_{z^{\prime}}^{\prime} & =\min _{z \in Y_{x, y}^{r}} d\left(z^{\prime}, z\right)+c_{z}, \\
\text { et pour } t^{\prime} \in Y_{y, x}^{s^{\prime}}, c_{t^{\prime}}^{\prime} & =\min _{t \in Y_{y, x}^{s}} d\left(t^{\prime}, t\right)+c_{t} .
\end{aligned}
$$


En d'autres termes, pour définir $\alpha_{r^{\prime} \leftarrow r}^{s^{\prime} \leftarrow s}$ on fait comme si la géodésique entre le point virtuel et tout point de $Y_{x, y}^{r^{\prime}}\left(\right.$ resp. $Y_{y, x}^{s^{\prime}}$ ) rencontrait $Y_{x, y}^{r}$ (resp. $Y_{y, x}^{s}$ ), comme dans le dessin ci-dessous.

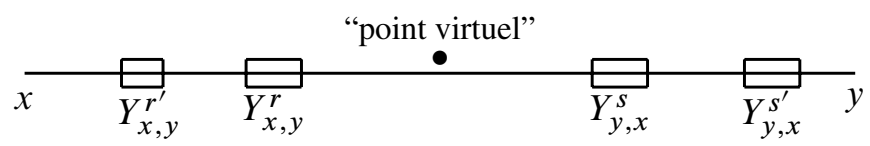

Le a) du lemme 3.42 montre que ces applications se composent bien : si $r, r^{\prime}, r^{\prime \prime}$, $s, s^{\prime}, s^{\prime \prime}$ vérifient

$$
0 \leq r^{\prime \prime} \leq r^{\prime} \leq r \leq \mathrm{E}\left(\frac{d(x, y)}{2}\right)-3 \delta \quad \text { et } \quad 0 \leq s^{\prime \prime} \leq s^{\prime} \leq s \leq \mathrm{E}\left(\frac{d(x, y)}{2}\right)-3 \delta,
$$

on a $\alpha_{r^{\prime \prime}}^{s^{\prime \prime \prime} \leftarrow r^{\prime}} \circ \alpha_{r^{\prime} \leftarrow r}^{s^{\prime} \leftarrow s}=\alpha_{r^{\prime \prime} \leftarrow r}^{s^{\prime \prime} \leftarrow s}$. Si on imposait en plus la condition évoquée cidessus, on ne posséderait plus de telles applications. On va imposer cette condition en d'autres entiers $r^{\prime}, s^{\prime}$, en utilisant précisément l'application $\alpha_{r^{\prime} \leftarrow r}^{s^{\prime} \leftarrow s}$, et dans l'astuce des ensembles emboîtés, $r$ et $r^{\prime}$, respectivement $s$ et $s^{\prime}$, varieront en sens inverse l'un de l'autre (c'est pourquoi on ne peut pas les réunir en une seule variable). Pour $r, r^{\prime}, s, s^{\prime}$ vérifiant (24) on définit donc $\Lambda_{x, r^{\prime}, r}^{y, s^{\prime}, s}$ comme l'ensemble des $c \in \Lambda_{x, r}^{y, s}$ dont l'image $c^{\prime}=\alpha_{r^{\prime} \leftarrow r}^{s^{\prime} \leftarrow s}(c) \in \Lambda_{x, r^{\prime}}^{y, s^{\prime}}$ vérifie la condition introduite ci-dessus : il existe $z^{\prime} \in Y_{x, y}^{r^{\prime}}$ et $t^{\prime} \in Y_{y, x}^{s^{\prime}}$ tels que $c_{z^{\prime}}^{\prime}+c_{t^{\prime}}^{\prime}=d\left(z^{\prime}, t^{\prime}\right)$.

Lemme 3.43. Pour $r, s \in\left\{0, \ldots, \mathrm{E}\left(\frac{d(x, y)}{2}\right)-3 \delta\right\}$ on a des inclusions

$$
\begin{aligned}
& \Lambda_{x, 0, r}^{y, 0, s} \subset \cdots \subset \Lambda_{x, r-1, r}^{y, 0, s} \subset \Lambda_{x, r, r}^{y, 0, s} \\
& \cap \cap \cap \cap \\
& \cap \cap \cap \cap \cap \\
& \Lambda_{x, 0, r}^{y, s-1, s} \subset \cdots \subset \Lambda_{x, r-1, r}^{y, s-1, s} \subset \Lambda_{x, r, r}^{y, s-1, s} \\
& \cap \cap \cdots \quad \cap \quad \cap \\
& \Lambda_{x, 0, r}^{y, s, s} \subset \cdots \subset \Lambda_{x, r-1, r}^{y, s, s} \subset \Lambda_{x, r, r}^{y, s, s}
\end{aligned}
$$

dans $\Lambda_{x, r}^{y, s}$ et toutes ces parties sont non vides.

Démonstration. Soit $r^{\prime} \in\{0, \ldots, r-1\}, s^{\prime} \in\{0, \ldots, s\}$ et montrons l'inclusion horizontale $\Lambda_{x, r^{\prime}, r}^{y, s^{\prime}, s} \subset \Lambda_{x, r^{\prime}+1, r}^{y, s^{\prime}, s}$. Soit $c \in \Lambda_{x, r^{\prime}, r}^{y, s^{\prime}, s}$. On note $c^{\prime}=\alpha_{r^{\prime} \leftarrow r}^{s^{\prime} \leftarrow s}(c) \in \Lambda_{x, r^{\prime}}^{y, s^{\prime}}$ et $c^{\prime \prime}=\alpha_{r^{\prime}+1 \leftarrow r}^{s^{\prime} \leftarrow s}(c) \in \Lambda_{x, r^{\prime}+1}^{y, s^{\prime}}$, si bien que $c^{\prime}=\alpha_{r^{\prime} \leftarrow r^{\prime}+1}^{s^{\prime} \leftarrow s^{\prime}}\left(c^{\prime \prime}\right)$. Par hypothèse il existe $u^{\prime} \in Y_{x, y}^{r^{\prime}}$ et $v^{\prime} \in Y_{y, x}^{s^{\prime}}$ tels que $c_{u^{\prime}}^{\prime}+c_{v^{\prime}}^{\prime}=d\left(u^{\prime}, v^{\prime}\right)$. Par définition de l'application 
$\alpha_{r^{\prime} \leftarrow r^{\prime}+1}^{s^{\prime} \leftarrow s^{\prime}}$ il existe $u^{\prime \prime} \in Y_{x, y}^{r^{\prime}+1}$ tel que $c_{u^{\prime}}^{\prime}=c_{u^{\prime \prime}}^{\prime \prime}+d\left(u^{\prime}, u^{\prime \prime}\right)$. Les inégalités

$$
\begin{aligned}
d\left(u^{\prime}, v^{\prime}\right) & =c_{u^{\prime}}^{\prime}+c_{v^{\prime}}^{\prime} \\
& =c_{u^{\prime \prime}}^{\prime \prime}+d\left(u^{\prime}, u^{\prime \prime}\right)+c_{v^{\prime}}^{\prime} \\
& =d\left(u^{\prime}, u^{\prime \prime}\right)+c_{u^{\prime \prime}}^{\prime \prime}+c_{v^{\prime}}^{\prime \prime} \\
& \geq d\left(u^{\prime}, u^{\prime \prime}\right)+d\left(u^{\prime \prime}, v^{\prime}\right) \geq d\left(u^{\prime}, v^{\prime}\right)
\end{aligned}
$$

sont toutes des égalités, donc $c_{u^{\prime \prime}}^{\prime \prime}+c_{v^{\prime}}^{\prime \prime}=d\left(u^{\prime \prime}, v^{\prime}\right)$ et $c$ appartient bien à $\Lambda_{x, r^{\prime}+1, r}^{y, s^{\prime}, s}$. Les inclusions verticales se démontrent de la même manière. Il reste à montrer que $\Lambda_{x, 0, r}^{y, 0, s}$ est non vide. Soit $t \in \operatorname{géod}(x, y)$ tel que $d(x, t) \in[r, d(x, y)-s]$ et soit $c \in \Lambda_{x, r}^{y, s}$ défini par $c_{z}=d(z, t)$ pour $z \in Y_{x, y}^{r} \cup Y_{y, x}^{s}$. Alors $c$ appartient à $\Lambda_{x, 0, r}^{y, 0, s}$ car $c^{\prime}=\alpha_{0 \leftarrow r}^{0 \leftarrow s}(c)$ vérifie $c_{x}^{\prime}=d(x, t)$ et $c_{y}^{\prime}=d(t, y)$ (puisque géod $(x, t)$ rencontre $Y_{x, y}^{r}$ et $\operatorname{géod}(t, y)$ rencontre $\left.Y_{y, x}^{s}\right)$ et donc $c_{x}^{\prime}+c_{y}^{\prime}=d(x, y)$. Par conséquent $\Lambda_{x, 0, r}^{y, 0, s}$ est non vide.

Enfin étant donné $r_{1}, r_{2}, r_{3}, s_{1}, s_{2}, s_{3}$ vérifiant

$$
0 \leq r_{1} \leq r_{2} \leq r_{3} \leq \mathrm{E}\left(\frac{d(x, y)}{2}\right)-3 \delta \quad \text { et } \quad 0 \leq s_{1} \leq s_{2} \leq s_{3} \leq \mathrm{E}\left(\frac{d(x, y)}{2}\right)-3 \delta,
$$

on définit $\Lambda_{x, r_{1}, r_{2}, r_{3}}^{y, s_{1}, s_{2}, s_{3}}$ comme l'image de $\Lambda_{x, r_{2}, r_{3}}^{y, s_{2}, s_{3}}$ dans $\Lambda_{x, r_{1}}^{y, s_{1}}$ par l'application $\alpha_{r_{1} \leftarrow r_{3}}^{s_{1} \leftarrow s_{3}}$. Pour tout entier $r \in\left\{0, \ldots, \mathrm{E}\left(\frac{d(x, y)}{2}\right)\right\}-3 \delta$ on a une application $\beta_{x, r}^{y, s}: \Lambda_{x, r}^{y, s} \rightarrow \mathbb{N}$ définie par

$$
\begin{aligned}
\beta_{x, r}^{y, s}(c) & =\min _{z \in Y_{x, y}^{r}, t \in Y_{y, x}^{s}} d(x, z)+c_{z}+c_{t}+d(t, y) \\
& =\min _{z \in Y_{x, y}^{r}}\left(d(x, z)+c_{z}\right)+\min _{t \in Y_{y, x}^{s}}\left(c_{t}+d(t, y)\right) .
\end{aligned}
$$

On doit comprendre $\min _{z \in Y_{x, y}^{r}}\left(d(x, z)+c_{z}\right)$, respectivement $\min _{t \in Y_{y, x}^{s}}\left(c_{t}+d(t, y)\right)$, comme la "distance" de $x$, respectivement $y$, au point virtuel. Si $r, r^{\prime}, s, s^{\prime}$ sont des entiers vérifiant (24), on a $\beta_{x, r^{\prime}}^{y, s^{\prime}} \circ \alpha_{r^{\prime} \leftarrow r}^{s^{\prime} \leftarrow s}=\beta_{x, r}^{y, s}$ par le b) du lemme 3.42. Cette application $\beta_{x, r}^{y, s}$ se factorise par $\tilde{\Lambda}_{x, r}^{y, s}$, où $\tilde{\Lambda}_{x, r}^{y, s}$ est le quotient de $\Lambda_{x, r}^{y, s}$ par la relation d'équivalence suivante : deux éléments $c$ et $c^{\prime}$ sont équivalents s'il existe $k \in \mathbb{Z}$ avec $c^{\prime}(z)=c(z)+k$ pour $z \in Y_{x, y}^{r}$ et $c^{\prime}(t)=c(t)-k$ pour $t \in Y_{y, x}^{s}$. Toutes les constructions ci-dessus passent au quotient de cette façon et on note en particulier $\tilde{\Lambda}_{x, r_{1}, r_{2}, r_{3}}^{y, s_{1}, s_{2}, s_{3}}$ l'image de $\tilde{\Lambda}_{x, r_{2}, r_{3}}^{y, s_{2}, s_{3}}$ dans $\tilde{\Lambda}_{x, r_{1}}^{y, s_{1}}$ (qui est aussi l'image de $\Lambda_{x, r_{2}, r_{3}}^{y, s_{2}, s_{3}}$ dans $\tilde{\Lambda}_{x, r_{1}}^{y, s_{1}}$ ). On remarque que si $r, r^{\prime}, s, s^{\prime}$ vérifient (24), $\tilde{\Lambda}_{x, r^{\prime}, r}^{y, s^{\prime}, s}$ est un ensemble fini, dont le cardinal est borné par une constante de la forme $C(\delta, K)$. Il en va donc de même pour $\tilde{\Lambda}_{x, r_{1}, r_{2}, r_{3}}^{y, s_{1}, s_{2}, s_{3}}$, lorsque $r_{1}, r_{2}, r_{3}, s_{1}, s_{2}, s_{3}$ vérifient (25). Grâce à $\alpha_{r_{1}-1 \leftarrow r_{1}}^{s_{1} \leftarrow s_{1}}, \alpha_{r_{1} \leftarrow r_{1}}^{s_{1}-1 \leftarrow s_{1}}, \alpha_{r_{3}-1 \leftarrow r_{3}}^{s_{3} \leftarrow s_{3}}, \alpha_{r_{3} \leftarrow r_{3}}^{s_{3}-1 \leftarrow s_{3}}$ et au lemme 3.43 on possède, pour $\left(r_{1}, r_{2}, r_{3}, s_{1}, s_{2}, s_{3}\right)$ vérifiant (25),

- une application surjective $\tilde{\Lambda}_{x, r_{1}, r_{2}, r_{3}}^{y, s_{1}, s_{2}, s_{3}} \rightarrow \tilde{\Lambda}_{x, r_{1}-1, r_{2}, r_{3}}^{y, s_{1}, s_{2}, s_{3}}$ si $r_{1} \geq 1$, 
- une application surjective $\tilde{\Lambda}_{x, r_{1}, r_{2}, r_{3}}^{y, s_{1}, s_{2}, s_{3}} \rightarrow \tilde{\Lambda}_{x, r_{1}, r_{2}, r_{3}}^{y, s_{1}-1, s_{2}, s_{3}}$ si $s_{1} \geq 1$,

- des applications injectives

$$
\tilde{\Lambda}_{x, r_{1}, r_{2}, r_{3}}^{y, s_{1}, s_{2}, s_{3}} \rightarrow \tilde{\Lambda}_{x, r_{1}, r_{2}+1, r_{3}}^{y, s_{1}, s_{2}, s_{3}} \quad \text { et } \quad \tilde{\Lambda}_{x, r_{1}, r_{2}, r_{3}}^{y, s_{1}, s_{2}, s_{3}} \rightarrow \tilde{\Lambda}_{x, r_{1}, r_{2}, r_{3}-1}^{y, s_{1}, s_{2}, s_{3}}
$$

si $r_{2}<r_{3}$,

- des applications injectives

$$
\tilde{\Lambda}_{x, r_{1}, r_{2}, r_{3}}^{y, s_{1}, s_{2}, s_{3}} \rightarrow \tilde{\Lambda}_{x, r_{1}, r_{2}, r_{3}}^{y, s_{1}, s_{2}+1, s_{3}} \quad \text { et } \quad \tilde{\Lambda}_{x, r_{1}, r_{2}, r_{3}}^{y, s_{1}, s_{2}, s_{3}} \rightarrow \tilde{\Lambda}_{x, r_{1}, r_{2}, r_{3}}^{y, s_{1}, s_{2}, s_{3}-1}
$$

si $s_{2}<s_{3}$.

De plus ces applications sont compatibles entre elles.

On est maintenant en mesure de construire $d^{b}$. On pose $d^{b}(x, y)=d(x, y)$ si $d(x, y)<6 \delta$ et si $d(x, y) \geq 6 \delta$ on pose $\Delta_{x, y}=\mathrm{E}(d(x, y) / 6)-\delta$ et on définit

$$
d^{b}(x, y)=\frac{1}{\left(\Delta_{x, y}+1\right)^{6}} \sum_{\substack{r_{1}, s_{1} \in\{0, \ldots, \Delta x, y\} \\ r_{2}, s_{2} \in\left\{\Delta x, y, \ldots, 2 \Delta_{x}, y\right\} \\ r_{3}, s_{3} \in\left\{2 \Delta x, y, 3 \Delta_{x, y\}}\right.}} \frac{1}{\sharp\left(\tilde{\Lambda}_{x, r_{1}, r_{2}, r_{3}}^{y, s_{1}, s_{2}, s_{3}}\right)} \sum_{c \in \tilde{\Lambda}_{x, r_{1}, r_{2}, r_{3}}^{y, s_{1}, s_{2}, r_{3}}} \beta_{x, r_{1}}^{y, s_{1}}(c) .
$$

Pour $u_{1}, u_{2}, u_{3}, v_{1}, v_{2}, v_{3} \in\left[0,1\left[\right.\right.$ et $x, y \in X$ on pose $d_{u_{1}, u_{2}, u_{3}}^{b_{1}, v_{2}, v_{3}}(x, y)=$ $d(x, y)$ si $d(x, y)<6 \delta$ et si $d(x, y) \geq 6 \delta$ on pose

$$
A_{x, u_{1}, u_{2}, u_{3}}^{y, v_{1}, v_{2}, v_{3}}=\tilde{\Lambda}_{x, \mathrm{E}\left(\left(\Delta_{x, y}+1\right) u_{1}\right), \Delta_{x, y}+\mathrm{E}\left(\left(\Delta_{x, y}+1\right) u_{2}\right), 2 \Delta_{x, y}+\mathrm{E}\left(\left(\Delta_{x, y}+1\right) u_{3}\right)}^{y, \mathrm{E}\left(\left(\Delta_{x, y}+1\right) v_{1}\right), \Delta_{x, y}+\mathrm{E}\left(\left(\Delta_{x, y}+1\right) v_{2}\right), 2 \Delta_{x, y}+\mathrm{E}\left(\left(\Delta_{x, y}+1\right) v_{3}\right)},
$$

et

$$
d^{\mathrm{b}}{ }_{u_{1}, u_{2}, u_{3}}^{v_{1}, v_{2}, v_{3}}(x, y)=\frac{1}{\sharp\left(A_{x, u_{1}, u_{2}, u_{3}}^{y, v_{1}, v_{2}, v_{3}}\right)} \sum_{c \in A_{x, u_{1}, u_{2}, u_{3}}^{y, v_{1}, v_{2}, v_{3}}} \beta_{x, \mathrm{E}\left(\left(\Delta_{x, y}+1\right) u_{1}\right)}^{y, \mathrm{E}\left(\left(\Delta_{x, y}+1\right) v_{1}\right)}(c)
$$

de sorte que d'après la formule (26) on a toujours

$$
d^{\mathrm{b}}(x, y)=\int_{u_{1}, u_{2}, u_{3}, v_{1}, v_{2}, v_{3} \in[0,1[} d_{u_{1}, u_{2}, u_{3}}^{v_{1}, v_{2}, v_{3}}(x, y) d u_{1} d u_{2} d u_{3} d v_{1} d v_{2} d v_{3}
$$

Dans la formule précédente on intègre sur $\left[0,1\left[^{6}\right.\right.$ au lieu de $[0,1]^{6}$ car $1^{\text {'expression }}$ pourrait ne pas avoir de sens pour $u_{1}=1$ et $u_{2}=0$ par exemple.

Lemme 3.44. a) Pour $r_{1}, s_{1} \in\left\{0, \ldots, \Delta_{x, y}\right\}, r_{2}, s_{2} \in\left\{\Delta_{x, y}, \ldots, 2 \Delta_{x, y}\right\}, r_{3}, s_{3} \in$ $\left\{2 \Delta_{x, y}, \ldots, 3 \Delta_{x, y}\right\}, c \in \tilde{\Lambda}_{x, r_{1}, r_{2}, r_{3}}^{y, s_{1}, s_{2}, s_{3}}$ on a $\beta_{x, r_{1}}^{y, s_{1}}(c) \in[d(x, y), d(x, y)+7 \delta]$.

b) Pour $u_{1}, u_{2}, u_{3}, v_{1}, v_{2}, v_{3} \in[0,1[$ et $x, y \in X$ on $a$

$$
d(x, y) \leq d_{u_{1}, u_{2}, u_{3}}^{v_{1}, v_{2}, v_{3}}(x, y) \leq d(x, y)+7 \delta .
$$


Démonstration. On montre seulement a) car b) en résulte immédiatement. Il existe $z \in Y_{x, y}^{r_{2}}$ et $t \in Y_{y, x}^{s_{2}}$ tels que $\beta_{x, r_{1}}^{y, s_{1}}(c) \leq d(x, z)+d(z, t)+d(t, y)$. On rappelle que $z, t$ appartiennent à $3 \delta$ - $\operatorname{géod}(x, y)$ et que $d(x, z) \leq \frac{d(x, y)}{2}$ et $d(t, y) \leq \frac{d(x, y)}{2}$. $\operatorname{Par}\left(H_{\delta}(z, x, t, y)\right)$ on a

$$
\begin{aligned}
d(z, t)+d(x, y) & \leq \max (d(x, z)+d(t, y), d(x, t)+d(z, y))+\delta \\
& =d(x, t)+d(z, y)+\delta
\end{aligned}
$$

d'où

$$
\begin{aligned}
d(x, z)+d(z, t)+d(t, y) & \leq d(x, z)+d(x, t)+d(z, y)+d(t, y)+\delta-d(x, y) \\
& \leq d(x, y)+7 \delta .
\end{aligned}
$$

Lemme 3.45. Pour tout $\rho \in \mathbb{N}$, il existe $C=C(\delta, K, \rho)$ tel que pour $x, x^{\prime}, y, y^{\prime} \in X$ verifiant $d\left(x, x^{\prime}\right) \leq \rho$ et $d\left(y, y^{\prime}\right) \leq \rho$ la mesure de l'ensemble des $\left(u_{1}, u_{2}, u_{3}, v_{1}\right.$, $\left.v_{2}, v_{3}\right) \in\left[0,1\left[{ }^{6}\right.\right.$ tels que

$$
\begin{aligned}
& d_{u_{1}, u_{2}, u_{3}}^{b^{v_{1}, v_{2}, v_{3}}}(x, y)-d^{\mathrm{b}}{ }_{u_{1}, u_{2}, u_{3}}^{v_{1}, v_{2}, v_{3}}\left(x^{\prime}, y\right)-d^{\mathrm{b}}{ }_{u_{1}, u_{2}, u_{3}}^{v_{1}, v_{2}, v_{3}}\left(x, y^{\prime}\right)+d^{\mathrm{b}}{ }_{u_{1}, u_{2}, u_{3}}^{v_{1}, v_{2}, v_{3}}\left(x^{\prime}, y^{\prime}\right) \neq 0 \\
& \text { est } \leq \frac{C}{1+d(x, y)} .
\end{aligned}
$$

Démonstration. La démonstration consiste encore en une astuce d'ensembles emboîtés.

Sous-lemme 3.46. Soient $x, y, \tilde{x}, \tilde{y} \in X$ et $d \geq 2(d(x, \tilde{x})+d(y, \tilde{y})+3 \delta)$.

a) Etant donnés $r, r^{\prime}, r^{\prime \prime} \in\left\{0, \ldots, \mathrm{E}\left(\frac{d(x, y)}{2}\right)-3 \delta\right\}$ tels que $r+d \leq r^{\prime} \leq r^{\prime \prime}-d$, $z \in Y_{x, y}^{r}$ et $z^{\prime \prime} \in Y_{x, y}^{r^{\prime \prime}}$, géod $\left(z, z^{\prime \prime}\right)$ rencontre $Y_{\tilde{x}, \tilde{y}}^{r^{\prime}}$.

b) Etant donnés $r, r^{\prime}, r^{\prime \prime} \in\left\{0, \ldots, \mathrm{E}\left(\frac{d(x, y)}{2}\right)-3 \delta\right\}$ tels que $r \leq r^{\prime} \leq r^{\prime \prime}-d$, $z \in Y_{\tilde{x}, \tilde{y}}^{r}$ et $z^{\prime \prime} \in Y_{x, y}^{r^{\prime \prime}}$, géod $\left(z, z^{\prime \prime}\right)$ rencontre $Y_{\tilde{x}, \tilde{y}}^{r^{\prime}}$.

c) Etant donnés $r, r^{\prime}, r^{\prime \prime} \in\left\{0, \ldots, \mathrm{E}\left(\frac{d(x, y)}{2}\right)-3 \delta\right\}$ tels que $r+d \leq r^{\prime} \leq r^{\prime \prime}$, $z \in Y_{\tilde{x}, \tilde{y}}^{r}$ et $z^{\prime \prime} \in Y_{x, y}^{r^{\prime \prime}}$, $\operatorname{géod}\left(z, z^{\prime \prime}\right)$ rencontre $Y_{x, y}^{r^{\prime}}$.

d) Etant donnés $r, r^{\prime} \in\left\{0, \ldots, \mathrm{E}\left(\frac{d(x, y)}{2}\right)-3 \delta\right\}$ tels que $r+d \leq r^{\prime}, z^{\prime} \in Y_{x, y}^{r^{\prime}}$, $\operatorname{géod}\left(\tilde{x}, z^{\prime}\right)$ rencontre $Y_{\tilde{x}, \tilde{y}}^{r}$.

Démonstration. Pour a) on prend $z^{\prime} \in \operatorname{géod}\left(z, z^{\prime \prime}\right)$ tel que $d\left(\tilde{x}, z^{\prime}\right)=r^{\prime}$. Cela est possible car $d(\tilde{x}, z) \leq d(x, \tilde{x})+r+3 \delta \leq r^{\prime}-\frac{d}{2}$ et $d\left(\tilde{x}, z^{\prime \prime}\right) \geq r^{\prime \prime}-d(x, \tilde{x}) \geq r^{\prime}+\frac{d}{2}$. On a $z, z^{\prime \prime} \in(d-3 \delta)$ - $\operatorname{géod}(\tilde{x}, \tilde{y})$ par le lemme 3.2 et $d\left(z, z^{\prime}\right) \geq \frac{d}{2}, d\left(z^{\prime}, z^{\prime \prime}\right) \geq \frac{d}{2}$ ce qui permet d'appliquer le lemme 3.40 avec $\alpha=\beta=d-3 \delta$. D'où $z^{\prime} \in Y_{\tilde{x}, \tilde{y}}^{r^{\prime}}$.

Pour b) on prend $z^{\prime}=z$ si $d(\tilde{x}, z) \geq r^{\prime}$ donc on suppose $d(\tilde{x}, z)<r^{\prime}$. On a

$$
d\left(\tilde{x}, z^{\prime \prime}\right) \geq d\left(x, z^{\prime \prime}\right)-d(x, \tilde{x}) \geq r^{\prime \prime}-\frac{d}{2}+3 \delta \geq r^{\prime}+\frac{d}{2}+3 \delta
$$

donc il existe $z^{\prime} \in \operatorname{géod}\left(z, z^{\prime \prime}\right)$ tel que $d\left(\tilde{x}, z^{\prime}\right)=r^{\prime}+3 \delta$. On a alors $z \in 3 \delta$ - $\operatorname{géod}(\tilde{x}, \tilde{y})$, $z^{\prime \prime} \in(d-3 \delta)-\operatorname{géod}(\tilde{x}, \tilde{y}), d\left(z, z^{\prime}\right) \geq 3 \delta$ et $d\left(z^{\prime}, z^{\prime \prime}\right) \geq \frac{d}{2}$, ce qui permet d'appliquer le lemme 3.40 avec $\alpha=3 \delta$ et $\beta=d-3 \delta$. D'où $z^{\prime} \in Y_{\tilde{x}, \tilde{y}}^{r^{\prime}}$. 
Pour c) on prend $z^{\prime}=z^{\prime \prime} \operatorname{si} d\left(x, z^{\prime \prime}\right) \leq r^{\prime}+3 \delta$ donc on suppose $d\left(x, z^{\prime \prime}\right)>r^{\prime}+3 \delta$. On a

$$
d(x, z) \leq d(x, \tilde{x})+d(\tilde{x}, z) \leq r+3 \delta+d(x, \tilde{x}) \leq r+\frac{d}{2} \leq r^{\prime}-\frac{d}{2} .
$$

Donc il existe $z^{\prime} \in \operatorname{géod}\left(z, z^{\prime \prime}\right)$ tel que $d\left(x, z^{\prime}\right)=r^{\prime}$. On a $z \in(d-3 \delta)$ - $\operatorname{géod}(x, y)$, $z^{\prime \prime} \in 3 \delta$ - $\operatorname{géod}(x, y), d\left(z, z^{\prime}\right) \geq \frac{d}{2}, d\left(z^{\prime}, z^{\prime \prime}\right) \geq 3 \delta$ ce qui permet d'appliquer le lemme 3.40 avec $\alpha=d-3 \delta$ et $\beta=3 \delta$. D'où $z^{\prime} \in Y_{x, y}^{r^{\prime}}$.

Pour d) on prend $z \in \operatorname{géod}\left(\tilde{x}, z^{\prime}\right)$ vérifiant $d(\tilde{x}, z)=r$. Cela est possible car $d\left(\tilde{x}, z^{\prime}\right) \geq d\left(x, z^{\prime}\right)-d(x, \tilde{x}) \geq r^{\prime}-\frac{d}{2} \geq r+\frac{d}{2}$. Comme $z^{\prime} \in(d-3 \delta)-\operatorname{géod}(\tilde{x}, \tilde{y})$, $z \in \operatorname{géod}\left(\tilde{x}, z^{\prime}\right)$ et $d\left(z, z^{\prime}\right) \geq \frac{d}{2}$, le lemme 3.10 appliqué à $\varepsilon=d-3 \delta$ montre $z \in 3 \delta-\operatorname{géod}(\tilde{x}, \tilde{y})$ d'où $z \in Y_{\tilde{x}, \tilde{y}}^{r}$.

Suite de la démonstration du lemme 3.45. Pour $x, y, \tilde{x}, \tilde{y} \in X$ et $d \geq 2(d(x, \tilde{x})+$ $d(y, \tilde{y})+3 \delta)$ comme dans le sous-lemme 3.46, et pour $r, s \in\left\{d, \ldots, \mathrm{E}\left(\frac{d(x, y)}{2}\right)-3 \delta\right\}$, on a une application $\gamma: \Lambda_{x, r}^{y, s} \rightarrow \Lambda_{\tilde{x}, r-d}^{\tilde{y}, s-d}$ qui envoie $c$ sur $c^{\prime}$ défini par

$$
c_{z^{\prime}}^{\prime}=\min _{z \in Y_{x, y}^{r}} c_{z}+d\left(z^{\prime}, z\right) \quad \text { pour } z^{\prime} \in Y_{\tilde{x}, \tilde{y}}^{r-d}
$$

et

$$
c_{t^{\prime}}^{\prime}=\min _{t \in Y_{y, x}^{s}} c_{t}+d\left(t^{\prime}, t\right) \quad \operatorname{pour} t^{\prime} \in Y_{\tilde{y}, \tilde{x}}^{s-d}
$$

On note $\gamma$ sans indices pour alléger les formules car les indices sont déterminés par l'ensemble de départ et l'ensemble d'arrivée. De la même façon, pour $r, s \in$ $\left\{d, \ldots, \mathrm{E}\left(\frac{d(\tilde{x}, \tilde{y})}{2}\right)-3 \delta\right\}$, on a une application $\gamma: \Lambda_{\tilde{x}, r}^{\tilde{y}, s} \rightarrow \Lambda_{x, r-d}^{y, s-d}$. Ces applications sont compatibles avec les applications $\alpha_{r^{\prime} \leftarrow r}^{s^{\prime} \leftarrow s}$ (associées à $(x, y)$ et $\left.(\tilde{x}, \tilde{y})\right)$. Par exemple, lorsque les applications ont un sens,

- la composée $\Lambda_{x, r}^{y, s} \stackrel{\gamma}{\rightarrow} \Lambda_{\tilde{x}, r-d}^{\tilde{y}, s-d} \stackrel{\gamma}{\rightarrow} \Lambda_{x, r-2 d}^{y, s-2 d}$ coïncide avec $\alpha_{r-2 d \leftarrow r}^{s-2 d \leftarrow s}$ (grâce à a) du sous-lemme 3.46),

- les deux composées

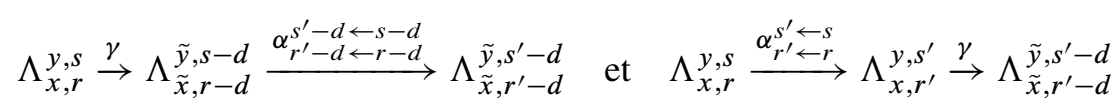

sont égales car, grâce à b) et c) du sous-lemme 3.46 l'image $c^{\prime} \in \Lambda_{\tilde{x}, r^{\prime}-d}^{\tilde{y}, s^{\prime}-d}$ de $c \in \Lambda_{x, r}^{y, s}$ par chacune de ces deux composées est donnée par

$$
c_{z^{\prime}}^{\prime}=\min _{z \in Y_{x, y}^{r}} c_{z}+d\left(z^{\prime}, z\right) \quad \text { pour } z^{\prime} \in Y_{\tilde{x}, \tilde{y}}^{r^{\prime}}
$$

et

$$
c_{t^{\prime}}^{\prime}=\min _{t \in Y_{y, x}^{s}} c_{t}+d\left(t^{\prime}, t\right) \quad \text { pour } t^{\prime} \in Y_{\tilde{y}, \tilde{x}}^{s^{\prime}-d}
$$


Plus généralement la compatibilité signifie que deux composées construites à partir des applications $\alpha$ et $\gamma$ sont égales lorsqu'elles possèdent le même ensemble de départ et le même ensemble d'arrivée. En effet on démontre que si l'ensemble de départ est $\Lambda_{\check{x}, \check{r}, \check{r}}^{\check{y}, \check{s}}$ et l'ensemble d'arrivée est $\Lambda_{\hat{x}, \hat{r}}^{\hat{y}, \hat{s}}(\operatorname{avec}(\check{x}, \check{y})$ et $(\hat{x}, \hat{y})$ égaux à $(x, y)$ ou $(\tilde{x}, \tilde{y}))$, les deux composées sont égales à l'application qui à $\check{c} \in \Lambda_{\check{x}, \check{r}}^{\check{y}, \check{s}}$ associe $\hat{c} \in \Lambda_{\hat{x}, \hat{r}}^{\hat{y}, \hat{s}}$ défini par les formules

$$
\hat{c}_{\hat{z}}=\min _{\check{z} \in Y_{\check{x}, \check{y}}^{\check{c}}} \check{c}_{\check{z}}+d(\hat{z}, \check{z}) \quad \text { pour } \hat{z} \in Y_{\hat{x}, \hat{y}}^{\hat{r}}
$$

et

$$
\hat{c}_{\hat{t}}=\min _{\breve{t} \in Y_{\grave{y}, \breve{x}}^{\grave{s}}} \check{c}_{\check{t}}+d(\hat{t}, \check{t}) \quad \operatorname{pour} \hat{t} \in Y_{\hat{y}, \hat{x}}^{\hat{s}}
$$

Sous-lemme 3.47. Pour $r, r^{\prime}, s, s^{\prime}$ comme dans (24) vérifiant $r^{\prime}+2 d \leq r$ et $s^{\prime}+2 d \leq s$, l'application $\Lambda_{x, r}^{y, s} \stackrel{\gamma}{\rightarrow} \Lambda_{\tilde{x}, r-d}^{\tilde{y}, s-d}$ envoie $\Lambda_{x, r^{\prime}, r}^{y, s^{\prime}, s}$ dans $\Lambda_{\tilde{x}, r^{\prime}+d, r-d}^{\tilde{y}, s^{\prime}+d, s-d}$.

Démonstration. D'après ce qui précède, $\alpha_{r^{\prime} \leftarrow r}^{s^{\prime} \leftarrow s}: \Lambda_{x, r}^{y, s} \rightarrow \Lambda_{x, r^{\prime}}^{y, s^{\prime}}$ est la composée

$$
\Lambda_{x, r}^{y, s} \stackrel{\gamma}{\rightarrow} \Lambda_{\tilde{x}, r-d}^{\tilde{y}, s-d} \stackrel{\alpha_{r^{\prime}+d \leftarrow r-d}^{s^{\prime}+d \leftarrow s-d}}{\longrightarrow} \Lambda_{\tilde{x}, r^{\prime}+d}^{\tilde{y}, s^{\prime}+d} \stackrel{\gamma}{\rightarrow} \Lambda_{x, r^{\prime}}^{y, s^{\prime}}
$$

De plus pour $c \in \Lambda_{\tilde{x}, r^{\prime}+d}^{\tilde{y}, s^{\prime}+d}$ d'image $c^{\prime}=\gamma(c) \in \Lambda_{x, r^{\prime}}^{y, s^{\prime}}$, s'il existe $z \in Y_{x, y}^{r^{\prime}}$ et $t \in Y_{y, x}^{s^{\prime}}$ tels que $c_{z}^{\prime}+c_{t}^{\prime}=d(z, t)$, alors il existe $\hat{z} \in Y_{\tilde{x}, \tilde{y}}^{r^{\prime}+d}$ et $\hat{t} \in Y_{\tilde{y}, \tilde{x}}^{s^{\prime}+d}$ tels que $c_{\hat{z}}+c_{\hat{t}}=d(\hat{z}, \hat{t})$ par le même argument que dans la preuve du lemme 3.43.

On déduit du sous-lemme 3.47 que pour $\left(r_{1}, r_{2}, r_{3}, s_{1}, s_{2}, s_{3}\right)$ vérifiant (25) et $\left(\tilde{r}_{1}, \tilde{r}_{2}, \tilde{r}_{3}, \tilde{s}_{1}, \tilde{s}_{2}, \tilde{s}_{3}\right)$ vérifiant

$$
\begin{aligned}
& 0 \leq \tilde{r}_{1} \leq \tilde{r}_{2} \leq \tilde{r}_{3} \leq \mathrm{E}\left(\frac{d(\tilde{x}, \tilde{y})}{2}\right)-3 \delta \text { et } 0 \leq \tilde{s}_{1} \leq \tilde{s}_{2} \leq \tilde{s}_{3} \leq \mathrm{E}\left(\frac{d(\tilde{x}, \tilde{y})}{2}\right)-3 \delta \\
& \text { - si } \tilde{r}_{1} \leq r_{1}-d, \tilde{s}_{1} \leq s_{1}-d, \tilde{r}_{2} \geq r_{2}+d, \tilde{s}_{2} \geq s_{2}+d, \tilde{r}_{3} \leq r_{3}-d, \tilde{s}_{3} \leq s_{3}-d \text {, on }
\end{aligned}
$$

$$
\beta_{\tilde{x}, \tilde{r}_{1}}^{\tilde{y}, \tilde{s}_{1}}(\tilde{c})=\min _{z \in Y_{x, y}^{r_{1}}}\left(d(\tilde{x}, z)+c_{z}\right)+\min _{t \in Y_{y, x}^{s_{1}}}\left(c_{t}+d(t, \tilde{y})\right),
$$

- si $r_{1} \leq \tilde{r}_{1}-d, s_{1} \leq \tilde{s}_{1}-d, r_{2} \geq \tilde{r}_{2}+d, s_{2} \geq \tilde{s}_{2}+d, r_{3} \leq \tilde{r}_{3}-d, s_{3} \leq \tilde{s}_{3}-d$, on a une application $\Lambda_{\tilde{x}, \tilde{r}_{1}, \tilde{r}_{2}, \tilde{r}_{3}}^{\tilde{y}, \tilde{r}_{3}, \tilde{r}_{3}, \tilde{r}_{3}} \stackrel{\gamma}{\rightarrow} \Lambda_{x, r_{1}, r_{2}, r_{3}}^{y, s_{1}, s_{2}, s_{3}}$ verifiant une propriété semblable à (27). 
On rappelle que $\Delta_{x, y}=\mathrm{E}(d(x, y) / 6)-\delta, \Delta_{\tilde{x}, \tilde{y}}=\mathrm{E}(d(\tilde{x}, \tilde{y}) / 6)-\delta$, et que pour $\left(u_{1}, u_{2}, u_{3}, v_{1}, v_{2}, v_{3}\right) \in\left[0,1\left[^{6}\right.\right.$,

$$
\begin{gathered}
A_{x, u_{1}, u_{2}, u_{3}}^{y, v_{1}, v_{2}, v_{3}}=\tilde{\Lambda}_{x, \mathrm{E}\left(\left(\Delta_{x, y}+1\right) u_{1}\right), \Delta_{x, y}+\mathrm{E}\left(\left(\Delta_{x, y}+1\right) u_{2}\right), 2 \Delta_{x, y}+\mathrm{E}\left(\left(\Delta_{x, y}+1\right) u_{3}\right)}^{y, \mathrm{E}\left(\left(\Delta_{x, y}+1\right) v_{1}\right), \Delta_{x, y}+\mathrm{E}\left(\left(\Delta_{x, y}+1\right) v_{2}\right), 2 \Delta_{x, y}+\mathrm{E}\left(\left(\Delta_{x, y}+1\right) v_{3}\right)}, \\
A_{\tilde{x}, u_{1}, u_{2}, u_{3}}^{\tilde{y}, v_{1}, v_{2}, v_{3}}=\tilde{\Lambda}_{\tilde{x}, \mathrm{E}\left(\left(\Delta_{\tilde{x}, \tilde{y}}+1\right) u_{1}\right), \Delta_{\tilde{x}, \tilde{y}}+\mathrm{E}\left(\left(\Delta_{\tilde{x}, \tilde{y}}+1\right) u_{2}\right), 2 \Delta_{\tilde{x}, \tilde{y}}+\mathrm{E}\left(\left(\Delta_{\tilde{x}, \tilde{y}}+1\right) u_{3}\right)}^{\tilde{y}\left(\left(\Delta_{\tilde{x}}\right)\right.} .
\end{gathered}
$$

Soit $\eta \in] 0, \frac{1}{2}\left[\right.$ tel que $d(x, y) \geq \frac{9 d}{\eta}+6 \delta$. On a alors pour tout $\left(u_{1}, u_{2}, u_{3}, v_{1}, v_{2}, v_{3}\right) \in$ $\left[\eta, 1-\eta\left[{ }^{6}\right.\right.$, les six inégalités

$$
\begin{aligned}
\mathrm{E}\left(\left(\Delta_{\tilde{x}, \tilde{y}}+1\right)\left(u_{1}-\eta\right)\right) & \leq \mathrm{E}\left(\left(\Delta_{x, y}+1\right) u_{1}\right)-d, \\
\Delta_{\tilde{x}, \tilde{y}}+\mathrm{E}\left(\left(\Delta_{\tilde{x}, \tilde{y}}+1\right)\left(u_{2}+\eta\right)\right) & \geq \Delta_{x, y}+\mathrm{E}\left(\left(\Delta_{x, y}+1\right) u_{2}\right)+d, \ldots, \\
2 \Delta_{\tilde{x}, \tilde{y}}+\mathrm{E}\left(\left(\Delta_{\tilde{x}, \tilde{y}}+1\right)\left(v_{3}-\eta\right)\right) & \leq 2 \Delta_{x, y}+\mathrm{E}\left(\left(\Delta_{x, y}+1\right) v_{3}\right)-d .
\end{aligned}
$$

Ces inégalités ont lieu car

et

$$
\left|\Delta_{x, y}-\Delta_{\tilde{x}, \tilde{y}}\right| \leq \frac{|d(x, y)-d(\tilde{x}, \tilde{y})|}{6}+1 \leq \frac{d(x, \tilde{x})+d(y, \tilde{y})}{6}+1 \leq \frac{d}{6}
$$

$$
\left(\Delta_{x, y}+1\right) \eta \geq \frac{d(x, y)-6 \delta}{6} \eta \geq \frac{3 d}{2} \geq d+3\left|\Delta_{x, y}-\Delta_{\tilde{x}, \tilde{y}}\right| .
$$

On montre aussi les 6 inégalités analogues obtenues en permutant les rôles de $x, y$ et $\tilde{x}, \tilde{y}$. On possède donc, pour $\left(u_{1}, u_{2}, u_{3}, v_{1}, v_{2}, v_{3}\right) \in\left[\eta, 1-\eta\left[{ }^{6}\right.\right.$, des applications

$$
A_{x, u_{1}, u_{2}, u_{3}}^{y, v_{1}, v_{2}, v_{3}} \stackrel{\gamma}{\rightarrow} A_{\tilde{x}, u_{1}-\eta, u_{2}+\eta, u_{3}-\eta}^{\tilde{y}, v_{1}-\eta, v_{2}+\eta, v_{3}-\eta} \quad \text { et } \quad A_{\tilde{x}, u_{1}, u_{2}, u_{3}}^{\tilde{y}, v_{1}, v_{2}, v_{3}} \stackrel{\gamma}{\rightarrow} A_{x, u_{1}-\eta, u_{2}+\eta, u_{3}-\eta}^{y, v_{1}-\eta, v_{2}+\eta, v_{3}-\eta} .
$$

Les phrases précédentes, le a) du lemme 3.42 et les parties a), b), c) du souslemme 3.46 garantissent que ces applications vérifient les conditions de compatibilité supposées dans le sous-lemme suivant (en prenant $A_{u_{1}, u_{2}, u_{3}}^{v_{1}, v_{2}, v_{3}}=A_{x, u_{1}, u_{2}, u_{3}}^{y, v_{1}, v_{2}, v_{3}}$ et $\tilde{A}_{u_{1}, u_{2}, u_{3}}^{v_{1}, v_{2}, v_{3}}=A_{\tilde{x}, u_{1}, u_{2}, u_{3}}^{\tilde{y}, v_{1}, v_{2}, v_{3}}$.

Sous-lemme 3.48. Soit $C \in \mathbb{N}^{*}$. Alors pour tout $\left.\eta \in\right] 0, \frac{1}{4}[$ et pour toutes familles d'ensembles finis non vides, de cardinaux inférieurs ou égaux à $C$,

$$
\left(A_{u_{1}, u_{2}, u_{3}}^{v_{1}, v_{2}, v_{3}}\right)_{\left(u_{1}, u_{2}, u_{3}, v_{1}, v_{2}, v_{3}\right) \in\left[0,1\left[{ }^{6}\right.\right.} \quad \text { et } \quad\left(\tilde{A}_{u_{1}, u_{2}, u_{3}}^{v_{1}, v_{2}, v_{3}}\right)_{\left(u_{1}, u_{2}, u_{3}, v_{1}, v_{2}, v_{3}\right) \in[0,1[6}
$$

\section{munis}

- d'applications surjectives $A_{u_{1}, u_{2}, u_{3}}^{v_{1}, v_{2}, v_{3}} \rightarrow A_{u_{1}^{\prime}, u_{2}, u_{3}}^{v_{1}^{\prime}, v_{2}, v_{3}}$ et $\tilde{A}_{u_{1}, u_{2}, u_{3}}^{v_{1}, v_{2}, v_{3}} \rightarrow \tilde{A}_{u_{1}^{\prime}, u_{2}, u_{3}}^{v_{1}^{\prime}, v_{2}, v_{3}}$ pour $u_{1}^{\prime} \leq u_{1}$ et $v_{1}^{\prime} \leq v_{1}$,

- d'applications injectives $A_{u_{1}, u_{2}, u_{3}}^{v_{1}, v_{2}, v_{3}} \rightarrow A_{u_{1}, u_{2}^{\prime}, u_{3}^{\prime}}^{v_{1}, v_{2}^{\prime}, v_{3}^{\prime}}$ et $\tilde{A}_{u_{1}, u_{2}, u_{3}}^{v_{1}, v_{2}, v_{3}} \rightarrow \tilde{A}_{u_{1}, u_{2}, u_{3}^{\prime}}^{v_{1}, v_{2}^{\prime}, v_{3}^{\prime}}$ pour $u_{2}^{\prime} \geq u_{2}, v_{2}^{\prime} \geq v_{2}, u_{3}^{\prime} \leq u_{3}$ et $v_{3}^{\prime} \leq v_{3}$, 
- et pour $\left(u_{1}, u_{2}, u_{3}, v_{1}, v_{2}, v_{3}\right) \in\left[\eta, 1-\eta\left[{ }^{6}\right.\right.$, d'applications

$$
A_{u_{1}, u_{2}, u_{3}}^{v_{1}, v_{2}, v_{3}} \rightarrow \tilde{A}_{u_{1}-\eta, u_{2}+\eta, u_{3}-\eta}^{v_{1}-\eta, v_{2}+\eta, v_{3}-\eta} \quad \text { et } \quad \tilde{A}_{u_{1}, u_{2}, u_{3}}^{v_{1}, v_{2}, v_{3}} \rightarrow A_{u_{1}-\eta, u_{2}+\eta, u_{3}-\eta}^{v_{1}-\eta, v_{2}+\eta, v_{3}-\eta}
$$

telles que toutes ces applications soient compatibles entre elles (c'est-à-dire que deux composées d'applications comme ci-dessus sont égales lorsqu'elles ont le même ensemble de départ et le même ensemble d'arrivée), alors la mesure de l'ensemble des $\left(u_{1}, u_{2}, u_{3}, v_{1}, v_{2}, v_{3}\right) \in\left[\eta, 1-\eta\left[{ }^{6}\right.\right.$ tels que l'application

$$
A_{u_{1}, u_{2}, u_{3}}^{v_{1}, v_{2}, v_{3}} \rightarrow \tilde{A}_{u_{1}-\eta, u_{2}+\eta, u_{3}-\eta}^{v_{1}-\eta, v_{2}+\eta, v_{3}-\eta}
$$

soit bijective est $\geq 1-50 C \eta$.

Démonstration. Pour $\left(u_{1}, u_{2}, u_{3}, v_{1}, v_{2}, v_{3}\right) \in\left[2 \eta, 1-2 \eta\left[{ }^{6}\right.\right.$ on considère les applications

$$
\tilde{A}_{u_{1}+\eta, u_{2}-\eta, u_{3}+\eta}^{v_{1}+\eta, v_{2}-\eta, v_{3}+\eta} \stackrel{f}{\rightarrow} A_{u_{1}, u_{2}, u_{3}}^{v_{1}, v_{2}, v_{3}} \stackrel{g}{\rightarrow} \tilde{A}_{u_{1}-\eta, u_{2}+\eta, u_{3}-\eta}^{v_{1}-\eta, v_{2}+\eta, v_{3}-\eta} \stackrel{h}{\rightarrow} A_{u_{1}-2 \eta, u_{2}+2 \eta, u_{3}-2 \eta}^{v_{1}-2 \eta, v_{2}+2 \eta, v_{3}-2 \eta} .
$$

Si $g \circ f$ et $h \circ g$ sont bijectives, $g$ est bijective. Or $g \circ f$ est égale à la composée

$$
\begin{gathered}
\tilde{A}_{u_{1}+\eta, u_{2}-\eta, u_{3}+\eta}^{v_{1}+\eta, v_{2}-\eta, v_{3}+\eta} \rightarrow \tilde{A}_{u_{1}-\eta, u_{2}-\eta, u_{3}+\eta}^{v_{1}+\eta, v_{2}-\eta, v_{3}+\eta} \rightarrow \tilde{A}_{u_{1}-\eta, u_{2}+\eta, u_{3}+\eta}^{v_{1}+\eta, v_{2}-\eta, v_{3}+\eta} \\
\rightarrow \tilde{A}_{u_{1}-\eta, u_{2}+\eta, u_{3}-\eta}^{v_{1}+\eta, v_{2}-\eta, u_{3}+\eta} \rightarrow \tilde{A}_{u_{1}-\eta, u_{2}+\eta, u_{3}-\eta}^{v_{1}-\eta, u_{2}-\eta, u_{3}+\eta} \\
\rightarrow \tilde{A}_{u_{1}-\eta, u_{2}+\eta, u_{3}-\eta}^{v_{1}-\eta, v_{2}+\eta, v_{3}+\eta} \rightarrow \tilde{A}_{u_{1}-\eta, u_{2}+\eta, u_{3}-\eta}^{v_{1}-\eta, v_{2}+\eta, u_{3}-\eta}
\end{gathered}
$$

et $h \circ g$ est égale à la composée

$$
\begin{aligned}
& A_{u_{1}, u_{2}, u_{3}}^{v_{1}, v_{2}, v_{3}} \rightarrow A_{u_{1}-2 \eta, u_{2}, u_{3}}^{v_{1}, v_{2}, v_{3}} \rightarrow A_{u_{1}-2 \eta, u_{2}+2 \eta, u_{3}}^{v_{1}, v_{2}, v_{3}} \rightarrow A_{u_{1}-2 \eta, u_{2}+2 \eta, u_{3}-2 \eta}^{v_{1}, v_{2}, v_{3}} \\
& \rightarrow A_{u_{1}-2 \eta, u_{2}+2 \eta, u_{3}-2 \eta}^{v_{1}-2 \eta, v_{3}-2 \eta} \rightarrow A_{u_{1}-2 \eta, u_{2}+2 \eta, u_{3}-2 \eta}^{v_{1}-2 \eta, v_{2}+2 \eta, v_{3}} \rightarrow A_{u_{1}-2 \eta, u_{2}+2 \eta, u_{3}-2 \eta}^{v_{1}-2 \eta, v_{2}+2 \eta, v_{3}-2 \eta}
\end{aligned}
$$

Pour $u_{2}, u_{3}, v_{1}, v_{2}, v_{3} \in\left[2 \eta, 1-2 \eta\left[\right.\right.$, la mesure de l'ensemble des $u_{1} \in[2 \eta, 1-2 \eta[$ tels que

$$
\tilde{A}_{u_{1}+\eta, u_{2}-\eta, u_{3}+\eta}^{v_{1}+\eta, v_{2}-\eta, v_{3}+\eta} \rightarrow \tilde{A}_{u_{1}-\eta, u_{2}-\eta, u_{3}+\eta}^{v_{1}+\eta, v_{2}-\eta, v_{3}+\eta}
$$

(qui est la première application dans (28)) ne soit pas une bijection est inférieure ou égale à $4 \eta(C-1)$. En effet l'application (30) est surjective donc est une bijection en cas d'égalité des cardinaux des deux parties et d'autre part l'application de [0, 1[ dans $\mathbb{N}$ qui à $u$ associe le cardinal de $\tilde{A}_{u, u_{2}-\eta, u_{3}+\eta}^{v_{1}+\eta, v_{3}+\eta}$ est décroissante et prend ses valeurs dans $\{1, \ldots, C\}$. On a 11 autres énoncés correspondant aux autres applications de (28) et (29). Donc la mesure de l'ensemble des

$$
\left(u_{1}, u_{2}, u_{3}, v_{1}, v_{2}, v_{3}\right) \in\left[2 \eta, 1-2 \eta\left[^{6}\right.\right.
$$

tels que $g$ soit une bijection est supérieure ou égale à

$$
(1-4 \eta)^{6}-48 \eta(C-1)(1-4 \eta)^{5} \geq 1-24 \eta-48 \eta(C-1) \geq 1-48 C \eta \geq 1-50 C \eta
$$

et le sous-lemme 3.48 est démontré. 
Fin de la démonstration du lemme 3.45. Soit $C=C(\delta, K)$ tel que pour $x, y \in X$ et $r_{1}, r_{2}, r_{3}, s_{1}, s_{2}, s_{3}$ vérifiant (25) on ait $\sharp\left(\tilde{\Lambda}_{x, r_{1}, r_{2}, r_{3}}^{y, s_{1}, s_{2}, s_{3}}\right) \leq C$. Soit $\rho \in \mathbb{N}$. On pose $d=4 \rho+6 \delta$. Soient $x, y, x^{\prime}, y^{\prime} \in X$ vérifiant $d\left(x, x^{\prime}\right) \leq \rho$ et $d\left(y, y^{\prime}\right) \leq \rho$. Il suffit de montrer le lemme 3.45 en supposant $d(x, y)>36 d+6 \delta$. On choisit alors $\eta \in] 0, \frac{1}{4}\left[\right.$ vérifiant $d(x, y)=\frac{9 d}{\eta}+6 \delta$, c'est-à-dire $\eta=\frac{9 d}{d(x, y)-6 \delta}$. On applique le sous-lemme 3.48 avec $(\tilde{x}, \tilde{y})$ égal à $(x, y),\left(x^{\prime}, y\right),\left(x, y^{\prime}\right)$ ou $\left(x^{\prime}, y^{\prime}\right)$ et avec les familles $\left(A_{u_{1}, u_{2}, u_{3}}^{v_{1}, v_{2}, v_{3}}\right)$ et $\left(\tilde{A}_{u_{1}, u_{2}, u_{3}}^{v_{1}, v_{2}, v_{3}}\right)$ égales à $\left(A_{x, u_{1}, u_{2}, u_{3}}^{y, v_{1}, v_{2}, v_{3}}\right)$ et $\left(A_{\tilde{x}, u_{1}, u_{2}, u_{3}}^{\tilde{y}, v_{1}, v_{2}, v_{3}}\right)$.

Il existe donc une partie $J \subset\left[\eta, 1-\eta\left[{ }^{6}\right.\right.$ de mesure $\geq 1-200 C \eta$ telle que pour $\left(u_{1}, u_{2}, u_{3}, v_{1}, v_{2}, v_{3}\right) \in J$ les applications

$$
\begin{gathered}
A_{x, u_{1}, u_{2}, u_{3}}^{y, v_{1}, v_{2}, v_{3}} \rightarrow A_{x, u_{1}-\eta, u_{2}+\eta, u_{3}-\eta}^{y, v_{1}-\eta, v_{2}+\eta, v_{3}-\eta}, \quad A_{x, u_{1}, u_{2}, u_{3}}^{y, v_{1}, v_{2}, v_{3}} \rightarrow A_{x^{\prime}, u_{1}-\eta, u_{2}+\eta, u_{3}-\eta}^{y, v_{1}-\eta, v_{2}+\eta, v_{3}-\eta}, \\
A_{x, u_{1}, u_{2}, u_{3}}^{y, v_{1}, v_{2}, v_{3}} \rightarrow A_{x, u_{1}-\eta, u_{2}+\eta, u_{3}-\eta}^{y^{\prime}, v_{1}-\eta, v_{2}+\eta, v_{3}-\eta}
\end{gathered}
$$

et

$$
A_{x, u_{1}, u_{2}, u_{3}}^{y, v_{1}, v_{2}, v_{3}} \rightarrow A_{x^{\prime}, u_{1}-\eta, u_{2}+\eta, u_{3}-\eta}^{y^{\prime}, v_{1}-\eta, v_{2}+\eta, v_{3}-\eta}
$$

soient bijectives.

Soient $\left(u_{1}, u_{2}, u_{3}, v_{1}, v_{2}, v_{3}\right) \in J, c \in A_{x, u_{1}, u_{2}, u_{3}}^{y, v_{1}, v_{2}, v_{3}}$ et

$c_{x, y} \in A_{x, u_{1}-\eta, u_{2}+\eta, u_{3}-\eta}^{y, v_{1}-\eta, v_{2}+\eta, v_{3}-\eta}, c_{x^{\prime}, y} \in A_{x^{\prime}, u_{1}-\eta, u_{2}+\eta, u_{3}-\eta}^{y, v_{1}-\eta, v_{2}+\eta, v_{3}-\eta}, c_{x, y^{\prime}} \in A_{x, u_{1}-\eta, u_{2}+\eta, u_{3}-\eta}^{y^{\prime}, v_{1}-\eta, v_{2}+\eta, v_{3}-\eta}$ et

$$
c_{x^{\prime}, y^{\prime}} \in A_{x^{\prime}, u_{1}-\eta, u_{2}+\eta, u_{3}-\eta}^{y^{\prime}, v_{1}-\eta, v_{2}+\eta, v_{3}-\eta}
$$

les images de $c$ par les quatre bijections ci-dessus. Alors

$$
\beta_{x, u_{1}-\eta}^{y, v_{1}-\eta}\left(c_{x, y}\right)-\beta_{x^{\prime}, u_{1}-\eta}^{y, v_{1}-\eta}\left(c_{x^{\prime}, y}\right)-\beta_{x, u_{1}-\eta}^{y^{\prime}, v_{1}-\eta}\left(c_{x, y^{\prime}}\right)+\beta_{x^{\prime}, u_{1}-\eta}^{y^{\prime}, v_{1}-\eta}\left(c_{x^{\prime}, y^{\prime}}\right)=0
$$

car par (27) on a

$$
\beta_{\tilde{x}, u_{1}-\eta}^{\tilde{y}, v_{1}-\eta}\left(c_{\tilde{x}, \tilde{y}}\right)=\min _{z \in Y_{x, y}^{\mathrm{E}\left((\Delta x, y+1) u_{1}\right)}}\left(d(\tilde{x}, z)+c_{z}\right)+\min _{t \in Y_{y, x}^{\left.\mathrm{E}(\Delta x, y+1) v_{1}\right)}}\left(c_{t}+d(t, \tilde{y})\right)
$$

pour $(\tilde{x}, \tilde{y})$ égal à $(x, y),\left(x^{\prime}, y\right),\left(x, y^{\prime}\right)$ ou $\left(x^{\prime}, y^{\prime}\right)$. Pour tout $\left(u_{1}, u_{2}, u_{3}, v_{1}, v_{2}, v_{3}\right) \in$ $J$ on a donc

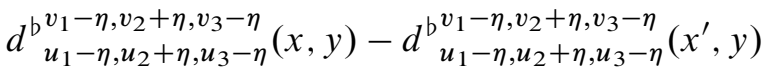

$$
\begin{aligned}
& -d^{\mathrm{b}}{ }_{u_{1}-\eta, u_{2}+\eta, u_{3}-\eta}^{v_{1}-\eta, v_{2}+\eta, v_{3}-\eta}\left(x, y^{\prime}\right)+d^{\mathrm{b}^{v_{1}-\eta, v_{2}+\eta, v_{3}-\eta}}{ }_{u_{1}-\eta, u_{2}+\eta, u_{3}-\eta}\left(x^{\prime}, y^{\prime}\right)=0 .
\end{aligned}
$$

Comme la mesure de $J$ est supérieure ou égale à

$$
1-200 C \eta=1-\frac{1800 C d}{d(x, y)-6 \delta}=1-\frac{1800 C(4 \rho+6 \delta)}{d(x, y)-6 \delta},
$$

cela termine la démonstration du lemme 3.45. 
Proposition 3.49. Pour tout $x, y \in X$ on a $d(x, y) \leq d^{b}(x, y) \leq d(x, y)+7 \delta$ et $d^{\text {b }}$ vérifie la condition (22).

Il résulte de la première assertion que l'on pourrait facilement remplacer $d^{\mathrm{b}}$ par une vraie distance (comme on avait obtenu $d^{\prime \prime}$ à partir de $d^{\prime}$ dans la preuve de la proposition 3.38), mais cela n'est pas nécessaire pour la suite.

Démonstration. La première assertion résulte du lemme 3.44. La seconde assertion résulte des lemmes 3.44 et 3.45 .

On définit alors, pour $x \in X$, la fonction $\rho_{x}^{b}: X \rightarrow \mathbb{R}_{+}$par

$$
\rho_{x}^{b}(a)=d^{\mathrm{b}}(x, a) .
$$

On l'étend ensuite en une fonction $\rho_{x}^{b}: \Delta \rightarrow \mathbb{R}_{+}$par la formule

$$
\rho_{x}^{b}(S)=\frac{\sum_{a \in S} \rho^{b}(a)}{\sharp S}
$$

si $S$ est non vide et $\rho_{x}^{b}(\emptyset)=0$.

\section{Construction des normes}

Soit $s \in] 0,1]$. Nous allons donner la formule pour la norme pré-hilbertienne $\|\cdot\|_{\mathscr{H}_{x, s}\left(\Delta_{p}\right)}$ sur $\mathbb{C}^{\left(\Delta_{p}\right)}$ qui servira pour la partie difficile de l'homotopie de 1 à $\gamma$. Nous montrerons d'abord que cette norme est bien définie. Nous montrerons ensuite la continuité des opérateurs, puis les propriétés d'équivariance de cette norme, et enfin l'équivariance à compacts près des opérateurs.

4.1. Formule pour la norme. On rappelle que la constante $F$ définie dans (21) est majorée par une constante de la forme $C(\delta, K, N)$. On fixe un entier $P \in \mathbb{N}^{*}$ tel que

$P$ soit divisible par 3 et assez grand en fonction de $\delta, K, N, Q$.

$\left(H_{P}\right)$

Dans la suite nous utiliserons un nombre fini de fois l'inégalité $P \geq C$ avec $C$ de la forme $C(\delta, K, N, Q)$.

Soient $p \in\left\{1, \ldots, p_{\max }\right\}$ et $k, m, l_{0}, \ldots, l_{m} \in \mathbb{N}$.

Définition 4.1. On note $Y_{x}^{p, k, m,\left(l_{0}, \ldots, l_{m}\right)}$ l'ensemble des $\left(p+m+1+\sum_{i=0}^{m} l_{i}\right)$-uplets

$$
\left(a_{1}, \ldots, a_{p}, S_{0}, \ldots, S_{m},\left(y_{i}^{j}\right)_{i \in\{0, \ldots, m\}, j \in\left\{1, \ldots, l_{i}\right\}}\right)
$$

tels que 
- i) $a_{1}, \ldots, a_{p} \in X$ sont deux à deux distincts, $S_{0}=\left\{a_{1}, \ldots, a_{p}\right\}, S_{0}$ appartient à $\Delta_{p}, S_{i} \in \Delta \backslash\{\emptyset\}$ pour $i \in\{1, \ldots, m\}$, et pour tout $i \in\{0, \ldots, m-1\}$, on a

$$
\begin{gathered}
\left.\left.S_{i+1} \subset S_{i} \cup \bigcup_{\substack{\tilde{x} \in B(x, k) \\
a \in S_{i}}}\{y \in 4 \delta-\operatorname{géod}(\tilde{x}, a) \mid d(y, a) \in] N-2 \delta, Q N\right]\right\} \\
\cup \bigcup_{\substack{\tilde{x} \in B(x, k) \\
a \in S_{i}}}\left\{z \in F-\operatorname{géod}(\tilde{x}, a) \mid d(z, a) \geq \frac{Q}{F}\right\},
\end{gathered}
$$

- ii) pour tout $i \in\{1, \ldots, m\}, d\left(x, S_{i}\right)>k+P$,

- iii) pour $i \in\{0, \ldots, m-1\}$ et $j \in\left\{1, \ldots, l_{i}\right\}, y_{i}^{j}$ est une partie non vide de $X$ de diamètre inférieur ou égal à $P$ et

$$
y_{i}^{j} \subset \bigcup_{y \in S_{i}, z \in S_{i+1}} P-\operatorname{géod}(y, z) .
$$

- iv) pour tout $j \in\left\{1, \ldots, l_{m}\right\}, y_{m}^{j}$ est une partie non vide de $X$ de diamètre inférieur ou égal à $P$ et

$$
y_{m}^{j} \subset \bigcup_{y \in S_{m}, \tilde{x} \in B(x, k)} 2 P-\operatorname{géod}(\tilde{x}, y) \quad \text { et } \quad d\left(x, y_{m}^{j}\right) \geq k+3 P \text {. }
$$

Remarque. La condition iv) implique que $l_{m}=0$ si $d\left(x, S_{m}\right) \leq k$ (ce qui ne peut se produire que si $m=0$ à cause de ii)). En effet pour $y \in B(x, k+N)$ et $\tilde{x} \in B(x, k)$, on a $2 P-\operatorname{géod}(\tilde{x}, y) \subset B(x, k+N+2 P+\delta)$ par le lemme 3.31 et on suppose $P>N+\delta$ (ce qui est permis par $\left.\left(H_{P}\right)\right)$.

Dans la définition précédente, la seule raison pour laquelle on veut connaître $\left(a_{1}, \ldots, a_{p}\right)$ en plus de $S_{0}$ est que cela détermine $e_{a_{1}} \wedge \cdots \wedge e_{a_{p}}$ alors que $S_{0}$ permet seulement de le connaître au signe près (le choix de $e_{S_{0}}= \pm e_{a_{1}} \wedge \cdots \wedge e_{a_{p}}$, qui avait pour but de simplifier certaines formules, ne doit pas être utilisé ici, bien sûr). En contrepartie, chaque partie $S_{0}$ est comptée $p$ ! fois, mais ce n'est pas grave car $p$ est borné par $p_{\max }$, qui est de la forme $C(\delta, K, N)$. Dans la définition précédente la donnée de $S_{0}$ est redondante mais nous préférons la garder car elle fournit une notation commode pour $\left\{a_{1}, \ldots, a_{p}\right\}$.

On fixe un entier $M \in \mathbb{N}^{*}$ tel que

$$
M \text { soit pair et soit assez grand en fonction de } \delta, K, N, Q, P \text {. }
$$

$\left(H_{M}\right)$

Dans la suite nous utiliserons un nombre fini de fois l'inégalité $M \geq C$ avec $C$ de la forme $C(\delta, K, N, Q, P)$.

On introduit maintenant une partition de $Y_{x}^{p, k, m,\left(l_{0}, \ldots, l_{m}\right)}$ pour la relation d'équivalence suivante :

$$
\left(a_{1}, \ldots, a_{p}, S_{0}, \ldots, S_{m},\left(\mathcal{Y}_{i}^{j}\right)_{i \in\{0, \ldots, m\}, j \in\left\{1, \ldots, l_{i}\right\}}\right)
$$


et

$$
\left(\hat{a}_{1}, \ldots, \hat{a}_{p}, \widehat{S}_{0}, \ldots, \widehat{S}_{m},\left(\widehat{\mathcal{Y}}_{i}^{j}\right)_{i \in\{0, \ldots, m\}, j \in\left\{1, \ldots, l_{i}\right\}}\right)
$$

sont en relation s'il existe une isométrie de

$$
\bigcup_{i \in\{0, \ldots, m\}} B\left(S_{i}, M\right) \cup \underset{\substack{i \in\{0, \ldots, m\} \\ j \in\left\{1, \ldots, l_{i}\right\}}}{\bigcup} B\left(\mathcal{Y}_{i}^{j}, M\right) \cup B(x, k+2 M)
$$

vers

$$
\bigcup_{i \in\{0, \ldots, m\}} B\left(\widehat{S}_{i}, M\right) \cup \underset{\substack{i \in\{0, \ldots, m\} \\ j \in\left\{1, \ldots, l_{i}\right\}}}{\bigcup} B\left(\hat{\mathcal{Y}}_{i}^{j}, M\right) \cup B(x, k+2 M)
$$

qui envoie $a_{i}$ sur $\hat{a}_{i}$ pour $i \in\{1, \ldots, p\}, S_{i}$ sur $\widehat{S}_{i}$ pour $i \in\{0, \ldots, m\}, y_{i}^{j}$ sur $\hat{y}_{i}^{j}$ pour $i \in\{0, \ldots, m\}, j \in\left\{1, \ldots, l_{i}\right\}$ et est l'identité sur $B(x, k+2 M)$. On rappelle que la notation $B(A, r)$ pour $A$ une partie de $X$ a été introduite dans (16).

On note $\bar{Y}_{x}^{p, k, m,\left(l_{0}, \ldots, l_{m}\right)}$ le quotient de $Y_{x}^{p, k, m,\left(l_{0}, \ldots, l_{m}\right)}$ pour cette relation d'équivalence, et $\pi_{x}^{p, k, m,\left(l_{0}, \ldots, l_{m}\right)} 1$ 'application quotient.

Notation. Pour $Z \in \bar{Y}_{x}^{p, k, m,\left(l_{0}, \ldots, l_{m}\right)}$ on note $r_{0}(Z), \ldots, r_{m}(Z), s_{0}(Z), \ldots, s_{m}(Z)$ les entiers tels que

$$
\begin{aligned}
& -r_{i}(Z)=d\left(x, S_{i}\right) \text { pour } i \in\{0, \ldots, m\}, \\
& -s_{i}(Z)=d\left(S_{i}, S_{i+1}\right)+2 M \text { pour } i \in\{0, \ldots, m-1\}, \\
& -s_{m}(Z)=d\left(x, S_{m}\right)-k
\end{aligned}
$$

pour tout

$$
\left(a_{1}, \ldots, a_{p}, S_{0}, \ldots, S_{m},\left(\mathcal{Y}_{i}^{j}\right)_{i \in\{0, \ldots, m\}, j \in\left\{1, \ldots, l_{i}\right\}}\right) \in\left(\pi_{x}^{p, k, m,\left(l_{0}, \ldots, l_{m}\right)}\right)^{-1}(Z) .
$$

On fixe $B \in \mathbb{R}_{+}^{*}$ et $\left.\alpha \in\right] 0,1[$ tels que

$$
B \text { soit assez grand en fonction de } \delta, K, N, Q, P, M, s
$$

et

$\alpha$ soit assez petit en fonction de $\delta, K, N, Q, P, M, s, B$.

Pour $Z \in \bar{Y}_{x}^{p, k, m,\left(l_{0}, \ldots, l_{m}\right)}$ on note $\xi_{Z}$ la forme linéaire sur $\mathbb{C}^{\left(\Delta_{p}\right)}$ définie par

$$
\xi_{Z}(f)=\sum_{\substack{\left(a_{1}, \ldots, a_{p}, S_{0}, \ldots, S_{m},\left(y_{i}^{j}\right)_{i \in\{0, \ldots, m\}, j \in\left\{1, \ldots, l_{i}\right\}}\right) \\ \in\left(\pi_{x}^{p, k, m,\left(l_{0}, \ldots, l_{m}\right)}\right)^{-1}(Z)}} f\left(a_{1}, \ldots, a_{p}\right) .
$$

Dans cette formule, comme dans la suite, pour $f \in \mathbb{C}^{\left(\Delta_{p}\right)}$ on note $f\left(a_{1}, \ldots, a_{p}\right)$ le coefficient de $e_{a_{1}} \wedge \cdots \wedge e_{a_{p}}$ lorsque qu'on écrit $f$ dans la base $\left( \pm e_{S}\right)_{S \in \Delta_{p}}$ (dont 
les vecteurs sont définis au signe près). On écrira aussi $f(S)= \pm f\left(a_{1}, \ldots, a_{p}\right)$ si $S=\left\{a_{1}, \ldots, a_{p}\right\}$.

On munit alors $\mathbb{C}^{\left(\Delta_{p}\right)}$ de la norme pré-hilbertienne, définie par la formule suivante :

$$
\begin{aligned}
\|f\|_{\mathcal{H}_{X, s}\left(\Delta_{p}\right)=}^{2} & \sum_{k, m, l_{0}, \ldots, l_{m} \in \mathbb{N}} B^{-\left(m+\sum_{i=0}^{m} l_{i}\right)} \sum_{Z \in \bar{Y}_{x}^{p, k, m,\left(l_{0}, \ldots, l_{m}\right)}} e^{2 s\left(r_{0}(Z)-k\right)} \\
& \left(\prod_{i=0}^{m} s_{i}(Z)^{-l_{i}}\right) \sharp\left(\left(\pi_{x}^{p, k, m,\left(l_{0}, \ldots, l_{m}\right)}\right)^{-1}(Z)\right)^{-\alpha}\left|\xi_{Z}(f)\right|^{2} .
\end{aligned}
$$

Remarque. On a vu que $l_{m}=0$ lorsque $s_{m}(Z) \leq 0$ (ce qui ne peut se produire que si $m=0)$ et dans ce cas on convient que $s_{m}(Z)^{l_{m}}=1$. En revanche pour $i \in\{0, \ldots, m-1\}$, on a toujours $s_{i}(Z) \geq 1$.

Remarque. On verra dans la démonstration des propositions 4.21 et 4.30 que l'on majore la partie de $\|\partial(f)\|_{\mathscr{H}_{x, s}\left(\Delta_{p}\right)}^{2}$ ou de $\left\|J_{x}(f)\right\|_{\mathcal{H}_{x, s}\left(\Delta_{p}\right)}^{2}$ correspondant à une valeur donnée de $m$ par la partie de $\|f\|_{\mathscr{H}_{x, s}\left(\Delta_{p}\right)}^{2}$ correspondant à $m+1$ (en fait c'est un peu plus compliqué mais on renvoie aux propositions 4.21 et 4.30 pour les détails). D'autre part la partie de $\|f\|_{\mathscr{H}_{x, s}\left(\Delta_{p}\right)}^{2}$ correspondant à $m=0$ et $l_{0}=0$ est essentiellement égale au carré de la norme introduite dans le premier paragraphe de [Laf08] pour montrer que les groupes hyperboliques n'ont pas la propriété (T) renforcée. Enfin les $y_{i}^{j}$ assurent la connaissance des points intermédiaires sur lesquels on moyenne dans la construction de $h_{x}, u_{x}, \rho_{x}^{\prime}$ (le but de ces moyennes est d'assurer l'équivariance à compacts près des opérateurs par l'astuce des ensembles emboîtés, comme dans [KS03]). On ne peut pas demander que les parties $y_{i}^{j}$ soient des singletons car ces parties seront construites de façon naturelle dans les preuves et la nécessité de choisir un point dans chacune empêcherait les preuves de fonctionner.

Remarque. Le facteur $e^{2 s\left(r_{0}(Z)-k\right)}$ est utile pour la continuité de $J_{x}$, comme on le verra dans la proposition 4.30. Le facteur $\prod_{i=0}^{m} s_{i}(Z)^{-l_{i}}$ est motivé par le fait qu'étant donnés $a_{1}, \ldots, a_{p}, S_{0}, \ldots, S_{m}$ le nombre de possibilités pour $\left(\mathcal{Y}_{i}^{j}\right)_{i \in\{0, \ldots, m\}, j \in\left\{1, \ldots, l_{i}\right\}}$ vérifiant

$$
\left(a_{1}, \ldots, a_{p}, S_{0}, \ldots, S_{m},\left(y_{i}^{j}\right)_{i \in\{0, \ldots, m\}, j \in\left\{1, \ldots, l_{i}\right\}}\right) \in Y_{x}^{p, k, m,\left(l_{0}, \ldots, l_{m}\right)}
$$

est borné par $C \sum_{i=0}^{m} l_{i} \prod_{i=0}^{m} s_{i}(Z)^{l_{i}}$, avec $C=C(\delta, K, N, Q, P)$, comme on le verra dans le lemme 4.15. La constante $2 M$ qui apparaît dans la définition de $s_{i}(Z)$ pour $i \in\{0, \ldots, m-1\}$ trouvera son utilité dans la démonstration du lemme 4.66. Le facteur $\sharp\left(\left(\pi_{x}^{p, k, m,\left(l_{0}, \ldots, l_{m}\right)}\right)^{-1}(Z)\right)^{-\alpha}$ servira pour montrer que les opérateurs $\partial$ et $J_{x}$ conjugués par $e^{\tau \theta_{x}^{\mathrm{b}}}$ sont équivariants à compact près. Enfin le facteur $B^{-\left(m+\sum_{i=0}^{m} l_{i}\right)}$ (avec $B$ assez grand) est nécessaire pour que la somme converge, comme le montre la preuve de la proposition 4.2. 
4.2. Enoncé des résultats. La proposition suivante montre que la norme pré-hilbertienne définie dans le sous-paragraphe précédent a bien un sens.

Proposition 4.2. Pour tout $p \in\left\{1, \ldots, p_{\max }\right\}$ et pour tout $f \in \mathbb{C}^{\left(\Delta_{p}\right)},\|f\|_{\mathscr{H}_{x, s}\left(\Delta_{p}\right)}$ est fini.

Cette proposition sera démontrée dans le sous-paragraphe 4.3 et la démonstration utilisera l'hypothèse (qui est une partie de $\left(H_{B}\right)$ ) selon laquelle $B$ est assez grand en fonction de $\delta, K, N, Q, P, M$ (sans cette hypothèse la proposition serait fausse).

On note $\mathscr{H}_{x, s}\left(\Delta_{p}\right)$ le complété de $\mathbb{C}^{\left(\Delta_{p}\right)}$ pour la norme pré-hilbertienne $\|\cdot\|_{\mathscr{H}_{x, s}\left(\Delta_{p}\right)}$. On note $\mathscr{H}_{x, s}=\bigoplus_{p=1}^{p_{\max }} \mathscr{H}_{x, s}\left(\Delta_{p}\right)$.

La proposition suivante sera démontrée dans le sous-paragraphe 4.7.

Proposition 4.3. L'action de $G$ sur $\bigoplus_{p=1}^{p_{\max }} \mathbb{C}^{\left(\Delta_{p}\right)} s^{\prime}$ 'étend en une action continue de $G$ sur $\mathscr{H}_{x, s}$ et il existe une constante $C$ telle que pour tout $g \in G$ on ait $\|\pi(g)\|_{\mathscr{L}\left(\mathcal{H}_{x, s}\right)} \leq$ $e^{2 s \ell(g)+C}$.

La démonstration de cette proposition utilisera entièrement l'hypothèse $\left(H_{B}\right)(\mathrm{y}$ compris la condition que $B$ est assez grand en fonction de $s$ ).

La proposition suivante est le résultat principal de ce paragraphe.

Pour $p \in\left\{1, \ldots, p_{\max }\right\}$, on définit $\theta_{x}^{b}: \mathbb{C}^{\left(\Delta_{p}\right)} \rightarrow \mathbb{C}^{\left(\Delta_{p}\right)} \operatorname{par} \theta_{x}^{b}\left(e_{S}\right)=\rho_{x}^{b}(S) e_{S}$ pour tout $S \in \Delta_{p}$. Pour tout $\tau \in \mathbb{R}$ on note $e^{\tau \theta_{x}^{\mathrm{b}}}: \mathbb{C}^{\left(\Delta_{p}\right)} \rightarrow \mathbb{C}^{\left(\Delta_{p}\right)}$ l'opérateur défini $\operatorname{par} e^{\tau \theta_{x}^{\mathrm{b}}}\left(e_{S}\right)=e^{\tau \rho_{x}^{\mathrm{b}}(S)} e_{S}$.

Proposition 4.4. Pour tout $T \in \mathbb{R}_{+}$, l'opérateur

$$
\left(e^{\tau \theta_{x}^{\mathrm{b}}}\left(\partial+J_{x} \partial J_{x}\right) e^{-\tau \theta_{x}^{\mathrm{b}}}\right)_{\tau \in[0, T]}
$$

s'étend en un opérateur continu sur le $\mathbb{C}[0, T]$-module hilbertien $\mathscr{H}_{x, s}[0, T]$, et

$$
\left(\mathscr{H}_{x, s}[0, T],\left(e^{\tau \theta_{x}^{\mathrm{b}}}\left(\partial+J_{x} \partial J_{x}\right) e^{-\tau \theta_{x}^{\mathrm{b}}}\right)_{\tau \in[0, T]}\right)
$$

appartient à $\mathrm{KK}_{G, 2 s \ell+C}(\mathbb{C}, \mathbb{C}[0, T])$.

En particulier $\left(\mathscr{H}_{x, s}, \partial+J_{x} \partial J_{x}\right)$ est un élément de $\mathrm{KK}_{G, 2 s \ell+C}(\mathbb{C}, \mathbb{C})$.

Proposition 4.5. Cet élément est égal à l'image de $1 \in \mathrm{KK}_{G}(\mathbb{C}, \mathbb{C})$.

Démonstration. La preuve est analogue à celle de la proposition 1.4.2 de [Laf02]. On reprend les arguments car on travaille ici dans un cadre hilbertien plutôt que banachique. Soit $\widehat{\mathscr{H}}_{x, s}=\mathbb{C} \oplus \mathscr{H}_{x, s}$ (avec une graduation inversée pour $\mathscr{H}_{x, s}$ ). On veut montrer que $\left(\hat{\mathscr{H}}_{x, s}, \partial+J_{x} \partial J_{x}\right)$ est nul dans $\mathrm{KK}_{G, 2 s \ell+C}(\mathbb{C}, \mathbb{C})$. Il résulte de l'égalité $\partial J_{x}+J_{x} \partial=$ Id que $\partial J_{x}$ et $J_{x} \partial$ commutent et $J_{x} \partial \partial J_{x}=0$ car $\partial^{2}=0$. 
Donc $\partial J_{x}$ et $J_{x} \partial$ sont deux projecteurs qui commutent, de somme Id, de produit nul, pairs. On pose $T=J_{x} \partial J_{x}$. Alors $T^{2}=0$. On a $\partial T=\partial J_{x}$ et $T \partial=J_{x} \partial$. D'autre part, $\partial$ et $\partial T$ ont même image, de même que $T$ et $T \partial$, d'où l'identification $\hat{\mathscr{H}}_{x, s}=\partial\left(\hat{\mathscr{H}}_{x, s}\right) \oplus T\left(\hat{\mathscr{H}}_{x, s}\right)$ en tant qu'espaces de Hilbert à équivalence des normes près (car les deux idempotents $\partial J_{x}$ et $J_{x} \partial$ ne sont pas nécessairement auto-adjoints). Dans cette décomposition, l'action de $g \in G$ s'écrit sous la forme $\left(\begin{array}{cc}c_{1,1}(g) & c_{1,2}(g) \\ 0 & c_{2,2}(g)\end{array}\right)$. Considérons alors le $\mathbb{C}[0,1]$-module hilbertien $\mathbb{Z} / 2 \mathbb{Z}$-gradué $\hat{\mathscr{H}}_{x, s}[0,1]$, où l'action de $g \in G$ est donnée par $\left(\left(\begin{array}{cc}c_{1,1}(g) & t c_{1,2}(g) \\ 0 & c_{2,2}(g)\end{array}\right)\right)_{t \in[0,1]}$. Alors $\left(\hat{\mathscr{H}}_{x, s}[0,1], \partial+T\right)$ fournit une homotopie entre $\left(\hat{\mathscr{H}}_{x, s}, \partial+T\right)$, en $t=1$ et un élément dégénéré, en $t=0$. En effet, quand l'action de $G$ est diagonale, les opérateurs $\partial$ et $T$ commutent exactement à cette action.

Donc $\left(\mathscr{H}_{x, s}[0, T],\left(e^{\tau \theta_{x}^{\mathrm{b}}}\left(\partial+J_{x} \partial J_{x}\right) e^{-\tau \theta_{x}^{\mathrm{b}}}\right)_{\tau \in[0, T]}\right)$ réalise une homotopie entre 1 et $\left(\mathscr{H}_{x, s}, e^{T \theta_{x}^{\mathrm{b}}}\left(\partial+J_{x} \partial J_{x}\right) e^{-T \theta_{x}^{\mathrm{b}}}\right)$ et montre donc l'égalité entre ces deux éléments dans $\mathrm{KK}_{G, 2 s \ell+C}(\mathbb{C}, \mathbb{C})$. On fixera $T$ assez grand et cela constituera la partie difficile de l'homotopie de 1 à $\gamma$. La partie facile (qui fera l'objet du paragraphe 5), sera une homotopie entre $\left(\mathscr{H}_{x, s}, e^{T \theta_{x}^{\mathrm{b}}}\left(\partial+J_{x} \partial J_{x}\right) e^{-T \theta_{x}^{\mathrm{b}}}\right)$ et $\gamma$ (qui montrera donc l'égalité entre ces deux éléments dans $\mathrm{KK}_{G, 2 s \ell+C}(\mathbb{C}, \mathbb{C})$ ).

4.3. Premières propriétés de la norme. Le but de ce sous-paragraphe est de montrer la proposition 4.2. Les lemmes 4.6, 4.7, 4.8, 4.9, 4.10, 4.11, 4.12 et 4.15 sont des préliminaires à la preuve de la proposition 4.2.

Nous commençons par rappeler le lemme d'approximation par les arbres.

Lemme 4.6. Soit $\left(Y, d_{Y}\right)$ un espace métrique fini et $\delta$-hyperbolique, et $w \in Y$ un point base. Soit $l \in \mathbb{N}$ tel que $\sharp Y \leq 2^{l}+2$. Alors il existe un arbre métrique fini $\left(T, d_{T}\right)$ et une application $\Psi: Y \rightarrow T$ telle que

$$
\begin{aligned}
& \text { - pour } y \in Y, d_{T}(\Psi w, \Psi y)=d_{Y}(w, y), \\
& \text { - pour } y, z \in Y, d_{Y}(y, z)-l \delta \leq d_{T}(\Psi y, \Psi z) \leq d_{Y}(y, z) .
\end{aligned}
$$

Démonstration. C'est exactement le (i) du théorème 12 du chapitre 2 de [GdlH90] car un espace métrique est $\delta$-hyperbolique au sens de la définition 0.1 si et seulement s'il est $\frac{\delta}{2}$-hyperbolique au sens de la définition 3 (reformulée dans 4 ) du chapitre 2 de [GdlH90].

Lemme 4.7. Dans les notations du lemme précédent, soient $y, z, t \in Y$.

a) Si $t \in \alpha-\operatorname{géod}(y, z)$, alors $\Psi t \in(\alpha+l \delta)-\operatorname{géod}(\Psi y, \Psi z)$ et si $y=w$, $\Psi t \in \alpha-\operatorname{géod}(\Psi y, \Psi z)$.

b) Si $\Psi t \in \alpha-\operatorname{géod}(\Psi y, \Psi z)$, alors $t \in(\alpha+2 l \delta)-\operatorname{géod}(y, z)$ et si $t=w$, $t \in \alpha-\operatorname{géod}(y, z)$.

c) On a $|d(t, \operatorname{géod}(y, z))-d(\Psi t, \operatorname{géod}(\Psi y, \Psi z))| \leq(l+1) \delta+1$. 
Démonstration. Seul c) demande une démonstration. On a

$$
d(\Psi t, \operatorname{géod}(\Psi y, \Psi z))=\frac{d(\Psi t, \Psi y)+d(\Psi t, \Psi z)-d(\Psi y, \Psi z)}{2} .
$$

Il est évident que $\frac{d(t, y)+d(t, z)-d(y, z)}{2} \leq d(t, \operatorname{géod}(y, z))$. Enfin

$$
d(t, \operatorname{géod}(y, z)) \leq \frac{d(t, y)+d(t, z)-d(y, z)}{2}+\delta+1
$$

car si $v \in \operatorname{géod}(y, z)$ est tel que $d(y, v)=\mathrm{E}\left(\frac{d(t, y)+d(y, z)-d(t, z)}{2}\right)$ on a $d(t, v) \leq$ $\frac{d(t, y)+d(t, z)-d(y, z)}{2}+\delta+1 \operatorname{par}\left(H_{\delta}^{0}(t, y, v, z)\right)$. Le c) en résulte facilement.

Lemme 4.8. Soient $\alpha, \beta \in \mathbb{N}, \rho \in \mathbb{Z}$ et $x, y, a, b, \in X$ tels que

$$
a \in \alpha-\operatorname{géod}(x, y), b \in \beta-\operatorname{géod}(x, y) \quad \text { et } \quad d(x, b) \leq d(x, a)+\rho .
$$

Alors $b \in(\max (\alpha+2 \rho, \beta)+\delta)-\operatorname{géod}(x, a)$.

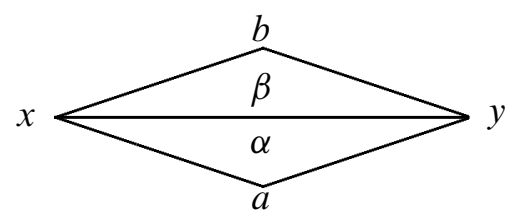

Démonstration. $\operatorname{Par}\left(H_{\delta}(a, x, b, y)\right)$ on a

$$
d(a, b) \leq \max (d(a, y)+d(x, b)-d(x, y), d(a, x)+d(b, y)-d(x, y))+\delta
$$

donc

$$
\begin{aligned}
& d(a, b)+d(b, x)-d(a, x) \\
& \leq \max (d(a, y)+2 d(x, b)-d(a, x)-d(x, y), d(b, x)+d(b, y)-d(x, y))+\delta \\
& \leq \max (\alpha+2 \rho, \beta)+\delta .
\end{aligned}
$$

Lemme 4.9. Soient $k \in \mathbb{N}$ et $x, y, z$, $t$ des points de $X$ tels que $z$ appartienne à $B(x, k)$ et que $t$ soit un point de $B(x, k)$ à distance minimale de $y$. Alors $t \in \delta$ - $\operatorname{géod}(z, y)$.

Démonstration. L'énoncé est clair si $y \in B(x, k)$ car alors $t=y$.

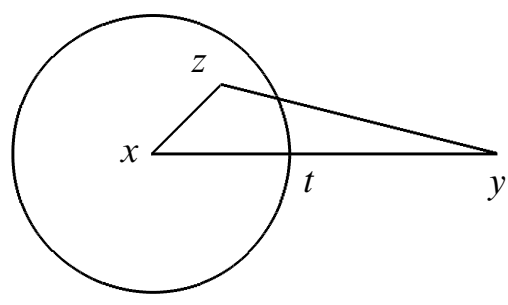


Sinon on a $t \in \operatorname{géod}(x, y), d(x, z) \leq k=d(x, t)$ et $d(y, z) \geq d(y, t)$ d'où $\operatorname{par}\left(H_{\delta}^{0}(z, x, t, y)\right), d(z, t) \leq \max (d(z, x)-d(x, t), d(z, y)-d(t, y))+\delta=$ $d(z, y)-d(t, y)+\delta$ d'où $t \in \delta$ - $\operatorname{géod}(z, y)$.

Lemme 4.10. Soient $k, \mu, v \in \mathbb{N}, x, y, y^{\prime} \in X$ et $z \in B(x, k)$ vérifiant

$-y^{\prime} \notin B\left(x, k+\frac{\mu+\delta}{2}\right)$,

- $y^{\prime} \in \mu-\operatorname{géod}(z, y)$ et $d\left(z, y^{\prime}\right) \leq d(z, y)-v$.

Alors

$$
d\left(x, y^{\prime}\right) \leq d(x, y)-v+\delta \quad \text { et } \quad y^{\prime} \in(\mu+\delta)-\operatorname{géod}(x, y) .
$$

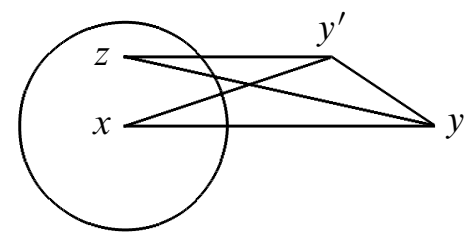

Démonstration. On applique le lemme 4.6 à $\left\{x, y, y^{\prime}, z\right\}$ avec $l=1$ et $y^{\prime}$ comme point base. Soit $T$ et $\Psi$ comme dans le lemme 4.6.

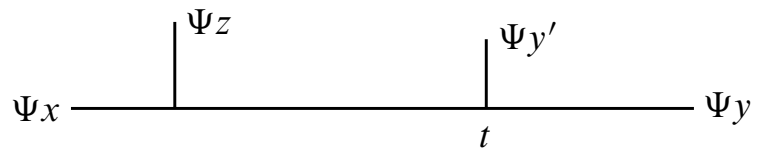

Soit $t$ le point de géod $(\Psi z, \Psi y)$ à distance minimale de $\Psi y^{\prime}$. On a donc

$$
t \in \operatorname{géod}(\Psi z, \Psi y) \quad \text { et } \quad t \in \operatorname{géod}\left(\Psi z, \Psi y^{\prime}\right)
$$

On a $\Psi y^{\prime} \in(\mu+\delta)$ - géod( $\left.\Psi z, \Psi y\right)$ par le a) du lemme 4.7, donc $d\left(t, \Psi y^{\prime}\right) \leq \frac{\mu+\delta}{2}$. Comme $d\left(\Psi x, \Psi y^{\prime}\right)=d\left(x, y^{\prime}\right)>k+\frac{\mu+\delta}{2}$ par hypothèse, on a $d(\Psi x, t)>k$ et comme $d(\Psi x, \Psi z) \leq k$,

$$
t \text { appartient à } \operatorname{géod}(\Psi x, \Psi y) \text { et à } \operatorname{géod}\left(\Psi x, \Psi y^{\prime}\right) \text {. }
$$

Il résulte de (32) et (33) que

$$
d\left(\Psi x, \Psi y^{\prime}\right)-d(\Psi x, \Psi y)=d\left(t, \Psi y^{\prime}\right)-d(t, \Psi y)=d\left(\Psi z, \Psi y^{\prime}\right)-d(\Psi z, \Psi y)
$$

et donc $d\left(x, y^{\prime}\right)-d(x, y) \leq d\left(z, y^{\prime}\right)-d(z, y)+\delta \leq-v+\delta$ où la dernière inégalité a lieu par hypothèse. Enfin par la première partie de (33), et comme $d\left(t, \Psi y^{\prime}\right) \leq \frac{\mu+\delta}{2}$, on a $\Psi y^{\prime} \in(\mu+\delta)$ - géod $(\Psi x, \Psi y)$, d'où $y^{\prime} \in(\mu+\delta)$ - géod $(x, y)$ par le b) du lemme 4.7 . 
Le lemme suivant est une conséquence du précédent.

Lemme 4.11. Soient $\mu_{1}, v_{1}, \mu_{2}, v_{2} \in \mathbb{N}$ vérifiant

$$
v_{1}>\mu_{1}+\frac{3 \delta}{2} \quad \text { et } \quad v_{2}>\mu_{2}+\frac{3 \delta}{2} \text {. }
$$

Soient $k \in \mathbb{N}$ et $x \in X$. Soit $y_{0}, \ldots, y_{j}$ une suite de points de $X$ telle que $y_{1}, \ldots, y_{j}$ $n$ 'appartiennent pas à $B\left(x, k+\frac{\max \left(\mu_{1}, \mu_{2}\right)+\delta}{2}\right)$ et que pour tout $i \in\{0, \ldots, j-1\}$,

- ou bien $y_{i+1}=y_{i}$,

- ou bien il existe $z \in B(x, k)$ tel que

$$
y_{i+1} \in \mu_{1}-\operatorname{géod}\left(z, y_{i}\right) \text { et } d\left(z, y_{i+1}\right) \leq d\left(z, y_{i}\right)-v_{1},
$$

- ou bien il existe $z \in B(x, k)$ tel que

$$
y_{i+1} \in \mu_{2}-\operatorname{géod}\left(z, y_{i}\right) \text { et } d\left(z, y_{i+1}\right) \leq d\left(z, y_{i}\right)-v_{2} .
$$

a) On a $d\left(x, y_{j}\right) \leq \cdots \leq d\left(x, y_{1}\right) \leq d\left(x, y_{0}\right)$ et pour $i \in\{0, \ldots, j-1\}$ on $a$ $d\left(x, y_{i+1}\right)<d\left(x, y_{i}\right)$ si $y_{i} \neq y_{i+1}$.

b) Soit h le nombre de valeurs prises par la suite $y_{0}, \ldots, y_{j}$. Alors

$$
y_{j} \in\left(\max \left(\mu_{1}, \mu_{2}\right)+2 \delta\right)-\operatorname{géod}\left(x, y_{0}\right)
$$

et

$$
d\left(x, y_{j}\right) \leq d\left(x, y_{0}\right)-(h-1)\left(\min \left(v_{1}, v_{2}\right)-\delta\right) .
$$

Démonstration. Montrons a). Soit $i \in\{0, \ldots, j-1\}$ tel que $y_{i} \neq y_{i+1}$. On va montrer $d\left(x, y_{i+1}\right)<d\left(x, y_{i}\right)$. Il existe $c \in\{1,2\}$ et $z \in B(x, k)$ tels que

$$
y_{i+1} \in \mu_{c}-\operatorname{géod}\left(z, y_{i}\right) \quad \text { et } \quad d\left(z, y_{i+1}\right) \leq d\left(z, y_{i}\right)-v_{c} .
$$

Comme $y_{i+1} \notin B\left(x, k+\frac{\mu_{c}+\delta}{2}\right)$, en appliquant le lemme 4.10 à $\left(y_{i}, y_{i+1}, z\right)$ au lieu $\operatorname{de}\left(y, y^{\prime}, z\right)$ et $\left(\mu_{c}, v_{c}\right)$ au lieu de $(\mu, v)$, on obtient $d\left(x, y_{i+1}\right) \leq d\left(x, y_{i}\right)-v_{c}+\delta<$ $d\left(x, y_{i}\right)$ puisque $v_{c}>\delta$.

Pour montrer $b$ ), on procède par récurrence ascendante. On pose

$$
\lambda=\max \left(\mu_{1}, \mu_{2}\right)+2 \delta .
$$

Pour $i \in\{0, \ldots, j\}$ on note $h_{i}$ le nombre de valeurs prises par la suite $y_{0}, \ldots, y_{i}$, de sorte que $1=h_{0} \leq h_{1} \leq \cdots \leq h_{j}=h$. Par l'hypothèse de récurrence on a

$$
y_{i} \in \lambda-\operatorname{géod}\left(x, y_{0}\right) \quad \text { et } \quad d\left(x, y_{i}\right) \leq d\left(x, y_{0}\right)-\left(h_{i}-1\right)\left(\min \left(v_{1}, v_{2}\right)-\delta\right) \text {. }
$$

Si $y_{i+1}=y_{i}$, on a $h_{i+1}=h_{i}$ et $y_{i+1}$ satisfait l'hypothèse de récurrence. Sinon, soit $c \in\{1,2\}$ et $z \in B(x, k)$ tel que

$$
y_{i+1} \in \mu_{c^{-}} \operatorname{géod}\left(z, y_{i}\right) \quad \text { et } \quad d\left(z, y_{i+1}\right) \leq d\left(z, y_{i}\right)-v_{c} .
$$


En appliquant le lemme 4.10 à

$$
\left(y_{i}, y_{i+1}, z\right) \text { au lieu de }\left(y, y^{\prime}, z\right) \text { et }\left(\mu_{c}, v_{c}\right) \text { au lieu de }(\mu, v)
$$

on obtient

$$
y_{i+1} \in\left(\mu_{c}+\delta\right)-\operatorname{géod}\left(x, y_{i}\right) \quad \text { et } \quad d\left(x, y_{i+1}\right) \leq d\left(x, y_{i}\right)-v_{c}+\delta \text {. }
$$

Comme $d\left(y_{i}, y_{i+1}\right) \geq d\left(z, y_{i}\right)-d\left(z, y_{i+1}\right) \geq v_{c}$ et grâce aux premières parties de (34) et (35), le lemme 3.21 appliqué à $\left(x, y_{0}, y_{i}, y_{i+1}\right)$ au lieu de $(x, a, b, c)$ et $\left(\lambda, \mu_{c}+\delta\right)$ au lieu de $(\alpha, \beta)$ montre que $y_{i+1} \in \lambda$ - $\operatorname{géod}\left(x, y_{0}\right)$ puisque

$$
\max \left(\lambda+2\left(\mu_{c}+\delta\right)-2 v_{c}, \mu_{c}+\delta\right)+\delta \leq \lambda \text {. }
$$

D'autre part les deuxièmes parties de (34) et (35) impliquent immédiatement

$$
d\left(x, y_{i+1}\right) \leq d\left(x, y_{0}\right)-\left(h_{i+1}-1\right)\left(\min \left(v_{1}, v_{2}\right)-\delta\right) .
$$

On rappelle que pour $x \in X$ et $S \in \Delta$, on note $d_{\max }(x, S)=\max _{y \in S} d(x, y)$.

Lemme 4.12. Il existe une constante $D=C(\delta, K, N, Q, P)$ telle que le résultat suivant soit vrai. Soit $S_{0} \in \Delta, x \in X, k \in \mathbb{N}$. Alors pour tout $m \in \mathbb{N}$ et pour toute suite $S_{1}, \ldots, S_{m}$ de $\Delta$ vérifiant les conditions i) et ii) de la définition 4.1, c'est-à-dire

i) pour tout $i \in\{0, \ldots, m-1\}$,

$$
\begin{gathered}
\left.\left.S_{i+1} \subset S_{i} \cup \underset{\substack{\tilde{x} \in B(x, k) \\
a \in S_{i}}}{\bigcup}\{y \in 4 \delta-\operatorname{géod}(\tilde{x}, a) \mid d(y, a) \in] N-2 \delta, Q N\right]\right\} \\
\cup \bigcup_{\substack{\tilde{x} \in B(x, k) \\
a \in S_{i}}}\left\{z \in F-\operatorname{géod}(\tilde{x}, a) \mid d(z, a) \geq \frac{Q}{F}\right\},
\end{gathered}
$$

ii) pour tout $i \in\{1, \ldots, m\}, d\left(x, S_{i}\right)>k+P$,

on $a$

$$
\begin{gathered}
S_{0} \cup \cdots \cup S_{m} \subset \bigcup_{a \in S_{0}}(F+2 \delta)-\operatorname{géod}(x, a), \\
d_{\max }\left(x, S_{0}\right) \geq d_{\max }\left(x, S_{1}\right) \geq \cdots \geq d_{\max }\left(x, S_{m}\right),
\end{gathered}
$$

et

$-\operatorname{sid}\left(x, S_{0}\right) \leq k$ on a $m=0$,

- $\operatorname{sid}\left(x, S_{0}\right)>k$, le nombre de valeurs prises par la suite $S_{0}, \ldots, S_{m}$ est inférieur ou égal à $D\left(d\left(x, S_{0}\right)-k\right)$ et le nombre de possibilités pour $\left(S_{1}, \ldots, S_{m}\right)$ est fini et majoré par $e^{D\left(d\left(x, S_{0}\right)-k+m\right)}$. 
Démonstration. On applique le lemme 4.11 à

$$
\mu_{1}=4 \delta, \quad v_{1}=N-6 \delta, \quad \mu_{2}=F \quad \text { et } \quad v_{2}=Q / F-F .
$$

Pour tout $i \in\{0, \ldots, m-1\}$ et tout $y_{i+1} \in S_{i+1}$ il existe $y_{i} \in S_{i}$ tel que

$$
\left(C_{i}\right) \begin{cases}\text {-ou bien } & y_{i+1}=y_{i}, \\ \text {-ou bien } & \text { il existe } \tilde{x} \in B(x, k) \text { tel que } \\ & y_{i+1} \in \mu_{1}-\operatorname{géod}\left(\tilde{x}, y_{i}\right) \text { et } d\left(\tilde{x}, y_{i+1}\right) \leq d\left(\tilde{x}, y_{i}\right)-v_{1}, \\ \text {-ou bien } & \text { il existe } \tilde{x} \in B(x, k) \text { tel que } \\ & y_{i+1} \in \mu_{2}-\operatorname{géod}\left(\tilde{x}, y_{i}\right) \text { et } d\left(\tilde{x}, y_{i+1}\right) \leq d\left(\tilde{x}, y_{i}\right)-v_{2} .\end{cases}
$$

On suppose $v_{1}>\mu_{1}+\frac{3 \delta}{2}, v_{2}>\mu_{2}+\frac{3 \delta}{2}$ et $P \geq \frac{\max \left(\mu_{1}, \mu_{2}\right)+\delta}{2}$, ce qui est permis par $\left(H_{N}\right),\left(H_{Q}\right)$ et $\left(H_{P}\right)$ respectivement. Soit $i \in\{1, \ldots, m\}$ et $y_{i} \in S_{i}$. Il existe $y_{i-1} \in S_{i-1}, \ldots, y_{0} \in S_{0}$ tels que les conditions $\left(C_{i-1}\right), \ldots,\left(C_{0}\right)$ soient satisfaites. Le a) du lemme 4.11 montre alors que $d\left(x, y_{i}\right) \leq d\left(x, y_{i-1}\right)$ et comme $y_{i} \in S_{i}$ est arbitraire il résulte que $d_{\max }\left(x, S_{i}\right) \leq d_{\max }\left(x, S_{i-1}\right)$ et on a montré (37). Comme $\max \left(\mu_{1}, \mu_{2}\right)+2 \delta=F+2 \delta$, le b) du lemme 4.11 montre que

$$
y_{i} \in \bigcup_{a \in S_{0}}(F+2 \delta)-\operatorname{géod}(x, a)
$$

et on a montré (36).

Pour montrer la suite de l'énoncé on suppose d'abord $d\left(x, S_{0}\right) \leq k$. Alors $m=0$ par (37) et par la condition ii), car $P \geq N$. On suppose $d\left(x, S_{0}\right)>k$ dans toute la suite de la démonstration. Soit $y_{m} \in S_{m}$ et soient $y_{m-1} \in S_{m-1}, \ldots, y_{0} \in S_{0}$ tels que les conditions $\left(C_{m-1}\right), \ldots,\left(C_{0}\right)$ soient satisfaites. On note $h$ le nombre de valeurs différentes prises par la suite $y_{0}, \ldots, y_{m}$. On suppose $\min \left(v_{1}, v_{2}\right)=N-6 \delta$, ce qui est permis par $\left(H_{Q}\right)$. Le b) du lemme 4.11 montre que

$$
y_{m} \in(F+2 \delta)-\operatorname{géod}\left(x, y_{0}\right) \quad \text { et } \quad d\left(x, y_{m}\right) \leq d\left(x, y_{0}\right)-(h-1)(N-7 \delta) .
$$

On a $d\left(x, y_{m}\right) \geq k+P$ puisque $m \geq 1$. On en déduit

$$
(h-1)(N-7 \delta) \leq d\left(x, S_{0}\right)+N-(k+P) .
$$

On suppose $N-7 \delta \geq 1$ et $P \geq N+1$, ce qui est permis par $\left(H_{N}\right)$ et $\left(H_{P}\right)$. Alors (38) implique $h \leq d\left(x, S_{0}\right)-k$. Il existe une constante $C_{1}=C(\delta, K, N)$ telle que tout point de $X$ appartienne au plus à $C_{1}$ éléments de $\Delta$. En notant $l$ le nombre de valeurs prises par la suite $S_{0}, \ldots, S_{m}$, on a $h \geq \frac{l}{C_{1}}$, d'où

$$
l \leq C_{1} h \leq C_{1}\left(d\left(x, S_{0}\right)-k\right) .
$$

Il existe une constante $C_{2}=C(\delta, K, N)$ telle que, pour $i \in\{0, \ldots, m-1\}$, connaissant $S_{i}$ et $d_{\max }\left(x, S_{i+1}\right)$ le nombre de possibilités pour $S_{i+1}$ vérifiant les 
conditions de l'énoncé est inférieur ou égal $C_{2}$. En effet pour $y_{i+1} \in S_{i+1}$ il existe $y_{i} \in S_{i}$ tel que $y_{i+1} \in(F+2 \delta)$ - $\operatorname{géod}\left(x, y_{i}\right)$ d'après (36) appliqué à $\left(S_{i}, \ldots, S_{m}\right)$, et on déduit l'existence de $C_{2}$ du lemme 3.13 appliqué à $\left(x, y_{i}\right)$ au lieu de $(x, y)$.

Etant donné $m$, le nombre de possibilités pour les entiers $d_{\max }\left(x, S_{i}\right)$ (pour $i=$ $1, \ldots, m)$ qui vérifient nécessairement

$$
d_{\max }\left(x, S_{0}\right) \geq d_{\max }\left(x, S_{1}\right) \geq \cdots \geq d_{\max }\left(x, S_{m}\right) \geq k+P
$$

est inférieur ou égal à

$$
\max \left(1,\left(\begin{array}{c}
m+d_{\max }\left(x, S_{0}\right)-k-P \\
m
\end{array}\right)\right) \leq 2^{d\left(x, S_{0}\right)-k+m}
$$

car $P \geq N$. Le nombre de possibilités pour $\left(S_{1}, \ldots, S_{m}\right)$ est donc inférieur ou égal à $\left(C_{2}\right)^{m} 2^{d\left(x, S_{0}\right)-k+m}$. Ceci termine la démonstration du lemme 4.12.

Les deux lemmes suivants sont des conséquences du lemme 4.12 et serviront ultérieurement.

Lemme 4.13. Pour $m, l_{0}, \ldots, l_{m} \in \mathbb{N}$ et

$$
\left(a_{1}, \ldots, a_{p}, S_{0}, \ldots, S_{m},\left(y_{i}^{j}\right)_{i \in\{0, \ldots, m\}, j \in\left\{1, \ldots, l_{i}\right\}}\right) \in Y_{x}^{p, k, m,\left(l_{0}, \ldots, l_{m}\right)}
$$

on a pour $i \in\{0, \ldots, m\}$ et $j \in\left\{1, \ldots, l_{i}\right\}$,

$$
d_{\max }\left(x, y_{i}^{j}\right) \leq d_{\max }\left(x, S_{i}\right)+2 P+\delta .
$$

Démonstration. Le lemme 3.31 appliqué à $\alpha=2 P$ donne

- pour $i \in\{0, \ldots, m-1\}$,

$$
d_{\max }\left(x, y_{i}^{j}\right) \leq \max \left(d_{\max }\left(x, S_{i}\right), d_{\max }\left(x, S_{i+1}\right)\right)+2 P+\delta,
$$

d'où le résultat puisque $d_{\max }\left(x, S_{i}\right) \geq d_{\max }\left(x, S_{i+1}\right)$ d'après le lemme 4.12,

- pour $i=m, d_{\max }\left(x, y_{m}^{j}\right) \leq \max \left(d_{\max }\left(x, S_{m}\right), k\right)+2 P+\delta$ d'où le résultat puisque $l_{m}=0$ si $d_{\max }\left(x, S_{m}\right) \leq k$, par la remarque qui suit la définition 4.1.

Lemme 4.14. Soient $p \in\left\{1, \ldots, p_{\max }\right\}, k, m, l_{0}, \ldots, l_{m} \in \mathbb{N}$ et

$$
\left(a_{1}, \ldots, a_{p}, S_{0}, \ldots, S_{m},\left(y_{i}^{j}\right)_{i \in\{0, \ldots, m\}, j \in\left\{1, \ldots, l_{i}\right\}}\right) \in Y_{x}^{p, k, m,\left(l_{0}, \ldots, l_{m}\right)} .
$$

Soit $b \in S_{0}$ et $u$ un point de $B(x, k)$ à distance minimale de $b$. Alors

a) $S_{0} \cup \cdots \cup S_{m} \subset 2 F$ - géod $(b, u) \subset P$ - $\operatorname{géod}(b, u)$,

b) $\bigcup_{j \in\left\{1, \ldots, l_{m}\right\}} y_{m}^{j} \in \bigcup_{a \in S_{m}}(2 P+\delta)-\operatorname{géod}(x, a)$,

c) pour tout $i \in\{0, \ldots, m\}, \bigcup_{j \in\left\{1, \ldots, l_{i}\right\}} y_{i}^{j} \subset 4 P-\operatorname{géod}(b, u)$.

d) pour tout $i \in\{0, \ldots, m-1\}$, et tout $j \in\left\{1, \ldots, l_{i}\right\}$,

$$
d\left(x, y_{i}^{j}\right) \geq d_{\max }\left(x, S_{i+1}\right)-4 P .
$$


Démonstration. On commence par traiter le cas où $b \in B(x, k)$. Alors $d\left(x, S_{0}\right) \leq k$, d'où $m=0$ par le lemme 4.12. De plus $l_{0}=0$ par la remarque qui suit la définition 4.1, et les assertions a), b), c) et d) sont évidentes. On suppose maintenant que $b \notin B(x, k)$. En particulier $d(x, u)=k$ et $d(u, b)=d(x, b)-k$.

Montrons a). D'après le lemme 4.12, on a

$$
S_{0} \cup \cdots \cup S_{m} \subset \bigcup_{a \in S_{0}}(F+2 \delta)-\operatorname{géod}(x, a) .
$$

Comme $d(a, b) \leq N$ pour tout $a \in S_{0}$, le lemme 3.2 montre que

$$
S_{0} \cup \cdots \cup S_{m} \subset(F+2 N+2 \delta)-\operatorname{géod}(x, b) \text {. }
$$

Pour tout $y \in S_{0}$ on a $d(y, b) \leq N$, donc $y \in 2 F$-géod $(b, u)$ car $F \geq N$ par (21). Soit donc $i \geq 1$ et $y \in S_{i}$, et montrons $y \in 2 F$-géod $(b, u)$. On a $y \in$ $(F+2 N+2 \delta)-\operatorname{géod}(x, b)$ et $d(x, y) \geq d(x, u)+P$.

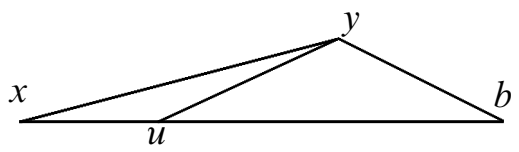

$\operatorname{Par}\left(H_{\delta}^{F+2 N+2 \delta}(u, x, y, b)\right)$ on a

$$
d(u, y) \leq \max (d(u, x)-d(x, y), d(u, b)-d(b, y))+F+2 N+3 \delta .
$$

Or $d(u, x)-d(x, y)+F+2 N+3 \delta \leq-P+F+2 N+3 \delta$ et on suppose $-P+F+2 N+3 \delta<0$, ce qui est permis par $\left(H_{P}\right)$. Donc $d(u, y) \leq d(u, b)-$ $d(b, y)+F+2 N+3 \delta$, c'est-à-dire $y \in(F+2 N+3 \delta)$ - géod $(u, b)$. On en déduit $y \in 2 F-\operatorname{géod}(u, b)$ puisque $F \geq 2 N+3 \delta$ par (21). Enfin on suppose $P \geq 2 F$, ce qui est permis par $\left(H_{P}\right)$.

On va montrer maintenant $\mathrm{b})$, ainsi que c) dans le cas où $i=m$. Soit $j \in$ $\left\{1, \ldots, l_{m}\right\}$ et $y \in y_{m}^{j}$. On a $d(x, y) \geq k+3 P$ et il existe $a \in S_{m}$ et $\tilde{x} \in B(x, k)$ tels que $y \in 2 P-\operatorname{géod}(\tilde{x}, a)$. On a $a \in P-\operatorname{géod}(u, b)$ par le a). Ensuite $\left(H_{\delta}^{2 P}(x, \tilde{x}, y, a)\right)$ implique $d(x, y) \leq \max (k, d(x, a)-d(a, y))+2 P+\delta$. Comme $d(x, y) \geq k+3 P$ et $P>\delta$ on en déduit $d(x, y) \leq d(x, a)-d(a, y)+2 P+\delta$, c'est-à-dire $y \in$ $(2 P+\delta)-\operatorname{géod}(x, a)$, ce qui montre déjà b). $\operatorname{Par}\left(H_{\delta}^{2 P+\delta}(u, x, y, a)\right)$ on a $d(u, y) \leq$ $\max (d(u, x)-d(x, y), d(u, a)-d(a, y))+2 P+2 \delta$ et comme $d(u, x)-d(x, y) \leq$ $-3 P$ et $P>2 \delta$ on en déduit $d(u, y) \leq d(u, a)-d(a, y)+2 P+2 \delta$, c'est-à-dire $y \in(2 P+2 \delta)-\operatorname{géod}(a, u)$.

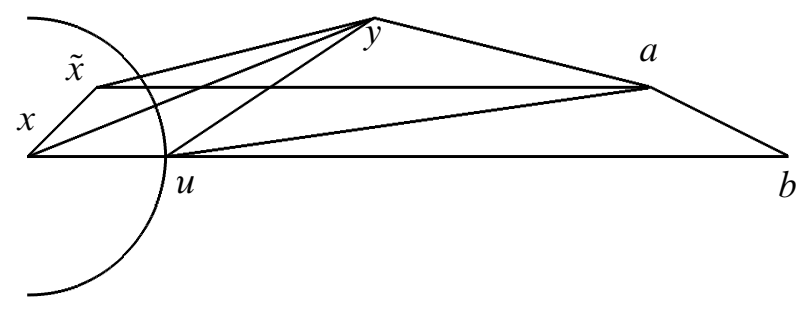


Comme $a \in P$-géod $(u, b)$, le a) du lemme 3.3 montre alors $y \in(3 P+2 \delta)$ - géod $(u, b)$ d'où $y \in 4 P$ - géod $(u, b)$ car $P \geq 2 \delta$.

On montre maintenant $\mathrm{c}$ ) dans le cas où $i \in\{0, \ldots, m-1\}$, ainsi que $\mathrm{d}$ ). Soit $j \in\left\{1, \ldots, l_{i}\right\}$ et $t \in y_{i}^{j}$. Il existe $y \in S_{i}$ et $z \in S_{i+1}$ tels que $t \in P$ - $\operatorname{géod}(y, z)$. Les hypothèses $y, z \in P$-géod $(u, b)$ et $t \in P$-géod $(y, z)$ suffisent à impliquer $t \in(3 P+\delta)-\operatorname{géod}(u, b)$.

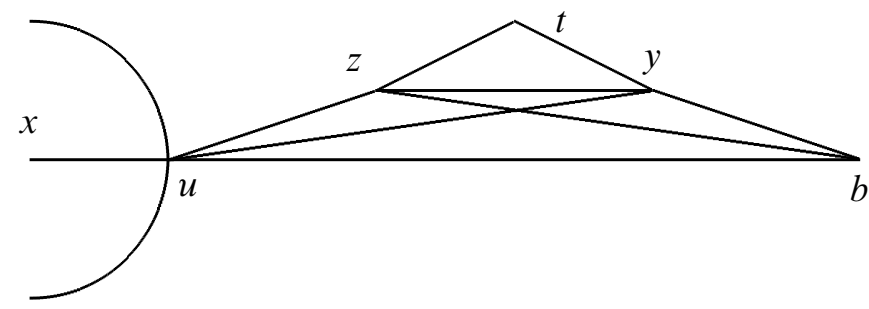

En effet quitte à permuter $y$ et $z$ on peut supposer

$$
d(u, z)+d(y, b) \leq d(u, y)+d(z, b) .
$$

Alors $\left(H_{\delta}(z, u, y, b)\right)$ implique $d(z, y) \leq d(z, b)+d(u, y)-d(u, b)+\delta$ donc

$$
\begin{aligned}
d(u, z)+d(z, y)+d(y, b) \leq & (d(u, z)+d(z, b))+(d(u, y)+d(y, b)) \\
& -d(u, b)+\delta \\
\leq & d(u, b)+2 P+\delta
\end{aligned}
$$

et

$$
\begin{aligned}
d(u, t)+d(t, b) & \leq d(u, z)+d(z, t)+d(t, y)+d(y, b) \\
& \leq d(u, z)+d(z, y)+P+d(y, b) \\
& \leq d(u, b)+3 P+\delta .
\end{aligned}
$$

On a donc $t \in(3 P+\delta)$ - $\operatorname{géod}(u, b)$ donc $t \in 4 P$ - $\operatorname{géod}(u, b)$ puisque $P \geq \delta$.

Il reste à montrer d). On garde les notations du dessin ci-dessus. Par (39) on a $d(x, u)+d(u, z)+d(z, t)+d(t, b) \leq d(x, b)+3 P+\delta$, d'où $d(x, z)+d(z, t) \leq$ $d(x, t)+3 P+\delta$. Comme $d(x, z) \geq d_{\max }\left(x, S_{i+1}\right)-N$, on en déduit $d(x, t) \geq$ $d_{\max }\left(x, S_{i}\right)-N-3 P-\delta \geq d_{\max }\left(x, S_{i+1}\right)-4 P$ car on suppose $P \geq N+\delta$.

Le lemme suivant sera utile pour la démonstration de la proposition 4.2.

Lemme 4.15. Il existe une constante $C=C(\delta, K, N, Q, P)$ telle que pour tous $x \in X, k, m, l_{0}, \ldots, l_{m} \in \mathbb{N}$, et $a_{1}, \ldots, a_{p}, S_{0}, \ldots, S_{m} \in \Delta$ vérifiant les conditions i) et ii) de la définition 4.1,

- pour tout $i \in\{0, \ldots, m\}$ et $j \in\left\{1, \ldots, l_{i}\right\}$, si on se donne $d\left(x, y_{i}^{j}\right)$, le nombre de possibilités pour $y_{i}^{j}$ vérifiant la condition iii) ou iv) de la définition 4.1 (selon que $i<m$ ou $i=m$ ) est inférieur ou égal à $C$, 
- pour tout $i \in\{0, \ldots, m\}$ et $j \in\left\{1, \ldots, l_{i}\right\}$ le nombre de possibilités pour $y_{i}^{j}$ vérifiant la condition iii) ou iv) de la définition 4.1 est inférieur ou égal à $C s_{i}(Z)$.

- le cardinal de l'ensemble des $\left.\left(y_{i}^{j}\right)_{i \in\{0, \ldots, m\}, j \in\left\{1, \ldots, l_{i}\right\}}\right)$ tels que

$$
\left(a_{1}, \ldots, a_{p}, S_{0}, \ldots, S_{m},\left(\mathcal{Y}_{i}^{j}\right)_{i \in\{0, \ldots, m\}, j \in\left\{1, \ldots, l_{i}\right\}}\right)
$$

appartienne à $Y_{x}^{p, k, m,\left(l_{0}, \ldots, l_{m}\right)}$ est majoré par $C^{\sum_{i=0}^{m} l_{i}} \prod_{i=0}^{m} s_{i}(Z)^{l_{i}}$.

Démonstration. La première assertion résulte immédiatement du c) du lemme 4.14, qui implique que $y_{i}^{j} \subset 4 P-\operatorname{géod}(x, b)$, du fait que $\operatorname{diam}\left(\mathcal{Y}_{i}^{j}\right) \leq P$, et du lemme 3.13 appliqué à $(x, b)$ au lieu de $(x, y)$. On montre d'abord la deuxième assertion pour $i \in\{0, \ldots, m-1\}$. Soit $a \in S_{i}$ et $b \in S_{i+1}$. Pour $j \in\left\{1, \ldots, l_{i}\right\}$ on a

$$
y_{i}^{j} \subset \bigcup_{y \in S_{i}, z \in S_{i+1}} P-\operatorname{géod}(y, z) \subset(P+4 N)-\operatorname{géod}(a, b)
$$

par le lemme 3.2. Comme $\operatorname{diam}\left(\mathcal{Y}_{i}^{j}\right) \leq P$, le lemme 3.13 appliqué à $(a, b)$ au lieu de $(x, y)$ montre alors la deuxième assertion. On montre maintenant la deuxième assertion pour $i=m$. Soit $a \in S_{m}$. On a

$$
y_{m}^{j} \subset \bigcup_{y \in S_{m}}(2 P+\delta)-\operatorname{géod}(x, y)
$$

par le b) du lemme 4.14, d'où $y_{m}^{j} \subset(2 P+\delta+2 N)$ - géod( $\left.x, a\right)$ par le lemme 3.2. Comme $\operatorname{diam}\left(y_{i}^{j}\right) \leq P$ et $d\left(x, y_{m}^{j}\right)>k+3 P$, le lemme 3.13 appliqué à $(x, a)$ au lieu de $(x, y)$ montre la deuxième assertion. Enfin la troisième assertion résulte facilement de la deuxième.

Démonstration de la proposition 4.2. Soit $p \in\left\{1, \ldots, p_{\max }\right\}$ et $f \in \mathbb{C}^{\left(\Delta_{p}\right)}$. Soit $R=\max d_{\max }(x, S)$ où le maximum est pris sur les $S$ tels que $e_{S}$ apparaisse dans $f$ avec un coefficient non nul.

Soit $k \geq R$. Si $m, l_{0}, \ldots, l_{m} \in \mathbb{N}$ et

$$
\left(a_{1}, \ldots, a_{p}, S_{0}, \ldots, S_{m},\left(\mathcal{Y}_{i}^{j}\right)_{i \in\{0, \ldots, m\}, j \in\left\{1, \ldots, l_{i}\right\}}\right) \in Y_{x}^{p, k, m,\left(l_{0}, \ldots, l_{m}\right)}
$$

sont tels que $f\left(a_{1}, \ldots, a_{p}\right) \neq 0$, alors $d_{\max }\left(x, S_{0}\right) \leq k$, donc $m=0$ et $l_{0}=0$ par le lemme 4.12 et la remarque qui suit la définition 4.1. De plus pour $k \geq R$ la relation d'équivalence sur la partie de $Y_{x}^{p, k, 0,(0)}$ telle que $e_{S_{0}}$ apparaisse dans $f$ avec un coefficient non nul, est triviale, donc la partie de (31) correspondant à $k \geq R$ se réécrit

$$
p ! \sum_{S_{0}, k \geq R} e^{2 s\left(d\left(x, S_{0}\right)-k\right)}\left|f\left(S_{0}\right)\right|^{2}
$$

et elle est finie et majorée par $p !\left(1-e^{-2 s}\right)^{-1}\|f\|_{\ell^{2}\left(\Delta_{p}\right)}^{2}$. 
On fixe $k \in\{0, \ldots, R-1\}$. Il reste donc à montrer que la partie correspondante de (31) est une somme convergente. La somme

$$
\begin{gathered}
\sum_{\substack{m \in \mathbb{N} \\
\left(l_{0}, \ldots, l_{m}\right) \in \mathbb{N}^{m}+1}} B^{-\left(m+\sum_{i=0}^{m} l_{i}\right)} \sum_{Z \in \bar{Y}_{x}^{p, k, m,\left(l_{0}, \ldots, l_{m}\right)}} e^{2 s\left(r_{0}(Z)-k\right)} \\
\left(\prod_{i=0}^{m} s_{i}(Z)^{-l_{i}}\right) \sharp\left(\left(\pi_{x}^{p, k, m,\left(l_{0}, \ldots, l_{m}\right)}\right)^{-1}(Z)\right)^{-\alpha}\left|\xi_{Z}(f)\right|^{2}
\end{gathered}
$$

est majorée par

$$
e^{2 s R} \sum_{\substack{m \in \mathbb{N} \\\left(l_{0}, \ldots, l_{m}\right) \in \mathbb{N} m+1}} B^{-\left(m+\sum_{i=0}^{m} l_{i}\right)} \sum_{Z \in \bar{Y}_{x}^{p, k, m,\left(l_{0}, \ldots, l_{m}\right)}}\left(\prod_{i=0}^{m} s_{i}(Z)^{-l_{i}}\right)\left|\xi_{Z}(f)\right|^{2}
$$

Soit $D$ comme dans le lemme 4.12. Grâce au lemme 4.12, pour tout $m \in \mathbb{N}$ et pour tout $S_{0} \in \Delta$ vérifiant $S_{0} \subset B(x, R)$, le nombre de possibilités pour $\left(S_{1}, \ldots, S_{m}\right)$ est inférieur ou égal à $\max \left(1, e^{D(R-k+m)}\right) \leq e^{D(R+m)}$. Soit $C$ égal à la constante $C$ du lemme 4.15 (qui est de la forme $C(\delta, K, N, Q, P)$ ). Pour tous $m,\left(l_{0}, \ldots, l_{m}\right)$, et pour tout $Z \in \bar{Y}_{x}^{p, k, m,\left(l_{0}, \ldots, l_{m}\right)}$ l'application de $\left(\pi_{x}^{p, k, m,\left(l_{0}, \ldots, l_{m}\right)}\right)^{-1}(Z)$ dans $\Delta_{p}$ qui à

$$
\left(a_{1}, \ldots, a_{p}, S_{0}, \ldots, S_{m},\left(\mathcal{Y}_{i}^{j}\right)_{i \in\{0, \ldots, m\}, j \in\left\{1, \ldots, l_{i}\right\}}\right)
$$

associe $S_{0}$ a des fibres de cardinal $\leq p ! e^{D(R+m)} C^{\sum_{i=0}^{m} l_{i}}$, car connaissant $S_{0}$ on a $p$ ! possibilités pour $\left(a_{1}, \ldots, a_{p}\right)$, au plus $e^{D(R+m)}$ possibilités pour $\left(S_{1}, \ldots, S_{m}\right)$, et grâce au lemme 4.15 , au plus $C^{\sum_{i=0}^{m} l_{i}}$ possibilités pour $\left(\mathcal{Y}_{i}^{j}\right)_{i \in\{0, \ldots, m\}, j \in\left\{1, \ldots, l_{i}\right\}}$, puisque $Z$ détermine, pour tous $i \in\{0, \ldots, m\}$ et $j \in\left\{1, \ldots, l_{i}\right\}$, l'entier $d\left(x, y_{i}^{j}\right)$. Donc dans (40) on a toujours

$$
\left|\xi_{Z}(f)\right| \leq p ! e^{D(R+m)} C^{\sum_{i=0}^{m} l_{i}}\|f\|_{\ell^{1}\left(\Delta_{p}\right)} .
$$

Donc la somme (40) est majorée par

$$
\begin{aligned}
& e^{2 s R} \sum_{\substack{m \in \mathbb{N} \\
\left(l_{0}, \ldots, l_{m}\right) \in \mathbb{N} m+1}} B^{-\left(m+\sum_{i=0}^{m} l_{i}\right)} \sum_{Z \in \bar{Y}_{x}^{p, k, m,\left(l_{0}, \ldots, l_{m}\right)}} \\
&\left(\prod_{i=0}^{m} s_{i}(Z)^{-l_{i}}\right)(p !)^{2} e^{2 D(R+m)} C^{2 \sum_{i=0}^{m} l_{i}}\|f\|_{\ell^{1}\left(\Delta_{p}\right)}^{2}
\end{aligned}
$$

Il existe $D^{\prime}=C(\delta, K)$ tel que le nombre de $S_{0} \in \Delta_{p}$ inclus dans $B(x, R)$ soit inférieur ou égal à $e^{D^{\prime} R}$ pour tout $R \in \mathbb{N}$. Par Cauchy-Schwarz, on en déduit $\|f\|_{\ell^{1}\left(\Delta_{p}\right)}^{2} \leq$ $e^{D^{\prime} R}\|f\|_{\ell^{2}\left(\Delta_{p}\right)}^{2}$. Pour tous $m,\left(l_{0}, \ldots, l_{m}\right)$ le cardinal de $Y_{x}^{p, k, m,\left(l_{0}, \ldots, l_{m}\right)}$, et donc a fortiori celui de $\bar{Y}_{x}^{p, k, m,\left(l_{0}, \ldots, l_{m}\right)}$, sont majorés par

$$
p ! e^{D(R+m)+D^{\prime} R} C^{\sum_{i=0}^{m} l_{i}}\left(\prod_{i=0}^{m} s_{i}(Z)^{l_{i}}\right)
$$


car on a au plus $e^{D^{\prime} R}$ possibilités pour $S_{0}, p$ ! possibilités pour $\left(a_{1}, \ldots, a_{p}\right)$, au plus $e^{D(R+m)}$ possibilités pour $\left(S_{1}, \ldots, S_{m}\right)$, et grâce au lemme 4.15 , au plus $C^{\sum_{i=0}^{m} l_{i}} \prod_{i=0}^{m} s_{i}(Z)^{l_{i}}$ possibilités pour $\left(\mathcal{Y}_{i}^{j}\right)_{i \in\{0, \ldots, m\}, j \in\left\{1, \ldots, l_{i}\right\}}$. Donc la somme (41) est majorée par

$$
(p !)^{3} e^{2 s R} \sum_{\substack{m \in \mathbb{N} \\\left(l_{0}, \ldots, l_{m}\right) \in \mathbb{N} m+1}} B^{-\left(m+\sum_{i=0}^{m} l_{i}\right)} e^{3 D(R+m)+2 D^{\prime} R} C^{3 \sum_{i=0}^{m} l_{i}}\|f\|_{\ell^{2}\left(\Delta_{p}\right)}^{2} .
$$

On suppose $B>C^{3}+e^{3 D}$, ce qui est permis par $\left(H_{B}\right)$. Donc cette somme converge et est majorée par

$$
\begin{gathered}
(p !)^{3} e^{\left(2 s+3 D+2 D^{\prime}\right) R} \sum_{m \in \mathbb{N}} \frac{B^{-m} e^{3 D m}}{\left(1-B^{-1} C^{3}\right)^{m+1}}\|f\|_{\ell^{2}\left(\Delta_{p}\right)}^{2} \\
=(p !)^{3} e^{\left(2 s+3 D+2 D^{\prime}\right) R} \frac{1}{1-B^{-1} C^{3}} \frac{1}{1-\frac{B^{-1} e^{3 D}}{1-B^{-1} C^{3}}}\|f\|_{\ell^{2}\left(\Delta_{p}\right)}^{2} \\
=\frac{(p !)^{3} e^{\left(2 s+3 D+2 D^{\prime}\right) R}}{1-B^{-1}\left(C^{3}+e^{3 D}\right)}\|f\|_{\ell^{2}\left(\Delta_{p}\right)}^{2} .
\end{gathered}
$$

Cela termine la démonstration de la proposition 4.2.

Au total, pour $p \in\left\{1, \ldots, p_{\max }\right\}$ et $f \in \mathbb{C}^{\left(\Delta_{p}\right)}$ et en notant $R=\max d_{\max }(x, S)$ où le maximum est pris sur les $S$ tels que $e_{S}$ apparaisse dans $f$ avec un coefficient non nul, on a

$$
\begin{aligned}
\|f\|_{\mathscr{H}_{x, s}\left(\Delta_{p}\right)}^{2} & \leq p !\left(1-e^{-2 s}\right)^{-1}\|f\|_{\ell^{2}\left(\Delta_{p}\right)}^{2}+\sum_{k=0}^{R-1} \frac{(p !)^{3} e^{\left(2 s+3 D+2 D^{\prime}\right) R}}{1-B^{-1}\left(C^{3}+e^{3 D}\right)}\|f\|_{\ell^{2}\left(\Delta_{p}\right)}^{2} \\
& \leq\left(p !\left(1-e^{-2 s}\right)^{-1}+\frac{(p !)^{3} R e^{\left(2 s+3 D+2 D^{\prime}\right) R}}{1-B^{-1}\left(C^{3}+e^{3 D}\right)}\right)\|f\|_{\ell^{2}\left(\Delta_{p}\right)}^{2} .
\end{aligned}
$$

Le lemme suivant donne au contraire une minoration de la norme de $\mathscr{H}_{x, s}\left(\Delta_{p}\right)$.

Lemme 4.16. Pour tout $p \in\left\{1, \ldots, p_{\max }\right\}$ et pour tout $f \in \mathbb{C}^{\left(\Delta_{p}\right)}$ on a

$$
\|f\|_{\mathscr{H}_{x, s}\left(\Delta_{p}\right)}^{2} \geq p !\left(1-e^{-2 s}\right)^{-1}\|f\|_{\ell^{2}\left(\Delta_{p}\right)}^{2} .
$$

Démonstration. Pour tout $S_{0} \in \Delta_{p}$, pour tout $k \geq d\left(x, S_{0}\right)$ et pour toute énumération $\left(a_{1}, \ldots, a_{p}\right)$ des points de $S_{0}, Y_{x}^{p, k, 0,(0)}$ contient $\left(a_{1}, \ldots, a_{p}, S_{0}\right)$ et comme $M \geq N$, le singleton $\left\{\left(a_{1}, \ldots, a_{p}, S_{0}\right)\right\}$ est une classe d'équivalence dans $\bar{Y}_{x}^{p, k, 0,(0)}$. On a donc $\|f\|_{\mathscr{H}_{x, s}\left(\Delta_{p}\right)}^{2} \geq p ! \sum_{S_{0} \in \Delta_{p}} \sum_{k \geq d\left(x, S_{0}\right)} e^{2 s\left(d\left(x, S_{0}\right)-k\right)}\left|f\left(S_{0}\right)\right|^{2}=p !\left(1-e^{-2 s}\right)^{-1}\|f\|_{\ell^{2}\left(\Delta_{p}\right)}^{2}$. 
4.4. Autres propriétés de la norme. Les propriétés que nous allons établir dans ce sous-paragraphe sont des préliminaires indispensables pour les sous-paragraphes suivants. De façon un peu imprécise nous allons montrer qu'il existe une constante $C$ de la forme $C(\delta, K, N, Q, P, M)$ telle que pour $p \in\left\{1, \ldots, p_{\max }\right\}, k, m, l_{0}, \ldots, l_{m} \in$ $\mathrm{N}$ et

$$
\left(a_{1}, \ldots, a_{p}, S_{0}, \ldots, S_{m},\left(\mathcal{Y}_{i}^{j}\right)_{i \in\{0, \ldots, m\}, j \in\left\{1, \ldots, l_{i}\right\}}\right) \in Y_{x}^{p, k, m,\left(l_{0}, \ldots, l_{m}\right)},
$$

pour connaître les distances entre les points de

$$
\bigcup_{i \in\{0, \ldots, m\}} B\left(S_{i}, M\right) \cup \underset{\substack{i \in\{0, \ldots, m\} \\ j \in\left\{1, \ldots, l_{i}\right\}}}{\bigcup} B\left(y_{i}^{j}, M\right) \cup B(x, k+2 M)
$$

il suffit de connaître certaines de ces distances, de telle sorte que pour chaque point de cet ensemble n'appartenant pas à $B(x, k+2 M)$, le nombre de distances à connaître depuis de ce point soit inférieur ou égal à $C$. La raison est que cet ensemble est une réunion de boules de grands rayons dont les centres sont à peu près alignés le long d'une géodésique (grâce au lemme 4.14) et qu'il suffit donc de connaître les distances entre les points de boules voisines.

Lemme 4.17. Soient $c, d \in X, I$ un ensemble fini, et pour $i \in I, \alpha_{i} \in \mathbb{N}, \rho_{i} \in \mathbb{N}^{*}$ et $w_{i} \in \alpha_{i}-\operatorname{géod}(c, d)$. On suppose que pour tout $i \in I, \rho_{i} \geq 4 \delta+\frac{\alpha_{i}}{2}$.

a) Pour connaître les distances entre les points de $\bigcup_{i \in I} B\left(w_{i}, \rho_{i}\right)$ il suffit de connaître les distances entre les points de $B\left(w_{i}, \rho_{i}\right)$ et $B\left(w_{j}, \rho_{j}\right)$ pour tous les couples $(i, j) \in \Lambda$ où $\Lambda \subset I^{2}$ est l'ensemble des couples $(i, j)$ tels qu'il n'existe pas de $k \in I$ vérifiant

$$
d\left(c, w_{j}\right)-d\left(c, w_{k}\right) \geq 2 \rho_{j}+\frac{\alpha_{j}}{2} \quad \text { et } \quad d\left(c, w_{k}\right)-d\left(c, w_{i}\right) \geq 2 \rho_{i}+\frac{\alpha_{i}}{2}+\frac{\alpha_{k}}{2} .
$$

b) Soit $R \in \mathbb{N}^{*}$ tel que $\alpha_{i} \leq R$ et $\rho_{i} \leq R$ pour tout $i \in I$. Soit $i \in I$. Alors l'ensemble des $d\left(c, w_{j}\right)$ pour $j \in I$ tel que $(i, j) \in \Lambda$ ou $(j, i) \in \Lambda$ est inclus dans la réunion de $\left[d\left(c, w_{i}\right)-3 R, d\left(c, w_{i}\right)+3 R\right]$ et de deux intervalles de longueur $\leq 3 R$.

c) Soit $R$ comme dans b). Il existe une constante $C=C(\delta, K, R)$ (indépendante de $\sharp I$ en particulier) telle que pour toute partie $J \subset I$, connaissant les distances entre les points de $\bigcup_{i \in I \backslash J} B\left(w_{i}, \rho_{i}\right)$, les distances entre les points de $\bigcup_{i \in J} B\left(w_{i}, \rho_{i}\right)$ et ceux de $\bigcup_{i \in I \backslash J} B\left(w_{i}, \rho_{i}\right)$ soient déterminées par la donnée des distances entre les points de $\bigcup_{i \in J} B\left(w_{i}, \rho_{i}\right)$ et les points d'une partie de $\bigcup_{i \in I \backslash J} B\left(w_{i}, \rho_{i}\right)$

- dont le cardinal est borné par $C(\sharp J)$,

- qui est déterminée par la connaissance des distances entre les points de

$$
\bigcup_{i \in I \backslash J} B\left(w_{i}, \rho_{i}\right) \cup\{c\}
$$

et par les entiers $d\left(c, w_{i}\right)$ pour $i \in J$. 
Remarque. Dans toutes les situations où on appliquera le c) de ce lemme, $\sharp J$ sera majoré par une constante de la forme $C(\delta, K, N, Q, P, M)$.

Démonstration. On commence par montrer a). On suppose $I=\{1, \ldots, r\}$ et $w_{1}, \ldots$, $w_{r}$ ordonnés de telle sorte que $d\left(c, w_{1}\right) \leq \cdots \leq d\left(c, w_{r}\right)$. Soit $i<k<j$ des entiers vérifiant (43). Soient $y \in B\left(w_{i}, \rho_{i}\right)$ et $z \in B\left(w_{j}, \rho_{j}\right)$. On a

et

$$
d(c, y) \leq d\left(c, w_{i}\right)+\rho_{i} \leq d\left(c, w_{k}\right)-\rho_{i}-\frac{\alpha_{i}}{2}-\frac{\alpha_{k}}{2}
$$

$$
d(c, z) \geq d\left(c, w_{j}\right)-\rho_{j} \geq d\left(c, w_{k}\right)+\rho_{j}+\frac{\alpha_{j}}{2} .
$$

Donc il existe $v \in \operatorname{géod}(y, z)$ tel que $d(c, v)=d\left(c, w_{k}\right)-\mathrm{E}\left(\frac{\alpha_{k}}{2}\right)$. On choisit un tel $v$.

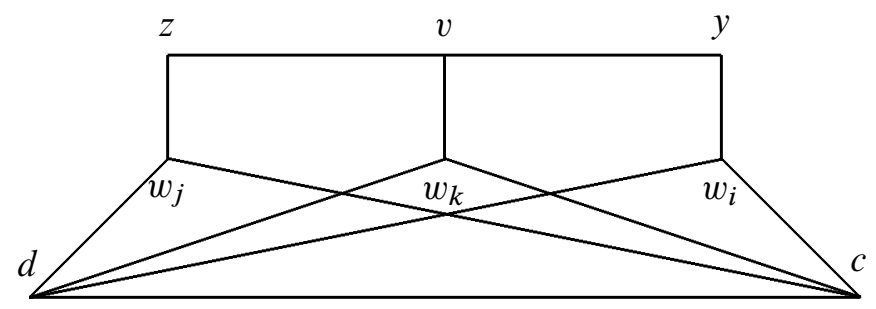

On a

$d(y, v) \geq d(c, v)-d(c, y) \geq \rho_{i}+\alpha_{i} / 2$ et $d(z, v) \geq d(c, z)-d(c, v) \geq \rho_{j}+\alpha_{j} / 2$.

D'après le lemme 3.2, on a $y \in\left(\alpha_{i}+2 \rho_{i}\right)$ - $\operatorname{géod}(c, d)$ et $z \in\left(\alpha_{j}+2 \rho_{j}\right)$ - $\operatorname{géod}(c, d)$. Le lemme 3.40 implique alors $v \in 3 \delta$-géod $(c, d)$. Comme $w_{k} \in \alpha_{k}$-géod $(c, d)$ et $d(c, v)=d\left(c, w_{k}\right)-\mathrm{E}\left(\frac{\alpha_{k}}{2}\right),\left(H_{\delta}\left(v, c, w_{k}, d\right)\right)$ implique

$$
\begin{aligned}
d\left(v, w_{k}\right) \leq & \max \left(d(c, v)+d\left(w_{k}, d\right)-d(c, d), d\left(c, w_{k}\right)+d(v, d)-d(c, d)\right)+\delta \\
\leq & \max \left(d\left(c, w_{k}\right)-\mathrm{E}\left(\frac{\alpha_{k}}{2}\right)+d\left(w_{k}, d\right)-d(c, d), d(c, v)\right. \\
& \left.+\mathrm{E}\left(\frac{\alpha_{k}}{2}\right)+d(v, d)-d(c, d)\right)+\delta \\
\leq & \max \left(\alpha_{k}-\mathrm{E}\left(\frac{\alpha_{k}}{2}\right), \mathrm{E}\left(\frac{\alpha_{k}}{2}\right)+3 \delta\right)+\delta \leq 4 \delta+\frac{\alpha_{k}}{2} \leq \rho_{k},
\end{aligned}
$$

donc $v \in B\left(w_{k}, \rho_{k}\right) \cap \operatorname{géod}(y, z)$. On a donc montré que pour $i<k<j$ vérifiant (43) toute géodésique entre un point de $B\left(w_{i}, \rho_{i}\right)$ et un point de $B\left(w_{j}, \rho_{j}\right)$ intersecte $B\left(w_{k}, \rho_{k}\right)$, ce qui implique que la connaissance des distances entre les points de $B\left(w_{k}, \rho_{k}\right)$ et ceux de $B\left(w_{i}, \rho_{i}\right) \cup B\left(w_{j}, \rho_{j}\right)$ permet de déterminer les distances entre les points de $B\left(w_{i}, \rho_{i}\right)$ et ceux de $B\left(w_{j}, \rho_{j}\right)$. Ceci termine la preuve du a). Le b) résulte facilement du a). Le cas particulier de c) où $\sharp J=1$ résulte du b) et du lemme 3.13. Pour montrer $\mathrm{c}$ ) dans le cas général on se ramène au cas particulier déjà démontré de la façon suivante. Pour tout $j \in J$ on note $I^{\prime}=I \backslash J \cup\{j\}$ et $J^{\prime}=\{j\}$ et on applique le cas particulier de c) déjà démontré avec $I^{\prime}$ et $J^{\prime}$ au lieu $I$ et $J$. 
Lemme 4.18. Soient $x, b \in X, l \in \mathbb{N}, \alpha \in \mathbb{N}$ et $u$ un point de $B(x, l)$ à distance minimale de $b$. Pour tout $z \in \alpha$-géod $(b, u)$, les distances entre $z$ et les points de $B(x, l)$ sont déterminées par les distances entre $z$ et les points de $B(u, \alpha+4 \delta) \cap$ $B(x, l)$. Plus précisément pour tout $\tilde{x} \in B(x, l)$ et pour tout $z \in \alpha$-géod $(b, u)$, $\operatorname{géod}(\tilde{x}, z)$ intersecte $B(u, \alpha+4 \delta) \cap B(x, l)$.

Démonstration. Le lemme est évident si $b \in B(x, l)$. On suppose donc $b \notin B(x, l)$. Soit $\tilde{x} \in B(x, l)$ et $z \in \alpha$ - $\operatorname{géod}(b, u)$. On veut montrer qu'il existe $w \in \operatorname{géod}(\tilde{x}, z) \cap$ $B(u, \alpha+4 \delta) \cap B(x, l)$. Si $d(u, \tilde{x}) \leq \alpha+4 \delta$ on prend $w=\tilde{x}$. On suppose donc $d(u, \tilde{x})>\alpha+4 \delta$. D'après le lemme 4.9 appliqué à $(x, b, u, \tilde{x})$ au lieu de $(x, y, t, z)$ et $l$ au lieu de $k$, on a $u \in \delta$-géod $(\tilde{x}, b)$. Donc $d(b, \tilde{x}) \geq d(b, u)+\alpha+3 \delta$ et comme de plus $d(b, z) \leq d(b, u)+\alpha$, il existe $w \in \operatorname{géod}(z, \tilde{x})$ tel que $d(b, w)=$ $d(b, u)+\alpha+2 \delta$. On choisit un tel $w$.

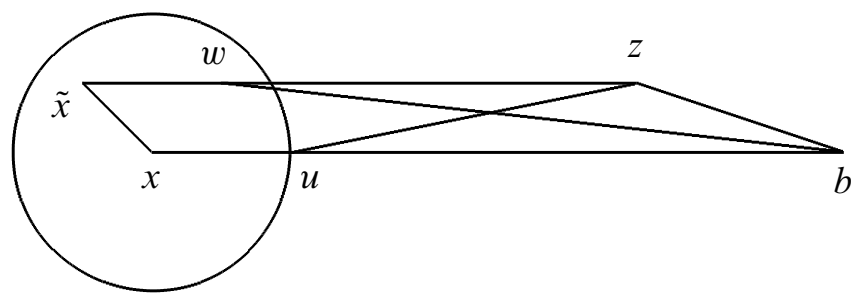

Comme $u \in \delta$-géod $(\tilde{x}, b)$ et $z \in \alpha$-géod $(b, u)$ le a) du lemme 3.3 montre que $z \in$ $(\alpha+\delta)-\operatorname{géod}(\tilde{x}, b)$. On en déduit que $w \in(\alpha+\delta)-\operatorname{géod}(\tilde{x}, b)$. Alors $\left(H_{\delta}(w, \tilde{x}, u, b)\right)$ montre que

$d(w, u) \leq \max (d(w, \tilde{x})-d(b, \tilde{x})+d(b, u)+\delta, d(w, b)-d(b, \tilde{x})+d(\tilde{x}, u)+\delta)$.

Or $d(w, \tilde{x})-d(b, \tilde{x})+d(b, u)+\delta \leq-d(b, w)+\alpha+\delta+d(b, u)+\delta=0$ et $d(w, b)-d(b, \tilde{x})+d(\tilde{x}, u)+\delta \leq d(\bar{w}, b)-d(b, u)+2 \delta=\alpha+4 \delta$. On en déduit $d(w, u) \leq \alpha+4 \delta$. De plus $\left(H_{\delta}(x, \tilde{x}, w, b)\right)$ montre que

$d(x, w) \leq \max (d(x, \tilde{x})-d(b, \tilde{x})+d(b, w)+\delta, d(x, b)-d(b, \tilde{x})+d(\tilde{x}, w)+\delta)$.

Or $d(x, \tilde{x})-d(b, \tilde{x})+d(b, w)+\delta \leq l \operatorname{car} d(x, \tilde{x}) \leq l$ et $d(b, \tilde{x}) \geq d(b, w)+\delta$ et $d(x, b)-d(b, \tilde{x})+d(\tilde{x}, w)+\delta \leq d(x, b)-d(b, w)+\alpha+2 \delta=l$ car $d(b, w)=$ $d(b, u)+\alpha+2 \delta$ et $d(x, b)=l+d(u, b)$. Donc on a bien $w \in B(x, l)$.

Le lemme suivant est une conséquence des deux précédents.

Lemme 4.19. Il existe une constante $C=C(\delta, K, N, Q, P, M)$ telle que pour $p \in\left\{1, \ldots, p_{\max }\right\}, k, m, l_{0}, \ldots, l_{m} \in \mathbb{N}$ et

$$
\left(a_{1}, \ldots, a_{p}, S_{0}, \ldots, S_{m},\left(\mathcal{Y}_{i}^{j}\right)_{i \in\{0, \ldots, m\}, j \in\left\{1, \ldots, l_{i}\right\}}\right) \in Y_{x}^{p, k, m,\left(l_{0}, \ldots, l_{m}\right)},
$$


les distances entre les points de

$$
B\left(S_{0}, M\right) \cup \underset{j \in\left\{1, \ldots, l_{0}\right\}}{\bigcup} B\left(y_{0}^{j}, M\right)
$$

et ceux de

$$
\bigcup_{i \in\{1, \ldots, m\}} B\left(S_{i}, M\right) \cup \underset{\substack{i \in\{1, \ldots, m\} \\ j \in\left\{1, \ldots, l_{i}\right\}}}{\bigcup} B\left(\mathcal{Y}_{i}^{j}, M\right) \cup B(x, k+2 M)
$$

sont déterminées par

a) les distances entre les points de (45),

b) les entiers $d\left(x, S_{0}\right)$ et $d\left(x, y_{0}^{j}\right)$,

c) les distances entre les points de (44) et $C\left(1+l_{0}\right)$ points de (45) (qui sont eux-mêmes déterminés par a) et b))

et de plus les distances entre les points de (44) et ceux de (45) sont déterminées à $C$ près par a) et b).

Remarque. Dans toutes les situations où on appliquera ce lemme, $l_{0}$ sera majoré par une constante de la forme $C(\delta, K, N, Q, P, M)$.

Démonstration. Soient $p \in\left\{1, \ldots, p_{\max }\right\}, k, m, l_{0}, \ldots, l_{m} \in \mathbb{N}$ et

$$
\left(a_{1}, \ldots, a_{p}, S_{0}, \ldots, S_{m},\left(\mathcal{Y}_{i}^{j}\right)_{i \in\{0, \ldots, m\}, j \in\left\{1, \ldots, l_{i}\right\}}\right) \in Y_{x}^{p, k, m,\left(l_{0}, \ldots, l_{m}\right)} .
$$

On applique le lemme 4.17 (complété par la remarque que, dans les notations de ce lemme, $4 P$ - géod $(u, b) \subset 4 P$ - géod $(x, b))$ avec

- $(x, b)$ au lieu de $(c, d)$,

- $\left\{w_{i}, i \in I\right\}$ égal à $\bigcup_{i \in\{0, \ldots, m\}} S_{i} \cup \bigcup_{i \in\{0, \ldots, m\}, j \in\left\{1, \ldots, l_{i}\right\}} y_{i}^{j}$,

- $J$ la partie de $I$ telle que $\left\{w_{j}, j \in J\right\}=S_{0} \cup \bigcup_{j \in\left\{1, \ldots, l_{0}\right\}} y_{0}^{j}$,

- et $\left(\alpha_{i}, \rho_{i}\right)$ égal à $(4 P, M)$ pour tout $i$ (les hypothèses sont satisfaites grâce au lemme 4.14).

Puis on applique le lemme 4.18 à $z$ parcourant (44), $\alpha=4 P+2 M$ et $l=k+2 M$ (grâce aux lemmes 4.14 et 3.2, (44) est inclus dans $(4 P+2 M)$ - géod $(b, u)$ ).

4.5. Continuité de $\boldsymbol{\partial}$ et $\boldsymbol{J}_{\boldsymbol{x}}$. On introduit d'abord une variante $\|\cdot\|_{\mathscr{H}_{\overrightarrow{x, s}}}$ de la norme $\|\cdot\|_{\mathscr{H}_{x, s}}$ et on montre que ces deux normes sont équivalentes.

Soient $p \in\left\{1, \ldots, p_{\max }\right\}$ et $k, m, l_{0}, \ldots, l_{m} \in \mathbb{N}$. On note $Y_{x} \rightarrow, p, k, m,\left(l_{0}, \ldots, l_{m}\right)$ l'ensemble défini de la même façon que $Y_{x}^{p, k, m,\left(l_{0}, \ldots, l_{m}\right)}$ mais en ajoutant la condition

- ou bien $d\left(x, S_{0}\right)>k+P$, ou bien $k=0, m=0, l_{0}=0, d\left(x, S_{0}\right) \leq P$. 
On définit le quotient $\bar{Y}_{x}^{\rightarrow}, p, k, m,\left(l_{0}, \ldots, l_{m}\right)$ et l'application

$$
\pi_{x}^{\rightarrow, p, k, m,\left(l_{0}, \ldots, l_{m}\right)}: Y_{x}^{\rightarrow, p, k, m,\left(l_{0}, \ldots, l_{m}\right)} \rightarrow \bar{Y}_{x} \rightarrow, p, k, m,\left(l_{0}, \ldots, l_{m}\right)
$$

de la même façon que $\bar{Y}_{x}^{p, k, m,\left(l_{0}, \ldots, l_{m}\right)}$ et $\pi_{x}^{p, k, m,\left(l_{0}, \ldots, l_{m}\right)}$. Cela fournit un diagramme commutatif

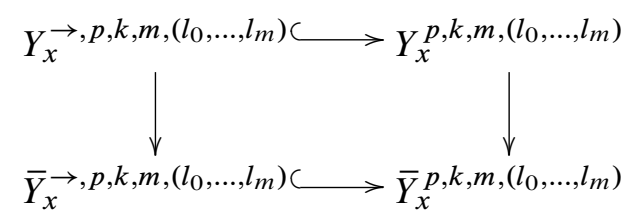

où les flèches verticales sont des surjections. Ce diagramme est cartésien au sens où les flèches horizontales induisent des bijections sur les fibres des flèches verticales.

On note $\|\cdot\|_{\mathscr{H}_{x, s}\left(\Delta_{p}\right)}$ la norme sur $\mathbb{C}^{\left(\Delta_{p}\right)}$ donnée par la formule (31) en remplaçant $Y_{x}^{p, k, m,\left(l_{0}, \ldots, l_{m}\right)}, \bar{Y}_{x}^{p, k, m,\left(l_{0}, \ldots, l_{m}\right)}$ et $\pi_{x}^{p, k, m,\left(l_{0}, \ldots, l_{m}\right)}$ par $Y_{x}^{\rightarrow, p, k, m,\left(l_{0}, \ldots, l_{m}\right)}$, $\bar{Y}_{x} \rightarrow, p, k, m,\left(l_{0}, \ldots, l_{m}\right)$ et $\pi_{x} \rightarrow, p, k, m,\left(l_{0}, \ldots, l_{m}\right)$. On remarque que les sous-espaces de $\mathbb{C}^{\left(\Delta_{p}\right)}$ engendrés par les $e_{S}$ pour $d(x, S) \leq P$, resp. $d(x, S)>P$ sont orthogonaux pour la norme pré-hilbertienne $\|\cdot\|_{\mathscr{H}_{\vec{x}, S}\left(\Delta_{p}\right)}$, et que sur le premier la norme est donnée par $\|f\|_{\mathscr{H}_{x, S}\left(\Delta_{p}\right)}^{2}=p ! \sum_{S} e^{2 s d(x, S)}|f(S)|^{2}$, car si $S \in \Delta_{p}$ vérifie $d(x, S) \leq P$, on a $S \subset B(x, M)$. En effet on suppose $M \geq P+N$, ce qui est permis par $\left(H_{M}\right)$.

Lemme 4.20. Les normes $\|\cdot\|_{\mathscr{H}_{x, s}\left(\Delta_{p}\right)}$ et $\|\cdot\|_{\mathscr{H}_{x, s}\left(\Delta_{p}\right)}$ sont équivalentes.

Démonstration. D'abord il est évident que $\|\cdot\|_{\mathscr{H}_{x, s}\left(\Delta_{p}\right)} \leq\|\cdot\|_{\mathscr{H}_{x, s}\left(\Delta_{p}\right)}$. Soit

$$
\begin{array}{r}
\left(a_{1}, \ldots, a_{p}, S_{0}, \ldots, S_{m},\left(\mathcal{Y}_{i}^{j}\right)_{\left.i \in\{0, \ldots, m\}, j \in\left\{1, \ldots, l_{i}\right\}\right)}\right. \\
\in Y_{x}^{p, k, m,\left(l_{0}, \ldots, l_{m}\right)}-Y_{x}^{\rightarrow, p, k, m,\left(l_{0}, \ldots, l_{m}\right)} .
\end{array}
$$

On a alors $d\left(x, S_{0}\right) \leq k+P$ et $d\left(x, S_{i}\right)>k+P$ pour $i \geq 1$. Le lemme 4.12 montre que $d_{\max }\left(x, S_{0}\right) \geq d_{\max }\left(x, S_{1}\right) \geq \cdots \geq d_{\max }\left(x, S_{m}\right)$. Donc

$$
S_{0} \cup \cdots \cup S_{m} \subset B(x, k+N+P) .
$$

Le lemme 4.13 implique alors

$$
\underset{\substack{i \in\{0, \ldots, m\} \\ j \in\left\{1, \ldots, l_{i}\right\}}}{\bigcup} y_{i}^{j} \subset B(x, k+N+3 P+\delta) .
$$

Grâce à $\left(H_{M}\right)$ on suppose $M \geq N+3 P+\delta$, d'où

$$
\bigcup_{i \in\{0, \ldots, m\}} B\left(S_{i}, M\right) \cup \underset{\substack{i \in\{0, \ldots, m\} \\ j \in\left\{1, \ldots, l_{i}\right\}}}{\bigcup} B\left(y_{i}^{j}, M\right) \subset B(x, k+2 M) .
$$


Donc l'ensemble des points entre lesquels on veut connaître les distances, à savoir

$$
\bigcup_{i \in\{0, \ldots, m\}} B\left(S_{i}, M\right) \cup \underset{\substack{i \in\{0, \ldots, m\} \\ j \in\left\{1, \ldots, l_{i}\right\}}}{\bigcup} B\left(y_{i}^{j}, M\right) \cup B(x, k+2 M)
$$

est simplement égal à $B(x, k+2 M)$. Il en résulte que le singleton

$$
\left\{\left(a_{1}, \ldots, a_{p}, S_{0}, \ldots, S_{m},\left(\mathcal{Y}_{i}^{j}\right)_{i \in\{0, \ldots, m\}, j \in\left\{1, \ldots, l_{i}\right\}}\right)\right\}
$$

est une classe d'équivalence dans $\bar{Y}_{x}^{p, k, m,\left(l_{0}, \ldots, l_{m}\right)}$. On a donc

$$
\begin{gathered}
\|f\|_{\mathcal{H}_{x, s}\left(\Delta_{p}\right)}^{2}-\|f\|_{\mathscr{H}_{x, s}\left(\Delta_{p}\right)}^{2} \\
\leq \sum_{S_{0} \in \Delta_{p}}\left(\sum_{k \geq d\left(x, S_{0}\right)-P} e^{2 s\left(d\left(x, S_{0}\right)-k\right)} \sum_{m, l_{0}, \ldots, l_{m} \in \mathbb{N}} B^{-\left(m+\sum_{i=0}^{m} l_{i}\right)}\right. \\
\qquad \sum_{\substack{\left(a_{1}, \ldots, a_{p}, S_{0}, \ldots, S_{m},\left(y_{i}^{j}\right)_{i \in\{0, \ldots, m\}, j \in\left\{1, \ldots, l_{i}\right\}}\right) \\
\in Y_{x}^{p, k, m,\left(l_{0}, \ldots, l_{m}\right)} \text { tel que } S_{0}=\left\{a_{1}, \ldots, a_{p}\right\}}}^{\left.\left(\prod_{i=0}^{m} s_{i}(Z)^{-l_{i}}\right)\right)\left|f\left(S_{0}\right)\right|^{2} .}
\end{gathered}
$$

Soient $D$ et $C$ comme dans les lemmes 4.12 et 4.15 (on rappelle que ce sont des constantes de la forme $C(\delta, K, N, Q, P))$. Etant donnés $S_{0}, k, m, l_{0}, \ldots, l_{m}$ tels que $k \geq d\left(x, S_{0}\right)-P$, le nombre de possibilités pour $\left(S_{1}, \ldots, S_{m}\right)$ est inférieur ou égal à $e^{D(P+m)}$ d'après le lemme 4.12 et le nombre de possibilités pour $\left(y_{i}^{j}\right)_{i \in\{0, \ldots, m\}, j \in\left\{1, \ldots, l_{i}\right\}}$ est inférieur ou égal à $C^{\sum_{i=0}^{m} l_{i}} \prod_{i=0}^{m} s_{i}(Z)^{l_{i}}$ d'après le lemme 4.15. Donc

$$
\begin{aligned}
& \|f\|_{\mathscr{H}_{x, s}\left(\Delta_{p}\right)}^{2}-\|f\|_{\mathscr{H}_{x, s}\left(\Delta_{p}\right)}^{2} \\
& \leq p ! \sum_{S_{0} \in \Delta_{p}}\left(\sum_{k \geq d\left(x, S_{0}\right)-P} e^{2 s\left(d\left(x, S_{0}\right)-k\right)} \sum_{m, l_{0}, \ldots, l_{m} \in \mathbb{N}} B^{-\left(m+\sum_{i=0}^{m} l_{i}\right)} e^{D(P+m)} C^{\sum_{i=0}^{m} l_{i}}\right)\left|f\left(S_{0}\right)\right|^{2} \\
& =p ! \frac{e^{D P}}{1-B^{-1}\left(C+e^{D}\right)} \sum_{S_{0} \in \Delta_{p}} \sum_{k \geq d\left(x, S_{0}\right)-P} e^{2 s\left(d\left(x, S_{0}\right)-k\right)}\left|f\left(S_{0}\right)\right|^{2} \\
& \leq p ! \frac{e^{(D+2 s) P}}{\left(1-B^{-1}\left(C+e^{D}\right)\right)\left(1-e^{-2 s}\right)}\|f\|_{\ell^{2}\left(\Delta_{p}\right)}^{2} .
\end{aligned}
$$

Pour $\left(a_{1}, \ldots, a_{p}, S_{0}\right) \in Y_{x}^{p, k, 0,(0)}$ tel que $S_{0}$ vérifie

- ou bien $d\left(x, S_{0}\right)=k+P+1$,

- ou bien $k=0$ et $d\left(x, S_{0}\right) \leq P$,

le singleton $\left\{\left(a_{1}, \ldots, a_{p}, S_{0}\right)\right\}$ est une classe d'équivalence dans $\bar{Y}_{x}^{\rightarrow, p, k, 0,(0)}$. En limitant la somme qui définit $\|f\|_{\mathscr{H}_{\overrightarrow{x, s}}\left(\Delta_{p}\right)}^{2}$ à ces éléments-là, on voit que

$$
\|f\|_{\mathscr{H}_{x, s}\left(\Delta_{p}\right)}^{2} \geq p !\|f\|_{\ell^{2}\left(\Delta_{p}\right)}^{2} .
$$


Maintenant on déduit des inégalités (46) et (47) qu'il existe une constante $C=$ $C(\delta, K, N, Q, P, M, s, B)$ telle que $\|f\|_{\mathcal{H}_{x, s}\left(\Delta_{p}\right)}^{2} \leq C\|f\|_{\mathscr{H}_{x, s}\left(\Delta_{p}\right)}^{2}$ pour tout $f \in$ $\mathbb{C}^{\left(\Delta_{p}\right)}$.

Soit $\mathcal{P}$ le projecteur orthogonal sur le sous-espace vectoriel de $\mathscr{H}_{x, s}\left(\Delta_{p}\right)$ engendré par les $e_{S}$ pour $S \in \Delta_{p}$ tel que $d(x, S) \leq P$, de sorte que $(\mathscr{P} f)(S)=f(S)$ si $d(x, S) \leq P$ et $(\mathcal{P} f)(S)=0$ sinon.

Pour $f \in \mathbb{C}^{\left(\Delta_{p}\right)}$ on a

$$
\begin{aligned}
\|(1-\mathcal{P}) f\|_{\mathscr{H}_{x, s}\left(\Delta_{p}\right)}^{2} & \sum_{k, m, l_{0}, \ldots, l_{m} \in \mathbb{N}} B^{-\left(m+\sum_{i=0}^{m} l_{i}\right)} \sum_{\substack{Z \in \bar{Y}_{x}^{p, k, m,\left(l_{0}, \ldots, l_{m}\right)} r_{0}(Z)>k+P}} e^{2 s\left(r_{0}(Z)-k\right)} \\
& \left(\prod_{i=0}^{m} s_{i}(Z)^{-l_{i}}\right) \sharp\left(\left(\pi_{x}^{p, k, m,\left(l_{0}, \ldots, l_{m}\right)}\right)^{-1}(Z)\right)^{-\alpha}\left|\xi_{Z}(f)\right|^{2} .
\end{aligned}
$$

Cette formule est la raison pour laquelle on a introduit la norme $\|\cdot\|_{\mathscr{H}_{x, s}\left(\Delta_{p}\right)}$. En effet pour montrer la continuité de $\partial$ ou de $J_{x}$ on cherchera à majorer $\left|\xi_{Z}(\partial f)\right|^{2}$ ou $\left|\xi_{Z}\left(J_{x} f\right)\right|^{2}$ par une combinaison de $\left|\xi_{\widetilde{Z}}(f)\right|^{2}$ avec $\widetilde{Z}$ vérifiant notamment $r_{1}(\widetilde{Z})=$ $r_{0}(Z)$. Comme la condition $r_{1}(\widetilde{Z})>k+P$ est imposée par la condition ii) de la définition 4.1 , il est très utile d'avoir $r_{0}(Z)>k+P$.

Proposition 4.21. Pour tout $p \in\left\{1, \ldots, p_{\max }\right\}$, d se prolonge en un opérateur continu de $\mathscr{H}_{x, s}\left(\Delta_{p}\right)$ dans $\mathscr{H}_{x, s}\left(\Delta_{p-1}\right)$.

Démonstration. Supposons d'abord $p=1$. On va montrer qu'il existe une constante $C=C(\delta, K, N, Q, P, M, s, B)$ telle que $\left|\sum_{a \in X} f(a)\right| \leq C\|f\|_{\mathscr{H}_{x, s}\left(\Delta_{1}\right)}$. D'après la formule (31), $\|f\|_{\mathscr{H}_{x, s}\left(\Delta_{1}\right)}^{2}$ est une somme sur $k, m, l_{0}, \ldots, l_{m}$ et en limitant cette somme à $k=0, m=0, l_{0}=0$ on voit que

$$
\|f\|_{\mathscr{H}_{x, s}\left(\Delta_{1}\right)}^{2} \geq \sum_{Z \in \bar{Y}_{x}^{1,0,0,(0)}} e^{2 s r_{0}(Z)} \sharp\left(\left(\pi_{x}^{1,0,0,(0)}\right)^{-1}(Z)\right)^{-\alpha}\left|\sum_{(a,\{a\}) \in\left(\pi_{x}^{1,0,0,(0)}\right)^{-1}(Z)} f(a)\right|^{2} .
$$

De plus $\bar{Y}_{x}^{1,0,0,(0)}$ s'identifie au quotient de $X$ pour la relation d'équivalence suivante : $a$ et $b$ sont équivalents s'il existe une isométrie de $B(a, M) \cup B(x, 2 M)$ vers $B(b, M) \cup B(x, 2 M)$ qui est l'identité sur $B(x, 2 M)$ et applique $a$ sur $b$. Cette relation d'équivalence détermine $d(x, a)$ et inversement il existe $C=C(\delta, K, N, Q, P, M)$ telle que pour tout $r \in \mathbb{N}$, l'ensemble $\{a \in X, d(x, a)=r\}$ est réunion d'au plus $C$ classes d'équivalences de $\bar{Y}_{x}^{1,0,0,(0)}$. Il existe $D^{\prime}=C(\delta, K)$ telle que pour tout $r \in \mathbb{N}$, le cardinal de l'ensemble $\{a \in X, d(x, a)=r\}$ soit inférieur ou égal à $e^{D^{\prime} r}$. 
On a donc, par Cauchy-Schwarz,

$$
\|f\|_{\mathscr{H}_{x, s}\left(\Delta_{1}\right)}^{2} \geq C^{-1} \sum_{r \in \mathbb{N}} e^{\left(2 s-\alpha D^{\prime}\right) r}\left|\sum_{\substack{a \in X \\ d(x, a)=r}} f(a)\right|^{2} .
$$

Grâce à $\left(H_{\alpha}\right)$ on suppose $2 s-\alpha D^{\prime} \geq s$. Par Cauchy-Schwarz,

$$
\begin{aligned}
\left|\sum_{a \in X} f(a)\right|^{2} & \leq\left(\sum_{r \in \mathbb{N}} e^{-\left(2 s-\alpha D^{\prime}\right) r}\right)\left(\sum_{r \in \mathbb{N}} e^{\left(2 s-\alpha D^{\prime}\right) r}\left|\sum_{\substack{a \in X \\
d(x, a)=r}} f(a)\right|^{2}\right) \\
& \leq \frac{C}{1-e^{-\left(2 s-\alpha D^{\prime}\right)}}\|f\|_{\mathscr{H}_{x, s}\left(\Delta_{1}\right)}^{2} \\
& \leq \frac{C}{1-e^{-s}}\|f\|_{\mathscr{H}_{x, s}\left(\Delta_{1}\right)}^{2}
\end{aligned}
$$

Soit maintenant $p \in\left\{2, \ldots, p_{\max }\right\}$. Grâce au lemme 4.20, il suffit de montrer qu'il existe une constante $C=C(\delta, K, N, Q, P, M, s, B)$ telle que

$$
\|\partial f\|_{\mathscr{H}_{x, s}\left(\Delta_{p-1}\right)} \leq C\|f\|_{\mathscr{H}_{x, s}\left(\Delta_{p}\right)}
$$

pour tout $f \in \mathbb{C}^{\left(\Delta_{p}\right)}$. Grâce à (42) et au lemme 4.16, il est clair que

$$
\|\mathcal{P}(\partial f)\|_{\mathscr{H}_{x, s}\left(\Delta_{p-1}\right)} \leq C\|f\|_{\mathscr{H}_{x, s}\left(\Delta_{p}\right)}
$$

pour une constante $C=C(\delta, K, N, Q, P, M, s, B)$. Il reste donc à montrer qu'il existe une constante $C=C(\delta, K, N, Q, P, M, s, B)$ telle que

$$
\|(1-\mathcal{P})(\partial f)\|_{\mathscr{H}_{x, s}\left(\Delta_{p-1}\right)} \leq C\|f\|_{\mathscr{H}_{x, s}\left(\Delta_{p}\right)}
$$

pour tout $f \in \mathbb{C}^{\left(\Delta_{p}\right)}$. Par (48) on a

$$
\begin{aligned}
\|(1-\mathcal{P})(\partial f)\|_{\mathcal{H}_{x, s}\left(\Delta_{p-1}\right)}^{2} & \sum_{k, m, l_{0}, \ldots, l_{m} \in \mathbb{N}} B^{-\left(m+\sum_{i=0}^{m} l_{i}\right)} \sum_{\substack{Z \in \bar{Y}_{x}^{p-1, k, m,\left(l_{0}, \ldots, l_{m}\right)} \\
r_{0}(Z)>k+P}} e^{2 s\left(r_{0}(Z)-k\right)} \\
& \left(\prod_{i=0}^{m} s_{i}(Z)^{-l_{i}}\right) \sharp\left(\left(\pi_{x}^{p-1, k, m,\left(l_{0}, \ldots, l_{m}\right)}\right)^{-1}(Z)\right)^{-\alpha}\left|\xi_{Z}(\partial f)\right|^{2}
\end{aligned}
$$

Soient $k, m, l_{0}, \ldots, l_{m} \in \mathbb{N}$ et $Z \in \bar{Y}_{x}^{p-1, k, m,\left(l_{0}, \ldots, l_{m}\right)}$ vérifiant $r_{0}(Z)>k+P$. On pose $\tilde{l}_{0}=0, \tilde{l}_{i}=l_{i-1}$ pour $i \in\{1, \ldots, m+1\}$. Alors on a

$$
\begin{aligned}
& \xi_{Z}(\partial f)=\sum_{\substack{\left(a_{1}, \ldots, a_{p-1}, S_{0}, \ldots, S_{m},\left(y_{i}^{j}\right)_{i \in\{0, \ldots, m\}, j \in\left\{1, \ldots, l_{i}\right\}}\right) \\
\in\left(\pi_{x}^{p-1, k, m,\left(l_{0}, \ldots, l_{m}\right)}\right)^{-1}(Z)}}(\partial f)\left(a_{1}, \ldots, a_{p-1}\right) \\
& =\sum_{\tilde{Z} \in \Lambda_{Z}} \sum_{\left(\tilde{a}_{1}, \ldots, \tilde{a}_{p}, \tilde{S}_{0}, \ldots, \tilde{S}_{m+1},\left(\tilde{y}_{i}^{j}\right)_{i \in\{0, \ldots, m+1\}, j \in\left\{1, \ldots, \tilde{l}_{i}\right\}}\right.} f\left(\tilde{a}_{1}, \ldots, \tilde{a}_{p}\right) \\
& \in\left(\pi_{x}^{p, k, m+1,\left(\tilde{l}_{0}, \ldots, \tilde{l}_{m+1}\right)}\right)^{-1}(\widetilde{Z}) \\
& =\sum_{\widetilde{Z} \in \Lambda_{Z}} \xi_{\widetilde{Z}}(f)
\end{aligned}
$$


où $\Lambda_{Z}$ est la partie de $\bar{Y}_{x}^{p, k, m+1,\left(\tilde{l}_{0}, \ldots, \tilde{l}_{m+1}\right)}$ formée des $\widetilde{Z}$ tels que pour tout

$$
\begin{array}{r}
\left(\tilde{a}_{1}, \ldots, \tilde{a}_{p}, \tilde{S}_{0}, \ldots, \widetilde{S}_{m+1},\left(\widetilde{\mathcal{Y}}_{i}^{j}\right)_{i \in\{0, \ldots, m+1\}, j \in\left\{1, \ldots, \tilde{l}_{i}\right\}}\right) \\
\in\left(\pi_{x}^{p, k, m+1,\left(\tilde{l}_{0}, \ldots, \tilde{l}_{m+1}\right)}\right)^{-1}(\widetilde{Z})
\end{array}
$$

on ait $\widetilde{S}_{1}=\left\{\tilde{a}_{2}, \ldots, \tilde{a}_{p}\right\}$ et

$\left(\tilde{a}_{2}, \ldots, \tilde{a}_{p}, \tilde{S}_{1}, \ldots, \tilde{S}_{m+1},\left(\tilde{\mathcal{Y}}_{i+1}^{j}\right)_{i \in\{0, \ldots, m\}, j \in\left\{1, \ldots, l_{i}\right\}}\right) \in\left(\pi_{x}^{p-1, k, m,\left(l_{0}, \ldots, l_{m}\right)}\right)^{-1}(Z)$.

Il existe $C_{1}=C(\delta, K, N, Q, P, M)$ telle que $\sharp \Lambda_{Z} \leq C_{1}$. En effet, grâce au lemme 4.19 , pour connaître les distances entre les points de $B\left(\widetilde{S}_{0}, M\right)$ et ceux de

$$
\bigcup_{i \in\{1, \ldots, m+1\}} B\left(\widetilde{S}_{i}, M\right) \cup \underset{\substack{i \in\{1, \ldots, m+1\} \\ j \in\left\{1, \ldots, \tilde{l}_{i}\right\}}}{\bigcup} B\left(\tilde{y}_{i}^{j}, M\right) \cup B(x, k+2 M)
$$

il suffit de connaître les distances entre les points de $B\left(\widetilde{S}_{0}, M\right)$ et $C$ points de $(51)$, avec $C=C(\delta, K, N, Q, P, M)$ et comme $\widetilde{S}_{0}=\widetilde{S}_{1} \cup\left\{\tilde{a}_{1}\right\}$ et $d\left(\tilde{a}_{1}, \tilde{a}_{2}\right) \leq N$, ces distances sont déterminées à $N+M$ près par les distances de $\tilde{a}_{2}$ à ces $C$ points (qui font partie de la donnée de $Z$ ). Comme $\sharp \Lambda_{Z} \leq C_{1}$, grâce à (50) et par CauchySchwarz, on obtient

$$
\left|\xi_{Z}(\partial f)\right|^{2} \leq C_{1} \sum_{\tilde{Z} \in \Lambda_{Z}}\left|\xi_{\tilde{Z}}(f)\right|^{2}
$$

De plus pour $\widetilde{Z} \in \Lambda_{Z}$ on a

$$
\begin{gathered}
\prod_{i=0}^{m+1} s_{i}(\widetilde{Z})^{-\tilde{l}_{i}}=\prod_{i=0}^{m} s_{i}(Z)^{-l_{i}}, \\
\sharp\left(\pi_{x}^{p, k, m+1,\left(\tilde{l}_{0}, \ldots, \tilde{l}_{m+1}\right)}\right)^{-1}(\widetilde{Z}) \leq C \sharp\left(\pi_{x}^{p-1, k, m,\left(l_{0}, \ldots, l_{m}\right)}\right)^{-1}(Z)
\end{gathered}
$$

avec $C=C(\delta, K, N)$ et $\underset{r_{0}}{\mid}(Z)-r_{0}(\widetilde{Z}) \mid \leq N$. Enfin $\widetilde{Z}$ détermine $Z$, donc quand on somme sur $Z$, chaque $\widetilde{Z}$ ne peut apparaître qu'une fois. L'inégalité (49) en résulte facilement et ceci termine la démonstration de la proposition 4.21.

Remarque. Le coeur de la démonstration ci-dessus est formé par

- l'égalité (50), que l'on peut mettre sous la forme ${ }^{t} \partial\left(\xi_{Z}\right)=\sum \tilde{Z}_{\in} \Lambda_{Z} \xi_{\tilde{Z}}$,

- l'application de Cauchy-Schwarz qui fournit l'inégalité (52),

- le fait que les normes sont des sommes pondérées des $\left|\xi_{Z}\right|^{2}$.

Dans la suite les arguments seront plus compliqués mais ils reposeront tous sur ce principe.

Avant de montrer la continuité de $J_{x}$ on va introduire des nouvelles normes préhilbertiennes $\|\cdot\|_{\mathscr{H}_{x, s}^{\natural, \mu_{0}, \mu_{1}}} \operatorname{sur} \mathbb{C}^{\left(\Delta_{p}\right)}$ (pour $\mu_{0}, \mu_{1} \in \mathbb{N}$ ) et montrer qu'elles sont 
équivalentes à $\|\cdot\|_{\mathscr{H}_{x, s}}$. Ces normes seront obtenues en ajoutant aux parties $\mathcal{Y}_{i}^{j}$ qui intervenaient dans la définition 4.1 de nouvelles parties, notées $\mathcal{Z}_{i}^{j}$ dans la définition ci-dessous. On verra dans la démonstration de la continuité de $J_{x}$ (proposition 4.30) que la connaissance des points de $B\left(\mathcal{Z}_{i}^{j}, M\right)$ détermine exactement les différentes moyennes intervenant dans la formule pour $J_{x}$, d'où l'intérêt de ces parties supplémentaires. On va voir dans la preuve de l'équivalence des normes $\|\cdot\|_{\mathscr{H}_{x, s}^{\natural, \mu_{0}, \mu_{1}}}$ et $\|\cdot\|_{\mathscr{H}_{x, s}}\left(\right.$ lemme 4.24) que chaque partie $\mathcal{Z}_{i}^{j}$ peut être oubliée ou reconsidérée comme une partie $\mathcal{Y}_{i^{\prime}}^{j^{\prime}}$ supplémentaire. Cependant $i^{\prime}$ est déterminé par $d\left(x, Z_{i}^{j}\right)$ d'une façon assez compliquée. Ces nouvelles normes rendent donc la preuve de la continuité de $J_{x}$ (proposition 4.30) beaucoup plus lisible et elles resserviront de plus pour montrer la continuité des autres opérateurs (proposition 4.46) et l'équivariance à compact près de tous les opérateurs (proposition 4.68). Inversement on n'a pas inclus ces parties $Z_{i}^{j}$ dans la définition 4.1 car elles auraient rendu beaucoup plus difficile la preuve des propriétés d'équivariance de la norme $\|\cdot\|_{\mathscr{H}_{x, s}}$ (proposition 4.3, démontrée dans le sous-paragraphe 4.7 ).

Soient $p \in\left\{1, \ldots, p_{\max }\right\}$ et $k, m, l_{0}, \ldots, l_{m}, \lambda_{0}, \lambda_{1} \in \mathbb{N}$ vérifiant $\lambda_{1}=0$ si $m=0$.

Définition 4.22. On note $Y_{x}^{\natural, p, k, m,\left(l_{0}, \ldots, l_{m}\right), \lambda_{0}, \lambda_{1}}$ l'ensemble des $(p+m+1+$ $\left.\sum_{i=0}^{m} l_{i}+\lambda_{0}+\lambda_{1}\right)$-uplets

$$
\left(a_{1}, \ldots, a_{p}, S_{0}, \ldots, S_{m},\left(\mathcal{Y}_{i}^{j}\right)_{i \in\{0, \ldots, m\}, j \in\left\{1, \ldots, l_{i}\right\}},\left(\mathcal{Z}_{i}^{j}\right)_{i \in\{0,1\}, j \in\left\{1, \ldots, \lambda_{i}\right\}}\right)
$$

tels que

- $\left(a_{1}, \ldots, a_{p}, S_{0}, \ldots, S_{m},\left(y_{i}^{j}\right)_{i \in\{0, \ldots, m\}, j \in\left\{1, \ldots, l_{i}\right\}}\right)$ appartient à $Y_{x}^{p, k, m,\left(l_{0}, \ldots, l_{m}\right)}$, c'est-à-dire vérifie les conditions i), ii), iii), iv) de la définition 4.1,

- pour $i \in\{0,1\}$ et $j \in\left\{1, \ldots, \lambda_{i}\right\}, Z_{i}^{j}$ est une partie non vide de $X$ de diamètre inférieur ou égal à $P / 3$ et $Z_{i}^{j} \subset \bigcup_{a \in S_{i}} \operatorname{géod}(x, a)$.

On introduit une partition de $Y_{x}^{\natural, p, k, m,\left(l_{0}, \ldots, l_{m}\right), \lambda_{0}, \lambda_{1}}$ pour la relation d'équivalence suivante :

$$
\left(a_{1}, \ldots, a_{p}, S_{0}, \ldots, S_{m},\left(\mathcal{Y}_{i}^{j}\right)_{i \in\{0, \ldots, m\}, j \in\left\{1, \ldots, l_{i}\right\}},\left(\mathcal{Z}_{i}^{j}\right)_{i \in\{0,1\}, j \in\left\{1, \ldots, \lambda_{i}\right\}}\right)
$$

et

$$
\left(\hat{a}_{1}, \ldots, \hat{a}_{p}, \widehat{S}_{0}, \ldots, \widehat{S}_{m},\left(\widehat{Y}_{i}^{j}\right)_{i \in\{0, \ldots, m\}, j \in\left\{1, \ldots, l_{i}\right\}},\left(\hat{\mathcal{Z}}_{i}^{j}\right)_{i \in\{0,1\}, j \in\left\{1, \ldots, \lambda_{i}\right\}}\right)
$$

sont en relation s'il existe une isométrie de

$$
\bigcup_{i \in\{0, \ldots, m\}} B\left(S_{i}, M\right) \cup \bigcup_{\substack{i \in\{0, \ldots, m\} \\ j \in\left\{1, \ldots, l_{i}\right\}}}^{\bigcup} B\left(\mathcal{Y}_{i}^{j}, M\right) \cup \bigcup_{\substack{i \in\{0,1\} \\ j \in\left\{1, \ldots, \lambda_{i}\right\}}} B\left(\mathcal{Z}_{i}^{j}, M\right) \cup B(x, k+2 M)
$$


vers

$$
\bigcup_{i \in\{0, \ldots, m\}} B\left(\hat{S}_{i}, M\right) \cup \bigcup_{\substack{i \in\{0, \ldots, m\} \\ j \in\left\{1, \ldots, l_{i}\right\}}}^{\cup} B\left(\hat{\mathcal{Y}}_{i}^{j}, M\right) \cup \bigcup_{\substack{i \in\{0,1\} \\ j \in\left\{1, \ldots, \lambda_{i}\right\}}} B\left(\hat{\mathcal{Z}}_{i}^{j}, M\right) \cup B(x, k+2 M)
$$

qui envoie $a_{i}$ sur $\hat{a}_{i}$ pour $i \in\{1, \ldots, p\}, S_{i}$ sur $\hat{S}_{i}$ pour $i \in\{0, \ldots, m\}, y_{i}^{j}$ sur $\hat{y}_{i}^{j}$ pour $i \in\{0, \ldots, m\}, j \in\left\{1, \ldots, l_{i}\right\}, \mathcal{Z}_{i}^{j}$ sur $\hat{Z}_{i}^{j}$ pour $i \in\{0,1\}, j \in\left\{1, \ldots, \lambda_{i}\right\}$ et est l'identité sur $B(x, k+2 M)$.

On note $\bar{Y}_{x}^{\natural, p, k, m,\left(l_{0}, \ldots, l_{m}\right), \lambda_{0}, \lambda_{1}}$ le quotient de $Y_{x}^{\natural, p, k, m,\left(l_{0}, \ldots, l_{m}\right), \lambda_{0}, \lambda_{1}}$ pour cette relation d'équivalence, et $\pi_{x}^{\natural, p, k, m,\left(l_{0}, \ldots, l_{m}\right), \lambda_{0}, \lambda_{1}}$ l'application quotient.

Notation. Pour $Z \in \bar{Y}_{x}^{\natural, p, k, m,\left(l_{0}, \ldots, l_{m}\right), \lambda_{0}, \lambda_{1}}$ on note $r_{0}(Z), \ldots, r_{m}(Z), s_{0}(Z), \ldots$, $s_{m}(Z),\left(r_{i, j}^{\max }(Z)\right)_{i \in\{0,1\}, j \in\{i, \ldots, m+1\}}$ et $\left(t_{i}^{j}(Z)\right)_{i \in\{0,1\}, j \in\left\{1, \ldots, \lambda_{i}\right\}}$ les entiers tels que

- $r_{i}(Z)=d\left(x, S_{i}\right)$ pour $i \in\{0, \ldots, m\}$,

$-s_{i}(Z)=d\left(S_{i}, S_{i+1}\right)+2 M$ pour $i \in\{0, \ldots, m-1\}$,

$-s_{m}(Z)=d\left(x, S_{m}\right)-k$,

- pour $i \in\{0,1\}$, si $d_{\max }\left(x, S_{i}\right) \leq k+3 P, r_{i, j}^{\max }(Z)=d_{\max }\left(x, S_{i}\right)$ pour tout $j \in\{i, \ldots, m+1\}$,

- pour $i \in\{0,1\}$, si $d_{\max }\left(x, S_{i}\right) \geq k+3 P, r_{i, j}^{\max }(Z)=\max \left(k+3 P, d_{\max }\left(x, S_{j}\right)\right)$ pour tout $j \in\{i, \ldots, m\}$ et $r_{i, m+1}^{\max }(Z)=k+3 P$,

$-t_{i}^{j}(Z)=d\left(x, Z_{i}^{j}\right)$ pour $i \in\{0,1\}, j \in\left\{1, \ldots, \lambda_{i}\right\}$

pour tout $\left(a_{1}, \ldots, a_{p}, S_{0}, \ldots, S_{m},\left(\mathcal{Y}_{i}^{j}\right)_{i \in\{0, \ldots, m\}, j \in\left\{1, \ldots, l_{i}\right\}},\left(\mathcal{Z}_{i}^{j}\right)_{i \in\{0,1\}, j \in\left\{1, \ldots, \lambda_{i}\right\}}\right) \in$ $\left(\pi_{x}^{\natural, p, k, m,\left(l_{0}, \ldots, l_{m}\right), \lambda_{0}, \lambda_{1}}\right)^{-1}(Z)$.

Pour clarifier le sens des notations ci-dessus, on rappelle que d'après le lemme 4.12, on a toujours

$$
d_{\max }\left(x, S_{0}\right) \geq d_{\max }\left(x, S_{1}\right) \geq \cdots \geq d_{\max }\left(x, S_{m}\right) .
$$

D'autre part, pour $i \in\{0,1\}$, on a toujours

$$
r_{i, i}^{\max }(Z)=d_{\max }\left(x, S_{i}\right) \quad \text { et } \quad r_{i, m+1}^{\max }(Z)=\min \left(k+3 P, d_{\max }\left(x, S_{i}\right)\right) .
$$

Le lemme suivant indique quelques propriétés de ces entiers, qui nous seront utiles ensuite.

Lemme 4.23. Pour $k, m, l_{0}, \ldots, l_{m}, \lambda_{0}, \lambda_{1} \in \mathbb{N}$ (vérifiant $\lambda_{1}=0$ si $m=0$ ) et $Z \in \bar{Y}_{x}^{\natural, p, k, m,\left(l_{0}, \ldots, l_{m}\right), \lambda_{0}, \lambda_{1}}$ on a a) $\begin{aligned} r_{0,0}^{\max }(Z) \geq r_{0,1}^{\max }(Z) \geq \cdots \geq r_{0, m+1}^{\max }(Z) \text { et } r_{1,1}^{\max }(Z) \geq r_{1,2}^{\max }(Z) \geq \cdots \geq \\ \\ r_{1, m+1}^{\max }(Z),\end{aligned}$ 
b) pour tout $i \in\{0,1\}, r_{i}(Z) \leq r_{i, i}^{\max }(Z) \leq r_{i}(Z)+N$,

c) pour $i \in\{0,1\}$ et $j \in\left\{1, \ldots, \lambda_{i}\right\}, t_{i}^{j}(Z) \leq r_{i, i}^{\max }(Z)$,

d) pour $i \in\{0,1\}, r_{i, m+1}^{\max }(Z)=\min \left(k+3 P, r_{i, i}^{\max }(Z)\right)$.

Démonstration. L'assertion a) découle de (53), b) est évidente et pour montrer c) on remarque que dans les notations précédentes on a $d\left(x, Z_{i}^{j}\right) \leq d_{\max }\left(x, S_{i}\right)$ par la dernière condition de la définition 4.22. Enfin d) résulte de (54).

Pour $Z \in \bar{Y}_{x}^{\natural, p, k, m,\left(l_{0}, \ldots, l_{m}\right), \lambda_{0}, \lambda_{1}}$ on note $\xi_{Z}$ la forme linéaire sur $\mathbb{C}^{\left(\Delta_{p}\right)}$ définie par

$$
\xi_{Z}(f)=\sum_{\left(a_{1}, \ldots, a_{p}, S_{0}, \ldots, S_{m},\left(y_{i}^{j}\right){\substack{i \in\{0, \ldots, m\} \\ j \in\left\{1, \ldots, l_{i}\right\}}},\left(Z_{i}^{j}\right)_{\substack{i \in\{0,1\} \\ j \in\left\{1, \ldots, \lambda_{i}\right\}}}\right)} f\left(a_{1}, \ldots, a_{p}\right) .
$$

Pour $\mu_{0}, \mu_{1} \in \mathbb{N}$ on munit alors $\mathbb{C}^{\left(\Delta_{p}\right)}$ de la norme pré-hilbertienne, définie par la formule suivante :

$$
\begin{aligned}
& \|f\|_{\mathcal{H}_{x, s}^{\natural, \mu_{0}, \mu_{1}}\left(\Delta_{p}\right)}^{2} \sum_{k, m, l_{0}, \ldots, l_{m}, \lambda_{0}, \lambda_{1}} B^{-\left(m+\sum_{i=0}^{m} l_{i}\right)} \sum_{Z \in \bar{Y}_{x}^{\natural, p, k, m,\left(l_{0}, \ldots, l_{m}\right), \lambda_{0}, \lambda_{1}} e^{2 s\left(r_{0}(Z)-k\right)}} \\
& \left(\prod_{i=0}^{m} s_{i}(Z)^{-l_{i}}\right)\left(r_{0}(Z)+1\right)^{-\lambda_{0}}\left(r_{1}(Z)+1\right)^{-\lambda_{1}} \\
& \sharp\left(\left(\pi_{x}^{\natural, p, k, m,\left(l_{0}, \ldots, l_{m}\right), \lambda_{0}, \lambda_{1}}\right)^{-1}(Z)\right)^{-\alpha}\left|\xi_{Z}(f)\right|^{2}
\end{aligned}
$$

où la première somme porte sur $k, m, l_{0}, \ldots, l_{m}, \lambda_{0}, \lambda_{1} \in \mathbb{N}$ vérifiant $\lambda_{1}=0$ si $m=0$ et satisfaisant les conditions

$$
\lambda_{0} \leq \mu_{0} \quad \text { et } \quad \lambda_{1} \leq \mu_{1} .
$$

En utilisant l'hypothèse $\left(H_{B}\right)$ nous allons montrer le lemme suivant.

Lemme 4.24. Il existe $C=C(\delta, K, N, Q, P, M, s, B)$ tel que pour $\mu_{0}, \mu_{1} \in \mathbb{N}$ et $f \in \mathbb{C}^{\left(\Delta_{p}\right)}$,

$$
\|f\|_{\mathscr{H}_{x, s}\left(\Delta_{p}\right)}^{2} \leq\|f\|_{\mathscr{H}_{x, s}^{\natural, \mu_{0}, \mu_{1}}\left(\Delta_{p}\right)}^{2} \leq C^{\mu_{0}+\mu_{1}}\|f\|_{\mathcal{H}_{x, s}\left(\Delta_{p}\right)}^{2} .
$$

Démonstration. L'inégalité de gauche est évidente, car la somme (31) qui donne $\|f\|_{\mathscr{H}_{x, s}\left(\Delta_{p}\right)}^{2}$ est une partie de la somme (55) qui donne $\|f\|_{\mathscr{H}_{x, s}^{\natural, \mu_{0}, \mu_{1}\left(\Delta_{p}\right)}}^{2}$ (c'est la partie qui correspond à $\lambda_{0}=\lambda_{1}=0$ ). Pour montrer l'inégalité de droite on a besoin de deux lemmes préliminaires. L'idée est simplement de reconsidérer chaque partie 
$Z_{i}^{j}$ comme une partie $Y_{i^{\prime}}^{j^{\prime}}$ supplémentaire, avec $i^{\prime}$ déterminé $\operatorname{par} t_{i}^{j}(Z)=d\left(x, Z_{i}^{j}\right)$, si $t_{i}^{j}(Z)>k+3 P$, et d'oublier $Z_{i}^{j}$ si $t_{i}^{j}(Z) \leq k+3 P$.

On prend $k, m, l_{0}, \ldots, l_{m}, \lambda_{0}, \lambda_{1} \in \mathbb{N}$ (vérifiant $\lambda_{1}=0$ si $m=0$ ). Soit $\sigma \in\{0,1\}$ (avec $\sigma=0$ si $m=0$ ). On pose

$$
-\left(\tilde{\lambda}_{0}, \tilde{\lambda}_{1}\right)=\left(\lambda_{0}+1, \lambda_{1}\right) \text { si } \sigma=0
$$

et

$$
-\left(\tilde{\lambda}_{0}, \tilde{\lambda}_{1}\right)=\left(\lambda_{0}, \lambda_{1}+1\right) \operatorname{si} \sigma=1 .
$$

Sous-lemme 4.25. Soit $Z \in \bar{Y}_{x}^{\natural, p, k, m,\left(l_{0}, \ldots, l_{m}\right), \tilde{\lambda}_{0}, \tilde{\lambda}_{1}}$ et

$$
\begin{gathered}
\left(a_{1}, \ldots, a_{p}, S_{0}, \ldots, S_{m},\left(\mathcal{Y}_{i}^{j}\right)_{i \in\{0, \ldots, m\}, j \in\left\{1, \ldots, l_{i}\right\}},\left(\mathcal{Z}_{i}^{j}\right)_{i \in\{0,1\}, j \in\left\{1, \ldots, \tilde{\lambda}_{i}\right\}}\right) \\
\in\left(\pi_{x}^{\natural, p, k, m,\left(l_{0}, \ldots, l_{m}\right), \tilde{\lambda}_{0}, \tilde{\lambda}_{1}}\right)^{-1}(Z) .
\end{gathered}
$$

Alors

- si $0 \leq t_{\sigma}^{\lambda \sigma+1}(Z) \leq r_{\sigma, m+1}^{\max }(Z)$, on $a$

$$
\begin{gathered}
\left(a_{1}, \ldots, a_{p}, S_{0}, \ldots, S_{m},\left(\mathcal{Y}_{i}^{j}\right)_{i \in\{0, \ldots, m\}, j \in\left\{1, \ldots, l_{i}\right\}},\left(\mathcal{Z}_{i}^{j}\right)_{i \in\{0,1\}, j \in\left\{1, \ldots, \lambda_{i}\right\}}\right) \\
\in Y_{x}^{\natural, p, k, m,\left(l_{0}, \ldots, l_{m}\right), \lambda_{0}, \lambda_{1}}
\end{gathered}
$$

- si $r_{\sigma, i+1}^{\max }(Z)<t_{\sigma}^{\lambda_{\sigma}+1}(Z) \leq r_{\sigma, i}^{\max }(Z)$, pour $i \in\{\sigma, \ldots, m\}$, en posant $\tilde{l}_{j}=l_{j}$ pour $j \in\{0, \ldots, m\} \backslash\{i\}, \tilde{l}_{i}=l_{i}+1$ et $y_{i}^{l_{i}+1}=\mathcal{Z}_{\sigma}^{\lambda_{\sigma}+1}$ on $a$

$$
\begin{gathered}
\left(a_{1}, \ldots, a_{p}, S_{0}, \ldots, S_{m},\left(\mathcal{Y}_{i}^{j}\right)_{i \in\{0, \ldots, m\}, j \in\left\{1, \ldots, \tilde{l}_{i}\right\}},\left(\mathcal{Z}_{i}^{j}\right)_{i \in\{0,1\}, j \in\left\{1, \ldots, \lambda_{i}\right\}}\right) \\
\in Y_{x}^{\natural, p, k, m,\left(\tilde{l}_{0}, \ldots, \tilde{l}_{m}\right), \lambda_{0}, \lambda_{1}}
\end{gathered}
$$

Remarque. Par le c) et le d) du lemme 4.23, la condition $t_{\sigma}^{\lambda_{\sigma}+1}(Z) \leq r_{\sigma, m+1}^{\max }(Z)$ qui détermine le premier cas est équivalente à $t_{\sigma}^{\lambda_{\sigma}+1}(Z) \leq k+3 P$.

Démonstration. D'après le c) du lemme 4.23, on a $t_{\sigma}^{\lambda_{\sigma}+1}(Z) \leq r_{\sigma, \sigma}^{\max }(Z)$ donc on se trouve toujours exactement dans l'un des cas ci-dessus. Il n'y a rien à montrer dans le premier cas. Supposons maintenant

$$
\left.\left.t_{\sigma}^{\lambda_{\sigma}+1}(Z) \in\right] r_{\sigma, m+1}^{\max }(Z), r_{\sigma, m}^{\max }(Z)\right] .
$$

Alors nécessairement $r_{\sigma, m+1}^{\max }(Z)<r_{\sigma, m}^{\max }(Z)$, ce qui implique

$$
r_{\sigma, m}^{\max }(Z)=d_{\max }\left(x, S_{m}\right) \quad \text { et } \quad r_{\sigma, m+1}^{\max }(Z)=k+3 P .
$$

On a donc $d\left(x, Z_{\sigma}^{\lambda_{\sigma}{ }^{+1}}\right)>k+3 P$. Montrons $\mathcal{Z}_{\sigma}^{\lambda_{\sigma}{ }^{+1}} \subset \bigcup_{y \in S_{m}} 2 P-\operatorname{géod}(x, y)$. Soit $z \in \mathcal{Z}_{\sigma}^{\lambda_{\sigma}+1}$. Il existe $a \in S_{0}$ tel que $z \in 2 F-\operatorname{géod}(x, a)$. Cela est clair si $\sigma=0$. Si 
$\sigma=1$ il existe $b \in S_{1}$ tel que $z \in \operatorname{géod}(x, b)$. Soit $a \in S_{0}$. Alors le a) du lemme 4.14 montre que $b \in 2 F$-géod $(x, a)$, d'où $z \in 2 F-\operatorname{géod}(x, a)$.

Soit $y \in S_{m}$ tel que $d(x, y)=d_{\max }\left(x, S_{m}\right)$. On a $y \in 2 F-\operatorname{géod}(x, a)$ d'après le a) du lemme 4.14. D'autre part $d(x, z) \leq d(x, y)+P / 3$ puisque $t_{\sigma}^{\lambda_{\sigma}+1}(Z) \leq$ $d_{\max }\left(x, S_{m}\right)$ et $Z_{\sigma}^{\lambda_{\sigma}+1}$ est de diamètre inférieur ou égal à $P / 3$. En appliquant le lemme 4.8 à $(x, a, y, z)$ au lieu de $(x, y, a, b)$ et $(2 F, 2 F, P / 3)$ au lieu de $(\alpha, \beta, \rho)$ on trouve

$$
z \in(2 F+2 P / 3+\delta)-\operatorname{géod}(x, y) \subset 2 P-\operatorname{géod}(x, y)
$$

car on suppose $2 F+2 P / 3+\delta \leq 2 P$, ce qui est permis par $\left(H_{P}\right)$. On a donc montré $Z_{\sigma}^{\lambda \sigma+1} \subset \bigcup_{y \in S_{m}} 2 P-\operatorname{géod}(x, y)$ et $y_{m}^{l_{m}+1}=Z_{\sigma}^{\lambda_{\sigma}+1}$ vérifie la condition iv) de la définition 4.1.

Supposons maintenant

$$
\left.\left.t_{\sigma}^{\lambda_{\sigma}+1}(Z) \in\right] r_{\sigma, i+1}^{\max }(Z), r_{\sigma, i}^{\max }(Z)\right] \text { avec } i \in\{\sigma, \ldots, m-1\} .
$$

Alors nécessairement $r_{\sigma, i+1}^{\max }(Z)<r_{\sigma, i}^{\max }(Z)$, ce qui implique

$$
r_{\sigma, i}^{\max }(Z)=d_{\max }\left(x, S_{i}\right) \quad \text { et } \quad r_{\sigma, i+1}^{\max }(Z)=\max \left(k+3 P, d_{\max }\left(x, S_{i+1}\right)\right) .
$$

On a donc $\left.\left.t_{\sigma}^{\lambda_{\sigma}+1}(Z) \in\right] d_{\max }\left(x, S_{i+1}\right), d_{\max }\left(x, S_{i}\right)\right]$ Soit $z \in Z_{\sigma}^{\lambda_{\sigma}+1}$. On a vu qu'il existe $a \in S_{0}$ tel que $z \in 2 F-\operatorname{géod}(x, a)$. Soit $y \in S_{i}$ tel que $d(x, y)=d_{\text {max }}\left(x, S_{i}\right)$ et soit $y^{\prime} \in S_{i+1}$. D'après le a) du lemme 4.14, $y$ et $y^{\prime}$ appartiennent à $2 F-\operatorname{géod}(x, a)$. Il est clair que $d\left(x, y^{\prime}\right) \leq d(x, z) \leq d(x, y)+P / 3$.

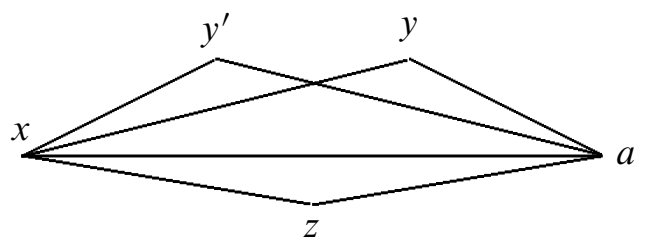

Le lemme 4.8 appliqué à $\left(x, a, z, y^{\prime}\right)$ au lieu de $(x, y, a, b)$ et $(2 F, 2 F, 0)$ au lieu de $(\alpha, \beta, \rho)$ montre

$$
y^{\prime} \in(2 F+\delta)-\operatorname{géod}(x, z) \text {. }
$$

Le lemme 4.8 appliqué à $(x, a, y, z)$ au lieu de $(x, y, a, b)$ et $(2 F, 2 F, P / 3)$ au lieu de $(\alpha, \beta, \rho)$ montre

$$
z \in(2 P / 3+2 F+\delta)-\operatorname{géod}(x, y) .
$$

Par le b) du lemme 3.3 on déduit de (56) et (57) que

$$
z \in(2 P / 3+4 F+2 \delta)-\operatorname{géod}\left(y^{\prime}, y\right) \subset P-\operatorname{géod}\left(y^{\prime}, y\right)
$$

car on suppose $2 P / 3+4 F+2 \delta \leq P$, ce qui est permis par $\left(H_{P}\right)$. On a donc montré $Z_{\sigma}^{\lambda_{\sigma}+1} \subset \bigcup_{y \in S_{i}, y^{\prime} \in S_{i+1}} P-\operatorname{géod}\left(y, y^{\prime}\right)$ et $y_{i}^{l_{i}+1}=Z_{\sigma}^{\lambda_{\sigma}+1}$ vérifie la condition iii) de la définition 4.1. 
Soit $\sigma \in\{0,1\}$ (avec $\sigma=0$ si $m=0$ ). On pose

$-\left(\tilde{\lambda}_{0}, \tilde{\lambda}_{1}\right)=\left(\lambda_{0}+1, \lambda_{1}\right)$ si $\sigma=0$

et

- et $\left(\tilde{\lambda}_{0}, \tilde{\lambda}_{1}\right)=\left(\lambda_{0}, \lambda_{1}+1\right)$ si $\sigma=1$.

On note

$$
\begin{gathered}
\kappa_{\sigma}: Y_{x}^{\natural, p, k, m,\left(l_{0}, \ldots, l_{m}\right), \tilde{\lambda}_{0}, \tilde{\lambda}_{1}} \rightarrow Y_{x}^{\natural, p, k, m,\left(l_{0}, \ldots, l_{m}\right), \lambda_{0}, \lambda_{1}} \\
\cup \bigcup_{i \in\{\sigma, \ldots, m\}} Y_{x}^{\natural, p, k, m,\left(l_{0}, \ldots, l_{i-1}, l_{i}+1, l_{i+1}, \ldots, l_{m}\right), \lambda_{0}, \lambda_{1}}
\end{gathered}
$$

l'application définie par le sous-lemme 4.25. On vérifie facilement que $\kappa_{\sigma}$ passe au quotient et définit

$$
\begin{aligned}
& \bar{\kappa}_{\sigma, \infty}:\left\{Z \in \bar{Y}_{x}^{\natural, p, k, m,\left(l_{0}, \ldots, l_{m}\right), \tilde{\lambda}_{0}, \tilde{\lambda}_{1}} \mid 0 \leq t_{\sigma}^{\lambda_{\sigma}+1}(Z) \leq k+3 P\right\} \\
& \rightarrow \bar{Y}_{x}^{\natural, p, k, m,\left(l_{0}, \ldots, l_{m}\right), \lambda_{0}, \lambda_{1}}
\end{aligned}
$$

et

$$
\begin{gathered}
\bar{\kappa}_{\sigma, i}:\left\{Z \in \bar{Y}_{x}^{\natural, p, k, m,\left(l_{0}, \ldots, l_{m}\right), \tilde{\lambda}_{0}, \tilde{\lambda}_{1}} \mid r_{\sigma, i+1}^{\max }(Z)<t_{\sigma}^{\lambda_{\sigma}+1}(Z) \leq r_{\sigma, i}^{\max }(Z)\right\} \\
\rightarrow \bar{Y}_{x}^{\natural, p, k, m,\left(l_{0}, \ldots, l_{i-1}, l_{i}+1, l_{i+1}, \ldots, l_{m}\right), \lambda_{0}, \lambda_{1}}
\end{gathered}
$$

pour $i \in\{\sigma, \ldots, m\}$.

Sous-lemme 4.26. Il existe $C=C(\delta, K, N, Q, P)$ tel que

a) pour tout $i \in\{\sigma, \ldots, m\}, \bar{\kappa}_{\sigma, i}$ est injective, et pour $Z \in \bar{Y}_{x}^{\natural, p, k, m,\left(l_{0}, \ldots, l_{m}\right), \tilde{\lambda}_{0}, \tilde{\lambda}_{1}}$ tel que $r_{\sigma, i+1}^{\max }(Z)<t_{\sigma}^{\lambda_{\sigma}+1}(Z) \leq r_{\sigma, i}^{\max }(Z), \kappa_{\sigma}$ induit une bijection de

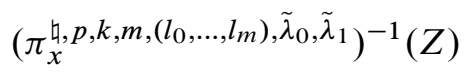

dans

$$
\left(\pi_{x}^{\natural, p, k, m,\left(l_{0}, \ldots, l_{i-1}, l_{i}+1, l_{i+1}, \ldots, l_{m}\right), \lambda_{0}, \lambda_{1}}\right)^{-1}\left(\bar{\kappa}_{\sigma, i}(Z)\right),
$$

b) pour tout élément $Z_{\infty} \in \bar{Y}_{x}^{\natural, p, k, m,\left(l_{0}, \ldots, l_{m}\right), \lambda_{0}, \lambda_{1}}, \bar{\kappa}_{\sigma, \infty}^{-1}\left(Z_{\infty}\right)$ est de cardinal inférieur ou égal à $C\left(r_{\sigma, m+1}^{\max }\left(Z_{\infty}\right)+1\right)$ et pour tout entier $t \leq k+3 P$ le nombre de $Z \in \bar{Y}_{x}^{\natural, p, k, m,\left(l_{0}, \ldots, l_{m}\right), \tilde{\lambda}_{0}, \tilde{\lambda}_{1}}$ tels que $t_{\sigma}^{\lambda_{\sigma}+1}(Z)=t$ et $\bar{\kappa}_{\sigma, \infty}(Z)=Z_{\infty}$ est inférieur ou égal à $C$. De plus pour tout $Z \in \bar{Y}_{x}^{\natural, p, k, m,\left(l_{0}, \ldots, l_{m}\right), \tilde{\lambda}_{0}, \tilde{\lambda}_{1}}$ tel que $t_{\sigma}^{\lambda_{\sigma}+1}(Z) \leq k+3 P, \kappa_{\sigma}$ induit une bijection de $\left(\pi_{x}^{\natural, p, k, m,\left(l_{0}, \ldots, l_{m}\right), \tilde{\lambda}_{0}, \tilde{\lambda}_{1}}\right)^{-1}(Z)$ dans $\left(\pi_{x}^{\natural, p, k, m,\left(\bar{l}_{0}, \ldots, l_{m}\right), \lambda_{0}, \lambda_{1}}\right)^{-1}\left(\bar{\kappa}_{\sigma, \infty}(Z)\right)$.

Démonstration. La preuve de a) est immédiate. 
Montrons b). Soit $Z \in \bar{Y}_{x}^{\natural, p, k, m,\left(l_{0}, \ldots, l_{m}\right), \tilde{\lambda}_{0}, \tilde{\lambda}_{1}}$ tel que $t_{\sigma}^{\lambda_{\sigma}+1}(Z) \leq k+3 P$. Pour tout

$$
\left(a_{1}, \ldots, a_{p}, S_{0}, \ldots, S_{m},\left(\mathcal{Y}_{i}^{j}\right)_{i \in\{0, \ldots, m\}, j \in\left\{1, \ldots, l_{i}\right\}},\left(\mathcal{Z}_{i}^{j}\right)_{i \in\{0,1\}, j \in\left\{1, \ldots, \tilde{\lambda}_{i}\right\}}\right)
$$

dans $\left(\pi_{x}^{\natural, p, k, m,\left(l_{0}, \ldots, l_{m}\right), \tilde{\lambda}_{0}, \tilde{\lambda}_{1}}\right)^{-1}(Z)$ on a $Z_{\sigma}^{\lambda_{\sigma}+1} \subset B(x, k+3 P+P / 3)$ donc $Z_{\sigma}^{\lambda_{\sigma}+1} \subset B(x, k+M)$ car on suppose $3 P+P / 3 \leq M$, ce qui est permis $\operatorname{par}\left(H_{M}\right)$, et donc $B\left(Z_{\sigma}^{\lambda_{\sigma}+1}, M\right) \subset B(x, k+2 M)$. Par conséquent, si on note $Z_{\infty}=\bar{\kappa}_{\sigma, \infty}(Z)$, la donnée de $Z$ est équivalente à celle de $Z_{\infty}$ et de $Z_{\sigma}^{\lambda_{\sigma}+1} \subset B(x, k+2 M)$. La condition $Z_{\sigma}^{\lambda_{\sigma}+1} \subset \bigcup_{a \in S_{\sigma}}$ géod $(x, a)$ s'exprime uniquement en termes des distances entre les points de $S_{\sigma}$ et ceux de $B(x, k+2 M)$ (qui font partie de la donnée de $Z_{\infty}$ ) et le nombre de possibilités pour $Z_{\sigma}^{\lambda_{\sigma}+1} \subset B(x, k+2 M)$ de diamètre $\leq P / 3$ vérifiant cette condition et $d\left(x, Z_{\sigma}^{\lambda_{\sigma}+1}\right) \leq k+3 P$ est borné par $C\left(r_{\sigma, m+1}^{\max }\left(Z_{\infty}\right)+1\right)$ avec $C=C(\delta, N, K, Q, P)$ à cause du lemme 3.13. Pour tout $t$, l'ensemble des $Z_{\sigma}^{\lambda_{\sigma}+1}$ vérifiant les conditions précédentes et $d\left(x, Z_{\sigma}^{\lambda_{\sigma}+1}\right)=t$ ne dépend que de $Z_{\infty}$ et de $t$ et le lemme 3.13 montre que le cardinal de cet ensemble est inférieur ou égal à $C=C(\delta, N, K, Q, P)$.

Suite de la démonstration du lemme 4.24. Grâce au sous-lemme 4.26, il existe $C=$ $C(\delta, K, N, Q, P, M)$ tel que pour $k, m, l_{0}, \ldots, l_{m}, \lambda_{0}, \lambda_{1} \in \mathbb{N}, \sigma \in\{0,1\}$ (vérifiant $\lambda_{1}=0$ et $\sigma=0$ si $m=0$ ), et en posant

$$
-\left(\tilde{\lambda}_{0}, \tilde{\lambda}_{1}\right)=\left(\lambda_{0}+1, \lambda_{1}\right) \text { si } \sigma=0
$$

et

$$
-\left(\tilde{\lambda}_{0}, \tilde{\lambda}_{1}\right)=\left(\lambda_{0}, \lambda_{1}+1\right) \operatorname{si} \sigma=1
$$

on ait l'inégalité suivante :

$$
\begin{gathered}
B^{-\left(m+\sum_{i=0}^{m} l_{i}\right)} \sum_{Z \in \bar{Y}_{x}^{\natural, p, k, m,\left(l_{0}, \ldots, l_{m}\right), \tilde{\lambda}_{0}, \tilde{\lambda}_{1}}} e^{2 s\left(r_{0}(Z)-k\right)}\left(\prod_{i=0}^{m} s_{i}(Z)^{-l_{i}}\right)\left(r_{0}(Z)+1\right)^{-\tilde{\lambda}_{0}} \\
\left(r_{1}(Z)+1\right)^{-\tilde{\lambda}_{1}} \sharp\left(\left(\pi_{x}^{\left.\left.\natural, p, k, m,\left(l_{0}, \ldots, l_{m}\right), \tilde{\lambda}_{0}, \tilde{\lambda}_{1}\right)^{-1}(Z)\right)^{-\alpha}\left|\xi_{Z}(f)\right|^{2}}\right.\right. \\
\leq C\left(B^{-\left(m+\sum_{i=0}^{m} l_{i}\right)} \sum_{Z \in \bar{Y}_{x}^{\natural, p, k, m,\left(l_{0}, \ldots, l_{m}\right), \lambda_{0}, \lambda_{1}}}\left(\frac{r_{\sigma, m+1}^{\max }(Z)+1}{r_{\sigma, \sigma}^{\max }(Z)+1}\right) e^{2 s\left(r_{0}(Z)-k\right)}\right. \\
\left(\prod_{i=0}^{m} s_{i}(Z)^{-l_{i}}\right)\left(r_{0}(Z)+1\right)^{-\lambda_{0}}\left(r_{1}(Z)+1\right)^{-\lambda_{1}} \\
\left.\sharp\left(\left(\pi_{x}^{\natural, p, k, m,\left(l_{0}, \ldots, l_{m}\right), \lambda_{0}, \lambda_{1}}\right)^{-1}(Z)\right)^{-\alpha}\left|\xi_{Z}(f)\right|^{2}\right)
\end{gathered}
$$




$$
\begin{aligned}
+ & C B \sum_{i=\sigma}^{m}\left(B^{-\left(m+\sum_{j=0}^{m} l_{j}+1\right)} \sum_{\left.Z \in \bar{Y}_{x}^{\natural, p, k, m,\left(l_{0}, \ldots, l_{i}-1\right.}, l_{i}+1, l_{i+1}, \ldots, l_{m}\right), \lambda_{0}, \lambda_{1}}\right. \\
& \left(\frac{r_{\sigma, i}^{\max }(Z)-r_{\sigma, i+1}^{\max }(Z)}{r_{\sigma, \sigma}^{\max }(Z)+1}\right) e^{2 s\left(r_{0}(Z)-k\right)}\left(s_{i}(Z)^{-\left(l_{i}+1\right)} \prod_{j \in\{0, \ldots, m\} \backslash\{i\}} s_{j}(Z)^{-l_{j}}\right) \\
& \left(r_{0}(Z)+1\right)^{-\lambda_{0}}\left(r_{1}(Z)+1\right)^{-\lambda_{1}} \\
& \left.\sharp\left(\left(\pi_{x}^{\natural, p, k, m,\left(l_{0}, \ldots, l_{i-1}, l_{i}+1, l_{i+1}, \ldots, l_{m}\right), \lambda_{0}, \lambda_{1}}\right)^{-1}(Z)\right)^{-\alpha}\left|\xi_{Z}(f)\right|^{2}\right) .
\end{aligned}
$$

En effet le b) du lemme 4.23 assure que $r_{\sigma, \sigma}^{\max }(Z)+1 \leq(N+1)\left(r_{\sigma}(Z)+1\right)$ d'où $\left(r_{\sigma}(Z)+1\right)^{-1} \leq(N+1)\left(r_{\sigma, \sigma}^{\max }(Z)+1\right)^{-1}$. De plus le $i^{\text {ème }}$ terme dans la somme qui constitue la deuxième moitié du membre de droite n'apparaît pas si $r_{\sigma, i}^{\max }(Z)=$ $r_{\sigma, i+1}^{\max }(Z)$ et si $r_{\sigma, i}^{\max }(Z)>r_{\sigma, i+1}^{\max }(Z)$ on a

$$
s_{i}(Z) \leq C\left(r_{\sigma, i}^{\max }(Z)-r_{\sigma, i+1}^{\max }(Z)\right)
$$

avec $C=C(\delta, K, N, Q, P)$.

Sous-lemme 4.27. Soient $C \in \mathbb{R}_{+}^{*}, m \in \mathbb{N}, \varepsilon_{0}, \ldots, \varepsilon_{m}, \eta_{1}, \ldots, \eta_{m} \in[0,1]$ vérifiant $\sum_{i=0}^{m} \varepsilon_{i} \leq 1$ et $\sum_{i=1}^{m} \eta_{i} \leq 1$. Soient $\mu_{0}, \mu_{1} \in \mathbb{N}$ et $\left(A_{l_{0}, \ldots, l_{m}, \lambda_{0}, \lambda_{1}}\right)$ une famille d'éléments de $\mathbb{R}_{+}$indexée par les $\left(l_{0}, \ldots, l_{m}, \lambda_{0}, \lambda_{1}\right) \in \mathbb{N}^{m+3}$ vérifiant $\lambda_{0} \leq \mu_{0}$ et $\lambda_{1} \leq \mu_{1}$. On suppose

- pour $\lambda_{0} \in\left\{0, \ldots, \mu_{0}-1\right\}$ et $\lambda_{1} \in\left\{0, \ldots, \mu_{1}\right\}$,

$$
A_{l_{0}, \ldots, l_{m}, \lambda_{0}+1, \lambda_{1}} \leq C\left(A_{l_{0}, \ldots, l_{m}, \lambda_{0}, \lambda_{1}}+\sum_{i=0}^{m} \varepsilon_{i} A_{l_{0}, \ldots, l_{i-1}, l_{i}+1, l_{i+1}, \ldots, l_{m}, \lambda_{0}, \lambda_{1}}\right),
$$

- et pour $\lambda_{0} \in\left\{0, \ldots, \mu_{0}\right\}$ et $\lambda_{1} \in\left\{0, \ldots, \mu_{1}-1\right\}$,

$$
A_{l_{0}, \ldots, l_{m}, \lambda_{0}, \lambda_{1}+1} \leq C\left(A_{l_{0}, \ldots, l_{m}, \lambda_{0}, \lambda_{1}}+\sum_{i=1}^{m} \eta_{i} A_{l_{0}, \ldots, l_{i-1}, l_{i}+1, l_{i+1}, \ldots, l_{m}, \lambda_{0}, \lambda_{1}}\right) .
$$

Alors

$$
\sum_{\substack{l_{0}, \ldots, l_{m} \in \mathbb{N} \\ \lambda_{0} \in\left\{0, \ldots, \mu_{0}\right\}, \lambda_{1} \in\left\{0, \ldots, \mu_{1}\right\}}} A_{l_{0}, \ldots, l_{m}, \lambda_{0}, \lambda_{1}} \leq(2 C+1)^{\mu_{0}+\mu_{1}} \sum_{l_{0}, \ldots, l_{m} \in \mathbb{N}} A_{l_{0}, \ldots, l_{m}, 0,0}
$$

où l'on sous-entend que si le membre de droite converge, le membre de gauche converge aussi.

Démonstration. Posons pour $\lambda_{0} \in\left\{0, \ldots, \mu_{0}\right\}$ et $\lambda_{1} \in\left\{0, \ldots, \mu_{1}\right\}$,

$$
\mathcal{A}_{\lambda_{0}, \lambda_{1}}=\sum_{l_{0}, \ldots, l_{m}} A_{l_{0}, \ldots, l_{m}, \lambda_{0}, \lambda_{1}} .
$$

Alors pour $\lambda_{0} \in\left\{0, \ldots, \mu_{0}-1\right\}$ et $\lambda_{1} \in\left\{0, \ldots, \mu_{1}\right\}$, on a

$$
\mathcal{A}_{\lambda_{0}+1, \lambda_{1}} \leq 2 C \mathcal{A}_{\lambda_{0}, \lambda_{1}}
$$


et de même pour $\lambda_{0} \in\left\{0, \ldots, \mu_{0}\right\}$ et $\lambda_{1} \in\left\{0, \ldots, \mu_{1}-1\right\}$,

$$
\mathcal{A}_{\lambda_{0}, \lambda_{1}+1} \leq 2 C \mathcal{A}_{\lambda_{0}, \lambda_{1}} .
$$

Le sous-lemme en résulte facilement car $1+(2 C)+\cdots+(2 C)^{\mu_{c}} \leq(2 C+1)^{\mu_{c}}$ pour $c=0,1$.

Fin de la démonstration du lemme 4.24. On applique le sous-lemme 4.27 de la façon suivante. On fixe $m, k$ et des entiers $R_{0,0} \geq \cdots \geq R_{0, m+1} \geq 0$ et $R_{1,1} \geq \cdots \geq$ $R_{1, m+1} \geq 0$. On applique le sous-lemme 4.27 en prenant

$$
\begin{gathered}
\varepsilon_{j}=\frac{R_{0, j}-R_{0, j+1}}{R_{0,0}+1} \quad \text { pour } j=0, \ldots, m, \\
\eta_{j}=\frac{R_{1, j}-R_{1, j+1}}{R_{1,1}+1} \quad \text { pour } j=1, \ldots, m
\end{gathered}
$$

et, pour $l_{0}, \ldots, l_{m} \in \mathbb{N}, \lambda_{0} \in\left\{0, \ldots, \mu_{0}\right\}$ et $\lambda_{1} \in\left\{0, \ldots, \mu_{1}\right\}$,

$$
A_{l_{0}, \ldots, l_{m}, \lambda_{0}, \lambda_{1}}=0 \text { si } m=0 \text { et } \lambda_{1}>0,
$$

et sinon

$$
\begin{aligned}
A_{l_{0}, \ldots, l_{m}, \lambda_{0}, \lambda_{1}=} & B^{-\left(m+\sum_{i=0}^{m} l_{i}\right)} \sum_{\substack{Z \in \bar{Y}_{x}^{\natural, p, k, m,\left(l_{0}, \ldots, l_{m}\right), \lambda_{0}, \lambda_{1}} \text { tel que } \\
r_{i, j}^{\max }(Z)=R_{i, j} \text { pour } i \in\{0,1\} \text { et } j \in\{i, \ldots, m+1\}}} \\
& e^{2 s\left(r_{0}(Z)-k\right)}\left(\prod_{i=0}^{m} s_{i}(Z)^{-l_{i}}\right)\left(r_{0}(Z)+1\right)^{-\lambda_{0}}\left(r_{1}(Z)+1\right)^{-\lambda_{1}} \\
& \sharp\left(\left(\pi_{x}^{\natural, p, k, m,\left(l_{0}, \ldots, l_{m}\right), \lambda_{0}, \lambda_{1}}\right)^{-1}(Z)\right)^{-\alpha}\left|\xi_{Z}(f)\right|^{2} .
\end{aligned}
$$

Grâce à (58) et comme $\frac{r_{\sigma, m+1}^{\max }(Z)+1}{r_{\sigma, \sigma}^{\max }(Z)+1} \leq 1$, les hypothèses du sous-lemme 4.27 sont satisfaites pour une constante $C=C(\delta, K, N, Q, P, M, s, B)$. Puis on somme l'inégalité (59) sur

$$
m, k,\left(R_{0,0}, \ldots, R_{0, m+1}\right),\left(R_{1,1}, \ldots, R_{1, m+1}\right) .
$$

Ceci termine la démonstration du lemme 4.24.

Le lemme suivant est une variante du lemme 4.19.

Lemme 4.28. Il existe une constante $C=C(\delta, K, N, Q, P, M)$ telle que pour $p \in\left\{1, \ldots, p_{\max }\right\}, k, m, l_{0}, \ldots, l_{m}, \lambda_{0}, \lambda_{1} \in \mathbb{N}$ (vérifiant $\lambda_{1}=0$ si $m=0$ ) et

$$
\begin{gathered}
\left(a_{1}, \ldots, a_{p}, S_{0}, \ldots, S_{m},\left(\mathcal{Y}_{i}^{j}\right)_{i \in\{0, \ldots, m\}, j \in\left\{1, \ldots, l_{i}\right\}},\left(\mathcal{Z}_{i}^{j}\right)_{i \in\{0,1\}, j \in\left\{1, \ldots, \lambda_{i}\right\}}\right) \\
\in Y_{x}^{\natural, p, k, m,\left(l_{0}, \ldots, l_{m}\right), \lambda_{0}, \lambda_{1}},
\end{gathered}
$$


les distances entre les points de

$$
B\left(S_{0}, M\right) \cup \bigcup_{j \in\left\{1, \ldots, l_{0}\right\}} B\left(y_{0}^{j}, M\right) \cup \bigcup_{\substack{i \in\{0,1\} \\ j \in\left\{1, \ldots, \lambda_{i}\right\}}} B\left(\mathcal{Z}_{i}^{j}, M\right)
$$

et ceux de

$$
\bigcup_{i \in\{1, \ldots, m\}} B\left(S_{i}, M\right) \cup \underset{\substack{i \in\{1, \ldots, m\} \\ j \in\left\{1, \ldots, l_{i}\right\}}}{\bigcup} B\left(y_{i}^{j}, M\right) \cup B(x, k+2 M)
$$

sont déterminées par

a) les distances entre les points de (61),

b) les entiers $d\left(x, S_{0}\right), d\left(x, y_{0}^{j}\right), d\left(x, Z_{0}^{j}\right)$ et $d\left(x, Z_{1}^{j}\right)$,

c) les distances entre les points de (60) et $C\left(1+l_{0}+\lambda_{0}+\lambda_{1}\right)$ points de (61) (qui sont eux-mêmes déterminés par a) et b))

et de plus les distances entre les points de (60) et ceux de (61) sont déterminées à $C$ près par a) et $\mathrm{b}$ ).

Remarque. Dans toutes les situations où on appliquera ce lemme, $l_{0}+\lambda_{0}+\lambda_{1}$ sera majoré par une constante de la forme $C(\delta, K, N, Q, P, M)$.

Démonstration. Soient $p \in\left\{1, \ldots, p_{\max }\right\}, k, m, l_{0}, \ldots, l_{m}, \lambda_{0}, \lambda_{1} \in \mathbb{N}$ (vérifiant $\lambda_{1}=0$ si $m=0$ ) et

$$
\begin{gathered}
\left(a_{1}, \ldots, a_{p}, S_{0}, \ldots, S_{m},\left(\mathcal{Y}_{i}^{j}\right)_{i \in\{0, \ldots, m\}, j \in\left\{1, \ldots, l_{i}\right\}},\left(\mathcal{Z}_{i}^{j}\right)_{i \in\{0,1\}, j \in\left\{1, \ldots, \lambda_{i}\right\}}\right) \\
\in Y_{x}^{\natural, p, k, m,\left(l_{0}, \ldots, l_{m}\right), \lambda_{0}, \lambda_{1}} .
\end{gathered}
$$

Soit $b \in S_{0}$ et $u$ un point de $B(x, k)$ à distance minimale de $b$. On commence par montrer que pour $\sigma \in\{0,1\}$ et $j \in\left\{1, \ldots, \lambda_{\sigma}\right\}$, on a

$$
Z_{\sigma}^{j} \subset 2 F-\operatorname{géod}(x, b)
$$

et

$$
B\left(\mathcal{Z}_{\sigma}^{j}, M\right) \subset B(x, k+2 M) \quad \text { ou } \quad Z_{\sigma}^{j} \subset(2 F+\delta)-\operatorname{géod}(u, b) .
$$

Soit $j \in\left\{1, \ldots, \lambda_{0}\right\}$. On a

$$
Z_{0}^{j} \subset \bigcup_{a \in S_{0}} \operatorname{géod}(x, a) \subset 2 N-\operatorname{géod}(x, b) \subset 2 F-\operatorname{géod}(x, b)
$$

$\operatorname{car} F \geq N$. Si $d\left(x, Z_{0}^{j}\right) \leq k+3 P$,

$$
B\left(Z_{0}^{j}, M\right) \subset B(x, k+2 M)
$$

car on suppose $3 P+P / 3 \leq M$, ce qui est permis $\operatorname{par}\left(H_{M}\right)$. Si $d\left(x, Z_{0}^{j}\right)>k+3 P$, pour $z \in Z_{0}^{j}$ on a

$$
z \in 2 N-\operatorname{géod}(x, b) \quad \text { et } \quad d(x, z)>d(x, u)+3 P .
$$


Soit $j \in\left\{1, \ldots, \lambda_{1}\right\}$. On a $\mathbb{Z}_{1}^{j} \subset \bigcup_{a \in S_{1}} \operatorname{géod}(x, a)$. Par le lemme 4.14, $S_{1} \subset$ $2 F-\operatorname{géod}(u, b)$, donc $Z_{1}^{j} \subset 2 F-\operatorname{géod}(x, b)$. On a déjà prouvé $(62)$. Si $d\left(x, Z_{1}^{j}\right) \leq$ $k+3 P$,

$$
B\left(\mathcal{Z}_{1}^{j}, M\right) \subset B(x, k+2 M)
$$

car $3 P+P / 3 \leq M$. Si $d\left(x, \mathbb{Z}_{1}^{j}\right)>k+3 P$, soit $z \in Z_{1}^{j}$. On a donc

$$
z \in 2 F-\operatorname{géod}(x, b) \text { et } d(x, z)>d(x, u)+3 P .
$$

Soit maintenant $z$ vérifiant (64) ou (65). Alors $z$ vérifie (65) car $F \geq N$ par (21).

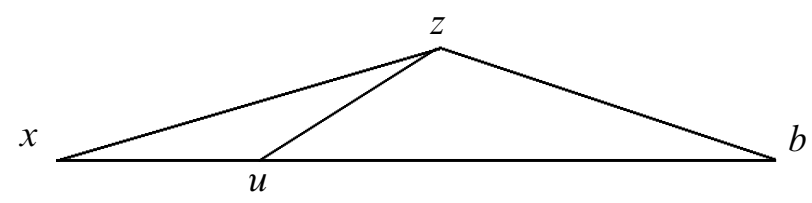

$\operatorname{Par}\left(H_{\delta}^{2 F}(u, x, z, b)\right)$ on a

$$
d(u, z) \leq \max (d(u, x)-d(x, z), d(u, b)-d(b, z))+2 F+\delta .
$$

Or $d(u, x)-d(x, z)+2 F+\delta \leq-3 P+2 F+\delta$ et on suppose $-3 P+2 F+\delta<0$, ce qui est permis par $\left(H_{P}\right)$. Donc $d(u, z) \leq d(u, b)-d(b, z)+2 F+\delta$, c'est-à-dire $z \in(2 F+\delta)$ - géod $(u, b)$. Ceci termine la preuve de (63). On suppose $2 F+\delta \leq 4 P$, ce qui est permis par $\left(H_{P}\right)$.

Pour montrer le lemme 4.28 on répète alors les arguments de la preuve du lemme 4.19.

Plus précisément on applique le lemme 4.17 avec

- $(x, b)$ au lieu de $(c, d)$,

- $\left\{w_{i}, i \in I\right\}$ égal à

$$
\bigcup_{i \in\{0, \ldots, m\}} S_{i} \cup \underset{\substack{i \in\{0, \ldots, m\} \\ j \in\left\{1, \ldots, l_{i}\right\}}}{\bigcup} y_{i}^{j} \cup \bigcup_{\substack{i \in\{0,1\} \\ j \in\left\{1, \ldots, \lambda_{i}\right\}}} Z_{i}^{j},
$$

- $J$ la partie de $I$ telle que

$$
\left\{w_{j}, j \in J\right\}=S_{0} \cup \underset{j \in\left\{1, \ldots, l_{0}\right\}}{\bigcup} y_{0}^{j} \cup \underset{\substack{i \in\{0,1\} \\ j \in\left\{1, \ldots, \lambda_{i}\right\}}}{\mathcal{Z}_{i}^{j},}
$$

- et $\left(\alpha_{i}, \rho_{i}\right)$ égal à $(4 P, M)$ pour tout $i$ (les hypothèses sont satisfaites grâce au lemme 4.14 et à (62) et car on a supposé $2 F \leq 4 P$ ).

Puis on applique le lemme 4.18 à $z$ parcourant

$$
B\left(S_{0}, M\right) \cup \bigcup_{j \in\left\{1, \ldots, l_{0}\right\}} B\left(\mathcal{Y}_{0}^{j}, M\right) \cup \bigcup_{\substack{i \in\{0,1\}, j \in\left\{1, \ldots, \lambda_{i}\right\} \\ B\left(\mathcal{Z}_{i}^{j}, M\right) \not \subset B(x, k+2 M)}} B\left(\mathcal{Z}_{i}^{j}, M\right)
$$


avec $\alpha=4 P+2 M$ et $l=k+2 M$ (grâce au lemme 4.14 et à (63) et comme on a supposé $2 F+\delta \leq 4 P$, cet ensemble est inclus dans $(4 P+2 M)$ - $\operatorname{géod}(b, u))$.

Le lemme suivant nous sera utile ensuite.

Lemme 4.29. Il existe une constante $C=C(\delta, K, N, Q, P)$ telle que pour $\mathrm{m}$, $l_{0}, \ldots, l_{m}, \lambda_{0}, \lambda_{1} \in \mathbb{N}\left(\right.$ avec $\lambda_{1}=0$ si $\left.m=0\right)$ et pour $Z \in \bar{Y}_{x}^{p, k, m,\left(l_{0}, \ldots, l_{m}\right)}$ et $\widetilde{Z} \in \bar{Y}_{x}^{\natural, p, k, m,\left(l_{0}, \ldots, l_{m}\right), \lambda_{0}, \lambda_{1}}$ tels que pour tout

$$
\begin{gathered}
\left(a_{1}, \ldots, a_{p}, S_{0}, \ldots, S_{m},\left(y_{i}^{j}\right)_{i \in\{0, \ldots, m\}, j \in\left\{1, \ldots, l_{i}\right\}},\left(\mathcal{Z}_{i}^{j}\right)_{i \in\{0,1\}, j \in\left\{1, \ldots, \lambda_{i}\right\}}\right) \\
\in\left(\pi_{x}^{\natural, p, k, m,\left(l_{0}, \ldots, l_{m}\right), \lambda_{0}, \lambda_{1}}\right)^{-1}(\tilde{Z})
\end{gathered}
$$

on ait

$$
\left(a_{1}, \ldots, a_{p}, S_{0}, \ldots, S_{m},\left(y_{i}^{j}\right)_{i \in\{0, \ldots, m\}, j \in\left\{1, \ldots, l_{i}\right\}}\right) \in\left(\pi_{x}^{p, k, m,\left(l_{0}, \ldots, l_{m}\right)}\right)^{-1}(Z)
$$

alors $\sharp\left(\left(\pi_{x}^{\natural, p, k, m,\left(l_{0}, \ldots, l_{m}\right), \lambda_{0}, \lambda_{1}}\right)^{-1}(\tilde{Z})\right) \leq C^{\lambda_{0}+\lambda_{1}} \sharp\left(\left(\pi_{x}^{p, k, m,\left(l_{0}, \ldots, l_{m}\right)}\right)^{-1}(Z)\right)$.

Démonstration. Soit

$$
\left(a_{1}, \ldots, a_{p}, S_{0}, \ldots, S_{m},\left(y_{i}^{j}\right)_{i \in\{0, \ldots, m\}, j \in\left\{1, \ldots, l_{i}\right\}}\right) \in\left(\pi_{x}^{p, k, m,\left(l_{0}, \ldots, l_{m}\right)}\right)^{-1}(Z)
$$

et soient $b_{0} \in S_{0}, b_{1} \in S_{1}$. Si $\left(\mathcal{Z}_{i}^{j}\right)_{i \in\{0,1\}, j \in\left\{1, \ldots, \lambda_{i}\right\}}$ sont tels que

$$
\begin{gathered}
\left(a_{1}, \ldots, a_{p}, S_{0}, \ldots, S_{m},\left(Y_{i}^{j}\right)_{i \in\{0, \ldots, m\}, j \in\left\{1, \ldots, l_{i}\right\}},\left(\mathcal{Z}_{i}^{j}\right)_{i \in\{0,1\}, j \in\left\{1, \ldots, \lambda_{i}\right\}}\right) \\
\in\left(\pi_{x}^{\natural, p, k, m,\left(l_{0}, \ldots, l_{m}\right), \lambda_{0}, \lambda_{1}}\right)^{-1}(\tilde{Z})
\end{gathered}
$$

on a $Z_{i}^{j} \subset 2 N-\operatorname{géod}\left(x, b_{i}\right)$ pour $i \in\{0,1\}, j \in\left\{1, \ldots, \lambda_{i}\right\}$. De plus la donnée de $\widetilde{Z}$ détermine $t_{i}^{j}(\tilde{Z})=d\left(x, Z_{i}^{j}\right)$ et le diamètre de $Z_{i}^{j}$ doit être inférieur ou égal à $P / 3$. On applique alors le lemme 3.13.

Voici quelques rappels et notations pour la proposition suivante. On a

$$
J_{x}=\widetilde{H}_{x}+u_{x} K_{x}=\widetilde{H}_{x}+\sum_{r=1}^{+\infty} u_{x, r} K_{x}
$$

en notant $u_{x, r}=\int_{0}^{1} u_{x, r, t} d t$ (de sorte que $u_{x}=\sum_{r=1}^{+\infty} u_{x, r}$ ). Pour $q \in\{1, \ldots, Q\}$ on note $\widetilde{H}_{x, q}=h_{x}\left(1-\partial h_{x}-h_{x} \partial\right)^{q-1}$, de sorte que

$$
\tilde{H}_{x, q}=\int_{\left(t_{1}, \ldots, t_{q}\right) \in[0,1]^{q}} \tilde{H}_{x, q,\left(t_{1}, \ldots, t_{q}\right)} d t_{1} \ldots d t_{q} \quad \text { et } \quad \tilde{H}_{x}=\sum_{q=1}^{Q} \widetilde{H}_{x, q} .
$$

On rappelle aussi que $K_{x}=\int_{\left(t_{1}, \ldots, t_{Q}\right) \in[0,1] Q} K_{x, Q,\left(t_{1}, \ldots, t_{Q}\right)} d t_{1} \ldots d t_{Q}$. 
Proposition 4.30. Pour tout $p \in\left\{1, \ldots, p_{\max }\right\}, J_{x}$ se prolonge en un opérateur continu de $\mathscr{H}_{x, s}\left(\Delta_{p-1}\right)$ dans $\mathscr{H}_{x, s}\left(\Delta_{p}\right)$. Plus précisément, pour $p \in\left\{2, \ldots, p_{\max }\right\}$ il existe $C=C(\delta, K, N, Q, P, M, s, B)$ tel que pour tout $q \in\{1, \ldots, Q\}$,

$$
\left\|\tilde{H}_{x, q}\right\|_{\mathscr{L}\left(\mathscr{H}_{x, s}\left(\Delta_{p-1}\right), \mathscr{H}_{x, s}\left(\Delta_{p}\right)\right)} \leq C
$$

et pour tout $r \in \mathbb{N}$,

$$
\left\|u_{x, r} K_{x}\right\|_{\mathscr{L}\left(\mathscr{H}_{x, s}\left(\Delta_{p-1}\right), \mathscr{H}_{x, s}\left(\Delta_{p}\right)\right)} \leq C e^{-\frac{s}{2} r} .
$$

On remarque que $\widetilde{H}_{x, 1}=h_{x}$ et grâce à la proposition 4.21 la continuité de $h_{x}$ implique celle de $\widetilde{H}_{x, q}$ pour tout $q \in\{1, \ldots, Q\}$. Cependant nous préférons montrer directement la continuité de $\widetilde{H}_{x, q}$ pour tout $q \in\{1, \ldots, Q\}$ car cela prépare à la démonstration de la continuité de $u_{x, r} K_{x}$.

Démonstration. Le cas où $p=1$ est trivial.

Soit $p \in\left\{2, \ldots, p_{\max }\right\}$ et $q \in\{1, \ldots, Q\}$. Comme dans la démonstration de la proposition 4.21, on note $\mathcal{P}$ le projecteur orthogonal sur le sous-espace vectoriel de $\mathscr{H}_{x, S}\left(\Delta_{p}\right)$ engendré par les $e_{S}$ pour $S \in \Delta_{p}$ tel que $d(x, S) \leq P$, de sorte que $(\mathcal{P} f)(S)=f(S)$ si $d(x, S) \leq P$ et $(\mathcal{P} f)(S)=0$ sinon. La proposition 4.30 résulte donc des lemmes $4.31,4.32,4.36$ et 4.40 que nous allons montrer successivement.

Lemme 4.31. Il existe $C=C(\delta, K, N, Q, P, M, s, B)$ tel que

$$
\left\|\mathcal{P} \tilde{H}_{x, q}\right\|_{\mathscr{L}\left(\mathscr{H}_{x, s}\left(\Delta_{p-1}\right), \mathscr{H}_{x, s}\left(\Delta_{p}\right)\right)} \leq C .
$$

Démonstration. Il suffit de montrer que pour tout $U \in \Delta_{p}$ vérifiant $d(x, U) \leq P$, il existe $C=C(\delta, K, N, Q, P, M, s, B)$ tel que pour $f \in \mathbb{C}^{\left(\Delta_{p}\right)}$,

$$
\left|\left(\widetilde{H}_{x, q}(f)\right)(U)\right| \leq C\|f\|_{\mathscr{H}_{x, s}\left(\Delta_{p-1}\right)} .
$$

Soit $U \in \Delta_{p}$ vérifiant $d(x, U) \leq P$. L'inégalité (66) est évidente, car, d'après le 1)a) de la proposition 3.37, pour tout $S \in \Delta_{p-1}, \widetilde{H}_{x, q}\left(e_{S}\right)$ est supporté par les $T \in \Delta_{p}$ tels que $T \subset \bigcup_{a \in S} B(a, Q N)$, donc $\left(\widetilde{H}_{x, q}\left(e_{S}\right)\right)(U)$ est nul sauf si $d(x, S) \leq Q N+P$ et le nombre de telles parties $S$ est majoré par une constante $C=C(\delta, K, N, Q, P)$. De plus pour une telle partie $S,\left|\left(\widetilde{H}_{x, q}\left(e_{S}\right)\right)(U)\right|$ est également majoré par une telle constante par le 3) de la proposition 3.37. On conclut en utilisant le lemme 4.16.

Lemme 4.32. Il existe $C=C(\delta, K, N, Q, P, M, s, B)$ tel que, pour tout $r \in \mathbb{N}$,

$$
\left\|\mathcal{P} u_{x, r} K_{x}\right\|_{\mathscr{L}\left(\mathscr{H}_{x, s}\left(\Delta_{p-1}\right), \mathscr{H}_{x, s}\left(\Delta_{p}\right)\right)} \leq C e^{-\frac{s}{2} r} .
$$

Démonstration. Il suffit de montrer que pour tout $U \in \Delta_{p}$ vérifiant $d(x, U) \leq P$, il existe $C=C(\delta, K, N, Q, P, M, s, B)$ tel que, pour $r \in \mathbb{N}$ et $f \in \mathbb{C}^{\left(\Delta_{p}\right)}$,

$$
\left|\left(u_{x, r} K_{x}(f)\right)(U)\right| \leq C e^{-\frac{s}{2} r}\|f\|_{\mathscr{H}_{x, s}\left(\Delta_{p-1}\right)} .
$$


Soit $U \in \Delta_{p}$ vérifiant $d(x, U) \leq P$ et $r \in \mathbb{N}$. Nous allons montrer (67). Soit $t, t_{1}, \ldots, t_{Q} \in[0,1]$. D'après le 2)a) de la proposition 3.37, pour $S \in \Delta_{p-1}$, $u_{x, r, t} K_{x, Q,\left(t_{1}, \ldots, t_{Q}\right)}\left(e_{S}\right)$ est une combinaison de $e_{T}$ pour $T$ vérifiant

$$
d(x, T) \in\left[d(x, S)-r-Q F, d(x, S)-r+N+F-\frac{Q}{F}\right] .
$$

On suppose $\frac{Q}{F} \geq F+N$, ce qui est permis par $\left(H_{Q}\right)$. Pour que

$$
\left(u_{x, r, t} K_{x, Q,\left(t_{1}, \ldots, t_{Q}\right)}\left(e_{S}\right)\right)(U)
$$

soit non nul il est donc nécessaire que

$$
d(x, S) \in[r, r+Q F+P] .
$$

On note $\Lambda_{t_{1}, \ldots, t_{Q}}$ la partie de $\bar{Y}_{x}^{\natural, p-1,0,0,(0), Q, 0}$ formée des $Z$ tels que

$-r_{0}(Z) \in[r, r+Q F+P]$,

- pour tout $\left(a_{1}, \ldots, a_{p-1}, S_{0},\left(Z_{0}^{j}\right)_{j \in\{1, \ldots, Q\}}\right) \in\left(\pi_{x}^{\natural, p-1,0,0,(0), Q, 0}\right)^{-1}(Z)$, et pour tout $j \in\{1, \ldots, Q\}$, on a

$$
Z_{0}^{j}=\bigcup_{b \in S_{0}}\left\{z \in \operatorname{géod}(x, b) \mid d(x, z)=\mathrm{E}\left(t_{j} r_{0}(Z)\right)\right\}
$$

(on rappelle que $r_{0}(Z)=d\left(x, S_{0}\right)$ ).

La condition (68) implique que pour $Z \in \Lambda_{t_{1}, \ldots, t_{Q}}$ et $j \in\{1, \ldots, Q\}$ on a $t_{0}^{j}(Z)=$ $\mathrm{E}\left(t_{j} r_{0}(Z)\right)$.

Sous-lemme 4.33. Soit $\left(a_{1}, \ldots, a_{p-1}, S_{0}\right) \in Y_{x}^{p-1,0,0,(0)}$ tel que $d\left(x, S_{0}\right) \in[r, r+$ $Q F+P]$. Pour $j \in\{1, \ldots, Q\}$ on définit $Z_{0}^{j}$ par (68). Alors $Z_{0}^{j}$ est de diamètre inférieur ou égal à $P / 3$ et il existe $Z \in \Lambda_{t_{1}, \ldots, t_{Q}}$ tel que

$$
\left(a_{1}, \ldots, a_{p-1}, S_{0},\left(\mathcal{Z}_{0}^{j}\right)_{j \in\{1, \ldots, q\}}\right) \in\left(\pi_{x}^{\natural, p-1,0,0,(0), Q, 0}\right)^{-1}(Z) .
$$

Démonstration. Soient $j \in\{1, \ldots, Q\}$ et $z, z^{\prime} \in Z_{0}^{j}$. Soit $b \in S_{0}$. On a $z, z^{\prime} \in$ $2 N$ - géod $(x, b)$ et $d(x, z)=d\left(x, z^{\prime}\right)$ donc par $\left(H_{\delta}\left(z, x, z^{\prime}, b\right)\right), d\left(z, z^{\prime}\right) \leq 2 N+\delta$ et on suppose $2 N+\delta \leq P / 3$, ce qui est permis par $\left(H_{P}\right)$. Comme les parties $Z_{0}^{j}$ sont non vides l'argument que nous venons de donner montre aussi que la condition (68) est vérifiée par les autres éléments de la classe d'équivalence $Z$ de $\left(a_{1}, \ldots, a_{p-1}, S_{0},\left(Z_{0}^{j}\right)_{j \in\{1, \ldots, q\}}\right)$ (car on suppose $P / 3 \leq M$, ce qui est permis par $\left.\left(H_{M}\right)\right)$ et donc que $Z \in \Lambda_{t_{1}, \ldots, t_{Q}}$.

Sous-lemme 4.34. Soit $Z \in \Lambda_{t_{1}, \ldots, t_{Q}}$ et

$$
\left(a_{1}, \ldots, a_{p-1}, S_{0},\left(\mathcal{Z}_{0}^{j}\right)_{j \in\{1, \ldots, Q\}}\right) \in\left(\pi_{x}^{\natural, p-1,0,0,(0), Q, 0}\right)^{-1}(Z) .
$$


Alors $u_{x, r, t} K_{x, Q,\left(t_{1}, \ldots, t_{Q}\right)}\left(e_{a_{1}} \wedge \cdots \wedge e_{a_{p-1}}\right)$ ne dépend que de la connaissance des points de

$$
B\left(S_{0}, M\right) \cup B(x, 2 M) \cup \underset{j \in\{1, \ldots, Q\}}{\bigcup} B\left(\mathcal{Z}_{0}^{j}, M\right)
$$

et des distances entre ces points.

Démonstration. Le 2 b) de la proposition 3.37 montre que

$$
u_{x, r, t} K_{x, Q,\left(t_{1}, \ldots, t_{Q}\right)}\left(e_{a_{1}} \wedge \cdots \wedge e_{a_{p-1}}\right)
$$

dépend seulement de la connaissance des points de

$$
\begin{aligned}
B(x, F) & \cup B\left(S_{0}, Q N\right) \cup \bigcup_{a \in S_{0}}\{y \in F-\operatorname{géod}(x, a) \mid d(y, a) \in[r, r+Q F]\} \\
& \cup \bigcup_{a \in S_{0}}\{y \in F-\operatorname{géod}(x, a)|| d(x, y)-(1-t)(d(x, a)-r) \mid \leq Q F\} \\
& \cup \bigcup_{\substack{a \in S_{0} \\
j \in\{1, \ldots, Q\}}}\left\{y \in F-\operatorname{géod}(x, a)|| d(x, y)-t_{j} d(x, a) \mid \leq Q F\right\}
\end{aligned}
$$

et des distances entre ces points. Il suffit donc de montrer que cet ensemble est inclus dans (69).

On a $B(x, F) \subset B(x, 2 M)$ et $B\left(S_{0}, Q N\right) \subset B\left(S_{0}, M\right)$ car on suppose $F \leq 2 M$ et $Q N \leq M$, ce qui est permis par $\left(H_{M}\right)$. Comme $d\left(x, S_{0}\right) \in[r, r+Q F+P]$ on a

$$
\begin{aligned}
& \bigcup_{a \in S_{0}}\{y \in F-\operatorname{géod}(x, a) \mid d(y, a) \in[r, r+Q F]\} \\
& \quad \subset B\left(x, d\left(x, S_{0}\right)+N+F-r\right) \subset B(x, Q F+P+N+F) \subset B(x, 2 M)
\end{aligned}
$$

car on suppose $Q F+P+N+F \leq 2 M$, ce qui est permis par $\left(H_{M}\right)$. Donc (70) est inclus dans (69).

Comme $d\left(x, S_{0}\right) \in[r, r+Q F+P]$ on a

$$
\begin{gathered}
\bigcup_{a \in S_{0}}\{y \in F-\operatorname{géod}(x, a)|| d(x, y)-(1-t)(d(x, a)-r) \mid \leq Q F\} \\
\subset B(x, N+2 Q F+P) \subset B(x, 2 M)
\end{gathered}
$$

car on suppose $N+2 Q F+P \leq 2 M$, ce qui est permis par $\left(H_{M}\right)$. Donc (71) est inclus dans (69).

Enfin, soit $j \in\{1, \ldots, Q\}, a \in S_{0}$, et $y \in F$ - $\operatorname{géod}(x, a)$ vérifiant $\mid d(x, y)-$ $t_{j} d(x, a) \mid \leq Q F$. Soit $z \in \operatorname{géod}(x, a)$ vérifiant $d(x, z)=\mathrm{E}\left(t_{j} d\left(x, S_{0}\right)\right)$, si bien que $z$ appartient à $Z_{0}^{j}$. Comme $y, z \in F$ - géod $(x, a)$ et $|d(x, y)-d(x, z)| \leq Q F+$ $N+1,\left(H_{\delta}(y, x, z, a)\right)$ montre que $d(y, z) \leq(Q F+N+1)+F+\delta$. On suppose $(Q F+N+1)+F+\delta \leq M$, ce qui est permis par $\left(H_{M}\right)$. Donc (72) est inclus dans $\bigcup_{j \in\{1, \ldots, Q\}} B\left(Z_{0}^{j}, M\right)$ et a fortiori dans (69). 
Sous-lemme 4.35. Le cardinal de $\Lambda_{t_{1}, \ldots, t_{Q}}$ est majoré par une constante de la forme $C(\delta, K, N, Q, P, M)$.

Démonstration. Cela résulte du lemme 4.28 (ou même d'un argument plus simple car le cardinal de l'ensemble (69) est borné par $C=C(\delta, K, N, Q, P, M)$ et les distances entre les points de (69) sont déterminées à $C^{\prime}=C(\delta, K, N, Q, P, M)$ près par la donnée de $r, t_{1}, \ldots, t_{Q}$ ).

Fin de la démonstration du lemme 4.32. On écrit $U=\left\{b_{1}, \ldots, b_{p}\right\}$ pour lever l'ambiguïté de signe. On rappelle, pour $Z \in \Lambda_{t_{1}, \ldots, t_{Q}}$, la notation

$$
\xi_{Z}(f)=\sum_{\left(a_{1}, \ldots, a_{p-1}, S_{0},\left(\mathcal{Z}_{0}^{j}\right)_{j \in\{1, \ldots, Q\}}\right) \in\left(\pi_{x}^{\natural, p-1,0,0,(0), Q, 0}\right)^{-1}(Z)} f\left(a_{1}, \ldots, a_{p-1}\right) .
$$

On a $f=\frac{1}{(p-1) !} \sum_{\left(a_{1}, \ldots, a_{p-1}\right)} f\left(a_{1}, \ldots, a_{p-1}\right) e_{a_{1}} \wedge \cdots \wedge e_{a_{p-1}}$ où la somme porte que les $\left(a_{1}, \ldots, a_{p-1}\right)$ tel que $\left\{a_{1}, \ldots, a_{p-1}\right\} \in \Delta_{p-1}$. Le sous-lemme 4.33 montre donc que

$$
\left(u_{x, r} K_{x, Q,\left(t_{1}, \ldots, t_{Q}\right)}(f)\right)\left(b_{1}, \ldots, b_{p}\right)=\frac{1}{(p-1) !} \sum_{Z \in \Lambda_{t_{1}, \ldots, t_{Q}}} \alpha_{Z,\left(t_{1}, \ldots, t_{Q}\right),\left(b_{1}, \ldots, b_{p}\right)} \xi_{Z}(f)
$$

où $\alpha_{Z,\left(t_{1}, \ldots, t_{Q}\right),\left(b_{1}, \ldots, b_{p}\right)} \in \mathbb{C}$ est défini de la façon suivante :

$$
\begin{aligned}
& \text { pour tout }\left(a_{1}, \ldots, a_{p-1}, S_{0},\left(\mathcal{Z}_{0}^{j}\right)_{j \in\{1, \ldots, Q\}}\right) \in\left(\pi_{x}^{\natural, p-1,0,0,(0), Q, 0}\right)^{-1}(Z), \\
& \alpha_{Z,\left(t_{1}, \ldots, t_{Q}\right),\left(b_{1}, \ldots, b_{p}\right)}=\left(u_{x, r} K_{x, Q,\left(t_{1}, \ldots, t_{Q}\right)}\left(e_{a_{1}} \wedge \cdots \wedge e_{a_{p-1}}\right)\right)\left(b_{1}, \ldots, b_{p}\right)
\end{aligned}
$$

(d'après le sous-lemme 4.34 ce nombre ne dépend que de $Z$ ). D'après le 3 ) de la proposition 3.37, $\left|\alpha_{Z,\left(t_{1}, \ldots, t_{Q}\right),\left(b_{1}, \ldots, b_{p}\right)}\right|$ est majoré par une constante de la forme $C(\delta, K, N, Q)$. Par Cauchy-Schwarz et grâce au sous-lemme 4.35, on a donc

$$
\left|\left(u_{x, r} K_{x, Q,\left(t_{1}, \ldots, t_{Q}\right)}(f)\right)(U)\right|^{2} \leq C \sum_{Z \in \Lambda_{t_{1}, \ldots, t_{Q}}}\left|\xi_{Z}(f)\right|^{2}
$$

pour une certaine constante $C=C(\delta, K, N, Q, P, M)$.

On en déduit, pour $t_{1}, \ldots, t_{Q} \in[0,1]$,

$$
\begin{aligned}
& \left|\left(u_{x, r} K_{x, Q,\left(t_{1}, \ldots, t_{Q}\right)}(f)\right)(U)\right|^{2} \\
& \quad \leq C e^{-s r} \sum_{Z \in \Lambda_{t_{1}, \ldots, t_{Q}}} e^{2 s r_{0}(Z)} \sharp\left(\left(\pi_{x}^{\natural, p-1,0,0,(0), Q, 0}\right)^{-1}(Z)\right)^{-\alpha}\left|\xi_{Z}(f)\right|^{2}
\end{aligned}
$$

pour une certaine constante $C=C(\delta, K, N, Q, P, M)$. En effet il existe une constante $D=C(\delta, K, N, Q, P, M)$ telle que pour tout $Z \in \Lambda_{t_{1}, \ldots, t_{Q}}$,

$$
\sharp\left(\left(\pi_{x}^{\natural, p-1,0,0,(0), Q, 0}\right)^{-1}(Z)\right) \leq e^{D\left(r_{0}(Z)+1\right)}
$$


et on suppose $\alpha D \leq s$, ce qui est permis par $\left(H_{\alpha}\right)$. De plus pour tout $Z \in \Lambda_{t_{1}, \ldots, t_{Q}}$ on a $r \leq r_{0}(Z)$, d'où $e^{-s r} \geq e^{-s r_{0}(Z)}$.

On a vu que $\Lambda_{t_{1}, \ldots, t_{Q}}$ est inclus dans la partie de $\bar{Y}_{x}^{\natural, p-1,0,0,(0), Q, 0}$ formée des $Z$ tels que

$-r_{0}(Z) \in[r, r+Q F+P]$,

- pour tout $j \in\{1, \ldots, Q\}$ on a $t_{0}^{j}(Z)=\mathrm{E}\left(t_{j} r_{0}(Z)\right)$.

Pour $r_{0} \in \mathbb{N}$, quand $\left(t_{1}, \ldots, t_{Q}\right)$ parcourt $[0,1]^{Q}$ muni de la mesure de Lebesgue, $\left(\mathrm{E}\left(t_{j} r_{0}\right)\right)_{j=1, \ldots, Q}$ parcourt $\left\{0, \ldots, \max \left(0, r_{0}-1\right)\right\}^{Q}$ avec la probabilité uniforme $\max \left(1, r_{0}\right)^{-Q}$ et comme

$$
\max \left(1, r_{0}\right)^{-1} \leq \frac{2}{r_{0}+1},
$$

par Cauchy-Schwarz on obtient l'inégalité

$$
\begin{gathered}
\left|\left(u_{x, r} K_{x}(f)\right)(U)\right|^{2} \leq C e^{-s r}\left(\sum_{Z \in \bar{Y}_{x}^{\natural, p-1,0,0,(0), Q, 0}} e^{2 s r_{0}(Z)}\left(r_{0}(Z)+1\right)^{-Q}\right. \\
\left.\sharp\left(\left(\pi_{x}^{\natural, p-1,0,0,(0), Q, 0}\right)^{-1}(Z)\right)^{-\alpha}\left|\xi_{Z}(f)\right|^{2}\right)
\end{gathered}
$$

pour une certaine constante $C=C(\delta, K, N, Q, P, M)$. A fortiori on a

$$
\left|\left(u_{x, r} K_{x}(f)\right)(U)\right|^{2} \leq C e^{-s r}\|f\|_{\mathcal{H}_{x, S}^{\natural, Q, 0}\left(\Delta_{p-1}\right)}^{2}
$$

puisque l'expression entre parenthèses dans (74) est la partie de la somme (55) donnant $\|f\|_{\mathcal{H}_{x, S}^{\natural, Q}, 0}^{2}$ qui correspond à $\bar{Y}_{x}^{\natural, p-1,0,0,(0), Q, 0}$. Grâce au lemme 4.24 ceci termine la démonstration du lemme 4.32 .

Lemme 4.36. Il existe $C=C(\delta, K, N, Q, P, M, s, B)$ tel que

$$
\left\|(1-\mathcal{P}) \tilde{H}_{x, q}\right\|_{\mathscr{L}\left(\mathscr{H}_{x, s}\left(\Delta_{p-1}\right), \mathscr{H}_{x, s}\left(\Delta_{p}\right)\right)} \leq C .
$$

Démonstration. Grâce au lemme 4.24, il suffit de montrer l'inégalité suivante : il existe $C=C(\delta, K, N, Q, P, M, B)$ tel que pour tout $f \in \mathbb{C}^{\left(\Delta_{p-1}\right)}$,

$$
\left\|(1-\mathcal{P})\left(\tilde{H}_{x, q} f\right)\right\|_{\mathscr{H}_{x, s}\left(\Delta_{p}\right)}^{2} \leq C\|f\|_{\mathcal{H}_{x, s}^{\natural, q, 0}\left(\Delta_{p-1}\right)}^{2} .
$$

On rappelle que

$$
\begin{aligned}
& \left\|(1-\mathcal{P})\left(\tilde{H}_{x, q} f\right)\right\|_{\mathscr{H}_{\vec{x}, S}}^{2}\left(\Delta_{p}\right) \\
& =\sum_{k, m, l_{0}, \ldots, l_{m} \in \mathbb{N}} B^{-\left(m+\sum_{i=0}^{m} l_{i}\right)} \sum_{\substack{Z \in \bar{Y}_{x}^{p, k, m,\left(l_{0}, \ldots, l_{m}\right)} \\
r_{0}(Z)>k+P}} e^{2 s\left(r_{0}(Z)-k\right)} \\
& \left(\prod_{i=0}^{m}\left(s_{i}(Z)\right)^{-l_{i}}\right) \sharp\left(\left(\pi_{x}^{p, k, m,\left(l_{0}, \ldots, l_{m}\right)}\right)^{-1}(Z)\right)^{-\alpha}\left|\xi_{Z}\left(\tilde{H}_{x, q} f\right)\right|^{2} .
\end{aligned}
$$


On va voir que l'inégalité (75) résulte de l'inégalité (76) ci-dessous.

Soient $k, m, l_{0}, \ldots, l_{m} \in \mathbb{N}$ et $Z \in \bar{Y}_{x}^{p, k, m,\left(l_{0}, \ldots, l_{m}\right)}$ vérifiant $r_{0}(Z)>k+P$. On pose $\tilde{l}_{0}=0$ et $\tilde{l}_{i}=l_{i-1}$ pour $i \in\{1, \ldots, m+1\}$. On va montrer qu'il existe $C=C(\delta, K, N, Q, P, M)$ tel que

$$
\left|\xi_{Z}\left(\tilde{H}_{x, q} f\right)\right|^{2} \leq C \sum_{\tilde{Z} \in \Lambda_{Z}}\left(r_{0}(\tilde{Z})+1\right)^{-q}\left|\xi_{\widetilde{Z}}(f)\right|^{2}
$$

où $\Lambda_{Z}$ est la partie de $\bar{Y}_{x}^{\natural, p-1, k, m+1,\left(\tilde{l}_{0}, \ldots, \tilde{l}_{m+1}\right), q, 0}$ formée des $\widetilde{Z}$ vérifiant

$$
\left|r_{0}(\tilde{Z})-r_{1}(\tilde{Z})\right| \leq(q+1) N
$$

et tels que pour tout

$$
\begin{gathered}
\left(\tilde{a}_{1}, \ldots, \tilde{a}_{p-1}, \widetilde{S}_{0}, \ldots, \widetilde{S}_{m+1},\left(\tilde{\mathcal{Y}}_{i}^{j}\right)_{i \in\{0, \ldots, m+1\}, j \in\left\{1, \ldots, \tilde{l}_{i}\right\}},\left(\widetilde{\mathcal{Z}}_{0}^{j}\right)_{j \in\{1, \ldots, q\}}\right) \\
\in\left(\pi_{x}^{\natural, p-1, k, m+1,\left(\tilde{l}_{0}, \ldots, \tilde{l}_{m+1}\right), q, 0}\right)^{-1}(\widetilde{Z})
\end{gathered}
$$

il existe une énumération $\left(a_{1}, \ldots, a_{p}\right)$ de $\widetilde{S}_{1}$ vérifiant

$$
\left(a_{1}, \ldots, a_{p}, \widetilde{S}_{1}, \ldots, \widetilde{S}_{m+1},\left(\tilde{y}_{i+1}^{j}\right)_{i \in\{0, \ldots, m\}, j \in\left\{1, \ldots, l_{i}\right\}}\right) \in\left(\pi_{x}^{p, k, m,\left(l_{0}, \ldots, l_{m}\right)}\right)^{-1}(Z) .
$$

On rappelle que

$$
\xi_{\tilde{Z}}(f)=\sum_{\left(\tilde{a}_{1}, \ldots, \tilde{a}_{p-1}, \widetilde{S}_{0}, \ldots, \widetilde{S}_{m+1},\left(\widetilde{y}_{i}^{j}\right)_{\substack{i \in\{0, \ldots, m+1\} \\ j \in\left\{1, \ldots, \tilde{l}_{i}\right\}}},\left(\widetilde{\mathcal{Z}}_{0}^{j}\right)_{j \in\{1, \ldots, q\}}\right)} f\left(\tilde{a}_{1}, \ldots, \tilde{a}_{p-1}\right) .
$$

On justifie maintenant le fait que (76) implique (75). D'abord $\widetilde{Z}$ détermine $Z$ à permutation près de $a_{1}, \ldots, a_{p}$ donc connaissant $\widetilde{Z}$ il y a au plus $p$ ! possibilités pour $Z$. Dans les notations précédentes, soit $b \in \widetilde{S}_{0}$. D'après (77), pour tout $y \in \widetilde{S}_{1}$ on a $|d(x, y)-d(x, b)| \leq(Q+2) N$ et on a $y \in 2 F-\operatorname{géod}(x, b)$ par le a) du lemme 4.14 , donc $d(y, b) \leq(Q+2) N+2 F$. Connaissant $\widetilde{S}_{1}$ on a donc au plus $C=C(\delta, K, N, Q)$ possibilités pour $\widetilde{S}_{0}$. En utilisant de plus le lemme 4.29 on en déduit que pour $\widetilde{Z} \in \Lambda_{Z}$ on a

$$
\sharp\left(\left(\pi_{x}^{\natural, p-1, k, m+1,\left(\tilde{l}_{0}, \ldots, \tilde{l}_{m+1}\right), q, 0}\right)^{-1}(\widetilde{Z})\right) \leq C \sharp\left(\left(\pi_{x}^{p, k, m,\left(l_{0}, \ldots, l_{m}\right)}\right)^{-1}(Z)\right)
$$

avec $C=C(\delta, K, N, Q, P, M)$. Il est clair que pour $\widetilde{Z} \in \Lambda_{Z}$ on a $\prod_{i=0}^{m+1} s_{i}(\widetilde{Z})^{-\tilde{l}_{i}}=$ $\prod_{i=0}^{m} s_{i}(Z)^{-l_{i}}$. Donc (76) implique (75).

L'inégalité (76) résulte de l'inégalité plus précise (79) ci-dessous.

Soient $t_{1}, \ldots, t_{q} \in[0,1]$. On va montrer qu'il existe $C=C(\delta, K, N, Q, P, M)$ tel que

$$
\left|\xi_{Z}\left(\tilde{H}_{x, q,\left(t_{1}, \ldots, t_{q}\right)} f\right)\right|^{2} \leq C \sum_{\tilde{Z} \in \Lambda_{Z,\left(t_{1}, \ldots, t_{q}\right)}}\left|\xi_{\tilde{Z}}(f)\right|^{2}
$$



où $\Lambda_{Z,\left(t_{1}, \ldots, t_{q}\right)}$ est l'ensemble des $\widetilde{Z} \in \Lambda_{Z}$ tels que pour tout

$$
\begin{gathered}
\left(\tilde{a}_{1}, \ldots, \tilde{a}_{p-1}, \widetilde{S}_{0}, \ldots, \widetilde{S}_{m+1},\left(\tilde{\mathcal{Y}}_{i}^{j}\right)_{i \in\{0, \ldots, m+1\}, j \in\left\{1, \ldots, \tilde{l}_{i}\right\}},\left(\widetilde{\mathcal{Z}}_{0}^{j}\right)_{j \in\{1, \ldots, q\}}\right) \\
\in\left(\pi_{x}^{\natural, p-1, k, m+1,\left(\tilde{l}_{0}, \ldots, \tilde{l}_{m+1}\right), q, 0}\right)^{-1}(\widetilde{Z})
\end{gathered}
$$

et pour tout $j \in\{1, \ldots, q\}$ on ait

$$
\widetilde{Z}_{0}^{j}=\bigcup_{b \in \widetilde{S}_{0}}\left\{z \in \operatorname{géod}(x, b) \mid d(x, z)=\mathrm{E}\left(t_{j} r_{0}(\widetilde{Z})\right)\right\} .
$$

La condition (80) implique que pour $\widetilde{Z} \in \Lambda_{Z,\left(t_{1}, \ldots, t_{q}\right)}$ et $j \in\{1, \ldots, q\}$ on a $t_{0}^{j}(\widetilde{Z})=$ $\mathrm{E}\left(t_{j} r_{0}(\widetilde{Z})\right)$.

Sous-lemme 4.37. Soit

$$
\left(\tilde{a}_{1}, \ldots, \tilde{a}_{p-1}, \widetilde{S}_{0}, \ldots, \widetilde{S}_{m+1},\left(\tilde{y}_{i}^{j}\right)_{i \in\{0, \ldots, m+1\}, j \in\left\{1, \ldots, \tilde{l}_{i}\right\}}\right) \in Y_{x}^{p-1, k, m+1,\left(\tilde{l}_{0}, \ldots, \tilde{l}_{m+1}\right)}
$$

tel que $\left|d\left(x, \widetilde{S}_{0}\right)-d\left(x, \widetilde{S}_{1}\right)\right| \leq(q+1) N$ et qu'il existe $\left(a_{1}, \ldots, a_{p}\right)$ vérifiant $(78)$. Pour $j \in\{1, \ldots, q\}$ on définit $\widetilde{Z}_{0}^{j}$ par (80). Alors $\widetilde{Z}_{0}^{j}$ est de diamètre inférieur ou égal à $P / 3$ et il existe $\widetilde{Z} \in \Lambda_{Z,\left(t_{1}, \ldots, t_{q}\right)}$ tel que

$$
\left(\tilde{a}_{1}, \ldots, \tilde{a}_{p-1}, \tilde{S}_{0}, \ldots, \widetilde{S}_{m+1},\left(\tilde{\mathcal{Y}}_{i}^{j}\right)_{i \in\{0, \ldots, m+1\}, j \in\left\{1, \ldots, \tilde{l}_{i}\right\}},\left(\widetilde{\mathcal{Z}}_{0}^{j}\right)_{j \in\{1, \ldots, q\}}\right)
$$

appartienne $\grave{a}\left(\pi_{x}^{\natural, p-1, k, m+1,\left(\tilde{l}_{0}, \ldots, \tilde{l}_{m+1}\right), q, 0}\right)^{-1}(\widetilde{Z})$.

Démonstration. Soient $j \in\{1, \ldots, q\}$ et $z, z^{\prime} \in \mathcal{Z}_{0}^{j}$. Soit $b \in \widetilde{S}_{0}$. On a $z, z^{\prime} \in$ $2 N-\operatorname{géod}(x, b)$ et $d(x, z)=d\left(x, z^{\prime}\right)$ donc $\operatorname{par}\left(H_{\delta}\left(z, x, z^{\prime}, b\right)\right), d\left(z, z^{\prime}\right) \leq 2 N+\delta \leq$ $P / 3$. Comme les parties $\tilde{Z}_{0}^{j}$ sont non vides et $P / 3 \leq M$, l'argument que nous venons de donner montre aussi que la condition (80) est vérifiée par les autres éléments de la classe d'équivalence $\widetilde{Z}$ de l'élément (81) et donc $\widetilde{Z} \in \Lambda_{Z,\left(t_{1}, \ldots, t_{q}\right)}$.

Sous-lemme 4.38. Soit $\widetilde{Z} \in \Lambda_{Z,\left(t_{1}, \ldots, t_{q}\right)}$, et

$$
\begin{gathered}
\left(\tilde{a}_{1}, \ldots, \tilde{a}_{p-1}, \widetilde{S}_{0}, \ldots, \widetilde{S}_{m+1},\left(\widetilde{\mathcal{Y}}_{i}^{j}\right)_{i \in\{0, \ldots, m+1\}, j \in\left\{1, \ldots, \tilde{l}_{i}\right\}},\left(\widetilde{Z}_{0}^{j}\right)_{j \in\{1, \ldots, q\}}\right) \\
\in\left(\pi_{x}^{\natural, p-1, k, m+1,\left(\tilde{l}_{0}, \ldots, \tilde{l}_{m+1}\right), q, 0}\right)^{-1}(\widetilde{Z}) .
\end{gathered}
$$

Alors $\tilde{H}_{x, q,\left(t_{1}, \ldots, t_{q}\right)}\left(e_{\tilde{a}_{1}} \wedge \cdots \wedge e_{\tilde{a}_{p-1}}\right)$ ne dépend que de la connaissance des points de

$$
B\left(\widetilde{S}_{0}, M\right) \cup B(x, k+2 M) \cup \underset{j \in\{1, \ldots, q\}}{\bigcup} B\left(\tilde{Z}_{0}^{j}, M\right)
$$

et des distances entre ces points. 
Démonstration. D'après le 1)b) de la proposition 3.37,

$$
\tilde{H}_{x, q,\left(t_{1}, \ldots, t_{q}\right)}\left(e_{\tilde{a}_{1}} \wedge \cdots \wedge e_{\tilde{a}_{p-1}}\right)
$$

ne dépend que de la connaissance des points de

$$
\cup \bigcup_{a \in \widetilde{S}_{0}, j \in\{1, \ldots, q\}} \begin{gathered}
B(x, 7 \delta) \cup B\left(\widetilde{S}_{0}, Q N\right) \\
\left\{y \in F-\operatorname{géod}(x, a)|| d(x, y)-t_{j} d(x, a) \mid \leq Q F\right\}
\end{gathered}
$$

et des distances entre ces points. Il suffit donc de montrer que cet ensemble est inclus dans (82). D'abord on suppose $7 \delta \leq 2 M$ et $Q N \leq M$, ce qui est permis par $\left(H_{M}\right)$, et (83) est inclus dans (82).

Soit $a \in \widetilde{S}_{0}, j \in\{1, \ldots, q\}$, et $y \in F$ - géod $(x, a)$ vérifiant $\left|d(x, y)-t_{j} d(x, a)\right| \leq$ $Q F$. Soit $z \in \operatorname{géod}(x, a)$ vérifiant $d(x, z)=\mathrm{E}\left(t_{j} r_{0}(\widetilde{Z})\right)$, si bien que $z$ appartient à $\widetilde{Z}_{0}^{j}$. On a $\left|t_{j} d(x, a)-\mathrm{E}\left(t_{j} r_{0}(\tilde{Z})\right)\right| \leq N+1$ puisque $\left|d(x, a)-r_{0}(\tilde{Z})\right| \leq N$, d'où $|d(x, y)-d(x, z)| \leq Q F+N+1$, et comme $y$ et $z$ appartiennent à $F$ - $\operatorname{géod}(x, a)$, $\left(H_{\delta}(y, x, z, a)\right)$ montre que

$$
d(y, z) \leq(Q F+N+1)+F+\delta .
$$

On suppose $(Q F+N+1)+F+\delta \leq M$, ce qui est permis par $\left(H_{M}\right)$. Donc (84) est inclus dans (82).

Sous-lemme 4.39. Le cardinal de $\Lambda_{Z,\left(t_{1}, \ldots, t_{q}\right)}$ est majoré par une constante de la forme $C(\delta, K, N, Q, P, M)$.

Démonstration. Les parties $\widetilde{\mathcal{Z}}_{0}^{j}$ sont déterminées de manière unique par (80) et grâce au lemme 4.28, pour connaître les distances entre les points de

$$
B\left(\widetilde{S}_{0}, M\right) \cup \bigcup_{j \in\{1, \ldots, q\}} B\left(\widetilde{Z}_{0}^{j}, M\right)
$$

et ceux de

$$
\bigcup_{i \in\{1, \ldots, m+1\}} B\left(\tilde{S}_{i}, M\right) \cup \underset{\substack{i \in\{1, \ldots, m+1\} \\ j \in\left\{1, \ldots, \tilde{I}_{i}\right\}}}{\bigcup} B\left(\tilde{y}_{i}^{j}, M\right) \cup B(x, k+2 M)
$$

il suffit de connaître les distances entre les points de (85) et $C$ points de (86), avec $C=C(\delta, K, N, Q, P, M)$ et grâce à (77) ces distances sont déterminées à $C^{\prime}=$ $C(\delta, K, N, Q, P, M)$ près par les distances de $\widetilde{S}_{1}$ à ces $C$ points (qui font partie de la donnée de $Z$ ) et les entiers $\left(t_{0}^{j}(\widetilde{Z})\right)_{j \in\{1, \ldots, q\}}$, qui grâce à (77) sont eux-mêmes déterminés à $C^{\prime \prime}=C(\delta, K, N, Q, P, M)$ près par $r_{0}(Z), t_{1}, \ldots, t_{q}$.

Fin de la démonstration du lemme 4.36. On termine la démonstration de (79). Pour $\widetilde{Z} \in \Lambda_{Z,\left(t_{1}, \ldots, t_{q}\right)}$ et

$$
\begin{gathered}
\left(\tilde{a}_{1}, \ldots, \tilde{a}_{p-1}, \tilde{S}_{0}, \ldots, \widetilde{S}_{m+1},\left(\widetilde{y}_{i}^{j}\right)_{i \in\{0, \ldots, m+1\}, j \in\left\{1, \ldots, \tilde{l}_{i}\right\}},\left(\widetilde{\mathcal{Z}}_{0}^{j}\right)_{j \in\{1, \ldots, q\}}\right) \\
\in\left(\pi_{x}^{\natural, p-1, k, m+1,\left(\tilde{l}_{0}, \ldots, \tilde{l}_{m+1}\right), q, 0}\right)^{-1}(\widetilde{Z})
\end{gathered}
$$


on considère

$$
\sum_{\left(b_{1}, \ldots, b_{p}\right)}\left(\tilde{H}_{x, q,\left(t_{1}, \ldots, t_{q}\right)}\left(e_{\tilde{a}_{1}} \wedge \cdots \wedge e_{\tilde{a}_{p-1}}\right)\right)\left(b_{1}, \ldots, b_{p}\right)
$$

où la somme porte sur les énumérations $\left(b_{1}, \ldots, b_{p}\right)$ de $\widetilde{S}_{1}$ telles que

$$
\left(b_{1}, \ldots, b_{p}, \widetilde{S}_{1}, \ldots, \widetilde{S}_{m+1},\left(\widetilde{\mathcal{Y}}_{i+1}^{j}\right)_{i \in\{0, \ldots, m\}, j \in\left\{1, \ldots, \tilde{l}_{i}\right\}}\right) \in\left(\pi_{x}^{p, k, m,\left(l_{0}, \ldots, l_{m}\right)}\right)^{-1}(Z) .
$$

Comme la somme (87) a au plus $p$ ! termes, le 3) de la proposition 3.37 montre qu'elle est majorée par une constante de la forme $C(\delta, K, N, Q, P, M)$. D'après le sous-lemme 4.38 la somme (87) ne dépend que de $\widetilde{Z}$ et on peut donc la noter $\alpha_{Z, \tilde{Z},\left(t_{1}, \ldots, t_{q}\right)}$. D'après le sous-lemme 4.37 on a

$$
\xi_{Z}\left(\tilde{H}_{x, q,\left(t_{1}, \ldots, t_{q}\right)} f\right)=\frac{1}{(p-1) !} \sum_{\tilde{Z} \in \Lambda_{Z,\left(t_{1}, \ldots, t_{q}\right)}} \alpha_{Z, \widetilde{Z},\left(t_{1}, \ldots, t_{q}\right)} \xi_{\widetilde{Z}}(f) .
$$

Par Cauchy-Schwarz et grâce au sous-lemme 4.39 on en déduit que

$$
\left|\xi_{Z}\left(\tilde{H}_{x, q,\left(t_{1}, \ldots, t_{q}\right)} f\right)\right|^{2} \leq C \sum_{\tilde{Z} \in \Lambda_{Z,\left(t_{1}, \ldots, t_{q}\right)}}\left|\xi_{\tilde{Z}}(f)\right|^{2}
$$

avec $C=C(\delta, K, N, Q, P, M)$. On a montré (79), donc (76) et (75). Ceci termine la preuve du lemme 4.36 .

Lemme 4.40. Il existe $C=C(\delta, K, N, Q, P, M, s, B)$ tel que, pour tout $r \in \mathbb{N}$,

$$
\left\|(1-\mathcal{P}) u_{x, r} K_{x}\right\|_{\mathscr{L}\left(\mathscr{H}_{x, s}\left(\Delta_{p-1}\right), \mathscr{H}_{\overrightarrow{x, s}}\left(\Delta_{p}\right)\right)} \leq C e^{-\frac{s r}{2}} .
$$

Démonstration. Grâce au lemme 4.24, il suffit de montrer l'inégalité (89) ci-dessous. Soit $r \in \mathbb{N}$. On va montrer qu'il existe $C=C(\delta, K, N, Q, P, M, B)$ tel que pour $f \in \mathbb{C}^{\left(\Delta_{p-1}\right)}$,

$$
\left\|(1-\mathcal{P})\left(u_{x, r} K_{x} f\right)\right\|_{\mathscr{H}_{x, s}\left(\Delta_{p}\right)}^{2} \leq C e^{-s r}\|f\|_{\mathcal{H}_{x, S}^{\natural, Q}\left(\Delta_{p-1}\right)}^{2} .
$$

On a

$$
\begin{aligned}
\left\|(1-\mathcal{P})\left(u_{x, r} K_{x} f\right)\right\|_{\substack{\mathcal{H}_{x, s}\left(\Delta_{p}\right) \\
2}} \sum_{k, m, l_{0}, \ldots, l_{m} \in \mathbb{N}} B^{-\left(m+\sum_{i=0}^{m} l_{i}\right)} \sum_{\substack{Z \in \bar{Y}_{x}^{p, k, m,\left(l_{0}, \ldots, l_{m}\right)} \\
r_{0}(Z)>k+P}} \\
\quad e^{2 s\left(r_{0}(Z)-k\right)}\left(\prod_{i=0}^{m} s_{i}(Z)^{-l_{i}}\right) \sharp\left(\left(\pi_{x}^{p, k, m,\left(l_{0}, \ldots, l_{m}\right)}\right)^{-1}(Z)\right)^{-\alpha}\left|\xi_{Z}\left(u_{x, r} K_{x} f\right)\right|^{2} .
\end{aligned}
$$

On va voir que l'inégalité (89) résulte de l'inégalité (90) ci-dessous. 
Soient $k, m, l_{0}, \ldots, l_{m} \in \mathbb{N}$ et $Z \in \bar{Y}_{x}^{p, k, m,\left(l_{0}, \ldots, l_{m}\right)}$ vérifiant $r_{0}(Z)>k+P$. On pose $\tilde{l}_{0}=0$ et $\tilde{l}_{i}=l_{i-1}$ pour $i \in\{1, \ldots, m+1\}$. On va montrer qu'il existe $C=C(\delta, K, N, Q, P, M)$ tel que

$$
\begin{gathered}
e^{2 s\left(r_{0}(Z)-k\right)} \sharp\left(\left(\pi_{x}^{p, k, m,\left(l_{0}, \ldots, l_{m}\right)}\right)^{-1}(Z)\right)^{-\alpha}\left|\xi_{Z}\left(u_{x, r} K_{x} f\right)\right|^{2} \\
\leq C e^{-s r} \sum_{\tilde{Z} \in \Lambda_{Z}}\left(r_{0}(\widetilde{Z})+1\right)^{-Q}\left(r_{1}(\tilde{Z})+1\right)^{-1} e^{2 s\left(r_{0}(\tilde{Z})-k\right)} \\
\sharp\left(\left(\pi_{x}^{\natural, p-1, k, m+1,\left(\tilde{l}_{0}, \ldots, \tilde{l}_{m+1}\right), Q, 1}\right)^{-1}(\tilde{Z})\right)^{-\alpha}\left|\xi_{\tilde{Z}}(f)\right|^{2}
\end{gathered}
$$

où $\Lambda_{Z}$ est la partie de $\bar{Y}_{x}^{\natural, p-1, k, m+1,\left(\tilde{l}_{0}, \ldots, \tilde{l}_{m+1}\right), Q, 1}$ formée des $\widetilde{Z}$ vérifiant

$$
\left|r_{0}(\widetilde{Z})-r_{1}(\widetilde{Z})-r\right| \leq Q F
$$

et tels que pour tout

$$
\begin{gathered}
\left(\tilde{a}_{1}, \ldots, \tilde{a}_{p-1}, \tilde{S}_{0}, \ldots, \tilde{S}_{m+1},\left(\tilde{\mathcal{Y}}_{i}^{j}\right)_{i \in\{0, \ldots, m+1\}, j \in\left\{1, \ldots, \tilde{l}_{i}\right\}},\left(\widetilde{\mathcal{Z}}_{0}^{j}\right)_{j \in\{1, \ldots, Q\}}, \widetilde{\mathcal{Z}}_{1}^{1}\right) \\
\in\left(\pi_{x}^{\natural, p-1, k, m+1,\left(\tilde{l}_{0}, \ldots, \tilde{l}_{m+1}\right), Q, 1}\right)^{-1}(\widetilde{Z})
\end{gathered}
$$

il existe une énumération $\left(a_{1}, \ldots, a_{p}\right)$ de $\widetilde{S}_{1}$ vérifiant

$$
\left(a_{1}, \ldots, a_{p}, \widetilde{S}_{1}, \ldots, \widetilde{S}_{m+1},\left(\tilde{y}_{i+1}^{j}\right)_{i \in\{0, \ldots, m\}, j \in\left\{1, \ldots, l_{i}\right\}}\right) \in\left(\pi_{x}^{p, k, m,\left(l_{0}, \ldots, l_{m}\right)}\right)^{-1}(Z) .
$$

Pour $\tilde{Z} \in \Lambda_{Z}$ on a $\prod_{i=0}^{m} s_{i}(Z)^{-l_{i}}=\prod_{i=0}^{m+1} s_{i}(\tilde{Z})^{-\tilde{l}_{i}}$. De plus $\tilde{Z}$ détermine $Z$ à permutation près de $a_{1}, \ldots, a_{p}$ donc connaissant $\widetilde{Z}$ il y a au plus $p$ ! possibilités pour $Z$ tels que $\tilde{Z} \in \Lambda_{Z}$. Donc en sommant sur $Z$ on voit que (90) implique (89).

L'inégalité (90) résulte de l'inégalité plus précise (93) ci-dessous (en reprenant les arguments de la fin de la démonstration du lemme 4.32).

Soient $t, t_{1}, \ldots, t_{Q} \in[0,1]$. On va montrer qu'il existe $C=C(\delta, K, N, Q, P, M)$ tel que

$$
\begin{aligned}
& e^{2 s\left(r_{0}(Z)-k\right)} \sharp\left(\left(\pi_{x}^{p, k, m,\left(l_{0}, \ldots, l_{m}\right)}\right)^{-1}(Z)\right)^{-\alpha}\left|\xi_{Z}\left(u_{x, r, t} K_{x, Q,\left(t_{1}, \ldots, t_{Q}\right)} f\right)\right|^{2} \\
& \quad \leq C e_{\tilde{Z} \in \Lambda_{Z, t,\left(t_{1}, \ldots, t_{Q}\right)}^{-s r}} \sum^{2 s\left(r_{0}(\widetilde{Z})-k\right)} \sharp\left(\left(\pi_{x}^{\natural, p-1, k, m+1,\left(\tilde{l}_{0}, \ldots, \tilde{l}_{m+1}\right), Q, 1}\right)^{-1}(\widetilde{Z})\right)^{-\alpha}\left|\xi_{\tilde{Z}}(f)\right|^{2}
\end{aligned}
$$

où $\Lambda_{Z, t,\left(t_{1}, \ldots, t_{Q}\right)}$ est l'ensemble des $\widetilde{Z} \in \Lambda_{Z}$ tels que pour tout

$$
\begin{gathered}
\left(\tilde{a}_{1}, \ldots, \tilde{a}_{p-1}, \tilde{S}_{0}, \ldots, \widetilde{S}_{m+1},\left(\tilde{y}_{i}^{j}\right)_{i \in\{0, \ldots, m+1\}, j \in\left\{1, \ldots, \tilde{l}_{i}\right\}},\left(\tilde{\mathcal{Z}}_{0}^{j}\right)_{j \in\{1, \ldots, Q\}}, \widetilde{\mathcal{Z}}_{1}^{1}\right) \\
\in\left(\pi_{x}^{\natural, p-1, k, m+1,\left(\tilde{l}_{0}, \ldots, \tilde{l}_{m+1}\right), Q, 1}\right)^{-1}(\widetilde{Z})
\end{gathered}
$$


on ait

$$
\widetilde{Z}_{0}^{j}=\bigcup_{b \in \widetilde{S}_{0}}\left\{z \in \operatorname{géod}(x, b) \mid d(x, z)=\mathrm{E}\left(t_{j} r_{0}(\tilde{Z})\right)\right\} \quad \text { pour } j \in\{1, \ldots, Q\}
$$

et

$$
\widetilde{Z}_{1}^{1}=\bigcup_{b \in \widetilde{S}_{1}}\left\{z \in \operatorname{géod}(x, b) \mid d(x, z)=\mathrm{E}\left((1-t) r_{1}(\widetilde{Z})\right)\right\} .
$$

Pour $\widetilde{Z} \in \Lambda_{Z, t,\left(t_{1}, \ldots, t_{Q}\right)}$, les conditions (94) et (95) impliquent

$$
t_{0}^{j}(\tilde{Z})=\mathrm{E}\left(t_{j} r_{0}(\tilde{Z})\right) \text { pour } j \in\{1, \ldots, Q\} \quad \text { et } \quad t_{1}^{1}(\widetilde{Z})=\mathrm{E}\left((1-t) r_{1}(\tilde{Z})\right) .
$$

\section{Sous-lemme 4.41. Soit}

$$
\left(\tilde{a}_{1}, \ldots, \tilde{a}_{p-1}, \tilde{S}_{0}, \ldots, \tilde{S}_{m+1},\left(\tilde{\mathcal{Y}}_{i}^{j}\right)_{i \in\{0, \ldots, m+1\}, j \in\left\{1, \ldots, \tilde{l}_{i}\right\}}\right) \in Y_{x}^{p-1, k, m+1,\left(\tilde{l}_{0}, \ldots, \tilde{l}_{m+1}\right)}
$$

tel que $\left|d\left(x, \widetilde{S}_{0}\right)-d\left(x, \widetilde{S}_{1}\right)-r\right| \leq Q F$ et qu'il existe $\left(a_{1}, \ldots, a_{p}\right)$ vérifiant (92). On définit $\left(\widetilde{Z}_{0}^{j}\right)_{j \in\{1, \ldots, Q\}}$ et $\widetilde{Z}_{1}^{1}$ par (94) et $(95)$. Alors les parties $\widetilde{Z}_{0}^{j}$ et $\widetilde{Z}_{1}^{1}$ sont de diamètre inférieur ou égal à $P / 3$ et il existe $\widetilde{Z} \in \Lambda_{Z, t,\left(t_{1}, \ldots, t_{Q}\right)}$ tel que

$$
\left(\tilde{a}_{1}, \ldots, \tilde{a}_{p-1}, \widetilde{S}_{0}, \ldots, \widetilde{S}_{m+1},\left(\tilde{\mathcal{Y}}_{i}^{j}\right)_{i \in\{0, \ldots, m+1\}, j \in\left\{1, \ldots, \tilde{l}_{i}\right\}},\left(\widetilde{\mathcal{Z}}_{0}^{j}\right)_{j \in\{1, \ldots, Q\}}, \widetilde{\mathcal{Z}}_{1}^{1}\right)
$$

appartienne $\grave{a}\left(\pi_{x}^{\natural, p-1, k, m+1,\left(\tilde{l}_{0}, \ldots, \tilde{l}_{m+1}\right), Q, 1}\right)^{-1}(\widetilde{Z})$.

Démonstration. Pour $\sigma \in\{0,1\}$ et $z, z^{\prime} \in \widetilde{Z}_{\sigma}^{j}$, on choisit $b \in \widetilde{S}_{\sigma}$, d'où $z, z^{\prime} \in$ $2 N-\operatorname{géod}(x, b)$ et comme $d(x, z)=d\left(x, z^{\prime}\right),\left(H_{\delta}\left(z, x, z^{\prime}, b\right)\right)$ donne $d\left(z, z^{\prime}\right) \leq$ $2 N+\delta \leq P / 3$. Comme les parties $\widetilde{\mathcal{Z}}_{0}^{j}$ et $\widetilde{Z}_{1}^{1}$ sont non vides et $P / 3 \leq M$, l'argument que nous venons de donner montre aussi que les conditions (94) et (95) sont vérifiées par les autres éléments de la classe d'équivalence $\widetilde{Z}$ de l'élément (96) et donc $\widetilde{Z} \in$ $\Lambda_{Z, t,\left(t_{1}, \ldots, t_{Q}\right)}$.

Le sous-lemme suivant explique d'où vient la condition (91).

Sous-lemme 4.42. Pour $S \in \Delta_{p-1}$ et $T \in \Delta_{p}$ tels que $e_{T}$ apparaisse avec un coefficient non nul dans $u_{x, r, t} K_{x, Q,\left(t_{1}, \ldots, t_{Q}\right)}\left(e_{S}\right)$, on a

$$
|d(x, S)-d(x, T)-r| \leq Q F \text {. }
$$

Démonstration. D'après le 2)a) de la proposition 3.37, on a

$$
T \subset \bigcup_{a \in S}\left\{y \in F-\operatorname{géod}(x, a) \mid d(y, a) \in\left[r+\frac{Q}{F}, r+Q F\right]\right\}
$$

d'où l'énoncé du sous-lemme car $\frac{Q}{F} \geq N+F$. 
Sous-lemme 4.43. Soit $\widetilde{Z} \in \Lambda_{Z, t,\left(t_{1}, \ldots, t_{Q}\right)}$, et

$$
\begin{gathered}
\left(\tilde{a}_{1}, \ldots, \tilde{a}_{p-1}, \widetilde{S}_{0}, \ldots, \widetilde{S}_{m+1},\left(\widetilde{\mathcal{Y}}_{i}^{j}\right)_{i \in\{0, \ldots, m+1\}, j \in\left\{1, \ldots, \tilde{l}_{i}\right\}},\left(\widetilde{Z}_{0}^{j}\right)_{j \in\{1, \ldots, Q\}}, \widetilde{Z}_{1}^{1}\right) \\
\in\left(\pi_{x}^{\natural, p-1, k, m+1,\left(\tilde{l}_{0}, \ldots, \tilde{l}_{m+1}\right), Q, 1}\right)^{-1}(\widetilde{Z}) .
\end{gathered}
$$

Alors $u_{x, r, t} K_{x, Q,\left(t_{1}, \ldots, t_{Q}\right)}\left(e_{\widetilde{S}_{0}}\right)$ ne dépend que de la connaissance des points de

$$
B(x, k+2 M) \cup B\left(\widetilde{S}_{0}, M\right) \cup B\left(\widetilde{S}_{1}, M\right) \cup \underset{j \in\{1, \ldots, Q\}}{\cup} B\left(\widetilde{\mathcal{Z}}_{0}^{j}, M\right) \cup B\left(\widetilde{\mathcal{Z}}_{1}^{1}, M\right)
$$

et des distances entre ces points.

Démonstration. D'après le $2 \mathrm{~b})$ de la proposition $3.37, u_{x, r, t} K_{x, Q,\left(t_{1}, \ldots, t_{Q}\right)}\left(e_{\widetilde{S}_{0}}\right)$ ne dépend que de la connaissance des points de

$$
\begin{gathered}
\mathcal{B}(x, F) \cup B\left(\widetilde{S}_{0}, Q N\right) \\
\cup \bigcup_{\substack{a \in \widetilde{S}_{0} \\
j \in\{1, \ldots, Q\}}}\left\{y \in F-\operatorname{géod}(x, a)|| d(x, y)-t_{j} d(x, a) \mid \leq Q F\right\} \\
\cup \bigcup_{a \in \widetilde{S}_{0}}\{z \in F-\operatorname{géod}(x, a) \mid d(z, a) \in[r, r+Q F]\} \\
\cup \bigcup_{a \in \widetilde{S}_{0}}\{z \in F-\operatorname{géod}(x, a)|| d(x, z)-(1-t)(d(x, a)-r) \mid \leq Q F\}
\end{gathered}
$$

et des distances entre ces points. Il suffit donc de montrer que cet ensemble est inclus dans (97).

On suppose $F \leq 2 M$ et $Q N \leq M$, ce qui est permis par $\left(H_{M}\right)$. Donc (98) est inclus dans (97).

Soient $a \in \widetilde{S}_{0}, j \in\{1, \ldots, Q\}, y \in F-\operatorname{géod}(x, a)$ vérifiant

$$
\left|d(x, y)-t_{j} d(x, a)\right| \leq Q F .
$$

Soit $z \in \operatorname{géod}(x, a)$ vérifiant $d(x, z)=\mathrm{E}\left(t_{j} r_{0}(\widetilde{Z})\right)$, si bien que $z$ appartient à $\widetilde{Z}_{0}^{j}$. On a

$$
\left|t_{j} d(x, a)-\mathrm{E}\left(t_{j} r_{0}(\tilde{Z})\right)\right| \leq N+1,
$$

d'où $|d(x, y)-d(x, z)| \leq Q F+N+1$ et grâce à $\left(H_{\delta}(y, x, z, a)\right), d(y, z) \leq$ $(Q F+N+1)+F+\delta$. On suppose $(Q F+N+1)+F+\delta \leq M$, ce qui est permis par $\left(H_{M}\right)$. Donc (99) est inclus dans $\bigcup_{j \in\{1, \ldots, Q\}} B\left(\widetilde{\mathcal{Z}}_{0}^{j}, M\right)$ et a fortiori dans (97).

Soit $a \in \widetilde{S}_{0}$ et $z \in F$ - $\operatorname{gé}$ od $(x, a)$ vérifiant $d(z, a) \in[r, r+Q F]$. Soit $y \in \widetilde{S}_{1}$. Par le a) du lemme 4.14, on a $y \in 2 F$ - géod $(a, x)$. La condition (91) implique

$$
|d(x, a)-d(x, y)-r| \leq Q F+N .
$$


Comme $d(x, a)-d(x, z) \in[r-F, r+Q F]$, on en déduit

$$
|d(x, y)-d(x, z)| \leq 2 Q F+N .
$$

Comme $z \in F$ - $\operatorname{géod}(x, a)$ et $y \in 2 F$ - géod $(x, a),\left(H_{\delta}(z, x, y, a)\right)$ montre

$$
d(y, z) \leq(2 Q F+N)+2 F+\delta .
$$

On suppose $(2 Q F+N)+2 F+\delta \leq M$, ce qui est permis par $\left(H_{M}\right)$. Donc (100) est inclus dans $B\left(\widetilde{S}_{1}, M\right)$ et a fortiori dans (97).

Enfin soit $a \in \widetilde{S}_{0}$ et $z \in F$ - géod $(x, a)$ vérifiant

$$
|d(x, z)-(1-t)(d(x, a)-r)| \leq Q F .
$$

Soit $b \in \widetilde{S}_{1}$ et $y \in \operatorname{géod}(x, b)$ vérifiant $d(x, y)=\mathrm{E}\left((1-t) r_{1}(\tilde{Z})\right)$, si bien que $y$ appartient à $\widetilde{Z}_{1}^{1}$. Comme $y \in \operatorname{géod}(x, b)$ et $b \in 2 F-\operatorname{géod}(x, a)$, on a $y \in$ $2 F-\operatorname{géod}(x, a)$. Comme $d(x, a) \in\left[r_{0}(\widetilde{Z}), r_{0}(\widetilde{Z})+N\right]$ et grâce à $(91)$, on a

$$
\left|d(x, z)-(1-t) r_{1}(\tilde{Z})\right| \leq 2 Q F+N,
$$

d'où

$$
|d(x, y)-d(x, z)| \leq 2 Q F+1+N .
$$

Comme $z \in F$ - $\operatorname{géod}(x, a)$ et $y \in 2 F$ - $\operatorname{géod}(x, a),\left(H_{\delta}(y, x, z, a)\right)$ montre

$$
d(y, z) \leq(2 Q F+1+N)+2 F+\delta .
$$

On suppose $(2 Q F+1+N)+2 F+\delta \leq M$, ce qui est permis par $\left(H_{M}\right)$. Donc (101) est inclus dans $B\left(\widetilde{Z}_{1}^{1}, M\right)$ et a fortiori dans (97).

Sous-lemme 4.44. Le cardinal de $\Lambda_{Z, t,\left(t_{1}, \ldots, t_{Q}\right)}$ est majoré par une constante de la forme $C(\delta, K, N, Q, P, M)$.

Démonstration. Grâce au lemme 4.28, pour connaître les distances entre les points de

$$
B\left(\widetilde{S}_{0}, M\right) \cup \bigcup_{j \in\{1, \ldots, Q\}} B\left(\widetilde{\mathcal{Z}}_{0}^{j}, M\right) \cup B\left(\widetilde{\mathcal{Z}}_{1}^{1}, M\right)
$$

et ceux de

$$
\bigcup_{i \in\{1, \ldots, m+1\}} B\left(\tilde{S}_{i}, M\right) \cup \bigcup_{\substack{i \in\{0, \ldots, m\} \\ j \in\left\{1, \ldots, l_{i}\right\}}} B\left(\widetilde{y}_{i+1}^{j}, M\right) \cup B(x, k+2 M)
$$

il suffit de connaître les distances entre les points de (102) et $C$ points de (103), avec $C=C(\delta, K, N, Q, P, M)$ et grâce à (91) ces distances sont déterminées à $C^{\prime}=C(\delta, K, N, Q, P, M)$ près par les distances de $\widetilde{S}_{1}$ à ces $C$ points (qui font partie de la donnée de $Z$ ) et les entiers $r,\left(t_{0}^{j}(\widetilde{Z})\right)_{j \in\{1, \ldots, Q\}}$ et $t_{1}^{1}(\widetilde{Z})$, qui sont euxmêmes déterminés à $C^{\prime \prime}=C(\delta, K, N, Q, P, M)$ près par $r_{0}(Z), r, t, t_{1}, \ldots, t_{Q}$. 
Suite de la démonstration du lemme 4.40. On termine maintenant la preuve de l'inégalité (93). Pour $\widetilde{Z} \in \Lambda_{Z, t,\left(t_{1}, \ldots, t_{Q}\right)}$, et

$$
\begin{gathered}
\left(\tilde{a}_{1}, \ldots, \tilde{a}_{p-1}, \widetilde{S}_{0}, \ldots, \widetilde{S}_{m+1},\left(\widetilde{y}_{i}^{j}\right)_{i \in\{0, \ldots, m+1\}, j \in\left\{1, \ldots, \tilde{l}_{i}\right\}},\left(\tilde{\mathcal{Z}}_{0}^{j}\right)_{j \in\{1, \ldots, Q\}}, \widetilde{Z}_{1}^{1}\right) \\
\in\left(\pi_{x}^{\natural, p-1, k, m+1,\left(\tilde{l}_{0}, \ldots, \tilde{l}_{m+1}\right), Q, 1}\right)^{-1}(\widetilde{Z})
\end{gathered}
$$

on considère

$$
\sum_{\left(b_{1}, \ldots, b_{p}\right)}\left(u_{x, r, t} K_{x, Q,\left(t_{1}, \ldots, t_{Q}\right)}\left(e_{\tilde{a}_{1}} \wedge \cdots \wedge e_{\tilde{a}_{p-1}}\right)\right)\left(b_{1}, \ldots, b_{p}\right),
$$

où la somme porte sur les énumérations de $\widetilde{S}_{1}=\left\{b_{1}, \ldots, b_{p}\right\}$ telles que

$$
\left(b_{1}, \ldots, b_{p}, \widetilde{S}_{1}, \ldots, \widetilde{S}_{m+1},\left(\tilde{y}_{i+1}^{j}\right)_{i \in\{0, \ldots, m\}, j \in\left\{1, \ldots, l_{i}\right\}}\right) \in\left(\pi_{x}^{p, k, m,\left(l_{0}, \ldots, l_{m}\right)}\right)^{-1}(Z) .
$$

Comme la somme (104) a au plus $p$ ! termes, le 3) de la proposition 3.37 montre qu'elle est majorée par une constante de la forme $C(\delta, K, N, Q, P, M)$. D'après le sous-lemme 4.43 la somme (104) ne dépend que de $\widetilde{Z}$ et on peut donc la noter $\alpha_{Z, \tilde{Z}, t,\left(t_{1}, \ldots, t_{Q}\right)}$. D'après les sous-lemmes 4.41 et 4.42 on a

$$
\xi_{Z}\left(u_{x, r, t} K_{x, Q,\left(t_{1}, \ldots, t_{Q}\right)} f\right)=\frac{1}{(p-1) !} \sum_{\tilde{Z} \in \Lambda_{Z, t_{,}\left(t_{1}, \ldots, t_{Q}\right)}} \alpha_{Z, \tilde{Z}, t,\left(t_{1}, \ldots, t_{Q}\right)} \xi_{\widetilde{Z}}(f) .
$$

Par Cauchy-Schwarz et grâce au sous-lemme 4.44, on en déduit que

$$
\left|\xi_{Z}\left(u_{x, r, t} K_{x, Q,\left(t_{1}, \ldots, t_{Q}\right)} f\right)\right|^{2} \leq C \sum_{\tilde{Z} \in \Lambda_{Z, t,\left(t_{1}, \ldots, t_{Q}\right)}}\left|\xi_{\widetilde{Z}}(f)\right|^{2}
$$

avec $C=C(\delta, K, N, Q, P, M)$.

Sous-lemme 4.45. Il existe $D^{\prime}=C(\delta, K)$ et $C=C(\delta, K, N, Q, P, M)$ tels que pour tout

$$
\left(a_{1}, \ldots, a_{p}, S_{0}, \ldots, S_{m},\left(y_{i}^{j}\right)_{i \in\{0, \ldots, m\}, j \in\left\{1, \ldots, l_{i}\right\}}\right) \in Y_{x}^{p, k, m,\left(l_{0}, \ldots, l_{m}\right)}
$$

le nombre de possibilités pour $\left(\tilde{a}_{1}, \ldots, \tilde{a}_{p-1}\right)$ tels que

$$
\left(\tilde{a}_{1}, \ldots, \tilde{a}_{p-1},\left\{\tilde{a}_{1}, \ldots, \tilde{a}_{p-1}\right\}, S_{0}, \ldots, S_{m},\left(y_{i-1}^{j}\right)_{i \in\{0, \ldots, m+1\}, j \in\left\{1, \ldots, \tilde{I}_{i}\right\}}\right)
$$

appartienne à $Y_{x}^{p-1, k, m+1,\left(\tilde{l}_{0}, \ldots, \tilde{l}_{m+1}\right)}$ et vérifie

$$
\left|d\left(x,\left\{\tilde{a}_{1}, \ldots, \tilde{a}_{p-1}\right\}\right)-d\left(x, S_{0}\right)-r\right| \leq Q F
$$

soit $\leq C e^{D^{\prime} r}$. 
Démonstration. Il existe $D^{\prime}=C(\delta, K)$ tel que tout $y \in X$ et pour tout $R \in \mathbb{N}$, $\sharp B(y, R) \leq e^{D^{\prime} R}$. Pour tout $a \in\left\{\tilde{a}_{1}, \ldots, \tilde{a}_{p-1}\right\}$, grâce à (107) et au fait que $S_{0} \subset$ $2 F-\operatorname{géod}(a, x)$ on a $d\left(a, S_{0}\right) \leq r+Q F+N+2 F$.

Fin de la démonstration du lemme 4.40. Grâce à (91), on peut appliquer le souslemme 4.45 et grâce au lemme 4.29 , on en déduit qu'il existe $D^{\prime}=C(\delta, K)$ et $C=C(\delta, K, N, Q, P, M)$ tels que pour $\widetilde{Z} \in \Lambda_{Z, t,\left(t_{1}, \ldots, t_{Q}\right)}$,

$$
\sharp\left(\left(\pi_{x}^{\natural, p-1, k, m+1,\left(\tilde{l}_{0}, \ldots, \tilde{l}_{m+1}\right), Q, 1}\right)^{-1}(\widetilde{Z})\right) \leq \sharp\left(\left(\pi_{x}^{p, k, m,\left(l_{0}, \ldots, l_{m}\right)}\right)^{-1}(Z)\right) C e^{D^{\prime} r} .
$$

Grâce à (91) on a

$$
e^{2 s\left(r_{0}(Z)-k\right)} \leq e^{2 s\left(r_{0}(\tilde{Z})-k\right)} e^{Q F} e^{-2 s r} .
$$

On suppose $\alpha D^{\prime} \leq s$, ce qui est permis par $\left(H_{\alpha}\right)$. Par conséquent il existe $C=$ $C(\delta, K, N, Q, P, M)$ tel que

$$
\begin{aligned}
& \left(e^{2 s\left(r_{0}(Z)-k\right)} \sharp\left(\left(\pi_{x}^{p, k, m,\left(l_{0}, \ldots, l_{m}\right)}\right)^{-1}(Z)\right)^{-\alpha}\right) \\
& \quad \leq C e^{-s r}\left(e^{2 s\left(r_{0}(\tilde{Z})-k\right)} \sharp\left(\left(\pi_{x}^{p-1, k, m+1,\left(\tilde{l}_{0}, \ldots, \tilde{l}_{m+1}\right)}\right)^{-1}(\widetilde{Z})\right)^{-\alpha}\right) .
\end{aligned}
$$

L'inégalité (93) résulte de (106) et (108). On a montré (93) et donc (90) et (89). Ceci termine la preuve du lemme 4.40 .

On a donc montré la proposition 4.30 .

4.6. Continuité de $e^{\tau \theta_{x}^{\mathrm{b}}} \partial \boldsymbol{e}^{-\tau \theta_{x}^{\mathrm{b}}}$ et $e^{\tau \theta_{x}^{\mathrm{b}}} J_{x} e^{-\tau \theta_{x}^{\mathrm{b}}}$. Le but de ce sous-paragraphe est de montrer la proposition 4.46 .

Proposition 4.46. Pour tout $T \in \mathbb{R}_{+}$et tout $r \in \mathbb{N}$,

$$
\left(e^{\tau \theta_{x}^{\mathrm{b}}} \partial e^{-\tau \theta_{x}^{\mathrm{b}}}\right)_{\tau \in[0, T]}, \quad\left(e^{\tau \theta_{x}^{\mathrm{b}}} J_{x} e^{-\tau \theta_{x}^{\mathrm{b}}}\right)_{\tau \in[0, T]}, \quad\left(e^{\tau \theta_{x}^{\mathrm{b}}} h_{x} e^{-\tau \theta_{x}^{\mathrm{b}}}\right)_{\tau \in[0, T]}
$$

et

$$
\left(e^{\tau \theta_{x}^{\mathrm{b}}} u_{x, r} K_{x} e^{-\tau \theta_{x}^{\mathrm{b}}}\right)_{\tau \in[0, T]}
$$

s'étendent en des opérateurs continus sur le $\mathbb{C}[0, T]$-module hilbertien $\mathscr{H}_{x, s}[0, T]$.

On a inclus les opérateurs $h_{x}$ et $u_{x, r} K_{x}$ dans l'énoncé de cette proposition pour un usage ultérieur.

On rappelle que pour tout $t \in \mathbb{R}_{+}$et pour $p \in\left\{1, \ldots, p_{\max }\right\}$, on a défini $\theta_{x}^{b}: \mathbb{C}^{\left(\Delta_{p}\right)} \rightarrow \mathbb{C}^{\left(\Delta_{p}\right)}$ par $\theta_{x}^{\mathrm{b}}\left(e_{S}\right)=\rho_{x}^{\mathrm{b}}(S) e_{S}$ pour tout $S \in \Delta_{p}$ et que la fonction $\rho_{x}^{b}: X \rightarrow \mathbb{R}_{+}$avait été définie par $\rho_{x}^{b}(a)=d^{b}(x, a)$ et étendue en une fonction $\rho_{x}^{b}: \Delta \rightarrow \mathbb{R}_{+}$par la formule

$$
\rho_{x}^{b}(S)=\frac{\sum_{a \in S} \rho_{x}^{b}(a)}{\sharp S} \quad \text { si } S \text { est non vide et } \rho_{x}^{b}(\emptyset)=0 .
$$


De façon analogue on définit $\rho_{x}: X \rightarrow \mathbb{R}_{+}$en posant $\rho_{x}(a)=d(x, a)$ et on étend cette fonction en $\rho_{x}: \Delta \rightarrow \mathbb{R}_{+}$par la formule

$$
\rho_{x}(S)=\frac{\sum_{a \in S} \rho_{x}(a)}{\sharp S} \quad \text { si } S \text { est non vide et } \rho_{x}(\emptyset)=0 .
$$

D'après la proposition 3.49, pour $x, y \in X$, on a $d(x, y) \leq d^{\mathrm{b}}(x, y) \leq d(x, y)+7 \delta$. Il en résulte que pour tout $S \in \Delta$ on a $\rho_{x}(S) \leq \rho_{x}^{b}(S) \leq \rho_{x}(S)+7 \delta$. On définit l'opérateur $\theta_{x}: \mathbb{C}^{\left(\Delta_{p}\right)} \rightarrow \mathbb{C}^{\left(\Delta_{p}\right)}$ par $\theta_{x}\left(e_{S}\right)=\rho_{x}(S) e_{S}$.

Démonstration de la proposition 4.46 en admettant les lemmes 4.47 et 4.48 . Pour $\tau \in \mathbb{R}_{+}$et $p \in\left\{1, \ldots, p_{\max }\right\}$, on introduit les opérateurs $e^{\tau\left(\theta_{x}^{\mathrm{b}}-\theta_{x}\right)}: \mathbb{C}^{\left(\Delta_{p}\right)} \rightarrow \mathbb{C}^{\left(\Delta_{p}\right)}$ et $e^{\tau \theta_{x}}: \mathbb{C}^{\left(\Delta_{p}\right)} \rightarrow \mathbb{C}^{\left(\Delta_{p}\right)}$ en posant

$$
e^{\tau\left(\theta_{x}^{\mathrm{b}}-\theta_{x}\right)}\left(e_{S}\right)=e^{\tau\left(\theta_{x}^{\mathrm{b}}(S)-\theta_{x}(S)\right)} e_{S} \quad \text { et } \quad e^{\tau \theta_{x}}\left(e_{S}\right)=e^{\tau \theta_{x}(S)} e_{S} .
$$

Ces opérateurs commutent entre eux et on a bien sûr $e^{\tau \theta_{x}^{b}}=e^{\tau\left(\theta_{x}^{b}-\theta_{x}\right)} e^{\tau \theta_{x}}$. Pour $p=0$ on définit $\theta_{x}^{\mathrm{b}}$ et $\theta_{x}$ comme 0 sur $\mathbb{C}^{\left(\Delta_{0}\right)}=\mathbb{C}$.

La proposition 4.46 résulte des lemmes 4.47 et 4.48 .

Lemme 4.47. Pour tout $T \in \mathbb{R}_{+}$et tout $r \in \mathbb{N}$,

$$
\left(e^{\tau \theta_{x}} \partial e^{-\tau \theta_{x}}\right)_{\tau \in[0, T]}, \quad\left(e^{\tau \theta_{x}} J_{x} e^{-\tau \theta_{x}}\right)_{\tau \in[0, T]}, \quad\left(e^{\tau \theta_{x}} h_{x} e^{-\tau \theta_{x}}\right)_{\tau \in[0, T]}
$$

et

$$
\left(e^{\tau \theta_{x}} u_{x, r} K_{x} e^{-\tau \theta_{x}}\right)_{\tau \in[0, T]}
$$

s'étendent en des opérateurs continus sur le $\mathbb{C}[0, T]$-module hilbertien $\mathscr{H}_{x, s}[0, T]$.

Pour la démonstration de ce lemme on a besoin de la notation suivante.

Notation. Pour $Z \in \bar{Y}_{x}^{p, k, m,\left(l_{0}, \ldots, l_{m}\right)}$ et

$$
\left(a_{1}, \ldots, a_{p}, S_{0}, \ldots, S_{m},\left(y_{i}^{j}\right)_{i \in\{0, \ldots, m\}, j \in\left\{1, \ldots, l_{i}\right\}}\right) \in\left(\pi_{x}^{p, k, m,\left(l_{0}, \ldots, l_{m}\right)}\right)^{-1}(Z),
$$

$\rho_{x}\left(S_{0}\right)$ et $\rho_{x}\left(S_{1}\right)$ ne dépendent que de $Z$ et on les note $\rho_{x}^{0}(Z)$ et $\rho_{x}^{1}(Z)$. On adopte une notation similaire pour $Z \in \bar{Y}_{x}^{\natural, p, k, m,\left(l_{0}, \ldots, l_{m}\right), \lambda_{0}, \lambda_{1}}$.

Remarque. Il est évidemment faux que $\rho_{x}^{b}\left(S_{0}\right)$ et $\rho_{x}^{b}\left(S_{1}\right)$ ne dépendent que de $Z$ et c'est pour cette raison que la preuve de la proposition 4.46 n'est pas aussi simple que celle du lemme 4.47.

Démonstration du lemme 4.47. On reprend la démonstration des propositions 4.21 et 4.30 du sous-paragraphe précédent, qui affirmaient la continuité de $\partial, J_{x}, h_{x}$ et $u_{x, r} K_{x}$. Les seuls ingrédients supplémentaires sont les faits suivants : 
- dans les notations ci-dessus $\rho_{x}\left(S_{0}\right)$ et $\rho_{x}\left(S_{1}\right)$ ne dépendent que de $Z$,

- d'après le 1a) et le 2a) de la proposition 3.37, il existe $C=C(\delta, K, N, Q)$ tel que si $e_{T}$ apparait dans $\partial\left(e_{S}\right)$ ou $J_{x}\left(e_{S}\right)$ avec un coefficient non nul, on a $d(x, T) \leq d(x, S)+C$, d'où $\rho_{x}(T) \leq \rho_{x}(S)+C+N$.

Voici de façon plus précise les modifications à apporter :

- pour la proposition 4.21, dans (50) on remplace $\xi_{\widetilde{Z}}(f) \operatorname{par} e^{\tau\left(\rho_{x}^{1}(\tilde{Z})-\rho_{x}^{0}(\tilde{Z})\right)} \xi_{\widetilde{Z}}(f)$ et on remarque que $\rho_{x}^{1}(\tilde{Z})-\rho_{x}^{0}(\tilde{Z}) \leq N$,

- pour le lemme 4.32, dans (73) on remplace $\xi_{Z}(f)$ par $e^{\tau\left(\rho_{x}(U)-\rho_{x}^{0}(Z)\right)} \xi_{Z}(f)$ et on remarque que $\rho_{x}(U) \leq P+N$,

- pour le lemme 4.36 , dans (88) on remplace $\xi_{\widetilde{Z}}(f)$ par $e^{\tau\left(\rho_{x}^{1}(\tilde{Z})-\rho_{x}^{0}(\widetilde{Z})\right)} \xi_{\widetilde{Z}}(f)$ et on remarque que $\rho_{x}^{1}(\widetilde{Z})-\rho_{x}^{0}(\widetilde{Z}) \leq(q+2) N$ grâce à $(77)$,

- pour le lemme 4.40, dans (105) on remplace $\xi_{\widetilde{Z}}(f) \operatorname{par} e^{\tau\left(\rho_{x}^{1}(\tilde{Z})-\rho_{x}^{0}(\widetilde{Z})\right)} \xi_{\widetilde{Z}}(f)$ et on remarque que $\rho_{x}^{1}(\widetilde{Z})-\rho_{x}^{0}(\widetilde{Z}) \leq Q F+N$ par (91)

et en plus on remplace les opérateurs par les opérateurs conjugués à de nombreux endroits (notamment dans les égalités (50), (73), (88) et (105)).

En utilisant le fait que les fonctions à support fini sont denses dans $\mathscr{H}_{x, s}$ on montre que les opérateurs du lemme 4.47 sont continus en $\tau$ pour la topologie forte et leurs adjoints aussi. Ceci justifie le fait qu'ils s'étendent en des morphismes de $\mathbb{C}[0, T]$-modules hilbertiens.

Lemme 4.48. Pour tout $T \in \mathbb{R}_{+}$, l'opérateur $\left(e^{\tau\left(\theta_{x}^{b}-\theta_{x}\right)}\right)_{\tau \in[0, T]}$ s'étend en un automorphisme du $\mathbb{C}[0, T]$-module hilbertien $\mathscr{H}_{x, s}[0, T]$.

Démonstration du lemme 4.48 en admettant le lemme 4.49. Soit $p \in\left\{1, \ldots, p_{\max }\right\}$. Le lemme 4.48 résulte immédiatement du lemme suivant.

Lemme 4.49. L'opérateur $\theta_{x}^{\mathrm{b}}-\theta_{x}$ s'étend en un opérateur continu sur $\mathscr{H}_{x, s}\left(\Delta_{p}\right)$, dont la norme est bornée par une constante du type $C(\delta, N, K, Q, P, M, s, B)$.

Bien entendu cet énoncé n'est pas vrai pour les opérateurs $\theta_{x}^{\text {b }}$ et $\theta_{x}$ séparément. Pour $u_{1}, u_{2}, u_{3}, v_{1}, v_{2}, v_{3} \in\left[0,1\left[\right.\right.$ on définit $\left(\rho_{x}^{b}\right)_{u_{1}, u_{2}, u_{3}}^{v_{1}, v_{2}, v_{3}}: X \rightarrow \mathbb{R}_{+}$en posant $\left(\rho_{x}^{b}\right)_{u_{1}, u_{2}, u_{3}}^{v_{1}, v_{2}, v_{3}}(a)=d^{\mathrm{b}}{ }_{u_{1}, u_{2}, u_{3}}^{v_{1}, v_{2}, v_{3}}(x, a)$ et on l'étend en une fonction $\left(\rho_{x}^{b}\right)_{u_{1}, u_{2}, u_{3}}^{v_{1}, v_{2}, v_{3}}: \Delta \rightarrow$ $\mathbb{R}_{+}$par la formule

$\left(\rho_{x}^{b}\right)_{u_{1}, u_{2}, u_{3}}^{v_{1}, v_{2}, v_{3}}(S)=\frac{\sum_{a \in S}\left(\rho_{x}^{b}\right)_{u_{1}, u_{2}, u_{3}}^{v_{1}, v_{2}, v_{3}}(a)}{\sharp S}$ si $S$ est non vide et $\left(\rho_{x}^{b}\right)_{u_{1}, u_{2}, u_{3}}^{v_{1}, v_{2}, v_{3}}(\emptyset)=0$.

Pour $u_{1}, u_{2}, u_{3}, v_{1}, v_{2}, v_{3} \in[0,1[$ et $S \in \Delta$ on a

$$
\rho_{x}(S) \leq\left(\rho_{x}^{\mathrm{b}}\right)_{u_{1}, u_{2}, u_{3}}^{v_{1}, v_{2}, v_{3}}(S) \leq \rho_{x}(S)+7 \delta .
$$


De plus, pour $S \in \Delta$,

$$
\left(\rho_{x}^{\mathrm{b}}\right)(S)=\int_{u_{1}, u_{2}, u_{3}, v_{1}, v_{2}, v_{3} \in[0,1[}\left(\rho_{x}^{\mathrm{b}}\right)_{u_{1}, u_{2}, u_{3}}^{v_{1}, v_{2}, v_{3}}(S) d u_{1} d u_{2} d u_{3} d v_{1} d v_{2} d v_{3} .
$$

Lemme 4.50. Pour $S \in \Delta_{p}$, $\left(\rho_{x}^{b}\right)_{u_{1}, u_{2}, u_{3}}^{v_{1}, v_{2}, v_{3}}(S)$ ne dépend que de la connaissance des points de

$$
\{x\} \cup S \cup \bigcup_{\substack{a \in S \\ j \in\{1, \ldots, 6\}}}\left\{y \in 3 \delta-\operatorname{géod}(x, a)|| d(x, y)-w_{j} \mid \leq N+6 \delta+4\right\}
$$

et des distances entre ces points, où l'on note

$$
\begin{aligned}
& w_{1}=\mathrm{E}\left(\frac{u_{1}}{6} d(x, S)\right), \\
& w_{2}=\mathrm{E}\left(\left(\frac{1}{6}+\frac{u_{2}}{6}\right) d(x, S)\right), \\
& w_{3}=\mathrm{E}\left(\left(\frac{2}{6}+\frac{u_{3}}{6}\right) d(x, S)\right), \\
& w_{4}=\mathrm{E}\left(\left(1-\frac{v_{1}}{6}\right) d(x, S)\right), \\
& w_{5}=\mathrm{E}\left(\left(\frac{5}{6}-\frac{v_{2}}{6}\right) d(x, S)\right), \\
& w_{6}=\mathrm{E}\left(\left(\frac{4}{6}-\frac{v_{3}}{6}\right) d(x, S)\right) .
\end{aligned}
$$

Démonstration. L'énoncé est évident si $d(x, S) \leq 6 \delta$ et on suppose donc $d(x, S)>$ $6 \delta$. Par la construction même de $d^{b},\left(\rho_{x}^{b}\right)_{u_{1}, u_{2}, u_{3}}^{v_{1}, v_{2}, v_{3}}(S)$ ne dépend que de la connaissance de $x$, de $S$ et de la réunion pour $a \in S$ de

$$
\begin{aligned}
& Y_{x, a}^{\mathrm{E}\left(\left(\Delta_{x, a}+1\right) u_{1}\right)} \cup Y_{x, a}^{\Delta_{x, a}+\mathrm{E}\left(\left(\Delta_{x, a}+1\right) u_{2}\right)} \cup Y_{x, a}^{2 \Delta_{x, a}+\mathrm{E}\left(\left(\Delta_{x, a}+1\right) u_{3}\right)} \\
& \quad \cup Y_{a, x}^{\mathrm{E}\left(\left(\Delta_{x, a}+1\right) v_{1}\right)} \cup Y_{a, x}^{\Delta_{x, a}+\mathrm{E}\left(\left(\Delta_{x, a}+1\right) v_{2}\right)} \cup Y_{a, x}^{2 \Delta_{x, a}+\mathrm{E}\left(\left(\Delta_{x, a}+1\right) v_{3}\right)} .
\end{aligned}
$$

Soit $a \in S$. On rappelle que $\Delta_{x, a}=\mathrm{E}\left(\frac{d(x, a)}{6}\right)-\delta$ et que pour $u, v \in X$ et $r \in$ $\left\{0, \ldots, \mathrm{E}\left(\frac{d(u, v)}{2}\right)-3 \delta\right\}$, on note $Y_{u, v}^{r}$ l'ensemble des points $z \in 3 \delta$ - $\operatorname{géod}(u, v)$ tels que $d(u, z) \in\{r, \ldots, r+3 \delta\}$. Il suffit donc de montrer que l'ensemble (110) est inclus dans l'ensemble

$$
\bigcup_{j \in\{1, \ldots, 6\}}\left\{y \in 3 \delta-\operatorname{géod}(x, a)|| d(x, y)-w_{j} \mid \leq N+6 \delta+4\right\} .
$$

On a $\left|\Delta_{x, a}-\frac{d(x, a)}{6}\right| \leq \delta+1$ et pour $i \in\{1, \ldots, 3\}$,

$\left|\mathrm{E}\left(\left(\Delta_{x, a}+1\right) u_{i}\right)-\frac{u_{i} d(x, a)}{6}\right| \leq \delta+1 \quad$ et $\quad\left|\mathrm{E}\left(\left(\Delta_{x, a}+1\right) v_{i}\right)-\frac{v_{i} d(x, a)}{6}\right| \leq \delta+1$.

Il en résulte facilement que pour $i \in\{1, \ldots, 3\}$ et $y \in Y_{x, a}^{(i-1) \Delta_{x, a}+\mathrm{E}\left(\left(\Delta_{x, a}+1\right) u_{i}\right)}$ on a

$$
\begin{aligned}
d(x, y) & \in\left[(i-1) \Delta_{x, a}+\mathrm{E}\left(\left(\Delta_{x, a}+1\right) u_{i}\right),(i-1) \Delta_{x, a}+\mathrm{E}\left(\left(\Delta_{x, a}+1\right) u_{i}\right)+3 \delta\right] \\
& \subset\left[\frac{\left((i-1)+u_{i}\right) d(x, a)}{6}-(3 \delta+3), \frac{\left((i-1)+u_{i}\right) d(x, a)}{6}+(6 \delta+3)\right] .
\end{aligned}
$$


D'autre part $\left|\frac{\left((i-1)+u_{i}\right) d(x, a)}{6}-w_{i}\right| \leq N+1$ car $|d(x, a)-d(x, S)| \leq N$. On en déduit que $y$ appartient à (111).

Soit maintenant $i \in\{1, \ldots, 3\}$ et $y \in Y_{a, x}^{(i-1) \Delta_{x, a}+\mathrm{E}\left(\left(\Delta_{x, a}+1\right) v_{i}\right)}$. Donc $d(a, y)$ appartient à

$$
\begin{gathered}
{\left[(i-1) \Delta_{x, a}+\mathrm{E}\left(\left(\Delta_{x, a}+1\right) v_{i}\right),(i-1) \Delta_{x, a}+\mathrm{E}\left(\left(\Delta_{x, a}+1\right) v_{i}\right)+3 \delta\right]} \\
\subset\left[\frac{\left((i-1)+v_{i}\right) d(x, a)}{6}-(3 \delta+3), \frac{\left((i-1)+v_{i}\right) d(x, a)}{6}+(6 \delta+3)\right] .
\end{gathered}
$$

Comme $y \in 3 \delta-\operatorname{géod}(x, a)$ on a $d(x, y) \in[d(x, a)-d(a, y), d(x, a)-d(a, y)+3 \delta]$, et comme $d(a, y)$ appartient à (112) on en déduit que $d(x, y)$ appartient à

$$
\left[\frac{\left(7-i-v_{i}\right) d(x, a)}{6}-(6 \delta+3), \frac{\left(7-i-v_{i}\right) d(x, a)}{6}+(6 \delta+3)\right] .
$$

D'autre part $\left|\frac{\left(7-i-v_{i}\right) d(x, a)}{6}-w_{i+3}\right| \leq N+1 \operatorname{car}|d(x, a)-d(x, S)| \leq N$. On en déduit que $y$ appartient à (111).

Enfin on note $\left(\theta_{x}^{b}\right)_{u_{1}, u_{2}, u_{3}}^{v_{1}, v_{2}, v_{3}}: \mathbb{C}^{\left(\Delta_{p}\right)} \rightarrow \mathbb{C}^{\left(\Delta_{p}\right)}$ l'opérateur défini par

$$
\left(\theta_{x}^{b}\right)_{u_{1}, u_{2}, u_{3}}^{v_{1}, v_{2}, v_{3}}\left(e_{S}\right)=\left(\rho_{x}^{b}\right)_{u_{1}, u_{2}, u_{3}}^{v_{1}, v_{2}, v_{3}}(S) e_{S},
$$

de sorte que

$$
\theta_{x}^{b}=\int_{u_{1}, u_{2}, u_{3}, v_{1}, v_{2}, v_{3} \in[0,1[}\left(\theta_{x}^{b}\right)_{u_{1}, u_{2}, u_{3}}^{v_{1}, v_{2}, v_{3}} d u_{1} d u_{2} d u_{3} d v_{1} d v_{2} d v_{3} .
$$

Démonstration du lemme 4.49. Soit $f \in \mathbb{C}^{\left(\Delta_{p}\right)}$. Par définition

$$
\begin{aligned}
\left\|\left(\theta_{x}^{b}-\theta_{x}\right)(f)\right\|_{\mathcal{H}_{x, s}\left(\Delta_{p}\right)}^{2} & \sum_{k, m, l_{0}, \ldots, l_{m} \in \mathbb{N}} B^{-\left(m+\sum_{i=0}^{m} l_{i}\right)} \sum_{Z \in \bar{Y}_{x}^{p, k, m,\left(l_{0}, \ldots, l_{m}\right)}} e^{2 s\left(r_{0}(Z)-k\right)} \\
& \quad\left(\prod_{i=0}^{m} s_{i}(Z)^{-l_{i}}\right) \sharp\left(\left(\pi_{x}^{p, k, m,\left(l_{0}, \ldots, l_{m}\right)}\right)^{-1}(Z)\right)^{-\alpha}\left|\xi_{Z}\left(\left(\theta_{x}^{b}-\theta_{x}\right)(f)\right)\right|^{2} .
\end{aligned}
$$

On va voir que le lemme 4.49 résulte de l'inégalité (113) ci-dessous.

Soient $k, m, l_{0}, \ldots, l_{m} \in \mathbb{N}$ et $Z \in \bar{Y}_{x}^{p, k, m,\left(l_{0}, \ldots, l_{m}\right)}$. On va montrer qu'il existe $C=C(\delta, K, N, Q, P, M)$ tel que

$$
\left|\xi_{Z}\left(\left(\theta_{x}^{b}-\theta_{x}\right)(f)\right)\right|^{2} \leq C \sum_{\tilde{Z} \in \Lambda_{Z}}\left(r_{0}(\tilde{Z})+1\right)^{-6}\left|\xi_{\tilde{Z}}(f)\right|^{2}
$$

où $\Lambda_{Z}$ est la partie de $\bar{Y}_{x}^{\natural, p, k, m,\left(l_{0}, \ldots, l_{m}\right), 6,0}$ formée des $\widetilde{Z}$ tels que pour tout

$$
\begin{gathered}
\left(\tilde{a}_{1}, \ldots, \tilde{a}_{p}, \tilde{S}_{0}, \ldots, \widetilde{S}_{m},\left(\tilde{\mathcal{Y}}_{i}^{j}\right)_{i \in\{0, \ldots, m\}, j \in\left\{1, \ldots, l_{i}\right\}},\left(\tilde{Z}_{0}^{j}\right)_{j \in\{1, \ldots, 6\}}\right) \\
\in\left(\pi_{x}^{\natural, p, k, m,\left(l_{0}, \ldots, l_{m}\right), 6,0}\right)^{-1}(\widetilde{Z})
\end{gathered}
$$


on ait

$$
\left(\tilde{a}_{1}, \ldots, \tilde{a}_{p}, \tilde{S}_{0}, \ldots, \widetilde{S}_{m},\left(\tilde{\mathcal{Y}}_{i}^{j}\right)_{i \in\{0, \ldots, m\}, j \in\left\{1, \ldots, l_{i}\right\}}\right) \in\left(\pi_{x}^{p, k, m,\left(l_{0}, \ldots, l_{m}\right)}\right)^{-1}(Z) .
$$

On justifie maintenant le fait que l'inégalité (113) implique l'énoncé du lemme. Le lemme 4.29 montre que pour $\widetilde{Z} \in \Lambda_{Z}$ on a

$$
\sharp\left(\pi_{x}^{\natural, p, k, m,\left(l_{0}, \ldots, l_{m}\right), 6,0}\right)^{-1}(\widetilde{Z}) \leq C \sharp\left(\pi_{x}^{p, k, m,\left(l_{0}, \ldots, l_{m}\right)}\right)^{-1}(Z)
$$

avec $C=C(\delta, K, N, Q, P, M)$. En sommant sur $Z$ et en appliquant le lemme 4.24 à $\mu_{0}=6$ et $\mu_{1}=0$, on voit que l'inégalité (113) implique l'énoncé du lemme.

L'inégalité (113) découle de l'inégalité (114) plus précise ci-dessous. Soient $u_{1}, u_{2}, u_{3}, v_{1}, v_{2}, v_{3} \in[0,1[$. On va montrer qu'il existe $C=C(\delta, K, N, Q, P, M)$ tel que

$$
\left|\xi_{Z}\left(\left(\left(\theta_{x}^{b}\right)_{u_{1}, u_{2}, u_{3}}^{v_{1}, v_{2}, v_{3}}-\theta_{x}\right)(f)\right)\right|^{2} \leq C \sum_{\tilde{Z} \in\left(\Lambda_{Z}\right) \sum_{u_{1}, u_{2}, u_{3}}^{v_{1}, v_{2}, v_{3}}}\left|\xi_{\widetilde{Z}}(f)\right|^{2}
$$

où $\left(\Lambda_{Z}\right)_{u_{1}, u_{2}, u_{3}}^{v_{1}, v_{2}, v_{3}}$ est l'ensemble des $\widetilde{Z} \in \Lambda_{Z}$ tels que, en notant

$$
\begin{aligned}
& w_{1}=\mathrm{E}\left(\frac{u_{1}}{6} r_{0}(\widetilde{Z})\right), \\
& w_{2}=\mathrm{E}\left(\left(\frac{1}{6}+\frac{u_{2}}{6}\right) r_{0}(\tilde{Z})\right), \\
& w_{3}=\mathrm{E}\left(\left(\frac{2}{6}+\frac{u_{3}}{6}\right) r_{0}(\widetilde{Z})\right), \\
& w_{4}=\mathrm{E}\left(\left(1-\frac{v_{1}}{6}\right) r_{0}(\widetilde{Z})\right), \\
& w_{5}=\mathrm{E}\left(\left(\frac{5}{6}-\frac{v_{2}}{6}\right) r_{0}(\widetilde{Z})\right), \\
& w_{6}=\mathrm{E}\left(\left(\frac{4}{6}-\frac{v_{3}}{6}\right) r_{0}(\widetilde{Z})\right)
\end{aligned}
$$

on ait, pour tout

$$
\begin{gathered}
\left(\tilde{a}_{1}, \ldots, \tilde{a}_{p}, \tilde{S}_{0}, \ldots, \tilde{S}_{m},\left(\tilde{\mathcal{Y}}_{i}^{j}\right)_{i \in\{0, \ldots, m\}, j \in\left\{1, \ldots, l_{i}\right\}},\left(\widetilde{Z}_{0}^{j}\right)_{j \in\{1, \ldots, 6\}}\right) \\
\in\left(\pi_{x}^{\natural, p, k, m,\left(l_{0}, \ldots, l_{m}\right), 6,0}\right)^{-1}(\widetilde{Z})
\end{gathered}
$$

et pour tout $j \in\{1, \ldots, 6\}$,

$$
\widetilde{\mathcal{Z}}_{0}^{j}=\bigcup_{b \in \widetilde{S}_{0}}\left\{z \in \operatorname{géod}(x, b) \mid d(x, z)=w_{j}\right\} .
$$

La condition (115) implique que pour $\widetilde{Z} \in\left(\Lambda_{Z}\right)_{u_{1}, u_{2}, u_{3}}^{v_{1}, v_{2}, v_{3}}$ et pour $j \in\{1, \ldots, 6\}$, on a $t_{0}^{j}(\widetilde{Z})=w_{j}$.

Sous-lemme 4.51. Soit

$$
\left(\tilde{a}_{1}, \ldots, \tilde{a}_{p}, \widetilde{S}_{0}, \ldots, \widetilde{S}_{m},\left(\tilde{y}_{i}^{j}\right)_{i \in\{0, \ldots, m\}, j \in\left\{1, \ldots, l_{i}\right\}}\right) \in\left(\pi_{x}^{p, k, m,\left(l_{0}, \ldots, l_{m}\right)}\right)^{-1}(Z) .
$$


On définit $\left(\widetilde{Z}_{0}^{j}\right)_{j \in\{1, \ldots, 6\}}$ par (115). Alors les parties $\widetilde{Z}_{0}^{j}$ sont de diamètre inférieur ou égal à $P / 3$ et il existe $\tilde{Z} \in\left(\Lambda_{Z}\right)_{u_{1}, u_{2}, u_{3}}^{v_{1}, v_{2}, v_{3}}$ tel que

$$
\left(\tilde{a}_{1}, \ldots, \tilde{a}_{p}, \tilde{S}_{0}, \ldots, \tilde{S}_{m},\left(\tilde{\mathcal{Y}}_{i}^{j}\right)_{i \in\{0, \ldots, m\}, j \in\left\{1, \ldots, l_{i}\right\}},\left(\tilde{\mathcal{Z}}_{0}^{j}\right)_{j \in\{1, \ldots, 6\}}\right)
$$

appartienne à $\left(\pi_{x}^{\natural, p, k, m,\left(l_{0}, \ldots, l_{m}\right), 6,0}\right)^{-1}(\tilde{Z})$.

Démonstration. Soit $j \in\{1, \ldots, 6\}$ et $z, z^{\prime} \in \tilde{\mathcal{Z}}_{0}^{j}$. Soit $b \in \widetilde{S}_{0}$. On a $z, z^{\prime} \in$ $2 N-\operatorname{géod}(x, b)$ et $d(x, z)=d\left(x, z^{\prime}\right)$ donc $\operatorname{par}\left(H_{\delta}\left(z, x, z^{\prime}, b\right)\right), d\left(z, z^{\prime}\right) \leq 2 N+\delta \leq$ $P / 3$. Comme les parties $\widetilde{Z}_{0}^{j}$ sont non vides et $P / 3 \leq M$, l'argument que nous venons de donner montre aussi que la condition (115) est vérifiée par les autres éléments de la classe d'équivalence $\widetilde{Z}$ de l'élément (116) et donc $\widetilde{Z} \in\left(\Lambda_{Z}\right)_{u_{1}, u_{2}, u_{3}}^{v_{1}, v_{2}, v_{3}}$.

Sous-lemme 4.52. Pour $\widetilde{Z} \in\left(\Lambda_{Z}\right)_{u_{1}, u_{2}, u_{3}}^{v_{1}, v_{2}, v_{3}}$ et

$$
\begin{gathered}
\left(\tilde{a}_{1}, \ldots, \tilde{a}_{p}, \tilde{S}_{0}, \ldots, \tilde{S}_{m},\left(\tilde{\mathcal{Y}}_{i}^{j}\right)_{i \in\{0, \ldots, m\}, j \in\left\{1, \ldots, l_{i}\right\}},\left(\tilde{Z}_{0}^{j}\right)_{j \in\{1, \ldots, 6\}}\right) \\
\in\left(\pi_{x}^{\natural, p, k, m,\left(l_{0}, \ldots, l_{m}\right), 6,0}\right)^{-1}(\widetilde{Z}),
\end{gathered}
$$

$\left(\rho_{x}^{b}\right)_{u_{1}, u_{2}, u_{3}}^{v_{1}, v_{2}, v_{3}}\left(\widetilde{S}_{0}\right)$ ne dépend que de la connaissance des points de

$$
B(x, k+2 M) \cup B\left(\widetilde{S}_{0}, M\right) \cup \underset{j \in\{1, \ldots, 6\}}{\bigcup} B\left(\tilde{Z}_{0}^{j}, M\right)
$$

et des distances entre ces points.

Démonstration. Il suffit de montrer que l'ensemble (109) figurant dans le lemme 4.50 (avec $\widetilde{S}_{0}$ au lieu de $S$ ) est inclus dans (117). Soit $a \in \widetilde{S}_{0}, j \in\{1, \ldots, 6\}$, et $y \in$ $3 \delta$ - $\operatorname{géod}(x, a)$ vérifiant

$$
\left|d(x, y)-w_{j}\right| \leq N+6 \delta+4
$$

Soit $z \in \operatorname{géod}(x, a)$ vérifiant $d(x, z)=w_{j}$, si bien que $z$ appartient à $\widetilde{Z}_{0}^{j}$. Comme $|d(x, y)-d(x, z)| \leq N+6 \delta+4$ et que $y, z \in 3 \delta-\operatorname{géod}(x, a),\left(H_{\delta}(y, x, z, a)\right)$ implique $d(y, z) \leq(N+6 \delta+4)+3 \delta+\delta$. On suppose $(N+6 \delta+4)+3 \delta+\delta \leq M$, ce qui est permis par $\left(H_{M}\right)$. On a donc $d\left(y, \widetilde{Z}_{0}^{j}\right) \leq M$.

Sous-lemme 4.53. Le cardinal de $\left(\Lambda_{Z}\right)_{u_{1}, u_{2}, u_{3}}^{v_{1}, v_{2}, v_{3}}$ est majoré par une constante de la forme $C(\delta, K, N, Q, P, M)$.

Démonstration. Grâce au lemme 4.28, pour connaître les distances entre les points de

$$
\bigcup_{j \in\{1, \ldots, 6\}} B\left(\widetilde{\mathcal{Z}}_{0}^{j}, M\right)
$$


et ceux de

$$
\bigcup_{i \in\{0, \ldots, m\}} B\left(\widetilde{S}_{i}, M\right) \cup \underset{\substack{i \in\{0, \ldots, m\} \\ j \in\left\{1, \ldots, l_{i}\right\}}}{\bigcup} B\left(\tilde{y}_{i}^{j}, M\right) \cup B(x, k+2 M)
$$

il est suffisant de connaître les distances entre les points de (118) et $C$ points de (119), avec $C=C(\delta, K, N, Q, P, M)$ et ces distances sont déterminées à $C^{\prime}=C(\delta, K, N, Q, P, M)$ par $Z$ et $u_{1}, u_{2}, u_{3}, v_{1}, v_{2}, v_{3}$ (en fait le lemme 4.28 fournit un énoncé légèrement différent où $B\left(\widetilde{S}_{0}, M\right)$ figure dans (118) et non dans (119), mais il est clair que cet énoncé implique le nôtre).

Fin de la démonstration du lemme 4.49. D'après le sous-lemme 4.52, pour $\widetilde{Z} \in\left(\Lambda_{Z}\right)_{u_{1}, u_{2}, u_{3}}^{v_{1}, v_{2}, v_{3}}$ on a

$$
\xi_{\widetilde{Z}}\left(\left(\left(\theta_{x}^{b}\right)_{u_{1}, u_{2}, u_{3}}^{v_{1}, v_{2}, v_{3}}-\theta_{x}\right)(f)\right)=\left(\alpha_{\tilde{Z}}\right)_{u_{1}, u_{2}, u_{3}}^{v_{1}, v_{2}, v_{3}} \xi_{\widetilde{Z}}(f)
$$

$\operatorname{avec}\left|\left(\alpha_{\tilde{Z}}\right)_{u_{1}, u_{2}, u_{3}}^{v_{1}, v_{2}, v_{3}}\right| \leq 7 \delta$. Le sous-lemme 4.51 montre que pour $Z \in \bar{Y}_{x}^{p, k, m,\left(l_{0}, \ldots, l_{m}\right)}$,

$$
\xi_{Z}\left(\left(\left(\theta_{x}^{b}\right)_{u_{1}, u_{2}, u_{3}}^{v_{1}, v_{2}, v_{3}}-\theta_{x}\right)(f)\right)=\sum_{\tilde{Z} \in\left(\Lambda_{Z}\right)_{u_{1}, u_{2}, u_{3}}^{v_{1}, v_{2}, v_{3}}} \xi_{\widetilde{Z}}\left(\left(\left(\theta_{x}^{b}\right)_{u_{1}, u_{2}, u_{3}}^{v_{1}, v_{2}, v_{3}}-\theta_{x}\right)(f)\right) .
$$

Par Cauchy-Schwarz et grâce au sous-lemme 4.53 on en déduit (114). On a montré (114) donc (113) et ceci termine la démonstration du lemme 4.49.

4.7. Propriétés d'équivariance de la norme. Ce sous-paragraphe a pour but de montrer la proposition 4.3. On recommande au lecteur de commencer par lire le premier paragraphe de [Laf08], car la démonstration de la proposition 4.3 repose sur les mêmes idées que celle de la proposition 1.10 de [Laf08] mais sur des calculs beaucoup plus compliqués.

On fixe $p \in\left\{1, \ldots, p_{\max }\right\}$ et $x, x^{\prime} \in X$. On rappelle qu'après la définition 4.1, pour $k, m \in \mathbb{N}$ et $\left(l_{0}, \ldots, l_{m}\right) \in \mathbb{N}^{m+1}$ on a introduit une relation d'équivalence sur $Y_{x}^{p, k, m,\left(l_{0}, \ldots, l_{m}\right)}$, et noté $\bar{Y}_{x}^{p, k, m,\left(l_{0}, \ldots, l_{m}\right)}$ le quotient, et $\pi_{x}^{p, k, m,\left(l_{0}, \ldots, l_{m}\right)}$ l'application quotient. On va introduire maintenant une relation d'équivalence plus fine

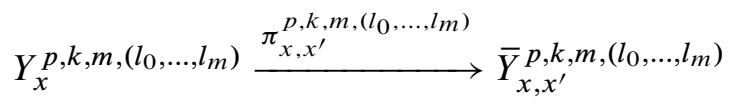

et une autre encore plus fine

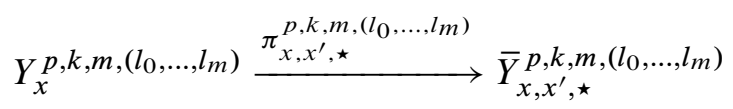

telles que tout élément de $\bar{Y}_{x}^{p, k, m,\left(l_{0}, \ldots, l_{m}\right)}$ a au plus $2 d\left(x, x^{\prime}\right)+1$ antécédents dans $\bar{Y}_{x, x^{\prime}}^{p, k, m,\left(l_{0}, \ldots, l_{m}\right)}$ et tout élément de $\bar{Y}_{x, x^{\prime}}^{p, k, m,\left(l_{0}, \ldots, l_{m}\right)}$ a au plus $C$ antécédents dans $\bar{Y}_{x, x^{\prime}, \star}^{p, k, m,\left(l_{0}, \ldots, l_{m}\right)}$, pour une certaine constante $C=C(\delta, K, N, Q, P, M)$. 
On définit la relation d'équivalence

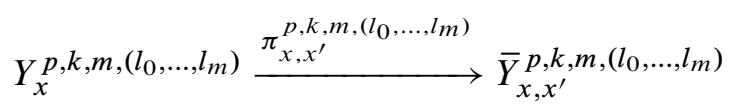

de la façon suivante :

et

$$
\left(a_{1}, \ldots, a_{p}, S_{0}, \ldots, S_{m},\left(\mathcal{Y}_{i}^{j}\right)_{i \in\{0, \ldots, m\}, j \in\left\{1, \ldots, l_{i}\right\}}\right)
$$

$$
\left(\hat{a}_{1}, \ldots, \hat{a}_{p}, \widehat{S}_{0}, \ldots, \widehat{S}_{m},\left(\widehat{\mathcal{Y}}_{i}^{j}\right)_{i \in\{0, \ldots, m\}, j \in\left\{1, \ldots, l_{i}\right\}}\right)
$$

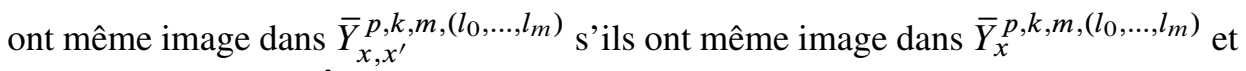
si $d\left(x^{\prime}, S_{0}\right)=d\left(x^{\prime}, \hat{S}_{0}\right)$. L'application

$$
\left(a_{1}, \ldots, a_{p}, S_{0}, \ldots, S_{m},\left(y_{i}^{j}\right)_{i \in\{0, \ldots, m\}, j \in\left\{1, \ldots, l_{i}\right\}}\right) \mapsto d\left(x^{\prime}, S_{0}\right)
$$

se factorise donc en une application $r_{0}^{\prime}: \bar{Y}_{x, x^{\prime}}^{p, k, m,\left(l_{0}, \ldots, l_{m}\right)} \rightarrow \mathbb{N}$. On définit une application

$$
k^{\prime}: \bar{Y}_{x, x^{\prime}}^{p, k, m,\left(l_{0}, \ldots, l_{m}\right)} \rightarrow \mathbb{N}
$$

en posant, pour $Z \in \bar{Y}_{x, x^{\prime}}^{p, k, m,\left(l_{0}, \ldots, l_{m}\right)}$

$$
k^{\prime}(Z)=\min \left(r_{0}^{\prime}(Z), \max \left(r_{0}^{\prime}(Z)-r_{0}(Z)+k, \mathrm{E}\left(\frac{r_{0}^{\prime}(Z)+d\left(x, x^{\prime}\right)-r_{0}(Z)}{2}\right)\right)\right)+\frac{M}{2} .
$$

Voici le dessin dans le cas où $X$ est un arbre et $p=1$ (si bien que $S_{0}=\left\{a_{1}\right\}$ et on note $b=a_{1}$ pour que le dessin serve de nouveau dans la suite) et où l'on prend $M=0$ dans la formule précédente. Dans ce dessin on a choisi

$$
(b,\{b\}) \in\left(\pi_{x, x^{\prime}}^{1, k, 0,(0)}\right)^{-1}(Z),
$$

on a représenté $B(x, k)$ par une moitié de boule et on a noté $u$ le point de $B(x, k)$ à distance minimale de $b$. On définit $t$ comme le point de

$$
\bigcap_{\tilde{x} \in B(x, k)} \operatorname{géod}(\tilde{x}, b)
$$

le plus proche de $x^{\prime}$ (ce point est unique et appartient à géod $\left(x^{\prime}, b\right)$ ). On remarque que $t$ est aussi le point central du triangle $u b x^{\prime}$. La formule précédente pour $k^{\prime}(Z)$ devient

$$
\begin{aligned}
k^{\prime}(Z) & =\min \left(d\left(x^{\prime}, b\right), \max \left(d\left(x^{\prime}, b\right)-d(x, b)+k, \frac{d\left(x^{\prime}, b\right)+d\left(x, x^{\prime}\right)-d(x, b)}{2}\right)\right) \\
& =d\left(x^{\prime}, t\right) .
\end{aligned}
$$

La boule $B\left(x^{\prime}, k^{\prime}(Z)\right)$ (dont le bord contient $t$ ) est la boule de centre $x^{\prime}$ de plus petit rayon telle que toute géodésique entre $b$ et un point de $B(x, k)$ la rencontre. On 
remarque que $t$ dépend de $S_{0}=\{b\}$ mais que $d\left(x^{\prime}, t\right)$ ne dépend que de $Z$. Dans le dessin ci-dessous, le premier cas est le cas où $b \in B(x, k)$ et le deuxième cas et le troisième cas sont distingués par l'appartenance ou non de $u$ à géod $\left(x, x^{\prime}\right)$ (dans le premier et le deuxième cas $x^{\prime}$ n'appartient pas forcément à $\left.B(x, k)\right)$.

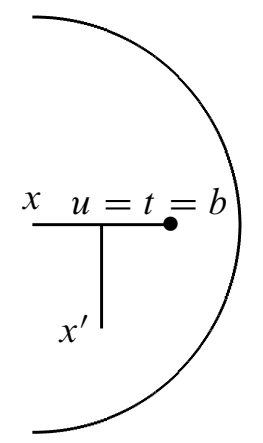

Premier cas

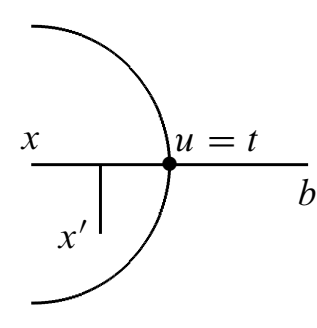

Deuxième cas

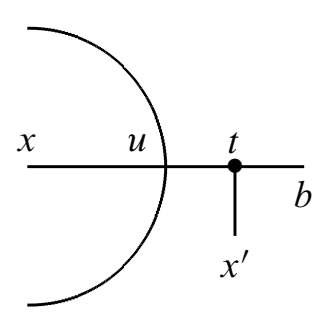

Troisième cas

On revient maintenant au cas général et on va distinguer trois cas comme dans le dessin pour les arbres. Soit $Z \in \bar{Y}_{x, x^{\prime}}^{p, m,\left(l_{0}, \ldots, l_{m}\right)}$

Premier cas. On suppose $r_{0}(Z) \leq k$.

Alors, par (120),

$$
k^{\prime}(Z)=r_{0}^{\prime}(Z)+\frac{M}{2} .
$$

Deuxième cas. On suppose $r_{0}(Z)>k$ et $r_{0}(Z)-r_{0}^{\prime}(Z)+d\left(x, x^{\prime}\right) \leq 2 k$.

Cette dernière condition implique

$$
r_{0}^{\prime}(Z)-r_{0}(Z)+k \geq \mathrm{E}\left(\frac{r_{0}^{\prime}(Z)-r_{0}(Z)+d\left(x, x^{\prime}\right)}{2}\right)
$$

d'où, par (120),

$$
k^{\prime}(Z)=r_{0}^{\prime}(Z)-r_{0}(Z)+k+\frac{M}{2} .
$$

Troisième cas. On suppose $r_{0}(Z)>k$ et $r_{0}(Z)-r_{0}^{\prime}(Z)+d\left(x, x^{\prime}\right)>2 k$.

Cette dernière condition implique

$$
r_{0}^{\prime}(Z)-r_{0}(Z)+k \leq \mathrm{E}\left(\frac{r_{0}^{\prime}(Z)-r_{0}(Z)+d\left(x, x^{\prime}\right)}{2}\right)
$$

et comme $d\left(x, x^{\prime}\right) \leq r_{0}(Z)+r_{0}^{\prime}(Z)+N$ on en déduit, par (120),

$$
\left|k^{\prime}(Z)-\left(E\left(\frac{r_{0}^{\prime}(Z)-r_{0}(Z)+d\left(x, x^{\prime}\right)}{2}\right)+\frac{M}{2}\right)\right| \leq \frac{N}{2} .
$$

On introduit maintenant une partition de $Y_{x}^{p, k, m,\left(l_{0}, \ldots, l_{m}\right)}$ pour la relation suivante :

$$
\left(a_{1}, \ldots, a_{p}, S_{0}, \ldots, S_{m},\left(y_{i}^{j}\right)_{i \in\{0, \ldots, m\}, j \in\left\{1, \ldots, l_{i}\right\}}\right)
$$


et

$$
\left(\hat{a}_{1}, \ldots, \hat{a}_{p}, \widehat{S}_{0}, \ldots, \widehat{S}_{m},\left(\widehat{\mathcal{Y}}_{i}^{j}\right)_{i \in\{0, \ldots, m\}, j \in\left\{1, \ldots, l_{i}\right\}}\right)
$$

sont en relation s'ils ont même image $Z$ dans $\bar{Y}_{x, x^{\prime}}^{p, k, m,\left(l_{0}, \ldots, l_{m}\right)} \operatorname{par} \pi_{x, x^{\prime}}^{p, k, m,\left(l_{0}, \ldots, l_{m}\right)}$ et s'il existe une isométrie de

$$
\bigcup_{i \in\{0, \ldots, m\}} B\left(S_{i}, M\right) \cup \underset{\substack{i \in\{0, \ldots, m\} \\ j \in\left\{1, \ldots, l_{i}\right\}}}{\bigcup} B\left(y_{i}^{j}, M\right) \cup B(x, k+2 M) \cup B\left(x^{\prime}, k^{\prime}(Z)+2 M\right)
$$

vers

$$
\bigcup_{i \in\{0, \ldots, m\}} B\left(\widehat{S}_{i}, M\right) \cup \underset{\substack{i \in\{0, \ldots, m\} \\ j \in\left\{1, \ldots, l_{i}\right\}}}{\bigcup} B\left(\hat{y}_{i}^{j}, M\right) \cup B(x, k+2 M) \cup B\left(x^{\prime}, k^{\prime}(Z)+2 M\right)
$$

qui envoie $a_{i}$ sur $\hat{a}_{i}$ pour $i \in\{1, \ldots, p\}, S_{i}$ sur $\hat{S}_{i}$ pour $i \in\{0, \ldots, m\}, y_{i}^{j}$ sur $\widehat{y}_{i}^{j}$ pour $i \in\{0, \ldots, m\}, j \in\left\{1, \ldots, l_{i}\right\}$ et est l'identité sur $B(x, k+2 M)$ et sur $B\left(x^{\prime}, k^{\prime}(Z)+2 M\right)$.

On note $\bar{Y}_{x, x^{\prime}, \star}^{p, k, m,\left(l_{0}, \ldots, l_{m}\right)}$ le quotient de $Y_{x}^{p, k, m,\left(l_{0}, \ldots, l_{m}\right)}$ pour cette relation d'équivalence, et $\pi_{x, x^{\prime}, \star}^{p, k, m,\left(l_{0}, \ldots, l_{m}\right)}$ l'application quotient.

Lemme 4.54. a) Les fibres de l'application surjective

$$
\bar{Y}_{x, x^{\prime}}^{p, k, m,\left(l_{0}, \ldots, l_{m}\right)} \rightarrow \bar{Y}_{x}^{p, k, m,\left(l_{0}, \ldots, l_{m}\right)}
$$

sont toutes de cardinal $\leq 2 d\left(x, x^{\prime}\right)+1$.

b) Il existe une constante $C=C(\delta, K, N, Q, P, M)$ telle que les fibres de l'application surjective

$$
\bar{Y}_{x, x^{\prime}, \star}^{p, k, m,\left(l_{0}, \ldots, l_{m}\right)} \rightarrow \bar{Y}_{x, x^{\prime}}^{p, k, m,\left(l_{0}, \ldots, l_{m}\right)}
$$

soient toutes de cardinal $\leq C$.

Avant de montrer ce lemme, expliquons la stratégie de la preuve de la proposition 4.3. On veut majorer $\|f\|_{\mathscr{H}_{x, s}\left(\Delta_{p}\right)}^{2}$ en fonction de $\|f\|_{\mathscr{H}_{x^{\prime}, s}\left(\Delta_{p}\right)}^{2}$. D'abord $\|f\|_{\mathscr{H}_{x, s}\left(\Delta_{p}\right)}^{2}$ est une somme pondérée indexée par certaines classes d'équivalence $Z \in \bar{Y}_{x}^{p, k, m,\left(l_{0}, \ldots, l_{m}\right)}$ des carrés des formes linéaires

$$
\xi_{Z}: f \mapsto \sum_{\substack{\left(a_{1}, \ldots, a_{p}, S_{0}, \ldots, S_{m},\left(y_{i}^{j}\right)_{\left.i \in\{0, \ldots, m\}, j \in\left\{1, \ldots, l_{i}\right\}\right)} \\ \in\left(\pi_{x}^{p, k, m,\left(l_{0}, \ldots, l_{m}\right)}\right)^{-1}(Z)\right.}} f\left(a_{1}, \ldots, a_{p}\right) .
$$

Grâce au lemme précédent on peut couper chaque classe d'équivalence $Z$ en morceaux $\widetilde{Z}$ (paramétrés par $\bar{Y}_{x, x^{\prime}, \star}^{p, k, m,\left(l_{0}, \ldots, l_{m}\right)}$ ) dont le nombre est inférieur ou égal à $C\left(2 d\left(x, x^{\prime}\right)+1\right)$. On a donc pour tout $f,\left|\xi_{Z}(f)\right|^{2} \leq C\left(2 d\left(x, x^{\prime}\right)+1\right) \sum_{\tilde{Z}}\left|\xi_{\tilde{Z}}(f)\right|^{2}$ (par Cauchy-Schwarz) où la somme porte sur les $\widetilde{Z} \in \bar{Y}_{x, x^{\prime}, \star}^{p, k,\left(l_{0}, \ldots, l_{m}\right)}$ dont l'image 
dans $\bar{Y}_{x}^{p, k, m,\left(l_{0}, \ldots, l_{m}\right)}$ est $Z$. On montrera ensuite (dans le lemme 4.61) que chaque forme linéaire $\xi_{\widetilde{Z}}$ est proportionnelle à une forme linéaire $\xi_{Z^{\prime}}$ apparaissant dans la formule pour $\|f\|_{\mathcal{H}_{x^{\prime}, s}\left(\Delta_{p}\right)}^{2}$. Par définition $\widetilde{Z}$ est la donnée des distances entre les points de $B(x, k+2 M)$, ceux de $B\left(x^{\prime}, k^{\prime}+2 M\right)$ et les points à distance $\leq M$ de la réunion de toutes les parties $S_{i}$ et $y_{i}^{j}$. Le dessin ci-dessous correspond au troisième cas du dessin pour les arbres (qui est le cas le plus intéressant) et les deux boules y représentent $B(x, k)$ et $B\left(x^{\prime}, k^{\prime}\right)$. Les parties $S_{i}$ sont représentées par des cercles plus grands que les parties $y_{i}^{j}$ dans un souci de clarté, bien que leur diamètre maximal soit plus petit ( $N$ au lieu de $P$ ).

parties éliminées parties conservées

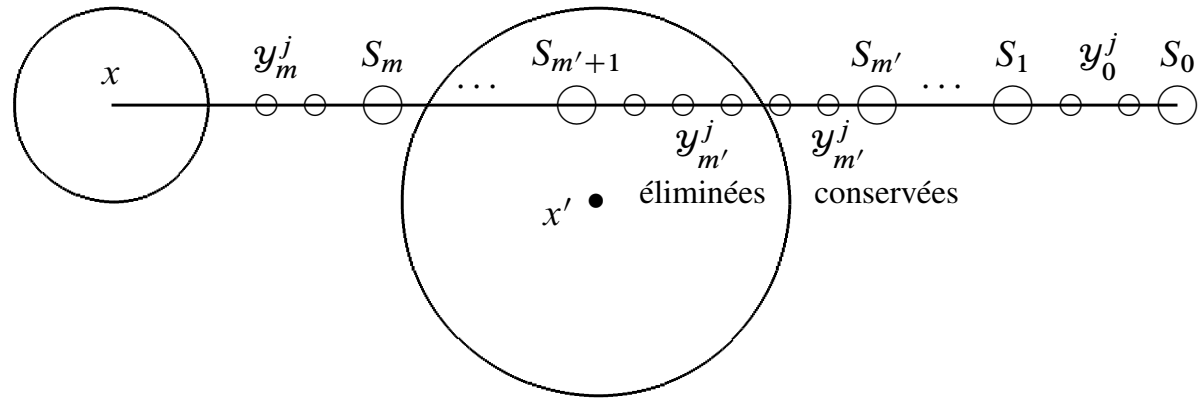

La classe d'équivalence $Z^{\prime}$ sera la donnée des distances entre les points de $B\left(x^{\prime}, k^{\prime}+\right.$ $2 M$ ) et les points à distance $\leq M$ de la réunion des parties $S_{i}$ et $y_{i}^{j}$ situées à droite de la boule $B\left(x^{\prime}, k^{\prime}\right)$, qui sont indiquées sur le dessin comme "parties conservées". On montrera qu'il existe $m^{\prime}$ tel que les parties conservées soient les $S_{i}$ pour $i \in$ $\left\{0, \ldots, m^{\prime}\right\}$, tous les $y_{i}^{j}$ pour $i \in\left\{0, \ldots, m^{\prime}-1\right\}$ et certains des ${\underset{Z}{m^{\prime}}}^{j}$. La raison pour laquelle $\xi_{\widetilde{Z}}$ est proportionnelle à $\xi_{Z^{\prime}}$ est que la connaissance de $\widetilde{Z}$ et des parties $S_{i}$ et $y_{i}^{j}$ éliminées (qui se trouvent dans $B(x, k)$ ou $B\left(x^{\prime}, k^{\prime}\right)$ ou entre ces deux boules) détermine $Z$, car si $\Gamma$ est une partie conservée et $\Delta$ une partie éliminée toute géodésique entre $B(\Gamma, M)$ et $B(\Delta, M) \cup B(x, k+2 M)$ traverse $B\left(x^{\prime}, k^{\prime}+\right.$ $2 M)$, comme on le verra dans le lemme 4.57. Par conséquent, pour connaître les distances entre $B(\Gamma, M)$ et $B(\Delta, M) \cup B(x, k+2 M)$, il suffit de connaître les distances entre $B(\Gamma, M)$ et $B\left(x^{\prime}, k^{\prime}+2 M\right)$, qui font partie de la donnée de $\widetilde{Z}$. La difficulté sera ensuite que chaque $\xi_{Z^{\prime}}$ est proportionnelle à $\xi_{\widetilde{Z}}$ pour une infinité de $\tilde{Z} \in \bigcup_{k, m,\left(l_{0}, \ldots, l_{m}\right)} \bar{Y}_{x, x^{\prime}, \star,}^{p, k,\left(l_{0}, \ldots, l_{m}\right)}$ et on devra vérifier que, compte tenu des pondérations et grâce à $\left(H_{B}\right)$, la somme sur $\widetilde{Z}$ n'introduit pas de divergence.

Démonstration du lemme 4.54. Le a) vient simplement du fait que pour

$$
\left(a_{1}, \ldots, a_{p}, S_{0}, \ldots, S_{m},\left(y_{i}^{j}\right)_{i \in\{0, \ldots, m\}, j \in\left\{1, \ldots, l_{i}\right\}}\right) \in Y_{x}^{p, k, m,\left(l_{0}, \ldots, l_{m}\right)}
$$


on a $d\left(x^{\prime}, S_{0}\right) \in\left[d\left(x, S_{0}\right)-d\left(x, x^{\prime}\right), d\left(x, S_{0}\right)+d\left(x, x^{\prime}\right)\right]$. Montrons b). Soit $Z \in \bar{Y}_{x, x^{\prime}}^{p, k, m,\left(l_{0}, \ldots, l_{m}\right)}$. On doit montrer que le nombre d'antécédents de $Z$ dans $\bar{Y}_{x, x^{\prime}, \star}^{\left.p, k, l_{0}, \ldots, l_{m}\right)}$ est majoré par une constante $C=C(\delta, K, N, Q, P, M)$. On note $u$ un point de $B(x, k)$ tel que pour tout

$$
\left(a_{1}, \ldots, a_{p}, S_{0}, \ldots, S_{m},\left(y_{i}^{j}\right)_{i \in\{0, \ldots, m\}, j \in\left\{1, \ldots, l_{i}\right\}}\right) \in\left(\pi_{x, x^{\prime}}^{p, k, m,\left(l_{0}, \ldots, l_{m}\right)}\right)^{-1}(Z)
$$

$u$ soit à distance minimale de $S_{0}$ (cela ne dépend que de $Z$ car les distances entre les points de $S_{0}$ et ceux de $B(x, k)$ font partie de la donnée de $Z$ ). On a alors $d\left(u, S_{0}\right)=\max \left(0, r_{0}(Z)-k\right)$. On rappelle que d'après le lemme 4.14 , pour tout

$$
\left(a_{1}, \ldots, a_{p}, S_{0}, \ldots, S_{m},\left(y_{i}^{j}\right)_{i \in\{0, \ldots, m\}, j \in\left\{1, \ldots, l_{i}\right\}}\right) \in\left(\pi_{x, x^{\prime}}^{p, k, m,\left(l_{0}, \ldots, l_{m}\right)}\right)^{-1}(Z)
$$

et pour $b \in S_{0}$ à distance minimale de $u$ (si bien que $u$ est un point de $B(x, k)$ à distance minimale de $b$ ) on a

$$
S_{0} \cup \cdots \cup S_{m} \cup \underset{\substack{i \in\{0, \ldots, m\} \\ j \in\left\{1, \ldots, l_{i}\right\}}}{\cup} y_{i}^{j} \subset 4 P-\operatorname{géod}(b, u)
$$

d'où par le lemme 3.2,

$$
\bigcup_{i \in\{0, \ldots, m\}} B\left(S_{i}, M\right) \cup \underset{\substack{i \in\{0, \ldots, m\} \\ j \in\left\{1, \ldots, l_{i}\right\}}}{\bigcup} B\left(y_{i}^{j}, M\right) \subset(2 M+4 P)-\operatorname{géod}(b, u) .
$$

On va distinguer trois cas qui correspondent à peu près aux trois cas envisagés dans le dessin pour les arbres (auquel le lecteur peut se reporter pour lire la démonstration). Premier cas. On suppose $r_{0}(Z) \leq k$.

Soit

$$
\left(a_{1}, \ldots, a_{p}, S_{0}, \ldots, S_{m},\left(y_{i}^{j}\right)_{i \in\{0, \ldots, m\}, j \in\left\{1, \ldots, l_{i}\right\}}\right) \in\left(\pi_{x, x^{\prime}}^{p, k, m,\left(l_{0}, \ldots, l_{m}\right)}\right)^{-1}(Z)
$$

et $b \in S_{0}$ à distance minimale de $u$. On a alors $b=u$ d'où

$$
\begin{aligned}
\bigcup_{i \in\{0, \ldots, m\}} B\left(S_{i}, M\right) \cup \bigcup_{\substack{i \in\{0, \ldots, m\} \\
j \in\left\{1, \ldots, l_{i}\right\}}} B\left(y_{i}^{j}, M\right) & \subset(2 M+4 P)-\operatorname{géod}(u, u) \\
& =B(u, M+2 P) \subset B(x, k+2 M)
\end{aligned}
$$

où la dernière inclusion vient de l'inégalité $2 P \leq M$ que l'on suppose grâce à $\left(H_{M}\right)$. Donc $\left(\pi_{x, x^{\prime}}^{p, k, m,\left(l_{0}, \ldots, l_{m}\right)}\right)^{-1}(Z)$ est un singleton et a fortiori l'image inverse de $Z$ par l'application $\bar{Y}_{x, x^{\prime}, \star}^{p, k, m},\left(l_{0}, \ldots, l_{m}\right) \rightarrow \bar{Y}_{x, x^{\prime}}^{p, m,\left(l_{0}, \ldots, l_{m}\right)}$ est un singleton.

Deuxième cas. On suppose $r_{0}(Z)>k$ et $r_{0}(Z)-r_{0}^{\prime}(Z)+d\left(x, x^{\prime}\right) \leq 2 k$.

Par (122) on a

$$
k^{\prime}(Z)=r_{0}^{\prime}(Z)-r_{0}(Z)+k+\frac{M}{2} .
$$


Soit

$$
\left(a_{1}, \ldots, a_{p}, S_{0}, \ldots, S_{m},\left(y_{i}^{j}\right)_{i \in\{0, \ldots, m\}, j \in\left\{1, \ldots, l_{i}\right\}}\right) \in\left(\pi_{x, x^{\prime}}^{p, k, m,\left(l_{0}, \ldots, l_{m}\right)}\right)^{-1}(Z)
$$

et $b \in S_{0}$ à distance minimale de $u$. On a donc

$$
\begin{gathered}
d(x, b)-d\left(x^{\prime}, b\right)+d\left(x, x^{\prime}\right) \leq 2 k+N, \quad d(x, b)>k, \\
\left|k^{\prime}(Z)-\left(d\left(x^{\prime}, b\right)-d(x, b)+k+\frac{M}{2}\right)\right| \leq N .
\end{gathered}
$$

Soit $t \in B\left(x^{\prime}, k^{\prime}(Z)+2 M\right)$ à distance minimale de $b$. On a $u \in \operatorname{géod}(x, b)$, et $d(x, u)=k, d(u, b)=d(x, b)-k$. Par $\left(H_{\delta}^{0}\left(x^{\prime}, x, u, b\right)\right)$ et (124) on a

$$
\begin{aligned}
d\left(x^{\prime}, u\right) & \leq \max \left(d\left(x, x^{\prime}\right)-k, d\left(x^{\prime}, b\right)-d(x, b)+k\right)+\delta \\
& \leq d\left(x^{\prime}, b\right)-d(x, b)+k+N+\delta \\
& =d\left(x^{\prime}, b\right)-d(u, b)+N+\delta,
\end{aligned}
$$

donc $u \in(N+\delta)$ - géod $\left(x^{\prime}, b\right)$. On a $t \in \operatorname{géod}\left(x^{\prime}, b\right)$,

$$
d\left(x^{\prime}, t\right)=\min \left(d\left(x^{\prime}, b\right), k^{\prime}(Z)+2 M\right)
$$

et $\left|k^{\prime}(Z)-\left(d\left(x^{\prime}, b\right)-d(u, b)+\frac{M}{2}\right)\right| \leq N$ donc

$$
\begin{aligned}
& d\left(x^{\prime}, t\right) \leq k^{\prime}(Z)+2 M \leq d\left(x^{\prime}, u\right)+\frac{5 M}{2}+N, \\
& d\left(x^{\prime}, t\right) \geq \min \left(d\left(x^{\prime}, b\right), d\left(x^{\prime}, u\right)+\frac{5 M}{2}-2 N-\delta\right),
\end{aligned}
$$

et comme $d\left(x^{\prime}, u\right) \leq d\left(x^{\prime}, b\right)+N+\delta$, on en déduit

$$
\left|d\left(x^{\prime}, t\right)-d\left(x^{\prime}, u\right)\right| \leq \frac{5 M}{2}+N
$$

car on suppose $N+\delta \leq \frac{5 M}{2}$, ce qui est permis grâce à $\left(H_{M}\right)$.

Comme $u \in(N+\delta)$ - $\operatorname{géod}\left(x^{\prime}, b\right)$ et $t \in \operatorname{géod}\left(x^{\prime}, b\right)$, et en utilisant l'inégalité (125), $\left(H_{\delta}\left(u, x^{\prime}, t, b\right)\right)$ implique alors

$$
d(u, t) \leq\left(\frac{5 M}{2}+N\right)+(N+\delta)+\delta=\frac{5 M}{2}+2 N+2 \delta .
$$

Cette inégalité reflète l'égalité $u=t$ dans le dessin pour les arbres qui correspond au deuxième cas.

On en déduit

$$
(2 M+4 P)-\operatorname{géod}(u, b) \subset \alpha \text { - géod }(t, b)
$$

avec $\alpha=2\left(\frac{5 M}{2}+2 N+2 \delta\right)+(2 M+4 P)$. Le lemme 4.18 montre que les distances entre un point de $\alpha$ - géod $(t, b)$ et les points de $B\left(x^{\prime}, k^{\prime}(Z)+2 M\right)$ sont déterminées par les distances entre ce point et les points de

$$
B\left(x^{\prime}, k^{\prime}(Z)+2 M\right) \cap B(t, \alpha+4 \delta) \subset B(t, \alpha+4 \delta) \subset B(u, \beta)
$$


avec $\beta=\left(\frac{5 M}{2}+2 N+2 \delta\right)+\alpha+4 \delta=3\left(\frac{5 M}{2}+2 N+2 \delta\right)+2 M+4 P+4 \delta$. Donc les distances entre les points de l'ensemble

$$
\bigcup_{i \in\{0, \ldots, m\}} B\left(S_{i}, M\right) \cup \underset{\substack{i \in\{0, \ldots, m\} \\ j \in\left\{1, \ldots, l_{i}\right\}}}{\bigcup} B\left(y_{i}^{j}, M\right)
$$

(qui est inclus dans $(4 P+2 M)$ - $\operatorname{géod}(u, b) \subset \alpha$-géod $(t, b))$ et les points de $B\left(x^{\prime}, k^{\prime}(Z)+2 M\right)$ sont déterminées par les distances entre les points de (126) et les points de $B(u, \beta)$. D'autre part le c) du lemme 4.17 , appliqué à

- $(u, b)$ au lieu de $(c, d)$,

- $\left\{w_{i}, i \in I\right\}$ égal à $\{u\} \cup \bigcup_{i \in\{0, \ldots, m\}} S_{i} \cup \bigcup_{i \in\{0, \ldots, m\}, j \in\left\{1, \ldots, l_{i}\right\}} y_{i}^{j}$,

- $J \subset I$ le singleton tel que $\left\{w_{j}, j \in J\right\}=\{u\}$,

- $\left(\alpha_{i}, \rho_{i}\right)$ égal à $(0, \beta)$ pour $i \in J$ et à $(4 P, M)$ pour $i \in I \backslash J$

montre que les distances entre les points de l'ensemble (126) et ceux de $B(u, \beta)$ sont déterminées par les distances entre $C=C(\delta, K, N, Q, P, M)$ points de (126) (déterminés par la donnée de $Z$ ) et les points de $B(u, \beta)$. De plus le cardinal de $B(u, \beta)$ est majoré par une constante de la forme $C(\delta, N, K, Q, P, M)$ et les distances entre ces $C$ points de (126) et les points de $B(u, \beta)$ sont déterminées à $\beta$ près par la donnée de $Z$. Cela termine l'étude du deuxième cas.

Troisième cas. On suppose $r_{0}(Z)>k$ et $r_{0}(Z)-r_{0}^{\prime}(Z)+d\left(x, x^{\prime}\right)>2 k$.

Par (123) on a

$$
\left|k^{\prime}(Z)-\left(E\left(\frac{r_{0}^{\prime}(Z)-r_{0}(Z)+d\left(x, x^{\prime}\right)}{2}\right)+\frac{M}{2}\right)\right| \leq \frac{N}{2} .
$$

Soit

$$
\left(a_{1}, \ldots, a_{p}, S_{0}, \ldots, S_{m},\left(y_{i}^{j}\right)_{i \in\{0, \ldots, m\}, j \in\left\{1, \ldots, l_{i}\right\}}\right) \in\left(\pi_{x, x^{\prime}}^{p, k, m,\left(l_{0}, \ldots, l_{m}\right)}\right)^{-1}(Z)
$$

et $b \in S_{0}$ à distance minimale de $u$. On a donc

$$
\begin{gathered}
d(x, b)-d\left(x^{\prime}, b\right)+d\left(x, x^{\prime}\right) \geq 2 k-N, \quad d(x, b)>k, \\
\left|k^{\prime}(Z)-\left(\mathrm{E}\left(\frac{d\left(x^{\prime}, b\right)-d(x, b)+d\left(x, x^{\prime}\right)}{2}\right)+\frac{M}{2}\right)\right| \leq N .
\end{gathered}
$$

Comme $d(x, b)-d\left(x^{\prime}, b\right)+d\left(x, x^{\prime}\right) \geq 2 k-N,\left(H_{\delta}^{0}\left(x^{\prime}, x, u, b\right)\right)$ donne

$$
\begin{aligned}
d\left(x^{\prime}, u\right) & \leq \max \left(d\left(x^{\prime}, x\right)-k, d\left(x^{\prime}, b\right)-d(x, b)+k\right)+\delta \\
& \leq d\left(x, x^{\prime}\right)-k+N+\delta \\
& =d\left(x, x^{\prime}\right)-d(x, u)+N+\delta
\end{aligned}
$$

donc

$$
u \in(N+\delta)-\operatorname{géod}\left(x^{\prime}, x\right)
$$


Donc

$$
\left|k^{\prime}(Z)-\left(\mathrm{E}\left(\frac{d\left(x^{\prime}, b\right)+d\left(x^{\prime}, u\right)-d(u, b)}{2}\right)+\frac{M}{2}\right)\right| \leq 2 N+\delta .
$$

Soit $u^{\prime} \in B\left(x^{\prime}, k^{\prime}(Z)+2 M\right)$ à distance minimale de $u$ et $b^{\prime} \in B\left(x^{\prime}, k^{\prime}(Z)+2 M\right)$ à distance minimale de $b$. Soit $w \in 4 P$ - géod $(u, b)$. On applique le lemme 4.6 à $u, b$, $x^{\prime}, u^{\prime}, b^{\prime}, w$ avec $l=2$ et $x^{\prime}$ comme point base. On note $t$ le point central du triangle de sommets $\Psi u, \Psi b, \Psi x^{\prime}$. Dans le dessin ci-dessous le point de géod $(\Psi u, \Psi b)$ le plus proche de $\Psi w$ est arbitraire.

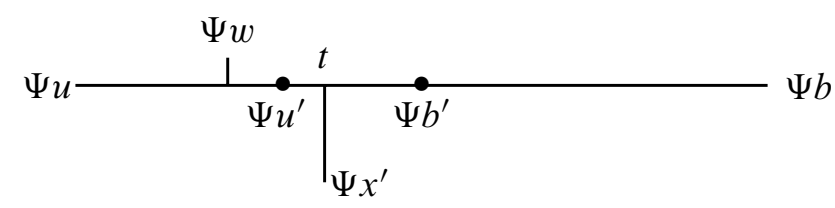

On a

$$
\begin{gathered}
d\left(\Psi x^{\prime}, t\right)=\frac{d\left(\Psi x^{\prime}, \Psi u\right)+d\left(\Psi x^{\prime}, \Psi b\right)-d(\Psi u, \Psi b)}{2} \\
\in\left[\frac{d\left(x^{\prime}, u\right)+d\left(x^{\prime}, b\right)-d(u, b)}{2}, \frac{d\left(x^{\prime}, u\right)+d\left(x^{\prime}, b\right)-d(u, b)}{2}+\delta\right] .
\end{gathered}
$$

Grâce au a) du lemme 4.7 on a de plus

$$
\Psi u^{\prime} \in \operatorname{géod}\left(\Psi x^{\prime}, \Psi u\right), \quad d\left(\Psi x^{\prime}, \Psi u^{\prime}\right)=\min \left(k^{\prime}(Z)+2 M, d\left(\Psi x^{\prime}, \Psi u\right)\right)
$$

et

$$
\Psi b^{\prime} \in \operatorname{géod}\left(\Psi x^{\prime}, \Psi b\right), \quad d\left(\Psi x^{\prime}, \Psi b^{\prime}\right)=\min \left(k^{\prime}(Z)+2 M, d\left(\Psi x^{\prime}, \Psi b\right)\right) .
$$

Par (127) on a $k^{\prime}(Z)+2 M \geq \frac{d\left(x^{\prime}, u\right)+d\left(x^{\prime}, b\right)-d(u, b)}{2}+\delta$ car on suppose $\frac{5 M}{2} \geq$ $2 N+2 \delta+1$, ce qui est permis par $\left(H_{M}\right)$. On déduit alors de $(128)$ que

et

$$
\Psi u^{\prime} \in \operatorname{géod}(t, \Psi u), \quad d\left(t, \Psi u^{\prime}\right) \leq \frac{5 M}{2}+2 N+\delta
$$

$$
\Psi b^{\prime} \in \operatorname{géod}(t, \Psi b), \quad d\left(t, \Psi b^{\prime}\right) \leq \frac{5 M}{2}+2 N+\delta .
$$

Grâce au a) du lemme 4.7 on a $\Psi w \in(4 P+2 \delta)$ - $\operatorname{géod}(\Psi b, \Psi u)$ donc $\Psi w$ est à distance $\leq 2 P+\delta$ de géod $(\Psi b, \Psi u)$. Donc l'une au moins des assertions suivante est vraie (suivant que le point de géod $(\Psi b, \Psi u)$ le plus proche de $\Psi w$ appartient à $\operatorname{géod}(\Psi b, t)$ ou géod $(t, \Psi u))$ :

$-\Psi w$ est à distance $\leq \frac{5 M}{2}+2 N+2 P+2 \delta$ de géod $\left(\Psi b^{\prime}, \Psi b\right)$,

- ou $\Psi w$ est à distance $\leq \frac{5 M}{2}+2 N+2 P+2 \delta$ de géod $\left(\Psi u^{\prime}, \Psi u\right)$.

Il résulte facilement de ce qui précède et du b) du lemme 4.7 que l'une au moins des assertions suivante est vraie 
$-w \in(5 M+4 N+4 P+8 \delta)-\operatorname{géod}\left(b^{\prime}, b\right)$,

- ou $w \in(5 M+4 N+4 P+8 \delta)$ - $\operatorname{géod}\left(u^{\prime}, u\right)$.

On suppose $4 N+4 P+8 \delta \leq M$, ce qui est possible par $\left(H_{M}\right)$. Il résulte de ce qui précède que $w \in 6 M$-géod $\left(b^{\prime}, b\right) \cup 6 M$ - $\operatorname{géod}\left(u^{\prime}, u\right)$. On applique ceci à $w \in \bigcup_{i \in\{0, \ldots, m\}} S_{i} \cup \bigcup_{i \in\{0, \ldots, m\}, j \in\left\{1, \ldots, l_{i}\right\}} y_{i}^{j} \subset 4 P$ - $\operatorname{géod}(u, b)$. Donc l'ensemble

$$
\bigcup_{i \in\{0, \ldots, m\}} B\left(S_{i}, M\right) \cup \underset{\substack{i \in\{0, \ldots, m\} \\ j \in\left\{1, \ldots, l_{i}\right\}}}{\bigcup} B\left(y_{i}^{j}, M\right)
$$

est inclus dans $8 M$ - géod $\left(b^{\prime}, b\right) \cup 8 M$ - géod $\left(u^{\prime}, u\right)$.

En appliquant le lemme 4.18 à $\alpha=8 M,\left(u, u^{\prime}\right)\left(\right.$ resp. $\left.\left(b, b^{\prime}\right)\right)$ au lieu de $(b, u)$ et $\left(x^{\prime}, k^{\prime}(Z)+2 M\right)$ au lieu de $(x, l)$ on voit que les distances entre n'importe quel point $y \in 8 M$ - $\operatorname{géod}\left(u^{\prime}, u\right)\left(\right.$ resp. $y \in 8 M$ - géod $\left.\left(b^{\prime}, b\right)\right)$ et les points de $B\left(x^{\prime}, k^{\prime}(Z)+2 M\right)$ sont déterminées par les distances entre $y$ et les points de

$$
B\left(x^{\prime}, k^{\prime}(Z)+2 M\right) \cap B\left(u^{\prime}, 8 M+4 \delta\right) \subset B\left(u^{\prime}, 8 M+4 \delta\right)
$$

et

$$
B\left(x^{\prime}, k^{\prime}(Z)+2 M\right) \cap B\left(b^{\prime}, 8 M+4 \delta\right) \subset B\left(b^{\prime}, 8 M+4 \delta\right)
$$

respectivement. De plus

$$
d\left(u^{\prime}, b^{\prime}\right) \leq d\left(\Psi u^{\prime}, \Psi b^{\prime}\right)+2 \delta \leq d\left(\Psi u^{\prime}, t\right)+d\left(t, \Psi b^{\prime}\right)+2 \delta \leq 5 M+4 N+4 \delta
$$

donc $B\left(b^{\prime}, 8 M+4 \delta\right) \subset B\left(u^{\prime}, 13 M+4 N+8 \delta\right)$. On a $\Psi u^{\prime} \in \operatorname{géod}(\Psi u, \Psi b)$ donc $u^{\prime} \in 4 \delta$ - géod $(u, b)$ par le b) du lemme 4.7. En appliquant le c) du lemme 4.17 à

- $(u, b)$ au lieu de $(c, d)$,

- $\left\{w_{i}, i \in I\right\}$ égal à $\left\{u^{\prime}\right\} \cup \bigcup_{i \in\{0, \ldots, m\}} S_{i} \cup \bigcup_{i \in\{0, \ldots, m\}, j \in\left\{1, \ldots, l_{i}\right\}} y_{i}^{j}$,

- $J \subset I$ le singleton tel que $\left\{w_{j}, j \in J\right\}=\left\{u^{\prime}\right\}$,

- $\left(\alpha_{i}, \rho_{i}\right)$ égal à $(4 \delta, 13 M+4 N+8 \delta)$ pour $i \in J$ et à $(4 P, M)$ pour $i \in I \backslash J$, on obtient que les distances entre les points de l'ensemble (129) et ceux de $B\left(u^{\prime}, 13 M+\right.$ $4 N+8 \delta)$ sont déterminées par les distances entre $C=C(\delta, K, N, Q, P, M)$ points de (129) (déterminés par la donnée de $Z$ ) et les points de $B\left(u^{\prime}, 13 M+4 N+8 \delta\right)$. De plus ces distances sont déterminées à $C^{\prime}=C(\delta, N, K, Q, P, M)$ près par la donnée de $Z \in \bar{Y}_{x, x^{\prime}}^{p, k, m,\left(l_{0}, \ldots, l_{m}\right)}$.

Cela termine l'étude du troisième cas et achève donc la démonstration du lemme 4.54.

On définit la norme pré-hilbertienne $\|\cdot\|_{\mathscr{H}_{x, x^{\prime}, \star, s}\left(\Delta_{p}\right)}$ sur $\mathbb{C}^{\left(\Delta_{p}\right)}$ de la même façon que $\|\cdot\|_{\mathscr{H}_{x, s}\left(\Delta_{p}\right)}$, mais en remplaçant $\bar{Y}_{x}^{p, k, m,\left(l_{0}, \ldots, l_{m}\right)}$ par $\bar{Y}_{x, x^{\prime}, \star}^{p, k},\left(l_{0}, \ldots, l_{m}\right)$. 
Plus précisément, pour $Z \in \bar{Y}_{x, x^{\prime}, \star}^{p, k, m,\left(l_{0}, \ldots, l_{m}\right)}$ on note $\xi_{Z}$ la forme linéaire sur $\mathbb{C}^{\left(\Delta_{p}\right)}$ définie par

$$
\xi_{Z}(f)=\sum_{\substack{\left(a_{1}, \ldots, a_{p}, S_{0}, \ldots, S_{m},\left(y_{i}^{j}\right)_{i \in\{0, \ldots, m\}, j \in\left\{1, \ldots, l_{i}\right\}}\right) \\ \in\left(\pi_{x, x^{\prime}, \star}^{p, k,\left(l_{0}, \ldots, l_{m}\right)}\right)^{-1}(Z)}} f\left(a_{1}, \ldots, a_{p}\right) .
$$

Puis pour $f \in \mathbb{C}^{\left(\Delta_{p}\right)}$ on pose

$$
\begin{aligned}
\|f\|_{\mathscr{H}_{x, x^{\prime}, \star, s}\left(\Delta_{p}\right)=}^{2} & \sum_{k, m, l_{0}, \ldots, l_{m} \in \mathbb{N}} B^{-\left(m+\sum_{i=0}^{m} l_{i}\right)} \sum_{\left.Z \in \bar{Y}_{x, x^{\prime}, \star}^{p, k}, l_{0}, \ldots, l_{m}\right)} e^{2 s\left(r_{0}(Z)-k\right)} \\
& \left(\prod_{i=0}^{m} s_{i}(Z)^{-l_{i}}\right) \sharp\left(\left(\pi_{x, x^{\prime}, \star}^{p, k, m,\left(l_{0}, \ldots, l_{m}\right)}\right)^{-1}(Z)\right)^{-\alpha}\left|\xi_{Z}(f)\right|^{2} .
\end{aligned}
$$

Lemme 4.55. En notant $C$ la constante qui apparaît dans le b) du lemme 4.54, on a pour tout $f \in \mathbb{C}^{\left(\Delta_{p}\right)}$,

$$
\|f\|_{\mathcal{H}_{x, s}\left(\Delta_{p}\right)}^{2} \leq C\left(2 d\left(x, x^{\prime}\right)+1\right)\|f\|_{\mathscr{H}_{x, x^{\prime}, \star, s}\left(\Delta_{p}\right)}^{2} .
$$

Démonstration. Cela résulte immédiatement du lemme 4.54, de l'inégalité de CauchySchwarz et du fait que pour $Z \in \bar{Y}_{x}^{p, k, m,\left(l_{0}, \ldots, l_{m}\right)}$ et $\widetilde{Z} \in \bar{Y}_{x, x^{\prime}, \star}^{p, k,\left(l_{0}, \ldots, l_{m}\right)}$ antécédent de $Z$, c'est-à-dire que

$$
\left(\pi_{x, x^{\prime}, \star}^{p, k, m,\left(l_{0}, \ldots, l_{m}\right)}\right)^{-1}(\tilde{Z}) \subset\left(\pi_{x}^{p, k, m,\left(l_{0}, \ldots, l_{m}\right)}\right)^{-1}(Z)
$$

on a évidemment $\sharp\left(\left(\pi_{x, x^{\prime}, \star}^{p, k, m,\left(l_{0}, \ldots, l_{m}\right)}\right)^{-1}(\widetilde{Z})\right) \leq \sharp\left(\left(\pi_{x}^{p, k, m,\left(l_{0}, \ldots, l_{m}\right)}\right)^{-1}(Z)\right)$.

Démonstration de la proposition 4.3 en admettant le lemme 4.56. Grâce au lemme 4.55 , pour montrer la proposition 4.3 on est ramené à montrer le lemme suivant.

Lemme 4.56. Il existe une constante $C=C(\delta, K, N, Q, P, M, s, B)$, tel que pour tout $f \in \mathbb{C}^{\left(\Delta_{p}\right)}$,

$$
\|f\|_{\mathscr{H}_{x, x^{\prime}, \star, s}\left(\Delta_{p}\right)}^{2} \leq C e^{3 s d\left(x, x^{\prime}\right)}\|f\|_{\mathcal{H}_{x^{\prime}, s}\left(\Delta_{p}\right)}^{2} .
$$

La preuve du lemme 4.56 occupe toute la suite de ce sous-paragraphe.

Afin de mieux comprendre le lemme suivant, on peut se référer au dessin pour les arbres, en remarquant que $\max \left(k, \frac{d\left(x, x^{\prime}\right)+r_{0}(Z)-r_{0}^{\prime}(Z)}{2}\right)$ vaut $k$ dans les deux premiers dessins et $d(x, t)$ dans le troisième.

Lemme 4.57. Soit

$$
\left(a_{1}, \ldots, a_{p}, S_{0}, \ldots, S_{m},\left(y_{i}^{j}\right)_{i \in\{0, \ldots, m\}, j \in\left\{1, \ldots, l_{i}\right\}}\right) \in Y_{x}^{p, k, m,\left(l_{0}, \ldots, l_{m}\right)} .
$$


a) Il existe un unique $m^{\prime} \in\{0, \ldots, m\}$ tel que

$$
d_{\max }\left(x, S_{i}\right) \leq \max \left(k, \frac{d\left(x, x^{\prime}\right)+r_{0}(Z)-r_{0}^{\prime}(Z)}{2}\right)+M \quad \text { pour } i>m^{\prime}
$$

et

$$
d_{\max }\left(x, S_{i}\right)>\max \left(k, \frac{d\left(x, x^{\prime}\right)+r_{0}(Z)-r_{0}^{\prime}(Z)}{2}\right)+M \quad \text { pour } 1 \leq i \leq m^{\prime} .
$$

b) Pour $i>m^{\prime}$ et $j \in\left\{1, \ldots, l_{i}\right\}$ on $a$

$$
d_{\max }\left(x, y_{i}^{j}\right) \leq \max \left(k, \frac{d\left(x, x^{\prime}\right)+r_{0}(Z)-r_{0}^{\prime}(Z)}{2}\right)+M+2 P+\delta .
$$

c) Soit $J \subset\left\{1, \ldots, l_{m^{\prime}}\right\}$ l'ensemble des $j$ tels que

$$
d_{\max }\left(x, y_{m^{\prime}}^{j}\right)>\max \left(k, \frac{d\left(x, x^{\prime}\right)+r_{0}(Z)-r_{0}^{\prime}(Z)}{2}\right)+M+2 P+\delta .
$$

On écrit $J=\left\{j_{1}, \ldots, j_{l_{m^{\prime}}^{\prime}}\right\}$ avec $l_{m^{\prime}}^{\prime}=\sharp J \in\left\{0, \ldots, l_{m^{\prime}}\right\}$ et $j_{1}<\cdots<j_{l_{m^{\prime}}^{\prime}}$ et on note $k^{\prime}=k^{\prime}(Z)$ où $Z \in \bar{Y}_{x, x^{\prime}}^{p, k, m,\left(l_{0}, \ldots, l_{m}\right)}$ est tel que

$$
\left(a_{1}, \ldots, a_{p}, S_{0}, \ldots, S_{m},\left(y_{i}^{j}\right)_{i \in\{0, \ldots, m\}, j \in\left\{1, \ldots, l_{i}\right\}}\right) \in\left(\pi_{x, x^{\prime}}^{p, k, m,\left(l_{0}, \ldots, l_{m}\right)}\right)^{-1}(Z) .
$$

Alors

$$
\left(a_{1}, \ldots, a_{p}, S_{0}, \ldots, S_{m^{\prime}},\left(y_{i}^{j}\right)_{i \in\left\{0, \ldots, m^{\prime}-1\right\}, j \in\left\{1, \ldots, l_{i}\right\}},\left(\mathcal{Y}_{m^{\prime}}^{j_{\lambda}}\right)_{\lambda \in\left\{1, \ldots, l_{m^{\prime}}^{\prime}\right\}}\right)
$$

appartient à $Y_{x^{\prime}}^{p, k^{\prime}, m^{\prime},\left(l_{0}, \ldots, l_{m^{\prime}-1}, l_{m^{\prime}}^{\prime}\right)}$.

d) Pour tout $z$ dans

$$
B(x, k+2 M) \cup \bigcup_{i \in\left\{m^{\prime}+1, \ldots, m\right\}} B\left(S_{i}, M\right) \cup \underset{\substack{i \in\left\{m^{\prime}+1, \ldots, m\right\} \\ j \in\left\{1, \ldots, l_{i}\right\}}}{\bigcup} B\left(\mathcal{Y}_{i}^{j}, M\right) \cup \bigcup_{j \notin J} B\left(y_{m^{\prime}}^{j}, M\right)
$$

et pour tout $z^{\prime}$ dans

$$
\bigcup_{i \in\left\{0, \ldots, m^{\prime}\right\}} B\left(S_{i}, M\right) \cup \underset{\substack{i \in\left\{0, \ldots, m^{\prime}-1\right\} \\ j \in\left\{1, \ldots, l_{i}\right\}}}{\bigcup} B\left(\mathcal{Y}_{i}^{j}, M\right) \cup \bigcup_{j \in J} B\left(y_{m^{\prime}}^{j}, M\right)
$$

géod $\left(z, z^{\prime}\right)$ rencontre $B\left(x^{\prime}, k^{\prime}+2 M-2 \delta\right)$.

e) Pour tout

$$
\left(\tilde{a}_{1}, \ldots, \tilde{a}_{p}, \tilde{S}_{0}, \ldots, \widetilde{S}_{m^{\prime}},\left(\tilde{\mathcal{Y}}_{i}^{j}\right)_{i \in\left\{0, \ldots, m^{\prime}-1\right\}, j \in\left\{1, \ldots, l_{i}\right\}},\left(\tilde{\mathscr{Y}}_{m^{\prime}}^{j \lambda}\right)_{\lambda \in\left\{1, \ldots, l_{m^{\prime}}^{\prime}\right\}}\right)
$$

appartenant à la même classe que

$$
\left(a_{1}, \ldots, a_{p}, S_{0}, \ldots, S_{m^{\prime}},\left(y_{i}^{j}\right)_{i \in\left\{0, \ldots, m^{\prime}-1\right\}, j \in\left\{1, \ldots, l_{i}\right\}},\left(\mathcal{Y}_{m^{\prime}}^{j_{\lambda}}\right)_{\lambda \in\left\{1, \ldots, l_{m^{\prime}}^{\prime}\right\}}\right)
$$


dans $\bar{Y}_{x^{\prime}}^{p, k^{\prime}, m^{\prime},\left(l_{0}, \ldots, l_{m^{\prime}-1}, l_{m^{\prime}}^{\prime}\right)}$, pour tout z dans

$B(x, k+2 M) \cup \bigcup_{i \in\left\{m^{\prime}+1, \ldots, m\right\}} B\left(S_{i}, M\right) \cup \underset{\substack{i \in\left\{m^{\prime}+1, \ldots, m\right\} \\ j \in\left\{1, \ldots, l_{i}\right\}}}{\bigcup} B\left(\mathcal{Y}_{i}^{j}, M\right) \cup \bigcup_{j \notin J} B\left(\mathcal{Y}_{m^{\prime}}^{j}, M\right)$

et pour tout $\tilde{z}^{\prime}$ dans

$$
\bigcup_{i \in\left\{0, \ldots, m^{\prime}\right\}} B\left(\widetilde{S}_{i}, M\right) \cup \underset{\substack{i \in\left\{0, \ldots, m^{\prime}-1\right\} \\ j \in\left\{1, \ldots, l_{i}\right\}}}{\bigcup} B\left(\tilde{y}_{i}^{j}, M\right) \cup \underset{\lambda \in\left\{1, \ldots, l_{m^{\prime}}^{\prime}\right\}}{\bigcup} B\left(\tilde{y}_{m^{\prime}}^{j_{\lambda}}, M\right),
$$

$\operatorname{géod}\left(z, \tilde{z}^{\prime}\right)$ rencontre $B\left(x^{\prime}, k^{\prime}+2 M\right)$.

Remarque. L'intérêt de e) est que la classe de

$$
\left(a_{1}, \ldots, a_{p}, S_{0}, \ldots, S_{m},\left(\mathcal{Y}_{i}^{j}\right)_{i \in\{0, \ldots, m\}, j \in\left\{1, \ldots, l_{i}\right\}}\right)
$$

dans $\bar{Y}_{x, x^{\prime}, \star}^{p, k,\left(l_{0}, \ldots, l_{m}\right)}$ est déterminée par celle de

$$
\left(a_{1}, \ldots, a_{p}, S_{0}, \ldots, S_{m^{\prime}},\left(y_{i}^{j}\right)_{i \in\left\{0, \ldots, m^{\prime}-1\right\}, j \in\left\{1, \ldots, l_{i}\right\}},\left(\mathcal{Y}_{m^{\prime}}^{j_{\lambda}}\right)_{\lambda \in\left\{1, \ldots, l_{m^{\prime}}^{\prime}\right\}}\right)
$$

dans $\bar{Y}_{x^{\prime}}^{p, k^{\prime}, m^{\prime},\left(l_{0}, \ldots, l_{m^{\prime}-1}, l_{m^{\prime}}^{\prime}\right)}$ et par la connaissance des parties "éliminées"

$$
\left(S_{i}\right)_{i \in\left\{m^{\prime}+1, \ldots, m\right\}},\left(\mathcal{Y}_{i}^{j}\right)_{i \in\left\{m^{\prime}+1, \ldots, m\right\}, j \in\left\{1, \ldots, l_{i}\right\}},\left(\mathcal{Y}_{m^{\prime}}^{j}\right)_{j \notin J},
$$

qui se trouvent en gros entre les boules $B(x, k+2 M)$ et $B\left(x^{\prime}, k^{\prime}+2 M\right)$.

Démonstration. Le a) et le b) résultent des lemmes 4.12 et 4.13. Comme la preuve de c) et d) est longue on commence par montrer que d) implique e). Cela résulte du sous-lemme suivant, appliqué à $r=k^{\prime}+2 M$, et $x^{\prime}$ au lieu de $x$.

Sous-lemme 4.58. Soit $r \geq 2 \delta$ et $z, z^{\prime}, \tilde{z}^{\prime} \in X$ tels que

- $\operatorname{géod}\left(z, z^{\prime}\right)$ rencontre $B(x, r-2 \delta)$,

- pour tout $y \in B(x, r), d\left(y, z^{\prime}\right)=d\left(y, \tilde{z}^{\prime}\right)$.

Alors géod $\left(z, \tilde{z}^{\prime}\right)$ rencontre $B(x, r)$.

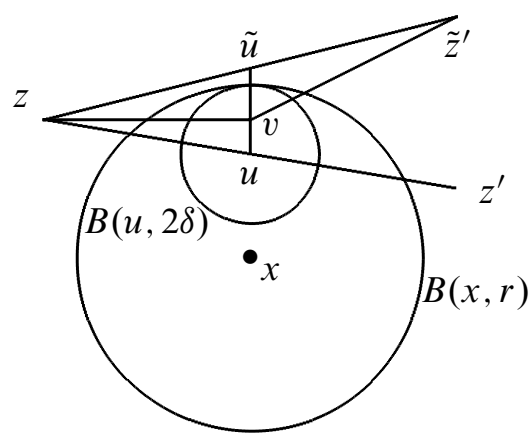


Démonstration. Soit $u \in \operatorname{géod}\left(z, z^{\prime}\right) \cap B(x, r-2 \delta)$. On veut montrer $u \in \operatorname{géod}\left(z, \tilde{z}^{\prime}\right)$. Supposons par l'absurde que cela ne soit pas vrai. Soit $\tilde{u} \in B(z, d(z, u))$ à distance minimale de $\tilde{z}^{\prime}$. On a alors $d\left(\tilde{u}, \tilde{z}^{\prime}\right)<d\left(u, \tilde{z}^{\prime}\right)=d\left(u, z^{\prime}\right) \leq d\left(\tilde{u}, z^{\prime}\right)$ donc $\tilde{u} \notin$ $B(x, r)$ et en particulier $d(u, \tilde{u}) \geq 2 \delta+1$. Soit $v \in \operatorname{géod}(u, \tilde{u})$ vérifiant $d(u, v)=\delta+$ 1. $\operatorname{Par}\left(H_{\delta}^{0}(z, u, v, \tilde{u})\right)$ on a $d(z, v) \leq d(z, u)$. $\operatorname{Par}\left(H_{\delta}^{0}\left(\tilde{z}^{\prime}, u, v, \tilde{u}\right)\right)$ on a $d\left(\tilde{z}^{\prime}, v\right)<$ $d\left(\tilde{z}^{\prime}, u\right)$. Comme $v \in B(x, r)$ on a $d\left(z^{\prime}, v\right)=d\left(\tilde{z}^{\prime}, v\right)$. On en déduit que $d(z, v)+$ $d\left(z^{\prime}, v\right)<d(z, u)+d\left(z^{\prime}, u\right)=d\left(z, z^{\prime}\right)$ ce qui est impossible.

Suite de la démonstration du lemme 4.57. Il reste à montrer c) et d). Pour le faire on distingue trois cas comme dans le dessin pour les arbres et dans la démonstration du lemme 4.54 .

Premier cas. On suppose $r_{0}(Z) \leq k$.

Alors $k^{\prime}=r_{0}^{\prime}(Z)+\frac{M}{2}$ par (121). D'après le lemme 4.12 et la remarque qui suit la définition 4.1 , on a $m=0$ et $l_{0}=0$, donc c) est évident. Comme $S_{0} \subset$ $B\left(x^{\prime}, r_{0}^{\prime}(Z)+N\right)$ on a $B\left(S_{0}, M\right) \subset B\left(x^{\prime}, k^{\prime}+2 M-2 \delta\right)$ car $M \geq N+2 \delta$ et d) en résulte, puisque pour $z^{\prime}$ comme dans d) on a $z^{\prime} \in B\left(x^{\prime}, k^{\prime}+2 M-2 \delta\right)$.

Deuxième cas. On suppose $r_{0}(Z)>k$ et $\frac{r_{0}(Z)-r_{0}^{\prime}(Z)+d\left(x, x^{\prime}\right)}{2} \leq k$.

Par (122) on a alors

$$
k^{\prime}=r_{0}^{\prime}(Z)-r_{0}(Z)+k+\frac{M}{2} .
$$

Soit $u \in B(x, k)$ à distance minimale de $S_{0}$ et $b \in S_{0}$ à distance minimale de $u$. Pour la suite on note le fait suivant. Comme $d(x, b)-d\left(x^{\prime}, b\right)+d\left(x, x^{\prime}\right) \leq 2 k+N$ et $u \in \operatorname{géod}(x, b),\left(H_{\delta}^{0}\left(x^{\prime}, x, u, b\right)\right)$ implique

$d\left(x^{\prime}, u\right) \leq \max \left(d\left(x, x^{\prime}\right)-k, d\left(x^{\prime}, b\right)-d(x, b)+k\right)+\delta \leq d\left(x^{\prime}, b\right)-d(b, u)+N+\delta$

et

$$
u \in(N+\delta)-\operatorname{géod}\left(x^{\prime}, b\right) \text {. }
$$

On a $k^{\prime} \geq d\left(x^{\prime}, b\right)-d(x, b)+k+\frac{M}{2}-N=d\left(x^{\prime}, b\right)-d(u, b)+\frac{M}{2}-N$ d'où, grâce à (130),

$$
k^{\prime} \geq d\left(x^{\prime}, u\right)+\frac{M}{2}-2 N-\delta .
$$

Comme $\max \left(k, \frac{d\left(x, x^{\prime}\right)+r_{0}(Z)-r_{0}^{\prime}(Z)}{2}\right)=k$, pour passer de

$$
\left(a_{1}, \ldots, a_{p}, S_{0}, \ldots, S_{m},\left(\mathcal{Y}_{i}^{j}\right)_{i \in\{0, \ldots, m\}, j \in\left\{1, \ldots, l_{i}\right\}}\right)
$$

à

$$
\left(a_{1}, \ldots, a_{p}, S_{0}, \ldots, S_{m^{\prime}},\left(y_{i}^{j}\right)_{i \in\left\{0, \ldots, m^{\prime}-1\right\}, j \in\left\{1, \ldots, l_{i}\right\}},\left(y_{m^{\prime}}^{j \lambda}\right)_{\lambda \in\left\{1, \ldots, l_{m^{\prime}}^{\prime}\right\}}\right)
$$

on enlève les $S_{i}$ et les $y_{i}^{j}$ pour $i \geq 1$ vérifiant $d_{\max }\left(x, S_{i}\right) \leq k+M$ et les $y_{m^{\prime}}^{j}$ vérifiant $d_{\max }\left(x, y_{m^{\prime}}^{j}\right) \leq k+M+2 P+\delta$. Pour vérifier c) on va montrer que les 
conditions i), ii), iii) et iv) de la définition 4.1 sont satisfaites par

$$
\left(a_{1}, \ldots, a_{p}, S_{0}, \ldots, S_{m^{\prime}},\left(y_{i}^{j}\right)_{i \in\left\{0, \ldots, m^{\prime}-1\right\}, j \in\left\{1, \ldots, l_{i}\right\}},\left(y_{m^{\prime}}^{j_{\lambda}}\right)_{\lambda \in\left\{1, \ldots, l_{m^{\prime}}^{\prime}\right\}}\right\} .
$$

On vérifie d'abord i). Soit $i \in\left\{0, \ldots, m^{\prime}-1\right\}, y \in S_{i+1}, a \in S_{i}$ et $\tilde{x} \in B(x, k)$ tels que

- $y \in 4 \delta$ - $\operatorname{géod}(\tilde{x}, a)$ et $d(y, a) \in] N-2 \delta, Q N]$,

- ou $y \in F-\operatorname{géod}(\tilde{x}, a)$ et $d(y, a) \geq \frac{Q}{F}$.

On note $\alpha=4 \delta$ ou $F$ suivant le cas, de sorte que $y \in \alpha$ - $\operatorname{géod}(\tilde{x}, a)$. Comme $d\left(x, S_{i+1}\right) \geq d_{\max }\left(x, S_{i+1}\right)-N$ et $i+1 \in\left\{1, \ldots, m^{\prime}\right\}$, on a

$$
d(x, y)>k+M-N .
$$

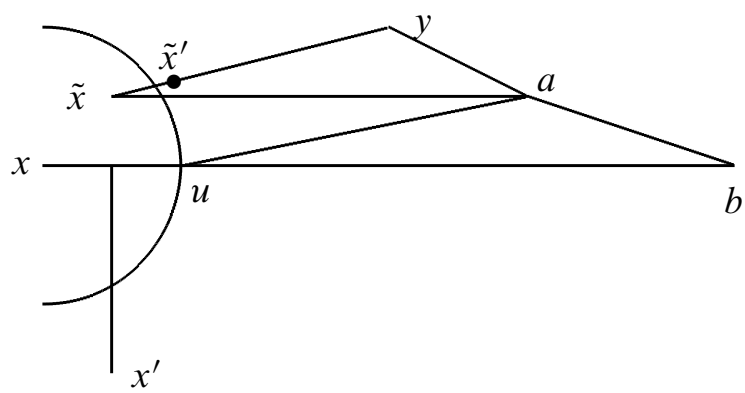

Pour vérifier i) il suffit de montrer qu'il existe $\tilde{x}^{\prime} \in B\left(x^{\prime}, k^{\prime}\right)$ tel que $y \in \alpha$-géod $\left(\tilde{x}^{\prime}, a\right)$. Cela résulte du sous-lemme suivant appliqué à $\alpha=4 \delta$ ou $\alpha=F$ et à $\beta=M-N$. On a $F \geq 4 \delta$ et on suppose $F+\frac{3 \delta+F}{2}<M-N$, ce qui est permis par $\left(H_{M}\right)$. Les hypothèses du sous-lemme suivant sont donc satisfaites et cela termine la preuve de i).

Sous-lemme 4.59. Soit $\alpha, \beta \in \mathbb{N}$ vérifiant $F+\frac{38+\alpha}{2}<\beta$. Soit $a \in 2 F$ - géod $(u, b)$, $\tilde{x} \in B(x, k)$ et $y \in \alpha-\operatorname{géod}(\tilde{x}, a)$ tel que $d(x, y) \geq k+\beta$. Alors il existe $\tilde{x}^{\prime} \in$ $B\left(x^{\prime}, k^{\prime}\right)$ tel que $y \in \alpha-\operatorname{géod}\left(\tilde{x}^{\prime}, a\right)$.

Démonstration du sous-lemme 4.59. Comme $a \in 2 F$ - $\operatorname{géod}(b, u)$ et $u \in \delta$ - $\operatorname{géod}(\tilde{x}, b)$ par le lemme 4.9 , on a $a \in(2 F+\delta)$ - $\operatorname{géod}(\tilde{x}, b)$ par le lemme 3.3 , et comme $y \in \alpha-\operatorname{géod}(\tilde{x}, a)$ on en déduit

$$
y \in(2 F+\delta+\alpha)-\operatorname{géod}(\tilde{x}, b) .
$$

On applique le lemme 4.6 à $\{x, u, b, \tilde{x}, y\}$ avec $l=2$ et $x$ comme point base. Soit $t$ le point de $\operatorname{géod}(\Psi \tilde{x}, \Psi b)$ le plus proche de $\Psi y$. 


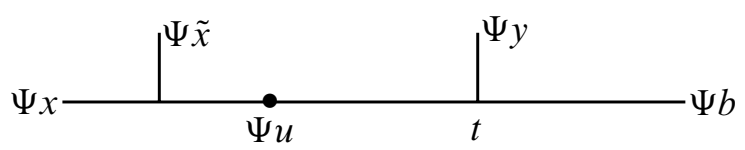

Grâce au a) du lemme 4.7 on a $\Psi y \in(2 F+3 \delta+\alpha)$ - géod $(\Psi \tilde{x}, \Psi b)$, d'où $d(\Psi y, t) \leq$ $F+\frac{3 \delta+\alpha}{2}$. De plus $d(\Psi x, \Psi y)=d(x, y) \geq k+\beta$. Comme $F+\frac{3 \delta+\alpha}{2}<\beta$ on a $d(\Psi x, t)>k=d(\Psi x, \Psi u)$ et comme $d(\Psi x, \Psi \tilde{x}) \leq k$ on en déduit $\Psi u \in$ $\operatorname{géod}(\Psi \tilde{x}, \Psi y)$ et $u \in 4 \delta$ - $\operatorname{géod}(\tilde{x}, y)$ par le b) du lemme 4.7 .

Soit $\tilde{x}^{\prime} \in \operatorname{géod}(\tilde{x}, y)$ vérifiant $d\left(y, \tilde{x}^{\prime}\right)=\min (d(y, u), d(y, \tilde{x}))$. On a alors, par $\left(H_{\delta}^{0}\left(u, y, \tilde{x}^{\prime}, \tilde{x}\right)\right), d\left(u, \tilde{x}^{\prime}\right) \leq 5 \delta$. Par (131) on a $k^{\prime} \geq d\left(x^{\prime}, u\right)+\frac{M}{2}-2 N-\delta$ et on suppose $2 N+6 \delta \leq \frac{M}{2}$, ce qui est permis par $\left(H_{M}\right)$. Alors $d\left(x^{\prime}, \tilde{x}^{\prime}\right) \leq k^{\prime}$ et ceci termine la démonstration du sous-lemme 4.59 .

Fin de l'étude du deuxième cas. On vérifie maintenant ii). Soit $i \in\left\{1, \ldots, m^{\prime}\right\}$. On a

$$
S_{i} \subset 2 F-\operatorname{géod}(b, u)
$$

par le a) du lemme 4.14. On a $d_{\max }\left(x, S_{i}\right)>k+M$ par hypothèse. Soit $a \in S_{i}$. On a alors $d(x, a)>k+M-N$ d'où $d(u, a)>M-N$. Par (130) et le b) du lemme 3.3, on a $u \in(2 F+N+\delta)$-géod $\left(x^{\prime}, a\right)$. D'où

$d\left(x^{\prime}, a\right) \geq d\left(x^{\prime}, u\right)+d(u, a)-(2 F+N+\delta)>d\left(x^{\prime}, u\right)+M-(2 F+2 N+\delta)$.

Or

$$
\begin{aligned}
k^{\prime} & \leq d\left(x^{\prime}, b\right)-d(x, b)+k+\frac{M}{2}+N \\
& =d\left(x^{\prime}, b\right)-d(b, u)+\frac{M}{2}+N \\
& \leq d\left(x^{\prime}, u\right)+\frac{M}{2}+N .
\end{aligned}
$$

D'où

$$
d\left(x^{\prime}, a\right)>k^{\prime}+\frac{M}{2}-(2 F+3 N+\delta) \geq k^{\prime}+P
$$

car on suppose $(2 F+3 N+\delta)+P \leq \frac{M}{2}$, ce qui est permis par $\left(H_{M}\right)$. Cela achève la preuve de ii).

La propriété iii) est immédiate.

On vérifie maintenant iv). Soit $j \in J$. On commence par montrer $d\left(x^{\prime}, y_{m^{\prime}}^{j}\right) \geq$ $k^{\prime}+3 P$. On rappelle que $y_{m^{\prime}}^{j} \subset 4 P-\operatorname{géod}(x, b)$ et $d\left(x, y_{m^{\prime}}^{j}\right) \geq k+M+P+\delta$. Soit $y \in y_{m^{\prime}}^{j}$. On a alors

$$
d(b, y) \leq d(x, b)-d(x, y)+4 P \leq d(b, x)-k-M-\delta+3 P
$$


et

$$
\begin{aligned}
d\left(x^{\prime}, y\right) & \geq d\left(x^{\prime}, b\right)-d(b, y) \\
& \geq d\left(x^{\prime}, b\right)-d(x, b)+k+M+\delta-3 P \\
& \geq k^{\prime}+\frac{M}{2}-N+\delta-3 P \geq k^{\prime}+3 P .
\end{aligned}
$$

car on suppose $\frac{M}{2}-N+\delta-3 P \geq 3 P$, ce qui est permis par $\left(H_{M}\right)$.

Il reste donc à montrer que pour tout $y \in y_{m^{\prime}}^{j}$ il existe $a \in S_{m^{\prime}}$ et $\tilde{x}^{\prime} \in B\left(x^{\prime}, k^{\prime}\right)$ tels que $y \in 2 P-\operatorname{géod}\left(\tilde{x}^{\prime}, a\right)$. Soit $y \in y_{m^{\prime}}^{j}$. On va distinguer deux cas.

On suppose d'abord $m^{\prime}<m$. Alors il existe $a \in S_{m^{\prime}}$ et $w \in S_{m^{\prime}+1}$ tels que $y \in P-\operatorname{géod}(w, a)$. Par le a) du lemme 4.14, $w$ et $a$ appartiennent à $2 F-\operatorname{géod}(u, b)$. Comme $u \in \operatorname{géod}(x, b)$ le b) du lemme 3.3 montre $u \in 2 F-\operatorname{géod}(x, w)$. Donc $d(u, w) \leq d(x, w)-k+2 F$.

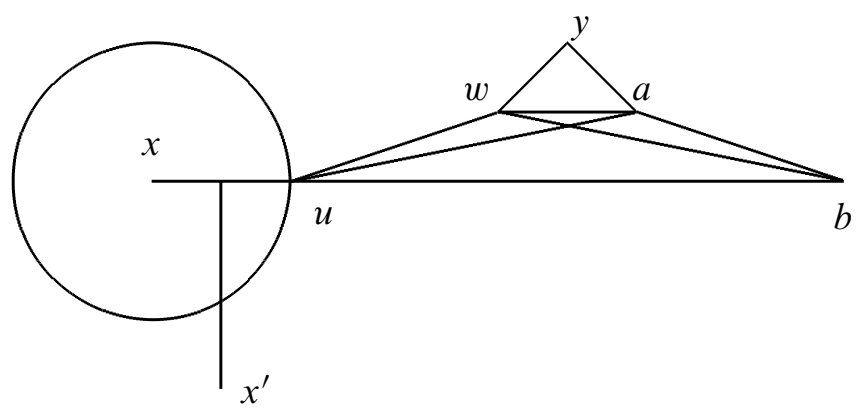

D'autre part $d(x, w) \leq d_{\max }\left(x, S_{m^{\prime}+1}\right) \leq d_{\max }\left(x, S_{m^{\prime}}\right) \leq d(x, a)+N$ et $d(x, a)-k \leq d(u, a)$. On en déduit $d(u, w) \leq d(u, a)+(2 F+N)$. Le lemme 4.8 appliqué à $(u, b, a, w)$ au lieu de $(x, y, a, b)$ et à $(2 F, 2 F, 2 F+N)$ au lieu de $(\alpha, \beta, \rho)$ montre alors $w \in(6 F+2 N+\delta)-\operatorname{géod}(u, a)$. On en déduit $y \in(6 F+2 N+$ $\delta+P)-\operatorname{géod}(u, a)$ par le lemme 3.3 et donc $y \in 2 P-\operatorname{géod}(u, a)$ car on suppose $P \geq 6 F+2 N+\delta$ grâce à $\left(H_{P}\right)$. On suppose $\frac{M}{2} \geq 2 N+\delta$ grâce à $\left(H_{M}\right)$. Par (131) on a alors $u \in B\left(x^{\prime}, k^{\prime}\right)$. En prenant $\tilde{x}^{\prime}=u$ on a fini.

On suppose maintenant $m^{\prime}=m$. Il existe $a \in S_{m}$ et $\tilde{x} \in B(x, k)$ tels que $y \in 2 P-\operatorname{géod}(\tilde{x}, a)$. On veut montrer qu'il existe $\tilde{x}^{\prime} \in B\left(x^{\prime}, k^{\prime}\right)$ tel que $y \in$ $2 P-\operatorname{géod}\left(\tilde{x}^{\prime}, a\right)$. On a $d(x, y) \geq k+M+P+\delta$. On applique le sous-lemme 4.59 avec $\alpha=2 P$ et $\beta=M+P+\delta$. On suppose $F+\frac{3 \delta+\alpha}{2}<\beta$, ce qui est permis par $\left(H_{M}\right)$.

Il reste à montrer d). Le premier ensemble de d) est inclus dans $B(x, k+2 M+$ $2 P+\delta)$ et le second ensemble de d) est inclus dans $(2 M+4 P)$ - géod $(u, b)$. On a $B\left(u, \frac{5 M}{2}-2 N-3 \delta\right) \subset B\left(x^{\prime}, k^{\prime}+2 M-2 \delta\right)$ grâce à (131). Il suffit donc de montrer que pour $z \in B(x, k+2 M+2 P+\delta)$ et $z^{\prime} \in(2 M+4 P)-\operatorname{géod}(u, b), \operatorname{géod}\left(z, z^{\prime}\right)$ rencontre $B\left(u, \frac{5 M}{2}-2 N-3 \delta\right)$. Par $\left(H_{\delta}^{0}(z, x, u, b)\right)$, on a $u \in \delta$ - $\operatorname{géod}(x, z)$ ou $u \in \delta-\operatorname{géod}(z, b)$. Si $u \in \delta$ - $\operatorname{géod}(x, z), d(u, z) \leq 2 M+2 P+2 \delta$, donc $z \in$ $\operatorname{géod}\left(z, z^{\prime}\right)$ appartient à $B\left(u, \frac{5 M}{2}-2 N-3 \delta\right)$ car on suppose $\frac{M}{2} \geq 2 P+2 N+5 \delta$ 
grâce à $\left(H_{M}\right)$. Si $u \in \delta$-géod $(z, b)$, comme $z^{\prime} \in(2 M+4 P)$-géod $(u, b)$, on a $u \in(2 M+4 P+\delta)$ - géod $\left(z, z^{\prime}\right)$ par le b) du lemme 3.3, donc $t \in \operatorname{géod}\left(z, z^{\prime}\right)$ vérifiant $d(z, t)=\min \left(d(z, u), d\left(z, z^{\prime}\right)\right)$ satisfait, grâce à $\left(H_{\delta}^{0}\left(u, z, t, z^{\prime}\right)\right)$,

$$
d(u, t) \leq 2 M+4 P+2 \delta \leq \frac{5 M}{2}-2 N-3 \delta
$$

car on suppose $2 M+4 P+2 \delta \leq \frac{5 M}{2}-2 N-3 \delta$ grâce à $\left(H_{M}\right)$. Cela termine la preuve de $\mathrm{d}$ ) et donc l'étude du deuxième cas.

Troisième cas. On suppose $r_{0}(Z)>k$ et $\frac{r_{0}(Z)-r_{0}^{\prime}(Z)+d\left(x, x^{\prime}\right)}{2}>k$.

Par (123) on a alors

$$
\left|k^{\prime}-\left(\mathrm{E}\left(\frac{r_{0}^{\prime}(Z)-r_{0}(Z)+d\left(x, x^{\prime}\right)}{2}\right)+\frac{M}{2}\right)\right| \leq \frac{N}{2} .
$$

Comme $\max \left(k, \frac{d\left(x, x^{\prime}\right)+r_{0}(Z)-r_{0}^{\prime}(Z)}{2}\right)=\frac{d\left(x, x^{\prime}\right)+r_{0}(Z)-r_{0}^{\prime}(Z)}{2}$, pour passer de

$$
\left(a_{1}, \ldots, a_{p}, S_{0}, \ldots, S_{m},\left(\mathcal{Y}_{i}^{j}\right)_{i \in\{0, \ldots, m\}, j \in\left\{1, \ldots, l_{i}\right\}}\right)
$$

à

$$
\left(a_{1}, \ldots, a_{p}, S_{0}, \ldots, S_{m^{\prime}},\left(\mathcal{Y}_{i}^{j}\right)_{i \in\left\{0, \ldots, m^{\prime}-1\right\}, j \in\left\{1, \ldots, l_{i}\right\}},\left(\mathcal{y}_{m^{\prime}}^{j_{\lambda}}\right)_{\lambda \in\left\{1, \ldots, l_{m^{\prime}}^{\prime}\right\}}\right)
$$

on enlève les $S_{i}$ et les $y_{i}^{j}$ pour $i \geq 1$ vérifiant

$$
d_{\max }\left(x, S_{i}\right) \leq \frac{r_{0}(Z)-r_{0}^{\prime}(Z)+d\left(x, x^{\prime}\right)}{2}+M
$$

ainsi que les $y_{m^{\prime}}^{j}$ vérifiant

$$
d_{\max }\left(x, y_{m^{\prime}}^{j}\right) \leq \frac{r_{0}(Z)-r_{0}^{\prime}(Z)+d\left(x, x^{\prime}\right)}{2}+M+2 P+\delta .
$$

Soit $u \in B(x, k)$ à distance minimale de $S_{0}$ et $b \in S_{0}$ à distance minimale de $u$. Il résulte de (132) que

$$
k^{\prime} \geq \frac{d\left(x^{\prime}, b\right)-d(x, b)+d\left(x, x^{\prime}\right)}{2}+\frac{M}{2}-N
$$

et

$$
k^{\prime} \leq \frac{d\left(x^{\prime}, b\right)-d(x, b)+d\left(x, x^{\prime}\right)}{2}+\frac{M}{2}+N .
$$

Pour vérifier c) on va montrer que les conditions i), ii), iii) et iv) de la définition 4.1 sont satisfaites par

$$
\left(a_{1}, \ldots, a_{p}, S_{0}, \ldots, S_{m^{\prime}},\left(y_{i}^{j}\right)_{i \in\left\{0, \ldots, m^{\prime}-1\right\}, j \in\left\{1, \ldots, l_{i}\right\}},\left(\mathcal{Y}_{m^{\prime}}^{j_{\lambda}}\right)_{\lambda \in\left\{1, \ldots, l_{m^{\prime}}^{\prime}\right\}}\right) .
$$

On vérifie d'abord i). Soit $i \in\left\{0, \ldots, m^{\prime}-1\right\}, y \in S_{i+1}, a \in S_{i}$ et $\tilde{x} \in B(x, k)$ tels que 
- $y \in 4 \delta-\operatorname{géod}(\tilde{x}, a)$ et $d(y, a) \in] N-2 \delta, Q N]$,

- ou $y \in F$ - $\operatorname{géod}(\tilde{x}, a)$ et $d(y, a) \geq \frac{Q}{F}$.

On note $\alpha=4 \delta$ ou $F$ suivant le cas, de sorte que $y \in \alpha$ - $\operatorname{géod}(\tilde{x}, a)$. Comme $d\left(x, S_{i+1}\right) \geq d_{\max }\left(x, S_{i+1}\right)-N$ et $i+1 \in\left\{1, \ldots, m^{\prime}\right\}$, on a

$$
\begin{aligned}
d(x, y) & \geq \frac{r_{0}(Z)-r_{0}^{\prime}(Z)+d\left(x, x^{\prime}\right)}{2}+M-N \\
& \geq \frac{d(x, b)-d\left(x^{\prime}, b\right)+d\left(x, x^{\prime}\right)}{2}+M-\frac{3 N}{2} .
\end{aligned}
$$

Pour vérifieri) il suffit de montrer qu'il existe $\tilde{x}^{\prime} \in B\left(x^{\prime}, k^{\prime}\right)$ tel que $y \in \alpha$-géod $\left(\tilde{x}^{\prime}, a\right)$.

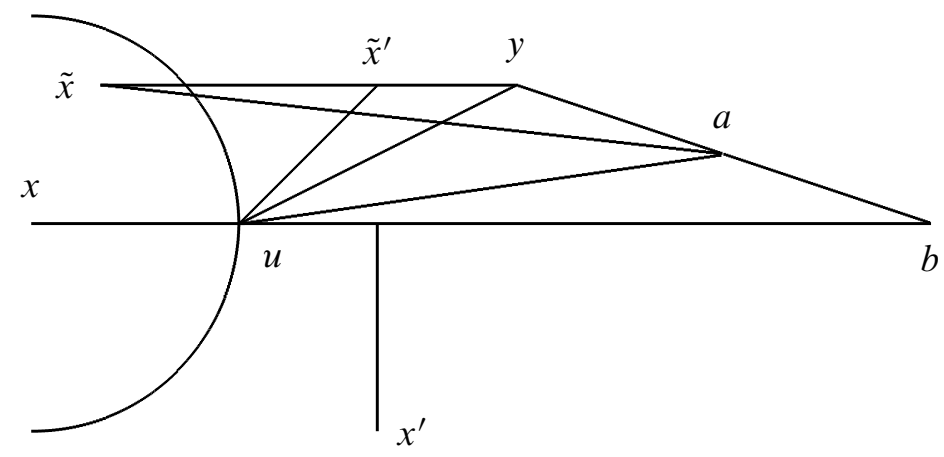

Cela résulte du sous-lemme suivant appliqué à $\alpha=4 \delta$ ou $\alpha=F$ et à $\beta=$ $M-\frac{3 N}{2}$. On a $F \geq 4 \delta$ et on suppose $F+\frac{3 \delta+F}{2}+\delta+N \leq M-\frac{3 N}{2}$, ce qui est permis par $\left(H_{M}\right)$. Les hypothèses du sous-lemme suivant sont donc satisfaites et cela termine la preuve de i).

Sous-lemme 4.60. Soit $\alpha, \beta \in \mathbb{N}$ vérifiant $F+\frac{38+\alpha}{2}+\delta+N \leq \beta$. Soit a $\in$ $2 F-\operatorname{géod}(u, b), \tilde{x} \in B(x, k)$ et $y \in \alpha-\operatorname{géod}(\tilde{x}, a)$ tel que

$$
d(x, y) \geq \frac{d(x, b)-d\left(x^{\prime}, b\right)+d\left(x, x^{\prime}\right)}{2}+\beta .
$$

Alors il existe $\tilde{x}^{\prime} \in B\left(x^{\prime}, k^{\prime}\right)$ tel que $y \in \alpha$-géod $\left(\tilde{x}^{\prime}, a\right)$.

Démonstration du sous-lemme 4.60. Comme $a \in 2 F$ - $\operatorname{géod}(b, u)$ et $u \in \delta$ - géod $(\tilde{x}, b)$ par le lemme 4.9 , on a $a \in(2 F+\delta)$ - $\operatorname{géod}(\tilde{x}, b)$ par le lemme 3.3, et comme $y \in \alpha-\operatorname{géod}(\tilde{x}, a)$ on en déduit $y \in(2 F+\delta+\alpha)-\operatorname{géod}(\tilde{x}, b)$. On applique le lemme 4.6 à $\left\{x, b, x^{\prime}, \tilde{x}, y\right\}$ avec $l=2$ et $x$ comme point base. Soit $t \in \operatorname{géod}(\Psi \tilde{x}, \Psi b)$ à distance minimale de $\Psi y$. Comme $y \in(2 F+\delta+\alpha)$ - $\operatorname{géod}(\tilde{x}, b)$, on a $\Psi y \in$ $(2 F+3 \delta+\alpha)-\operatorname{géod}(\Psi \tilde{x}, \Psi b)$ par le a) du lemme 4.7, donc $d(\Psi y, t) \leq F+\frac{3 \delta+\alpha}{2}$. 
Or

$$
\begin{aligned}
d(\Psi x, \Psi y)=d(x, y) \geq & \frac{d(x, b)-d\left(x^{\prime}, b\right)+d\left(x, x^{\prime}\right)}{2}+\beta \\
& \geq k-\frac{N}{2}+\beta>k+\left(F+\frac{3 \delta+\alpha}{2}\right)
\end{aligned}
$$

par hypothèse. Donc $d(\Psi x, t)>k$ et comme $d(\Psi x, \Psi \tilde{x}) \leq k, t \in \operatorname{géod}(\Psi x, \Psi b)$.

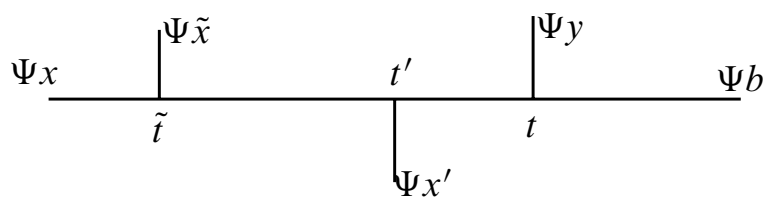

De ce qui précède on retient aussi que

$$
d(\Psi x, t) \geq \frac{d(x, b)-d\left(x^{\prime}, b\right)+d\left(x, x^{\prime}\right)}{2}+\beta-\left(F+\frac{3 \delta+\alpha}{2}\right) .
$$

Soit $t^{\prime}$ le point de géod $(\Psi x, \Psi b)$ à distance minimale de $\Psi x^{\prime}$. On a donc

$$
\begin{gathered}
d\left(\Psi x, t^{\prime}\right)=\frac{d(\Psi x, \Psi b)-d\left(\Psi x^{\prime}, \Psi b\right)+d\left(\Psi x, \Psi x^{\prime}\right)}{2} \\
\in\left[\frac{d(x, b)-d\left(x^{\prime}, b\right)+d\left(x, x^{\prime}\right)}{2}, \frac{d(x, b)-d\left(x^{\prime}, b\right)+d\left(x, x^{\prime}\right)}{2}+\delta\right]
\end{gathered}
$$

par le lemme 4.6. Comme $F+\frac{3 \delta+\alpha}{2}+\delta+N \leq \beta$ par hypothèse, il résulte de (135) et (136) que $d\left(\Psi x, t^{\prime}\right) \leq d(\Psi x, t)$. Par ailleurs $d\left(\Psi x, t^{\prime}\right) \geq \frac{d(x, b)-d\left(x^{\prime}, b\right)+d\left(x, x^{\prime}\right)}{2}>$ $k-N$. Si $\tilde{t}$ désigne le point de géod $(\Psi x, \Psi b)$ à distance minimale de $\Psi \tilde{x}$, comme $d(\Psi x, \Psi \tilde{x}) \leq k$, on a $d(\Psi x, \tilde{t}) \leq k$, donc $d\left(\Psi x, t^{\prime}\right) \geq d(\Psi x, \tilde{t})-N($ dans le dessin ci-dessus $t^{\prime}$ pourrait être entre $\Psi x$ et $\tilde{t}$, mais dans ce cas à distance $\leq N$ de $\tilde{t}$ ). En tous cas on a $d\left(t^{\prime}, \operatorname{géod}(\tilde{t}, t)\right) \leq N$, d'où $d\left(t^{\prime}, \operatorname{géod}(\Psi \tilde{x}, \Psi y)\right) \leq N$. On en déduit

$$
\begin{aligned}
d\left(\Psi x^{\prime}, \operatorname{géod}(\Psi \tilde{x}, \Psi y)\right) & \leq d\left(\Psi x^{\prime}, t^{\prime}\right)+d\left(t^{\prime}, \operatorname{géod}(\Psi \tilde{x}, \Psi y)\right) \\
& \leq d\left(\Psi x^{\prime}, t^{\prime}\right)+N \\
& =\frac{d\left(\Psi x, \Psi x^{\prime}\right)+d\left(\Psi x^{\prime}, \Psi b\right)-d(\Psi x, \Psi b)}{2}+N \\
& \leq \frac{d\left(x, x^{\prime}\right)+d\left(x^{\prime}, b\right)-d(x, b)}{2}+N .
\end{aligned}
$$

Par le c) du lemme 4.7,

$$
\begin{aligned}
d\left(x^{\prime}, \operatorname{géod}(\tilde{x}, y)\right) & \leq d\left(\Psi x^{\prime}, \operatorname{géod}(\Psi \tilde{x}, \Psi y)\right)+3 \delta+1 \\
& \leq \frac{d\left(x, x^{\prime}\right)+d\left(x^{\prime}, b\right)-d(x, b)}{2}+N+3 \delta+1 \leq k^{\prime}
\end{aligned}
$$

grâce à (133) et car on suppose $2 N+3 \delta+1 \leq \frac{M}{2}$, ce qui est permis par $\left(H_{M}\right)$. Il existe donc $\tilde{x}^{\prime} \in \operatorname{géod}(\tilde{x}, y) \cap B\left(x^{\prime}, k^{\prime}\right)$. On a alors $y \in \alpha-\operatorname{géod}\left(\tilde{x}^{\prime}, a\right)$. 
Fin de l'étude du troisième cas. On vérifie maintenant ii). Soit $i \in\left\{1, \ldots, m^{\prime}\right\}$. On a

$$
S_{i} \subset 2 F \text {-géod }(b, u) \subset 2 F \text { - géod }(x, b)
$$

par le a) du lemme 4.14. On a $d_{\max }\left(x, S_{i}\right)>\frac{r_{0}(Z)-r_{0}^{\prime}(Z)+d\left(x, x^{\prime}\right)}{2}+M$ par hypothèse.

Soit $a \in S_{i}$. On a donc

$$
\begin{aligned}
d(x, a) & >\frac{r_{0}(Z)-r_{0}^{\prime}(Z)+d\left(x, x^{\prime}\right)}{2}+M-N \\
& \geq \frac{d(x, b)-d\left(x^{\prime}, b\right)+d\left(x, x^{\prime}\right)}{2}+M-2 N .
\end{aligned}
$$

Donc $d(b, a) \leq d(x, b)-d(x, a)+2 F<\frac{d(x, b)+d\left(x^{\prime}, b\right)-d\left(x, x^{\prime}\right)}{2}-M+(2 N+2 F)$ et

$$
d\left(x^{\prime}, a\right) \geq d\left(x^{\prime}, b\right)-d(b, a)>\frac{d\left(x^{\prime}, b\right)+d\left(x, x^{\prime}\right)-d(x, b)}{2}+M-(2 N+2 F) .
$$

Grâce à (134) on en déduit

$$
d\left(x^{\prime}, a\right)>k^{\prime}+\frac{M}{2}-(3 N+2 F) \geq k^{\prime}+P
$$

car on suppose $\frac{M}{2} \geq(3 N+2 F)+P$, ce qui est permis par $\left(H_{M}\right)$. Ceci achève la preuve de ii).

La propriété iii) est immédiate.

On vérifie maintenant iv). On note $b$ et $u$ comme dans la preuve de i). Soit $j \in J$. On commence par montrer $d\left(x^{\prime}, y_{m^{\prime}}^{j}\right) \geq k^{\prime}+3 P$. On rappelle que $y_{m^{\prime}}^{j} \subset$ $4 P-\operatorname{géod}(x, b)$ et

$$
\begin{aligned}
d\left(x, y_{m^{\prime}}^{j}\right) & \geq \frac{r_{0}(Z)-r_{0}^{\prime}(Z)+d\left(x, x^{\prime}\right)}{2}+M+P+\delta \\
& \geq \frac{d(x, b)-d\left(x^{\prime}, b\right)+d\left(x, x^{\prime}\right)}{2}+M+P+\delta-\frac{N}{2} .
\end{aligned}
$$

Soit $y \in y_{m^{\prime}}^{j}$. On a alors, grâce à (138),

et

$$
\begin{aligned}
d(b, y) & \leq d(x, b)-d(x, y)+4 P \\
& \leq \frac{d(x, b)+d\left(x^{\prime}, b\right)-d\left(x, x^{\prime}\right)}{2}-M+3 P-\delta+\frac{N}{2}
\end{aligned}
$$

$$
\begin{aligned}
d\left(x^{\prime}, y\right) & \geq d\left(x^{\prime}, b\right)-d(b, y) \\
& \geq \frac{d\left(x^{\prime}, b\right)-d(x, b)+d\left(x, x^{\prime}\right)}{2}+M+\delta-3 P-\frac{N}{2} \\
& \geq k^{\prime}+\frac{M}{2}+\delta-3 P-\frac{3 N}{2} \geq k^{\prime}+3 P
\end{aligned}
$$


où l'avant-dernière inégalité a lieu par (134) et où, pour la dernière, on a supposé $\frac{M}{2}+\delta-3 P-\frac{3 N}{2} \geq 3 P$, ce qui est permis par $\left(H_{M}\right)$.

Il reste donc à montrer que pour tout $y \in y_{m^{\prime}}^{j}$ il existe $a \in S_{m^{\prime}}$ et $\tilde{x}^{\prime} \in B\left(x^{\prime}, k^{\prime}\right)$ tels que $y \in 2 P-\operatorname{géod}\left(\tilde{x}^{\prime}, a\right)$. Soit $y \in \mathcal{Y}_{m^{\prime}}^{j}$. On va distinguer deux cas.

On suppose d'abord $m^{\prime}<m$. Alors il existe $a \in S_{m^{\prime}}$ et $w \in S_{m^{\prime}+1}$ tels que $y \in P-\operatorname{géod}(w, a)$. On a $w, a \in 2 F-\operatorname{géod}(u, b)$. D'autre part

$$
d(x, w) \leq d_{\max }\left(x, S_{m^{\prime}+1}\right) \leq d_{\max }\left(x, S_{m^{\prime}}\right) \leq d(x, a)+N .
$$

Le lemme 4.8 appliqué à $(x, b, a, w)$ au lieu de $(x, y, a, b)$ et $(2 F, 2 F, N)$ au lieu de $(\alpha, \beta, \rho)$ montre alors $w \in(2 F+2 N+\delta)-\operatorname{géod}(x, a)$.

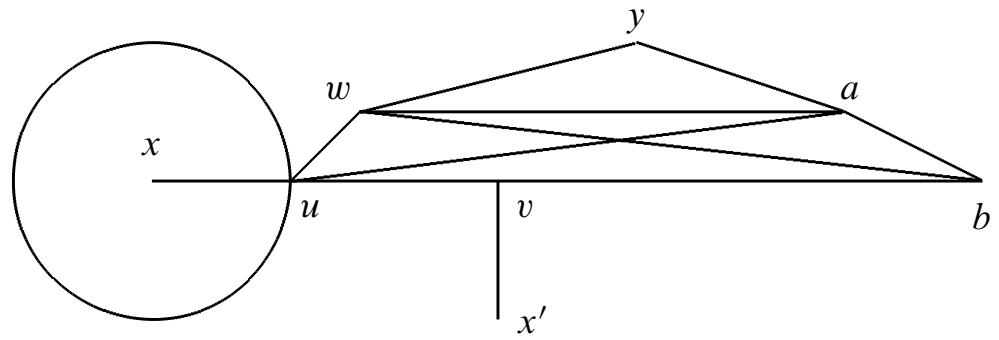

Soit maintenant $v \in \operatorname{géod}(x, b)$ vérifiant $d(x, v)=\mathrm{E}\left(\frac{d(x, b)+d\left(x, x^{\prime}\right)-d\left(x^{\prime}, b\right)}{2}\right)$. Par $\left(H_{\delta}^{0}\left(x^{\prime}, x, v, b\right)\right)$ on a

$$
v \in \operatorname{géod}(x, b) \cap(\delta+1)-\operatorname{géod}\left(x, x^{\prime}\right) \cap(\delta+1)-\operatorname{géod}\left(x^{\prime}, b\right)
$$

et

$$
d\left(x^{\prime}, v\right) \leq \frac{d\left(x^{\prime}, b\right)-d(x, b)+d\left(x, x^{\prime}\right)}{2}+\delta+1 \leq k^{\prime}
$$

où la dernière égalité a lieu grâce à (133) car on suppose $N+\delta+1 \leq \frac{M}{2}$, ce qui est permis par $\left(H_{M}\right)$. On a donc $v \in B\left(x^{\prime}, k^{\prime}\right)$.

Comme $y \in P-\operatorname{géod}(w, a)$ et $w \in(2 F+2 N+\delta)-\operatorname{géod}(x, a)$ on a $y \in(2 F+$ $2 N+\delta+P)-\operatorname{géod}(x, a)$. Par (137) on a $d(x, a) \geq d(x, v)+M-2 N$, et comme $a \in 2 F-\operatorname{géod}(x, b)$ on en déduit $d(b, a)-d(b, v) \leq-M+2 N+2 F<-\delta$ car on suppose $-M+2 N+2 F<-\delta$ grâce à $\left(H_{M}\right)$. Alors $\left(H_{\delta}^{0}(a, x, v, b)\right)$ donne $v \in \delta$ - géod $(x, a)$. Grâce à (138) on a

$$
\begin{aligned}
d(x, y) & \geq \frac{d(x, b)-d\left(x^{\prime}, b\right)+d\left(x, x^{\prime}\right)}{2}+M+P+\delta-\frac{N}{2} \\
& \geq d(x, v)-\frac{N}{2}+M+P+\delta .
\end{aligned}
$$

$\operatorname{Par}\left(H_{\delta}^{2 F+2 N+\delta+P}(v, x, y, a)\right)$ on a

$$
d(v, y) \leq \max (d(v, x)-d(x, y), d(v, a)-d(a, y))+2 F+2 N+2 \delta+P .
$$


Or $d(v, x)-d(x, y)+2 F+2 N+2 \delta+P<0$ par (139) et car on suppose $2 F+2 N+2 \delta+P<-\frac{N}{2}+M+P+\delta$, ce qui est permis par $\left(H_{M}\right)$. D'où $y \in(2 F+2 N+2 \delta+P)-\operatorname{géod}(v, a)$. Grâce à $\left(H_{P}\right)$ on suppose $P \geq 2 F+2 N+2 \delta$, d'où $y \in 2 P-\operatorname{géod}(v, a)$. En prenant $\tilde{x}^{\prime}=v$ on a fini.

On suppose maintenant $m^{\prime}=m$. Il existe $a \in S_{m^{\prime}}$ et $\tilde{x} \in B(x, k)$ tels que $y \in 2 P-\operatorname{géod}(\tilde{x}, a)$. On veut montrer qu'il existe $\tilde{x}^{\prime} \in B\left(x^{\prime}, k^{\prime}\right)$ tel que $y \in$ $2 P$ - géod $\left(\tilde{x}^{\prime}, a\right)$. Grâce à (138) on peut appliquer le sous-lemme 4.60 avec $\alpha=2 P$ et $\beta=M+P+\delta-\frac{N}{2}$. On suppose $F+\frac{3 \delta+\alpha}{2}+\delta+N \leq \beta$, ce qui est permis par $\left(H_{M}\right)$. Donc les hypothèses du sous-lemme 4.60 sont satisfaites et l'existence de $\tilde{x}^{\prime}$ est démontrée.

Il reste à montrer d). Soit $v \in \operatorname{géod}(x, b)$ vérifiant

$$
d(x, v)=\mathrm{E}\left(\frac{d(x, b)+d\left(x, x^{\prime}\right)-d\left(x^{\prime}, b\right)}{2}\right) .
$$

$\operatorname{Par}\left(H_{\delta}^{0}\left(x^{\prime}, x, v, b\right)\right)$ on a $v \in \operatorname{géod}(x, b) \cap(\delta+1)-\operatorname{géod}\left(x, x^{\prime}\right) \cap(\delta+1)-\operatorname{géod}\left(x^{\prime}, b\right)$ et

$$
d\left(x^{\prime}, v\right) \leq \frac{d\left(x^{\prime}, b\right)-d(x, b)+d\left(x, x^{\prime}\right)}{2}+\delta+1 .
$$

Il résulte alors de (133) que $k^{\prime} \geq d\left(x^{\prime}, v\right)+\frac{M}{2}-(N+\delta+1)$, donc

$$
B\left(v, \frac{5 M}{2}-(N+3 \delta+1)\right) \subset B\left(x^{\prime}, k^{\prime}+2 M-2 \delta\right) .
$$

Le premier ensemble de d) est inclus dans

$$
B\left(x, \frac{d\left(x, x^{\prime}\right)+r_{0}(Z)-r_{0}^{\prime}(Z)}{2}+2 M+2 P+\delta\right) \subset B(x, d(x, v)+2 M+2 P+N+\delta) .
$$

$\mathrm{Si}$

$$
r_{0}(Z) \leq \frac{d\left(x, x^{\prime}\right)+r_{0}(Z)-r_{0}^{\prime}(Z)}{2}+M
$$

on a $k^{\prime} \geq \frac{r_{0}^{\prime}(Z)-r_{0}(Z)+d\left(x, x^{\prime}\right)}{2}+\frac{M}{2}-N \geq r_{0}^{\prime}(Z)-\frac{M}{2}-N$, d'où $B\left(S_{0}, M\right) \subset$ $B\left(x^{\prime}, k^{\prime}+2 M\right)$.

Grâce au d) du lemme 4.14 le deuxième ensemble de d) (privé dans le cas où (140) a lieu, de $B\left(S_{0}, M\right)$ que l'on a déjà traité dans ce cas), est inclus dans

$$
\left\{z^{\prime} \in(2 M+4 P)-\operatorname{géod}(x, b) \mid d\left(x, z^{\prime}\right) \geq \frac{d\left(x, x^{\prime}\right)+r_{0}(Z)-r_{0}^{\prime}(Z)}{2}-N-4 P\right\} .
$$

Comme $\frac{d\left(x, x^{\prime}\right)+r_{0}(Z)-r_{0}^{\prime}(Z)}{2}-N-4 P \geq d(x, v)-2 N-4 P$, il suffit de montrer que pour

$$
z \in B(x, d(x, v)+2 M+2 P+N+\delta)
$$

et

$$
z^{\prime} \in(2 M+4 P) \text { - géod }(x, b) \text { vérifiant } d\left(x, z^{\prime}\right) \geq d(x, v)-2 N-4 P,
$$


$\operatorname{géod}\left(z, z^{\prime}\right)$ rencontre $B\left(v, \frac{5 M}{2}-(N+3 \delta+1)\right)$. Par $\left(H_{\delta}^{0}(z, x, v, b)\right)$, on a

$v \in \delta$ - $\operatorname{géod}(x, z) \quad$ ou $v \in \delta$ - $\operatorname{géod}(z, b)$.

Si $v \in \delta$ - géod $(x, z), d(v, z) \leq 2 M+2 P+N+2 \delta$, donc $z \in \operatorname{géod}\left(z, z^{\prime}\right)$ appartient à $B\left(v, \frac{5 M}{2}-(N+3 \delta+1)\right)$ car on suppose $\frac{M}{2} \geq(2 P+N+2 \delta)+(N+3 \delta+1)$ grâce à $\left(H_{M}\right)$. Supposons donc $v \in \delta-\operatorname{géod}(z, b)$.

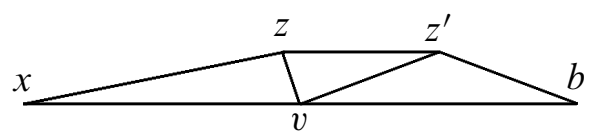

Comme

$$
z^{\prime} \in(2 M+4 P)-\operatorname{géod}(x, b) \text { et } d\left(x, z^{\prime}\right) \geq d(x, v)-4 P-2 N,
$$

on a

$$
d\left(b, z^{\prime}\right) \leq d(b, v)+2 M+8 P+2 N .
$$

Le lemme 4.8 appliqué à $\left(b, x, v, z^{\prime}\right)$ au lieu de $(x, y, a, b)$ et $(0,2 M+4 P, 2 M+$ $8 P+2 N)$ au lieu de $(\alpha, \beta, \rho)$ donne $z^{\prime} \in(4 M+16 P+4 N+\delta)$ - $\operatorname{géod}(v, b)$. Comme $v \in \delta$-géod $(z, b)$ le b) du lemme 3.3 montre

$$
v \in(4 M+16 P+4 N+2 \delta)-\operatorname{géod}\left(z, z^{\prime}\right) .
$$

Soit $t \in \operatorname{géod}\left(z, z^{\prime}\right)$ vérifiant $d(z, t)=\mathrm{E}\left(\frac{d\left(z, z^{\prime}\right)+d(z, v)-d\left(z^{\prime}, v\right)}{2}\right)$. Alors $\left(H_{\delta}^{0}\left(v, z, t, z^{\prime}\right)\right)$ montre $d(v, t) \leq 2 M+8 P+2 N+3 \delta+1 \leq \frac{5 M}{2}-(N+3 \delta+1)$ car on suppose $2 M+8 P+2 N+3 \delta+1 \leq \frac{5 M}{2}-(N+3 \delta+1)$ grâce à $\left(H_{M}\right)$. Cela termine la preuve de d) et donc l'étude du troisième cas. On a donc montré le lemme 4.57.

Lemme 4.61. Soit

$$
\left(a_{1}, \ldots, a_{p}, S_{0}, \ldots, S_{m},\left(\mathcal{Y}_{i}^{j}\right)_{i \in\{0, \ldots, m\}, j \in\left\{1, \ldots, l_{i}\right\}}\right) \in Y_{x}^{p, k, m,\left(l_{0}, \ldots, l_{m}\right)} .
$$

Dans les notations du lemme 4.57, les entiers $k^{\prime}, m^{\prime}, l_{m^{\prime}}^{\prime}$, la partie $J \subset\left\{1, \ldots, l_{m^{\prime}}\right\}$ et l'image $Z^{\prime}$ de

$$
\left(a_{1}, \ldots, a_{p}, S_{0}, \ldots, S_{m^{\prime}},\left(\mathcal{Y}_{i}^{j}\right)_{i \in\left\{0, \ldots, m^{\prime}-1\right\}, j \in\left\{1, \ldots, l_{i}\right\}},\left(\mathcal{Y}_{m^{\prime}}^{j \lambda}\right)_{\lambda \in\left\{1, \ldots, l_{m^{\prime}}^{\prime}\right\}}\right)
$$

dans $\bar{Y}_{x^{\prime}}^{p, k^{\prime}, m^{\prime},\left(l_{0}, \ldots, l_{m^{\prime}-1}, l_{m^{\prime}}^{\prime}\right)}$ par $\pi_{x^{\prime}}^{p, k^{\prime}, m^{\prime},\left(l_{0}, \ldots, l_{m^{\prime}-1}, l_{m^{\prime}}^{\prime}\right)}$ ne dépendent que de l'image $Z$ de

$$
\left(a_{1}, \ldots, a_{p}, S_{0}, \ldots, S_{m},\left(\mathcal{Y}_{i}^{j}\right)_{i \in\{0, \ldots, m\}, j \in\left\{1, \ldots, l_{i}\right\}}\right)
$$

dans $\bar{Y}_{x, k, \ldots}^{p, x^{\prime},\left(_{0}, \ldots, l_{m}\right)}$ par $\pi_{x, x^{\prime}, \star}^{p, k, m,\left(l_{0}, \ldots, l_{m}\right)}$.

De plus il existe $L$ ne dépendant que de $Z$ tel que tout élément de $Z^{\prime}$ a L antécédents par l'application de

$$
\left(\pi_{x, x^{\prime}, \star}^{p, k, m,\left(l_{0}, \ldots, l_{m}\right)}\right)^{-1}(Z)
$$


dans

$$
\left(\pi_{x^{\prime}}^{p, k^{\prime}, m^{\prime},\left(l_{0}, \ldots, l_{m^{\prime}-1}, l_{m^{\prime}}^{\prime}\right)}\right)^{-1}\left(Z^{\prime}\right)
$$

qui à

$$
\left(a_{1}, \ldots, a_{p}, S_{0}, \ldots, S_{m},\left(y_{i}^{j}\right)_{i \in\{0, \ldots, m\}, j \in\left\{1, \ldots, l_{i}\right\}}\right)
$$

associe

$$
\left(a_{1}, \ldots, a_{p}, S_{0}, \ldots, S_{m^{\prime}},\left(y_{i}^{j}\right)_{i \in\left\{0, \ldots, m^{\prime}-1\right\}, j \in\left\{1, \ldots, l_{i}\right\}},\left(y_{m^{\prime}}^{j_{\lambda}}\right)_{\lambda \in\left\{1, \ldots, l_{m^{\prime}}\right\}}\right)
$$

et il existe $C=C(\delta, N, K, Q, P, M)$ tel que l'on ait toujours

$$
1 \leq L \leq C^{\left(m+\sum_{i=m^{\prime}}^{m} l_{i}\right)-\left(m^{\prime}+l_{m^{\prime}}^{\prime}\right)}
$$

Démonstration. La première partie du lemme est évidente. L'existence de $L$ vient du e) du lemme 4.57, qui est une propriété très importante car elle "découple" les parties éliminées et les parties conservées en rendant superflue la connaissance des distances entre les points à distance $\leq M$ d'une partie éliminée et ceux à distance $\leq M$ d'une partie conservée. De plus $L$ est égal au nombre de possibilités pour les parties éliminées (dont la liste est rappelée dans (142) ci-dessous) telles que les distances entre les points de $B(x, k+2 M), B\left(x^{\prime}, k^{\prime}+2 M\right)$ et ceux à distance $\leq M$ des parties éliminées prennent les valeurs prescrites par la donnée de $Z$. Il reste à montrer (141). Les cardinaux des parties $S_{i}$ et $y_{i}^{j}$ sont bornés par une constante de la forme $C(\delta, K, N, Q, P)$. Comme le nombre des parties éliminées est $(m+$ $\left.\sum_{i=m^{\prime}}^{m} l_{i}\right)-\left(m^{\prime}+l_{m^{\prime}}^{\prime}\right)$, il suffit de montrer que tout point $y$ d'une partie $S_{i}$ ou $y_{i}^{j}$ éliminée ne peut prendre que $C$ positions avec $C=C(\delta, K, N, Q, P, M)$. Soit donc

$$
y \in \bigcup_{i \in\left\{m^{\prime}+1, \ldots, m\right\}} S_{i} \cup \underset{\substack{i \in\left\{m^{\prime}+1, \ldots, m\right\} \\ j \in\left\{1, \ldots, l_{i}\right\}}}{\bigcup} y_{i}^{j} \cup \bigcup_{j \notin J} y_{m^{\prime}}^{j} .
$$

Soit $b \in S_{0}$ et $u \in B(x, k)$ à distance minimale de $b$. On a alors $y \in 4 P$ - $\operatorname{géod}(x, b)$ par le a) et le c) du lemme 4.14, et

$$
d(x, y) \leq \max \left(k, \frac{d\left(x, x^{\prime}\right)+r_{0}(Z)-r_{0}^{\prime}(Z)}{2}\right)+M+2 P+\delta .
$$

Si $d(x, y) \leq k+M+2 P+\delta$ on a $y \in B(x, k+2 M)$ car on peut supposer $M \geq 2 P+\delta \operatorname{par}\left(H_{M}\right)$ donc $y$ était déjà déterminé par la donnée de $Z$. Supposons donc

$$
d(x, y) \leq \frac{d\left(x, x^{\prime}\right)+r_{0}(Z)-r_{0}^{\prime}(Z)}{2}+M+2 P+\delta .
$$

Cela implique immédiatement

$$
d(x, y) \leq \frac{d\left(x, x^{\prime}\right)+d(x, b)-d\left(x^{\prime}, b\right)}{2}+M+2 P+\delta+N .
$$


On a alors

$$
\begin{aligned}
d\left(x^{\prime}, y\right) & \leq \max \left(d\left(x^{\prime}, x\right)-d(b, x)+d(y, b), d\left(x^{\prime}, b\right)-d(b, x)+d(x, y)\right)+\delta \\
& \leq \max \left(d\left(x^{\prime}, x\right)-d(x, y)+4 P, d\left(x^{\prime}, b\right)-d(b, x)+d(x, y)\right)+\delta \\
& \leq d\left(x^{\prime}, x\right)-d(x, y)+2 M+4 P+2 N+3 \delta
\end{aligned}
$$

où la première inégalité vient de $\left(H_{\delta}\left(x^{\prime}, x, y, b\right)\right)$, la deuxième utilise le fait que $y \in 4 P-\operatorname{géod}(x, b)$ et la dernière résulte de (143). On en déduit $y \in(2 M+$ $4 P+2 N+3 \delta)-\operatorname{géod}\left(x, x^{\prime}\right)$. Comme $d(x, y)$ fait partie de la donnée de $Z$, le lemme 3.13 montre que le nombre de possibilités pour $y$ est borné par une constante $C=C(\delta, N, K, Q, P, M)$.

Dans les notations du lemme 4.61 on désigne par

$$
\theta: \bigcup_{k, m, l_{0}, \ldots, l_{m}} Y_{x, x^{\prime}, \star}^{p, k, m,\left(l_{0}, \ldots, l_{m}\right)} \rightarrow \bigcup_{k^{\prime}, m^{\prime}, l_{0}^{\prime}, \ldots, l_{m^{\prime}}^{\prime}} Y_{x^{\prime}}^{p, k^{\prime}, m^{\prime},\left(l_{0}^{\prime}, \ldots, l_{m^{\prime}}^{\prime}\right)}
$$

l'application qui à

$$
\left(a_{1}, \ldots, a_{p}, S_{0}, \ldots, S_{m},\left(\mathcal{Y}_{i}^{j}\right)_{i \in\{0, \ldots, m\}, j \in\left\{1, \ldots, l_{i}\right\}}\right)
$$

associe

$$
\left(a_{1}, \ldots, a_{p}, S_{0}, \ldots, S_{m^{\prime}},\left(\mathcal{Y}_{i}^{j}\right)_{i \in\left\{0, \ldots, m^{\prime}-1\right\}, j \in\left\{1, \ldots, l_{i}\right\}},\left(\mathcal{Y}_{m^{\prime}}^{j_{\lambda}}\right)_{\lambda \in\left\{1, \ldots, l_{m^{\prime}}^{\prime}\right.}\right)
$$

et on note

$$
\bar{\theta}: \bigcup_{k, m, l_{0}, \ldots, l_{m}} \bar{Y}_{x, x^{\prime}, \star}^{p, k,\left(l_{0}, \ldots, l_{m}\right)} \rightarrow \bigcup_{k^{\prime}, m^{\prime}, l_{0}^{\prime}, \ldots, l_{m^{\prime}}^{\prime}} \bar{Y}_{x^{\prime}}^{p, k^{\prime}, m^{\prime},\left(l_{0}^{\prime}, \ldots, l_{m^{\prime}}^{\prime}\right)}
$$

l'application induite par $\theta$, qui à $Z$ associe $Z^{\prime}$ et dont l'existence résulte du lemme 4.61.

Pour $k^{\prime}, m^{\prime}, l_{0}^{\prime}, \ldots, l_{m^{\prime}}^{\prime} \in \mathbb{N}$ et $Z^{\prime} \in \bar{Y}_{x^{\prime}}^{p, k^{\prime}, m^{\prime},\left(l_{0}^{\prime}, \ldots, l_{m^{\prime}}^{\prime}\right)}$ on note $\xi_{Z^{\prime}}$ la forme linéaire sur $\mathbb{C}^{\left(\Delta_{p}\right)}$ définie par

$$
\xi_{Z^{\prime}}(f)=\sum_{\substack {\left(a_{1}^{\prime}, \ldots, a_{p}^{\prime}, S_{0}^{\prime}, \ldots, S_{m^{\prime}}^{\prime},\left(y_{i}^{\prime}\right)_{i \in\left\{0, \ldots, m^{\prime}\right\}, j \in\left\{1, \ldots, l_{i}^{\prime}\right\}}\right) \\
\begin{subarray}{c}{p, k^{\prime}, m^{\prime},\left(l_{0}^{\prime}, \ldots, l_{m^{\prime}}^{\prime}\right)^{-1}\left(Z^{\prime}\right) \\
x_{x^{\prime}}{ ( a _ { 1 } ^ { \prime } , \ldots , a _ { p } ^ { \prime } , S _ { 0 } ^ { \prime } , \ldots , S _ { m ^ { \prime } } ^ { \prime } , ( y _ { i } ^ { \prime } ) _ { i \in \{ 0 , \ldots , m ^ { \prime } \} , j \in \{ 1 , \ldots , l _ { i } ^ { \prime } \} } ) \\
\begin{subarray} { c } { p , k ^ { \prime } , m ^ { \prime } , ( l _ { 0 } ^ { \prime } , \ldots , l _ { m ^ { \prime } } ^ { \prime } ) ^ { - 1 } ( Z ^ { \prime } ) \\
x _ { x ^ { \prime } } } }\end{subarray}} f\left(a_{1}^{\prime}, \ldots, a_{p}^{\prime}\right)
$$

Grâce au lemme 4.61, on a pour $Z^{\prime}=\theta(Z)$ et $f \in \mathbb{C}^{\left(\Delta_{p}\right)}$,

$$
\begin{aligned}
& \sharp\left(\left(\pi_{x, x^{\prime}, \star}^{p, k, m,\left(l_{0}, \ldots, l_{m}\right)}\right)^{-1}(Z)\right)^{-\alpha}\left|\xi_{Z}(f)\right|^{2} \\
& \quad=\left(L^{2-\alpha}\right) \sharp\left(\left(\pi_{x^{\prime}}^{p, k^{\prime}, m^{\prime},\left(l_{0}^{\prime}, \ldots, l_{m^{\prime}}^{\prime}\right)}\right)^{-1}\left(Z^{\prime}\right)\right)^{-\alpha}\left|\xi_{Z^{\prime}}(f)\right|^{2}
\end{aligned}
$$


avec $L \leq C^{\left(m+\sum_{i=m^{\prime}}^{m} l_{i}\right)-\left(m^{\prime}+l_{m^{\prime}}^{\prime}\right)}$, où $C$ est la constante du lemme 4.61. Il en résulte que pour tout $f \in \mathbb{C}^{\left(\Delta_{p}\right)}$, on a

$$
\begin{aligned}
& \|f\|_{\mathscr{H}_{x, x^{\prime}, \star, s}\left(\Delta_{p}\right)}^{2}
\end{aligned}
$$

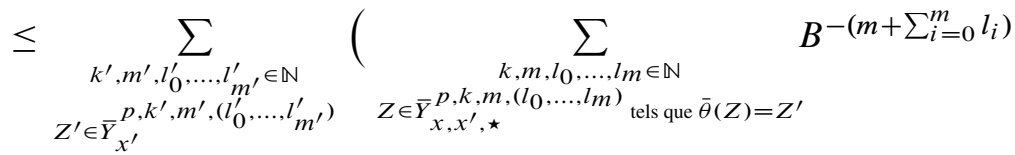

$$
\begin{aligned}
& \left.\left(C^{2-\alpha}\right)^{\left(m+\sum_{i=m^{\prime}}^{m} l_{i}\right)-\left(m^{\prime}+l_{m^{\prime}}^{\prime}\right)} e^{2 s\left(r_{0}(Z)-k\right)}\left(\prod_{i=0}^{m} s_{i}(Z)^{-l_{i}}\right)\right) \\
& \sharp\left(\left(\pi_{x^{\prime}}^{p, k^{\prime}, m^{\prime},\left(l_{0}^{\prime}, \ldots, l_{m^{\prime}}^{\prime}\right)}\right)^{-1}\left(Z^{\prime}\right)\right)^{-\alpha}\left|\xi_{Z^{\prime}}(f)\right|^{2} .
\end{aligned}
$$

D'autre part

$$
\begin{aligned}
& \|f\|_{\mathcal{H}_{x^{\prime}, s}\left(\Delta_{p}\right)}^{2}=\sum_{k^{\prime}, m^{\prime}, l_{0}^{\prime}, \ldots, l_{m^{\prime}}^{\prime} \in \mathbb{N}} B^{-\left(m^{\prime}+\sum_{i=0}^{m^{\prime}} l_{i}^{\prime}\right)} e^{2 s\left(r_{0}\left(Z^{\prime}\right)-k^{\prime}\right)} \\
& Z^{\prime} \in \bar{Y}_{x^{\prime}}^{p, k^{\prime}, m^{\prime},\left(l_{0}^{\prime}, \ldots, l_{m^{\prime}}^{\prime}\right)} \\
& \left(\prod_{i=0}^{m^{\prime}} s_{i}\left(Z^{\prime}\right)^{-l_{i}^{\prime}}\right) \sharp\left(\left(\pi_{x^{\prime}}^{p, k^{\prime}, m^{\prime},\left(l_{0}^{\prime}, \ldots, l_{m^{\prime}}^{\prime}\right)}\right)^{-1}\left(Z^{\prime}\right)\right)^{-\alpha}\left|\xi_{Z^{\prime}}(f)\right|^{2}
\end{aligned}
$$

où les entiers $r_{0}\left(Z^{\prime}\right), s_{0}\left(Z^{\prime}\right), \ldots, s_{m^{\prime}}\left(Z^{\prime}\right)$ sont tels que pour tout

$$
\left(a_{1}^{\prime}, \ldots, a_{p}^{\prime}, S_{0}^{\prime}, \ldots, S_{m^{\prime}}^{\prime},\left(y_{i}^{\prime j}\right)_{i \in\left\{0, \ldots, m^{\prime}\right\}, j \in\left\{1, \ldots, l_{i}^{\prime}\right\}}\right) \in\left(\pi_{x^{\prime}}^{p, k^{\prime}, m^{\prime},\left(l_{0}^{\prime}, \ldots, l_{m}^{\prime}\right)}\right)^{-1}\left(Z^{\prime}\right)
$$

on a $r_{0}\left(Z^{\prime}\right)=d\left(x^{\prime}, S_{0}^{\prime}\right), s_{i}\left(Z^{\prime}\right)=d\left(S_{i}^{\prime}, S_{i+1}^{\prime}\right)+2 M$ pour $i \in\left\{0, \ldots, m^{\prime}-1\right\}$, et $s_{m^{\prime}}\left(Z^{\prime}\right)=d\left(x^{\prime}, S_{m^{\prime}}^{\prime}\right)-k^{\prime}$. On remarque que pour $Z^{\prime}=\theta(Z)$ on a $r_{0}\left(Z^{\prime}\right)=r_{0}^{\prime}(Z)$ et pour $i \in\left\{0, \ldots, m^{\prime}-1\right\}, l_{i}^{\prime}=l_{i}$ et $s_{i}\left(Z^{\prime}\right)=s_{i}(Z)$.

Démonstration du lemme 4.56 en admettant le lemme 4.62. On pose $C_{0}=C^{2-\alpha}$ où $C$ est comme dans le lemme 4.61. Donc $C_{0} \leq C(\delta, N, K, Q, P, M)$. Pour montrer le lemme 4.56 on est ramené à montrer le lemme suivant.

Lemme 4.62. Il existe une constante $C=C(\delta, K, N, P, Q, M, s, B)$ telle que pour tous $k^{\prime}, m^{\prime}, l_{0}^{\prime}, \ldots, l_{m^{\prime}}^{\prime}$ et $Z^{\prime} \in \bar{Y}_{x^{\prime}}^{p, k^{\prime}, m^{\prime},\left(l_{0}^{\prime}, \ldots, l_{m^{\prime}}^{\prime}\right)}$ on ait

$$
\begin{aligned}
& \sum_{k, m, l_{0}, \ldots, l_{m} \in \mathbb{N}} \quad\left(C_{0} B^{-1}\right)^{m+\sum_{i=m^{\prime}}^{m} l_{i}} e^{2 s\left(r_{0}(Z)-k\right)}\left(\prod_{i=m^{\prime}}^{m} s_{i}(Z)^{-l_{i}}\right) \\
& Z \in \bar{Y}_{x, x^{\prime}, \star}^{\left.p, k, m, l_{0}, \ldots, l m\right)}{ }_{\text {tels que }} \bar{\theta}(Z)=Z^{\prime} \\
& \leq C e^{3 s d\left(x, x^{\prime}\right)}\left(C_{0} B^{-1}\right)^{m^{\prime}+l_{m^{\prime}}^{\prime}} e^{2 s\left(r_{0}\left(Z^{\prime}\right)-k^{\prime}\right)} s_{m^{\prime}}\left(Z^{\prime}\right)^{-l_{m^{\prime}}^{\prime}} .
\end{aligned}
$$

Démonstration du lemme 4.62 en admettant lemme 4.63. On rappelle que pour $Z^{\prime}=$ $\theta(Z), r_{0}\left(Z^{\prime}\right)=r_{0}^{\prime}(Z)$. Par (120) on a

$$
k^{\prime}-r_{0}^{\prime}(Z)=\min \left(0, \max \left(k-r_{0}(Z), \mathrm{E}\left(\frac{d\left(x, x^{\prime}\right)-r_{0}(Z)-r_{0}^{\prime}(Z)}{2}\right)\right)\right)+\frac{M}{2}
$$


d'où

$$
k^{\prime}-r_{0}^{\prime}(Z) \leq \max \left(k-r_{0}(Z), \mathrm{E}\left(\frac{d\left(x, x^{\prime}\right)-r_{0}(Z)-r_{0}^{\prime}(Z)}{2}\right)\right)+\frac{M}{2} .
$$

Comme $\left|r_{0}(Z)-r_{0}^{\prime}(Z)\right| \leq d\left(x, x^{\prime}\right)$ on a

$$
\frac{d\left(x, x^{\prime}\right)-r_{0}(Z)-r_{0}^{\prime}(Z)}{2} \leq d\left(x, x^{\prime}\right)-r_{0}(Z) \leq d\left(x, x^{\prime}\right)+\left(k-r_{0}(Z)\right)
$$

d'où $\left(r_{0}(Z)-k\right) \leq\left(r_{0}^{\prime}(Z)-k^{\prime}\right)+d\left(x, x^{\prime}\right)+\frac{M}{2}$. Les valeurs de $k$ pour lesquelles il existe $m, l_{0}, \ldots, l_{m} \in \mathbb{N}$ et $Z \in \bar{Y}_{x, x^{\prime}, \star}^{p, k, m},\left(l_{0}, \ldots, l_{m}\right)$ tels que $\theta(Z)=Z^{\prime}$ sont donc incluses dans un intervalle $\left[k_{0},+\infty\left[\right.\right.$ avec $k_{0} \in \mathbb{N}$ vérifiant

$$
\left(r_{0}(Z)-k_{0}\right) \leq\left(r_{0}^{\prime}(Z)-k^{\prime}\right)+d\left(x, x^{\prime}\right)+\frac{M}{2} .
$$

Comme la série $1+e^{-2 s}+e^{-4 s}+\cdots$ converge, pour montrer le lemme 4.62 il suffit d'établir le lemme suivant.

Lemme 4.63. Pour tous $k, k^{\prime}, m^{\prime}, l_{0}^{\prime}, \ldots, l_{m^{\prime}}^{\prime}$ et $Z^{\prime} \in \bar{Y}_{x^{\prime}}^{p, k^{\prime}, m^{\prime},\left(l_{0}^{\prime}, \ldots, l_{m^{\prime}}^{\prime}\right)}$ on a

$$
\begin{aligned}
& \sum_{\substack{m, l_{0}, \ldots, l_{m} \in \mathbb{N} \\
Z \in \bar{Y}_{x, x^{\prime}, \star}^{p, k,\left(l_{0}, \ldots, l_{m}\right)}}}\left(C_{0} B^{-1}\right)^{m+\sum_{\text {tels } q u e} \bar{\theta}(Z)=Z^{\prime}} \sum_{i=m^{\prime}}^{m} l_{i}\left(\prod_{i=m^{\prime}}^{m} s_{i}(Z)^{-l_{i}}\right)
\end{aligned}
$$

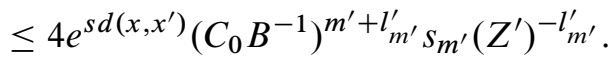

Démonstration du lemme 4.63 en admettant le lemme 4.64. D'après le lemme 4.61, pour $Z^{\prime}=\bar{\theta}(Z), \theta$ induit une surjection de

$$
\left(\pi_{x, x^{\prime}, \star}^{p, k, m,\left(l_{0}, \ldots, l_{m}\right)}\right)^{-1}(Z)
$$

dans

$$
\left(\pi_{x^{\prime}}^{p, k^{\prime}, m^{\prime},\left(l_{0}^{\prime}, \ldots, l_{m^{\prime}}^{\prime}\right)}\right)^{-1}\left(Z^{\prime}\right) .
$$

Pour montrer le lemme 4.63 il suffit donc d'établir le lemme suivant.

Lemme 4.64. Pour tous $k, k^{\prime}, m^{\prime}, l_{0}^{\prime}, \ldots, l_{m^{\prime}}^{\prime}$ et

$$
\left(a_{1}^{\prime}, \ldots, a_{p}^{\prime}, S_{0}^{\prime}, \ldots, S_{m^{\prime}}^{\prime},\left(\mathcal{Y}_{i}^{\prime}{ }^{j}\right)_{i \in\left\{0, \ldots, m^{\prime}\right\}, j \in\left\{1, \ldots, l_{i}^{\prime}\right\}}\right) \in Y_{x^{\prime}}^{p, k^{\prime}, m^{\prime},\left(l_{0}^{\prime}, \ldots, l_{m^{\prime}}^{\prime}\right)}
$$

on $a$

$$
\begin{aligned}
& \sum_{S_{m}}\left(C_{0} B^{-1}\right)^{\left(m-m^{\prime}\right)+\left(\sum_{i=m^{\prime}}^{m} l_{i}\right)-l_{m^{\prime}}^{\prime}} \\
& m, l_{0}, \ldots, l_{m},\left(a_{1}, \ldots, a_{p}, S_{0}, \ldots, S_{m},\left(y_{i}^{j}\right)_{i \in\{0, \ldots, m\}, j \in\left\{1, \ldots, l_{i}\right\}}\right) \\
& \left(\prod_{i=m^{\prime}}^{m-1}\left(d\left(S_{i}, S_{i+1}\right)+2 M\right)^{-l_{i}}\right)\left(d\left(x, S_{m}\right)-k\right)^{-l_{m}}\left(d\left(x^{\prime}, S_{m^{\prime}}^{\prime}\right)-k^{\prime}\right)^{l_{m^{\prime}}^{\prime}} \leq 4 e^{s d\left(x, x^{\prime}\right)}
\end{aligned}
$$


où la somme porte sur les $m, l_{0}, \ldots, l_{m} \in \mathbb{N}$ et les

$$
\left(a_{1}, \ldots, a_{p}, S_{0}, \ldots, S_{m},\left(y_{i}^{j}\right)_{i \in\{0, \ldots, m\}, j \in\left\{1, \ldots, l_{i}\right\}}\right) \in Y_{x}^{p, k, m,\left(l_{0}, \ldots, l_{m}\right)}
$$

tels que $k^{\prime}, m^{\prime}, l_{0}^{\prime}, \ldots, l_{m^{\prime}}^{\prime}$ et

$$
\left(a_{1}^{\prime}, \ldots, a_{p}^{\prime}, S_{0}^{\prime}, \ldots, S_{m^{\prime}}^{\prime},\left(y_{i}^{\prime j}\right)_{i \in\left\{0, \ldots, m^{\prime}\right\}, j \in\left\{1, \ldots, l_{i}^{\prime}\right\}}\right)
$$

soient associés à

$$
\left(a_{1}, \ldots, a_{p}, S_{0}, \ldots, S_{m},\left(y_{i}^{j}\right)_{i \in\{0, \ldots, m\}, j \in\left\{1, \ldots, l_{i}\right\}}\right)
$$

comme dans le lemme 4.57 (donc en particulier $a_{1}^{\prime}=a_{1}, \ldots, a_{p}^{\prime}=a_{p}$, $S_{0}^{\prime}=$ $S_{0}, \ldots, S_{m^{\prime}}^{\prime}=S_{m^{\prime}}, k^{\prime}=k^{\prime}(Z), l_{i}^{\prime}=l_{i}$ pour $i \in\left\{0, \ldots, m^{\prime}-1\right\}, y_{i}^{\prime j}=y_{i}^{j}$ pour $i \in\left\{0, \ldots, m^{\prime}-1\right\}$ et $j \in\left\{1, \ldots, l_{i}\right\}$ et $y_{m^{\prime}}^{\prime \lambda}=y_{m^{\prime}}^{j_{\lambda}}$ pour $\left.\lambda \in\left\{1, \ldots, l_{m^{\prime}}^{\prime}\right\}\right)$.

Démonstration du lemme 4.64 en admettant les lemmes 4.65 et $4.66 . \quad$ D'abord on suppose $l_{0}^{\prime}=\cdots=l_{m^{\prime}-1}^{\prime}=0$ car en supprimant les $\mathcal{Y}_{i}^{\prime} j$ pour $i \in\left\{0, \ldots, m^{\prime}-1\right\}$ et $j \in\left\{1, \ldots, l_{i}^{\prime}\right\}$ le nouvel énoncé est strictement équivalent à l'ancien. D'après le lemme 4.15 il existe une constante $C_{1}=C(\delta, K, N, Q, P)$ telle que, connaissant $S_{0}, \ldots, S_{m}$, pour $i \in\left\{m^{\prime}+1, \ldots, m\right\}$ et $j \in\left\{1, \ldots, l_{i}\right\}$ le nombre de possibilités pour $y_{i}^{j}$ est borné par $C_{1}\left(d\left(S_{i}, S_{i+1}\right)+2 M\right)$ si $i<m$ et par $C_{1}\left(d\left(x, S_{m}\right)-k\right)$ si $i=m$. Comme

$$
\sum_{l_{m^{\prime}+1}, \ldots, l_{m} \in \mathbb{N}}\left(C_{1} C_{0} B^{-1}\right)^{\sum_{i=m^{\prime}+1}^{m} l_{i}}=\left(1-C_{1} C_{0} B^{-1}\right)^{-\left(m-m^{\prime}\right)},
$$

pour montrer le lemme 4.64 il suffit de montrer les deux lemmes suivants, qui distinguent les cas $m^{\prime}=m$ et $m^{\prime}<m$. Dans les deux lemmes on note $l$ et $l^{\prime}$ au lieu de $l_{m^{\prime}}$ et $l_{m^{\prime}}^{\prime}$, puisque que les $l_{i}$ et $l_{i}^{\prime}$ pour $i \neq m^{\prime}$ ont disparu. Dans les deux lemmes suivants on a supposé $J=\left\{1, \ldots, l^{\prime}\right\}$ et remplacé la somme sur les parties $J \subset\{1, \ldots, l\}$ de cardinal $l^{\prime}$ par la multiplication $\operatorname{par}\left(\begin{array}{l}l \\ l^{\prime}\end{array}\right)$.

Lemme 4.65. Pour tous $k, k^{\prime}, m^{\prime}, l^{\prime} \in \mathbb{N}$ et

$$
\left(a_{1}, \ldots, a_{p}, S_{0}, \ldots, S_{m^{\prime}},\left(y_{m^{\prime}}^{j}\right)_{j \in\left\{1, \ldots, l^{\prime}\right\}}\right) \in Y_{x^{\prime}}^{p, k^{\prime}, m^{\prime},\left(0, \ldots, 0, l^{\prime}\right)}
$$

on $a$

$$
\sum_{l \geq l^{\prime}}\left(\begin{array}{l}
l \\
l^{\prime}
\end{array}\right) \sum_{\substack{y^{l^{\prime}+1}, \ldots, y \\
m^{\prime}}}\left(C_{0} B^{-1}\right)^{l-l^{\prime}}\left(d\left(x, S_{m^{\prime}}\right)-k\right)^{-l}\left(d\left(x^{\prime}, S_{m^{\prime}}\right)-k^{\prime}\right)^{l^{\prime}} \leq 2
$$

où la somme porte sur les $y_{m^{\prime}}^{l^{\prime}+1}, \ldots, y_{m^{\prime}}^{l}$ tels que

$$
\left(a_{1}, \ldots, a_{p}, S_{0}, \ldots, S_{m^{\prime}},\left(y_{m^{\prime}}^{j}\right)_{j \in\{1, \ldots, l\}}\right) \in Y_{x}^{p, k, m^{\prime},(0, \ldots, 0, l)}
$$


et, en notant $T=\frac{d\left(x, x^{\prime}\right)+d\left(x, S_{0}\right)-d\left(x^{\prime}, S_{0}\right)}{2}$ on ait

$$
\begin{aligned}
& d_{\max }\left(x, S_{m^{\prime}}\right)>\max (k, T)+M \text { si } m^{\prime}>0, \\
& d_{\max }\left(x, y_{m^{\prime}}^{j}\right)>\max (k, T)+M+2 P+\delta \text { pour } j \in\left\{1, \ldots, l^{\prime}\right\}
\end{aligned}
$$

et

$$
d_{\max }\left(x, y_{m^{\prime}}^{j}\right) \leq \max (k, T)+M+2 P+\delta \quad \text { pour } j \in\left\{l^{\prime}+1, \ldots, l\right\}
$$

On rappelle que les constantes $C_{0}$ et $C_{1}$ (qui apparaissent avant les énoncés des lemmes 4.62 et 4.65$)$ sont majorées $\operatorname{par} C(\delta, K, N, Q, P, M)$.

Lemme 4.66. Pour tous $k, k^{\prime}, m^{\prime}, l^{\prime} \in \mathbb{N}$ et

$$
\left(a_{1}, \ldots, a_{p}, S_{0}, \ldots, S_{m^{\prime}},\left(y_{m^{\prime}}^{j}\right)_{j \in\left\{1, \ldots, l^{\prime}\right\}}\right) \in Y_{x^{\prime}}^{p, k^{\prime}, m^{\prime},\left(0, \ldots, 0, l^{\prime}\right)}
$$

on $a$

$$
\begin{gathered}
\sum_{m>m^{\prime}, l \geq l^{\prime}}\left(\begin{array}{c}
l \\
l^{\prime}
\end{array}\right)\left(\frac{C_{0} B^{-1}}{1-C_{0} C_{1} B^{-1}}\right)^{m-m^{\prime}} \sum_{S_{m^{\prime}+1}, \ldots, S_{m}, y_{m^{\prime}}^{l^{\prime}+1}, \ldots, y_{m^{\prime}}^{l^{\prime}}}\left(C_{0} B^{-1}\right)^{l-l^{\prime}} \\
\left(d\left(S_{m^{\prime}}, S_{m^{\prime}+1}\right)+2 M\right)^{-l}\left(d\left(x^{\prime}, S_{m^{\prime}}\right)-k^{\prime}\right)^{l^{\prime}} \leq 4 e^{s d\left(x, x^{\prime}\right)}
\end{gathered}
$$

où la deuxième somme porte sur les $S_{m^{\prime}+1}, \ldots, S_{m}, y_{m^{\prime}}^{l^{\prime}+1}, \ldots, y_{m^{\prime}}^{l}$ tels que

$$
\left(a_{1}, \ldots, a_{p}, S_{0}, \ldots, S_{m},\left(\mathcal{Y}_{m^{\prime}}^{j}\right)_{j \in\{1, \ldots, l\}}\right) \in Y_{x}^{p, k, m,(0, \ldots, 0, l, 0, \ldots, 0)}
$$

et, en notant $T=\frac{d\left(x, x^{\prime}\right)+d\left(x, S_{0}\right)-d\left(x^{\prime}, S_{0}\right)}{2}$ on ait

$$
\begin{aligned}
d_{\max }\left(x, S_{m^{\prime}}\right) & >\max (k, T)+M \text { si } m^{\prime}>0, \\
d_{\max }\left(x, S_{m^{\prime}+1}\right) & \leq \max (k, T)+M, \\
d_{\max }\left(x, y_{m^{\prime}}^{j}\right) & >\max (k, T)+M+2 P+\delta \quad \text { pour } j \in\left\{1, \ldots, l^{\prime}\right\}
\end{aligned}
$$

$e t$

$$
d_{\max }\left(x, y_{m^{\prime}}^{j}\right) \leq \max (k, T)+M+2 P+\delta \quad \text { pour } j \in\left\{l^{\prime}+1, \ldots, l\right\}
$$

Dans la démonstration des lemmes 4.65 et 4.66 le petit calcul suivant servira plusieurs fois.

Lemme 4.67. Soit $\mathcal{A}, \mathscr{B} \in \mathbb{R}_{+}, 2 \mathcal{A}+\mathscr{B} \leq 1$. Alors pour tout $l^{\prime} \in \mathbb{N}$,

$$
\sum_{l \geq l^{\prime}}\left(\begin{array}{l}
l \\
l^{\prime}
\end{array}\right) \mathcal{A}^{l-l^{\prime}} \mathcal{B}^{l^{\prime}} \leq 2 \text {. }
$$


Démonstration. On a la formule générale, pour $x \in \mathbb{C}$ avec $|x|<1$ et $k \in \mathbb{N}$,

$$
\sum_{n \in \mathbb{N}}\left(\begin{array}{c}
n+k \\
k
\end{array}\right) x^{n}=(1-x)^{-(k+1)} \text {. }
$$

Donc $\sum_{l \geq l^{\prime}}\left(\begin{array}{l}l \\ l^{\prime}\end{array}\right) \mathcal{A}^{l-l^{\prime}} \mathscr{B}^{l^{\prime}}=\frac{\mathscr{B}^{l^{\prime}}}{(1-\mathcal{A})^{l^{\prime}+1}} \leq \frac{1}{1-\mathcal{A}} \leq 2$ puisque $\mathcal{B} \leq 1-\mathcal{A}$ et $1-\mathcal{A} \geq$ $1 / 2$.

Démonstration du lemme 4.65. On va distinguer trois cas comme dans la démonstration du lemme 4.57, mais une partie de la démonstration est commune aux trois cas. On note $T=\frac{d\left(x, S_{0}\right)-d\left(x^{\prime}, S_{0}\right)+d\left(x, x^{\prime}\right)}{2}$ comme dans l'énoncé du lemme. On fixe $b \in S_{0}$. Soit $y \in S_{m^{\prime}}$. Alors

$$
d(x, y) \geq \frac{d(x, b)+d\left(x, x^{\prime}\right)-d\left(x^{\prime}, b\right)}{2}-N
$$

En effet cela est vrai si $m^{\prime}=0$ car $d(x, y) \geq d\left(x, S_{0}\right) \geq d(x, b)-N$ et $d\left(x, x^{\prime}\right) \leq$ $d(x, b)+d\left(x^{\prime}, b\right)$, et cela est vrai si $m^{\prime}>0$ car la condition $d_{\max }\left(x, S_{m^{\prime}}\right)>$ $\max (k, T)+M$ implique

$$
\begin{aligned}
d(x, y) & \geq d_{\max }\left(x, S_{m^{\prime}}\right)-N \geq T+M-N \\
& \geq \frac{d(x, b)+d\left(x, x^{\prime}\right)-d\left(x^{\prime}, b\right)}{2}+M-2 N
\end{aligned}
$$

et on suppose $M \geq N$, ce qui est permis par $\left(H_{M}\right)$. On en déduit

$$
d\left(x^{\prime}, y\right)-d\left(x^{\prime}, b\right)+d(x, b) \leq d(x, y)+P+2 N+\delta .
$$

En effet

$$
\begin{aligned}
d\left(x^{\prime}, y\right) & \leq \max \left(d\left(x^{\prime}, b\right)-d(x, b)+d(x, y), d\left(x^{\prime}, x\right)-d(x, b)+d(b, y)\right)+\delta \\
& \leq \max \left(d\left(x^{\prime}, b\right)-d(x, b)+d(x, y), d\left(x^{\prime}, x\right)-d(x, y)+P\right)+\delta \\
& \leq d\left(x^{\prime}, b\right)-d(x, b)+d(x, y)+P+2 N+\delta
\end{aligned}
$$

où la première inégalité a lieu par $\left(H_{\delta}\left(x^{\prime}, x, y, b\right)\right)$, la deuxième inégalité utilise $y \in P$ - $\operatorname{géod}(x, b)$ et la dernière inégalité résulte de (148).

Premier cas. On suppose $d\left(x, S_{0}\right) \leq k$.

Alors $m^{\prime}=0$ et $l=0$ d'après le lemme 4.12 et la remarque qui suit la définition 4.1. Donc la somme est vide ou réduite à un élément et l'inégalité est triviale.

Deuxième cas. On suppose $d\left(x, S_{0}\right)>k$ et $T \leq k$.

Alors pour $j \in\left\{l^{\prime}+1, \ldots, l\right\}$ on a

$$
k+3 P \leq d_{\max }\left(x, y_{m^{\prime}}^{j}\right) \leq k+M+2 P+\delta
$$


où l'inégalité de gauche vient de la condition iv) de la définition 4.1. Comme $y_{m^{\prime}}^{j} \subset$ $4 P$-géod $(x, b)$ le lemme 3.13 montre que le nombre de possibilités pour $y_{m^{\prime}}^{j}$ est borné par $C_{2}=C(\delta, K, N, Q, P, M)$. On a

$$
k^{\prime}=d\left(x^{\prime}, S_{0}\right)-d\left(x, S_{0}\right)+k+\frac{M}{2}
$$

par (122) donc

$$
k^{\prime} \geq d\left(x^{\prime}, b\right)-d(x, b)+k+\frac{M}{2}-N .
$$

Soit $y \in S_{m^{\prime}}$. On a

$$
\begin{aligned}
d\left(x^{\prime}, y\right)-k^{\prime} & \leq d\left(x^{\prime}, y\right)-d\left(x^{\prime}, b\right)+d(x, b)+N-k-\frac{M}{2} \\
& \leq d(x, y)-k-\frac{M}{2}+P+3 N+\delta
\end{aligned}
$$

où la première inégalité vient de (150) et la deuxième de (149). Comme cela est vrai pour tout $y \in S_{m^{\prime}}$ on en déduit

d'où

$$
\left(d\left(x^{\prime}, S_{m^{\prime}}\right)-k^{\prime}\right) \leq\left(d\left(x, S_{m^{\prime}}\right)-k\right)-\frac{M}{2}+P+3 N+\delta
$$

$$
\left(d\left(x^{\prime}, S_{m^{\prime}}\right)-k^{\prime}\right) \leq\left(d\left(x, S_{m^{\prime}}\right)-k\right)-1
$$

car on suppose $\frac{M}{2} \geq P+3 N+\delta+1$, ce qui est permis par $\left(H_{M}\right)$. Le membre de gauche de (145) est donc majoré par

$$
\sum_{l \geq l^{\prime}}\left(\begin{array}{l}
l \\
l^{\prime}
\end{array}\right)\left(\frac{C_{0} C_{2} B^{-1}}{d\left(x, S_{m^{\prime}}\right)-k}\right)^{\left(l-l^{\prime}\right)}\left(\frac{d\left(x^{\prime}, S_{m^{\prime}}\right)-k^{\prime}}{d\left(x, S_{m^{\prime}}\right)-k}\right)^{l^{\prime}} \leq 2
$$

par le lemme 4.67. Les hypothèses du lemme 4.67 sont satisfaites car on suppose $2 C_{0} C_{2} B^{-1} \leq 1$ grâce à $\left(H_{B}\right)$ ce qui implique

$$
2 C_{2} C_{0} B^{-1}+\left(d\left(x^{\prime}, S_{m^{\prime}}\right)-k^{\prime}\right) \leq\left(d\left(x^{\prime}, S_{m^{\prime}}\right)-k\right)+1 \leq\left(d\left(x, S_{m^{\prime}}\right)-k\right) .
$$

Troisième cas. On suppose $d\left(x, S_{0}\right)>k$ et $T>k$.

Pour $j \in\left\{l^{\prime}+1, \ldots, l\right\}$ on a

$$
k+3 P \leq d_{\max }\left(x, y_{m^{\prime}}^{j}\right) \leq T+M+2 P+\delta .
$$

Comme $y_{m^{\prime}}^{j} \subset 4 P$-géod $(x, b)$ le lemme 3.13 montre que le nombre de possibilités pour $y_{m^{\prime}}^{j}$ est borné par $C_{2}(T-k)$ avec $C_{2}=C(\delta, K, N, Q, P, M)$. Par (123) on a

$$
\left|k^{\prime}-\left(\frac{d\left(x^{\prime}, S_{0}\right)+d\left(x, x^{\prime}\right)-d\left(x, S_{0}\right)}{2}+\frac{M}{2}\right)\right| \leq \frac{N}{2}+1 .
$$


Soit $y \in S_{m^{\prime}}$. Il résulte de (149) que

$$
d\left(x^{\prime}, y\right)-d\left(x^{\prime}, S_{0}\right)+d\left(x, S_{0}\right) \leq d(x, y)+P+3 N+\delta .
$$

Donc

$$
\begin{aligned}
d\left(x^{\prime}, y\right)-k^{\prime} & \leq d\left(x^{\prime}, y\right)-\frac{d\left(x^{\prime}, S_{0}\right)+d\left(x, x^{\prime}\right)-d\left(x, S_{0}\right)}{2}-\frac{M}{2}+\frac{N}{2}+1 \\
& \leq d(x, y)-T-\frac{M}{2}+(P+7 N / 2+\delta+1)
\end{aligned}
$$

où la première inégalité a lieu par (151) et la deuxième par (152). Comme cela est vrai pour tout $y \in S_{m^{\prime}}$ on en déduit

$$
\left(d\left(x^{\prime}, S_{m^{\prime}}\right)-k^{\prime}\right) \leq\left(d\left(x, S_{m^{\prime}}\right)-k\right)-(T-k)-\frac{M}{2}+(P+7 N / 2+\delta+1) .
$$

Le membre de gauche de (145) est alors majoré par

$$
\sum_{l \geq l^{\prime}}\left(\begin{array}{l}
l \\
l^{\prime}
\end{array}\right)\left(\frac{C_{0} C_{2} B^{-1}(T-k)}{d\left(x, S_{m^{\prime}}\right)-k}\right)^{\left(l-l^{\prime}\right)}\left(\frac{d\left(x^{\prime}, S_{m^{\prime}}\right)-k^{\prime}}{d\left(x, S_{m^{\prime}}\right)-k}\right)^{l^{\prime}} \leq 2
$$

par le lemme 4.67. Les hypothèses du lemme 4.67 sont satisfaites car on suppose $2 C_{0} C_{2} B^{-1} \leq 1$ grâce à $\left(H_{B}\right)$ et $\frac{M}{2} \geq P+\frac{7 N}{2}+\delta+1$ grâce à $\left(H_{M}\right)$ d'où $(T-k)+\left(d\left(x^{\prime}, S_{m^{\prime}}\right)-k^{\prime}\right) \leq\left(d\left(x, S_{m^{\prime}}\right)-k\right)$ grâce à (153).

Démonstration du lemme 4.66. On distingue trois cas comme dans les démonstrations des lemmes 4.57 et 4.65 . On note $T=\frac{d\left(x, S_{0}\right)-d\left(x^{\prime}, S_{0}\right)+d\left(x, x^{\prime}\right)}{2}$ comme dans l'énoncé du lemme 4.66. On fixe $b \in S_{0}$. En reprenant mot pour mot la preuve de (149), c'est-à-dire le début de la démonstration du lemme 4.65 jusqu'à la distinction des trois cas, on obtient (149), c'est-à-dire que pour tout $y \in S_{m^{\prime}}$,

$$
d\left(x^{\prime}, y\right)-d\left(x^{\prime}, b\right)+d(x, b) \leq d(x, y)+P+2 N+\delta .
$$

Premier cas. On suppose $d\left(x, S_{0}\right) \leq k$.

Alors $m=0$ d'après le lemme 4.12, et cela est impossible, puisque $m>m^{\prime}$.

Deuxième cas. On suppose $d\left(x, S_{0}\right)>k$ et $T \leq k$.

Pour $j \in\left\{l^{\prime}+1, \ldots, l\right\}$ on a

$$
k+3 P \leq d_{\max }\left(x, y_{m^{\prime}}^{j}\right) \leq k+M+2 P+\delta .
$$

Comme $y_{m^{\prime}}^{j} \subset 4 P$-géod $(x, b)$ le lemme 3.13 montre que le nombre de possibilités pour $y_{m^{\prime}}^{j}$ est borné par $C_{2}=C(\delta, K, N, Q, P, M)$. Pour $i \in\left\{m^{\prime}+1, \ldots, m\right\}$ on a

$$
k+P \leq d\left(x, S_{i}\right) \leq k+M .
$$


En effet l'inégalité de gauche a lieu par la condition ii) de la définition 4.1. Comme $S_{i} \subset P$-géod $(x, b)$ le lemme 3.13 montre que le nombre de possibilités pour $S_{i}$ est borné par $C_{3}=C(\delta, K, N, Q, P, M)$. On a

$$
k^{\prime}=d\left(x^{\prime}, S_{0}\right)-d\left(x, S_{0}\right)+k+\frac{M}{2}
$$

d'après (122) donc

$$
k^{\prime} \geq d\left(x^{\prime}, b\right)-d(x, b)+k+\frac{M}{2}-N
$$

Soit $y \in S_{m^{\prime}}$. On a

$$
\begin{aligned}
d\left(x^{\prime}, y\right)-k^{\prime} & \leq d\left(x^{\prime}, y\right)-d\left(x^{\prime}, b\right)+d(x, b)+N-k-\frac{M}{2} \\
& \leq d(x, y)-k-\frac{M}{2}+P+3 N+\delta
\end{aligned}
$$

où la première inégalité vient de (155) et la deuxième de (154). Comme cela est vrai pour tout $y \in S_{m^{\prime}}$ on en déduit

$$
\left(d\left(x^{\prime}, S_{m^{\prime}}\right)-k^{\prime}\right) \leq\left(d\left(x, S_{m^{\prime}}\right)-k\right)-\frac{M}{2}+P+3 N+\delta
$$

On a $d\left(x, S_{m^{\prime}+1}\right) \leq k+M \operatorname{donc} d\left(S_{m^{\prime}}, S_{m^{\prime}+1}\right) \geq d\left(x, S_{m^{\prime}}\right)-k-M-N$ et

$$
\begin{aligned}
d\left(S_{m^{\prime}}, S_{m^{\prime}+1}\right)+2 M & \geq d\left(x, S_{m^{\prime}}\right)-k+M-N \\
& \geq\left(d\left(x^{\prime}, S_{m^{\prime}}\right)-k^{\prime}\right)+3 M / 2-(P+4 N+\delta) \\
& \geq\left(d\left(x^{\prime}, S_{m^{\prime}}\right)-k^{\prime}\right)+1
\end{aligned}
$$

où l'avant-dernière inégalité a lieu par (156) et où la dernière inégalité a lieu car on suppose $3 M / 2 \geq P+4 N+\delta+1$, ce qui est permis par $\left(H_{M}\right)$.

Le membre de gauche de (146) est majoré par

$$
\begin{gathered}
\sum_{\substack{m>m^{\prime} \\
l \geq l^{\prime}}}\left(\frac{C_{0} C_{3} B^{-1}}{1-C_{0} C_{1} B^{-1}}\right)^{m-m^{\prime}}\left(\begin{array}{c}
l \\
l^{\prime}
\end{array}\right)\left(\frac{C_{0} C_{2} B^{-1}}{d\left(S_{m^{\prime}}, S_{m^{\prime}+1}\right)+2 M}\right)^{l-l^{\prime}}\left(\frac{d\left(x^{\prime}, S_{m^{\prime}}\right)-k^{\prime}}{d\left(S_{m^{\prime}}, S_{m^{\prime}+1}\right)+2 M}\right)^{l^{\prime}} \\
\leq 2 \sum_{m>m^{\prime}}\left(\frac{C_{0} C_{3} B^{-1}}{1-C_{0} C_{1} B^{-1}}\right)^{m-m^{\prime}}
\end{gathered}
$$

grâce au lemme 4.67. En effet les hypothèses du lemme 4.67 sont satisfaites car $2 C_{0} C_{2} B^{-1} \leq 1$ par $\left(H_{B}\right)$ donc

$$
2 C_{0} C_{2} B^{-1}+\left(d\left(x^{\prime}, S_{m^{\prime}}\right)-k^{\prime}\right) \leq d\left(S_{m^{\prime}}, S_{m^{\prime}+1}\right)+2 M
$$


grâce à (157). Ensuite on calcule

$$
\sum_{m>m^{\prime}}\left(\frac{C_{0} C_{3} B^{-1}}{1-C_{0} C_{1} B^{-1}}\right)^{m-m^{\prime}}=\frac{C_{0} C_{3} B^{-1}}{1-C_{0}\left(C_{1}+C_{3}\right) B^{-1}} \leq 1
$$

où la dernière inégalité a lieu car on suppose $B \geq 2 C_{0}\left(C_{1}+C_{3}\right)$ grâce à $\left(H_{B}\right)$.

Troisième cas. On suppose $d\left(x, S_{0}\right)>k$ et $T>k$.

Soit $j \in\left\{l^{\prime}+1, \ldots, l\right\}$ et $w \in y_{m^{\prime}}^{j}$. Il existe $y \in S_{m^{\prime}}$ et $z \in S_{m^{\prime}+1}$ tels que $w \in P-\operatorname{géod}(y, z)$. On a $d(x, w) \geq d(x, y)-d(y, w)$ et $d(y, w) \leq d(y, z)+P$ donc $d(x, w) \geq d(x, y)-d(y, z)-P$. On en déduit

$$
d_{\max }\left(x, y_{m^{\prime}}^{j}\right) \geq d\left(x, S_{m^{\prime}}\right)-d\left(S_{m^{\prime}}, S_{m^{\prime}+1}\right)-2 N-P .
$$

D'autre part comme $j \in\left\{l^{\prime}+1, \ldots, l\right\}$ on a $d_{\max }\left(x, y_{m^{\prime}}^{j}\right) \leq T+M+2 P+\delta$. Donc $d_{\max }\left(x, y_{m^{\prime}}^{j}\right)$ appartient à l'intervalle

$$
\left[d\left(x, S_{m^{\prime}}\right)-d\left(S_{m^{\prime}}, S_{m^{\prime}+1}\right)-2 N-P, T+M+2 P+\delta\right]
$$

qui est de longueur

$$
(M+3 P+2 N+\delta)+d\left(S_{m^{\prime}}, S_{m^{\prime}+1}\right)+T-d\left(x, S_{m^{\prime}}\right) .
$$

On suppose $3 P+2 N+\delta+1 \leq M$, ce qui est permis par $\left(H_{M}\right)$. Comme $y_{m^{\prime}}^{j} \subset$ $4 P$ - $\operatorname{géod}(x, b)$ le lemme 3.13 montre que le nombre de possibilités pour $y_{m^{\prime}}^{j}$ est borné par

$$
C_{2}\left(2 M+d\left(S_{m^{\prime}}, S_{m^{\prime}+1}\right)+T-d\left(x, S_{m^{\prime}}\right)\right)
$$

avec $C_{2}=C(\delta, K, N, Q, P)$. Pour éviter des absurdités vérifions que

$$
2 M+d\left(S_{m^{\prime}}, S_{m^{\prime}+1}\right)+T-d\left(x, S_{m^{\prime}}\right) \geq 1 .
$$

Cela résulte des inégalités $d\left(S_{m^{\prime}}, S_{m^{\prime}+1}\right) \geq d\left(x, S_{m^{\prime}}\right)-d\left(x, S_{m^{\prime}+1}\right)-N$ et $d\left(x, S_{m^{\prime}+1}\right) \leq d_{\max }\left(x, S_{m^{\prime}+1}\right) \leq T+M$ car on suppose $M \geq N+1$ grâce à $\left(H_{M}\right)$.

Par (123) on a

$$
\left|k^{\prime}-\left(\frac{d\left(x^{\prime}, S_{0}\right)+d\left(x, x^{\prime}\right)-d\left(x, S_{0}\right)}{2}+\frac{M}{2}\right)\right| \leq \frac{N}{2}+1 .
$$

Soit $y \in S_{m^{\prime}}$. Il résulte de (154) que

$$
d\left(x^{\prime}, y\right)-d\left(x^{\prime}, S_{0}\right)+d\left(x, S_{0}\right) \leq d(x, y)+P+3 N+\delta .
$$

Donc

$$
\begin{aligned}
d\left(x^{\prime}, y\right)-k^{\prime} & \leq d\left(x^{\prime}, y\right)-\frac{d\left(x^{\prime}, S_{0}\right)+d\left(x, x^{\prime}\right)-d\left(x, S_{0}\right)}{2}-\frac{M}{2}+\frac{N}{2}+1 \\
& \leq d(x, y)-T-\frac{M}{2}+(P+7 N / 2+\delta+1)
\end{aligned}
$$


où la première inégalité a lieu par (158) et la deuxième par (159). Comme cela est vrai pour tout $y \in S_{m^{\prime}}$ on en déduit

$$
d\left(x^{\prime}, S_{m^{\prime}}\right)-k^{\prime} \leq d\left(x, S_{m^{\prime}}\right)-T-\frac{M}{2}+(P+7 N / 2+\delta+1) .
$$

D'autre part on a

$$
k+P \leq d_{\max }\left(x, S_{m}\right) \leq \cdots \leq d_{\max }\left(x, S_{m^{\prime}+1}\right) \leq T+M
$$

donc le nombre de possibilités pour ces entiers est borné par $\left(\begin{array}{c}(T-k)+M+\left(m-m^{\prime}\right) \\ m-m^{\prime}\end{array}\right)$. Comme $S_{m^{\prime}+1}, \ldots, S_{m}$ sont inclus dans $P-\operatorname{géod}(x, b)$, le lemme 3.13 montre que, connaissant les entiers $d_{\max }\left(x, S_{m}\right), \ldots, d_{\max }\left(x, S_{m^{\prime}+1}\right)$, le nombre de possibilités pour $S_{m^{\prime}+1}, \ldots, S_{m}$ est borné par $C_{3}^{m-m^{\prime}}$ avec $C_{3}=C(\delta, K, N, Q, P)$.

Le membre de gauche de (146) est alors majoré par

$$
\begin{gathered}
\sum_{m>m^{\prime}}\left(\frac{C_{0} C_{3} B^{-1}}{1-C_{0} C_{1} B^{-1}}\right)^{m-m^{\prime}}\left(\begin{array}{c}
(T-k)+M+\left(m-m^{\prime}\right) \\
m-m^{\prime}
\end{array}\right) \\
\sum_{l \geq l^{\prime}}\left(\begin{array}{c}
l \\
l^{\prime}
\end{array}\right)\left(\frac{C_{0} C_{2} B^{-1}\left(2 M+d\left(S_{m^{\prime}}, S_{m^{\prime}+1}\right)+T-d\left(x, S_{m^{\prime}}\right)\right)}{d\left(S_{m^{\prime}}, S_{m^{\prime}+1}\right)+2 M}\right)^{l-l^{\prime}} \\
\left(\frac{d\left(x^{\prime}, S_{m^{\prime}}\right)-k^{\prime}}{d\left(S_{m^{\prime}}, S_{m^{\prime}+1}\right)+2 M}\right)^{l^{\prime}} \\
\leq 2 \sum_{m>m^{\prime}}\left(\frac{C_{0} C_{3} B^{-1}}{1-C_{0} C_{1} B^{-1}}\right)^{m-m^{\prime}}\left(\begin{array}{c}
(T-k)+M+\left(m-m^{\prime}\right) \\
m-m^{\prime}
\end{array}\right)
\end{gathered}
$$

où la dernière inégalité résulte du lemme 4.67. Les hypothèses du lemme 4.67 sont satisfaites car on suppose $2 C_{0} C_{2} B^{-1} \leq 1$ grâce à $\left(H_{B}\right)$ et car

$$
\begin{aligned}
(2 M & \left.+d\left(S_{m^{\prime}}, S_{m^{\prime}+1}\right)+T-d\left(x, S_{m^{\prime}}\right)\right)+\left(d\left(x^{\prime}, S_{m^{\prime}}\right)-k^{\prime}\right) \\
& \leq d\left(S_{m^{\prime}}, S_{m^{\prime}+1}\right)+\frac{3 M}{2}+(2 P+7 N / 2+\delta+1) \leq d\left(S_{m^{\prime}}, S_{m^{\prime}+1}\right)+2 M
\end{aligned}
$$

où la première inégalité a lieu par (160) et la deuxième a lieu car on suppose $\frac{M}{2} \geq$ $2 P+7 N / 2+\delta+1$, ce qui est permis par $\left(H_{M}\right)$. Or grâce à (147),

$$
\begin{gathered}
\sum_{m \geq m^{\prime}}\left(\frac{C_{0} C_{3} B^{-1}}{1-C_{0} C_{1} B^{-1}}\right)^{m-m^{\prime}}\left(\begin{array}{c}
(T-k)+M+\left(m-m^{\prime}\right) \\
m-m^{\prime}
\end{array}\right) \\
=\left(1-\left(\frac{C_{0} C_{3} B^{-1}}{1-C_{0} C_{1} B^{-1}}\right)\right)^{-((T-k)+M+1)} \leq 2 e^{s d\left(x, x^{\prime}\right)}
\end{gathered}
$$

$\operatorname{car}(T-k) \leq T \leq d\left(x, x^{\prime}\right)$ et car on suppose

$$
1-\left(\frac{C_{0} C_{3} B^{-1}}{1-C_{0} C_{1} B^{-1}}\right) \geq \max \left(e^{-s}, 2^{-(M+1)^{-1}}\right)
$$

grâce à $\left(H_{B}\right)$. Ceci termine l'étude du troisième cas. 
On a démontré les lemmes 4.65 et 4.66 et donc aussi les lemmes 4.64, 4.63, 4.62, 4.56, et la proposition 4.3.

4.8. Equivariance des opérateurs à compacts près. Pour terminer la démonstration de la proposition 4.4, il suffit de montrer la proposition suivante.

Proposition 4.68. Soit $T \in \mathbb{R}_{+}$et $x, x^{\prime} \in X$ vérifiant $d\left(x, x^{\prime}\right)=1$. Alors

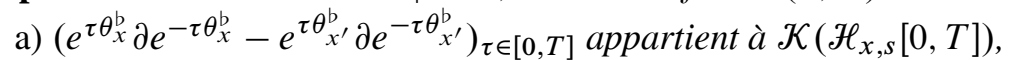

b) $\left(e^{\tau \theta_{x}^{\mathrm{b}}} h_{x} e^{-\tau \theta_{x}^{\mathrm{b}}}-e^{\tau \theta_{x^{\prime}}^{\mathrm{b}}} h_{x^{\prime}} e^{-\tau \theta_{x^{\prime}}^{\mathrm{b}}}\right)_{\tau \in[0, T]}$ appartient à $\mathcal{K}\left(\mathscr{H}_{x, s}[0, T]\right)$,

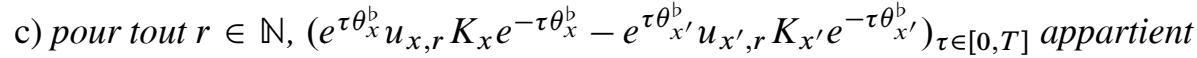
à $\mathcal{K}\left(\mathscr{H}_{x, s}[0, T]\right)$.

La proposition 4.3, qui a été établie au sous-paragraphe précédent, assure l'équivalence des normes de $\mathscr{H}_{x, s}$ et $\mathscr{H}_{x^{\prime}, s}$. On en déduit, grâce à la proposition 4.46, que tous les opérateurs apparaissant dans la proposition 4.68 sont continus. La proposition 4.68 est donc seulement un énoncé de compacité.

Démonstration de la proposition 4.4 en admettant la proposition 4.68. Comme $X$ est géodésique, l'énoncé de la proposition 4.68 implique évidemment le même énoncé pour $x, x^{\prime}$ quelconques (c'est-à-dire sans l'hypothèse $d\left(x, x^{\prime}\right)=1$ ). Comme $J_{x}=$ $\sum_{q=1}^{Q} h_{x}\left(1-\partial h_{x}-h_{x} \partial\right)^{q-1}+\sum_{r=1}^{\infty} u_{x, r} K_{x}$, on déduit des propositions 4.46 et 4.68 que pour $T \in \mathbb{R}_{+}$et $x, x^{\prime} \in X$,

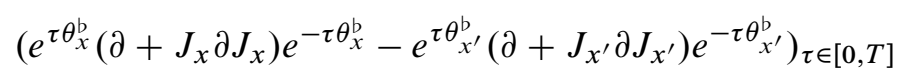

est un opérateur compact sur le $\mathbb{C}[0, T]$-module hilbertien $\mathscr{H}_{x, s}[0, T]$.

On va voir que la proposition 4.68 résulte du lemme suivant. On commence par remarquer que $\theta_{x^{\prime}}-\theta_{x}$ est un opérateur borné (et même de norme $\leq 1$ ) sur $\mathscr{H}_{x, s}$ : en effet pour tous $p, k, m, l_{0}, \ldots, l_{m}$, pour $Z \in \bar{Y}_{x}^{p, k, m,\left(l_{0}, \ldots, l_{m}\right)}$ et pour

$$
\left(a_{1}, \ldots, a_{p}, S_{0}, \ldots, S_{m},\left(y_{i}^{j}\right)_{i \in\{0, \ldots, m\}, j \in\left\{1, \ldots, l_{i}\right\}}\right) \in\left(\pi_{x}^{p, k, m,\left(l_{0}, \ldots, l_{m}\right)}\right)^{-1}(Z)
$$

$d\left(x, S_{0}\right)-d\left(x^{\prime}, S_{0}\right)$ appartient à $\{-1,0,1\}$ et est déterminé par $Z$ (on rappelle que $\left.S_{0}=\left\{a_{1}, \ldots, a_{p}\right\}\right)$. D'autre part $\theta_{x}^{b}-\theta_{x}$ est un opérateur borné sur $\mathscr{H}_{x, s}$ d'après le lemme 4.49 et de même $\theta_{x^{\prime}}^{b}-\theta_{x^{\prime}}$ est un opérateur borné sur $\mathscr{H}_{x^{\prime}, s}$. Grâce à l'équivalence des normes de $\mathscr{H}_{x, s}$ et $\mathscr{H}_{x^{\prime}, s}$ on en déduit que l'opérateur $\theta_{x}^{\mathrm{b}}-\theta_{x^{\prime}}^{\mathrm{b}}$ est borné sur $\mathscr{H}_{x, s}$. On note $[u, v]=u v-v u$.

Lemme 4.69. Soit $T \in \mathbb{R}_{+}$et $x, x^{\prime} \in X$ vérifiant $d\left(x, x^{\prime}\right)=1$. Alors

a) $\left(e^{\tau \theta_{x}}\left(h_{x}-h_{x^{\prime}}\right) e^{-\tau \theta_{x}}\right)_{\tau \in[0, T]}$ appartient à $\mathcal{K}\left(\mathscr{H}_{x, s}[0, T]\right)$,

b) pour tout $r \in \mathbb{N},\left(e^{\tau \theta_{x}}\left(u_{x, r} K_{x}-u_{x^{\prime}, r} K_{x^{\prime}}\right) e^{-\tau \theta_{x}}\right)_{\tau \in[0, T]}$ appartient à $\mathcal{K}\left(\mathscr{H}_{x, s}[0, T]\right)$, 
c) $\left(\left[\left(\theta_{x}^{b}-\theta_{x^{\prime}}^{b}\right), e^{\tau \theta_{x}} \partial e^{-\tau \theta_{x}}\right]\right)_{\tau \in[0, T]}$ appartient à $\mathcal{K}\left(\mathscr{H}_{x, s}[0, T]\right)$,

d) $\left(\left[\left(\theta_{x}^{\mathrm{b}}-\theta_{x^{\prime}}^{\mathrm{b}}\right), e^{\tau \theta_{x}} h_{x} e^{\left.-\tau \theta_{x}\right]}\right)_{\tau \in[0, T]}\right.$ appartient à $\mathcal{K}\left(\mathcal{H}_{x, s}[0, T]\right)$,

e) pour tout $r \in \mathbb{N},\left(\left[\left(\theta_{x}^{b}-\theta_{x^{\prime}}^{b}\right), e^{\tau \theta_{x}} u_{x, r} K_{x} e^{-\tau \theta_{x}}\right]\right)_{\tau \in[0, T]}$ appartient à $\mathcal{K}\left(\mathscr{H}_{x, s}[0, T]\right)$.

Démonstration de la proposition 4.68 en admettant le lemme 4.69. Nous montrons d'abord que le c) du lemme 4.69 implique le a) de la proposition 4.68. Pour tout $i \in \mathbb{N},\left(\left[\left(\theta_{x}^{\mathrm{b}}-\theta_{x^{\prime}}^{\mathrm{b}}\right)^{i}, e^{\tau \theta_{x}} \partial e^{-\tau \theta_{x}}\right]\right)_{\tau \in[0, T]}$ appartient à $\mathcal{K}\left(\mathscr{H}_{x, s}[0, T]\right)$ et donc

$$
\left(\left[e^{\tau\left(\theta_{x}^{\mathrm{b}}-\theta_{x^{\prime}}^{\mathrm{b}}\right)}, e^{\tau \theta_{x}} \partial e^{-\tau \theta_{x}}\right]\right)_{\tau \in[0, T]} \in \mathcal{K}\left(\mathscr{H}_{x, s}[0, T]\right) .
$$

Or on a

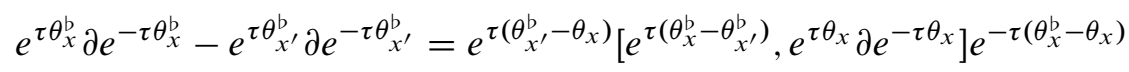

ce qui montre le a) de la proposition 4.68. Ensuite le a) et le d) du lemme 4.69 impliquent le b) de la proposition 4.68. En effet, on a

$$
\begin{aligned}
& e^{\tau \theta_{x}^{\mathrm{b}}} h_{x} e^{-\tau \theta_{x}^{\mathrm{b}}}-e^{\tau \theta_{x^{\prime}}^{\mathrm{b}}} h_{x^{\prime}} e^{-\tau \theta_{x^{\prime}}^{\mathrm{b}}} \\
& \quad=\left(e^{\tau \theta_{x^{\prime}}^{\mathrm{b}}}\left(h_{x}-h_{x^{\prime}}\right) e^{-\tau \theta_{x^{\prime}}^{\mathrm{b}}}\right)+\left(e^{\tau \theta_{x}^{\mathrm{b}}} h_{x} e^{-\tau \theta_{x}^{\mathrm{b}}}-e^{\tau \theta_{x^{\prime}}^{\mathrm{b}}} h_{x} e^{-\tau \theta_{x^{\prime}}^{\mathrm{b}}}\right)
\end{aligned}
$$

donc la compacité de $\left(e^{\tau \theta_{x}^{\mathrm{b}}} h_{x} e^{-\tau \theta_{x}^{\mathrm{b}}}-e^{\tau \theta_{x^{\prime}}^{\mathrm{b}}} h_{x^{\prime}} e^{-\tau \theta_{x^{\prime}}^{\mathrm{b}}}\right)_{\tau \in[0, T]}$ que l'on cherche à établir résulte de la compacité des deux termes du membre de droite. D'abord en conjugant $\operatorname{par} e^{\tau\left(\theta_{x^{\prime}}^{\mathrm{b}}-\theta_{x}\right)}$ on voit que le a) du lemme 4.69 implique que l'opérateur $\left(e^{\tau \theta_{x^{\prime}}^{\mathrm{b}}}\left(h_{x}-\right.\right.$ $\left.\left.h_{x^{\prime}}\right) e^{-\tau \theta_{x^{\prime}}^{\mathrm{b}}}\right)_{\tau \in[0, T]}$ est compact. Ensuite on a l'égalité

$$
e^{\tau \theta_{x}^{\mathrm{b}}} h_{x} e^{-\tau \theta_{x}^{\mathrm{b}}}-e^{\tau \theta_{x^{\prime}}^{\mathrm{b}}} h_{x} e^{-\tau \theta_{x^{\prime}}^{\mathrm{b}}}=e^{\tau\left(\theta_{x^{\prime}}^{\mathrm{b}}-\theta_{x}\right)}\left[e^{\tau\left(\theta_{x}^{\mathrm{b}}-\theta_{x^{\prime}}^{\mathrm{b}}\right)}, e^{\tau \theta_{x}} h_{x} e^{-\tau \theta_{x}}\right] e^{-\tau\left(\theta_{x}^{\mathrm{b}}-\theta_{x}\right)}
$$

donc le d) du lemme 4.69 montre la compacité de

$$
\left(e^{\tau \theta_{x}^{\mathrm{b}}} h_{x} e^{-\tau \theta_{x}^{\mathrm{b}}}-e^{\tau \theta_{x^{\prime}}^{\mathrm{b}}} h_{x} e^{-\tau \theta_{x^{\prime}}^{\mathrm{b}}}\right)_{\tau \in[0, T]} .
$$

Ceci termine la preuve du b) de la proposition 4.68. Enfin par un argument similaire (en remplaçant $h_{x}$ par $u_{x, r} K_{x}$ et $h_{x^{\prime}}$ par $u_{x^{\prime}, r} K_{x^{\prime}}$ ) le b) et le e) du lemme 4.69 impliquent le c) de la proposition 4.68.

Démonstration du lemme 4.69 en admettant le lemme 4.70. Pour tout $p \in\{1, \ldots$, $\left.p_{\max }\right\}$ et $n \in \mathbb{N}$ on note $\mathcal{P}_{n}$ le projecteur orthogonal sur le sous-espace vectoriel de $\mathscr{H}_{x, S}\left(\Delta_{p}\right)$ engendré par les $e_{S}$ pour $S \in \Delta_{p}$ tel que $d(x, S) \leq n$, de sorte que

$$
\left(\mathcal{P}_{n} f\right)(S)=f(S) \text { si } d(x, S) \leq n \quad \text { et } \quad\left(\mathcal{P}_{n} f\right)(S)=0 \text { si } d(x, S)>n .
$$

Dans les notations adoptées jusqu'ici on a donc $\mathcal{P}=\mathcal{P}_{P}$. On supposera toujours $n \geq P$, de sorte que $\left(1-\mathcal{P}_{n}\right)(1-\mathcal{P})=1-\mathcal{P}_{n}$. Il est évident que le lemme 4.69 résulte du lemme suivant. 
Lemme 4.70. Soit $T \in \mathbb{R}_{+}$et $x, x^{\prime} \in X$ vérifiant $d\left(x, x^{\prime}\right)=1$ et $p \in\left\{2, \ldots, p_{\max }\right\}$. Alors

a) $\sup _{\tau \in[0, T]}\left\|\left(1-\mathcal{P}_{n}\right) e^{\tau \theta_{x}}\left(h_{x}-h_{x^{\prime}}\right) e^{-\tau \theta_{x}}\right\|_{\mathscr{L}\left(\mathscr{H}_{x, s}\left(\Delta_{p-1}\right), \mathscr{H}_{x, s}\left(\Delta_{p}\right)\right)}$ tend vers 0 quand $n \rightarrow \infty$,

b) pour tout $r \in \mathbb{N}$,

$$
\sup _{\tau \in[0, T]}\left\|\left(1-\mathcal{P}_{n}\right) e^{\tau \theta_{x}}\left(u_{x, r} K_{x}-u_{x^{\prime}, r} K_{x^{\prime}}\right) e^{-\tau \theta_{x}}\right\|_{\mathscr{L}\left(\mathscr{H}_{x, s}\left(\Delta_{p-1}\right), \mathscr{H}_{x, s}\left(\Delta_{p}\right)\right)}
$$

tend vers 0 quand $n \rightarrow \infty$,

c) $\sup _{\tau \in[0, T]}\left\|\left(1-\mathcal{P}_{n}\right)\left[\left(\theta_{x}^{b}-\theta_{x^{\prime}}^{b}\right), e^{\tau \theta_{x}} \partial e^{-\tau \theta_{x}}\right]\right\|_{\mathscr{L}\left(\mathscr{H}_{x, s}\left(\Delta_{p}\right), \mathscr{H}_{x, s}\left(\Delta_{p-1}\right)\right)}$ tend vers 0 quand $n \rightarrow \infty$,

d) $\sup _{\tau \in[0, T]}\left\|\left(1-\mathcal{P}_{n}\right)\left[\left(\theta_{x}^{b}-\theta_{x^{\prime}}^{b}\right), e^{\tau \theta_{x}} h_{x} e^{-\tau \theta_{x}}\right]\right\|_{\mathscr{L}\left(\mathscr{H}_{x, s}\left(\Delta_{p-1}\right), \mathscr{H}_{x, S}\left(\Delta_{p}\right)\right)}$ tend vers 0 quand $n \rightarrow \infty$,

e) pour tout $r \in \mathbb{N}$,

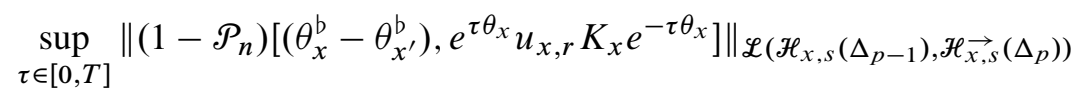

tend vers 0 quand $n \rightarrow \infty$.

Démonstration $d u$ a) du lemme 4.70. La preuve qui suit est assez voisine de la démonstration du lemme 4.36.

On déduira a) de (165) qui est une variante de l'inégalité (79).

Sous-lemme 4.71. Il existe $C_{1}=C(\delta, K, N)$ tel que pour tout $S \in \Delta_{p-1}$, la mesure de l'ensemble des $t \in[0,1]$ tels que $\left(h_{x, t}-h_{x^{\prime}, t}\right)\left(e_{S}\right) \neq 0$ est $\leq \frac{C_{1}}{1+d(x, S)}$.

Démonstration. C'est une conséquence immédiate du lemme 3.15.

Le sous-lemme suivant est une conséquence évidente du lemme 3.20.

Sous-lemme 4.72. Pour tout $S \in \Delta_{p-1}, h_{x, t}\left(e_{S}\right)$ et $h_{x^{\prime}, t}\left(e_{S}\right)$ ne dépendent que de la connaissance des points de

$$
\begin{aligned}
& B(x, 2 \delta+1) \cup B(S, N) \cup \bigcup_{a \in S}\{y \in(5 \delta+2)-\operatorname{géod}(x, a) \mid \\
&d(x, y) \in[\operatorname{td}(x, S)-2 N-3, t d(x, S)+2]\}
\end{aligned}
$$

et des distances entre ces points.

Démonstration. On applique le lemme 3.20 à $x$ et $x^{\prime}$ et on remarque que $5 \delta$ - $\operatorname{gé} o d\left(x^{\prime}, a\right) \subset(5 \delta+2)$ - géod $(x, a)$ et $\left|d(x, S)-d\left(x^{\prime}, S\right)\right| \leq 1$. 
Suite de la démonstration $d u$ a). Soient $k, m, l_{0}, \ldots, l_{m} \in \mathbb{N}$ et

$$
Z \in \bar{Y}_{x}^{p, k, m,\left(l_{0}, \ldots, l_{m}\right)} \text { vérifiant } r_{0}(Z)>k+P \text {. }
$$

On pose $\tilde{l}_{0}=0$ et $\tilde{l}_{i}=l_{i-1}$ pour $i \in\{1, \ldots, m+1\}$ et on note $\Lambda_{Z}$ la partie de $\bar{Y}_{x}^{\natural, p-1, k, m+1,\left(\tilde{l}_{0}, \ldots, \tilde{l}_{m+1}\right), 1,0}$ formée des $\tilde{Z}$ vérifiant

$$
r_{0}(Z) \leq r_{0}(\tilde{Z}) \leq r_{0}(Z)+N
$$

et tels que pour tout

$$
\begin{gathered}
\left(\tilde{a}_{1}, \ldots, \tilde{a}_{p-1}, \tilde{S}_{0}, \ldots, \widetilde{S}_{m+1},\left(\tilde{y}_{i}^{j}\right)_{i \in\{0, \ldots, m+1\}, j \in\left\{1, \ldots, \tilde{l}_{i}\right\}}, \widetilde{\mathcal{Z}}_{0}^{1}\right) \\
\in\left(\pi_{x}^{\natural, p-1, k, m+1,\left(\tilde{l}_{0}, \ldots, \tilde{l}_{m+1}\right), 1,0}\right)^{-1}(\widetilde{Z})
\end{gathered}
$$

il existe une énumération $\left(a_{1}, \ldots, a_{p}\right)$ de $\widetilde{S}_{1}$ vérifiant

$$
\left(a_{1}, \ldots, a_{p}, \widetilde{S}_{1}, \ldots, \widetilde{S}_{m+1},\left(\tilde{Y}_{i+1}^{j}\right)_{i \in\{0, \ldots, m\}, j \in\left\{1, \ldots, l_{i}\right\}}\right) \in\left(\pi_{x}^{p, k, m,\left(l_{0}, \ldots, l_{m}\right)}\right)^{-1}(Z) .
$$

Soit $t \in[0,1]$. On note $\Lambda_{Z, t}$ l'ensemble des $\tilde{Z} \in \Lambda_{Z}$ tels que pour tout

$$
\begin{gathered}
\left(\tilde{a}_{1}, \ldots, \tilde{a}_{p-1}, \widetilde{S}_{0}, \ldots, \tilde{S}_{m+1},\left(\widetilde{\mathcal{Y}}_{i}^{j}\right)_{i \in\{0, \ldots, m+1\}, j \in\left\{1, \ldots, \tilde{l}_{i}\right\}}, \widetilde{\mathcal{Z}}_{0}^{1}\right) \\
\in\left(\pi_{x}^{\natural, p-1, k, m+1,\left(\tilde{l}_{0}, \ldots, \tilde{l}_{m+1}\right), 1,0}\right)^{-1}(\widetilde{Z})
\end{gathered}
$$

on ait

$$
\widetilde{Z}_{0}^{1}=\bigcup_{b \in \widetilde{S}_{0}}\left\{z \in \operatorname{géod}(x, b), d(x, z)=\mathrm{E}\left(t r_{0}(\tilde{Z})\right)\right\}
$$

La condition (163) implique que pour $\tilde{Z} \in \Lambda_{Z, t}$ on a $t_{0}^{1}(\tilde{Z})=\mathrm{E}\left(\operatorname{tr}_{0}(\tilde{Z})\right)$.

Sous-lemme 4.73. Soit

$\left(\tilde{a}_{1}, \ldots, \tilde{a}_{p-1}, \tilde{S}_{0}, \ldots, \tilde{S}_{m+1},\left(\tilde{\mathscr{Y}}_{i}^{j}\right)_{i \in\{0, \ldots, m+1\}, j \in\left\{1, \ldots, \tilde{l}_{i}\right\}}\right) \in Y_{x}^{p-1, k, m+1,\left(\tilde{l}_{0}, \ldots, \tilde{l}_{m+1}\right)}$

tel que $d\left(x, \widetilde{S}_{1}\right) \leq d\left(x, \widetilde{S}_{0}\right) \leq d\left(x, \widetilde{S}_{1}\right)+N$ et qu'il existe $\left(a_{1}, \ldots, a_{p}\right)$ vérifiant (162). On définit $\widetilde{\widetilde{Z}}_{0}^{1}$ par (163). Alors $\widetilde{Z}_{0}^{1}$ est de diamètre inférieur ou égal à $P / 3$ et il existe $\tilde{Z} \in \Lambda_{Z, t}$ tel que

$$
\left(\tilde{a}_{1}, \ldots, \tilde{a}_{p-1}, \tilde{S}_{0}, \ldots, \tilde{S}_{m+1},\left(\tilde{y}_{i}^{j}\right)_{i \in\{0, \ldots, m+1\}, j \in\left\{1, \ldots, \tilde{l}_{i}\right\}}, \widetilde{\mathcal{Z}}_{0}^{1}\right)
$$

appartienne $\grave{a}\left(\pi_{x}^{\natural, p-1, k, m+1,\left(\tilde{l}_{0}, \ldots, \tilde{l}_{m+1}\right), 1,0}\right)^{-1}(\widetilde{Z})$.

Démonstration. Soient $z, z^{\prime} \in \widetilde{Z}_{0}^{1}$. Soit $b \in S$. On a $z, z^{\prime} \in 2 N$-géod $(x, b)$ et $d(x, z)=d\left(x, z^{\prime}\right)$ donc par $\left(H_{\delta}\left(z, x, z^{\prime}, b\right)\right), d\left(z, z^{\prime}\right) \leq 2 N+\delta \leq P / 3$. Comme $\widetilde{Z}_{0}^{1}$ est non vide et $P / 3 \leq M$, l'argument que nous venons de donner montre aussi que la condition (163) est vérifiée par les autres éléments de la classe d'équivalence $\widetilde{Z}$ de l'élément (164) et donc $\widetilde{Z} \in \Lambda_{Z, t}$. 
Suite de la démonstration $d u$ a). Notre but est de montrer l'inégalité suivante, qui est une variante de (79) : il existe une constante $C_{2}=C(\delta, K, N, Q, P, M, T)$ telle que

$$
\sup _{\tau \in[0, T]}\left|\xi_{Z}\left(e^{\tau \theta_{x}}\left(h_{x, t}-h_{x^{\prime}, t}\right) e^{-\tau \theta_{x}} f\right)\right|^{2} \leq C_{2} \sum_{\tilde{Z} \in \Lambda_{Z, t}^{\neq}}\left|\xi_{\widetilde{Z}}(f)\right|^{2}
$$

où $\Lambda_{Z, t}^{\neq}$est l'ensemble des $\widetilde{Z} \in \Lambda_{Z, t}$ tels que pour tout

$$
\begin{gathered}
\left(\tilde{a}_{1}, \ldots, \tilde{a}_{p-1}, \tilde{S}_{0}, \ldots, \widetilde{S}_{m+1},\left(\tilde{y}_{i}^{j}\right)_{i \in\{0, \ldots, m+1\}, j \in\left\{1, \ldots, \tilde{l}_{i}\right\}}, \tilde{Z}_{0}^{1}\right) \\
\in\left(\pi_{x}^{\natural, p-1, k, m+1,\left(\tilde{l}_{0}, \ldots, \tilde{l}_{m+1}\right), 1,0}\right)^{-1}(\tilde{Z})
\end{gathered}
$$

on ait

$$
\left(h_{x, t}-h_{x^{\prime}, t}\right)\left(e_{\widetilde{S}_{0}}\right) \neq 0 .
$$

Nous allons montrer (165) et en même temps justifier que la condition (166) ne dépend que de $\widetilde{Z}$ (c'est-à-dire que pour $\widetilde{Z} \in \Lambda_{Z, t}$ elle est vérifiée ou non simultanément pour tous les éléments de $\left.\left(\pi_{x}^{\natural, p-1, k, m+1,\left(\tilde{l}_{0}, \ldots, \tilde{l}_{m+1}\right), 1,0}\right)^{-1}(\widetilde{Z})\right)$.

Sous-lemme 4.74. Soit $\widetilde{Z} \in \Lambda_{Z, t}$ et

$$
\begin{gathered}
\left(\tilde{a}_{1}, \ldots, \tilde{a}_{p-1}, \widetilde{S}_{0}, \ldots, \widetilde{S}_{m+1},\left(\widetilde{y}_{i}^{j}\right)_{i \in\{0, \ldots, m+1\}, j \in\left\{1, \ldots, \tilde{l}_{i}\right\}}, \tilde{Z}_{0}^{1}\right) \\
\in\left(\pi_{x}^{\natural, p-1, k, m+1,\left(\tilde{l}_{0}, \ldots, \tilde{l}_{m+1}\right), 1,0}\right)^{-1}(\widetilde{Z}) .
\end{gathered}
$$

Alors $h_{x, t}\left(e_{\widetilde{S}_{0}}\right)$ et $h_{x^{\prime}, t}\left(e_{\widetilde{S}_{0}}\right)$ ne dépendent que de la connaissance des points de

$$
B\left(\tilde{S}_{0}, M\right) \cup B(x, k+2 M) \cup B\left(\tilde{\mathcal{Z}}_{0}^{1}, M\right) .
$$

et des distances entre ces points.

Remarque. On devrait plutôt noter $h_{x, t}\left(e_{\tilde{a}_{1}} \wedge \cdots \wedge e_{\tilde{a}_{p-1}}\right)$ au lieu de $h_{x, t}\left(e_{\tilde{S}_{0}}\right)$ mais à partir de maintenant nous commettrons cet abus.

Démonstration. Grâce au sous-lemme $4.72, h_{x, t}\left(e_{\widetilde{S}_{0}}\right)$ et $h_{x^{\prime}, t}\left(e_{\widetilde{S}_{0}}\right)$ ne dépendent que de la connaissance des points de

$$
\begin{aligned}
B(x, 2 \delta+1) \cup B\left(\widetilde{S}_{0}, N\right) \cup \bigcup_{a \in \widetilde{S}_{0}}\{y \in(5 \delta+2)-\operatorname{géod}(x, a) \mid \\
\left.\qquad(x, y) \in\left[\operatorname{tr}_{0}(\widetilde{Z})-2 N-3, t r_{0}(\widetilde{Z})+2\right]\right\}
\end{aligned}
$$

et des distances entre ces points. Il suffit donc de montrer que (168) est inclus dans (167). Il est évident que $B(x, 2 \delta+1) \cup B\left(\widetilde{S}_{0}, N\right)$ est inclus dans (167). Soient $a \in \widetilde{S}_{0}$, $y \in(5 \delta+2)-\operatorname{géod}(x, a)$ vérifiant

$$
d(x, y) \in\left[\operatorname{tr}_{0}(\widetilde{Z})-2 N-3, \operatorname{tr}_{0}(\widetilde{Z})+2\right] .
$$


Soit $z \in \operatorname{géod}(x, a)$ vérifiant $d(x, z)=\mathrm{E}\left(\operatorname{tr}_{0}(\widetilde{Z})\right)$, si bien que $z$ appartient à $\widetilde{Z}_{0}^{1}$. Alors $|d(x, y)-d(x, z)| \leq 2 N+3$ donc $\left(H_{\delta}(y, x, z, a)\right)$ montre $d(y, z) \leq(2 N+$ $3)+(5 \delta+2)+\delta$ et on suppose $(2 N+3)+(5 \delta+2)+\delta \leq M$ grâce à $\left(H_{M}\right)$. Donc l'ensemble (168) est inclus dans (167).

Le sous-lemme 4.74 implique immédiatement que la condition (166) ne dépend que de $\tilde{Z}$.

Sous-lemme 4.75. Le cardinal de $\Lambda_{Z, t}$ est majoré par une constante de la forme $C(\delta, K, N, Q, P, M)$.

Démonstration. Grâce au lemme 4.28, pour connaître les distances entre les points de

$$
B\left(\widetilde{S}_{0}, M\right) \cup B\left(\widetilde{\mathcal{Z}}_{0}^{1}, M\right)
$$

et ceux de

$$
\bigcup_{i \in\{1, \ldots, m+1\}} B\left(\widetilde{S}_{i}, M\right) \cup \bigcup_{\substack{i \in\{1, \ldots, m+1\} \\ j \in\left\{1, \ldots, \tilde{I}_{i}\right\}}} B\left(\widetilde{y}_{i}^{j}, M\right) \cup B(x, k+2 M)
$$

il est suffisant de connaitre les distances entre les points de (169) et $C^{\prime}$ points de (170), avec $C^{\prime}=C(\delta, K, N, Q, P, M)$ et ces distances sont déterminées à $C^{\prime \prime}=$ $C(\delta, K, N, Q, P, M)$ près par les distances de $\widetilde{S}_{1}$ à ces $C^{\prime}$ points (qui font partie de la donnée de $Z$ ) et l'entier $t_{0}^{1}(\widetilde{Z})$, qui est lui-même déterminé à $C^{\prime \prime \prime}=$ $C(\delta, K, N, Q, P, M)$ près par $r_{0}(Z)$ et $t$.

Suite de la démonstration $d u$ a). On termine maintenant la preuve de (165). Pour

$$
\begin{gathered}
\left(\tilde{a}_{1}, \ldots, \tilde{a}_{p-1}, \tilde{S}_{0}, \ldots, \tilde{S}_{m+1},\left(\tilde{y}_{i}^{j}\right)_{i \in\{0, \ldots, m+1\}, j \in\left\{1, \ldots, \tilde{I}_{i}\right\}}, \tilde{Z}_{0}^{1}\right) \\
\in\left(\pi_{x}^{\natural, p-1, k, m+1,\left(\tilde{l}_{0}, \ldots, \tilde{l}_{m+1}\right), 1,0}\right)^{-1}(\tilde{Z})
\end{gathered}
$$

on considère

$$
\sum_{\left(b_{1}, \ldots, b_{p}\right)}\left(\left(h_{x, t}-h_{x^{\prime}, t}\right)\left(e_{\tilde{a}_{1}} \wedge \cdots \wedge e_{\tilde{a}_{p-1}}\right)\right)\left(b_{1}, \ldots, b_{p}\right),
$$

où la somme porte sur les énumérations $\left(b_{1}, \ldots, b_{p}\right)$ de $\widetilde{S}_{1}$ telles que

$$
\left(b_{1}, \ldots, b_{p}, \widetilde{S}_{1}, \ldots, \widetilde{S}_{m+1},\left(\tilde{y}_{i+1}^{j}\right)_{i \in\{0, \ldots, m\}, j \in\left\{1, \ldots, \tilde{l}_{i}\right\}}\right) \in\left(\pi_{x}^{p, k, m,\left(l_{0}, \ldots, l_{m}\right)}\right)^{-1}(Z) .
$$

Comme la somme (171) a au plus $p$ ! termes, le 3) de la proposition 3.37 montre qu'elle est majorée par une constante de la forme $C(\delta, K, N, Q, P, M)$. D'après le sous-lemme 4.74 la somme (171) ne dépend que de $\widetilde{Z}$ et on peut donc la noter $\alpha_{Z, \widetilde{Z}, t}$. D'après le sous-lemme 4.73 on a

$$
\xi_{Z}\left(e^{\tau \theta_{x}}\left(h_{x, t}-h_{x^{\prime}, t}\right) e^{-\tau \theta_{x}} f\right)=\frac{1}{(p-1) !} \sum_{\tilde{Z} \in \Lambda_{Z, t}^{\neq}} e^{\tau\left(\rho_{x}^{1}(\tilde{Z})-\rho_{x}^{0}(\tilde{Z})\right)} \alpha_{Z, \tilde{Z}, t} \xi_{\widetilde{Z}}(f) .
$$


Grâce à (161) on a $\rho_{x}^{1}(\widetilde{Z})-\rho_{x}^{0}(\widetilde{Z}) \leq 2 N$. Par Cauchy-Schwarz et grâce au souslemme 4.75 on en déduit (165).

Montrons maintenant a) à l'aide de (165). Soit $C_{2}$ comme dans (165). Soient $k, m, l_{0}, \ldots, l_{m} \in \mathbb{N}$. On pose $\tilde{l}_{0}=0$ et $\tilde{l}_{i}=l_{i-1}$ pour $i \in\{1, \ldots, m+1\}$ comme précédemment.

Sous-lemme 4.76. Il existe $C_{3}=C(\delta, K, N, Q, P, M)$ tel que pour tout

$$
\left(a_{1}, \ldots, a_{p}, S_{0}, \ldots, S_{m},\left(\mathcal{Y}_{i}^{j}\right)_{i \in\{0, \ldots, m\}, j \in\left\{1, \ldots, l_{i}\right\}}\right) \in Y_{x}^{p, k, m,\left(l_{0}, \ldots, l_{m}\right)}
$$

le nombre de possibilités pour $\left(\tilde{a}_{1}, \ldots, \tilde{a}_{p-1}\right)$ tels que

$$
\left(\tilde{a}_{1}, \ldots, \tilde{a}_{p-1},\left\{\tilde{a}_{1}, \ldots, \tilde{a}_{p-1}\right\}, S_{0}, \ldots, S_{m},\left(\mathcal{Y}_{i-1}^{j}\right)_{i \in\{0, \ldots, m+1\}, j \in\left\{1, \ldots, \tilde{l}_{i}\right\}}\right)
$$

appartienne à $Y_{x}^{p-1, k, m+1,\left(\tilde{l}_{0}, \ldots, \tilde{l}_{m+1}\right)}$ et vérifie $d\left(x, S_{0}\right) \leq d\left(x,\left\{\tilde{a}_{1}, \ldots, \tilde{a}_{p-1}\right\}\right) \leq$ $d\left(x, S_{0}\right)+N$ soit $\leq C_{3}$.

Démonstration. Pour $a \in S_{0}$ et $i \in\{1, \ldots, p-1\}$ on a $a \in 2 F$-géod $\left(x, \tilde{a}_{i}\right)$ et $\left|d(x, a)-d\left(x, \tilde{a}_{i}\right)\right| \leq 2 N$, d'où $d\left(a, \tilde{a}_{i}\right) \leq 2 F+2 N$.

Fin de la démonstration $d u$ a). Soit

$$
Z \in \bar{Y}_{x}^{p, k, m,\left(l_{0}, \ldots, l_{m}\right)} \text { vérifiant } r_{0}(Z)>k+P \text {. }
$$

Grâce au sous-lemmes 4.71 et 4.76 , et comme $\widetilde{Z}_{0}^{1}$ est déterminé par (163) et $r_{0}(Z) \leq$ $r_{0}(\tilde{Z})$, on a alors

$$
\begin{array}{r}
\int_{0}^{1}\left(\sum_{\tilde{Z} \in \Lambda_{Z, t}^{\neq}} \sharp\left(\left(\pi_{x}^{\natural, p-1, k, m+1,\left(\tilde{l}_{0}, \ldots, \tilde{l}_{m+1}\right), 1,0}\right)^{-1}(\tilde{Z})\right)\right) d t \\
\leq \frac{C_{1} C_{3}}{r_{0}(Z)+1} \sharp\left(\left(\pi_{x}^{p, k, m,\left(l_{0}, \ldots, l_{m}\right)}\right)^{-1}(Z)\right) .
\end{array}
$$

Notons $I_{Z}$ l'ensemble des $t \in[0,1]$ tels qu'il existe $\widetilde{Z} \in \Lambda_{Z, t}^{\neq}$vérifiant

$$
\sharp\left(\left(\pi_{x}^{\natural, p-1, k, m+1,\left(\tilde{l}_{0}, \ldots, \tilde{l}_{m+1}\right), 1,0}\right)^{-1}(\tilde{Z})\right) \geq\left(r_{0}(Z)+1\right)^{-\frac{1}{2}} \sharp\left(\left(\pi_{x}^{p, k, m,\left(l_{0}, \ldots, l_{m}\right)}\right)^{-1}(Z)\right) .
$$

La mesure de $I_{Z}$ est donc $\leq C_{1} C_{3}\left(r_{0}(Z)+1\right)^{-\frac{1}{2}}$. Grâce à Cauchy-Schwarz et à (165) on obtient que

$$
\begin{aligned}
& \sup _{\tau \in[0, T]}\left|\xi_{Z}\left(e^{\tau \theta_{x}}\left(\int_{t \in I_{Z}}\left(h_{x, t}-h_{x^{\prime}, t}\right) d t\right) e^{-\tau \theta_{x}} f\right)\right|^{2} \\
& \leq C_{1} C_{3}\left(r_{0}(Z)+1\right)^{-\frac{1}{2}} \sup _{\tau \in[0, T]} \int_{t \in[0,1]}\left|\xi_{Z}\left(e^{\tau \theta_{x}}\left(h_{x, t}-h_{x^{\prime}, t}\right) e^{-\tau \theta_{x}} f\right)\right|^{2} d t \\
& \leq C_{1} C_{2} C_{3}\left(r_{0}(Z)+1\right)^{-\frac{1}{2}} \sum_{\tilde{Z} \in \Lambda_{Z}}\left(r_{0}(\widetilde{Z})+1\right)^{-1}\left|\xi_{\tilde{Z}}(f)\right|^{2} .
\end{aligned}
$$


D'après le sous-lemme 4.76 et le lemme 4.29 , il existe une constante $C_{4}=$ $C(\delta, K, N, Q, P, M)$ telle que pour $\widetilde{Z} \in \Lambda_{Z}$ on ait

$$
\sharp\left(\left(\pi_{x}^{\natural, p-1, k, m+1,\left(\tilde{l}_{0}, \ldots, \tilde{l}_{m+1}\right), 1,0}\right)^{-1}(\tilde{Z})\right) \leq C_{4} \sharp\left(\left(\pi_{x}^{p, k, m,\left(l_{0}, \ldots, l_{m}\right)}\right)^{-1}(Z)\right) .
$$

D'autre part pour $t \notin I_{Z}$ et $\tilde{Z} \in \Lambda_{Z, t}^{\neq}$on a $\sharp\left(\left(\pi_{x}^{\natural, p-1, k, m+1,\left(\tilde{l}_{0}, \ldots, \tilde{l}_{m+1}\right), 1,0}\right)^{-1}(\widetilde{Z})\right) \leq\left(r_{0}(Z)+1\right)^{-\frac{1}{2}} \sharp\left(\left(\pi_{x}^{p, k, m,\left(l_{0}, \ldots, l_{m}\right)}\right)^{-1}(Z)\right)$.

Par Cauchy-Schwarz on déduit alors de (165) que

$$
\begin{gathered}
\sup _{\tau \in[0, T]}\left(\sharp\left(\pi_{x}^{p, k, m,\left(l_{0}, \ldots, l_{m}\right)}\right)^{-1}(Z)\right)^{-\alpha}\left|\xi_{Z}\left(e^{\tau \theta_{x}}\left(\int_{t \notin I_{Z}}\left(h_{x, t}-h_{x^{\prime}, t}\right) d t\right) e^{-\tau \theta_{x}} f\right)\right|^{2} \\
\leq C_{2}\left(r_{0}(Z)+1\right)^{-\frac{\alpha}{2}} \sum_{\tilde{Z} \in \Lambda_{Z}}\left(r_{0}(\tilde{Z})+1\right)^{-1} \\
\left(\sharp\left(\pi_{x}^{\natural, p-1, k, m+1,\left(\tilde{l}_{0}, \ldots, \tilde{l}_{m+1}\right), 1,0}\right)^{-1}(\tilde{Z})\right)^{-\alpha}\left|\xi_{\tilde{Z}}(f)\right|^{2} .
\end{gathered}
$$

Comme

$$
h_{x}-h_{x^{\prime}}=\int_{t \in I_{Z}}\left(h_{x, t}-h_{x^{\prime}, t}\right) d t+\int_{t \notin I_{Z}}\left(h_{x, t}-h_{x^{\prime}, t}\right) d t,
$$

en combinant les inégalités (172), (173) et (174) et par Cauchy-Schwarz on obtient que

$$
\begin{gathered}
\sup _{\tau \in[0, T]}\left(\sharp\left(\pi_{x}^{p, k, m,\left(l_{0}, \ldots, l_{m}\right)}\right)^{-1}(Z)\right)^{-\alpha}\left|\xi_{Z}\left(e^{\tau \theta_{x}}\left(h_{x}-h_{x^{\prime}}\right) e^{-\tau \theta_{x}} f\right)\right|^{2} \\
\leq 2\left(C_{1} C_{2} C_{3} C_{4}^{\alpha}\left(r_{0}(Z)+1\right)^{-\frac{1}{2}}+C_{2}\left(r_{0}(Z)+1\right)^{-\frac{\alpha}{2}}\right) \sum_{\tilde{Z} \in \Lambda_{Z}}\left(r_{0}(\tilde{Z})+1\right)^{-1} \\
\left(\sharp\left(\pi_{x}^{\natural, p-1, k, m+1,\left(\tilde{l}_{0}, \ldots, \tilde{l}_{m+1}\right), 1,0}\right)^{-1}(\tilde{Z})\right)^{-\alpha}\left|\xi_{\tilde{Z}}(f)\right|^{2} .
\end{gathered}
$$

De plus pour $\tilde{Z} \in \Lambda_{Z}$ on a $\prod_{i=0}^{m} s_{i}(Z)^{-l_{i}}=\prod_{i=0}^{m+1} s_{i}(\tilde{Z})^{-\tilde{l}_{i}}$. Pour calculer la norme de $\left(1-\mathcal{P}_{n}\right) e^{\tau \theta_{x}}\left(h_{x}-h_{x^{\prime}}\right) e^{-\tau \theta_{x}}$ on peut se limiter aux $Z$ tels que $r_{0}(Z) \geq n$ et on déduit donc de (175) que

$$
\begin{array}{r}
\sup _{\tau \in[0, T]}\left\|\left(1-\mathcal{P}_{n}\right) e^{\tau \theta_{x}}\left(h_{x}-h_{x^{\prime}}\right) e^{-\tau \theta_{x}}\right\|_{\mathscr{L}\left(\mathscr{H}_{x, s}^{\natural, 1,0}\left(\Delta_{p-1}\right), \mathscr{H}_{x, s}\left(\Delta_{p}\right)\right)}^{2} \\
\leq 2 p ! B\left(C_{1} C_{2} C_{3} C_{4}^{\alpha}(n+1)^{-\frac{1}{2}}+C_{2}(n+1)^{-\frac{\alpha}{2}}\right)
\end{array}
$$

où le facteur $p$ ! est dû au fait que $\widetilde{Z}$ détermine $Z$ à permutation près de $a_{1}, \ldots, a_{p}$. Ceci termine la preuve de a). 
Démonstration $d u$ b) du lemme 4.70. La preuve de b) n'introduit aucune idée nouvelle par rapport à celle de a), donc on sera bref. Soit $r \in \mathbb{N}$. On déduira b) de (180) qui est une variante de l'inégalité (93), de la même façon que l'on avait montré le lemme 4.40 à l'aide de (93).

Sous-lemme 4.77. Il existe $C=C(\delta, K, N, r)$ tel que pour tout $a \in X$, la mesure de l'ensemble des $t \in[0,1]$ tels que $\mu_{r, t}(x, a) \neq \mu_{r, t}\left(x^{\prime}, a\right)$ est $\leq \frac{C}{1+d(x, a)}$.

Démonstration. Cela résulte du lemme 3.27.

Sous-lemme 4.78. Il existe $C_{1}=C(\delta, K, N, Q, r)$ tel que pour tout $S \in \Delta_{p-1}$, la mesure de l'ensemble des $\left(t, t_{1}, \ldots, t_{Q}\right) \in[0,1]^{Q+1}$ tels que

$$
u_{x, r, t} K_{x, Q,\left(t_{1}, \ldots, t_{Q}\right)}\left(e_{S}\right) \neq u_{x^{\prime}, r, t} K_{x^{\prime}, Q,\left(t_{1}, \ldots, t_{Q}\right)}\left(e_{S}\right)
$$

est $\leq \frac{C_{1}}{1+d(x, S)}$.

Démonstration. Il existe $C=C(\delta, K, N, Q, r)$ tel que pour $S \in \Delta_{p-1}$ la connaissance de $u_{x, r, t} K_{x, Q,\left(t_{1}, \ldots, t_{Q}\right)}\left(e_{S}\right)$ et celle de $u_{x^{\prime}, r, t} K_{x^{\prime}, Q,\left(t_{1}, \ldots, t_{Q}\right)}\left(e_{S}\right)$ ne dépendent que de la connaissance des $h_{x, t_{i}}\left(e_{S^{\prime}}\right)$ et $h_{x^{\prime}, t_{i}}\left(e_{S^{\prime}}\right)$ pour $i=1, \ldots, Q$ et $S^{\prime} \in \Delta$ vérifiant $d\left(S, S^{\prime}\right) \leq C$ et des $\mu_{r, t}(x, a)$ et $\mu_{r, t}\left(x^{\prime}, a\right)$ pour $a \in X$ vérifiant $d(a, S) \leq C$. On applique alors le sous-lemme 4.71 à ces parties $S^{\prime}$ et le sous-lemme 4.77 à ces points $a$.

Suite de la démonstration $d u \mathrm{~b})$. Soient $k, m, l_{0}, \ldots, l_{m} \in \mathbb{N}$ et $Z \in \bar{Y}_{x}^{p, k, m,\left(l_{0}, \ldots, l_{m}\right)}$ vérifiant $r_{0}(Z)>k+P$. On pose $\tilde{l}_{0}=0$ et $\tilde{l}_{i}=l_{i-1}$ pour $i \in\{1, \ldots, m+1\}$ et on note $\Lambda_{Z}$ la partie de $\bar{Y}_{x}^{\natural, p-1, k, m+1,\left(\tilde{l}_{0}, \ldots, \tilde{l}_{m+1}\right), Q, 1}$ formée des $\widetilde{Z}$ vérifiant

$$
\left|r_{0}(\tilde{Z})-r_{1}(\tilde{Z})-r\right| \leq Q F
$$

et tels que pour tout

$$
\begin{gathered}
\left(\tilde{a}_{1}, \ldots, \tilde{a}_{p-1}, \widetilde{S}_{0}, \ldots, \widetilde{S}_{m+1},\left(\tilde{y}_{i}^{j}\right)_{i \in\{0, \ldots, m+1\}, j \in\left\{1, \ldots, \tilde{l}_{i}\right\}},\left(\tilde{\mathcal{Z}}_{0}^{j}\right)_{j \in\{1, \ldots, Q\}}, \widetilde{Z}_{1}^{1}\right) \\
\in\left(\pi_{x}^{\natural, p-1, k, m+1,\left(\tilde{l}_{0}, \ldots, \tilde{l}_{m+1}\right), Q, 1}\right)^{-1}(\widetilde{Z})
\end{gathered}
$$

il existe une énumération $\left(a_{1}, \ldots, a_{p}\right)$ de $\widetilde{S}_{1}$ vérifiant

$$
\left(a_{1}, \ldots, a_{p}, \widetilde{S}_{1}, \ldots, \widetilde{S}_{m+1},\left(\tilde{y}_{i+1}^{j}\right)_{i \in\{0, \ldots, m\}, j \in\left\{1, \ldots, l_{i}\right\}}\right) \in\left(\pi_{x}^{p, k, m,\left(l_{0}, \ldots, l_{m}\right)}\right)^{-1}(Z) .
$$

Soient $t, t_{1}, \ldots, t_{Q} \in[0,1]$. On note $\Lambda_{Z, t,\left(t_{1}, \ldots, t_{Q}\right)}$ l'ensemble des $\tilde{Z} \in \Lambda_{Z}$ tels que pour tout

$$
\begin{gathered}
\left(\tilde{a}_{1}, \ldots, \tilde{a}_{p-1}, \widetilde{S}_{0}, \ldots, \widetilde{S}_{m+1},\left(\tilde{y}_{i}^{j}\right)_{i \in\{0, \ldots, m+1\}, j \in\left\{1, \ldots, \tilde{l}_{i}\right\}},\left(\tilde{Z}_{0}^{j}\right)_{j \in\{1, \ldots, Q\}}, \widetilde{Z}_{1}^{1}\right) \\
\in\left(\pi_{x}^{\natural, p-1, k, m+1,\left(\tilde{l}_{0}, \ldots, \tilde{l}_{m+1}\right), Q, 1}\right)^{-1}(\widetilde{Z})
\end{gathered}
$$


on ait

$$
\widetilde{Z}_{0}^{j}=\bigcup_{b \in \widetilde{S}_{0}}\left\{z \in \operatorname{géod}(x, b) \mid d(x, z)=\mathrm{E}\left(t_{j} r_{0}(\widetilde{Z})\right)\right\} \quad \text { pour } j \in\{1, \ldots, Q\}
$$

et

$$
\widetilde{Z}_{1}^{1}=\bigcup_{b \in \widetilde{S}_{1}}\left\{z \in \operatorname{géod}(x, b) \mid d(x, z)=\mathrm{E}\left((1-t) r_{1}(\widetilde{Z})\right)\right\} .
$$

Pour $\widetilde{Z} \in \Lambda_{Z, t,\left(t_{1}, \ldots, t_{Q}\right)}$, les conditions (178) et (179) impliquent

$$
t_{0}^{j}(\tilde{Z})=\mathrm{E}\left(t_{j} r_{0}(\tilde{Z})\right) \text { pour } j \in\{1, \ldots, Q\}, \quad \text { et } \quad t_{1}^{1}(\tilde{Z})=\mathrm{E}\left((1-t) r_{1}(\tilde{Z})\right) .
$$

Remarque. Les notations $\Lambda_{Z}$ et $\Lambda_{Z, t,\left(t_{1}, \ldots, t_{Q}\right)}$ que nous venons d'introduire sont les mêmes que dans la preuve du lemme 4.40 et les conditions (176), (177), (178) et (179) coïncident avec les conditions (91), (92), (94) et (95).

Sous-lemme 4.79. Soit

$$
\left(\tilde{a}_{1}, \ldots, \tilde{a}_{p-1}, \tilde{S}_{0}, \ldots, \tilde{S}_{m+1},\left(\tilde{\mathscr{Y}}_{i}^{j}\right)_{i \in\{0, \ldots, m+1\}, j \in\left\{1, \ldots, \tilde{l}_{i}\right\}}\right) \in Y_{x}^{p-1, k, m+1,\left(\tilde{l}_{0}, \ldots, \tilde{l}_{m+1}\right)}
$$

tel que $\left|d\left(x, \widetilde{S}_{0}\right)-d\left(x, \widetilde{S}_{1}\right)-r\right| \leq Q F$ et qu'il existe $\left(a_{1}, \ldots, a_{p}\right)$ vérifiant $(177)$. On définit $\left(\widetilde{Z}_{0}^{j}\right)_{j \in\{1, \ldots, Q\}}$ et $\widetilde{Z}_{1}^{1}$ par (178) et (179). Alors les parties $\widetilde{\mathcal{Z}}_{0}^{j}$ et $\widetilde{Z}_{1}^{1}$ sont de diamètre inférieur ou égal à $P / 3$ et il existe $\widetilde{Z} \in \Lambda_{Z, t,\left(t_{1}, \ldots, t_{Q}\right)}$ tel que

$$
\left(\tilde{a}_{1}, \ldots, \tilde{a}_{p-1}, \tilde{S}_{0}, \ldots, \widetilde{S}_{m+1},\left(\tilde{\mathcal{Y}}_{i}^{j}\right)_{i \in\{0, \ldots, m+1\}, j \in\left\{1, \ldots, \tilde{l}_{i}\right\}},\left(\tilde{\mathcal{Z}}_{0}^{j}\right)_{j \in\{1, \ldots, Q\}}, \widetilde{\mathcal{Z}}_{1}^{1}\right)
$$

appartienne $\grave{a}\left(\pi_{x}^{\natural, p-1, k, m+1,\left(\tilde{l}_{0}, \ldots, \tilde{l}_{m+1}\right), Q, 1}\right)^{-1}(\widetilde{Z})$.

Démonstration. C'est exactement le sous-lemme 4.41 .

Suite de la démonstration $d u \mathrm{~b})$. Notre but est maintenant de montrer l'inégalité suivante, qui est une variante de (93) : il y a une constante $C_{2}=C(\delta, K, N, Q, P, M, r, T)$ telle que

$$
\begin{gathered}
\sup _{\tau \in[0, T]}\left|\xi_{Z}\left(e^{\tau \theta_{x}}\left(u_{x, r, t} K_{x, Q,\left(t_{1}, \ldots, t_{Q}\right)}-u_{x^{\prime}, r, t} K_{x^{\prime}, Q,\left(t_{1}, \ldots, t_{Q}\right)}\right) e^{-\tau \theta_{x}} f\right)\right|^{2} \\
\leq C_{2} \sum_{\tilde{Z} \in \Lambda_{Z, t,\left(t_{1}, \ldots, t_{Q}\right)}^{\neq}}\left|\xi_{\tilde{Z}}(f)\right|^{2} .
\end{gathered}
$$

où $\Lambda_{Z, t,\left(t_{1}, \ldots, t_{Q}\right)}^{\neq}$est l'ensemble des $\widetilde{Z} \in \Lambda_{Z, t,\left(t_{1}, \ldots, t_{Q}\right)}$ tels que pour tout

$$
\begin{gathered}
\left(\tilde{a}_{1}, \ldots, \tilde{a}_{p-1}, \tilde{S}_{0}, \ldots, \widetilde{S}_{m+1},\left(\widetilde{\mathcal{Y}}_{i}^{j}\right)_{i \in\{0, \ldots, m+1\}, j \in\left\{1, \ldots, \tilde{l}_{i}\right\}},\left(\tilde{\mathcal{Z}}_{0}^{j}\right)_{j \in\{1, \ldots, Q\}}, \widetilde{\mathcal{Z}}_{1}^{1}\right) \\
\in\left(\pi_{x}^{\natural, p-1, k, m+1,\left(\tilde{l}_{0}, \ldots, \tilde{l}_{m+1}\right), Q, 1}\right)^{-1}(\widetilde{Z})
\end{gathered}
$$


on ait

$$
\left(u_{x, r, t} K_{x, Q,\left(t_{1}, \ldots, t_{Q}\right)}-u_{x^{\prime}, r, t} K_{x^{\prime}, Q,\left(t_{1}, \ldots, t_{Q}\right)}\right)\left(e_{\widetilde{S}_{0}}\right) \neq 0 .
$$

Le sous-lemme suivant indique d'où vient la condition (176).

Sous-lemme 4.80. Pour $S \in \Delta_{p-1}$ et $T \in \Delta_{p}$ tels que $e_{T}$ apparaisse avec un coefficient non nul dans $u_{x, r, t} K_{x, Q,\left(t_{1}, \ldots, t_{Q}\right)}\left(e_{S}\right)$ ou dans $u_{x^{\prime}, r, t} K_{x^{\prime}, Q,\left(t_{1}, \ldots, t_{Q}\right)}\left(e_{S}\right)$ on $a$

$$
|d(x, S)-d(x, T)-r| \leq Q F .
$$

Démonstration. D'après le 2)a) de la proposition 3.37 on a

$$
T \subset \bigcup_{a \in S}\left\{y \in(F+2)-\operatorname{géod}(x, a) \mid d(y, a) \in\left[r+\frac{Q}{F}, r+Q F\right]\right\}
$$

et on suppose $\frac{Q}{F}+Q F \geq N+(F+2)$, ce qui est permis $\operatorname{par}\left(H_{Q}\right)$.

Sous-lemme 4.81. Soit $\tilde{Z} \in \Lambda_{Z, t,\left(t_{1}, \ldots, t_{Q}\right)}^{\neq}$et

$$
\begin{gathered}
\left(\tilde{a}_{1}, \ldots, \tilde{a}_{p-1}, \widetilde{S}_{0}, \ldots, \widetilde{S}_{m+1},\left(\tilde{y}_{i}^{j}\right)_{i \in\{0, \ldots, m+1\}, j \in\left\{1, \ldots, \tilde{l}_{i}\right\}},\left(\widetilde{Z}_{0}^{j}\right)_{j \in\{1, \ldots, Q\}}, \tilde{Z}_{1}^{1}\right) \\
\in\left(\pi_{x}^{\natural, p-1, k, m+1,\left(\tilde{l}_{0}, \ldots, \tilde{l}_{m+1}\right), Q, 1}\right)^{-1}(\tilde{Z}) .
\end{gathered}
$$

Alors $u_{x, r, t} K_{x, Q,\left(t_{1}, \ldots, t_{Q}\right)}\left(e_{\widetilde{S}_{0}}\right)$ et $u_{x^{\prime}, r, t} K_{x^{\prime}, Q,\left(t_{1}, \ldots, t_{Q}\right)}\left(e_{\widetilde{S}_{0}}\right)$ ne dépendent que de la connaissance des points de

$$
B(x, k+2 M) \cup B\left(\tilde{S}_{0}, M\right) \cup B\left(\tilde{S}_{1}, M\right) \cup \underset{j \in\{1, \ldots, Q\}}{\cup} B\left(\widetilde{\mathcal{Z}}_{0}^{j}, M\right) \cup B\left(\widetilde{\mathcal{Z}}_{1}^{1}, M\right)
$$

et des distances entre ces points.

Démonstration. La réunion des ensembles figurant dans le 2)b) de la proposition 3.37 avec $x$ et $x^{\prime}$ au lieu de $x$ et $\widetilde{S}_{0}$ au lieu de $S$ (et qui possède la propriété que $u_{x, r, t} K_{x, Q,\left(t_{1}, \ldots, t_{Q}\right)}\left(e_{\widetilde{S}_{0}}\right)$ et $u_{x^{\prime}, r, t} K_{x^{\prime}, Q,\left(t_{1}, \ldots, t_{Q}\right)}\left(e_{\widetilde{S}_{0}}\right)$ ne dépendent que de la connaissance des distances entre les points de cette réunion) est inclus dans

$$
\begin{aligned}
& B(x, F+1) \cup B\left(\tilde{S}_{0}, Q N\right) \\
& \cup \bigcup_{\substack{a \in \widetilde{S}_{0} \\
j \in\{1, \ldots, Q\}}}\left\{y \in(F+2)-\operatorname{géod}(x, a)|| d(x, y)-t_{j} d(x, a) \mid \leq Q F+2\right\} \\
& \cup \bigcup_{a \in \widetilde{S}_{0}}\{z \in(F+2)-\operatorname{géod}(x, a) \mid d(z, a) \in[r, r+Q F]\} \\
& \cup \bigcup_{a \in \widetilde{S}_{0}}\{z \in(F+2)-\operatorname{géod}(x, a)|| d(x, z)-(1-t)(d(x, a)-r) \mid \leq Q F+2\} .
\end{aligned}
$$

Il suffit donc de montrer que cet ensemble est inclus dans (182). 
On suppose $F+1 \leq 2 M$ et $Q N \leq M$, ce qui est permis par $\left(H_{M}\right)$. Donc (183) est inclus dans (182).

Soit $a \in \widetilde{S}_{0}, j \in\{1, \ldots, Q\}$ et $y \in(F+2)$ - géod $(x, a)$ vérifiant

$$
\left|d(x, y)-t_{j} d(x, a)\right| \leq Q F+2 .
$$

Soit $z \in \operatorname{géod}(x, a)$ vérifiant $d(x, z)=\mathrm{E}\left(t_{j} r_{0}(\widetilde{Z})\right)$, si bien que $z$ appartient à $\widetilde{Z}_{0}^{j}$. On a

$$
\left|t_{j} d(x, a)-\mathrm{E}\left(t_{j} r_{0}(\widetilde{Z})\right)\right| \leq N+1,
$$

d'où $|d(x, y)-d(x, z)| \leq Q F+N+3$ et grâce à $\left(H_{\delta}(y, x, z, a)\right)$,

$$
d(y, z) \leq(Q F+N+3)+(F+2)+\delta .
$$

On suppose $(Q F+N+3)+(F+2)+\delta \leq M$, ce qui est permis par $\left(H_{M}\right)$. Par conséquent (184) est inclus dans $\bigcup_{j \in\{1, \ldots, Q\}} B\left(\widetilde{\mathcal{Z}}_{0}^{j}, M\right)$ et donc il est inclus dans (182).

Par le a) du lemme 4.14 , on a pour tout $a \in \widetilde{S}_{0}$,

$$
\widetilde{S}_{1} \subset 2 F-\operatorname{géod}(a, x) \text {. }
$$

Soit $a \in \widetilde{S}_{0}$ et $y \in \widetilde{S}_{1}$. L'inégalité (176) implique

$$
|d(x, a)-d(x, y)-r| \leq Q F+N .
$$

Soit $z \in(F+2)$ - géod $(x, a)$ vérifiant $d(z, a) \in[r, r+Q F]$. Cela implique $d(x, a)-$ $d(x, z) \in[r-F-2, r+Q F]$. On en déduit

$$
|d(x, y)-d(x, z)| \leq 2 Q F+N \text {. }
$$

Comme $z \in(F+2)-\operatorname{géod}(x, a), y \in 2 F-\operatorname{géod}(x, a)$ et $2 F \geq F+2,\left(H_{\delta}(z, x, y, a)\right)$ montre

$$
d(y, z) \leq(2 Q F+N)+2 F+\delta
$$

d'où

$$
\begin{gathered}
\bigcup_{a \in S}\{z \in(F+2)-\operatorname{géod}(x, a) \mid d(z, a) \in[r, r+Q F]\} \\
\subset B\left(\widetilde{S}_{1}, 2 Q F+N+2 F+\delta\right) \subset B\left(\widetilde{S}_{1}, M\right)
\end{gathered}
$$

car on suppose $2 Q F+N+2 F+\delta \leq M$, ce qui est permis par $\left(H_{M}\right)$. Donc (185) est inclus dans (182).

Enfin soit $a \in \widetilde{S}_{0}$ et $z \in(F+2)$ - géod $(x, a)$ vérifiant

$$
|d(x, z)-(1-t)(d(x, a)-r)| \leq Q F+2 .
$$


Soit $b \in \widetilde{S}_{1}$ et $\underset{\widetilde{Z}}{y} \in \operatorname{géod}(x, b)$ vérifiant $d(x, y)=\mathrm{E}\left((1-t) r_{1}(\widetilde{Z})\right)$, si bien que $y$ appartient à $\widetilde{Z}_{1}^{1}$. Comme $y \in \operatorname{géod}(x, b)$ et $b \in 2 F-\operatorname{géod}(x, a)$, on a $y \in$ $2 F-\operatorname{géod}(x, a)$. Comme $d(x, a) \in\left[r_{0}(\widetilde{Z}), r_{0}(\widetilde{Z})+N\right]$ et grâce à $(91)$, on a

$$
\left|d(x, z)-(1-t) r_{1}(\tilde{Z})\right| \leq 2 Q F+2+N,
$$

d'où

$$
|d(x, y)-d(x, z)| \leq 2 Q F+3+N .
$$

Comme $z \in(F+2)$ - géod $(x, a), y \in 2 F$ - géod $(x, a)$ et $F+2 \leq 2 F,\left(H_{\delta}(y, x, z, a)\right)$ montre

$$
d(y, z) \leq(2 Q F+3+N)+2 F+\delta .
$$

On suppose $(2 Q F+3+N)+2 F+\delta \leq M$, ce qui est permis par $\left(H_{M}\right)$. Par conséquent (186) est inclus dans $B\left(\widetilde{Z}_{1}^{1}, M\right)$ et donc dans (182). Ceci termine la preuve du sous-lemme 4.81 .

Le sous-lemme 4.81 justifie le fait que la condition (181) ne dépend que de $\widetilde{Z}$ (c'est-à-dire que pour $\widetilde{Z} \in \Lambda_{Z, t,\left(t_{1}, \ldots, t_{Q}\right)}$ elle est vérifiée ou non simultanément pour tous les éléments de $\left.\left(\pi_{x}^{\natural, p-1, k, m+1,\left(\tilde{l}_{0}, \ldots, \tilde{l}_{m+1}\right), Q, 1}\right)^{-1}(\tilde{Z})\right)$.

Sous-lemme 4.82. Le cardinal de $\Lambda_{Z, t,\left(t_{1}, \ldots, t_{Q}\right)}$ est majoré par une constante de la forme $C(\delta, K, N, Q, P, M)$.

Démonstration. Grâce au lemme 4.28, pour connaître les distances entre les points de

$$
B\left(\widetilde{S}_{0}, M\right) \cup \bigcup_{j \in\{1, \ldots, Q\}} B\left(\widetilde{\mathcal{Z}}_{0}^{j}, M\right) \cup B\left(\widetilde{\mathcal{Z}}_{1}^{1}, M\right)
$$

et ceux de

$$
\bigcup_{i \in\{1, \ldots, m+1\}} B\left(\tilde{S}_{i}, M\right) \cup \underset{\substack{i \in\{0, \ldots, m\} \\ j \in\left\{1, \ldots, l_{i}\right\}}}{\bigcup} B\left(\tilde{y}_{i+1}^{j}, M\right) \cup B(x, k+2 M)
$$

il suffit de connaître les distances entre les points de (187) et $C$ points de (188), avec $C=C(\delta, K, N, Q, P, M)$ et grâce à (176) ces distances sont déterminées à $C^{\prime}=C(\delta, K, N, Q, P, M)$ près par les distances de $\widetilde{S}_{1}$ à ces $C$ points (qui font partie de la donnée de $Z$ ) et les entiers $\left(t_{0}^{j}(\widetilde{Z})\right)_{j \in\{1, \ldots, Q\}}$ et $t_{1}^{1}(\widetilde{Z})$.

Suite de la démonstration $d u$ b). On termine maintenant la preuve de l'inégalité (180). Pour

$$
\begin{gathered}
\left(\tilde{a}_{1}, \ldots, \tilde{a}_{p-1}, \widetilde{S}_{0}, \ldots, \tilde{S}_{m+1},\left(\tilde{\mathcal{Y}}_{i}^{j}\right)_{i \in\{0, \ldots, m+1\}, j \in\left\{1, \ldots, \tilde{l}_{i}\right\}},\left(\tilde{\mathcal{Z}}_{0}^{j}\right)_{j \in\{1, \ldots, Q\}}, \widetilde{Z}_{1}^{1}\right) \\
\in\left(\pi_{x}^{\natural, p-1, k, m+1,\left(\tilde{l}_{0}, \ldots, \tilde{l}_{m+1}\right), Q, 1}\right)^{-1}(\widetilde{Z})
\end{gathered}
$$


on considère

$\sum_{\left(b_{1}, \ldots, b_{p}\right)}\left(\left(u_{x, r, t} K_{x, Q,\left(t_{1}, \ldots, t_{Q}\right)}-u_{x^{\prime}, r, t} K_{x^{\prime}, Q,\left(t_{1}, \ldots, t_{Q}\right)}\right)\left(e_{\tilde{a}_{1}} \wedge \cdots \wedge e_{\tilde{a}_{p-1}}\right)\right)\left(b_{1}, \ldots, b_{p}\right)$,

où la somme porte sur les énumérations $\widetilde{S}_{1}=\left\{b_{1}, \ldots, b_{p}\right\}$ telles que

$$
\left(b_{1}, \ldots, b_{p}, \widetilde{S}_{1}, \ldots, \widetilde{S}_{m+1},\left(\tilde{\mathcal{Y}}_{i+1}^{j}\right)_{i \in\{0, \ldots, m\}, j \in\left\{1, \ldots, l_{i}\right\}}\right) \in\left(\pi_{x}^{p, k, m,\left(l_{0}, \ldots, l_{m}\right)}\right)^{-1}(Z) .
$$

Comme la somme (189) a au plus $p$ ! termes, le 3) de la proposition 3.37 montre qu'elle est majorée par une constante de la forme $C(\delta, K, N, Q, P, M)$. D'après le sous-lemme 4.81 la somme (189) ne dépend que de $\widetilde{Z}$ et on peut donc la noter $\alpha_{Z, \tilde{Z}, t,\left(t_{1}, \ldots, t_{Q}\right)}$. D'après les sous-lemmes 4.79 et 4.80 on a

$$
\begin{aligned}
\xi_{Z}\left(e^{\tau \theta_{x}}\left(u_{x, r, t} K_{x, Q,\left(t_{1}, \ldots, t_{Q}\right)}-u_{x^{\prime}, r, t} K_{x^{\prime}, Q,\left(t_{1}, \ldots, t_{Q}\right)}\right) e^{-\tau \theta_{x}} f\right) & \frac{1}{(p-1) !} \sum_{\tilde{Z} \in \Lambda_{Z, t,\left(t_{1}, \ldots, t_{Q}\right)}^{\neq}} \alpha_{Z, \tilde{Z}, t,\left(t_{1}, \ldots, t_{Q}\right)} e^{\tau\left(\rho_{x}^{1}(\tilde{Z})-\rho_{x}^{0}(\tilde{Z})\right)} \xi_{\tilde{Z}}(f) .
\end{aligned}
$$

Grâce à (176) on a $\rho_{x}^{1}(\widetilde{Z})-\rho_{x}^{0}(\widetilde{Z}) \leq Q F+N$. Par Cauchy-Schwarz et grâce au sous-lemme 4.82 on en déduit (180).

Montrons maintenant b) à l'aide de (180). Soit $C_{2}$ comme dans (180). Soient $k, m, l_{0}, \ldots, l_{m} \in \mathbb{N}$. On pose $\tilde{l}_{0}=0$ et $\tilde{l}_{i}=l_{i-1}$ pour $i \in\{1, \ldots, m+1\}$ comme précédemment.

Sous-lemme 4.83. Il existe $C_{3}=C(\delta, K, N, Q, P, M, r)$ tel que pour tout

$$
\left(a_{1}, \ldots, a_{p}, S_{0}, \ldots, S_{m},\left(\mathcal{Y}_{i}^{j}\right)_{i \in\{0, \ldots, m\}, j \in\left\{1, \ldots, l_{i}\right\}}\right) \in Y_{x}^{p, k, m,\left(l_{0}, \ldots, l_{m}\right)}
$$

le nombre de possibilités pour $\left(\tilde{a}_{1}, \ldots, \tilde{a}_{p-1}\right)$ tels que

$$
\left(\tilde{a}_{1}, \ldots, \tilde{a}_{p-1},\left\{\tilde{a}_{1}, \ldots, \tilde{a}_{p-1}\right\}, S_{0}, \ldots, S_{m},\left(\mathcal{Y}_{i-1}^{j}\right)_{i \in\{0, \ldots, m+1\}, j \in\left\{1, \ldots, \tilde{l}_{i}\right\}}\right)
$$

appartienne à $Y_{x}^{p-1, k, m+1,\left(\tilde{l}_{0}, \ldots, \tilde{l}_{m+1}\right)}$ et vérifie

$$
\left|d\left(x,\left\{\tilde{a}_{1}, \ldots, \tilde{a}_{p-1}\right\}\right)-d\left(x, S_{0}\right)-r\right| \leq Q F
$$

soit $\leq C_{3}$.

Démonstration. C'est une conséquence immédiate du sous-lemme 4.45.

Fin de la démonstration $d u \mathrm{~b})$. Soit $Z \in \bar{Y}_{x}^{p, k, m,\left(l_{0}, \ldots, l_{m}\right)}$ vérifiant $r_{0}(Z)>k+P$. Grâce aux sous-lemmes 4.78 et 4.83 , on a

$$
\begin{gathered}
\int_{\left(t, t_{1}, \ldots, t_{Q}\right) \in[0,1]}\left(\sum_{\tilde{Z} \in \Lambda_{Z, t,\left(t_{1}, \ldots, t_{Q}\right)}^{\neq}} \sharp\left(\left(\pi_{x}^{\natural, p-1, k, m+1,\left(\tilde{l}_{0}, \ldots, \tilde{l}_{m+1}\right), Q, 1}\right)^{-1}(\tilde{Z})\right)\right) d t d t_{1} \ldots d t_{Q} \\
\leq \frac{C_{1} C_{3}}{r_{0}(Z)+1} \sharp\left(\left(\pi_{x}^{p, k, m,\left(l_{0}, \ldots, l_{m}\right)}\right)^{-1}(Z)\right) .
\end{gathered}
$$


Notons $I_{Z}$ l'ensemble des

$$
\left(t, t_{1}, \ldots, t_{Q}\right) \in[0,1]^{Q+1}
$$

tels qu'il existe $\widetilde{Z} \in \Lambda_{Z, t,\left(t_{1}, \ldots, t_{Q}\right)}^{\neq}$vérifiant

$\sharp\left(\left(\pi_{x}^{\natural, p-1, k, m+1,\left(\tilde{l}_{0}, \ldots, \tilde{l}_{m+1}\right), Q, 1}\right)^{-1}(\tilde{Z})\right) \geq\left(r_{0}(Z)+1\right)^{-\frac{1}{2}} \sharp\left(\left(\pi_{x}^{p, k, m,\left(l_{0}, \ldots, l_{m}\right)}\right)^{-1}(Z)\right)$.

La mesure de $I_{Z}$ est donc $\leq C_{1} C_{3}\left(r_{0}(Z)+1\right)^{-\frac{1}{2}}$. Grâce à Cauchy-Schwarz et à (180) on obtient que

$$
\begin{gathered}
\sup _{\tau \in[0, T]} \mid \xi_{Z}\left(e ^ { \tau \theta _ { x } } \left(\int _ { ( t , t _ { 1 } , \ldots , t _ { Q } ) \in I _ { Z } } \left(u_{x, r, t} K_{x, Q,\left(t_{1}, \ldots, t_{Q}\right)}\right.\right.\right. \\
\left.\left.\left.\quad-u_{x^{\prime}, r, t} K_{x^{\prime}, Q,\left(t_{1}, \ldots, t_{Q}\right)}\right) d t d t_{1} \ldots d t_{Q}\right) e^{-\tau \theta_{x}} f\right)\left.\right|^{2} \\
\leq C_{1} C_{3}\left(r_{0}(Z)+1\right)^{-\frac{1}{2}} \sup _{\tau \in[0, T]} \int_{\left(t, t_{1}, \ldots, t_{Q}\right) \in[0,1]} \mid \xi_{Z}\left(e ^ { \tau \theta _ { x } } \left(u_{x, r, t} K_{x, Q,\left(t_{1}, \ldots, t_{Q}\right)}\right.\right. \\
\left.\left.\quad-u_{x^{\prime}, r, t} K_{x^{\prime}, Q,\left(t_{1}, \ldots, t_{Q}\right)}\right) e^{-\tau \theta_{x}} f\right)\left.\right|^{2} d t d t_{1} \ldots d t_{Q} \\
\leq 2^{Q+1} C_{1} C_{2} C_{3}\left(r_{0}(Z)+1\right)^{-\frac{1}{2}} \sum_{\tilde{Z} \in \Lambda_{Z}}\left(r_{0}(\tilde{Z})+1\right)^{-Q}\left(r_{1}(\widetilde{Z})+1\right)^{-1}\left|\xi_{\tilde{Z}}(f)\right|^{2} .
\end{gathered}
$$

D'après le sous-lemme 4.83 et le lemme 4.29 , il existe $C_{4}=C(\delta, K, N, Q, P, M, r)$ tel que pour $\widetilde{Z} \in \Lambda_{Z}$ on ait

$$
\sharp\left(\left(\pi_{x}^{\natural, p-1, k, m+1,\left(\tilde{l}_{0}, \ldots, \tilde{l}_{m+1}\right), Q, 1}\right)^{-1}(\tilde{Z})\right) \leq C_{4} \sharp\left(\left(\pi_{x}^{p, k, m,\left(l_{0}, \ldots, l_{m}\right)}\right)^{-1}(Z)\right) .
$$

D'autre part pour $\left(t, t_{1}, \ldots, t_{Q}\right) \notin I_{Z}$ et $\widetilde{Z} \in \Lambda_{Z, t,\left(t_{1}, \ldots, t_{Q}\right)}^{\neq}$on a

$$
\sharp\left(\left(\pi_{x}^{\natural, p-1, k, m+1,\left(\tilde{l}_{0}, \ldots, \tilde{l}_{m+1}\right), Q, 1}\right)^{-1}(\tilde{Z})\right) \leq\left(r_{0}(Z)+1\right)^{-\frac{1}{2}} \sharp\left(\left(\pi_{x}^{p, k, m,\left(l_{0}, \ldots, l_{m}\right)}\right)^{-1}(Z)\right) .
$$

Par Cauchy-Schwarz on déduit alors de (180) que

$$
\begin{gathered}
\sup _{\tau \in[0, T]}\left(\sharp\left(\pi_{x}^{p, k, m,\left(l_{0}, \ldots, l_{m}\right)}\right)^{-1}(Z)\right)^{-\alpha} \mid \xi_{Z}\left(e ^ { \tau \theta _ { x } } \left(\int _ { ( t , t _ { 1 } , \ldots , t _ { Q } ) \notin I _ { Z } } \left(u_{x, r, t} K_{x, Q,\left(t_{1}, \ldots, t_{Q}\right)}\right.\right.\right. \\
\left.\left.\left.-u_{x^{\prime}, r, t} K_{x^{\prime}, Q,\left(t_{1}, \ldots, t_{Q}\right)}\right) d t d t_{1} \ldots d t_{Q}\right) e^{-\tau \theta_{x}} f\right)\left.\right|^{2} \\
\leq 2^{Q+1} C_{2}\left(r_{0}(Z)+1\right)^{-\frac{\alpha}{2}} \sum_{\tilde{Z} \in \Lambda_{Z}}\left(r_{0}(\widetilde{Z})+1\right)^{-Q}\left(r_{1}(\widetilde{Z})+1\right)^{-1} \\
\left(\sharp\left(\pi_{x}^{\natural, p-1, k, m+1,\left(\tilde{l}_{0}, \ldots, \tilde{l}_{m+1}\right), Q, 1}\right)^{-1}(\widetilde{Z})\right)^{-\alpha}\left|\xi_{\tilde{Z}}(f)\right|^{2} .
\end{gathered}
$$




\section{Comme}

$$
\begin{aligned}
u_{x, r} & K_{x}-u_{x^{\prime}, r} K_{x^{\prime}} \\
= & \int_{\left(t, t_{1}, \ldots, t_{Q}\right) \in I_{Z}}\left(u_{x, r, t} K_{x, Q,\left(t_{1}, \ldots, t_{Q}\right)}-u_{x^{\prime}, r, t} K_{x^{\prime}, Q,\left(t_{1}, \ldots, t_{Q}\right)}\right) d t d t_{1} \ldots d t_{Q} \\
& +\int_{\left(t, t_{1}, \ldots, t_{Q}\right) \notin I_{Z}}\left(u_{x, r, t} K_{x, Q,\left(t_{1}, \ldots, t_{Q}\right)}-u_{x^{\prime}, r, t} K_{x^{\prime}, Q,\left(t_{1}, \ldots, t_{Q}\right)}\right) d t d t_{1} \ldots d t_{Q},
\end{aligned}
$$

en combinant les inégalités (190), (191) et (192) et par Cauchy-Schwarz on obtient que

$$
\begin{gathered}
\sup _{\tau \in[0, T]}\left(\sharp\left(\pi_{x}^{p, k, m,\left(l_{0}, \ldots, l_{m}\right)}\right)^{-1}(Z)\right)^{-\alpha}\left|\xi_{Z}\left(e^{\tau \theta_{x}}\left(u_{x, r} K_{x}-u_{x^{\prime}, r} K_{x^{\prime}}\right) e^{-\tau \theta_{x}} f\right)\right|^{2} \\
\leq 2^{Q+2}\left(C_{1} C_{2} C_{3} C_{4}^{\alpha}\left(r_{0}(Z)+1\right)^{-\frac{1}{2}}+C_{2}\left(r_{0}(Z)+1\right)^{-\frac{\alpha}{2}}\right) \sum_{\tilde{Z} \in \Lambda_{Z}}\left(r_{0}(\widetilde{Z})+1\right)^{-Q} \\
\left(r_{1}(\tilde{Z})+1\right)^{-1}\left(\sharp\left(\pi_{x}^{\natural, p-1, k, m+1,\left(\tilde{l}_{0}, \ldots, \tilde{l}_{m+1}\right), Q, 1}\right)^{-1}(\widetilde{Z})\right)^{-\alpha}\left|\xi_{\tilde{Z}}(f)\right|^{2} .
\end{gathered}
$$

De plus pour $\tilde{Z} \in \Lambda_{Z}$ on a $\prod_{i=0}^{m} s_{i}(Z)^{-l_{i}}=\prod_{i=0}^{m+1} s_{i}(\tilde{Z})^{-\tilde{l}_{i}}$. Pour calculer la norme de $\left(1-\mathcal{P}_{n}\right) e^{\tau \theta_{x}}\left(u_{x, r} K_{x}-u_{x^{\prime}, r} K_{x^{\prime}}\right) e^{-\tau \theta_{x}}$ on peut se limiter aux $Z$ tels que $r_{0}(Z) \geq n$ et on déduit donc de (193) que

$$
\begin{gathered}
\sup _{\tau \in[0, T]}\left\|\left(1-\mathcal{P}_{n}\right) e^{\tau \theta_{x}}\left(u_{x, r} K_{x}-u_{x^{\prime}, r} K_{x^{\prime}}\right) e^{-\tau \theta_{x}}\right\|_{\mathscr{L}\left(\mathcal{H}_{x, S}^{\natural, Q, 1}\left(\Delta_{p-1}\right), \mathscr{H}_{x, s}\left(\Delta_{p}\right)\right)}^{2} \\
\leq 2^{Q+2} p ! B e^{2(Q F-r) s}\left(C_{1} C_{2} C_{3} C_{4}^{\alpha}(n+1)^{-\frac{1}{2}}+C_{2}(n+1)^{-\frac{\alpha}{2}}\right)
\end{gathered}
$$

où le facteur $p$ ! est dû au fait que $\widetilde{Z}$ détermine $Z$ à permutation près de $a_{1}, \ldots, a_{p}$. Ceci termine la preuve de b).

Démonstration $d u$ c) du lemme 4.70. La preuve de c) est quasiment identique à celle de $\mathrm{d}$ ) et légèrement plus simple (du fait que $\partial$ ne fait pas intervenir une moyenne, contrairement à $h_{x}$ ). Nous choisissons donc de montrer d) seulement.

Démonstration $d u$ d) du lemme 4.70. Soient $k, m, l_{0}, \ldots, l_{m} \in \mathbb{N}$ et

$$
Z \in \bar{Y}_{x}^{p, k, m,\left(l_{0}, \ldots, l_{m}\right)} \text { vérifiant } r_{0}(Z)>k+P \text {. }
$$

On pose $\tilde{l}_{0}=0$ et $\tilde{l}_{i}=l_{i-1}$ pour $i \in\{1, \ldots, m+1\}$. On note $\Lambda_{Z}$ la partie de $\bar{Y}_{x}^{\natural, p-1, k, m+1,\left(\tilde{l}_{0}, \ldots, \tilde{l}_{m+1}\right), 7,0}$ formée des $\widetilde{Z}$ vérifiant

$$
r_{0}(Z) \leq r_{0}(\tilde{Z}) \leq r_{0}(Z)+N
$$

et tels que pour tout

$$
\begin{gathered}
\left(\tilde{a}_{1}, \ldots, \tilde{a}_{p-1}, \tilde{S}_{0}, \ldots, \widetilde{S}_{m+1},\left(\widetilde{\mathcal{Y}}_{i}^{j}\right)_{i \in\{0, \ldots, m+1\}, j \in\left\{1, \ldots, \tilde{l}_{i}\right\}},\left(\widetilde{\mathcal{Z}}_{0}^{j}\right)_{j \in\{1, \ldots, 7\}}\right) \\
\in\left(\pi_{x}^{\natural, p-1, k, m+1,\left(\tilde{l}_{0}, \ldots, \tilde{l}_{m+1}\right), 7,0}\right)^{-1}(\widetilde{Z})
\end{gathered}
$$


il existe une énumération $\left(a_{1}, \ldots, a_{p}\right)$ de $\widetilde{S}_{1}$ vérifiant

$$
\left(a_{1}, \ldots, a_{p}, \widetilde{S}_{1}, \ldots, \widetilde{S}_{m+1},\left(\tilde{y}_{i+1}^{j}\right)_{i \in\{0, \ldots, m\}, j \in\left\{1, \ldots, l_{i}\right\}}\right) \in\left(\pi_{x}^{p, k, m,\left(l_{0}, \ldots, l_{m}\right)}\right)^{-1}(Z) .
$$

Soient $t \in[0,1]$ et $u_{1}, u_{2}, u_{3}, v_{1}, v_{2}, v_{3} \in\left[0,1\left[\right.\right.$. On note $\left(\Lambda_{Z, t}\right)_{u_{1}, u_{2}, u_{3}}^{v_{1}, v_{2}, v_{3}}$ l'ensemble des $\tilde{Z} \in \Lambda_{Z}$ tels que, en notant

$$
\begin{array}{lll}
w_{1}=\mathrm{E}\left(\frac{u_{1}}{6} r_{0}(\tilde{Z})\right), & w_{2}=\mathrm{E}\left(\left(\frac{1}{6}+\frac{u_{2}}{6}\right) r_{0}(\tilde{Z})\right), & w_{3}=\mathrm{E}\left(\left(\frac{2}{6}+\frac{u_{3}}{6}\right) r_{0}(\tilde{Z})\right), \\
w_{4}=\mathrm{E}\left(\left(1-\frac{v_{1}}{6}\right) r_{0}(\tilde{Z})\right), & w_{5}=\mathrm{E}\left(\left(\frac{5}{6}-\frac{v_{2}}{6}\right) r_{0}(\widetilde{Z})\right), & w_{6}=\mathrm{E}\left(\left(\frac{4}{6}-\frac{v_{3}}{6}\right) r_{0}(\tilde{Z})\right)
\end{array}
$$

on ait, pour tout

$$
\begin{gathered}
\left(\tilde{a}_{1}, \ldots, \tilde{a}_{p-1}, \tilde{S}_{0}, \ldots, \widetilde{S}_{m+1},\left(\tilde{\mathcal{Y}}_{i}^{j}\right)_{i \in\{0, \ldots, m+1\}, j \in\left\{1, \ldots, \tilde{l}_{i}\right\}},\left(\tilde{\mathcal{Z}}_{0}^{j}\right)_{j \in\{1, \ldots, 7\}}\right) \\
\in\left(\pi_{x}^{\natural, p-1, k, m+1,\left(\tilde{l}_{0}, \ldots, \tilde{l}_{m+1}\right), 7,0}\right)^{-1}(\widetilde{Z}),
\end{gathered}
$$

les égalités

$$
\widetilde{Z}_{0}^{j}=\bigcup_{b \in \widetilde{S}_{0}}\left\{z \in \operatorname{géod}(x, b) \mid d(x, z)=w_{j}\right\} \quad \text { pour } j \in\{1, \ldots, 6\}
$$

et

$$
\widetilde{Z}_{0}^{7}=\bigcup_{b \in \widetilde{S}_{0}}\left\{z \in \operatorname{géod}(x, b) \mid d(x, z)=\mathrm{E}\left(\operatorname{tr}_{0}(\tilde{Z})\right)\right\} .
$$

Pour $\widetilde{Z} \in\left(\Lambda_{Z, t}\right)_{u_{1}, u_{2}, u_{3}}^{v_{1}, v_{2}, v_{3}}$, les conditions (196) et (197) impliquent

$$
t_{0}^{j}(\widetilde{Z})=w_{j} \text { pour } j \in\{1, \ldots, 6\}, \quad \text { et } \quad t_{0}^{7}(\widetilde{Z})=\mathrm{E}\left(t_{0}(\tilde{Z})\right) .
$$

Sous-lemme 4.84. Soit

$$
\left(\tilde{a}_{1}, \ldots, \tilde{a}_{p-1}, \widetilde{S}_{0}, \ldots, \widetilde{S}_{m+1},\left(\tilde{y}_{i}^{j}\right)_{i \in\{0, \ldots, m+1\}, j \in\left\{1, \ldots, \tilde{l}_{i}\right\}}\right) \in Y_{x}^{p-1, k, m+1,\left(\tilde{l}_{0}, \ldots, \tilde{l}_{m+1}\right)}
$$

tel que $d\left(x, \widetilde{S}_{1}\right) \leq d\left(x, \widetilde{S}_{0}\right) \leq d\left(x, \widetilde{S}_{1}\right)+N$ et qu'il existe $\left(a_{1}, \ldots, a_{p}\right)$ vérifiant (195). On définit $\left(\widetilde{Z}_{0}^{j}\right)_{j \in\{1, \ldots, 7\}}$ par (196) et (197). Alors les parties $\widetilde{Z}_{0}^{j}$ sont de diamètre inférieur ou égal à $P / 3$ et il existe $\widetilde{Z} \in\left(\Lambda_{Z, t}\right)_{u_{1}, u_{2}, u_{3}}^{v_{1}, v_{2}, v_{3}}$ tel que

$$
\left(\tilde{a}_{1}, \ldots, \tilde{a}_{p-1}, \tilde{S}_{0}, \ldots, \tilde{S}_{m+1},\left(\tilde{y}_{i}^{j}\right)_{i \in\{0, \ldots, m+1\}, j \in\left\{1, \ldots, \tilde{l}_{i}\right\}},\left(\tilde{Z}_{0}^{j}\right)_{j \in\{1, \ldots, 7\}}\right)
$$

appartienne $\grave{a}\left(\pi_{x}^{\natural, p-1, k, m+1,\left(\tilde{l}_{0}, \ldots, \tilde{l}_{m+1}\right), 7,0}\right)^{-1}(\widetilde{Z})$.

Démonstration. En effet soit $b \in \widetilde{S}_{0}$. Pour $z, z^{\prime} \in \widetilde{\mathcal{Z}}_{0}^{j}$, on a $z, z^{\prime} \in 2 N$ - $\operatorname{géod}(x, b)$ et comme $d(x, z)=d\left(x, z^{\prime}\right),\left(H_{\delta}\left(z, x, z^{\prime}, b\right)\right)$ donne $d\left(z, z^{\prime}\right) \leq 2 N+\delta \leq P / 3$. Comme les parties $\tilde{z}_{0}^{j}$ sont non vides et $P / 3 \leq M$, l'argument que nous venons de donner montre aussi que les conditions (196) et (197) sont vérifiées par les autres éléments de la classe d'équivalence $\widetilde{Z}$ de l'élément (198) et donc $\widetilde{Z} \in\left(\Lambda_{Z, t}\right)_{u_{1}, u_{2}, u_{3}}^{v_{1}, v_{2}, v_{3}}$. 
Suite de la démonstration $d u \mathrm{~d})$. On déduira d) de l'inégalité suivante : il existe $C_{2}=$ $C(\delta, K, N, Q, P, M, T)$ tel que

$$
\begin{gathered}
\sup _{\tau \in[0, T]}\left|\xi_{Z}\left(\left[\left(\theta_{x}^{b}\right)_{u_{1}, u_{2}, u_{3}}^{v_{1}, v_{2}, v_{3}}-\left(\theta_{x^{\prime}}^{b}\right)_{u_{1}, u_{2}, u_{3}}^{v_{1}, v_{2}, v_{3}}, e^{\tau \theta_{x}} h_{x, t} e^{-\tau \theta_{x}}\right] f\right)\right|^{2} \\
\leq C_{2} \sum_{\tilde{Z} \in\left(\Lambda_{Z, t}^{\neq}\right)_{u_{1}, u_{2}, u_{3}}^{v_{1}, v_{2}, v_{3}}}\left|\xi_{\widetilde{Z}}(f)\right|^{2}
\end{gathered}
$$

où $\left(\Lambda_{Z, t}^{\neq}\right)_{u_{1}, u_{2}, u_{3}}^{v_{1}, v_{2}, v_{3}}$ est l'ensemble des $\widetilde{Z} \in\left(\Lambda_{Z, t}\right)_{u_{1}, u_{2}, u_{3}}^{v_{1}, v_{2}, v_{3}}$ tels que pour tout

$$
\begin{gathered}
\left(\tilde{a}_{1}, \ldots, \tilde{a}_{p-1}, \tilde{S}_{0}, \ldots, \widetilde{S}_{m+1},\left(\tilde{\mathcal{Y}}_{i}^{j}\right)_{i \in\{0, \ldots, m+1\}, j \in\left\{1, \ldots, \tilde{l}_{i}\right\}},\left(\widetilde{\mathcal{Z}}_{0}^{j}\right)_{j \in\{1, \ldots, 7\}}\right) \\
\in\left(\pi_{x}^{\natural, p-1, k, m+1,\left(\tilde{l}_{0}, \ldots, \tilde{l}_{m+1}\right), 7,0}\right)^{-1}(\widetilde{Z})
\end{gathered}
$$

on ait

$$
\left(\rho_{x}^{b}\right)_{u_{1}, u_{2}, u_{3}}^{v_{1}, v_{2}, v_{3}}\left(\widetilde{S}_{0}\right)-\left(\rho_{x^{\prime}}^{b}\right)_{u_{1}, u_{2}, u_{3}}^{v_{1}, v_{2}, v_{3}}\left(\widetilde{S}_{0}\right)-\left(\rho_{x}^{b}\right)_{u_{1}, u_{2}, u_{3}}^{v_{1}, v_{2}, v_{3}}\left(\widetilde{S}_{1}\right)+\left(\rho_{x^{\prime}}^{b}\right)_{u_{1}, u_{2}, u_{3}}^{v_{1}, v_{2}, v_{3}}\left(\widetilde{S}_{1}\right) \neq 0 .
$$

Nous allons maintenant montrer (199) et justifier que la condition (200) ne dépend que de $\widetilde{Z}$ (c'est-à-dire que pour $\widetilde{Z} \in\left(\Lambda_{Z, t}\right)_{u_{1}, u_{2}, u_{3}}^{v_{1}, v_{2}, v_{3}}$ elle est vérifiée ou non simultanément pour tous les éléments de $\left.\left(\pi_{x}^{\natural, p-1, k, m+1,\left(\tilde{l}_{0}, \ldots, \tilde{l}_{m+1}\right), 7,0}\right)^{-1}(\widetilde{Z})\right)$.

Sous-lemme 4.85. Pour $\widetilde{Z} \in\left(\Lambda_{Z, t}\right)_{u_{1}, u_{2}, u_{3}}^{v_{1}, v_{2}, v_{3}}$ et

$$
\begin{gathered}
\left(\tilde{a}_{1}, \ldots, \tilde{a}_{p-1}, \tilde{S}_{0}, \ldots, \widetilde{S}_{m+1},\left(\tilde{\mathcal{Y}}_{i}^{j}\right)_{i \in\{0, \ldots, m+1\}, j \in\left\{1, \ldots, \tilde{l}_{i}\right\}},\left(\tilde{Z}_{0}^{j}\right)_{j \in\{1, \ldots, 7\}}\right) \\
\in\left(\pi_{x}^{\natural, p-1, k, m+1,\left(\tilde{l}_{0}, \ldots, \tilde{l}_{m+1}\right), 7,0}\right)^{-1}(\widetilde{Z})
\end{gathered}
$$

le coefficient de $e_{\widetilde{S}_{1}}$ dans $\left[\left(\theta_{x}^{b}\right)_{u_{1}, u_{2}, u_{3}}^{v_{1}, v_{2}, v_{3}}-\left(\theta_{x^{\prime}}^{b}\right)_{u_{1}, u_{2}, u_{3}}^{v_{1}, v_{2}, v_{3}}, e^{\tau \theta_{x}} h_{x, t} e^{-\tau \theta_{x}}\right]\left(e_{\widetilde{S}_{0}}\right)$ et le membre de gauche de (200) ne dépendent que de la connaissance des points de

$$
B\left(\tilde{S}_{0}, M\right) \cup B\left(\tilde{S}_{1}, M\right) \cup B(x, k+2 M) \cup \bigcup_{j \in\{1, \ldots, 7\}} B\left(\widetilde{Z}_{0}^{j}, M\right)
$$

et des distances entre ces points.

Démonstration. Le sous-lemme 4.74 (que l'on applique avec $\widetilde{Z}_{0}^{7}$ au lieu de $\widetilde{Z}_{0}^{1}$ et en oubliant $\widetilde{Z}_{0}^{j}$ pour $\left.j \in\{1, \ldots, 6\}\right)$ montre que $h_{x, t}\left(e_{\widetilde{S}_{0}}\right)$ ne dépend que de la connaissance des points de

$$
B\left(\widetilde{S}_{0}, M\right) \cup B(x, k+2 M) \cup B\left(\tilde{Z}_{0}^{7}, M\right) .
$$

Pour montrer le sous-lemme, il suffit donc de montrer que pour $\tilde{x} \in B(x, 1)$, $\left(\rho_{\tilde{x}}^{b}\right)_{u_{1}, u_{2}, u_{3}}^{v_{1}, v_{2}, v_{3}}\left(\widetilde{S}_{0}\right)$ et $\left(\rho_{\tilde{x}}^{b}\right)_{u_{1}, u_{2}, u_{3}}^{v_{1}, v_{2}, v_{3}}\left(\widetilde{S}_{1}\right)$ ne dépendent que de la connaissance des points de (201) et des distances entre ces points (en effet on applique ceci à $\tilde{x}=x$ et $\tilde{x}=x^{\prime}$ ). 
En vertu du lemme 4.50, il suffit de montrer que pour $\tilde{x} \in B(x, 1), \sigma \in\{0,1\}, a \in \widetilde{S}_{\sigma}$ et $j \in\{1, \ldots, 6\}$, l'ensemble

$$
\left\{y \in 3 \delta-\operatorname{géod}(\tilde{x}, a)|| d(\tilde{x}, y)-w_{j}(\sigma, \tilde{x}) \mid \leq N+6 \delta+4\right\}
$$

est inclus dans (201), où l'on note

$$
\begin{array}{ll}
w_{1}(\sigma, \tilde{x})=\mathrm{E}\left(\frac{u_{1}}{6} d\left(\tilde{x}, \tilde{S}_{\sigma}\right)\right), & w_{2}(\sigma, \tilde{x})=\mathrm{E}\left(\left(\frac{1}{6}+\frac{u_{2}}{6}\right) d\left(\tilde{x}, \widetilde{S}_{\sigma}\right)\right), \\
w_{3}(\sigma, \tilde{x})=\mathrm{E}\left(\left(\frac{2}{6}+\frac{u_{3}}{6}\right) d\left(\tilde{x}, \widetilde{S}_{\sigma}\right)\right), & w_{4}(\sigma, \tilde{x})=\mathrm{E}\left(\left(1-\frac{v_{1}}{6}\right) d\left(\tilde{x}, \widetilde{S}_{\sigma}\right)\right), \\
w_{5}(\sigma, \tilde{x})=\mathrm{E}\left(\left(\frac{5}{6}-\frac{v_{2}}{6}\right) d\left(\tilde{x}, \widetilde{S}_{\sigma}\right)\right), & w_{6}(\sigma, \tilde{x})=\mathrm{E}\left(\left(\frac{4}{6}-\frac{v_{3}}{6}\right) d\left(\tilde{x}, \widetilde{S}_{\sigma}\right)\right) .
\end{array}
$$

Soit $\tilde{x} \in B(x, 1), \sigma \in\{0,1\}, a \in \widetilde{S}_{\sigma}$ et $j \in\{1, \ldots, 6\}$.

Soit $b \in \widetilde{S}_{0}$. On a

$$
d(a, b) \leq 2 N+2 F .
$$

En effet c'est évident si $\sigma=0$ et si $\sigma=1$ cela résulte du fait que

$$
d\left(x, \widetilde{S}_{1}\right)=r_{0}(Z) \in\left[d\left(x, \widetilde{S}_{0}\right)-N, d\left(x, \widetilde{S}_{0}\right)\right] \subset[d(x, b)-2 N, d(x, b)]
$$

et $\widetilde{S}_{1} \subset 2 F-\operatorname{géod}(x, b)$.

Soit $y$ dans l'ensemble (202). Comme $y \in 3 \delta$ - $\operatorname{géod}(\tilde{x}, a)$ et $\tilde{x} \in B(x, 1)$ et grâce à (203), le lemme 3.2 montre que

$$
y \in(3 \delta+4 N+4 F+2)-\operatorname{géod}(x, b) .
$$

D'autre part il résulte de (204) que $\left|w_{j}(\sigma, \tilde{x})-w_{j}\right| \leq N+1$. On a donc

$$
\left|d(x, y)-w_{j}\right| \leq 2 N+6 \delta+6 .
$$

Soit $z \in \operatorname{géod}(x, b)$ vérifiant $d(x, z)=w_{j}$, si bien que $z$ appartient à $\widetilde{Z}_{0}^{j}$. Comme $y$ et $z$ appartiennent à $(3 \delta+4 N+4 F+2)$ - $\operatorname{géod}(x, b)$ et que

$$
|d(x, y)-d(x, z)| \leq 2 N+6 \delta+6,
$$

$\left(H_{\delta}(y, x, z, b)\right)$ implique

$$
d(y, z) \leq(2 N+6 \delta+6)+(3 \delta+4 N+4 F+2)+\delta .
$$

On suppose $(2 N+6 \delta+6)+(3 \delta+4 N+4 F+2)+\delta \leq M$, ce qui est permis $\operatorname{par}\left(H_{M}\right)$. Donc $d(y, z) \leq M$ et $y$ appartient à (201). Ceci termine la preuve du sous-lemme 4.85 .

Le sous-lemme 4.85 implique immédiatement que la condition (200) ne dépend que de $\tilde{Z}$.

Sous-lemme 4.86. Le cardinal de $\left(\Lambda_{Z, t}^{\neq}\right)_{u_{1}, u_{2}, u_{3}}^{v_{1}, v_{2}, v_{3}}$ est majoré par une constante de la forme $C(\delta, K, N, Q, P, M)$. 
Démonstration. Grâce au lemme 4.28, pour connaître les distances entre les points de

$$
B\left(\tilde{S}_{0}, M\right) \cup \bigcup_{j \in\{1, \ldots, 7\}} B\left(\tilde{Z}_{0}^{j}, M\right)
$$

et ceux de

$$
\bigcup_{i \in\{1, \ldots, m+1\}} B\left(\widetilde{S}_{i}, M\right) \cup \underset{\substack{i \in\{0, \ldots, m\} \\ j \in\left\{1, \ldots, l_{i}\right\}}}{\bigcup} B\left(\widetilde{y}_{i+1}^{j}, M\right) \cup B(x, k+2 M)
$$

il suffit de connaître les distances entre les points de (205) et $C$ points de (206), avec $C=C(\delta, K, N, Q, P, M)$ et grâce à (194) ces distances sont déterminées à $C^{\prime}=C(\delta, K, N, Q, P, M)$ près par les distances de $\widetilde{S}_{1}$ à ces $C$ points (qui font partie de la donnée de $Z$ ) et les entiers $\left(t_{0}^{j}(\widetilde{Z})\right)_{j \in\{1, \ldots, 7\}}$.

Fin de la démonstration $d u$ d). On termine maintenant la preuve de (199). Pour

$$
\begin{gathered}
\left(\tilde{a}_{1}, \ldots, \tilde{a}_{p-1}, \tilde{S}_{0}, \ldots, \tilde{S}_{m+1},\left(\tilde{y}_{i}^{j}\right)_{i \in\{0, \ldots, m+1\}, j \in\left\{1, \ldots, \tilde{l}_{i}\right\}},\left(\tilde{\mathcal{Z}}_{0}^{j}\right)_{j \in\{1, \ldots, 7\}}\right) \\
\in\left(\pi_{x}^{\natural, p-1, k, m+1,\left(\tilde{l}_{0}, \ldots, \tilde{l}_{m+1}\right), 7,0}\right)^{-1}(\tilde{Z})
\end{gathered}
$$

on considère

$$
\sum_{\left(b_{1}, \ldots, b_{p}\right)}\left(\left[\left(\theta_{x}^{b}\right)_{u_{1}, u_{2}, u_{3}}^{v_{1}, v_{2}, v_{3}}-\left(\theta_{x^{\prime}}^{b}\right)_{u_{1}, u_{2}, u_{3}}^{v_{1}, v_{2}, v_{3}}, e^{\tau \theta_{x}} h_{x, t} e^{-\tau \theta_{x}}\right]\left(e_{\tilde{a}_{1}} \wedge \cdots \wedge e_{\tilde{a}_{p-1}}\right)\right)\left(b_{1}, \ldots, b_{p}\right),
$$

où la somme porte sur les énumérations $\widetilde{S}_{1}=\left\{b_{1}, \ldots, b_{p}\right\}$ telles que

$$
\left(b_{1}, \ldots, b_{p}, \widetilde{S}_{1}, \ldots, \widetilde{S}_{m+1},\left(\tilde{y}_{i+1}^{j}\right)_{i \in\{0, \ldots, m\}, j \in\left\{1, \ldots, \tilde{l}_{i}\right\}}\right) \in\left(\pi_{x}^{p, k, m,\left(l_{0}, \ldots, l_{m}\right)}\right)^{-1}(Z) .
$$

Comme la somme (207) a au plus $p$ ! termes, le 3) de la proposition 3.37 montre qu'elle est majorée par une constante de la forme $C(\delta, K, N, Q, P, M, T)$. D'après le sous-lemme 4.85 la somme (207) ne dépend que de $\widetilde{Z}$ et on peut donc la noter $\left(\alpha_{Z, \tilde{Z}, t, \tau}\right)_{u_{1}, u_{2}, u_{3}}^{v_{1}, v_{2}, v_{3}}$. D'après le sous-lemme 4.84 on a

$$
\begin{aligned}
& \xi_{Z}\left(\left[\left(\theta_{x}^{b}\right)_{u_{1}, u_{2}, u_{3}}^{v_{1}, v_{2}, v_{3}}-\left(\theta_{x^{\prime}}^{b}\right)_{u_{1}, u_{2}, u_{3}}^{v_{1}, v_{2}, v_{3}}, e^{\tau \theta_{x}} h_{x, t} e^{-\tau \theta_{x}}\right] f\right) \\
& \quad=\frac{1}{(p-1) !} \sum_{\tilde{Z} \in\left(\Lambda_{Z, t}^{f_{t}}\right)_{u_{1}, u_{2}, u_{3}}^{v_{1}, v_{2}, v_{3}}}\left(\alpha_{Z, \widetilde{Z}, t, \tau}\right)_{u_{1}, u_{2}, u_{3}}^{v_{1}, v_{2}, v_{3}} \xi_{\widetilde{Z}}(f) .
\end{aligned}
$$

Par Cauchy-Schwarz et grâce au sous-lemme 4.86 on en déduit (199).

Montrons maintenant d) à l'aide de (199). Il résulte de (203) que pour $\widetilde{S}_{0}, \widetilde{S}_{1}$ comme ci-dessus on a $d\left(\widetilde{S}_{0}, \widetilde{S}_{1}\right) \leq 2 N+2 F$. Donc le lemme 3.45 implique facilement qu'il existe $C_{1}=C(\delta, K, N)$ tel que pour $\widetilde{S}_{0}, \widetilde{S}_{1}$ comme ci-dessus la mesure de l'ensemble des $\left(u_{1}, u_{2}, u_{3}, v_{1}, v_{2}, v_{3}\right) \in\left[0,1\left[{ }^{6}\right.\right.$ vérifiant $(200)$ est $\leq \frac{C_{1}}{1+r_{0}(Z)}$. Soit $C_{2}$ comme dans (165). Soient $k, m, l_{0}, \ldots, l_{m} \in \mathbb{N}$. On pose $\tilde{l}_{0}=0$ et $\tilde{l}_{i}=l_{i-1}$ pour 
$i \in\{1, \ldots, m+1\}$ comme précédemment. Soit $C_{3}=C(\delta, K, N, Q, P, M)$ comme dans le sous-lemme 4.76. Soit

$$
Z \in \bar{Y}_{x}^{p, k, m,\left(l_{0}, \ldots, l_{m}\right)} \text { vérifiant } r_{0}(Z)>k+P .
$$

On a alors

$$
\begin{aligned}
& \left.\int_{\left(u_{1}, u_{2}, u_{3}, v_{1}, v_{2}, v_{3}\right) \in\left[0,1\left[{ }^{6}\right.\right.}\left(\sum_{\tilde{Z} \in\left(\Lambda_{Z, t}^{\neq}\right)_{u_{1}, u_{2}, u_{3}}^{v_{1}, v_{2}, v_{3}}} \sharp\left(\pi^{\natural, p-1, k, m+1,\left(\tilde{l}_{0}, \ldots, \tilde{l}_{m+1}\right), 7,0}\right)^{-1}(\tilde{Z})\right)\right) d u_{1} \ldots d v_{3} \\
& \leq \frac{C_{1} C_{3}}{r_{0}(Z)+1} \sharp\left(\left(\pi_{x}^{p, k, m,\left(l_{0}, \ldots, l_{m}\right)}\right)^{-1}(Z)\right) .
\end{aligned}
$$

Notons $I_{Z}$ l'ensemble des

$$
\left(t, u_{1}, u_{2}, u_{3}, v_{1}, v_{2}, v_{3}\right) \in[0,1] \times\left[0,1\left[^{6}\right.\right.
$$

tels qu'il existe $\tilde{Z} \in\left(\Lambda_{Z, t}^{\neq}\right)_{u_{1}, u_{2}, u_{3}}^{v_{1}, v_{2}, v_{3}}$ vérifiant

$\sharp\left(\left(\pi_{x}^{\natural, p-1, k, m+1,\left(\tilde{l}_{0}, \ldots, \tilde{l}_{m+1}\right), 7,0}\right)^{-1}(\tilde{Z})\right) \geq\left(r_{0}(Z)+1\right)^{-\frac{1}{2}} \sharp\left(\left(\pi_{x}^{p, k, m,\left(l_{0}, \ldots, l_{m}\right)}\right)^{-1}(Z)\right)$.

La mesure de $I_{Z}$ est donc $\leq C_{1} C_{3}\left(r_{0}(Z)+1\right)^{-\frac{1}{2}}$. Grâce à Cauchy-Schwarz on déduit de (199) que

$$
\begin{aligned}
& \sup _{\tau \in[0, T]} \mid \xi_{Z}\left(\left(\int _ { ( t , u _ { 1 } , u _ { 2 } , u _ { 3 } , v _ { 1 } , v _ { 2 } , v _ { 3 } ) \in I _ { Z } } \left[\left(\theta_{x}^{b}\right)_{u_{1}, u_{2}, u_{3}}^{v_{1}, v_{2}, v_{3}}\right.\right.\right. \\
&\left.\left.\left.-\left(\theta_{x^{\prime}}^{b}\right)_{u_{1}, u_{2}, u_{3}}^{v_{1}, v_{2}, v_{3}}, e^{\tau \theta_{x}} h_{x, t} e^{-\tau \theta_{x}}\right] d t \ldots d v_{3}\right) f\right)\left.\right|^{2} \\
& \leq 2^{7} 6^{6} C_{1} C_{2} C_{3}\left(r_{0}(Z)+1\right)^{-\frac{1}{2}} \sum_{\widetilde{Z} \in \Lambda_{Z}}\left(r_{0}(\widetilde{Z})+1\right)^{-7}\left|\xi_{\widetilde{Z}}(f)\right|^{2} .
\end{aligned}
$$

Grâce au sous-lemme 4.76 et au lemme 4.29 , il existe $C_{4}=C(\delta, K, N, Q, P, M)$ tel que pour $\widetilde{Z} \in \Lambda_{Z}$ on ait

$$
\sharp\left(\left(\pi_{x}^{\natural, p-1, k, m+1,\left(\tilde{l}_{0}, \ldots, \tilde{l}_{m+1}\right), 7,0}\right)^{-1}(\tilde{Z})\right) \leq C_{4} \sharp\left(\left(\pi_{x}^{p, k, m,\left(l_{0}, \ldots, l_{m}\right)}\right)^{-1}(Z)\right) .
$$

D'autre part pour $\left(t, u_{1}, u_{2}, u_{3}, v_{1}, v_{2}, v_{3}\right) \notin I_{Z}$ et $\tilde{Z} \in\left(\Lambda_{Z, t}^{\neq}\right)_{u_{1}, u_{2}, u_{3}}^{v_{1}, v_{2}, v_{3}}$ on a $\sharp\left(\left(\pi_{x}^{\natural, p-1, k, m+1,\left(\tilde{l}_{0}, \ldots, \tilde{l}_{m+1}\right), 7,0}\right)^{-1}(\tilde{Z})\right) \leq\left(r_{0}(Z)+1\right)^{-\frac{1}{2}} \sharp\left(\left(\pi_{x}^{p, k, m,\left(l_{0}, \ldots, l_{m}\right)}\right)^{-1}(Z)\right)$.

Par Cauchy-Schwarz on déduit alors de (199) que

$$
\begin{aligned}
& \left(\sharp\left(\pi_{x}^{p, k, m,\left(l_{0}, \ldots, l_{m}\right)}\right)^{-1}(Z)\right)^{-\alpha} \sup _{\tau \in[0, T]} \mid \xi_{Z}\left(\left(\int _ { ( t , u _ { 1 } , u _ { 2 } , u _ { 3 } , v _ { 1 } , v _ { 2 } , v _ { 3 } ) \notin I _ { Z } } \left[\left(\theta_{x}^{b}\right)_{u_{1}, u_{2}, u_{3}}^{v_{1}, v_{2}, v_{3}}\right.\right.\right. \\
& \left.\left.\left.-\left(\theta_{x^{\prime}}^{b}\right)_{u_{1}, u_{2}, u_{3}}^{v_{1}, v_{2}, v_{3}}, e^{\tau \theta_{x}} h_{x, t} e^{-\tau \theta_{x}}\right] d t \ldots d v_{3}\right) f\right)\left.\right|^{2} \\
& \leq 2^{7} 6^{6} C_{2}\left(r_{0}(Z)+1\right)^{-\frac{\alpha}{2}} \sum_{\tilde{Z} \in \Lambda_{Z}}\left(r_{0}(\tilde{Z})+1\right)^{-7} \\
& \left(\sharp\left(\pi_{x}^{\natural, p-1, k, m+1,\left(\tilde{l}_{0}, \ldots, \tilde{l}_{m+1}\right), 7,0}\right)^{-1}(\widetilde{Z})\right)^{-\alpha}\left|\xi_{\widetilde{Z}}(f)\right|^{2} .
\end{aligned}
$$


En combinant les inégalités (208), (209) et (210) et par Cauchy-Schwarz on obtient que

$$
\begin{gathered}
\left(\sharp\left(\pi_{x}^{p, k, m,\left(l_{0}, \ldots, l_{m}\right)}\right)^{-1}(Z)\right)^{-\alpha} \sup _{\tau \in[0, T]}\left|\xi_{Z}\left(\left(\left[\left(\theta_{x}^{b}-\theta_{x^{\prime}}^{b}\right), e^{\tau \theta_{x}} h_{x} e^{-\tau \theta_{x}}\right]\right) f\right)\right|^{2} \\
\leq 2^{8} 6^{6}\left(C_{1} C_{2} C_{3} C_{4}^{\alpha}\left(r_{0}(Z)+1\right)^{-\frac{1}{2}}+C_{2}\left(r_{0}(Z)+1\right)^{-\frac{\alpha}{2}}\right) \sum_{\tilde{Z} \in \Lambda_{Z}}\left(r_{0}(\widetilde{Z})+1\right)^{-7} \\
\left(\sharp\left(\pi_{x}^{\natural, p-1, k, m+1,\left(\tilde{l}_{0}, \ldots, \tilde{l}_{m+1}\right), 7,0}\right)^{-1}(\tilde{Z})\right)^{-\alpha}\left|\xi_{\tilde{Z}}(f)\right|^{2} .
\end{gathered}
$$

De plus pour $\tilde{Z} \in \Lambda_{Z}$ on a $\prod_{i=0}^{m} s_{i}(Z)^{-l_{i}}=\prod_{i=0}^{m+1} s_{i}(\tilde{Z})^{-\tilde{l}_{i}}$. Pour calculer la norme de $\left(1-\mathcal{P}_{n}\right)\left(\left[\left(\theta_{x}^{\mathrm{b}}-\theta_{x^{\prime}}^{\mathrm{b}}\right), e^{\tau \theta_{x}} h_{x} e^{-\tau \theta_{x}}\right]\right)$ on peut se limiter aux $Z$ tels que $r_{0}(Z) \geq n$ et on déduit donc de 211 que

$$
\begin{gathered}
\sup _{\tau \in[0, T]}\left\|\left(1-\mathcal{P}_{n}\right)\left(\left[\left(\theta_{x}^{\mathrm{b}}-\theta_{x^{\prime}}^{\mathrm{b}}\right), e^{\tau \theta_{x}} h_{x} e^{-\tau \theta_{x}}\right]\right)\right\|_{\mathscr{L}\left(\mathscr{H}_{x, s}^{\mathrm{\natural}, 7,0}\left(\Delta_{p-1}\right), \mathscr{H}_{x, S}\left(\Delta_{p}\right)\right)}^{2} \\
\leq 2^{8} 6^{6} p !\left(C_{1} C_{2} C_{3} C_{4}^{\alpha}(n+1)^{-\frac{1}{2}}+C_{2}(n+1)^{-\frac{\alpha}{2}}\right)
\end{gathered}
$$

où le facteur $p$ ! est dû au fait que $\widetilde{Z}$ détermine $Z$ à permutation près de $a_{1}, \ldots, a_{p}$. Ceci termine la preuve de $\mathrm{d}$ ).

Démonstration $d u$ e) du lemme 4.70. La preuve de e) est plus subtile que celle de d) pour la raison suivante. L'entier $r \in \mathbb{N}$ est fixé mais peut être beaucoup plus grand que $M$. On ne peut donc pas espérer que, dans les notations de la preuve de d), $\left(\rho_{x}^{b}\right) v_{u_{1}, u_{2}, u_{3}}^{v_{1}, v_{2}, v_{3}}\left(e_{\widetilde{S}_{0}}\right)$ et $\left(\rho_{x}^{b}\right) v_{u_{1}, u_{2}, u_{3}}^{v_{1}, v_{2}, v_{3}}\left(e_{\widetilde{S}_{1}}\right)$ soit déterminés par la connaissance des points de

$$
B\left(\tilde{S}_{0}, M\right) \cup B(x, k+2 M) \cup \underset{j \in\{1, \ldots, 6\}}{\bigcup} B\left(\tilde{\mathcal{Z}}_{0}^{j}, M\right)
$$

et des distances entre ces points, pour certaines parties $\widetilde{\mathcal{Z}}_{0}^{j}$. Au contraire si des parties $\left(\widetilde{Z}_{1}^{j}\right)_{j \in\{1, \ldots, 6\}}$ sont choisies de telle sorte qu'elles déterminent $\left(\rho_{x}^{b}\right)_{u_{1}, u_{2}, u_{3}}^{v_{1}, v_{2}, v_{3}}\left(e_{\tilde{S}_{1}}\right)$, on peut affirmer (en utilisant de nouveau $\left.\left(H_{M}\right)\right)$ qu'elles déterminent $\left(\rho_{x}^{b}\right)_{\hat{u}_{1}, \hat{u}_{2}, \hat{u}_{3}}\left(e_{\widetilde{S}_{0}}\right)$ pour certains $\hat{u}_{1}, \hat{u}_{2}, \hat{u}_{3}, \hat{v}_{1}, \hat{v}_{2}, \hat{v}_{3}$ que nous allons calculer. D'abord $\widetilde{S}_{1}$ est situé en gros (c'est-à-dire modulo des constantes de la forme $C(\delta, K, N, Q, P))$ sur une géodésique entre $x$ et $\widetilde{S}_{0}$, à distance $r$ de $\widetilde{S}_{0}$. Pour que les parties $\left(\widetilde{\mathcal{Z}}_{1}^{j}\right)_{j \in\{1, \ldots, 6\}}$ déterminent $\left(\rho_{x}^{b}\right)_{u_{1}, u_{2}, u_{3}}^{v_{1}, v_{2}, v_{3}}\left(e_{\widetilde{S}_{1}}\right)$, elles doivent être situées en gros sur une géodésique entre $x$ et $\widetilde{S}_{1}$, à des distances de $x$ égales à

$$
\begin{array}{lll}
\frac{u_{1}}{6} d\left(x, \widetilde{S}_{1}\right), & \frac{1+u_{2}}{6} d\left(x, \widetilde{S}_{1}\right), & \frac{2+u_{3}}{6} d\left(x, \widetilde{S}_{1}\right), \\
\frac{6-v_{1}}{6} d\left(x, \widetilde{S}_{1}\right), & \frac{5-v_{2}}{6} d\left(x, \widetilde{S}_{1}\right), & \frac{4-v_{3}}{6} d\left(x, \widetilde{S}_{1}\right) .
\end{array}
$$


Mais pour déterminer $\left(\rho_{x}^{b}\right)_{\hat{u}_{1}, \hat{u}_{2}, \hat{u}_{3}}^{\hat{v}_{1}, \hat{v}_{2}, \hat{v}_{3}}\left(e_{\widetilde{S}_{0}}\right)$ les parties $\left(\widetilde{Z}_{1}^{j}\right)_{j \in\{1, \ldots, 6\}}$ doivent également être situées en gros sur une géodésique entre $x$ et $\widetilde{S}_{0}$, à des distances de $x$ égales à

$$
\begin{array}{lll}
\frac{\hat{u}_{1}}{6} d\left(x, \widetilde{S}_{0}\right), & \frac{1+\hat{u}_{2}}{6} d\left(x, \widetilde{S}_{0}\right), & \frac{2+\hat{u}_{3}}{6} d\left(x, \widetilde{S}_{0}\right), \\
\frac{6-\hat{v}_{1}}{6} d\left(x, \widetilde{S}_{0}\right), & \frac{5-\hat{v}_{2}}{6} d\left(x, \widetilde{S}_{0}\right), & \frac{4-\hat{v}_{3}}{6} d\left(x, \widetilde{S}_{0}\right) .
\end{array}
$$

On pose $\kappa=\frac{r}{1+d\left(x, \widetilde{S}_{1}\right)}$ de sorte que $d\left(x, \widetilde{S}_{0}\right)$ est en gros égal à $(1+\kappa) d\left(x, \widetilde{S}_{1}\right)$. On obtient donc les relations

$$
\begin{array}{lll}
\hat{u}_{1}=\frac{u_{1}}{1+\kappa}, & \hat{u}_{2}=\frac{u_{2}-\kappa}{1+\kappa}, & \hat{u}_{3}=\frac{u_{3}-2 \kappa}{1+\kappa}, \\
\hat{v}_{1}=\frac{v_{1}+6 \kappa}{1+\kappa}, & \hat{v}_{2}=\frac{v_{2}+5 \kappa}{1+\kappa}, & \hat{v}_{3}=\frac{v_{3}+4 \kappa}{1+\kappa} .
\end{array}
$$

Pour adapter la preuve de d) on utilisera la variante suivante du lemme 3.45 (que l'on appliquera avec $\rho$ en gros égal à $r, \kappa$ comme ci-dessus, $y \in \widetilde{S}_{1}$ et $y^{\prime} \in \widetilde{S}_{0}$ ).

Sous-lemme 4.87. Pour tout $\rho \in \mathbb{N}$, il existe $C=C(\delta, K, \rho)$ tel que pour $x, x^{\prime}, y, y^{\prime} \in$ $X$ verifiant $d\left(x, x^{\prime}\right) \leq \rho$ et $d\left(y, y^{\prime}\right) \leq \rho$ et pour $\kappa \in\left[0, \frac{1}{10}\right]$ vérifiant $\kappa d(x, y) \leq \rho$, la mesure de l'ensemble des $\left(u_{1}, u_{2}, u_{3}, v_{1}, v_{2}, v_{3}\right) \in\left[5 \kappa, 1-5 \kappa\left[{ }^{6}\right.\right.$ tels que, en définissant $\hat{u}_{1}, \hat{u}_{2}, \hat{u}_{3}, \hat{v}_{1}, \hat{v}_{2}, \hat{v}_{3}$ comme dans (212),

$$
\begin{aligned}
& d^{b^{2}}{ }_{u_{1}, u_{2}, u_{3}}^{v_{2}, u_{3}}(x, y)-d^{\mathrm{b}}{ }_{u_{1}, u_{2}, u_{3}}^{v_{1}, v_{2}, v_{3}}\left(x^{\prime}, y\right)-d^{\mathrm{b}}{ }_{\hat{u}_{1}, \hat{u}_{2}, \hat{u}_{3}}^{\hat{v}_{1}, \hat{v}_{2}, \hat{v}_{3}}\left(x, y^{\prime}\right)+d^{\mathrm{b}}{ }_{\hat{u}_{1}, \hat{u}_{2}, \hat{u}_{3}}^{\hat{v}_{1}, \hat{v}_{2}, \hat{v}_{3}}\left(x^{\prime}, y^{\prime}\right) \neq 0 \\
& \text { est } \leq \frac{C}{1+d(x, y)} .
\end{aligned}
$$

Démonstration. La démonstration est une adaptation de celle du lemme 3.45. En particulier on applique le sous-lemme 3.48 aux familles

$$
\tilde{A}_{a_{1}, a_{2}, a_{3}}^{b_{1}, b_{2}, b_{3}}=A_{\tilde{x}, u_{1}, u_{2}, u_{3}}^{\tilde{y}, v_{1}, v_{2}, v_{3}} \text { si } \tilde{y}=y \quad \text { et } \quad \tilde{A}_{a_{1}, a_{2}, a_{3}}^{b_{1}, b_{2}, b_{3}}=A_{\tilde{x}, \hat{u}_{1}, \hat{u}_{2}, \hat{u}_{3}}^{\tilde{y}, \hat{u}_{1}, \hat{v}_{2}, \hat{v}_{3}} \text { si } \tilde{y}=y^{\prime},
$$

pour $a_{1}, a_{2}, a_{3}, b_{1}, b_{2}, b_{3} \in[0,1[$, avec

$$
u_{i}=5 \kappa+(1-10 \kappa) a_{i} \quad \text { et } \quad v_{i}=5 \kappa+(1-10 \kappa) b_{i} \quad \text { pour } i=1,2,3
$$

et $\hat{u}_{1}, \hat{u}_{2}, \hat{u}_{3}, \hat{v}_{1}, \hat{v}_{2}, \hat{v}_{3}$ comme dans (212).

Suite de la démonstration $d u \mathrm{e})$. Soient $k, m, l_{0}, \ldots, l_{m} \in \mathbb{N}$ et

$$
Z \in \bar{Y}_{x}^{p, k, m,\left(l_{0}, \ldots, l_{m}\right)} \text { vérifiant } r_{0}(Z)>k+P .
$$

On pose $\tilde{l}_{0}=0$ et $\tilde{l}_{i}=l_{i-1}$ pour $i \in\{1, \ldots, m+1\}$. On note $\Lambda_{Z}$ la partie de $\bar{Y}_{x}^{\natural, p-1, k, m+1,\left(\tilde{l}_{0}, \ldots, \tilde{l}_{m+1}\right), Q, 7}$ formée des $\widetilde{Z}$ vérifiant

$$
\left|r_{0}(\tilde{Z})-r_{1}(\tilde{Z})-r\right| \leq Q F,
$$


et tels que pour tout

$$
\begin{gathered}
\left(\tilde{a}_{1}, \ldots, \tilde{a}_{p-1}, \tilde{S}_{0}, \ldots, \tilde{S}_{m+1},\left(\tilde{\mathcal{Y}}_{i}^{j}\right)_{\substack{i \in\{0, \ldots, m+1\} \\
j \in\left\{1, \ldots, \tilde{l}_{i}\right\}}},\left(\tilde{\mathcal{Z}}_{0}^{j}\right)_{j \in\{1, \ldots, Q\}},\left(\tilde{\mathcal{Z}}_{1}^{j}\right)_{j \in\{1, \ldots, 7\}}\right) \\
\in\left(\pi_{x}^{\natural, p-1, k, m+1,\left(\tilde{l}_{0}, \ldots, \tilde{l}_{m+1}\right), Q, 7}\right)^{-1}(\widetilde{Z})
\end{gathered}
$$

il existe une énumération $\left(a_{1}, \ldots, a_{p}\right)$ de $\widetilde{S}_{1}$ vérifiant

$$
\left(a_{1}, \ldots, a_{p}, \widetilde{S}_{1}, \ldots, \widetilde{S}_{m+1},\left(\tilde{\mathcal{Y}}_{i+1}^{j}\right)_{i \in\{0, \ldots, m\}, j \in\left\{1, \ldots, l_{i}\right\}}\right) \in\left(\pi_{x}^{p, k, m,\left(l_{0}, \ldots, l_{m}\right)}\right)^{-1}(Z) .
$$

On pose $\kappa=\frac{r}{1+r_{0}(Z)}$. Soient $t, t_{1}, \ldots, t_{Q} \in[0,1]$ et $u_{1}, u_{2}, u_{3}, v_{1}, v_{2}, v_{3} \in$ $\left[5 \kappa, 1-5 \kappa\left[\text {. On note }\left(\Lambda_{Z, t,\left(t_{1}, \ldots, t_{Q}\right)}\right)\right)_{u_{1}, u_{2}, u_{3}}^{v_{1}, v_{2}, v_{3}}\right.$ l'ensemble des $\widetilde{Z} \in \Lambda_{Z}$ tels que, en notant

$$
\begin{array}{lll}
w_{1}=\mathrm{E}\left(\frac{u_{1}}{6} r_{1}(\tilde{Z})\right), & w_{2}=\mathrm{E}\left(\left(\frac{1}{6}+\frac{u_{2}}{6}\right) r_{1}(\tilde{Z})\right), & w_{3}=\mathrm{E}\left(\left(\frac{2}{6}+\frac{u_{3}}{6}\right) r_{1}(\tilde{Z})\right), \\
w_{4}=\mathrm{E}\left(\left(1-\frac{v_{1}}{6}\right) r_{1}(\tilde{Z})\right), & w_{5}=\mathrm{E}\left(\left(\frac{5}{6}-\frac{v_{2}}{6}\right) r_{1}(\tilde{Z})\right), & w_{6}=\mathrm{E}\left(\left(\frac{4}{6}-\frac{v_{3}}{6}\right) r_{1}(\tilde{Z})\right)
\end{array}
$$

on ait, pour tout

$$
\begin{gathered}
\left(\tilde{a}_{1}, \ldots, \tilde{a}_{p-1}, \widetilde{S}_{0}, \ldots, \widetilde{S}_{m+1},\left(\tilde{\mathcal{Y}}_{i}^{j}\right)_{\substack{i \in\{0, \ldots, m+1\} \\
j \in\left\{1, \ldots, \tilde{l}_{i}\right\}}},\left(\tilde{\mathcal{Z}}_{0}^{j}\right)_{j \in\{1, \ldots, Q\}},\left(\tilde{\mathcal{Z}}_{1}^{j}\right)_{j \in\{1, \ldots, 7\}}\right) \\
\in\left(\pi_{x}^{\natural, p-1, k, m+1,\left(\tilde{l}_{0}, \ldots, \tilde{l}_{m+1}\right), Q, 7}\right)^{-1}(\widetilde{Z})
\end{gathered}
$$

les égalités

$$
\begin{aligned}
& \widetilde{Z}_{0}^{j}=\bigcup_{b \in \widetilde{S}_{0}}\left\{z \in \operatorname{géod}(x, b) \mid d(x, z)=\mathrm{E}\left(t_{j} r_{0}(\widetilde{Z})\right)\right\} \text { pour } j \in\{1, \ldots, Q\}, \\
& \widetilde{Z}_{1}^{j}=\bigcup_{b \in \widetilde{S}_{1}}\left\{z \in \operatorname{géod}(x, b) \mid d(x, z)=w_{j}\right\} \text { pour } j \in\{1, \ldots, 6\},
\end{aligned}
$$

et

$$
\widetilde{Z}_{1}^{7}=\bigcup_{b \in \widetilde{S}_{1}}\left\{z \in \operatorname{géod}(x, b) \mid d(x, z)=\mathrm{E}\left((1-t) r_{1}(\tilde{Z})\right)\right\} .
$$

Pour $\left.\widetilde{Z} \in\left(\Lambda_{Z, t,\left(t_{1}, \ldots, t_{Q}\right.}\right)\right)_{u_{1}, u_{2}, u_{3}}^{v_{1}, v_{2}, v_{3}}$, les conditions (215), (216) et (217) impliquent

$$
\begin{aligned}
& t_{0}^{j}(\widetilde{Z})=\mathrm{E}\left(t_{j} r_{0}(\widetilde{Z})\right) \quad \text { pour } j \in\{1, \ldots, Q\}, \\
& t_{1}^{j}(\widetilde{Z})=w_{j} \quad \text { pour } j \in\{1, \ldots, 6\}
\end{aligned}
$$

et

$$
t_{1}^{7}(\tilde{Z})=\mathrm{E}\left((1-t) r_{1}(\tilde{Z})\right)
$$

Sous-lemme 4.88. Soit

$$
\left(\tilde{a}_{1}, \ldots, \tilde{a}_{p-1}, \tilde{S}_{0}, \ldots, \tilde{S}_{m+1},\left(\tilde{y}_{i}^{j}\right)_{i \in\{0, \ldots, m+1\}, j \in\left\{1, \ldots, \tilde{l}_{i}\right\}}\right) \in Y_{x}^{p-1, k, m+1,\left(\tilde{l}_{0}, \ldots, \tilde{l}_{m+1}\right)}
$$


tel que $\left|d\left(x, \widetilde{S}_{0}\right)-d\left(x, \widetilde{S}_{1}\right)-r\right| \leq Q F$ et qu'il existe $\left(a_{1}, \ldots, a_{p}\right)$ vérifiant (214). On définit $\left(\widetilde{\mathcal{Z}}_{0}^{j}\right)_{j \in\{1, \ldots, Q\}}$ et $\left(\widetilde{\mathcal{Z}}_{1}^{j}\right)_{j \in\{1, \ldots, 7\}}$ par (215), (216) et (217). Alors les parties $\widetilde{\mathcal{Z}}_{0}^{j}$ et $\widetilde{Z}_{1}^{j}$ sont de diamètre inférieur ou égal à $P / 3$ et il existe $\widetilde{Z} \in\left(\Lambda_{Z, t,\left(t_{1}, \ldots, t_{Q}\right)}\right)_{u_{1}, u_{2}, u_{3}}^{v_{1}, v_{2}, v_{3}}$ tel que

$$
\left(\tilde{a}_{1}, \ldots, \tilde{a}_{p-1}, \widetilde{S}_{0}, \ldots, \widetilde{S}_{m+1},\left(\tilde{\mathcal{Y}}_{i}^{j}\right)_{\substack{i \in\{0, \ldots, m+1\} \\ j \in\left\{1, \ldots, \tilde{l}_{i}\right\}}},\left(\widetilde{\mathcal{Z}}_{0}^{j}\right)_{j \in\{1, \ldots, Q\}},\left(\tilde{\mathcal{Z}}_{1}^{j}\right)_{j \in\{1, \ldots, 7\}}\right)
$$

appartienne $\grave{a}\left(\pi_{x}^{\natural, p-1, k, m+1,\left(\tilde{l}_{0}, \ldots, \tilde{l}_{m+1}\right), Q, 7}\right)^{-1}(\widetilde{Z})$.

Démonstration. Pour $\sigma \in\{0,1\}$ et $z, z^{\prime} \in \widetilde{\mathcal{Z}}_{\sigma}^{j}$, on choisit $b \in \widetilde{S}_{\sigma}$, d'où $z, z^{\prime} \in$ $2 N-\operatorname{géod}(x, b)$ et comme $d(x, z)=d\left(x, z^{\prime}\right),\left(H_{\delta}\left(z, x, z^{\prime}, b\right)\right)$ donne $d\left(z, z^{\prime}\right) \leq$ $2 N+\delta \leq P / 3$. Comme les parties $\widetilde{\mathcal{Z}}_{0}^{j}$ et $\widetilde{\mathcal{Z}}_{1}^{j}$ sont non vides et $P / 3 \leq M$, l'argument que nous venons de donner montre aussi que les conditions (215), (216) et (217) sont vérifiées par les autres éléments de la classe d'équivalence $\widetilde{Z}$ de l'élément (218) et donc $\left.\widetilde{Z} \in\left(\Lambda_{Z, t,\left(t_{1}, \ldots, t_{Q}\right)}\right)\right)_{u_{1}, u_{2}, u_{3}}^{v_{1}, v_{2}, v_{3}}$.

Suite de la démonstration $d u$ e). On déduira e) de l'inégalité suivante : il existe $C_{2}=$ $C(\delta, K, N, Q, P, M, r, T)$ tel que en notant $\hat{u}_{1}, \hat{u}_{2}, \hat{u}_{3}, \hat{v}_{1}, \hat{v}_{2}, \hat{v}_{3}$ comme dans (212), on ait

$$
\begin{aligned}
& \sup _{\tau \in[0, T]} \mid \xi_{Z}\left(\left(\left(\left(\theta_{x}^{b}\right)_{u_{1}, u_{2}, u_{3}}^{v_{1}, v_{2}, v_{3}}-\left(\theta_{x^{\prime}}^{b}\right)_{u_{1}, u_{2}, u_{3}}^{v_{1}, v_{2}, v_{3}}\right) e^{\tau \theta_{x}} u_{x, r, t} K_{x, Q,\left(t_{1}, \ldots, t_{Q}\right)} e^{-\tau \theta_{x}}\right.\right. \\
& \left.-e^{\tau \theta_{x}} u_{x, r, t} K_{x, Q,\left(t_{1}, \ldots, t_{Q}\right)} e^{-\tau \theta_{x}}\left(\left(\theta_{x}^{b}\right)_{\hat{u}_{1}, \hat{u}_{2}, \hat{u}_{3}}^{\hat{u}_{2} \hat{v}_{3}}-\left(\theta_{x^{\prime}}^{b}\right)_{\hat{u}_{1}, \hat{u}_{2}, \hat{u}_{3}}^{\hat{u}_{1} \hat{v}_{3}}\right) f\right)\left.\right|^{2} \\
& \leq C_{2} \sum_{\tilde{Z} \in\left(\Lambda_{Z, t,\left(t_{1}, \ldots, t_{Q}\right)}^{\neq}\right)}\left|\xi_{u_{1}, u_{2}, u_{3}}^{v_{1}, v_{2}, v_{3}}(f)\right|^{2}
\end{aligned}
$$

où $\left.\left(\Lambda_{Z, t,\left(t_{1}, \ldots, t_{Q}\right)}^{\neq}\right)\right)_{u_{1}, u_{2}, u_{3}}^{v_{1}, v_{2}, v_{3}}$ est l'ensemble des $\left.\widetilde{Z} \in\left(\Lambda_{Z, t,\left(t_{1}, \ldots, t_{Q}\right)}\right)\right)_{u_{1}, u_{2}, u_{3}}^{v_{1}, v_{2}, v_{3}}$ tels que pour tout

$$
\begin{gathered}
\left(\tilde{a}_{1}, \ldots, \tilde{a}_{p-1}, \widetilde{S}_{0}, \ldots, \widetilde{S}_{m+1},\left(\widetilde{\mathcal{Y}}_{i}^{j}\right)_{i \in\{0, \ldots, m+1\}, j \in\left\{1, \ldots, \tilde{l}_{i}\right\}},\left(\widetilde{\mathcal{Z}}_{0}^{j}\right)_{j \in\{1, \ldots, Q\}},\left(\widetilde{\mathcal{Z}}_{1}^{j}\right)_{j \in\{1, \ldots, 7\}}\right) \\
\in\left(\pi_{x}^{\natural, p-1, k, m+1,\left(\tilde{l}_{0}, \ldots, \tilde{l}_{m+1}\right), Q, 7}\right)^{-1}(\widetilde{Z})
\end{gathered}
$$

on ait

$$
\left(\rho_{x}^{b}\right)_{\hat{u}_{1}, \hat{u}_{2}, \hat{u}_{3}}^{\hat{v}_{2}, \hat{S}_{3}}\left(\widetilde{S}_{0}\right)-\left(\rho_{x^{\prime}}^{b}\right)_{\hat{u}_{1}, \hat{u}_{2}, \hat{u}_{3}}^{\hat{v}_{1} \hat{v}_{2}, \hat{v}_{3}}\left(\widetilde{S}_{0}\right)-\left(\rho_{x}^{b}\right)_{u_{1}, u_{2}, u_{3}}^{v_{1}, v_{2}, v_{3}}\left(\tilde{S}_{1}\right)+\left(\rho_{x^{\prime}}^{b}\right)_{u_{1}, u_{2}, u_{3}}^{v_{1}, v_{2}, v_{3}}\left(\tilde{S}_{1}\right) \neq 0 .
$$

Nous allons maintenant montrer (219). Nous allons justifier aussi que la condition (220) ne dépend que de $\widetilde{Z}$ (c'est-à-dire que pour $\widetilde{Z} \in\left(\Lambda_{Z, t,\left(t_{1}, \ldots, t_{Q}\right)}\right)_{u_{1}, u_{2}, u_{3}}^{v_{1}, v_{2}, v_{3}}$ elle est vérifiée ou non simultanément pour tous les éléments de $\left.\left(\pi_{x}^{\natural, p-1, k, m+1,\left(\tilde{l}_{0}, \ldots, \tilde{l}_{m+1}\right), Q, 7}\right)^{-1}(\widetilde{Z})\right)$. 
Sous-lemme 4.89. Pour $\widetilde{Z} \in\left(\Lambda_{Z, t,\left(t_{1}, \ldots, t_{Q}\right)}\right)_{u_{1}, u_{2}, u_{3}}^{v_{1}, v_{2}, v_{3}}$ et

$$
\begin{gathered}
\left(\tilde{a}_{1}, \ldots, \tilde{a}_{p-1}, \widetilde{S}_{0}, \ldots, \widetilde{S}_{m+1},\left(\widetilde{\mathcal{Y}}_{i}^{j}\right)_{\substack{i \in\{0, \ldots, m+1\} \\
j \in\left\{1, \ldots, \tilde{l}_{i}\right\}}},\left(\widetilde{\mathcal{Z}}_{0}^{j}\right)_{j \in\{1, \ldots, Q\}},\left(\widetilde{\mathcal{Z}}_{1}^{j}\right)_{j \in\{1, \ldots, 7\}}\right) \\
\left.\in\left(\widetilde{\mathcal{Z}}_{1}^{j}\right)_{j \in\{1, \ldots, 7\}}\right)\left(\pi_{x}^{\natural, p-1, k, m+1,\left(\tilde{l}_{0}, \ldots, \tilde{l}_{m+1}\right), Q, 7}\right)^{-1}(\widetilde{Z})
\end{gathered}
$$

le coefficient de $e_{\widetilde{S}_{1}}$ dans

$$
\begin{aligned}
& \left(\left(\left(\theta_{x}^{b}\right)_{u_{1}, u_{2}, u_{3}}^{v_{1}, v_{2}, v_{3}}-\left(\theta_{x^{\prime}}^{b}\right)_{u_{1}, u_{2}, u_{3}}^{v_{1}, v_{2}, v_{3}}\right) e^{\tau \theta_{x}} u_{x, r, t} K_{x, Q,\left(t_{1}, \ldots, t_{Q}\right)} e^{-\tau \theta_{x}}\right. \\
& \left.\quad-e^{\tau \theta_{x}} u_{x, r, t} K_{x, Q,\left(t_{1}, \ldots, t_{Q}\right.} e^{-\tau \theta_{x}}\left(\left(\theta_{x}^{b}\right)_{\hat{u}_{1}, \hat{u}_{2}, \hat{u}_{3}}^{\hat{v}_{1}, \hat{u}_{3}}-\left(\theta_{x^{\prime}}^{b}\right)_{\hat{u}_{1}, \hat{u}_{2}, \hat{u}_{3}}^{\hat{v}_{1}, \hat{v}_{3}}\right)\right)\left(e_{\tilde{S}_{0}}\right)
\end{aligned}
$$

et le membre de gauche de (220) ne dépendent que de la connaissance des points de

$$
B(x, k+2 M) \cup B\left(\tilde{S}_{0}, M\right) \cup B\left(\tilde{S}_{1}, M\right) \cup \underset{j \in\{1, \ldots, Q\}}{\bigcup} B\left(\widetilde{\mathcal{Z}}_{0}^{j}, M\right) \cup \underset{j \in\{1, \ldots, 7\}}{\bigcup} B\left(\widetilde{\mathcal{Z}}_{1}^{j}, M\right)
$$

et des distances entre ces points.

Démonstration. Le coefficient de $e_{\widetilde{S}_{1}}$ dans $e^{\tau \theta_{x}} u_{x, r, t} K_{x, Q,\left(t_{1}, \ldots, t_{Q}\right)} e^{-\tau \theta_{x}}\left(e_{\widetilde{S}_{0}}\right)$ ne dépend que de la connaissance des points de (221) et de leurs distances mutuelles. Cela résulte du sous-lemme 4.43 ou du sous-lemme 4.81 (que l'on applique avec $\widetilde{\mathcal{Z}}_{0}^{7}$ au lieu de $\widetilde{\mathcal{Z}}_{0}^{1}$ et en oubliant $\widetilde{\mathcal{Z}}_{0}^{j}$ pour $\left.j \in\{1, \ldots, 6\}\right)$.

Pour montrer le sous-lemme il suffit donc de montrer que pour $\tilde{x} \in B(x, 1)$, $\left(\rho_{\tilde{x}}^{b}\right)_{u_{1}, u_{2}, u_{3}}^{v_{1}, v_{2}, v_{3}}\left(\widetilde{S}_{1}\right)$ et $\left(\rho_{\tilde{x}}^{b}\right)_{\hat{u}_{1}, \hat{u}_{2}, \hat{u}_{3}}^{\hat{v}_{1}, \hat{S}_{2}, \hat{S}_{3}}\left(\widetilde{S}_{0}\right)$ ne dépendent que de la connaissance des points de (221) et des distances entre ces points (en effet on applique ceci à $\tilde{x}=x$ et $\tilde{x}=x^{\prime}$ ). En vertu du lemme 4.50 il suffit de montrer que pour $\tilde{x} \in B(x, 1), \sigma \in\{0,1\}, a \in \widetilde{S}_{\sigma}$ et $j \in\{1, \ldots, 6\}$, l'ensemble

$$
\left\{y \in 3 \delta-\operatorname{géod}(\tilde{x}, a)|| d(\tilde{x}, y)-w_{j}(\sigma, \tilde{x}) \mid \leq N+6 \delta+4\right\}
$$

est inclus dans (221), où l'on note

$$
\begin{gathered}
\lambda_{1}=\frac{u_{1}}{6}, \lambda_{2}=\frac{1+u_{2}}{6}, \lambda_{3}=\frac{2+u_{3}}{6}, \lambda_{4}=\frac{6-v_{1}}{6}, \lambda_{5}=\frac{5-v_{2}}{6}, \lambda_{6}=\frac{4-v_{3}}{6}, \\
\hat{\lambda}_{1}=\frac{\hat{u}_{1}}{6}, \hat{\lambda}_{2}=\frac{1+\hat{u}_{2}}{6}, \hat{\lambda}_{3}=\frac{2+\hat{u}_{3}}{6}, \hat{\lambda}_{4}=\frac{6-\hat{v}_{1}}{6}, \hat{\lambda}_{5}=\frac{5-\hat{v}_{2}}{6}, \hat{\lambda}_{6}=\frac{4-\hat{v}_{3}}{6}, \\
w_{j}(0, \tilde{x})=\mathrm{E}\left(\hat{\lambda}_{j} d\left(\tilde{x}, \tilde{S}_{0}\right)\right) \text { et } w_{j}(1, \tilde{x})=\mathrm{E}\left(\lambda_{j} d\left(\tilde{x}, \tilde{S}_{1}\right)\right) .
\end{gathered}
$$

On rappelle que $w_{j}=\mathrm{E}\left(\lambda_{j} d\left(x, \widetilde{S}_{1}\right)\right)$.

On commence par le cas où $\sigma=1$. Soit $\tilde{x} \in B(x, 1), a \in \widetilde{S}_{1}, j \in\{1, \ldots, 6\}$ et $y$ dans l'ensemble (222). Soit $z \in \operatorname{géod}(x, a)$ vérifiant $d(x, z)=w_{j}$, si bien que $z$ appartient à $\tilde{Z}_{1}^{j}$. Comme $\left|d\left(x, \widetilde{S}_{1}\right)-d\left(\tilde{x}, \widetilde{S}_{1}\right)\right| \leq 1$ on a $\left|w_{j}(1, \tilde{x})-w_{j}\right| \leq 1$ et donc $|d(x, y)-d(x, z)| \leq N+6 \delta+6$. Comme $y$ et $z$ appartiennent à $(3 \delta+2)-\operatorname{géod}(x, a)$, 
$\left(H_{\delta}(y, x, z, a)\right)$ implique $d(y, z) \leq(N+6 \delta+6)+(3 \delta+2)+\delta$. On suppose $(N+6 \delta+6)+(3 \delta+2)+\delta \leq M$, ce qui est permis par $\left(H_{M}\right)$. On a donc $d(y, z) \leq M$ et $y$ appartient à (221).

On considère maintenant le cas où $\sigma=0$. Soit $\tilde{x} \in B(x, 1), a \in \widetilde{S}_{0}, j \in$ $\{1, \ldots, 6\}$ et $y$ dans l'ensemble (222). On commence par montrer

$$
\left|w_{j}(0, \tilde{x})-w_{j}\right| \leq Q F+2 .
$$

On a $\hat{\lambda}_{j}=\frac{\lambda_{j}}{1+\kappa}$ et $\frac{r_{1}(\tilde{Z})+1+r}{1+\kappa}=r_{1}(\tilde{Z})+1$, d'où

$$
\hat{\lambda}_{j}\left(r_{1}(\tilde{Z})+1+r\right)=\lambda_{j}\left(r_{1}(\tilde{Z})+1\right) .
$$

D'après (213) on a $\left|\left(r_{0}(\tilde{Z})+1\right)-\left(r_{1}(\tilde{Z})+1+r\right)\right| \leq Q F$. Donc

$$
\left|\hat{\lambda}_{j}\left(r_{0}(\tilde{Z})+1\right)-\lambda_{j}\left(r_{1}(\tilde{Z})+1\right)\right| \leq Q F,
$$

et on en déduit immédiatement

$$
\left|\hat{\lambda}_{j} r_{0}(\tilde{Z})-\lambda_{j} r_{1}(\tilde{Z})\right| \leq Q F+1 .
$$

Comme $r_{0}(\widetilde{Z})=d\left(x, \widetilde{S}_{0}\right)$ et $r_{1}(\tilde{Z})=d\left(x, \widetilde{S}_{1}\right),(223)$ en résulte aisément.

Soit $b \in \widetilde{S}_{1}$ et $z \in \operatorname{géod}(x, b)$ vérifiant $d(x, z)=w_{j}$ si bien que $z$ appartient à $\widetilde{Z}_{1}^{j}$. Il résulte facilement de (223) que

$$
|d(x, y)-d(x, z)| \leq Q F+N+6 \delta+7 .
$$

Comme $b \in 2 F$ - géod $(x, a)$ on a $z \in 2 F$ - $\operatorname{géod}(x, a)$. On a $y \in(3 \delta+2)$ - géod $(x, a)$ et comme $2 F \geq 3 \delta+2, y$ et $z$ appartiennent à $2 F$ - géod $(x, a)$. Grâce à (224), $\left(H_{\delta}(y, x, z, a)\right)$ implique $d(y, z) \leq(Q F+N+6 \delta+7)+2 F+\delta$. On suppose $(Q F+N+6 \delta+7)+2 F+\delta \leq M$, ce qui est permis par $\left(H_{M}\right)$. On a donc $d(y, z) \leq$ $M$ et $y$ appartient à (221). Ceci termine la démonstration du sous-lemme 4.89.

Une conséquence immédiate du sous-lemme 4.89 est que la condition (220) ne dépend que de $\widetilde{Z}$.

Sous-lemme 4.90. Le cardinal de $\left.\left(\Lambda_{Z, t,\left(t_{1}, \ldots, t_{Q}\right)}\right)\right)_{u_{1}, u_{2}, u_{3}}^{v_{1}, v_{2}, v_{3}}$ est majoré par une constante de la forme $C(\delta, K, N, Q, P, M)$.

Démonstration. En effet, grâce au lemme 4.28, pour connaître les distances entre les points de

$$
B\left(\widetilde{S}_{0}, M\right) \cup \underset{j \in\{1, \ldots, Q\}}{\bigcup} B\left(\widetilde{\mathcal{Z}}_{0}^{j}, M\right) \cup \bigcup_{j \in\{1, \ldots, 7\}} B\left(\widetilde{\mathcal{Z}}_{1}^{j}, M\right)
$$

et ceux de

$$
\bigcup_{i \in\{1, \ldots, m+1\}} B\left(\widetilde{S}_{i}, M\right) \cup \underset{\substack{i \in \in\{, \ldots, m\} \\ j \in\left\{1, \ldots, l_{i}\right\}}}{\bigcup} B\left(\widetilde{y}_{i+1}^{j}, M\right) \cup B(x, k+2 M)
$$


il suffit de connaître les distances entre les points de (225) et $C$ points de (226), avec $C=C(\delta, K, N, Q, P, M)$ et grâce à (213) ces distances sont déterminées à $C^{\prime}=C(\delta, K, N, Q, P, M)$ près par les distances de $\widetilde{S}_{1}$ à ces $C$ points (qui font

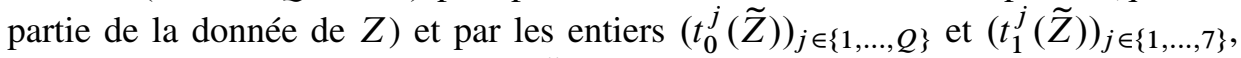
qui sont eux-mêmes déterminés à $C^{\prime \prime}=C(\delta, K, N, Q, P, M)$ près par $r_{0}(Z), r, t$, $t_{1}, \ldots, t_{Q}, u_{1}, u_{2}, u_{3}, v_{1}, v_{2}, v_{3}$.

Suite de la démonstration $d u$ e). On termine maintenant la preuve de (219). Pour

$$
\begin{gathered}
\left(\tilde{a}_{1}, \ldots, \tilde{a}_{p-1}, \tilde{S}_{0}, \ldots, \tilde{S}_{m+1},\left(\tilde{y}_{i}^{j}\right)_{i \in\{0, \ldots, m+1\}, j \in\left\{1, \ldots, \tilde{l}_{i}\right\}},\left(\tilde{Z}_{0}^{j}\right)_{j \in\{1, \ldots, Q\}},\right. \\
\left.\left(\tilde{\mathcal{Z}}_{1}^{j}\right)_{j \in\{1, \ldots, 7\}}\right) \in\left(\pi_{x}^{\natural, p-1, k, m+1,\left(\tilde{l}_{0}, \ldots, \tilde{l}_{m+1}\right), Q, 7}\right)^{-1}(\tilde{Z})
\end{gathered}
$$

on considère

$$
\begin{aligned}
\sum_{\left(b_{1}, \ldots, b_{p}\right)} & \left(\left(\left(\left(\theta_{x}^{b}\right)_{u_{1}, u_{2}, u_{3}}^{v_{1}, v_{2}, v_{3}}-\left(\theta_{x^{\prime}}^{b}\right)_{u_{1}, u_{2}, u_{3}}^{v_{1}, v_{2}, v_{3}}\right) e^{\tau \theta_{x}} u_{x, r, t} K_{x, Q,\left(t_{1}, \ldots, t_{Q}\right)} e^{-\tau \theta_{x}}\right.\right. \\
& \left.-e^{\tau \theta_{x}} u_{x, r, t} K_{x, Q,\left(t_{1}, \ldots, t_{Q}\right)} e^{-\tau \theta_{x}}\left(\left(\theta_{x}^{b}\right)_{\hat{u}_{1}, \hat{u}_{2}, \hat{u}_{3}}^{\hat{v}_{1}, \hat{v}_{3}, \hat{u}_{3}}-\left(\theta_{x^{\prime}}^{b}\right)_{\hat{u}_{1}, \hat{u}_{2}, \hat{u}_{3}}^{\hat{v}_{1}, \hat{v}_{2}, \hat{v}_{3}}\right)\right) \\
& \left.\left(e_{\tilde{a}_{1}} \wedge \cdots \wedge e_{\tilde{a}_{p-1}}\right)\right)\left(b_{1}, \ldots, b_{p}\right)
\end{aligned}
$$

où la somme porte sur les énumérations $\left(b_{1}, \ldots, b_{p}\right)$ de $\widetilde{S}_{1}$ telles que

$$
\left(b_{1}, \ldots, b_{p}, \widetilde{S}_{1}, \ldots, \widetilde{S}_{m+1},\left(\tilde{y}_{i+1}^{j}\right)_{i \in\{0, \ldots, m\}, j \in\left\{1, \ldots, \tilde{l}_{i}\right\}}\right) \in\left(\pi_{x}^{p, k, m,\left(l_{0}, \ldots, l_{m}\right)}\right)^{-1}(Z) .
$$

Comme la somme 227 a au plus $p$ ! termes, le 3) de la proposition 3.37 montre qu'elle est majorée par une constante de la forme $C(\delta, K, N, Q, P, M, T)$. D'après le sous-lemme 4.89 la somme (227) ne dépend que de $\widetilde{Z}$ et on peut donc la noter $\left(\alpha_{Z, \tilde{Z}, t, \tau,\left(t_{1}, \ldots, t_{Q}\right)}\right){ }_{u_{1}, u_{2}, u_{3}}^{v_{1}, v_{2}, v_{3}}$. D'après le sous-lemme 4.88 et le sous-lemme 4.42 (ou 4.80), on a

$$
\begin{aligned}
& \xi_{Z}\left(\left(\left(\left(\theta_{x}^{b}\right)_{u_{1}, u_{2}, u_{3}}^{v_{1}, v_{2}, v_{3}}-\left(\theta_{x^{\prime}}^{b}\right)_{u_{1}, u_{2}, u_{3}}^{v_{1}, v_{2}, v_{3}}\right) e^{\tau \theta_{x}} u_{x, r, t} K_{x, Q,\left(t_{1}, \ldots, t_{Q}\right)} e^{-\tau \theta_{x}}\right.\right. \\
& \left.\left.-e^{\tau \theta_{x}} u_{x, r, t} K_{x, Q,\left(t_{1}, \ldots, t_{Q}\right)} e^{-\tau \theta_{x}}\left(\left(\theta_{x}^{b}\right)_{\hat{u}_{1}, \hat{u}_{2}, \hat{u}_{3}}^{\hat{v}_{1}, \hat{v}_{2}, \hat{v}_{3}}-\left(\theta_{x^{\prime}}^{b}\right)_{\hat{u}_{1}, \hat{u}_{2}, \hat{u}_{3}}^{\hat{v}_{1}, \hat{v}_{2}, \hat{v}_{3}}\right)\right) f\right)
\end{aligned}
$$

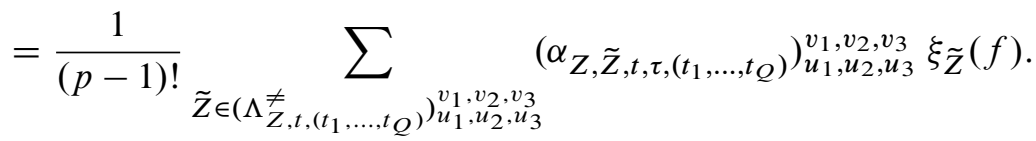

Par Cauchy-Schwarz et grâce au sous-lemme 4.90 on en déduit (219).

Montrons maintenant l'assertion e) à l' aide de (219). Le sous-lemme 4.87 appliqué à $\rho=r+C$ avec $C=C(\delta, K, N, Q)$ implique facilement qu'il existe $C_{1}=$ $C(\delta, K, N, Q, r)$ tel que pour $\widetilde{S}_{0}, \widetilde{S}_{1}$ comme ci-dessus la mesure de l'ensemble des $\left(u_{1}, u_{2}, u_{3}, v_{1}, v_{2}, v_{3}\right) \in\left[5 \kappa, 1-5 \kappa\left[{ }^{6}\right.\right.$ vérifiant (220) est $\leq \frac{C_{1}}{1+r_{0}(Z)}$. Soit $C_{2}$ comme dans (219). Soient $k, m, l_{0}, \ldots, l_{m} \in \mathbb{N}$ et posons $\tilde{l}_{0}=0$ et $\tilde{l}_{i}=l_{i-1}$ pour $i \in$ 
$\{1, \ldots, m+1\}$ comme précédemment. Soit $C_{3}=C(\delta, K, N, Q, P, M, r)$ comme dans le sous-lemme 4.83. Soit

$$
Z \in \bar{Y}_{x}^{p, k, m,\left(l_{0}, \ldots, l_{m}\right)} \text { vérifiant } r_{0}(Z)>k+P .
$$

On a alors

$$
\begin{aligned}
& \int_{\left(u_{1}, u_{2}, u_{3}, v_{1}, v_{2}, v_{3}\right) \in[5 \kappa, 1-5 \kappa[6} \\
& \left(\sum_{\tilde{Z} \in\left(\Lambda_{Z, t, u_{1}}{ }\right.} \sharp\left(\left(\pi_{x}^{\natural, p-1, k, m+1,\left(\tilde{l}_{0}, \ldots, \tilde{l}_{m+1}\right), Q, 7}\right)^{-1}(\tilde{Z})\right)\right) d u_{1} \ldots d v_{3} \\
& \leq \frac{C_{1} C_{3}}{r_{0}(Z)+1} \sharp\left(\left(\pi_{x}^{p, k, m,\left(l_{0}, \ldots, l_{m}\right)}\right)^{-1}(Z)\right),
\end{aligned}
$$

Notons $I_{Z}$ l'ensemble des

$$
\left(t, t_{1}, \ldots, t_{Q}, u_{1}, u_{2}, u_{3}, v_{1}, v_{2}, v_{3}\right) \in[0,1]^{Q+1} \times\left[5 \kappa, 1-5 \kappa\left[^{6}\right.\right.
$$

tels qu'il existe $\widetilde{Z} \in\left(\Lambda_{Z, t,\left(t_{1}, \ldots, t_{Q}\right)}^{\neq}\right)_{u_{1}, u_{2}, u_{3}}^{v_{1}, v_{2}, v_{3}}$ vérifiant

$\sharp\left(\left(\pi_{x}^{\natural, p-1, k, m+1,\left(\tilde{l}_{0}, \ldots, \tilde{l}_{m+1}\right), Q, 7}\right)^{-1}(\tilde{Z})\right) \geq\left(r_{0}(Z)+1\right)^{-\frac{1}{2}} \sharp\left(\left(\pi_{x}^{p, k, m,\left(l_{0}, \ldots, l_{m}\right)}\right)^{-1}(Z)\right)$.

La mesure de $I_{Z}$ est donc $\leq C_{1} C_{3}\left(r_{0}(Z)+1\right)^{-\frac{1}{2}}$. Grâce à Cauchy-Schwarz on déduit de (219) que

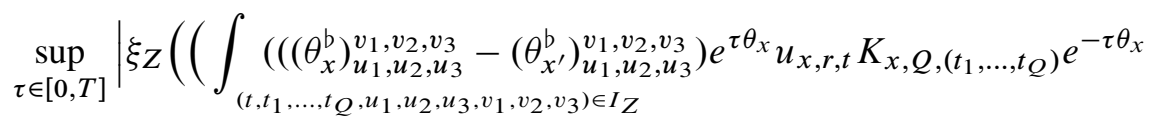

$$
\begin{aligned}
& \left.\left.\left.-e^{\tau \theta_{x}} u_{x, r, t} K_{x, Q,\left(t_{1}, \ldots, t_{Q}\right)} e^{-\tau \theta_{x}}\left(\left(\theta_{x}^{b}\right)_{\hat{u}_{1}, \hat{u}_{2}, \hat{u}_{3}}^{\hat{u}_{3}}-\left(\theta_{x^{\prime}}^{b}\right)_{\hat{u}_{1}, \hat{u}_{2}, \hat{u}_{3}}^{\hat{u}_{1}, \hat{v}_{3}}\right)\right) d t \ldots d v_{3}\right) f\right)\left.\right|^{2} \\
& \leq 2^{Q+7} 6^{6} C_{1} C_{2} C_{3}\left(r_{0}(Z)+1\right)^{-\frac{1}{2}} \sum_{\tilde{Z} \in \Lambda_{Z}}\left(r_{0}(\tilde{Z})+1\right)^{-Q}\left(r_{1}(\tilde{Z})+1\right)^{-7}\left|\xi_{\tilde{Z}}(f)\right|^{2} \text {. }
\end{aligned}
$$

D'après le sous-lemme 4.83 et le lemme 4.29, il existe $C_{4}=C(\delta, K, N, Q, P, M, r)$ tel que pour $\widetilde{Z} \in \Lambda_{Z}$ on ait

$$
\sharp\left(\left(\pi_{x}^{\natural, p-1, k, m+1,\left(\tilde{l}_{0}, \ldots, \tilde{l}_{m+1}\right), Q, 7}\right)^{-1}(\tilde{Z})\right) \leq C_{4} \sharp\left(\left(\pi_{x}^{p, k, m,\left(l_{0}, \ldots, l_{m}\right)}\right)^{-1}(Z)\right) .
$$

D'autre part pour $\left(t, t_{1}, \ldots, t_{Q}, u_{1}, u_{2}, u_{3}, v_{1}, v_{2}, v_{3}\right) \in\left([0,1]^{Q+1} \times\left[5 \kappa, 1-5 \kappa\left[{ }^{6} \backslash I_{Z}\right)\right.\right.$ et $\left.\widetilde{Z} \in\left(\Lambda_{Z, t,\left(t_{1}, \ldots, t_{Q}\right)}^{\neq}\right)\right)_{u_{1}, u_{2}, u_{3}}^{v_{1}, v_{2}, v_{3}}$ on a

$$
\sharp\left(\left(\pi_{x}^{\natural, p-1, k, m+1,\left(\tilde{l}_{0}, \ldots, \tilde{l}_{m+1}\right), Q, 7}\right)^{-1}(\tilde{Z})\right) \leq\left(r_{0}(Z)+1\right)^{-\frac{1}{2}} \sharp\left(\left(\pi_{x}^{p, k, m,\left(l_{0}, \ldots, l_{m}\right)}\right)^{-1}(Z)\right) .
$$


Par Cauchy-Schwarz on déduit alors de (219) que

$$
\begin{aligned}
& \left(\sharp\left(\pi_{x}^{p, k, m,\left(l_{0}, \ldots, l_{m}\right)}\right)^{-1}(Z)\right)^{-\alpha}
\end{aligned}
$$

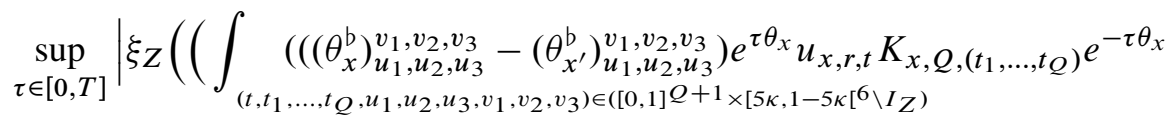

$$
\begin{aligned}
& \left.\left.-e^{\tau \theta_{x}} u_{x, r, t} K_{x, Q,\left(t_{1}, \ldots, t_{Q}\right)} e^{-\tau \theta_{x}}\left(\left(\theta_{x}^{b}\right)_{\hat{u}_{1}, \hat{u}_{2}, \hat{u}_{3}}^{\hat{v}_{1} \hat{v}_{2} \hat{v}_{3}}-\left(\theta_{x^{\prime}}^{b}\right)_{\hat{u}_{1}, \hat{u}_{2}, \hat{u}_{3}}^{\hat{v}_{1} \hat{v}_{2} \hat{v}_{3}}\right) d t \ldots d v_{3}\right) f\right)\left.\right|^{2} \\
& \leq 2^{Q+7} 6^{6} C_{2}\left(r_{0}(Z)+1\right)^{-\frac{\alpha}{2}} \sum_{\widetilde{Z} \in \Lambda_{Z}}\left(r_{0}(\widetilde{Z})+1\right)^{-Q}\left(r_{1}(\tilde{Z})+1\right)^{-7} \\
& \left(\sharp\left(\pi_{x}^{\natural, p-1, k, m+1,\left(\tilde{l}_{0}, \ldots, \tilde{l}_{m+1}\right), Q, 7}\right)^{-1}(\widetilde{Z})\right)^{-\alpha}\left|\xi_{\tilde{Z}}(f)\right|^{2} .
\end{aligned}
$$

En combinant les inégalités (228), (229) et (230) et par Cauchy-Schwarz on obtient que

$$
\begin{aligned}
& \left(\sharp\left(\pi_{x}^{p, k, m,\left(l_{0}, \ldots, l_{m}\right)}\right)^{-1}(Z)\right)^{-\alpha} \sup _{\tau \in[0, T]} \\
& \mid \xi_{Z}\left(\left(\int _ { ( u _ { 1 } , u _ { 2 } , u _ { 3 } , v _ { 1 } , v _ { 2 } , v _ { 3 } ) \in [ 5 \kappa , 1 - 5 \kappa [ 6 } \left(\left(\left(\theta_{x}^{b}\right)_{u_{1}, u_{2}, u_{3}}^{v_{1}, v_{2}, v_{3}}-\left(\theta_{x^{\prime}}^{b}\right)_{u_{1}, u_{2}, u_{3}}^{v_{1}, v_{2}, v_{3}}\right) e^{\tau \theta_{x}} u_{x, r} K_{x} e^{-\tau \theta_{x}}\right.\right.\right. \\
& \left.\left.\quad-e^{\tau \theta_{x}} u_{x, r} K_{x} e^{-\tau \theta_{x}}\left(\left(\theta_{x}^{b}\right)_{\hat{u}_{1}, \hat{v}_{2}, \hat{u}_{2}, \hat{u}_{3}}^{\hat{u}_{3}}-\left(\theta_{x^{\prime}}^{b}\right)_{\hat{u}_{1}, \hat{u}_{2}, \hat{u}_{3}}^{\hat{v}_{2}, \hat{v}_{3}}\right) d u_{1} \ldots d v_{3}\right) f\right)\left.\right|^{2} \\
& \leq 2^{Q+8} 6^{6}\left(C_{1} C_{2} C_{3} C_{4}^{\alpha}\left(r_{0}(Z)+1\right)^{-\frac{1}{2}}+C_{2}\left(r_{0}(Z)+1\right)^{-\frac{\alpha}{2}}\right) \sum_{\widetilde{Z} \in \Lambda_{Z}}\left(r_{0}(\widetilde{Z})+1\right)^{-Q} \\
& \quad\left(r_{1}(\widetilde{Z})+1\right)^{-7}\left(\sharp\left(\pi_{x}^{\natural, p-1, k, m+1,\left(\tilde{l}_{0}, \ldots, \tilde{l}_{m+1}\right), Q, 7}\right)^{-1}(\widetilde{Z})\right)^{-\alpha}\left|\xi_{\widetilde{Z}}(f)\right|^{2} .
\end{aligned}
$$

De plus pour $\tilde{Z} \in \Lambda_{Z}$ on a $\prod_{i=0}^{m} s_{i}(Z)^{-l_{i}}=\prod_{i=0}^{m+1} s_{i}(\tilde{Z})^{-\tilde{l}_{i}}$. En sommant sur les $Z$ tels que $r_{0}(Z)=n$, et en posant $\kappa=\frac{r}{1+n}$, on déduit donc de (231) que pour tout $n>P$,

$$
\begin{aligned}
& \sup _{\tau \in[0, T]} \|\left(\mathcal{P}_{n}-\mathcal{P}_{n-1}\right)\left(\left(\int_{[5 \kappa, 1-5 \kappa[6}\left(\left(\theta_{x}^{b}\right)_{u_{1}, u_{2}, u_{3}}^{v_{1}, v_{2}, v_{3}}-\left(\theta_{x^{\prime}}^{b}\right)_{u_{1}, u_{2}, u_{3}}^{v_{1}, v_{3}}\right) d u_{1} \ldots d v_{3}\right)\right. \\
& e^{\tau \theta_{x}} u_{x, r} K_{x} e^{-\tau \theta_{x}}-e^{\tau \theta_{x}} u_{x, r} K_{x} e^{-\tau \theta_{x}} \\
& \left.\left(\int_{[5 \kappa, 1-5 \kappa[6}\left(\left(\theta_{x}^{b}\right)_{\hat{u}_{1}, \hat{u}_{2}, \hat{v}_{3}, \hat{u}_{3}}-\left(\theta_{x^{\prime}}^{b}\right)_{\hat{u}_{1}, \hat{u}_{2}, \hat{u}_{3}}^{\hat{v}_{1}, \hat{v}_{2}, \hat{v}_{3}}\right) d u_{1} \ldots d v_{3}\right)\right) \|_{\mathscr{L}\left(\mathscr{H}_{x, S}^{\natural, Q}, 7\right.}^{2} \\
& \quad \leq 2^{Q+8} 6^{6} p ! B e^{2(Q F-r) s}\left(C_{1} C_{2} C_{3} C_{4}^{\alpha}(n+1)^{-\frac{1}{2}}+C_{2}(n+1)^{-\frac{\alpha}{2}}\right)
\end{aligned}
$$

où $\left(\hat{u}_{1}, \hat{u}_{2}, \hat{u}_{3}, \hat{v}_{1}, \hat{v}_{2}, \hat{v}_{3}\right)$ est défini par (212). Le facteur $p$ ! est dû au fait que $\tilde{Z}$ détermine $Z$ à permutation près de $a_{1}, \ldots, a_{p}$. On rappelle que $\mathcal{P}_{n}-\mathcal{P}_{n-1}$ est le projecteur orthogonal défini par

$$
\left(\mathcal{P}_{n}-\mathcal{P}_{n-1}\right)\left(e_{S}\right)=e_{S} \text { si } d(x, S)=n \quad \text { et } \quad\left(\mathcal{P}_{n}-\mathcal{P}_{n-1}\right)\left(e_{S}\right)=0 \text { sinon. }
$$


D'après les lemmes 4.24 et 4.20 , les normes de $\mathscr{H}_{x, s}^{\natural, Q, 7}\left(\Delta_{p-1}\right)$ et $\mathscr{H}_{x, s}^{\rightarrow}\left(\Delta_{p}\right)$ sont équivalentes à celles de $\mathscr{H}_{x, s}\left(\Delta_{p-1}\right)$ et $\mathscr{H}_{x, s}\left(\Delta_{p}\right)$. Donc il existe

$$
C=C(\delta, K, N, Q, P, M, s, B, r, T)
$$

tel que pour tout $n>P$,

$$
\begin{aligned}
& \sup _{\tau \in[0, T]} \|\left(\mathcal{P}_{n}-\mathcal{P}_{n-1}\right)\left(\left(\int_{[5 \kappa, 1-5 \kappa[6}\left(\left(\theta_{x}^{b}\right)_{u_{1}, u_{2}, u_{3}}^{v_{1}, v_{2}, v_{3}}-\left(\theta_{x^{\prime}}^{b}\right)_{u_{1}, u_{2}, u_{3}}^{v_{1}, v_{2}, v_{3}}\right) d u_{1} \ldots d v_{3}\right)\right. \\
& \quad e^{\tau \theta_{x}} u_{x, r} K_{x} e^{-\tau \theta_{x}}-e^{\tau \theta_{x}} u_{x, r} K_{x} e^{-\tau \theta_{x}} \\
& \left.\quad\left(\int_{[5 \kappa, 1-5 \kappa[6}\left(\left(\theta_{x}^{b}\right)_{\hat{u}_{1}, \hat{u}_{2}, \hat{u}_{3}, \hat{u}_{3}}-\left(\theta_{x^{\prime}}^{b}\right)_{\hat{u}_{1}, \hat{u}_{2}, \hat{u}_{3}}^{\hat{v}_{1}, \hat{v}_{2}, \hat{v}_{3}}\right) d u_{1} \ldots d v_{3}\right)\right) \|_{\mathscr{L}\left(\mathcal{H}_{x, s}\left(\Delta_{p-1}\right), \mathscr{H}_{x, s}\left(\Delta_{p}\right)\right)}^{2} \\
& \leq C(n+1)^{-\frac{\alpha}{2}}
\end{aligned}
$$

où $\kappa=\frac{r}{1+n}$ et où $\left(\hat{u}_{1}, \hat{u}_{2}, \hat{u}_{3}, \hat{v}_{1}, \hat{v}_{2}, \hat{v}_{3}\right)$ est défini par (212).

Sous-lemme 4.91. Il existe $C=C(\delta, K, N, Q, P, M, s, B)$ tel que pour $p \in\{1, \ldots$, $\left.p_{\max }\right\}$ et $\left.\kappa \in\right] 0, \frac{1}{10}[$,

$$
\begin{aligned}
& \| \int_{[5 \kappa, 1-5 \kappa[6}\left(\left(\theta_{x}^{b}\right)_{\hat{u}_{1}, \hat{u}_{2}, \hat{u}_{3}}^{\hat{v}_{2}, \hat{v}_{3}}-\left(\theta_{x^{\prime}}^{b}\right)_{\hat{u}_{1}, \hat{u}_{2}, \hat{u}_{3}}^{\hat{v}_{1}, \hat{v}_{2}, \hat{v}_{3}}\right) d u_{1} \ldots d v_{3} \\
& \quad-\int_{[0,1[6}\left(\left(\theta_{x}^{b}\right)_{u_{1}, u_{2}, u_{3}}^{v_{1}, v_{2}, v_{3}}-\left(\theta_{x^{\prime}}^{b}\right)_{u_{1}, u_{2}, u_{3}}^{v_{1}, v_{2}, v_{3}}\right) d u_{1} \ldots d v_{3} \|_{\mathscr{L}\left(\mathscr{H}_{x, s}\left(\Delta_{p}\right), \mathscr{H}_{x, s}\left(\Delta_{p}\right)\right)}^{2} \leq C \kappa \\
& \qquad \int_{[5 \kappa, 1-5 \kappa[6}\left(\left(\theta_{x}^{b}\right)_{u_{1}, u_{2}, u_{3}, v_{3}}^{v_{1}, v_{3}}-\left(\theta_{x^{\prime}}^{b}\right)_{u_{1}, u_{2}, u_{3}}^{v_{1}, v_{2}, v_{3}}\right) d u_{1} \ldots d v_{3} \\
& \quad-\int_{[0,1[6}\left(\left(\theta_{x}^{b}\right)_{u_{1}, u_{2}, u_{3}}^{v_{1}, v_{3}, v_{3}}-\left(\theta_{x^{\prime}}^{b}\right)_{u_{1}, u_{2}, u_{3}}^{v_{1}, v_{2}, v_{3}}\right) d u_{1} \ldots d v_{3} \|_{\mathscr{L}\left(\mathscr{H}_{x, s}\left(\Delta_{p}\right), \mathscr{H}_{x, s}\left(\Delta_{p}\right)\right)}^{2} \leq C \kappa .
\end{aligned}
$$

Démonstration. Comme $\left\|\theta_{x}-\theta_{x^{\prime}}\right\|_{\mathscr{L}\left(\mathscr{H}_{x, s}\left(\Delta_{p}\right), \mathscr{H}_{x, s}\left(\Delta_{p}\right)\right)} \leq 1$, il suffit de montrer qu'il existe $C=C(\delta, K, N, Q, P, M, s, B)$ tel que pour $\tilde{x} \in\left\{x, x^{\prime}\right\}$ on ait

$$
\begin{aligned}
& \| \int_{[5 \kappa, 1-5 \kappa[6}\left(\left(\theta_{\tilde{x}}^{b}\right)_{\hat{u}_{1}, \hat{u}_{2}, \hat{u}_{3}}^{\hat{v}_{2}, \hat{v}_{3}}-\theta_{\tilde{x}}\right) d u_{1} \ldots d v_{3} \\
& \quad-\int_{\left[0,1\left[^{6}\right.\right.}\left(\left(\theta_{\tilde{x}}^{b}\right)_{u_{1}, u_{2}, u_{3}}^{v_{1}, v_{2}, v_{3}}-\theta_{\tilde{x}}\right) d u_{1} \ldots d v_{3} \|_{\mathscr{L}\left(\mathscr{H}_{x, s}\left(\Delta_{p}\right), \mathcal{H}_{x, s}\left(\Delta_{p}\right)\right)}^{2} \leq C \kappa
\end{aligned}
$$

et

$$
\left\|\int_{\left[0,1\left[{ }^{6} \backslash[5 \kappa, 1-5 \kappa[6\right.\right.}^{6}\left(\left(\theta_{\tilde{x}}^{b}\right)_{u_{1}, u_{2}, u_{3}}^{v_{1}, v_{2}, v_{3}}-\theta_{\tilde{x}}\right) d u_{1} \ldots d v_{3}\right\|_{\mathscr{L}\left(\mathscr{H}_{x, s}\left(\Delta_{p}\right), \mathscr{H}_{x, s}\left(\Delta_{p}\right)\right)}^{2} \leq C \kappa .
$$

Grâce à l'équivalence des normes de $\mathscr{H}_{x, s}\left(\Delta_{p}\right)$ et $\mathscr{H}_{x^{\prime}, s}\left(\Delta_{p}\right)$, il suffit de montrer (233) et (234) pour $\tilde{x}=x$. 
Soient $h_{\kappa, 1}$ et $h_{\kappa, 2}$ les fonctions mesurables sur $\left[0,1\left[^{6}\right.\right.$ définies de la manière suivante : $h_{\kappa, 1}$ est telle que pour toute fonction continue $f$ sur $[0,1]^{6}$ on a

$$
\begin{aligned}
& \int_{[5 \kappa, 1-5 \kappa[6} f\left(\hat{u}_{1}, \hat{u}_{2}, \hat{u}_{3}, \hat{v}_{1}, \hat{v}_{2}, \hat{v}_{3}\right) d u_{1} \ldots d v_{3} \\
& \quad-\int_{[0,1[6} f\left(u_{1}, u_{2}, u_{3}, v_{1}, v_{2}, v_{3}\right) d u_{1} \ldots d v_{3}=\int_{[0,1[6} f h_{\kappa, 1} d u_{1} \ldots d v_{3}
\end{aligned}
$$

et $h_{\kappa, 2}$ est la fonction caractéristique de $\left[0,1\left[{ }^{6} \backslash\left[5 \kappa, 1-5 \kappa\left[{ }^{6}\right.\right.\right.\right.$.

Il suffit donc de montrer qu'il existe $C=C(\delta, K, N, Q, P, M, s, B)$ tel que pour $i \in\{1,2\}$ on ait

$$
\begin{aligned}
& \| \int_{[0,1[6}\left(\left(\theta_{x}^{b}\right)_{u_{1}, u_{2}, u_{3}}^{v_{1}, v_{2}, v_{3}}-\theta_{x}\right) \\
& \quad h_{\kappa, i}\left(u_{1}, u_{2}, u_{3}, v_{1}, v_{2}, v_{3}\right) d u_{1} \ldots d v_{3} \|_{\mathscr{L}\left(\mathcal{H}_{x, s}\left(\Delta_{p}\right), \mathcal{H}_{x, s}\left(\Delta_{p}\right)\right)}^{2} \leq C \kappa .
\end{aligned}
$$

A partir de maintenant on reprend les notations $\Lambda_{Z}$ et $\left(\Lambda_{Z}\right)_{u_{1}, u_{2}, u_{3}}^{v_{1}, v_{2}, v_{3}}$ de la démonstration du lemme 4.49. Ces notations sont définies entre les formules (113) et (115).

Pour montrer (235) il suffit de montrer qu'il existe $C=C(\delta, K, N, Q, P, M, s, B)$ tel que pour $i \in\{1,2\}, k, m, l_{0}, \ldots, l_{m} \in \mathbb{N}, Z \in \bar{Y}_{x}^{p, k, m,\left(l_{0}, \ldots, l_{m}\right)}$ et $f \in \mathbb{C}^{\left(\Delta_{p}\right)}$ on ait

$$
\begin{aligned}
& \left|\xi_{Z}\left(\left(\int_{[0,1[6}\left(\left(\theta_{x}^{b}\right)_{u_{1}, u_{2}, u_{3}}^{v_{1}, v_{2}, v_{3}}-\theta_{x}\right) h_{\kappa, i}\left(u_{1}, u_{2}, u_{3}, v_{1}, v_{2}, v_{3}\right) d u_{1} \ldots d v_{3}\right)(f)\right)\right|^{2} \\
& \quad \leq C \kappa \sum_{\tilde{Z} \in \Lambda_{Z}}\left(r_{0}(\widetilde{Z})+1\right)^{-6}\left|\xi_{\tilde{Z}}(f)\right|^{2}
\end{aligned}
$$

Or (114) montre qu'il existe $C=C(\delta, K, N, Q, P, M, s, B)$ tel que

$$
\int_{[0,1[6}\left|\xi_{Z}\left(\left(\left(\theta_{x}^{b}\right)_{u_{1}, u_{2}, u_{3}}^{v_{1}, v_{2}, v_{3}}-\theta_{x}\right)(f)\right)\right|^{2} d u_{1} \ldots d v_{3} \leq C \sum_{\widetilde{Z} \in \Lambda_{Z}}\left(r_{0}(\tilde{Z})+1\right)^{-6}\left|\xi_{\widetilde{Z}}(f)\right|^{2} .
$$

D'autre part on vérifie facilement qu'il existe une constante $C$ telle que pour $i \in\{1,2\}$, on ait

$$
\int_{[0,1[6}\left|h_{\kappa, i}\left(u_{1}, u_{2}, u_{3}, v_{1}, v_{2}, v_{3}\right)\right|^{2} d u_{1} \ldots d v_{3} \leq C \kappa .
$$

Par Cauchy-Schwarz et grâce à (237) et (238), on obtient (236). Ceci termine la démonstration du sous-lemme 4.91 .

Fin de la démonstration $d u$ e). D’après le lemme 4.47, il existe

$$
C=C(\delta, K, N, Q, P, M, s, B, T)
$$


tel que

$$
\sup _{\tau \in[0, T]}\left\|e^{\tau \theta_{x}} u_{x, r} K_{x} e^{-\tau \theta_{x}}\right\|_{\mathscr{L}\left(\mathscr{H}_{x, s}\left(\Delta_{p-1}\right), \mathscr{H}_{x, s}\left(\Delta_{p}\right)\right)} \leq C .
$$

En combinant (232), le sous-lemme 4.91 et (239) on voit qu'il existe $C=$ $C(\delta, K, N, Q, P, M, s, B, r, T)$ tel que pour tout $n>P$,

$$
\begin{aligned}
& \sup _{\tau \in[0, T]}\left\|\left(\mathcal{P}_{n}-\mathcal{P}_{n-1}\right)\left[\left(\theta_{x}^{b}-\theta_{x^{\prime}}^{b}\right), e^{\tau \theta_{x}} u_{x, r} K_{x} e^{-\tau \theta_{x}}\right]\right\|_{\mathscr{L}\left(\mathscr{H}_{x, s}\left(\Delta_{p-1}\right), \mathscr{H}_{x, s}\left(\Delta_{p}\right)\right)}^{2} \\
& \quad \leq C(n+1)^{-\frac{\alpha}{2}} .
\end{aligned}
$$

Pour tout $i \in \mathbb{Z}$ soit $T_{i} \in \mathscr{L}\left(\mathscr{H}_{x, s}\left(\Delta_{p-1}\right), \mathscr{H}_{x, s}\left(\Delta_{p}\right)\right)$ défini par

$$
\begin{aligned}
\left(\mathcal{P}_{n}-\mathcal{P}_{n-1}\right) T_{i}\left(\mathcal{P}_{n^{\prime}}-\mathcal{P}_{n^{\prime}-1}\right)= & \left(\mathcal{P}_{n}-\mathcal{P}_{n-1}\right)\left[\left(\theta_{x}^{\mathrm{b}}-\theta_{x^{\prime}}^{\mathrm{b}}\right), e^{\tau \theta_{x}} u_{x, r} K_{x} e^{-\tau \theta_{x}}\right] \\
& \left(\mathcal{P}_{n^{\prime}}-\mathcal{P}_{n^{\prime}-1}\right) \operatorname{si} n^{\prime}-n=i
\end{aligned}
$$

et

$$
\left(\mathcal{P}_{n}-\mathcal{P}_{n-1}\right) T_{i}\left(\mathcal{P}_{n^{\prime}}-\mathcal{P}_{n^{\prime}-1}\right)=0 \text { sinon. }
$$

D'après le sous-lemme 4.42 (ou 4.80) on a $T_{i}=0$ sauf si $|i-r| \leq Q F$. Il est clair que $\left[\left(\theta_{x}^{\mathrm{b}}-\theta_{x^{\prime}}^{\mathrm{b}}\right), e^{\tau \theta_{x}} u_{x, r} K_{x} e^{-\tau \theta_{x}}\right]=\sum_{i} T_{i}$.

D'après (240) il existe donc $C=C(\delta, K, N, Q, P, M, s, B, r, T)$ tel que pour tout $i \in \mathbb{Z}$,

$$
\left\|\left(1-\mathcal{P}_{n}\right) T_{i}\right\|_{\mathscr{L}\left(\mathscr{H}_{X, s}\left(\Delta_{p-1}\right), \mathscr{H}_{x, s}\left(\Delta_{p}\right)\right)}^{2} \leq C(n+1)^{-\frac{\alpha}{2}} .
$$

En sommant sur $i \in\{r-Q F, \ldots, r+Q F\}$ on en déduit qu'il existe $C=$ $C(\delta, K, N, Q, P, M, s, B, r, T)$ tel que pour tout $n>P$,

$$
\left\|\left(1-\mathcal{P}_{n}\right)\left[\left(\theta_{x}^{\mathrm{b}}-\theta_{x^{\prime}}^{\mathrm{b}}\right), e^{\tau \theta_{x}} u_{x, r} K_{x} e^{-\tau \theta_{x}}\right]\right\|_{\mathscr{L}\left(\mathscr{H}_{x, s}\left(\Delta_{p-1}\right), \mathscr{H}_{x, s}\left(\Delta_{p}\right)\right)}^{2} \leq C(n+1)^{-\frac{\alpha}{2}} .
$$

Ceci termine la preuve de e) et donc celle du lemme 4.70 .

On a donc montré les lemmes 4.69 et 4.68 , et la proposition 4.4 qui était l'énoncé principal de ce paragraphe.

\section{Fin de l'homotopie}

Ce paragraphe n'offre guère d'intérêt parce qu'il recopie quasiment la fin du paragraphe 2.3.4 et le paragraphe 2.3.5 de [Laf02].

Lemme 5.1. Pour T assez grand

a) on a $\left\|e^{T \theta_{x}^{\mathrm{b}}} K_{x} e^{-T \theta_{x}^{\mathrm{b}}}\right\|_{\mathscr{L}\left(\oplus_{p=1}^{p_{\max }} \ell^{2}\left(\Delta_{p}\right)\right)} \leq \frac{1}{2}$, 
b) il existe $C$ tel que $\left\|e^{T \theta_{x}^{\mathrm{b}}} \widetilde{H}_{x} e^{-T \theta_{x}^{\mathrm{b}}}\right\|_{\mathscr{L}\left(\oplus_{p=1}^{p_{\max }} \ell^{2}\left(\Delta_{p}\right)\right)} \leq C$ et

$$
\left\|e^{T \theta_{x}^{\mathrm{b}}} u_{x, r} e^{-T \theta_{x}^{\mathrm{b}}}\right\|_{\mathscr{L}\left(\oplus_{p=1}^{p_{\max }} \ell^{2}\left(\Delta_{p}\right)\right)} \leq C 2^{-r} .
$$

Démonstration. Grâce à 2) a) du lemme 3.34, $K_{x}\left(e_{S}\right)$ est une combinaison de $e_{T}$ où $T$ vérifie $d(x, T) \leq d(x, S)-\left(Q \frac{N-6 \partial t a}{p_{\max }}-2 N-4 \delta\right)$, d'où $\rho_{x}^{b}(T) \leq \rho_{x}^{b}(S)-$ $\left(Q \frac{N-6 \delta}{p_{\max }}-3 N-11 \delta\right)$. On suppose $\left(Q \frac{N-6 \delta}{p_{\max }}-3 N-11 \delta\right) \geq 1$, ce qui est permis par $\left(H_{Q}\right)$. Ceci permet de montrer que la condition a) est réalisée pour $T$ assez grand et pour b) on utilise 1)a) et 3) de la proposition 3.37, la proposition 3.29 et le lemme 3.33.

On prend $T$ assez grand pour que les conditions du lemme 5.1 soient satisfaites. En particulier $e^{T \theta_{x}^{\mathrm{b}}}\left(\partial+J_{x} \partial J_{x}\right) e^{-T \theta_{x}^{\mathrm{b}}}$ est continu sur $\bigoplus_{p=1}^{p_{\max }} \ell^{2}\left(\Delta_{p}\right)$. On peut relier les espaces de Hilbert $\mathscr{H}_{x, s}$ et $\bigoplus_{p=1}^{p_{\max }} \ell^{2}\left(\Delta_{p}\right)$ par un champ continu d'espaces de Hilbert $\left(\mathscr{H}_{x, s, \alpha}\right)_{\alpha \in[0,1]}$, défini par $\|\cdot\|_{\mathscr{H}_{x, s, \alpha}}^{2}=\alpha\|\cdot\|_{\ell^{2}}+(1-\alpha)\|\cdot\|_{\mathscr{H}_{x, s}} \operatorname{sur} \bigoplus_{p=1}^{p_{\max }} \mathbb{C}^{\left(\Delta_{p}\right)}$.

Lemme 5.2. L'élément $\left(\left(\mathscr{H}_{x, s, \alpha}\right)_{\alpha \in[0,1]}, e^{T \theta_{x}^{b}}\left(\partial+J_{x} \partial J_{x}\right) e^{-T \theta_{x}^{b}}\right)$ appartient à $\mathrm{KK}_{G, 2 s \ell+C}(\mathbb{C}, \mathbb{C}[0,1])$ et réalise donc une homotopie entre

$$
\left(\mathscr{H}_{x, s}, e^{T \theta_{x}^{\mathrm{b}}}\left(\partial+J_{x} \partial J_{x}\right) e^{-T \theta_{x}^{\mathrm{b}}}\right)
$$

et

$$
\left(\bigoplus_{p=1}^{p_{\max }} \ell^{2}\left(\Delta_{p}\right), e^{T \theta_{x}^{\mathrm{b}}}\left(\partial+J_{x} \partial J_{x}\right) e^{-T \theta_{x}^{\mathrm{b}}}\right)
$$

Démonstration. La continuité de $e^{T \theta_{x}^{\mathrm{b}}}\left(\partial+J_{x} \partial J_{x}\right) e^{-T \theta_{x}^{\mathrm{b}}}$ résulte de la proposition 4.46, du lemme 5.1 et du fait que pour tout opérateur $U$ on a

$$
\|U\|_{\mathscr{L}\left(\mathscr{H}_{x, s, \alpha}\right)} \leq \max \left(\|U\|_{\mathscr{L}\left(\mathscr{H}_{x, s}\right)},\|U\|_{\mathscr{L}\left(\ell^{2}\right)}\right) .
$$

Pour montrer que $e^{T \theta_{x}^{\mathrm{b}}}\left(\partial+J_{x} \partial J_{x}\right) e^{-T \theta_{x}^{\mathrm{b}}}$ est équivariant à compact près, on voit, en reprenant l'argument de la démonstration de la proposition 4.68 et du lemme 4.69, qu'il suffit de montrer l'énoncé analogue à celui du lemme 4.70 obtenu en prenant $\tau=T$ mais en remplaçant les normes $\|\cdot\|_{\mathscr{H}_{x, s}}$ par les normes $\|\cdot\|_{\mathscr{H}_{x, s, \alpha}}$ et en prenant le supremum sur $\alpha$. Par exemple l'énoncé analogue à a) du lemme 4.70 est que

$$
\sup _{\alpha \in[0,1]}\left\|\left(1-\mathcal{P}_{n}\right) e^{T \theta_{x}}\left(h_{x}-h_{x^{\prime}}\right) e^{-T \theta_{x}}\right\|_{\mathscr{L}\left(\mathscr{H}_{x, s, \alpha}\left(\Delta_{p-1}\right), \mathscr{H}_{x, s, \alpha}\left(\Delta_{p}\right)\right)}
$$

tend vers 0 quand $n \rightarrow \infty$. Par (242) il suffit de montrer que

$$
\left\|\left(1-\mathcal{P}_{n}\right) e^{T \theta_{x}}\left(h_{x}-h_{x^{\prime}}\right) e^{-T \theta_{x}}\right\|_{\mathscr{L}\left(\ell^{2}\left(\Delta_{p-1}\right), \ell^{2}\left(\Delta_{p}\right)\right)} \rightarrow 0 \quad \text { quand } n \rightarrow \infty .
$$

Cela résulte du lemme 3.14 (et même simplement du fait, mentionné avant le lemme 3.14, que $\left\|\psi_{S, x}-\psi_{S, x^{\prime}}\right\|_{1}$ tend vers 0 en dehors des parties finies de $\Delta$ ). Il reste 
à montrer les énoncés analogues à (243) correspondant aux opérateurs de b), c), d) et e) du lemme 4.70. Pour cela on utilise de plus la propriété (9) et le lemme 3.45 (ou même l'énoncé plus faible qui est la propriété (22) avec $d^{b}$ au lieu de $d^{\prime \prime}$ ).

En fait (241) appartient à $\mathrm{KK}_{G, 0}(\mathbb{C}, \mathbb{C})$ (c'est-à-dire qu'il vérifie les mêmes conditions qu'un élément de $\mathrm{KK}_{G}(\mathbb{C}, \mathbb{C})$ sauf celle qui assure que l'opérateur est autoadjoint à compact près). De plus l'image de $(241)$ dans $\mathrm{KK}_{G, 2 s \ell+C}(\mathbb{C}, \mathbb{C})$ est égale à 1 d'après le lemme 5.2 et les propositions 4.4 et 4.5 . Pour terminer la preuve du théorème 1.5 , il suffit donc de montrer le lemme suivant, dont la preuve suit de très près le paragraphe 2.3.5 de [Laf02].

Lemme 5.3. L'élément (241) du lemme 5.2 est égal à l'image de $\gamma \in \mathrm{KK}_{G}(\mathbb{C}, \mathbb{C})$ dans $\mathrm{KK}_{G, 0}(\mathbb{C}, \mathbb{C})$.

Démonstration. D’après le a) du lemme 5.1 et comme

$$
K_{x}=\left(1-\partial h_{x}-h_{x} \partial\right)^{Q},
$$

on a

$$
\rho\left(e^{T \theta_{x}^{\mathrm{b}}}\left(1-\partial h_{x}-h_{x} \partial\right) e^{-T \theta_{x}^{\mathrm{b}}}\right) \leq 2^{-Q^{-1}}
$$

où $\rho$ désigne le rayon spectral dans $\mathscr{L}\left(\ell^{2}\left(\Delta_{p}\right)\right)$. On rappelle que

$$
H_{x}=h_{x}\left(\partial h_{x}+h_{x} \partial\right)^{-1} \text {. }
$$

Donc $e^{T \theta_{x}^{\mathrm{b}}} H_{x} e^{-T \theta_{x}^{\mathrm{b}}}$ agit continûment $\operatorname{sur} \bigoplus_{p=1}^{p_{\max }} \ell^{2}\left(\Delta_{p}\right)$.

Sous-lemme 5.4. L'élément

$$
\left(\bigoplus_{p=1}^{p_{\max }} \ell^{2}\left(\Delta_{p}\right), e^{T \theta_{x}^{\mathrm{b}}}\left(\partial+H_{x}\right) e^{-T \theta_{x}^{\mathrm{b}}}\right)
$$

appartient à $\mathrm{KK}_{G, 0}(\mathbb{C}, \mathbb{C})$ et (241) est homotope à (245).

Démonstration. La continuité de $e^{T \theta_{x}^{b}}\left(\partial+H_{x}\right) e^{-T \theta_{x}^{b}}$ a été justifiée avant le lemme et l'équivariance à compact près résulte de (243). On a $H_{x}^{2}=0$ car on a vu dans le lemme 3.16 que $h_{x}^{2}=0$. L'homotopie entre (241) et (245) résulte du lemme 1.4.1 de [Laf02].

On pose $D_{x}=\partial\left(\partial h_{x}+h_{x} \partial\right)^{-1}$.

Sous-lemme 5.5. L'élément

$$
\left(\bigoplus_{p=1}^{p_{\max }} \ell^{2}\left(\Delta_{p}\right), e^{T \theta_{x}^{\mathrm{b}}}\left(h_{x}+D_{x}\right) e^{-T \theta_{x}^{\mathrm{b}}}\right)
$$

appartient à $\mathrm{KK}_{G, 0}(\mathbb{C}, \mathbb{C})$ et (245) est homotope à (246). 
Démonstration. La continuité de $e^{T \theta_{x}^{\mathrm{b}}}\left(\partial+D_{x}\right) e^{-T \theta_{x}^{\mathrm{b}}}$ vient de nouveau de (244) et son équivariance à compact près résulte de (243). Pour réaliser l'homotopie entre (245) et (246) on procède comme dans le lemme 2.3.11 de [Laf02]. Pour $\alpha \in[0,1]$ on définit grâce à (244) et en utilisant la détermination principale du logarithme,

$$
H_{x}^{\alpha}=h_{x}\left(\partial h_{x}+h_{x} \partial\right)^{-\alpha} \quad \text { et } \quad D_{x}^{\alpha}=\partial\left(\partial h_{x}+h_{x} \partial\right)^{-\alpha} .
$$

Alors

$$
\left(\left(\bigoplus_{p=1}^{p_{\max }} \ell^{2}\left(\Delta_{p}\right)\right)[0,1],\left(e^{T \theta_{x}^{\mathrm{b}}}\left(H_{x}^{1-\alpha}+D_{x}^{\alpha}\right) e^{-T \theta_{x}^{\mathrm{b}}}\right)_{\alpha \in[0,1]}\right)
$$

appartient à $\mathrm{KK}_{G, 0}(\mathbb{C}, \mathbb{C}[0,1])$ et réalise une homotopie entre (245) et (246).

On rappelle maintenant quelques notations de [Laf02]. On note $\phi_{S, x}=\sqrt{\psi_{S, x}}$. On note $f, h^{\prime}$ les opérateurs de $\ell^{2}\left(\Delta_{p}\right)$ dans $\ell^{2}\left(\Delta_{p+1}\right)$ donnés par

$$
f\left(e_{S}\right)=\phi_{S, x} \wedge e_{S} \quad \text { et } \quad h^{\prime}\left(e_{S}\right)=\frac{1}{\left\|\phi_{S, x}^{3}\right\|_{1}} \psi_{S, x} \wedge e_{S}
$$

On note $g, g^{\prime}$ les opérateurs de $\ell^{2}\left(\Delta_{p}\right)$ dans $\ell^{2}\left(\Delta_{p-1}\right)$ donnés par

$$
\left.\left.g\left(e_{S}\right)=\phi_{S, x}\right\lrcorner e_{S} \quad \text { et } \quad g^{\prime}\left(e_{S}\right)=\frac{1}{\left\|\phi_{S, x}^{3}\right\|_{1}} \phi_{S, x}\right\lrcorner e_{S} .
$$

On note aussi $h=h_{x}$ pour être cohérent avec [Laf02].

Alors (246) est homotope à

$$
\left(\bigoplus_{p=1}^{p_{\max }} \ell^{2}\left(\Delta_{p}\right), e^{T \theta_{x}^{b}}\left(h+g^{\prime}\right) e^{-T \theta_{x}^{b}}\right)
$$

grâce au lemme 1.4.1 de [Laf02]. Puis (247) est homotope à

$$
\left(\bigoplus_{p=1}^{p_{\max }} \ell^{2}\left(\Delta_{p}\right), h+g^{\prime}\right)
$$

par l'homotopie évidente

$$
\left(\left(\bigoplus_{p=1}^{p_{\max }} \ell^{2}\left(\Delta_{p}\right)\right)[0, T],\left(e^{\tau \theta_{x}^{\mathrm{b}}}\left(h+g^{\prime}\right) e^{-\tau \theta_{x}^{\mathrm{b}}}\right)_{\tau \in[0, T]}\right) .
$$

On note que l'homotopie entre (246) et (248) correspond au lemme 2.3.12 de [Laf02].

Enfin (248) est homotope à $\left(\bigoplus_{p=1}^{p_{\max }} \ell^{2}\left(\Delta_{p}\right), h^{\prime}+g\right)$ puis à

$$
\left(\bigoplus_{p=1}^{p_{\max }} \ell^{2}\left(\Delta_{p}\right), f+g\right)
$$


(voir les lemmes 2.3.13 et 2.3.14 de [Laf02]).

Or (249) est égal à l'image de $\gamma \in \mathrm{KK}_{G}(\mathbb{C}, \mathbb{C})$ dans $\mathrm{KK}_{G, 0}(\mathbb{C}, \mathbb{C})$. On renvoie à [KS03] pour la construction complète de $\gamma$, qui est rappelée au début de la section 2 de [Laf02]. Il y a une toute petite subtilité due au fait que dans [KS03] la construction utilise des mesures $\psi_{S, x}^{K S}$ qui sont légèrement différentes des mesures $\psi_{S, x}$ que nous avons définies. Cependant on peut les relier par une homotopie $\alpha \mapsto(1-\alpha) \psi_{S, x}+$ $\alpha \psi_{S, x}^{K S}$. En effet grâce au lemme 3.7 et au lemme 6.3 de [KS03], pour tout $T$ tel que $e_{T}$ apparaisse dans $\left.\psi_{S, x} \wedge e_{S}, \psi_{S, x}\right\lrcorner e_{S}, \psi_{S, x}^{K S} \wedge e_{S}$ ou $\left.\psi_{S, x}^{K S}\right\lrcorner e_{S}$, on a $\psi_{S, x}=\psi_{T, x}$ et $\psi_{S, x}^{K S}=\psi_{T, x}^{K S}$. Donc les opérateurs analogues à $f, g, g^{\prime}, h, h^{\prime}$ construits à l'aide de $(1-\alpha) \psi_{S, x}+\alpha \psi_{S, x}^{K S}$ sont de carré nul. Ceci termine la preuve du lemme 5.3.

On a donc terminé la preuve de théorème 1.5 .

\section{Références}

[BC82] P. Baum and A. Connes, Geometric $K$-theory for Lie groups and foliations. Enseign. Math. (2) 46 (2000), 3-42. Zbl 0985.46042 MR 1769535

[BCH94] P. Baum, A. Connes, and N. Higson, Classifying space for proper actions and $K$ theory of group $C^{*}$-algebras. In $C^{*}$-algebras : 1943-1993 (San Antonio, TX, 1993), Contemp. Math. 167, Amer. Math. Soc., Providence, RI 1994, 240-291. Zbl 0830.46061 MR 1292018

[CDP90] M. Coornaert, T. Delzant, and A. Papadopoulos, Géométrie et théorie des groupes. Lecture Notes in Math. 1441, Springer-Verlag, Berlin 1990. Zbl 0727.20018 MR 1075994

[GdlH90] E. Ghys and P. de la Harpe (eds.), Sur les groupes hyperboliques d'après Mikhael Gromov. Progr. Math. 83, Birkhäuser, Boston 1990. and P. de la Harpe. Zbl 0731.20025 MR 1086648

[Gro87] M. Gromov, Hyperbolic groups. In Essays in group theory, Math. Sci. Res. Inst. Publ. 8, Springer-Verlag, New York 1987, 75-263. Zbl 0634.20015 MR 0919829

[Gro03] M. Gromov, Random walk in random groups. Geom. Funct. Anal. 13 (2003), 73-146. Zbl 1122.20021 MR 1978492

[HK01] N. Higson and G. Kasparov, $E$-theory and $K K$-theory for groups which act properly and isometrically on Hilbert space. Invent. Math. 144 (2001), 23-74. Zbl 0988.19003 MR 1821144

[HLS02] N. Higson, V. Lafforgue, and G. Skandalis, Counterexamples to the Baum-Connes conjecture. Geom. Funct. Anal. 12 (2002), 330-354. Zbl 1014.46043 MR 1911663

[Ju197] P. Julg, Remarks on the Baum-Connes conjecture and Kazhdan's property $T$. In Operator algebras and their applications (Waterloo, ON, 1994/1995), Fields Inst. Commun. 13, Amer. Math. Soc., Providence, RI, 1997, 145-153. Zbl 0884.19006 MR 1424959 
[Jul02] P. Julg, La conjecture de Baum-Connes à coefficients pour le groupe $\operatorname{Sp}(n, 1) . C$. R. Acad. Sci. Paris Sér. I Math. 334 (2002), 533-538. Zbl 1004.19003 MR 1903759

[JV84] P. Julg and A. Valette, $K$-theoretic amenability for $S L_{2}\left(\mathbb{Q}^{p}\right)$, and the action on the associated tree. J. Funct. Anal. 58 (1984), 194-215. Zbl 0559.46030 MR 757995

[Kas88] G. G. Kasparov, Equivariant $K K$-theory and the Novikov conjecture. Invent. Math. 91 (1988), 147-201. Zbl 0647.46053 MR 918241

[KS94] G. Kasparov and G. Skandalis, Groupes "boliques" et conjecture de Novikov. $C$. $R$. Acad. Sci. Paris Sér. I Math. 319 (1994), 815-820. Zbl 0839.19003 MR 1300949

[KS03] G. Kasparov and G. Skandalis, Groups acting properly on "bolic" spaces and the Novikov conjecture. Ann. of Math. (2) 158 (2003), 165-206. Zbl 1029.19003 MR 1998480

[Laf02] V. Lafforgue, $K$-théorie bivariante pour les algèbres de Banach et conjecture de Baum-Connes. Invent. Math. 149 (2002), 1-95. Zbl 1084.19003 MR 1914617

[Laf07] V. Lafforgue, $K$-théorie bivariante pour les algèbres de Banach, groupoïdes et conjecture de Baum-Connes. Avec un appendice d'Hervé Oyono-Oyono. J. Inst. Math. Jussieu 6 (2007), 415-451. Zbl 1135.19002 MR 2329760

[Laf08] V. Lafforgue, Un renforcement de la propriété (T). Duke Math. J. 143 (2008), 559-602. Zbl 1158.46049 MR 2423763

[Laf09] V. Lafforgue, Propriété (T) renforcée et conjecture de Baum-Connes. In Quanta of maths, Clay Math. Proc. 11, Amer. Math. Soc., Providence, RI, 2010, 323-345. Zbl 1216.19007 MR 2732057

[MY02] I. Mineyev and G. Yu, The Baum-Connes conjecture for hyperbolic groups. Invent. Math. 149 (2002), 97-122. Zbl 1038.20030 MR 1914618

[Oza08] N. Ozawa, Weak amenability of hyperbolic groups. Groups Geom. Dyn. 2 (2008), 271-280. Zbl 1147.43003 MR 2393183

[Tu99] J. L. Tu, La conjecture de Novikov pour les feuilletages hyperboliques. $K$-Theory 16 (1999), 129-184. Zbl 0932.19005 MR 1671260

[Vai05] J. Väisälä, Gromov hyperbolic spaces. Expo. Math. 23 (2005), 187-231. Zbl 1087.53039 MR 2164775

Received January 12, 2010; revised February 12, 2011

V. Lafforgue Laboratoire de Mathématiques - Analyse, Probabilités, Modélisation - Orléans (MAPMO), UMR CNRS 6628, Université d'Orléans, Rue de Chartres, B.P. 6759, 45067 Orléans cedex 2, France

E-mail: vlafforg@math.jussieu.fr 